Florian Blank, Claus Schäfer,

Dorothee Spannagel (Hg.)

Grundsicherung

weiterdenken

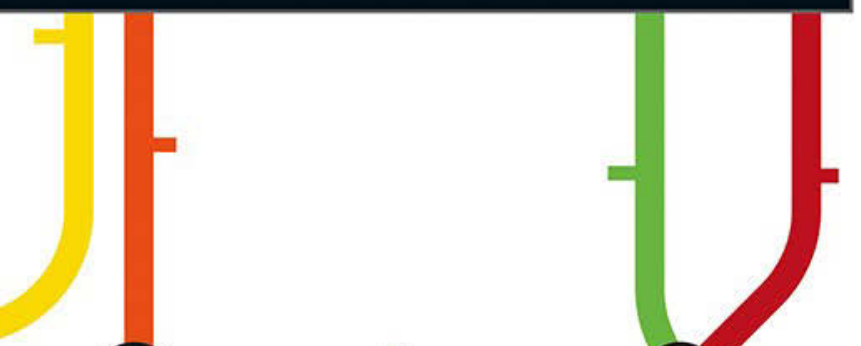


Florian Blank, Claus Schäfer, Dorothee Spannagel (Hg.)

Grundsicherung weiterdenken 


\section{Editorial}

Die Reihe Forschung aus der Hans-Böckler-Stiftung bietet einem breiten Leserkreis wissenschaftliche Expertise aus Forschungsprojekten, die die Hans-BöcklerStiftung gefördert hat. Die Hans-Böckler-Stiftung ist das Mitbestimmungs-, Forschungs- und Studienförderungswerk des DGB. Die Bände erscheinen in den drei Bereichen »Arbeit, Beschäftigung, Bildung«, »Transformationen im Wohlfahrtsstaat« und »Mitbestimmung und wirtschaftlicher Wandel«.

Forschung aus der Hans-Böckler-Stiftung bei transcript führt mit fortlaufender Zählung die bislang bei der edition sigma unter gleichem Namen erschienene Reihe weiter.

Die Reihe wird herausgegeben von der Hans-Böckler-Stiftung.

Florian Blank (Dr. phil.), geb. 1978, ist Leiter des Referats Sozialpolitik am Wirtschafts- und Sozialwissenschaftlichen Institut (WSI) der Hans-Böckler-Stiftung. Er beschäftigt sich $u$. a. mit aktuellen Problemen der Sozialversicherung und der Alterssicherung und geht Fragen der vergleichenden Wohlfahrtsstaatsforschung nach.

Claus Schäfer (Dr. rer. pol.), geb. 1948, hat von 1972 bis 2013 am Wirtschafts- und Sozialwissenschaftlichen Institut (WSI) der Hans-Böckler-Stiftung geforscht mit den Schwerpunkten Öffentliche Finanzen und vor allem Verteilung; dazu hat er insbesondere den jährlichen WSI-Verteilungsbericht begründet. Von 2009 bis 2013 war er Leiter der Abteilung WSI in der Hans-Böckler-Stiftung.

Dorothee Spannagel (Dr. rer. pol.), geb. 1980, ist Referatsleiterin für Verteilungsanalyse und Verteilungspolitik am Wirtschafts- und Sozialwissenschaftlichen Institut (WSI) der Hans-Böckler-Stiftung. Ihre Arbeitsschwerpunkte liegen auf dem Gebiet der sozialen Ungleichheit und der Verteilung von Armut und Reichtum. 
Florian Blank, Claus Schäfer, Dorothee Spannagel (Hg.)

\section{Grundsicherung weiterdenken}




\section{Bibliografische Information der Deutschen Nationalbibliothek}

Die Deutsche Nationalbibliothek verzeichnet diese Publikation in der Deutschen Nationalbibliografie; detaillierte bibliografische Daten sind im Internet über http://dnb.d-nb.de abrufbar.

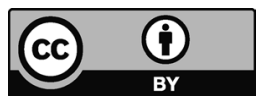

Dieses Werk ist lizenziert unter der Creative Commons Attribution 4.0 Lizenz (BY). Diese Lizenz erlaubt unter Voraussetzung der Namensnennung des Urhebers die Bearbeitung, Vervielfältigung und Verbreitung des Materials in jedem Format oder Medium für beliebige Zwecke, auch kommerziell. (Lizenztext:

https://creativecommons.org/licenses/by/4.o/deed.de)

Die Bedingungen der Creative-Commons-Lizenz gelten nur für Originalmaterial. Die Wiederverwendung von Material aus anderen Quellen (gekennzeichnet mit Quellenangabe) wie z.B. Schaubilder, Abbildungen, Fotos und Textauszüge erfordert ggf. weitere Nutzungsgenehmigungen durch den jeweiligen Rechteinhaber.

\section{Erschienen 2022 im transcript Verlag, Bielefeld \\ (ㄷ) Florian Blank, Claus Schäfer, Dorothee Spannagel (Hg.)}

Umschlaggestaltung: Maria Arndt, Bielefeld

Lektorat: Jürgen Hahnemann

Satz: Michael Rauscher, Bielefeld

Druck: Majuskel Medienproduktion GmbH, Wetzlar

Print-ISBN 978-3-8376-5594-0

PDF-ISBN 978-3-8394-5594-4

https://doi.org/10.14361/9783839455944

Buchreihen-ISSN: 2702-9255

Buchreihen-eISSN: 2702-9263

Gedruckt auf alterungsbeständigem Papier mit chlorfrei gebleichtem Zellstoff. Besuchen Sie uns im Internet: https://www.transcript-verlag.de Unsere aktuelle Vorschau finden Sie unter www.transcript-verlag.de/vorschaudownload 


\section{Inhalt}

\section{Einleitung}

Die Grundsicherung weiterdenken

Vorwort der Herausgeber:innen

Florian Blank, Claus Schäfer, Dorothee Spannagel | 11

Warum die herkömmliche Grundsicherung nicht reicht

Claus Schäfer | 17

\section{Teil I}

\section{Aktuelle Probleme der Grundsicherung}

Sozialversicherung und Grundsicherung im Spannungsverhältnis Umbrüche und Perspektiven des Systems der sozialen Sicherung Gerhard Bäcker | 37

Sicherung des Existenzminimums mit Regelleistungen Kritische Anmerkungen und Reformüberlegungen zu Hartz IV und zum Familienlastenausgleich

Irene Becker | 61

\section{Grundsicherung für alle Bedürftigen?}

Die Stellung von Ausländer:innen im System der Grundsicherung Florian Blank | 85 


\section{Teil II}

\section{Grundsicherung für Teilhabe durch Arbeit}

Grundsicherung "für Arbeitsuchende"

Arbeitsförderung, Teilhabe und Sozialer Arbeitsmarkt

Matthias Knuth | 109

Grundsicherung trotz Arbeit statt Integration durch Arbeit

Erwerbsarmut und das Problem der Aufstocker:innen

Dorothee Spannagel | 133

\section{Teil III}

Zum Konzept einer erweiterten Grundsicherung

Digitale Daseinsvorsorge

Voraussetzung für soziale und kulturelle Teilhabe im 21. Jahrhundert Tanja Klenk | 155

Öffentlicher Wohnungsbau als Element einer breit verstandenen Grundsicherung

Sebastian Dullien, Tom Krebs $\mid 171$

Daseinsvorsorge in der Gesundheitspolitik

Thomas Gerlinger | 189

Grundsicherung und rechtliche Beratung

Matthias Meißner | 209

Gesellschaftliche Teilhabe durch Bildung?

Ein sozial inklusives Bildungssystem braucht Alternativen zum Prinzip der Meritokratie

Ulf Banscherus | 227 


\section{Teil IV}

Zur Grundsicherung in Europa

Mindestsicherung im europäischen Vergleich

Thomas Bable 245

Grundsicherung und europäische Regulierung

Benjamin Benz | 265

Europäische Kindergarantie und Grundsicherung

Walter Hanesch $\mid 285$

\section{Fazit}

Den Sozialstaat komplett machen

Die Grundsicherung weiterdenken und "weiter denken"

Florian Blank, Claus Schäfer, Dorothee Spannagel | 307

Autorinnen und Autoren | 313 

Einleitung 



\title{
Die Grundsicherung weiterdenken
}

\author{
Vorwort der Herausgeber:innen
}

Florian Blank, Claus Schäfer, Dorothee Spannagel

Grundsicherung ist in Deutschland - und vielen anderen Sozialstaaten - sehr ambivalent zu bewerten, denn sie ist Versprechen und Enttäuschung zugleich.

Ein großes Versprechen ist Grundsicherung zunächst vom normativen Wortsinn her: »Hier seid ihr Bürger:innen gut aufgehoben. Ihr werdet gegen elementare Gefahren grundsätzlich geschützt." Im sozialwissenschaftlichen Duktus bedeutet das vor dem Hintergrund des grundgesetzlichen Sozialstaatsgebots: Grundsicherung sollte sowohl monetäre Armut als auch sozialen Ausschluss aufgrund von Risikolagen oder Chancenhürden vermeiden - zumindest nach unserem Verständnis. Dabei ist die Vermeidung von sozialem Ausschluss und gesellschaftlicher Benachteiligung - oder anders formuliert die Herstellung von sozialer Inklusion und gesellschaftlicher Teilhabe - in einer hoch entwickelten Gesellschaft wie der deutschen mindestens ebenso wichtig wie die Vermeidung von materieller Not.

Beide Umstände können im Lebensverlauf tendenziell jede und jeden treffen, und zwar in unterschiedlicher Ausprägung und durchaus mehr als einmal. Entsprechend breit muss das sozialstaatliche Konzept der Grundsicherung angelegt sein, wenn diese in der Lage sein soll, mit monetären Ersatzleistungen für mangelndes Erwerbseinkommen (z. B. bei Arbeitslosigkeit oder familiärer Pflegearbeit) und mit infrastrukturellen Angeboten an öffentlichen Gütern und Diensten (z. B. bei Bildungsdefiziten) wirksam gegenzuhalten.

Zur Wirksamkeit gehören nicht nur ausreichende Leistungshöhen und Angebotsstrukturen. Auch möglichst geringe Zugangsbarrieren zur Grundsicherung (wie etwa Anträge), soweit diese nicht ohnehin automatisch gewährt werden kann, und eine niedrigschwellige Möglichkeit zur Überprüfung administrativer Entscheidungen müssen mitgedacht werden. Denn viele individuelle oder familiäre Schutzbedürfnisse brauchen unserer Überzeugung nach keine 
Überprüfung durch einen formalen Prozess, sondern können a priori vorausgesetzt werden.

Mit dieser normativen Zielsetzung und einer entsprechenden Umsetzung sollte niemand davor zurückschrecken, im Fall des Falles Grundsicherung in Anspruch zu nehmen, oder gar Angst vor monetärem und sozialem Abstieg haben, schlimmstenfalls verbunden mit gesellschaftlicher Diskriminierung oder sogar Stigmatisierung. Sozialstaatliche Grundsicherung als im kollektiven Bewusstsein prominent verankerte öffentliche Einrichtung sollte vielmehr die beruhigende Gewissheit vermitteln, dass man darin individuell gut aufgefangen und gesellschaftlich akzeptiert wird. Solch eine wohlwollende allgemeine Akzeptanz könnte sich schließlich auch darauf gründen, dass das hier beschriebene System der Grundsicherung im Lebensverlauf von jeder und jedem als selbstverständlich genutzt wird.

In diesem idealen Sinne ist Grundsicherung also weit mehr als nur »Nothilfe« (z. B. bei Arbeitslosigkeit) für relativ wenige, sondern vielmehr ein System von Institutionen und Regeln für eine Basisförderung aller Bürger:innen und darüber hinaus auch vieler Menschen ohne deutsche Staatsbürgerschaft, die teils schon lange in Deutschland leben. Damit wird deutlich, dass der Ausgangspunkt unserer Überlegungen zwar die »klassische« Grundsicherung ist, wie sie im Sozialgesetzbuch und anderen Gesetzen behandelt wird, dass wir dieses Sicherungsnetz aber als Teil eines erweiterten Konzepts verstehen, in dessen Rahmen andere Bestandteile auf die herkömmliche Grundsicherung aufbauen und sie ergänzen.

Die Grundsicherung, wie wir sie bisher kennen, ist bei der beschriebenen Weiterentwicklung einer der legitimen Kerne eines ausgebauten Systems öffentlicher Sozialleistungen und Infrastrukturen, die als Ensemble wirken und aufeinander bezogen sind. Aus Gründen, die im vorliegenden Band dargestellt werden, sind Wissenschaft, Politik und Gesellschaft aufgerufen, Grundsicherung viel weiter zu denken als bisher üblich. Es geht darum, die Grenzen zwischen der Grundsicherung "im engeren Sinne « - also dem bisherigen System der sozialen Mindestsicherung - zu überschreiten und die Sozialversicherung ebenso wie andere sozialstaatliche Leistungen und die öffentlichen Infrastrukturen in die Überlegungen miteinzubeziehen.

Allerdings gilt heute noch: Die faktische Grundsicherung in Deutschland - und anderen Ländern - ist von einem solch weiten Verständnis noch weit entfernt, genau wie von dem skizzierten Versprechen samt dem damit verbundenen Anspruch, ja selbst von einer systematischen Weiterentwicklung im gegebenen System der öffentlichen Daseinsvorsorge. Dies zeigen auch die Inhal- 
te dieses Buches, die sich die Perspektive einer Grundsicherung »im weiteren Sinne« zu eigen machen.

Grundsicherung wird hierzulande im öffentlichen Diskurs allzu häufig mit dem System von Hartz IV gleichgesetzt oder in viel zu engem Zusammenhang damit gesehen. In diesem System befinden sich aber nur rund drei Viertel aller Leistungsempfänger:innen ${ }^{1}$ eines größeren Unterstützungssystems mit tendenziell gleichen Leistungsvoraussetzungen und Leistungshöhen - nämlich für Arbeitsuchende und ihre Familien, erwerbsgeminderte Jüngere, Ältere über 65 und (mit Abstrichen bei den Leistungen) Asylbewerber:innen. Immerhin enthalten die im Rahmen dieses Systems offiziellen Bezeichnungen »Grundsicherung für Arbeitsuchende« und »Grundsicherung im Alter und bei Erwerbsminderung« das Versprechen einer Grundsicherung.

Aber selbst dieses über 7 Millionen Menschen betreffende Gesamtsystem ist nach weitverbreiteter wissenschaftlicher und politischer Einschätzung materiell und sozial unzureichend: Vor allem die Regelsatzermittlung mit ihrem Bezug nur auf die untersten Einkommensschichten direkt oberhalb der Leistungsempfänger:innen - als vorgebliche Referenzgruppe für materielle und soziale "Standards« in der Gesellschaft - führt neben der heute nicht armutsfesten Regelsatzhöhe unvermeidlich auch zu sozialem Ausschluss, weil dabei die Einkommensentwicklung der darüberliegenden Einkommensschichten bzw. der Bevölkerungsmehrheit außer Acht bleibt, während die oberen Einkommensschichten den unteren mit wachsender Ungleichheit enteilen.

Die Vermeidung von sozialem Ausschluss - der als sozialwissenschaftlicher Begriff häufig genug eine beschönigende Bezeichnung für sozialen Absturz ist - wurde vom Gesetzgeber offenbar in der bestehenden »Grundsicherung für Arbeitsuchende« und für die anderen genannten Gruppen nie als wesentliche sozialstaatliche Herausforderung ernst genommen. Die problematischen $\mathrm{Zu}$ gangsbedingungen - allen voran die Anträge, die »Bedarfsgemeinschaft", die Bedürftigkeitsprüfung und die Vermögensprüfung - tun ein Übriges, um viele der eigentlich Anspruchsberechtigten von der Nutzung abzuhalten; immerhin gibt es eine Dunkelziffer von schätzungsweise bis zu 50 Prozent (so Bäcker/Naegele/ Bispinck 2020, S. 259).

Potenzielle wie tatsächliche Nutzer:innen insbesondere von Hartz IV haben begreiflicherweise Angst vor dem ökonomischen wie sozialen Absturz und Zweifel an einer künftigen Wiederherstellung ihrer sozialen Inklusion. Oft bleiben die Aussichten angesichts langjähriger ernüchternder Systemerfahrungen gering, durch staatliche Vermittlung in den regulären Arbeitsmarkt aus dem

1 | Vgl. Sozialpolitik-aktuell o.J. (Angaben für 2019). 
Hartz-IV-Bezug wieder herauszukommen, obwohl die Reintegration neben der materiellen und sozialen Absicherung ein Hauptziel des Systems war und ist.

Schließlich droht - unter Status-quo-Bedingungen bei der Rentenversicherung - die absehbar massiv wachsende Altersarmut aufgrund jahrelang niedriger Löhne und prekärer Arbeitsbedingungen das herkömmliche Grundsicherungssystem gänzlich infrage zu stellen, das dann viele Millionen Menschen zusätzlich mehr schlecht als recht »versorgen« müsste.

Nun wollen wir hier keine weitere ausführliche und kritische Auseinandersetzung mit Hartz IV und den anderen herkömmlichen Grundsicherungssystemen führen, wie es sie bereits in genügender Zahl gibt. Aber erwartungsgemäß kann auch dieser Band diese Frage nicht gänzlich ausklammern.

Deshalb widmet sich Teil I ausgewählten grundsätzlichen Problemen der »klassischen « Mindestsicherung in Deutschland. Diese Probleme betreffen das Spannungsverhältnis zwischen herkömmlicher Grundsicherung und gesetzlicher Sozialversicherung, den Umgang mit Ausländer:innen im System der Grundsicherung und vor allem zwei kritische Kernbestandteile: die Regelsatzermittlung und den Familienlastenausgleich, sprich die defizitäre Förderung von Kindern und Jugendlichen im Rahmen von Hartz IV vor dem Hintergrund anderer sozialer wie steuerlicher Förderinstrumente. Insbesondere der letztgenannte Beitrag von Irene Becker entwickelt auch die Grundprinzipien von Alternativen - in Form einer »inklusiven « Regelsatzermittlung und einer allumfassenden Kindergrundsicherung - und bildet damit eine inhaltliche Brücke zu den weiteren Beiträgen.

Die Beiträge in Teil II zur Grundsicherung für Teilhabe durch Arbeit beschäftigen sich ebenfalls mit etablierten Grundsicherungsmechanismen. Sie stellen aber das Problem der Erwerbsintegration in den Vordergrund, da in der deutschen Gesellschaft Erwerbstätigkeit nach wie vor ein zentraler Mechanismus zur Herstellung materieller und sozialer Teilhabe ist.

Teil III zum Konzept einer erweiterten Grundsicherung widmet sich dem eigentlichen Schwerpunkt dieser Veröffentlichung, nämlich der Betrachtung von Schutzbereichen im Leben aller Bürger:innen, die unserer Überzeugung nach mit wirksamen zusätzlichen Grundsicherungsleistungen ausgestattet werden müssen. Hier geht es um »Grundsicherung für die soziale und kulturelle Teilhabe « - also um diejenigen Leistungen und Infrastrukturen, die über finanzielle Transfers und die Teilhabe an Erwerbsarbeit hinausgehen.

Wir gehen davon aus, dass die »Grundsicherung im weiteren Sinne« auch mit den in Teil III behandelten Schutzbereichen nicht abschließend beschrieben ist. So lassen sich mindestens zwei weitere Bereiche hinzunehmen: 
- die Sicherung einer ausreichenden und günstigen Mobilität, solange diese aufgrund der Entfernung zwischen Wohnort und Arbeitsplatz bzw. Bildungseinrichtungen realistischerweise erforderlich ist, und

- die Sicherung der Vereinbarkeit von Erwerbsarbeit und Privatleben (Familie, Kinder, Pflege, Weiterbildung etc.) durch diverse rechtliche Ansprüche auf private Zeit während des gesamten Erwerbslebens samt dazugehöriger Lohnersatzleistungen - sozusagen eine »bedingungslose Grundzeit«.

Weitere Ausführungen zu diesen beiden Bereichen finden sich im Anschluss an dieses Vorwort im Beitrag von Claus Schäfer.

Die Beiträge in Teil IV zur Grundsicherung in Europa schließlich problematisieren für verschiedene Felder die vorhandenen Schutzdefizite und skizzieren Wege zu deren Abbau, sei es durch gänzlich neue Institutionen oder »nur" durch die Beseitigung von Widersprüchlichkeiten und Ungerechtigkeiten bei vorhandenen Schutzleistungen.

Die Coronapandemie hat ein weiteres Mal gezeigt, wie viele grundsätzliche Schutzvorkehrungen in weiteren Bereichen noch fehlen - für die ganze Bevölkerung z. B. medizinische Vorsorge und Versorgung, die nicht nur die gesellschaftlich alltäglichen Bedarfe deckt; für prekär bzw. nicht sozialversicherungspflichtig Beschäftigte ein den Normalbeschäftigten vergleichbares Kurzarbeitergeld; für (Solo-)Selbstständige ein adäquates »Ausfallgeld«.

Trotz vieler weiterhin offener Fragen sind wir überzeugt, dass sozialwissenschaftliche Indizien und Belege dem deutschen Sozialstaat im Zukunftsinteresse seiner Gesellschaft eine Handlungsstrategie nach der Maxime »Besser ist besser« nahelegen - im Gegensatz zu wieder laut werdenden Stimmen mit dem Impetus "Weniger ist mehr« (vgl. Schöb 2020), aber auch in Abgrenzung zu einem angeblich alle Probleme lösenden »bedingungslosen Grundeinkommen«.

Die Beiträge in diesem Buch sind unter anderem das Ergebnis von Debatten und Referaten im Arbeitskreis Grundsicherung am Wirtschafts- und Sozialwissenschaftlichen Institut (WSI) der Hans-Böckler-Stiftung, der seit 2018 besteht und seitdem mehrere themenbezogene Workshops mit internen und externen Teilnehmer:innen und Referent:innen aus verschiedenen Bereichen veranstaltet hat. Ihnen allen gilt unser Dank für ihre Anregungen, ebenso wie den hier versammelten Autor:innen, die teilweise schon im Arbeitskreis präsent waren. 


\section{Literatur}

Bäcker, G./Naegele, G./Bispinck, R. (2020): Sozialpolitik und soziale Lage in Deutschland, 6. Auflage, Wiesbaden.

Schöb, R. (2020): Der starke Sozialstaat. Weniger ist mehr, Frankfurt/New York.

Sozialpolitik-aktuell (o. J.): Grundsicherungssysteme in Deutschland, www.sozial politik-aktuell.de/files/sozialpolitik-aktuell/_Politikfelder/Sozialstaat/Daten sammlung/PDF-Dateien/abbIII200.pdf (Abruf am 23.6.2021). 


\section{Warum die herkömmliche Grundsicherung nicht reicht}

Claus Schäfer

\section{Einleitung}

Was Grundsicherung heute nicht ist, aber eigentlich sein sollte, haben die Herausgeber:innen dieses Bandes im Vorwort deutlich gemacht. Diese Sichtweise auf Grundsicherung in einem weiteren Sinn soll in diesem Beitrag durch die Problematisierung vorhandener Sicherungs- oder Schutzdefizite vertieft werden. Mit einer exemplarischen Betrachtung verschiedener politischer Handlungsmöglichkeiten wird gezeigt, in welche Richtungen ein weiteres Verständnis von Grundsicherung entwickelt werden kann, damit diese weit mehr und deutlich bessere staatliche Geldleistungen sowie öffentliche Infrastrukturangebote als beim herkömmlichen Verständnis enthält.

Im Rahmen dieser Problematisierung werden einige prominente Lücken der herkömmlichen Grundsicherung wie auch der Sozialversicherung mit der Skizzierung von eigentlich notwendigen umfassenderen Sicherungs- und Teilhaberechten konfrontiert. Die verwendeten Beispiele richten den Blick bei Kindern und Jugendlichen vor allem auf regionale und soziale Mobilität, bei Erwachsenen auf ausreichend Geld und Zeit innerhalb wie außerhalb der Erwerbstätigkeit.

Stärker ins Detail gehen schließlich die nachfolgenden Buchbeiträge, die auch auf mögliche Wege zum Defizitabbau hinweisen. Sie alle verdeutlichen, dass sich eine Reform der bisherigen Grundsicherung nicht auf eine "einfache" (und dennoch politisch schwer umsetzbare) Forderung nach höheren Regelleistungen im Sozialgesetzbuch beschränken kann.

\section{Grundsicherung (und Sozialversicherung) im defizitären Status quo}

Im offiziellen Sprachgebrauch der staatlichen Sozialpolitik werden die Begriffe Grundsicherung und Mindestsicherung oft nebeneinander gebraucht, wobei ihre Abgrenzung untereinander wie zu anderen sozialstaatlichen Leistungen nicht 
immer trennscharf ist. Zum Beispiel zählen die Statistischen Ämter des Bundes und der Länder (2019) in ihrer Berichterstattung in Anlehnung an das Sozialgesetzbuch (SGB) vor allem die zahlenmäßig bedeutende »Grundsicherung für Arbeitsuchende" und deren Familien bzw. Bedarfsgemeinschaften (sprich Arbeitslosengeld II und Sozialgeld bzw. Hartz IV) zu den Systemen der »Sozialen Mindestsicherung«, außerdem die öffentlichen »Sozialhilfen« für eingeschränkt oder generell nicht erwerbsfähige Personen, altersarme Personen und Asylbewerber:innen. Unter »Weiteren Sozialleistungen« der Mindestsicherung werden dort aufgeführt: Leistungen für Kriegsopfer, Wohngeld, Kinderzuschlag, Leistungen der Bundesausbildungsförderung (BAföG) und »Besondere Leistungen der Sozialhilfe« (nach SGB XII).

Die Leistungen für Hartz IV und Sozialhilfe gehen nach den letzten verfügbaren Zahlen an fast 7 Millionen tatsächliche Leistungsempfänger:innen in Deutschland; inklusive Kriegsopferfürsorge, Wohngeld, Kinderzuschlag und BAföG sogar an etwa 10 Millionen Personen oder rund 12 Prozent der Gesamtbevölkerung (wobei nur eine niedrige Zahl an Mehrfachempfänger:innen dieser Leistungen unterstellt werden kann). ${ }^{1}$ Doch viel zu wenig ist gesellschaftlich bewusst und wird problematisiert, dass die Zahl der maximal möglichen Leistungsbezieher:innen deutlich höher liegt, weil ein Teil der prinzipiell Berechtigten keinen Leistungsantrag stellt bzw. seine Ansprüche nicht kennt oder wegen bürokratischer Antragshürden oder sozialer Scham nicht ausschöptt (vgl. z.B. Friedrichsen/Schmacker 2019).

Zählt man diese Dunkelziffer zu den tatsächlichen Leistungsempfänger:innen hinzu, so sind von der herkömmlichen Grundsicherung nach verschiedenen vorliegenden Schätzungen eigentlich 16 bis 18 Millionen Personen oder mehr als 20 Prozent der Bevölkerung betroffen. ${ }^{2}$ Neben der heutigen Dunkelziffer droht zukünttig eine weitere Verdunkelungstendenz den Problemkreis deutlich

1 | Vgl. das Statistikportal und die Sozialberichterstattung der Statistischen Ämter des Bundes und der Länder: www.statistikportal.de; www.amtliche-sozialberichterstat tung.de.

2 | Hochgerechnet mit der in der Literatur häufig genannten Dunkelziffer-Spannbreite von 40 bis 50 Prozent; vgl. Bäcker/Naegele/Bispinck 2020, S. 259. Für das Untersystem „Grundsicherung im Alter« speziell wird die Dunkelziffer auf bis zu 60 Prozent der Antragsberechtigten veranschlagt; der dadurch entstehende Verzicht von Rentnerhaushalten auf eigentlich zustehende Transferleistung wird mit durchschnittlich 200 Euro im Monat oder ungefähr 30 Prozent ihres Nettoeinkommens beziffert (vgl. Hahn 2019).

Bei der ergänzenden Hartz-IV-Leistung »Bildungs- und Teilhabepaket« für Kinder bis 14 Jahre betrug die Nicht-Inanspruchnahme im Mai 2019 sogar 85 Prozent (Paritätischer Gesamtverband 2019); auch eine Nachbesserung im Herbst 2019 durch das »Star- 
zu vergrößern, weil nach vielen Prognosen aufgrund des sinkenden Niveaus der gesetzlichen Renten eine erhebliche Zunahme der Altersarmut - und entsprechend aufstockender Grundsicherungsleistungen - absehbar ist. ${ }^{3}$ Perspektivisch sind deswegen über 20 Millionen Personen als Bedürftigtenkreis im Rahmen der oben genannten Systeme wahrscheinlich, was diese tendenziell von Ausnahmesystemen zu »Regelsystemen« für viele Menschen machen würde.

Allein die hohe Anzahl dieser tatsächlichen und erst recht der »verhinderten« Grundsicherungsempfänger:innen ist eigentlich schon problematisch genug, weil sie dem Sozialstaat und der Wohlstandsverteilung in Deutschland kein gutes Zeugnis ausstellt. Doch noch bedenklicher ist, dass die Leistungen selbst und ihre Anspruchsbedingungen seit Langem aus Sicht von großen Teilen der Sozialwissenschaft, der Wohlfahrtsverbände und der Politik als unzulänglich gelten.

Ein eindeutiges Indiz dafür ist, dass parallel zum jahrelangen Sinken der Zahl faktischer Grundsicherungsempfänger:innen (Statistisches Bundesamt 2020b) die messbare Armutsquote in der deutschen Bevölkerung im Zeitverlauf leicht steigt - auf 15,9 Prozent oder rund 14 Millionen Personen in 2019 (Statistisches Bundesamt 2020a). In dieser Diskrepanz spiegelt sich allerdings nicht nur die Dunkelziffer; auch die individuellen Leistungen der Grundsicherung sind nicht armutsfest.

Wäre laut Paritätischem Gesamtverband (2020b) aktuell ein Regelsatz für alleinlebende Erwachsene von 644 Euro nötig, um Einkommensarmut zu vermeiden, werden ab Januar 2021 aufgrund der letzten offiziellen Erhöhung der entsprechenden Leistungen in Hartz IV und Sozialhilfe lediglich 446 Euro gezahlt. Das heißt, die pauschalierten Transfers für den laufenden Lebensunterhalt von Erwachsenen und Kindern in Hartz IV und Sozialhilfe (»Regelsätze«) fallen vor allem aufgrund einer problematischen Berechnungsmethode ( $\gg$ Regelbedarfsermittlung«) seit Langem entschieden zu niedrig aus. ${ }^{4}$

ke-Familien-Gesetz« hat entsprechend einer erneuten Erhebung im April 2020 diese Quote nicht verändert (Paritätischer Gesamtverband 2020a).

3 | Schon in der Vergangenheit stieg die Armuts(gefährdungs)quote der über 64-Jährigen innerhalb der Gesamtbevölkerung am stärksten - seit 2005 um 4,7 Prozentpunkte auf 15,7 Prozent -, während die der Gesamtbevölkerung "nur« um 1,2 Prozentpunkte auf 15,9 Prozent zunahm (Statistisches Bundesamt 2020a). Vorboten bzw. Frühindikatoren einer weiter zunehmenden Altersarmut sind die aktuell bereits stark zunehmende Verschuldung und die Überschuldung von Älteren (laut Creditreform-Schuldneratlas 2020; vgl. WirtschaftsWoche 2020).

4 | Haben wegen dieser Methode viele Wohlfahrtsverbände und andere Institutionen noch im Frühjahr 2020 zum wiederholten Mal in einem gemeinsamen Brief an die 
Fragwürdig sind ebenfalls die bei Regelverstößen von Leistungsempfänger:innen verhängten Sanktionen bei Bezug von Hartz IV, sprich Leistungskürzungen, unter anderem weil sie mit der höchstrichterlichen Rechtsprechung zum Existenzminimum schwer vereinbar sind. ${ }^{5}$ Schließlich funktioniert im Rahmen von Hartz IV die (Wieder-)Integration der immer noch vielen Langzeitarbeitslosen in den Arbeitsmarkt durch das gegenwärtige Vermittlungssystem mehr schlecht als recht, obwohl dies neben der materiellen Sicherung durch das System das zweite Hauptziel war und ist. ${ }^{6}$ Aber auch die Leistungen für Wohngeld, Kinderzuschlag und BAföG sind über Jahre hinweg bezüglich Preissteigerungen und Bedarfen nicht ausreichend dynamisiert worden und haben so den Berechtigtenkreis schrumpfen lassen.

Nun wäre für die Funktion dieser herkömmlichen Grundsicherung schon viel gewonnen - insbesondere zur Verringerung ihrer Fallzahlen - wenn andere sozialstaatliche Unterstützungssysteme, allen voran die gesetzliche Sozialversicherung, besser aufgestellt wären. Diese zielen teilweise ebenso auf die Sicherung der Grundbedürfnisse nach materieller Sicherheit und sozialen Dienstleistungen ab, auch wenn dies im Fall der Lohnersatzleistungen hauptsächlich nach anderen Kriterien erfolgt - insbesondere nach dem Äquivalenzprinzip, also der Maßgabe dass spätere Leistungen tendenziell früheren Beiträgen entsprechen und nicht dem Grad der Bedürftigkeit. Jedenfalls sollten die Leistungen der Sozialversicherung nach Möglichkeit so hoch sein, dass sie den Betroffenen einen zusätzlichen oder gar alternativen Rückgriff auf die herkömmlichen Grundsicherungsleistungen als »Rückfallsystemen« ersparen.

Dies gilt in erster Linie für die gesetzliche Rentenversicherung, in die nicht umsonst nach langen politischen Debatten eine teilweise bedarfsorientierte "Grundrente« eingezogen ist mit dem Motiv, dass auch nach dem lebenslangen Bezug von Niedriglöhnen oder auch nach wenigen Jahren Erwerbsarbeit oder vielen Jahren Teilzeit eine »lebensleistungsgerechte« und armutsfeste Rente zu

Bundesregierung »kein Weiter-so« gefordert (Nationale Armutskonferenz 2020), so mussten sie ihre Änderungshoffnungen nach der im Herbst 2020 vom Bundestag beschlossenen Novellierung des »Regelbedarfsermittlungsgesetzes« erneut aufgeben (vgl. Deutscher Bundestag 2020 und die darin enthaltenen Verbands-Stellungnahmen).

5 | Sogar der UN-Ausschuss für wirtschaftliche, soziale und kulturelle Rechte (2018) hat im Rahmen des Prüfverfahrens zum völkerrechtlich verbindlichen UN-Sozialpakt die Leistungshöhe und die Sanktionen in der deutschen Grundsicherung kritisiert.

6 | Das inzwischen eingeleitete Vorhaben der aktuellen Großen Koalition, einen »sozialen Arbeitsmarkt « zu schaffen, ist zwar ein weiteres indirektes Eingeständnis dieses Vermittlungsversagens, bleibt aber den Nachweis seiner Wirksamkeit noch - oder wieder? - schuldig (siehe den Beitrag von Matthias Knuth in diesem Band). 
erzielen ist. Es gilt aber ohne Zweifel, dass diese Grundrente nur relativ wenige zukünftige Rentner:innen begünstigt und den Großteil der erwartbaren Altersarmut nicht wird verhindern können. ${ }^{7}$

In der gesetzlichen Krankenversicherung ist zwar die Grundsicherung im Rahmen des systemimmanenten Solidarprinzips generell enthalten, weil Hartz-IVEmpfänger:innen automatisch und ohne Beitrag krankenversichert sind und weil verheiratete Partner:innen und Kinder von (auch ehemals) erwerbstätigen Beitragspflichtigen unabhängig von deren einkommensabhängiger Beitragshöhe immer ohne eigenen Beitrag mitversichert sind. Die gesetzliche Krankenversicherung steht auch Selbstständigen und ihren Familien offen (in Hamburg seit einiger Zeit auch Beamt:innen), und das auch schon vor Einführung der gesetzlichen Verpflichtung zum Abschluss einer Krankenversicherung für jedermann.

Aber Selbstständige mussten in der gesetzlichen Krankenversicherung jahrelang auf Basis eines fiktiven pauschalen »Grundeinkommens« einen entsprechenden »Grundbeitrag« zahlen, dessen Höhe viele Hunderttausend Selbstständige faktisch vom Beitritt und damit vom entsprechenden Schutzschirm abgehalten hat, bis der Gesetzgeber zuletzt nach großer Kritik beide Zugangshürden deutlich senken musste. Ob die niedrigeren Hürden nun von allen bedürftigen Selbstständigen überwunden werden, ist noch nicht bekannt. Immerhin trägt die Senkung der Tatsache Rechnung, dass unter den Selbstständigen - insbesondere den Solo-Selbstständigen, die teilweise immer schon prekär erwerbstätig waren - die Prekarität in der letzten Zeit zahlenmäßig merklich zugenommen hat, unter anderem durch Clickworker, Paketbot:innen und weitere neue, von der Digitalisierung angestoßene Tätigkeiten.

In der Arbeitslosenversicherung schließlich entscheidet die Dauer des Leistungsbezugs und die Höhe der Leistung, wann die ehemals abhängig beschäftigten Leistungsempfänger:innen ergänzend oder vollständig an das Grundsicherungssystem Hartz IV »weitergereicht« werden. Selbstständige dagegen müssen im Risikofall ohne abfedernden »Umweg" über die Arbeitslosenversicherung sofort auf das »Rückfallsystem« Hartz IV zugreifen - wenn sie sich nicht, wie schon geschildert, durch die Zutrittsbedingungen abschrecken lassen. Die aufgrund der Coronakrise nach und nach beschlossenen Ad-hoc-Hilfen der Politik

7 | Die Grundrente schließt nicht nur a priori Personen mit weniger als 35 bzw. 33 Arbeitsjahren vom Bezug aus; nach Auskunft des Bundesarbeitsministeriums haben auch Rentner:innen mit durchschnittlich 0,5 oder weniger Entgeltpunkten, also höchstens der Hälfte des Durchschnittseinkommens, trotz 35 Beitragsjahren nur eine Nettorente unterhalb des aktuellen Bruttobedarfs der Grundsicherung im Alter bzw. der Sozialhilfe (FAZ 2020). 
für Selbstständige signalisieren aktuell überdeutlich, dass es hier ein erhebliches institutionelles Sicherungsdefizit gibt.

Es ist also nicht zu übersehen, dass es auch Übergangsprobleme zwischen Sozialversicherungs- und Grundsicherungssystemen gibt, die entweder in einem der beiden Systeme oder gar auf beiden Seiten gleichzeitig und adäquat gelöst werden müssten, wenn Grundsicherung besser, sprich effektiver und inklusiver wirken soll.

\section{Erweiterte Teilhabe durch Aufstiegs- und Mobilitätsrechte}

Unabhängig von diesen Übergangsproblemen sind viele Netze der herkömmlichen Grundsicherung viel zu weitmaschig, löchrig oder nicht zielgenau, um einen ausreichenden Schutz zu gewährleisten. Dies soll hier am Beispiel von Kindern und Jugendlichen verdeutlicht werden, deren Entfaltungspotenziale am größten sind, aber nur mit der Unterstützung oder dem Anschub einer entsprechend spezifischen Grundsicherung zum Vorteil des Individuums wie der Gesellschaft ausgeschöpft werden können. Dies gelänge für Kinder und Jugendliche insbesondere durch eine Grundsicherung für soziale und regionale Mobilität - die derzeit beide mehr oder weniger defizitär ausfallen.

Soziale Mobilität soll vor allem über Bildung und das entsprechende Bildungssystem in Deutschland vermittelt werden. Doch die damit verbundenen Chancen werden nicht gleichmäßig verteilt und können auch nicht immer optimal genutzt werden. So ist das sogenannte Bildungspaket zugunsten von Kindern in Hartz-IV-Familien von der Höhe her völlig unbefriedigend und wegen der Antragshürden weitgehend unzugänglich, sodass es - wie schon gesagt - von nur 15 Prozent der Anspruchsberechtigten genutzt wird.

Auch das Angebot von Krippen- und Kindergartenplätzen ist immer noch unzureichend oder wird für einkommensschwache Haushalte durch Zutrittsbarrieren, sprich Gebühren beeinträchtigt. In den Schulen gibt es zwar ausreichend Lernplätze, aber gleichzeitig viele Qualitätsmängel - zuallererst eine zu geringe Zahl an Lehrkräften -, was das Lernen wiederum vor allem für einkommensbedingt oder anderweitig benachteiligte Schüler:innen erschwert. Ähnliches gilt für Universitäten und andere Hochschulen, wo teilweise sogar Plätze im Masterstudiengang fehlen.

Zwar verlangen allgemeinbildende Schulen und Hochschulen kaum Gebühren, aber für die Ausbildung in vielen Gesundheitsberufen muss z.B. immer noch über mehrere Jahre ein relativ hohes Schulgeld seitens der Auszubildenden gezahlt werden. Das Berufsziel Ärzt:in kann "gebührenbefreit« verfolgt wer- 
den, aber das Ziel Hebamme bzw. Entbindungspfleger oder Physiotherapeut:in nicht, obwohl alle Gesundheitsberufe nicht erst seit Corona als »systemrelevant« anzusehen sind.

Schließlich ist der Zutritt zu bildungsnahen Einrichtungen wie Museen für Kinder und Jugendliche trotz ermäßigter Eintrittspreise immer noch relativ teuer, während er z. B. in Frankreich kostenlos ist (dies gilt neuerdings auch für Studierende der Frankfurter Hochschulen in den städtischen Museen) und in Großbritannien bis vor Kurzem prinzipiell - auch für Erwachsene - kostenlos war.

Ähnlich ungereimt sieht die Grundsicherung zur regionalen Mobilität von Kindern und Jugendlichen aus, die bei vielen und häufigen Wegen zur öffentlichen Infrastruktur, aber auch bei privaten Zielen gefragt ist. Insbesondere zu ihren Schul- und Ausbildungsstätten müssen Kinder und Jugendliche teilweise weite Wege zurücklegen, was vor allem für regional nicht gleichmäßig verteilte weiterbildende Einrichtungen gilt und auch im Zusammenhang mit der wachsenden Berufspendlerzahl in Deutschland bzw. dem wachsenden Umzugsdruck ins preiswertere stadtnahe Umland steht.

Das zunehmende Gefälle zwischen Land und Stadt bei Infrastruktureinrichtungen erzeugt ebenfalls einen höheren Mobilitätsdruck. Gemessen daran ist das Netz des öffentlichen Verkehrs häufig zu dünn, die Taktzeiten zu lang, Busse und Bahnen zu alt etc. Zugleich muss für die Mobilität von Kindern und Jugendlichen trotz vorhandener, meist länderspezifisch unterschiedlicher ErmäBigungstarife im öffentlichen Verkehr relativ viel gezahlt werden.

Im Vergleich dazu fallen für abhängig Beschäftigte die steuerlich absetzbaren Fahrtkosten und die zumindest verbilligten, manchmal sogar allein von den Arbeitgebern bezahlten Jobtickets - meist in größeren und großen Unternehmen - relativ großzügig aus und führen zu weit höheren Belastungen der Allgemeinheit. Die Ticketkosten vermindern die Steuern aufseiten von Arbeitgeber wie Arbeitnehmer:in, je nach Finanzierungsgestaltung des Tickets können zusätzlich Sozialversicherungsbeiträge gespart werden, und unter bestimmten Bedingungen sind Steuervorteile durch Jobticket und Pendlerpauschale kombinierbar.

Passt es zusammen, wenn der Staat allein den 11,6 Millionen Nutzer:innen der steuerlichen Pendlerpauschale 2019 laut Bundesfinanzministerium im Durchschnitt 440 Euro Ersparnis »spendiert« (vgl. ADAC 2021), dagegen aber Schüler:innen und Senior:innen in Hessen und anderen Bundesländern für ein - schon verbilligtes - ÖPNV-Jahresticket immer noch 365 Euro »abverlangt«? Schwer verständlich ist erst recht, wenn zwar Landesbeschäftigte in Hessen seit Kurzem generell kostenlos und unabhängig von ihrem jeweiligen Einkommen den öffentlichen Nahverkehr nutzen können, aber Beschäftigte in Privatfirmen 
mit öffentlichen Aufgaben nicht und alle anderen Beschäftigten schon gar nicht. Dabei tragen Letztere mit ihren Steuern zum Haushalt des Arbeitsgebers Hessen bei und finanzieren den Vorteil des öffentlichen Personals in Hessen mit.

Politische Reformen, die die aufgezeigten Defizite und Widersprüche vermeiden, zielen im Sinne des hier vertretenen erweiterten Begriffs von Grundsicherung wohlgemerkt nicht (nur) auf die Bekämpfung der Armut von relativ wenigen, sondern auf die Bereitstellung von Transfers und Infrastrukturen für viele bzw. tendenziell alle.

\section{Erweiterte Teilhabe durch Zeit- und Geldrechte}

Wurde bisher am Beispiel von einzelnen Bevölkerungsgruppen und deren Lebenslage argumentiert, so lassen sich mit Blick auf den Lebensverlauf auch Schutzdefizite für alle Bevölkerungsmitglieder identifizieren, die hier am Beispiel von zwei zentralen Ressourcen für die lebenslange individuelle Entfaltung jedes Gesellschaftsmitglieds benannt werden sollen: der Grundsicherung für individuelle Zeit und der Grundsicherung für individuelles Einkommen in einer von Erwerbsarbeit geprägten Gesellschaft.

Kindern und Jugendlichen wird individuelle Zeit zur persönlichen Entwicklung nach den historischen Erfahrungen der Kinderarbeit und nach wissenschaftlichen Erkenntnissen zur Bildungsnotwendigkeit nicht nur durch öffentliche (und private) Schulen angeboten, sondern durch die Schulpflicht auch besonders geschützt. Aber warum gibt es bis heute trotz des Wissens um die besonders wichtigen »frühen Jahre« von Kindern und deren Potenzial für die gesellschaftliche Zukunft keine Kindergartenpflicht, wie sie z. B. in Frankreich 2019 für ab Dreijährige eingeführt wurde?

Auch in der späteren Erwerbsphase wird die individuelle Zeit jenseits der Erwerbszeit durch die prinzipielle Beschränkung der Erwerbstätigkeit auf den Achtstundentag und weitere Regularien im Rahmen der gesetzlichen Arbeitszeitordnung besonders geschützt. Es steht allerdings außer Frage, dass die $\mathrm{Zu}$ verlässigkeit dieser Regularien angesichts der ausfransenden Arbeitszeit über acht Stunden hinaus und der »Verdichtung« von Arbeitszeit unterhalb von acht Stunden kritisch zu bewerten ist.

Dies gilt angesichts vieler bezahlter und unbezahlter Überstunden, wachsender Schicht- und Nachtarbeit, Wochenend- und Feiertagsarbeit sowie allgemein ständiger Versuche von Arbeitgeberseite, die Arbeitszeit in ihrem Sinne noch flexibler zu gestalten. Dazu zählt auch die »verborgene« Arbeitszeit aufgrund von OnlineVerfügbarkeit der Beschäftigten auch außerhalb von Betriebs- und Bürozeiten. 
Für abhängig Erwerbstätige gab es lange Zeit nur die Möglichkeit, die »Individualisierungs-« bzw. Flexibilisierungsinteressen ihres jeweiligen Arbeitgebers beim Zuschnitt von Arbeitszeitlage und Arbeitszeitdauer akzeptieren zu müssen. Auch die Wahl von Teilzeit zugunsten von mehr individueller Zeit, insbesondere für Kinder und Familie, war und ist bei Weitem nicht immer freiwillig.

Immerhin aber gibt es seit etlichen Jahren den gesetzlichen Anspruch auf individuelle Elternzeit für das Aufziehen von Kindern und seit einiger Zeit auch den Anspruch auf individuelle Pflegezeit für die Versorgung von kranken oder gebrechlichen Familienmitgliedern. Beide Rechte sind mit einem Rückkehrrecht auf die ursprüngliche Arbeitsstelle verbunden, weisen aber ansonsten unterschiedliche Anspruchsdauern und Anspruchskonditionen auf. Reicht die Anspruchsdauer nicht aus oder gibt es neben Kindern und Pflege andere Motive für individuelle Zeit, können viele Beschä̈tigte ab bestimmten Betriebsgrößen viel leichter als früher Vollzeit in Teilzeit umwandeln.

Nur die Rückkehr von Teilzeit auf Vollzeit war bis vor Kurzem kein Rechtsanspruch. Diesen gibt es nun zwar prinzipiell, aber faktisch nur für Beschäftigte in größeren und großen Betrieben, in denen allerdings nicht die Mehrzahl der Beschäftigten tätig ist. Noch gibt es aber keinen generellen Anspruch auf individuelle Qualifizierungszeit, der mit einem Übergang in Teilzeit oder auch mit einem vorübergehenden Ausstieg aus dem Erwerbsleben verbunden wäre - abgesehen von einzelnen Tarifverträgen und dem sehr rudimentären gesetzlichen Bildungsurlaub.

Die unterschiedlichen Motive, Voraussetzungen, Zeitdauern und Rückkehrmöglichkeiten für die jeweiligen Ansprüche machen deutlich, dass es hier noch vieler Nachbesserungen bei der Gewichtung von Motiven, der Verbreiterung von Voraussetzungen, der Verlängerung von Anspruchsdauern etc. bedarf, bis man die Grundsicherung für individuelle Zeit - sozusagen eine »bedingungslose Grundzeit ${ }^{8}$ - auch bei Erwachsenen befriedigend nennen kann.

Die individuelle Zeit kann man nur dann weitgehend optimal nutzen, wenn dafür auch genügend Geld zur Verfügung steht. Demnach sollte die Grundsicherung für individuelle Zeit durch eine Grundsicherung für individuelles Einkommen auch jenseits von Erwerbseinkommen flankiert sein, zumindest für eine begrenzte Zeitdauer. Diese Flankierung ist im Status quo gerade bei Kindern und Jugendlichen besonders problematisch bzw. verbesserungsbedürftig.

Die finanzielle Grundsicherung für Kinder, die in der deutschen Politik begrifflich unter »Familienlastenausgleich" geführt wird, ist institutionell zersplit-

8 | Zum Begriff und Konzept der bedingungslosen Grundzeit vgl. Schäfer 2011. 
tert und im Verhältnis der entsprechenden Institutionen nicht ausgewogen: Es gibt die Leistungssätze für Kinder im Rahmen von Hartz IV nach Kindesalter gestaffelt; es gibt den Kinderzuschlag, der ein Abrutschen der Familie in Hartz IV verhindern soll, wenn das Elterneinkommen als nur für die Erwachsenen ausreichend bzw. als nicht bedürftig klassifiziert wird; es gibt das Kindergeld, das nach Zahl der Kinder im Haushalt gestaffelt ist und allen erwerbstätigen Vätern und Müttern gewährt wird, und es gibt den günstigeren steuerlichen Kinderfreibetrag, der anstelle des Kindergelds vom Finanzamt automatisch eingeräumt wird, wenn das Elterneinkommen eine bestimmte Höhe überschreitet.

Unverständlicherweise ist die Geldleistung für Kinder in Hartz IV am niedrigsten, der geldwerte Vorteil des Kinderfreibetrags am höchsten - mit anderen Worten: Die am meisten Bedürftigen bekommen am wenigsten. Diese Diskrepanz vergrößert sich noch durch weitere steuerliche Vorteile, die Eltern mit hohen Einkommen für die häusliche und auch außerhäusliche Betreuung oder Erziehung ihrer Kinder (etwa für Schulgeld insbesondere in Privatschulen) nutzen können. Eine wachsende Diskrepanz gibt es aber auch schon dann, wenn das Kindergeld für alle Kinder außerhalb von Hartz IV erhöht wird, ohne dass gleichzeitig auch die Hartz-IV-Leistungssätze für Kinder angehoben werden. Eine befriedigende Grundsicherung für die finanzielle Förderung von Kindern muss also erst noch geschaffen werden.

Hierfür scheint sich nach vielen Jahren der Kritik immerhin eine möglicherweise umwälzende Einsicht der Politik in die entsprechende Problematik zu entwickeln: Viele Sozialverbände im »Bündnis Kindergrundsicherung (vgl. Bündnis Kindergrundsicherung 2021), verschiedene Parteien (SPD 2019; Bündnis 90/ Die Grünen 2019; Fraktion Die Linke im Bundestag o.J.), der DGB (2020) und selbst ein bundesweiter Arbeitskreis der Sozialminister der Länder (Arbeitsgruppe Kindergrundsicherung der ASMK 2018) nähern sich mehr oder weniger stark der Idee einer alternativen, alle bisherigen Förderinstrumente ablösenden Kindergrundsicherung. Die entsprechenden Konzepte unterscheiden sich im Detail und werden hier nicht weiter aufgegriffen.'

Auch bei Erwerbstätigen in »individuellen Zeiten« jenseits von Erwerbsarbeit lässt die Absicherung zu wünschen übrig. Für sie ist die »Grundsicherung für individuelles Geld« bei Verkürzung oder Aussetzung von Erwerbsarbeit heute faktisch nur durch das Elterngeld gegeben. Ob dessen Höhe, Deckelungsgrenze und Bezugsdauer ausreichen, soll hier nicht diskutiert werden, obwohl es

9 | Zu den unumgänglichen und grundlegenden Gestaltungsprinzipien dieser allumfassenden Kindergrundsicherung siehe den Beitrag von Irene Becker in diesem Band, die zu den »Erfinder:innen« dieses Konzepts gehört. 
auch hierzu Kritisches zu sagen gäbe - insbesondere im Hinblick auf Anreize für Männer zum Bezug von Elterngeld bzw. ihre Bereitschaft zur Elternzeit.

Aber warum gibt es bis heute kein paralleles Pflegegeld, obwohl die Pflege von kranken oder gebrechlichen Familienangehörigen der zeitlichen, physischen und psychischen Belastung durch das Aufziehen von Kindern durchaus gleichkommt? Und warum gibt es für die Weiterbildung oder Umqualifizierung während der Erwerbstätigkeit auf Basis von Teilzeit oder Auszeit keine vergleichbare Regelung für einen finanziellen Lohnersatz?

Schließlich ist auch das durch Erwerbstätigkeit zu erzielende Einkommen angesichts der zunehmenden Prekarisierung von Arbeitsverhältnissen nicht per se existenzsichernd. Zwar gibt es inzwischen nach langem Widerstand einen gesetzlichen Mindestlohn in Deutschland, aber dieser ist bekanntlich schon in der Erwerbsphase von der Höhe her nicht ausreichend, erst recht nicht bei kurzen wöchentlichen Arbeitszeiten oder angesichts der verbreiteten Umgehungsversuchen durch die Arbeitgeber. Außerdem ist er wegen seines heutigen Stundenlohnniveaus in Verbindung mit einem festgeschriebenen Erhöhungsmechanismus nicht geeignet, Altersarmut nach der Erwerbsphase zu verhindern.

Noch weniger gesichert ist die finanzielle Existenz von vielen Selbstständigen, die vor allem als klassische Solo-Selbstständige oder auch als neue »WebArbeiter:innen« häufig prekären Bedingungen ausgesetzt sind. Wie schon gesagt fehlt insbesondere ihnen eine Auffanglinie, die dem Arbeitslosengeld I oder dem Kurzarbeitergeld vergleichbar wäre, bevor sie in das heutige oder zukünftige Hartz-IV-System »fallen" (vgl. dazu auch den Vorschlag von Schoukens/Weber 2020). Auch ein Mindestgewinn bzw. Mindestunternehmerlohn ist für SoloSelbstständige bei prekärer Erwerbssituation analog zum gesetzlichen Mindestlohn für abhängig Beschäftigte naheliegend.

\section{Fazit: Den Sozialstaat optimieren statt durchs bedingungslose Grundeinkommen ruinieren}

Diese Beispiele für heutige Defizite - mehr können es hier nicht sein - und im Umkehrschluss für Ansätze einer optimierten Grundsicherung belegen vor allem: Grundsicherung muss viel mehr sein als Hartz IV und die anderen herkömmlichen Grundsicherungsbausteine in den Sozialgesetzbüchern II und XII. Grundsicherung betrifft alle Menschen vor, während und nach der Erwerbsphase, um ihnen mittels grundlegender Ansprüche auf materielle und immaterielle öffentliche Unterstützung einen mindestens existenzsichernden und zugleich auch hindernisarmen bzw. chancenreichen Lebensweg zu eröffnen. Entspre- 
chend vielfältig und ausreichend müssten die nötigen staatlichen Geldleistungen und öffentlichen Infrastrukturangebote ausfallen.

Zugleich ist durch die Beispiele aber auch deutlich geworden, dass die Konturen und Strukturen einer erweiterten Grundsicherung in Deutschland noch unscharf sind. Hier gibt es zweifellos noch Diskussionsbedarf, aber die Leitideen und Handlungskriterien dieser Grundsicherung stehen fest. Zu diesen gehört der Grundsatz, dass bei einer erweiterten Grundsicherung Zugangshürden möglichst unterbleiben bzw. Zugangswege so weit wie möglich automatisch eröffnet werden - wie das etwa bei einer allumfassenden Kindergrundsicherung über die Familienkassen oder Finanzämter möglich wäre und deshalb entsprechend von den oben in Abschnitt 4 genannten Akteur:innen gefordert wird.

Ach ja: Es gibt eine Sichtweise, die mit dem Instrument eines bedingungslosen Grundeinkommens (BGE) für jede und jeden schon eine einfache und zugleich umfassende Lösung aller Probleme gefunden zu haben meint: $\mathrm{Ob}$ Kind oder Greis:in, arm oder reich, alle Bürger:innen sollen unbeschadet von Herkunft, Erwerbseinkommen und Vermögen, ohne jegliche Bedingung und automatisch den gleichen monatlichen "Grundbetrag « vom Sozialstaat erhalten. Der reicht bei den Protagonist:innen diese Konzepts - die teilweise aus weit entfernten politischen Lagern mit diverser Zielsetzung kommen - von 800 bis 1500 Euro monatlich und mehr, wobei manche den Grundbetrag für Kinder absenken wollen.

Die Hauptzielsetzung dabei ist, dass die Leistungshöhe ausreichen soll, um den heutigen Druck speziell zur Annahme auch prekärer Beschäftigungsverhältnisse zu neutralisieren und individuelle Muße und Kreativität unabhängig von weitgehend fremdbestimmter Erwerbsarbeit generell zu ermöglichen. Die Bedingungslosigkeit soll die heutigen Hürden für Grundsicherung wie insbesondere Antragsscham, Antragsmühen, Ausforschung des familiären Zusammenlebens, Bedürftigkeits- und Vermögensprüfung, gesellschaftliche Stigmatisierung etc. obsolet machen.

Die polarisierenden positiven und negativen, aber auch die abwägenden Reaktionen auf dieses schon alte, aber nach diversen Arbeitsmarktkrisen und »-reformen« aktualisierte Konzept sind inzwischen Legion, ${ }^{10}$ und erstaunlicherweise versiegen sie immer noch nicht. ${ }^{11} \mathrm{Zu}$ diesem BGE-Konzept soll hier nur

10 | Vgl. die prägnante Zusammenfassung von Pros und Cons bei Bäcker/Naegele/ Bispinck 2020, S. 303 ff.; ausführlicher z. B. bei Butterwegge/Rinke (2018) oder früher schon Schäfer (2006).

11 | Ein aktueller Anlass für ein Aufflammen ist das vom Deutschen Institut für Wirtschaftsforschung (DIW) und dem Verein »Mein Grundeinkommen« angekündigte 
kurz festgehalten werden, dass diese Idee nicht nur »Opium fürs Volk« ist, sondern zudem auch »Manna für die Reichen« sein wird:

- Seine Finanzierung wird selbst bei einem bescheidenen Grundeinkommensniveau von 800 Euro pro Monat und trotz Einsparungen bei anderen Sozialleistungen, vor allem beim zu ersetzenden Hartz IV, tendenziell eine Verdoppelung der Steuerlast oder ein erhebliches Abschmelzen anderer Sozialstaatsangebote bedeuten müssen - Letzteres ist durchaus im Sinn auch neoliberaler Verfechter:innen dieser Idee. Mit einem höherem BGE von über 800 Euro monatlich wird der Sozialstaat tendenziell gesprengt bzw. das heutige Sozialstaatsbudget rechnerisch - und gesellschaftlich unrealistisch - verdoppelt.

- Ein BGE wird aber auch »Manna für die Reichen« sein, denn das Grundeinkommen wird tendenziell auf die Löhne direkt oder indirekt angerechnet und zwar umso mehr, je höher das BGE ausfällt - und senkt so die Lohnkosten. Die besonders propagierte Finanzierung des BGE durch überwiegend oder ausschließlich indirekte Steuern schont die Unternehmen ein weiteres Mal; die Verteilung von Einkommen und Vermögen wird folglich noch einmal ungleicher. Und bei besonderen Wechselfällen oder Notlagen im Leben werden das Angebot an sozialstaatlichen Leistungen und das mögliche Ausmaß an sozialer Inklusion angesichts der drohenden finanziellen Überforderung des Sozialstaats durch das BGE wesentlich beschränkter ausfallen als heute. Die behaupteten Vorteile des BGE sind also weitgehend Illusion, wirken aber als »süßes Gift«.

Die Relevanz des BGE in manchen deutschen Debatten ist zweifellos berechtigter Kritik, Frust und auch Verzweiflung über den Zustand zentraler sozialstaatlicher Elemente in Deutschland entsprungen. Aber als Lösungskonzept für die kritisierten Probleme ist es nicht nur untauglich, sondern kontraproduktiv: Systemimmanent bedient es - teils ungewollt, teils beabsichtigt - als Trojanisches Pferd die Interessen für mehr Marktliberalität und weniger Sozialstaat.

Wir dagegen halten die in diesem Buch skizzierte erweiterte Grundsicherung für den eindeutig erfolgversprechenden Weg, weil er realitätsnäher ist bzw. an Vorhandenem anknüpft, schnell begehbar ist, materielles Auskommen sowie

Feld - Experiment mit etwa 1000 zufällig ausgewählten Teilnehmer:innen, die für drei Jahre mit einem gestifteten Grundeinkommen ausgestattet werden sollen (s. Schupp 2020) - und die überwiegend kritische Reaktion darauf etwa von Krämer (2020) oder von Steinhardt (2020). 
soziale Inklusion wirksam fördert und schließlich finanziell vom deutschen Sozialstaat gestemmt werden kann. Die »neue« Grundsicherung käme im Lebensverlauf der Bürger:innen immer wieder positiv in unterschiedlichen Lebenslagen zum Tragen und könnte teilweise - wie die Grundsicherung für regionale Mobilität - zum ständigen Begleiter werden. Der Kindergrundsicherung würden die Mitglieder dieser Gesellschaft sogar als allererstes Sicherungsinstrument begegnen und von diesem über einen sehr langen, zugleich Individuen wie Gesellschaft prägenden Zeitraum gefördert werden.

Deshalb neigen wir dazu, diese erweiterte Grundsicherung als »erstes soziales Netz« zu betrachten, während die herkömmliche Grundsicherung in der sozialwissenschaftlichen Literatur oft als »letztes soziales Netz« bezeichnet wird (so auch bei Bäcker/Naegele/Bispinck 2020, S. 246). Um keinerlei Missverständnisse aufkommen zu lassen: Die »neue« Grundsicherung schließt die »alte« bzw. herkömmliche selbstverständlich mit ein; die Forderung nach einer erweiterten Grundsicherung soll auf keinen Fall die Defizite in der traditionellen Grundsicherung überdecken. Auch diese gehören rasch und gründlich reformiert, allen voran das in fast jeder Hinsicht kritikwürdige Hartz-IV-System.

Dass die Politik zu solchen Reformen durchaus bereit sein kann, haben die Coronapandemie und die dafür - in begrenztem Ausmaß und mit befristeter Wirkung - kurzfristig geschaffenen zusätzlichen Hilfsmaßnahmen des Staates gezeigt. Und schließlich knüpft die hier präsentierte erweiterte Grundsicherung nicht nur an vorhandenen Institutionen, sondern zum Teil auch an schon länger bestehenden Vorstellungen von sozialstaatlichen Aufgaben an. Dazu gehört z. B. die in der Rechtsprechung insbesondere durch das Bundesverfassungsgericht, aber auch in der Politik seit Jahren betonte Verpflichtung des Staates zur $» D a-$ seinsvorsorge«, die die Bedeutung und Verknüpfung von sozialen Leistungen und komplementärer Infrastruktur kennt (siehe den Beitrag von Tanja Klenk in diesem Band).

Eine erweiterte Grundsicherung kann durchaus als die konzeptionelle und strukturierte Umsetzung dieses Auftrags zur Daseinsvorsorge interpretiert werden. Inhaltliche Bezüge zu einer so verstandenen Grundsicherung sind in der Politik insbesondere auch auf EU-Ebene zu finden (siehe hierzu den Beitrag von Walter Hanesch in diesem Band): Dort wird seit 2013 - anfänglich »nur« für arme Kinder und Jugendliche - die Kommissionsinitiative für eine "Kindergarantie« entwickelt, die die EU-Mitgliedstaaten nach dem Ende geplanter Pilotprojekte in ausgewählten Ländern flächendeckend umsetzen sollen. Auch die "Europäische Säule sozialer Rechte«, die 2017 vom EU-Parlament, dem Rat der Europäischen Union und der EU-Kommission proklamiert wurde, weist vergleichbare Intentionen für in diesem Fall alle Bevölkerungsgruppen auf. 
Doch bekanntlich besitzt die EU für Fragen der sozialen Sicherung nur eine Empfehlungs-, aber keine Handlungskompetenz, sodass für das Konzept der hier vorgestellten Grundsicherung im weiteren Sinne nach wie vor der deutsche Sozialstaat initiativ werden - und für die EU vielleicht auch initialisierend wirken - muss.

\section{Literatur}

ADAC (2021): Die Entfernungspauschale - was Arbeitnehmer dazu wissen müssen, www.adac.de/rund-ums-fahrzeug/auto-kaufen-verkaufen/autokosten/ent fernungspauschale/ (Abruf am 17.6.2021).

Arbeitsgruppe Kindergrundsicherung der ASMK (2018): Wege zu einer Kindergrundsicherung. Grobkonzept, Länderoffene Arbeitsgruppe der Konferenz der Ministerinnen und Minister, Senatorinnen und Senatoren für Arbeit und Soziales (ASMK), Hannover.

Bäcker, G./Naegele, G./Bispinck, R. (2020): Sozialpolitik und soziale Lage in Deutschland. Ein Handbuch, 6. Auflage, Wiesbaden.

Bündnis Kindergrundsicherung (2021): Kinderarmut hat Folgen, www.kinder armut-hat-folgen.de (Abruf am 17.6.2021).

Bündnis 90/Die Grünen (2019): Antrag der Fraktion Bündnis 90/Die Grünen. Faire Chancen für jedes Kind - Kindergrundsicherung einführen, in: Deutscher Bundestag, Drucksache 19/14326 vom 22.10.2019, http://dipbt.bundes tag.de/doc/btd/19/143/1914326.pdf (Abruf am 17.6.2021).

Butterwegge, C./Rinke, K. (Hrsg.) (2018): Grundeinkommen kontrovers. Plädoyers für und gegen ein neues Sozialmodell, Weinheim.

Deutscher Bundestag, Ausschuss für Arbeit und Soziales (2020): Materialien zur öffentlichen Anhörung von Sachverständigen in Berlin am 2. November 2020. Ausschussdrucksache 19(11)822 vom 30. Oktober 2020, http://portalsozialpolitik.de/uploads/sopo/pdf/2020/2020-10-30_RBEG_2021_Stellung nahmen.pdf (Abruf am 17.6.2021).

DGB = Deutscher Gewerkschaftsbund (2020): Kindergrundsicherung: Geringverdienende unterstützen - soziale Teilhabe für alle Kinder ermöglichen! arbeitsmarkt aktuell 3/2020, www.dgb.de/downloadcenter/++co++0465b9c29507-11ea-a727-52540088cada (Abruf am 17.6.2021).

FAZ = Frankfurter Allgemeine Zeitung (2020): Enttäuschende Grundrente. Viele Geringverdiener unter Grundsicherungsniveau, in: FAZ vom 28. Oktober 2020, https://zeitung.faz.net/faz/Wirtschaft/2020-10-28/437a17a718ec22cf00e 2462f8b7754b6?GEPC=s9 (Abruf am 17.6.2021). 
Fraktion Die Linke im Bundestag (o.J.): Kinderarmut/Kindergrundsicherung, www.linksfraktion.de/themen/a-z/detailansicht/kinderarmut-kindergrund sicherung/ (Abruf am 17.6.2021).

Friedrichsen, J./Schmacker, R. (2019): Die Angst vor Stigmatisierung hindert Menschen daran, Transferleistungen in Anspruch zu nehmen, in: DIW Wochenbericht 26/2019, S. 456-463.

Hahn, P. (2019): Bis zu 60\% aller Berechtigten nehmen Grundsicherung (im Alter) nicht in Anspruch. Interview, in: DIW Wochenbericht 49/2019, S. 918.

Krämer, R. (2020): Wieso das BGE-Pilotprojekt die Missverständnisse zum Grundeinkommen noch vergrößert, Beitrag vom 17. September 2020, https://makronom.de/wieso-das-bge-pilotprojekt-die-missverstaendnissezum-grundeinkommen-noch-vergroessert-37079 (Abruf am 17.6.2021).

Nationale Armutskonferenz (2020): Spaltungen verhindern, Zusammenhalt stärken - kein "Weiter-so « bei den Regelsätzen, www.nationale-armutskon ferenz.de/2020/03/11/spaltungen-verhindern-zusammenhalt-staerken-keinweiter-so-bei-den-regelsaetzen/ (Abruf am 17.6.2021).

Paritätischer Gesamtverband = Deutscher Paritätischer Wohlfahrtsverband Gesamtverband e.V. (2019): Bildungs- und Teilhabepaket: Nur jedes siebte Kind der 6- bis unter 15-Jährigen profitiert von Teilhabeleistungen, Pressemitteilung vom 8. Oktober 2019, www.der-paritaetische.de/alle-meldungen/ bildungs-und-teilhabepaket-nur-jedes-siebte-kind-der-6-bis-unter-15-jaehrigenprofitiert-von-teilh/ (Abruf am 17.6.2021).

Paritätischer Gesamtverband = Deutscher Paritätischer Wohlfahrtsverband Gesamtverband e.V. (2020a): Bildungs- und Teilhabepaket: Paritätische Expertise zeigt, dass Teilhabeleistungen nur jedes siebte benachteiligte Kind erreichen, Pressemitteilung vom 12. November 2020, www.der-paritaetische. de/alle-meldungen/bildungs-und-teilhabepaket-paritaetische-expertise-zeigtdass-teilhabeleistungen-nur-jedes-siebte/ (Abruf am 17.6.2021).

Paritätischer Gesamtverband = Deutscher Paritätischer Wohlfahrtsverband Gesamtverband e.V. (2020b): Paritätischer kritisiert Erosion der sozialen Sicherungssysteme angesichts wachsender Armut, Pressemitteilung vom 30. Oktober 2020, www.paritaet-bayern.de/nc/pressemitteilung/news/pari taetischer-kritisiert-erosion-der-sozialen-sicherungssysteme-angesichts-wach sender-armut/ (Abruf am 17.6.2021).

Schäfer, C. (2006): Bedingungsloses Grundeinkommen - Absurde Utopie oder reale Möglichkeit?, in: Schäfer, C./Seifert, H. (Hrsg): Kein bisschen leise: 60 Jahre WSI, Hamburg, S. 297-312.

Schäfer, C. (2011): Wir brauchen eine Bedingungslose Grundzeit, in: WSI-Mitteilungen 2/2011, S. 91-94. 
Schoukens, P./ Weber, E. (2020): Lehre aus der Coronakrise. Auch Selbstständige brauchen Kurzarbeit, in: Der Spiegel vom 9. November 2020, www.spiegel.de/ wirtschaft/soziales/corona-lehre-aus-der-krise-auch-selbststaendige-brauchenkurzarbeit-a-232ea75b-0251-4ce3-bf64-89bebbb02548 (Abruf am 17.6.2021).

Schupp, J. (2020): Bedingungsloses Grundeinkommen. Was das BGE-Pilotprojekt leisten kann - und was nicht, Beitrag vom 31. August 2020, https://mak ronom.de/was-das-bge-pilotprojekt-leisten-kann-und-was-nicht-36874 (Abruf am 17.6.2021).

SPD (2019): Unser Konzept für eine sozialdemokratische Kindergrundsicherung, Beschluss Nr. 4 des Bundesparteitags 2019. www.spd.de/fileadmin/Doku mente/Beschluesse/B4_Beschluss_Unser_Konzept_fuer_eine_sozialdemo kratische_Kindergrundsicherung.pdf (Abruf am 17.6.2021).

Statistische Ämter des Bundes und der Länder (2019): Soziale Mindestsicherung in Deutschland 2017, Wiesbaden.

Statistisches Bundesamt (2020a): Tag der älteren Menschen: Armutsgefährdung stieg seit 2005 am stärksten in der Generation 65 plus, Pressemitteilung Nr. N 062 vom 30. September 2020, www.destatis.de/DE/Presse/Pressemit teilungen/2020/09/PD20_N062_634.html (Abruf am 17.6.2021).

Statistisches Bundesamt (2020b): Zahl der Empfängerinnen und Empfänger sozialer Mindestsicherung 2019 um 4,7\% gesunken, Pressemitteilung Nr. 434 vom 30. Oktober 2020, www.destatis.de/DE/Presse/Pressemitteilungen/2020/10/ PD20_434_228.html (Abruf am 17.6.2021).

Steinhardt, P. (2020): Wunderland BGE, Beitrag vom 7. November 2020, https:// makroskop.eu/spotlight/k-eine-zukunft-fuer-die-arbeit/wunderland-bge/ (Abruf am 17.6.2021).

UN-Ausschuss für wirtschaftliche, soziale und kulturelle Rechte (2018): Abschließende Bemerkungen im Rahmen des Prüfverfahrens zum völkerrechtlich verbindlichen UN-Sozialpakt gegenüber Deutschland, Oktober 2018, Randziffer 46 und 47, aus dem Englischen übersetzt von Maskenfall, www. maskenfall.de/?p=12950 (Abruf am 17.6.2021).

WirtschaftsWoche (2020): Schuldneratlas 2020. Altersüberschuldung wächst: "Perspektiven sind besorgniserregend «, in: WirtschaftsWoche vom 10. November 2020, www.wiwo.de/finanzen/geldanlage/schuldneratlas-2020-alters ueberschuldung-waechst-perspektiven-sind-besorgniserregend/26608798. html (Abruf am 17.6.2021). 



\section{Teil I}

Aktuelle Probleme der Grundsicherung 



\title{
Sozialversicherung und Grundsicherung im Spannungsverhältnis
}

\author{
Umbrüche und Perspektiven des Systems der sozialen Sicherung
}

\section{Gerhard Bäcker}

\section{Einführung}

"Der Sozialstaat Deutschland ist ein Sozialversicherungsstaat«, so lauten zugespitzt die Befunde der vergleichenden Wohlfahrtsstaatsforschung. Tatsächlich prägt die gegen Ende des 19. Jahrhunderts einsetzende Bismarck'sche Sozialversicherungspolitik, mit der von der reinen Armenfürsorge abgewichen und eine zentralstaatliche Verantwortung für die Absicherung der sogenannten sozialen Standardrisiken der Arbeiterschaft übernommen wurde, bis heute das Erscheinungsbild des deutschen Sozialstaats. Mehr als 60 Prozent aller im Sozialbudget erfassten Sozialausgaben entfallen aktuell auf die beitragsfinanzierten Sozialversicherungssysteme, die neben der Kranken-, Renten- und Unfallversicherung auch die Arbeitslosenversicherung und die Pflegeversicherung umfassen (BMAS 2020).

Im Zeitverlauf sind das Spektrum wie auch das Niveau der Leistungen, die im Versicherungsfall gewährt werden und sich nach Geld- sowie Sach- und Dienstleistungen unterscheiden lassen, erheblich ausgeweitet worden. Hinzu kommt eine sukzessive Verbreiterung des Kreises der Anspruchsberechtigten und Leistungsempfänger:innen. So sind in der Renten- sowie in der Kranken- und Pflegeversicherung rund 90 Prozent der Bevölkerung abgesichert. Aber nach wie vor gilt, dass die einzelnen Zweige der Sozialversicherung »lohnarbeitszentriert « sind; ein universelles Versicherungssystem - umschrieben mit Begriffen wie Bürgerversicherung oder Erwerbstätigenversicherung - gibt es nicht, abgesichert sind im Wesentlichen die abhängig Beschäftigten und ihre Familienangehörigen.

Der Anteil der Sozialversicherung an den Gesamtausgaben des Sozialbudgets in Höhe von knapp zwei Dritteln signalisiert allerdings auch, dass sich die soziale Sicherung keineswegs auf die Zweige der Sozialversicherung beschränkt. Denn zu berücksichtigen sind neben den steuerlichen Entlastungen, der Beam- 
tenversorgung (Pensionen und Beihilfen) und den Arbeitgeberleistungen (Entgeltfortzahlung, betriebliche Altersversorgung) vor allem die im Sozialbudget so benannten "Förder- und Fürsorgesysteme", die rund 18 Prozent der Gesamtausgaben ausmachen und im Unterschied zu den Leistungen der Sozialversicherung vorleistungsunabhängig und ausschließlich steuerfinanziert sind.

Im Mittelpunkt dieses Beitrags stehen neben der Sozialversicherung die fürsorgeförmigen, d.h. einkommensgeprüften Leistungen dieses Komplexes. Es handelt sich um die Grundsicherung für Arbeitsuchende (SGB II), die Grundsicherung für Ältere und Erwerbsgeminderte (SGB XII), die Sozialhilfe (SGB XII), das Asylbewerberleistungsgesetz (AsylbLG) sowie - allerdings nur eingeschränkt das Wohngeld und die Ausbildungsförderung. ${ }^{1}$

Sie fungieren hinsichtlich der Existenzsicherung der Bevölkerung als eine Grund- oder Mindestsicherung, die das soziokulturelle Existenzminimum garantieren soll. Unterschreitet das verfügbare Einkommen das Minimum, so besteht grundsätzlich Anspruch sowohl auf aufstockende Geldleistungen als auch auf flankierende Sach- und Dienstleistungen. Gleichzeitig sind diese Leistungen nicht nur einkommensgeprüft, sie orientieren sich in einem gewissen Rahmen auch an spezifischen Bedarfen der Leistungsempfänger:innen.

Aufgabe soll es sein, das Wechselspiel von Grundsicherung und Sozialversicherung näher zu analysieren und die Grundelemente der beiden Systeme zu skizzieren. Die zu untersuchende Frage stellt sich aus zwei Richtungen: Welche Rückwirkung haben Bedingungen und Niveau der Grundsicherung auf die Leistungen der Sozialversicherung? Oder andersherum gefragt: Wie wirken sich Bedingungen, Umfang und Niveau der Leistungen der Sozialversicherung auf das Angewiesensein auf Leistungen der Grundsicherung aus?

$\mathrm{Zu}$ zeigen ist, dass die ab der Jahrtausendwende vom Neoliberalismus geprägte Sozialpolitik ${ }^{2}$ einen Trend in Richtung einer wachsenden Bedeutung der Grundsicherung eingeleitet hat. Zugleich haben sich hierarchisierte Teilsysteme der Grundsicherung herausgebildet, und es ist - so z. B. in der Rentenversiche-

1 | Neben den genannten Leistungen umfassen die »Förder- und Fürsorgesysteme« laut Sozialbudget auch die Bereiche »Kindergeld und Familienleistungsausgleich«, »Elterngeld « und »Kinder- und Jugendhilfe«.

2 | Verfolgt man die Debatten jener Zeit, so wurde die Sozialversicherung als zu teuer, statusbewahrend und zu starr bezeichnet. Statt soziale Probleme zu lösen, sei sie zu einem Problemverursacher geworden, dies vor allem hinsichtlich der Entstehung und Verhärtung von Arbeitslosigkeit. Private Vorsorge einerseits und die Grundsicherung andererseits seien hingegen die ökonomisch, fiskalisch und sozialpolitisch vorteilhafteren Modelle (vgl. Bäcker/Naegele/Bispinck 2020, S. 137 ff.). 
rung - zu Vermischungen zwischen Versicherungs- und Grundsicherungsleistungen gekommen.

Mit anderen Worten: Die Sozialversicherung ist zwar nach wie vor für den Großteil der sozialen Sicherung zuständig und umfasst den weitaus größten Teil der Bevölkerung. Die »Grundversorgung« beim Eintritt sozialer Risiken wird aber zunehmend an die eigentlich als Ausnahmelösung konzipierte Grundsicherung übertragen, die damit immer stärker in die Rolle einer »Regelleistung« hineinwächst.

Um diese Trends erkennen zu können, sollen in einem ersten Schritt die Systeme der Sozialversicherung (Abschnitt 2 und Abschnitt 3) und der Grundsicherung (Abschnitt 4) in Deutschland skizziert, bewertet und verglichen werden. Wir begrenzen uns dabei auf die Geldleistungen und lassen in diesem Beitrag die Frage nach den Schnittstellen zwischen Sozialversicherung und Grundsicherung bei den Sach- und Dienstleistungen unberücksichtigt. ${ }^{3}$ In einem zweiten Schritt werden dann in den Sicherungsfeldern "Alter« (Abschnitt 5) und "Arbeitslosigkeit« (Abschnitt 6) detailliertere Analysen vorgenommen. Der Beitrag schließt mit dem Hinweis auf Reformperspektiven und weist darauf hin, dass ein radikaler Systembruch in Richtung eines bedingungslosen Grundeinkommens den Sozialstaat aushöhlt, statt ihn zu stärken (Abschnitt 7).

\section{Charakteristika der Sozialversicherung}

In Deutschland steht die Absicherung der abhängig Beschäftigten im Mittelpunkt des sozialen Schutzes durch die Sozialversicherung. Die Zweige der Sozialversicherung gelten nach wir vor als »lohnarbeitszentriert«; ein universelles

3 | Seit 2009 muss jede Einwohnerin und jeder Einwohner in einer gesetzlichen oder wenn die Voraussetzungen dafür gegeben sind - einer privaten Krankenversicherung versichert sein. Dasselbe gilt für die Pflegeversicherung. Insofern gibt es hier - mit Ausnahmen - keine personellen Lücken, die durch die Grundsicherung aufgefangen werden müssten.

Allerdings ist die soziale Pflegeversicherung im Unterschied zur gesetzlichen Krankenversicherung nicht als Vollkosten-, sondern nur als Teilkostenversicherung ausgestaltet. Stationär versorgte Pflegebedürftige müssen die sogenannten Hotelkosten allein aufbringen, und die reinen Pflegekosten werden - nach Pflegegraden gestaffelt - nur teilweise übernommen. Diese Absicherungslücke führt dazu, dass eine wachsende Zahl von Pflegebedürftigen Sozialhilfe (Hilfe zur Pflege) beantragen muss, da die ungedeckten Kosten nicht aus dem eigenen Einkommen bestritten werden können (vgl. dazu im Detail Bäcker/Naegele/Bispinck 2020, S. 820 ff.). 
Versicherungssystem - im Sinne einer Bürgerversicherung oder Erwerbstätigenversicherung - gibt es nicht. Für Beamt:innen gelten Sondersysteme, und die selbstständig Erwerbstätigen sind weitgehend ausgeklammert.

Ausnahmen von der Versicherungspflicht bestehen - mit Ausnahme der Unfallversicherung - bei jenen Beschäftigungsverhältnissen, die nur kurzzeitig andauern oder bei denen nur ein geringes Einkommen erzielt wird (bis zu 450 Euro im Monat). Für die Beschäftigten fallen dann keine Beiträge in der Kranken-, Pflege- und Arbeitslosenversicherung an, im Gegenzug entstehen aber auch keine Leistungsansprüche. Die geringfügig Beschäftigten unterliegen zwar seit 2013 der Rentenversicherungspflicht, haben allerdings die Möglichkeit, sich von der Versicherungspflicht befreien zu lassen (Opt-out-Regelung).

Auf der anderen Seite ist zu berücksichtigen, dass die Zweige der Sozialversicherung auch Personen in den Versicherungsschutz einbeziehen, die nicht erwerbstätig sind, sondern sich in einer besonderen Lebenslage bzw. Lebensphase befinden. Die Zahl der Beispiele für derartige Regelungen ist lang; versicherungspflichtig sind unter anderem

- in der Kranken- und Pflegeversicherung: Studierende, Bezieher:innen von Elterngeld, Empfänger:innen von Arbeitslosengeld I (ALG I) und Arbeitslosengeld II (ALG II) und Rentner:innen;

- in der Rentenversicherung: Eltern, für die eine Kindererziehungszeit anzurechnen ist, Empfänger:innen von Lohnersatzleistungen nach SGB III, Bezieher:innen von Krankengeld und Personen, die eine nicht erwerbsmäßige Pflege ausüben.

Hinsichtlich der Leistungsansprüche beruht die Sozialversicherung auf dem Kausalprinzip. Fehlendes oder unzureichendes Einkommen bzw. eine allgemeine Bedarfslage als solche begründen noch keinen Anspruch auf Leistungen. Ein Anspruch entsteht erst dann, wenn der jeweils spezielle Risikofall eingetreten ist und Mitgliedschaft vorliegt. In der Arbeitslosen- und Rentenversicherung wird die Leistung zusätzlich noch an eine Mindestversicherungszeit (Wartezeit) gebunden. Wird diese Zeit - fünf Jahre in der Rentenversicherung, ein Jahr beim ALG I - nicht erreicht, bestehen keine Leistungsansprüche.

Die Geldleistungen in der Sozialversicherung werden nach dem (modifizierten) Äquivalenzprinzip berechnet. Danach hängt die (relative) Höhe der monetären Ansprüche unmittelbar von der Höhe des individuellen versicherungs- und beitragspflichtigen Arbeitsentgelts bzw. der zuvor eingezahlten Beiträge ab. Die durch das Arbeitsentgelt erzielte Einkommensposition soll zumindest teilweise beibehalten werden können (Lohnersatzfunktion). Ob jedoch diese Leistungen 
so hoch sind, dass tatsächlich von einem Lohnersatz gesprochen werden kann, hängt von den Berechnungsmaßstäben und vom Sicherungsniveau ab.

Dabei bleibt unberücksichtigt, in welcher Arbeitszeit die Einkommenshöhe erreicht worden ist. Die Höhe des Haushaltseinkommens oder Maßstäbe von Bedarf und Bedürftigkeit spielen bei der Leistungsberechnung keine Rolle. Eine Mindestleistung gibt es nicht.

Die Rentenversicherung beschränkt sich allerdings nicht auf die Abdeckung der sozialen Risiken (finanzielle Konsequenzen eines langen Lebens, von Tod und Invalidität), sondern bewirkt auch vielfältige Umverteilungseffekte. $\mathrm{Zu}$ berücksichtigen sind insbesondere die einkommens- und nicht risikobezogene Beitragsbemessung, die Hinterbliebenenversorgung, die Rentenberechnung bei einer Erwerbsminderung (Zurechnungszeiten), die Berücksichtigung beitragsfreier und beitragsgeminderter Zeiten, die Aufwertung von Anwartschaften während der Kindererziehungszeit und die Zuschläge an Entgeltpunkten bei der Grundrente.

\section{Leistungsfähigkeit und Leistungsgrenzen der Sozialversicherung}

Allgemein kann festgestellt werden, dass die Sozialversicherung sich in ihren einzelnen Zweigen als stabil und zugleich anpassungsfähig erwiesen hat und ein zentraler Faktor für die hohe Akzeptanz des Sozialstaatsprinzips in der Bevölkerung ist (vgl. u. a. Kohl 2017). Dafür ist eine Reihe von Gründen ausschlaggebend:

- Aus dem Versicherungsprinzip folgt, dass die Menschen ihre Ansprüche an den Sozialstaat aus ihren Beitragszahlungen ableiten können. Sie stehen dem Staat nicht als Bittsteller:in gegenüber, sondern als Bürger:innen, die sich ihren Rechtsanspruch erarbeitet und verdient haben. Die Beitragszahlungen begründen eigentumsrechtlich geschützte Anwartschaften. Durch das Prinzip von Leistung und Gegenleistung und den Verzicht auf Einkommens- und Bedürftigkeitsprüfungen kommt es nicht zur Diskriminierung der Leistungsempfänger:innen. Die für die vorleistungsunabhängigen Grundsicherungssysteme typischen Debatten über Missbrauch werden weitgehend vermieden.

- Der versicherungsförmige Lohnersatz führt zu einer Verstetigung des Einkommens im Lebenslauf und ermöglicht eine längerfristige Lebensplanung. Wenn lediglich eine Leistung auf dem Niveau des Existenzminimums gezahlt würde, hätte der Eintritt von Krankheiten, Unfällen, Arbeitslosigkeit oder Invalidität für die große Gruppe der Beschäftigten mit mittlerem und höherem Einkommen einen drastischen Rückgang des Lebensstandards zur Folge. 
Es geht auch und gerade für die Mittelschicht der Gesellschaft um Einkommenskontinuität im Lebensverlauf und die Möglichkeit einer verlässlichen Einkommensplanung. Selbst bei einer angehobenen, tatsächlich das soziokulturelle Existenzminimum sichernden Grundsicherungsleistung müsste der Lebensstand erheblich eingeschränkt werden, weil z. B. die Mieten nicht mehr bezahlt werden könnten.

- Einbezogen in die Sozialversicherung sind nicht nur die sogenannten »wirklich Bedürftigen«, sondern die Mehrheit der Bevölkerung. Dies ist einerseits notwendig, um den Solidarausgleich finanzieren zu können, bedeutet andererseits aber auch, dass über die Mittelschicht hinaus ein großes Interesse an der Stabilität und Leistungsfähigkeit des Sozialstaats besteht.

- Die Systeme haben ökonomische, gesellschaftliche und politische Umbrüche gemeistert und sich in Anpassung an neue Problemlagen als entwicklungsfähig erwiesen. Sie sind in ihrer gegliederten Organisationsstruktur effizient und durch die Selbstverwaltung im besonderen Maße demokratisch legitimiert.

- Die Sozialversicherungsbeiträge fließen in die autonomen Haushalte der Versicherungsträger und nicht in den allgemeinen Staatshaushalt. Fiskalisch motivierte Leistungskürzungen sind bei rein steuerfinanzierten, über die öffentlichen Haushalte abgewickelten Transfers deshalb sehr viel leichter möglich. Da die Beitragseinnahmen zweckgebunden sind und zwischen Beiträgen und Geldleistungen ein Entsprechungsverhältnis besteht, ist der Abgabenwiderstand bei Beitragszahlungen geringer als bei Steuerzahlungen.

Die Sozialversicherung in ihrer gegenwärtigen Struktur weist aber auch Leistungslücken und -grenzen auf, denn der Schutz vor sozialen Risiken ist an die Erfüllung bestimmter Voraussetzungen gebunden, bei deren Fehlen es zu folgenden Problemen kommen kann:

- Ohne eigenständige Leistungsansprüche bleiben diejenigen Personen, die kein (versicherungspflichtiges) Beschäftigungsverhältnis (haben) aufnehmen können - etwa Jugendliche oder junge Erwachsene, die nach Beendigung ihrer Schul- oder Hochschulausbildung keine Beschäftigung finden, oder Frauen, die aus familiären Gründen ihre Erwerbstätigkeit für längere Zeit unterbrochen oder ganz aufgegeben haben. ${ }^{4}$ Auch Arbeitsmigrant:innen sowie Flüchtlinge und Schutzsuchende, die (noch) nicht in den Arbeitsmarkt integriert

4 | Zu einem sozialen Ausgleich in der Rentenversicherung führen die Anerkennung von Kindererziehungs- und -berücksichtigungszeiten sowie die Anerkennung von Zeiten nicht erwerbsmäßiger Pflege. 
sind, gehen leer aus. Lücken im Versicherungsschutz entstehen schließlich bei einer nur kurzfristigen Beschäftigung oder bei durchbrochenen Erwerbsbiografien, da Leistungsbezug, -dauer und -höhe an die Versicherungs- bzw. Beitragsdauer geknüptt sein können.

- Problematisch ist die Situation für diejenigen Personen, die zwar erwerbstätig sind, aber nicht der Versicherungspflicht unterliegen, wie die geringfügig Beschäftigten und die (nicht anderweitig abgesicherten) Selbstständigen. Der Gedanke, dass gerade jene Selbstständigen, die keinem Pflichtversicherungsschutz unterliegen, am besten und ehesten in der Lage sind, freiwillig und privat vorzusorgen, erweist sich empirisch als falsch.

- Unzureichend abgesichert sind alle Bezieher:innen von Niedrigeinkommen, darunter auch Teilzeitbeschäftigte. Da die Lohnersatzleistungen das vorherige Arbeitseinkommen immer nur anteilig abdecken, geraten aus niedrigen Arbeitseinkommen abgeleitete Ansprüche auf Rente, Krankengeld oder ALG I sehr schnell in eine prekäre Zone. Eine Einkommenseinbuße von z. B. gut 40 Prozent (beim ALG I) bei einem Arbeitseinkommen, das zwar niedrig, aber gerade noch auskömmlich ist, ist gleichbedeutend mit einem Absinken unter das Existenzminimum.

- Unzureichend abgesichert sind diejenigen, die nicht nur kurzfristig, sondern längerfristig arbeitslos oder krank sind, denn der Versicherungsschutz dünnt sich in dem Maße aus, je länger das Risiko andauert. So ist Langzeitarbeitslosigkeit gleichbedeutend mit einem Verlust des Arbeitslosengeldanspruchs. Eine mehrjährige Krankheit bedeutet, dass der Krankengeldanspruch ausläuft.

Diese hier skizzierten Problemlagen und Absicherungslücken lassen erkennen, dass neben der Sozialversicherung ein »letztes soziales Netz«, nämlich eine Grundsicherung, unverzichtbar ist, um das Existenzminimum zu garantieren und Armutslagen zu verhindern. Gleichwohl ist das Ausmaß des Angewiesenseins auf Grundsicherungsleistungen in hohem Maße abhängig von der konkreten Ausgestaltung der jeweiligen Sozialversicherungszweige und damit auch Ansatzpunkt möglicher Reformen.

Die Ausweitung des Kreises der versicherten Personen auf Selbstständige in Richtung einer Erwerbstätigen- bzw. Bürgerversicherung und die Eindämmung der Minijobs sind hier als erste Schritte zu benennen. Auch lassen sich Anspruchsvoraussetzungen (Vorversicherungs- und Wartezeiten) entschärfen und die Dauer von befristeten Leistungen verlängern. Schließlich hat das Leistungsniveau der Lohnersatzleistungen (Rente, ALG I) eine Auswirkung auf die Höhe der Geldleistungen. Dadurch lässt sich verhindern, dass Renten wie auch ALG I 
trotz langjähriger Versicherungspflicht und Beitragszahlung noch nicht einmal das Bedarfsniveau der vorleistungsunabhängigen Grundsicherung erreichen oder nur wenig darüber liegen (vgl. Hönig 2020).

Ein Versicherungssystem ist aber nicht in der Lage, in jedem Fall Armutslagen zu vermeiden. Renten wie Arbeitslosengeld sind Ersatz für ein entfallenes Arbeitseinkommen. Wer nur kurzzeitig sozialversicherungspflichtig beschäftigt war oder ein nur sehr niedriges (Teilzeit-)Einkommen erzielt hat, kann in einem beitragsbasierten System nicht automatisch eine Zahlung erhalten, die das Existenzminimum abdeckt. Die Sozialversicherung wäre überfordert, wenn von ihr erwartet würde, die Defizite der Beschäftigungspolitik der vergangenen Jahrzehnte und die Folgen des politisch durchgesetzten Niedriglohnsektors auszugleichen. Dies ist die Aufgabe einer Grundsicherungsleistung.

Insofern gilt auch für die Alterssicherung von Frauen, dass die eigenständige Absicherung mit der ausreichenden Teilhabe am Arbeitsmarkt steht und fällt. Wenn ein (Teilzeit-)Lohn - und hier erst recht ein Minijob - kaum das individuelle Existenzminimum sichert und der Lebensunterhalt nur im Partnerkontext gewährleistet werden kann, kann keine Altersrente erwartet werden, die höher ist als die Grundsicherung. Es ist ein Widerspruch, dass zwar regelmäBig auf die niedrigen Frauenrenten (Stichwort gender pension gap) hingewiesen wird, dass aber auf der anderen Seite das vorgelagerte Problem, nämlich die Ausweitung der Teilzeitarbeit insbesondere auf der Basis von Minijobs, eher hingenommen, ja noch durch steuer- und sozialrechtliche Regelungen gefördert wird.

\section{Charakteristika der Grundsicherung}

Die Grundsicherungssysteme lassen sich als »fürsorgeförmig « bezeichnen, da sie aus der Tradition der kommunalen Armenfürsorge entstammen und auf den Prinzipien von Nachrang bzw. Bedürftigkeit und Bedarfsdeckung fußen. Sie greifen bei jenen Notlagen, die akut weder durch eigenes Bemühen noch durch vorgelagerte Sozialleistungen noch durch Leistungsansprüche gegenüber Angehörigen abgedeckt werden (können). Die Leistung erfolgt unabhängig von einer Vorleistung und unabhängig vom vormaligen Erwerbseinkommen und berücksichtigt die individuellen Lebensumstände wie die Haushaltskonstellation.

Die aktuelle Ausgestaltung der Systeme lässt jedoch - ausgehend vom Bundessozialhilfegesetz (BSHG) von 1962 und später seit der Einführung von Sozialgesetzbuch II und Sozialgesetzbuch XII (SGB II und XII) - eine qualitative 
Weiterentwicklung erkennen, die sie von der Praxis der traditionellen Armenfürsorge deutlich unterscheidet. Qualitativ unterschiedlich ist vor allem die Postulierung eines rechtlich garantierten Anspruchs auf eine die menschenwürdige Lebensführung sicherstellende Leistung (sozial-kulturelles Existenzminimum), die ein Mindestmaß an Teilhabe am gesellschaftlichen, kulturellen und politischen Leben garantiert.

Gesetzliche Leitmaxime ist es, Menschen die Führung eines Lebens zu ermöglichen, »das der Würde des Menschen entspricht« ( $\$ 1,1$ SGB II und SGB XII). 5 Dieser Leitsatz bezieht sich auf Artikel 1 des Grundgesetzes: „Die Würde des Menschen ist unantastbar. Sie zu achten und zu schützen ist Verpflichtung aller staatlicher Gewalt.«

Als eine qualitative Weiterentwicklung ist darüber hinaus die Begrenzung des vorrangigen Verweises auf unterhaltsverpflichtete Angehörige zu bezeichnen, nämlich auf (Ehe-)Partner:in sowie auf Eltern minderjähriger Kinder. Und auch hinsichtlich der Anrechnungsfreistellung von Vermögen (Schonvermögen und Vermögensfreibeträge) ist die Tendenz hin zu (etwas) höheren Freibeträgen sichtbar.

"Die« Grundsicherung gibt es allerdings nicht. Die Leistungen der Grundsicherung haben sich im Zeitverlauf in mehrere, auf einzelne Personenkreise zielende Teilsysteme und -gesetze ausdifferenziert. Während das 1962 kodifizierte und bis 2004 geltende BSHG ein universelles System war, das keine Differenzierung nach einzelnen Personengruppen vornahm, muss mittlerweile zwischen folgenden Teilsystemen unterschieden werden:

- Für erwerbsfähige Menschen - dazu zählen unter anderem Arbeitslose, Niedrigverdienende und teilweise Erwerbsgeminderte - und ihre Angehörigen ist die Grundsicherung für Arbeitsuchende (SGB II) zuständig. Anspruch besteht auf Geldleistungen (ALG II und Sozialgeld) sowie auf Sach- und Dienstleistungen zur Eingliederung in Arbeit. Kinder haben zusätzlich Anspruch auf Leistungen für Bildung und Teilhabe.

- Ältere Menschen und dauerhaft Erwerbsgeminderte sind die Zielgruppe von Grundsicherung im Alter und bei Erwerbsminderung (SGB XII).

- Kinder und Erwachsene unterhalb der Regelaltersgrenze, die zeitweise voll erwerbsgemindert sind, können Geldleistungen der Sozialhilfe (Hilfe zum Lebensunterhalt) nach SGB XII beantragen.

5 | Eine stets strittige Frage, die auch das Bundesverfassungsgericht mehrfach beschäftigt hat, ist und bleibt, ob dieses Postulat von der Politik auch tatsächlich umgesetzt wird. 
- Die Sozialhilfe (SGB XII) stellt besondere Sach- und Dienstleistungen bereit, so vor allem Hilfe zur Pflege und Eingliederungshilfe für Menschen mit Behinderungen.

- Asylbewerber:innen und Flüchtlinge/Schutzsuchende können Leistungen nach dem Asylbewerberleistungsgesetz erhalten.

- Das einkommensabhängige Wohngeld kann als eine ergänzende Grundsicherung interpretiert werden - da allerdings der Fürsorgecharakter (keine Bedarfsdeckung, keine strenge Nachrangigkeit) fehlt, wird das Wohngeld häufig als gesonderte Leistung dargestellt.

- Der einkommensabhängige Kinderzuschlag ist der Grundsicherung vorgelagert. Er stockt das einkommensunabhängige Kindergeld auf und soll vermeiden, dass Haushalte allein wegen des Unterhaltsbedarfs für ihre Kinder Anspruch auf ALG II und/oder Sozialgeld haben.

Art und Ausmaß des Wechselverhältnisses der Grundsicherungssysteme mit den Zweigen der Sozialversicherung hängen entscheidend von der Höhe der Leistung ab, denn je höher das Bedarfsniveau, desto eher kommt es zu einer Überschneidung mit niedrigen Renten oder einem niedrigen ALG I. Und je höher das Bedarfsniveau, desto mehr Leistungsberechtigte der Grundsicherung gibt es (Bedürftigkeit vorausgesetzt!), da Personen, die z.B. mit ihrer Rente bislang unter dem Schwellenwert des Bedarfsniveaus lagen, nun ihre Rente aufstocken können. Dasselbe gilt für Bezieher:innen von ALG I, auch hier kommt es zu einer vermehrten Aufstockung der Versicherungsleistungen durch die Grundsicherung.

Es ist nun keineswegs einfach, diese Effekte quantitativ zu berechnen. Die Bedarfe der Grundsicherung werden zwar durch Pauschalsätze (Regelbedarfe) bestimmt, beziehen sich aber auf die Bedarfs- und Haushaltsgemeinschaft, in der die Anspruchsberechtigten leben, und berücksichtigen die Zahl der Personen in der Bedarfsgemeinschaft und zusätzlich bei Kindern deren Lebensalter. Diese über Äquivalenzskalen vorgenommene Abstufung der Regelbedarfe soll einerseits dem mit dem Lebensalter variierenden Bedarf Rechnung tragen, andererseits aber auch berücksichtigen, dass mit einem größeren Haushalt Kostenvorteile bei der Haushaltsführung verbunden sind.

Hinzu kommen mögliche Ansprüche auf Mehrbedarfe, und die Kosten der Unterkunft werden in jedem Einzelfall individuell berücksichtigt. Da die Mieten einschließlich Nebenkosten regional und auch lokal erheblich voneinander abweichen, sind bundesweite Durchschnittswerte wenig aussagefähig (vgl. Kaltenborn 2019). 
Im Ergebnis handelt es sich also um ein von Haushaltskonstellation und Lebenssituation sowie von den Wohnverhältnissen abhängiges breites Band unterschiedlicher Grundsicherungsniveaus. Noch unübersichtlicher wird es, wenn die Ausgestaltung des Nachrangprinzips bei den einzelnen Grundsicherungssystemen berücksichtigt wird. Zwar gilt allgemein, dass erst dann geleistet wird, wenn sämtliche Einkommen und Einkommensarten wie Arbeits- und Gewinneinkommen, sozialversicherungsrechtliche Lohnersatzleistungen, Transfers und private Übertragungen nicht ausreichen, um das jeweilige Grundsicherungsniveau abzudecken. Aber durch die Anerkennung von Freibeträgen bei bestimmten Einkommensarten wird das Bedarfsniveau der Grundsicherung durch das anrechnungsfreie Einkommen noch aufgestockt. Freibeträge gibt es unter anderem

- im SGB II (Grundsicherung für Arbeitsuchende) für Leistungsempfänger:innen, die erwerbstätig sind oder eine Erwerbstätigkeit aufnehmen. Ziel ist es, Anreize für eine Aufnahme bzw. Beibehaltung einer Erwerbstätigkeit zu geben. Erwerbstätige Hilfeempfänger:innen stellen sich dadurch in ihrem Gesamteinkommen immer etwas besser als nicht erwerbstätige Hilfeempfänger:innen - Erwerbstätigkeit soll sich lohnen.

- im SGB XII (Grundsicherung im Alter und bei Erwerbsminderung) für Rentner:innen, die Leistungen aus einer zusätzlichen Altersvorsorge erhalten (seit 2018) und für Rentner:innen, die eine gesetzliche Altersrente beziehen (ab $2021)^{6}$. Ziel dieser Regelungen ist es, jene älteren oder erwerbsgeminderten Menschen, die vorgesorgt und Beiträge entrichtet haben, besserzustellen als jene, die dies nicht getan haben - Altersvorsorge soll sich lohnen.

Hier deutet sich an, dass die eigentlich vorleistungsunabhängige Grundsicherung faktisch zu einer vorleistungsabhängigen Leistung mutiert und das Existenzminimum unterschiedlich hoch ausfällt. Zudem kommt es je nach Grundsicherungszweig zu unterschiedlichen Vermögensfreibeträgen und damit zu einer unterschiedlich starken Anrechnung von Vermögen.

Im Ergebnis lassen sich die Teilsysteme der Grundsicherung hinsichtlich des anerkannten soziokulturellen Existenzminimums als sozial hierarchisiert bezeichnen: Am oberen Ende der Hierarchie stehen die Empfänger:innen von Grundsicherung im Alter und hier insbesondere jene, die Vorleistungen erbracht haben;

6 | Im Unterschied zur zusätzlichen Altersvorsorge ist der Anspruch hier allerdings auf Personen begrenzt, die auf 33 Jahre Grundrentenzeiten (Pflichtbeitragszeiten bei Erwerbstätigkeit und u.a. Zeiten der Kindererziehung und Pflege) kommen. 
am unteren Ende stehen die Empfänger:innen von Leistungen nach dem Asylbewerberleistungsgesetz, deren Leistungen gegenüber den Regelbedarfen noch abgesenkt sind.

Grundsicherung muss beantragt werden. Dies erfolgt aber nicht flächendeckend, sondern hoch selektiv. Über die Dunkelziffer der Nicht-Inanspruchnahme, also die Größenordnung der Personen, die aufgrund ihres niedrigen Einkommens zwar einen Leistungsanspruch haben, diesen aber nicht wahrnehmen, gibt es empirisch fundierte Schätzungen (zu neueren Berechnung vgl. Buslei/ Geyer et al 2019).

Die Ursachen für diese Nicht-Inanspruchnahme sind vielfältig: Die Betroffenen sind oft unzureichend informiert, ob sie noch einen Anspruch auf aufstockende Leistungen haben - dies insbesondere dann, wenn der Anspruch eher gering ist. Und nach wie vor herrschen Angst und Sorge, dass der Gang zum zuständigen Amt und die Einkommens- und Vermögensprüfung zum sozialen Stigma werden. Auch ist fraglich, ob der weitgehende Wegfall des Rückgriffs auf die Kinder den Betroffenen tatsächlich bekannt ist.

\section{Grundsicherung im Alter und Rentenversicherung}

In der wissenschaftlichen und politischen Diskussion über das Verhältnis zwischen Rentenversicherung und Grundsicherung spielt das Problem der Altersarmut eine entscheidende Rolle. Es dominiert die Befürchtung, dass das Risiko steigt, am Ende des Erwerbslebens nur eine niedrige Rente zu erhalten und auf aufstockende Leistung der Grundsicherung im Alter angewiesen zu sein. Eine niedrige Rente ist allerdings noch kein Indikator für eine finanzielle Notlage und für einen Anspruch auf Grundsicherung, da es auf das gesamte Alterseinkommen und bei (Ehe-)Paaren auch auf das Partnereinkommen ankommt.

Gleichwohl haben sich die Empfängerzahlen der Grundsicherung in den zurückliegenden Jahren deutlich erhöht. Ende 2019 erhielten knapp 1,1 Millionen Personen Leistungen der Grundsicherung im Alter und bei Erwerbsminderung - dies entspricht einem Anstieg um rund 150 Prozent gegenüber 2003 (Daten laut DRV 2020).

Die Empfänger:innen sind ungefähr je zur Hälfte Personen, die die Regelaltersgrenze erreicht haben, und Personen im Alter zwischen 18 Jahren und der Regelaltersgrenze mit einer dauerhaften vollen Erwerbsminderung. 14,7 Prozent aller Erwerbsminderungsrentner:innen und 2,6 Prozent der Altersrentner:innen erhalten eine Aufstockung durch die Grundsicherung. 
Setzt man die Zahlen der Grundsicherungsempfänger:innen allerdings ins Verhältnis zur entsprechenden Bevölkerung, so ergeben sich bislang lediglich geringe Empfängerquoten. Im Jahr 2019 mussten insgesamt nur 3,2 Prozent der älteren Bevölkerung (jenseits der Regelaltersgrenze) auf die Grundsicherung zurückgreifen. Die Daten zum aktuellen Ausmaß des Grundsicherungsbezugs sagen jedoch noch nichts über die zukünttigen Entwicklungen aus. Die (noch) niedrige Betroffenheit kann verdecken, dass ein Trend eingesetzt hat, der auf mittlere und längere Frist zu einem deutlichen Anstieg der absoluten Zahlen und Quoten des Grundsicherungsbezugs führt (vgl. Geyer et al. 2019).

In welchem Maße dies eintritt, hängt im Wesentlichen von zwei Faktoren ab. Zum einen kommt es darauf an, wie sich die Erwerbsbiografien und damit die individuellen Rentenanwartschaften der nachrückenden Kohorten entwickeln; zum anderen spielen die Auswirkungen eine Rolle, wenn die Renten infolge der Dämpfungsfaktoren in der Rentenanpassungsformel nur noch begrenzt der Lohnentwicklung folgen und dementsprechend das Rentenniveau kontinuierlich sinkt.

Hinsichtlich der Entwicklungsrichtung der Rentenanwartschaften lässt sich erkennen, dass sich in den zurückliegenden Jahren aufgrund der hohen Arbeitslosigkeit und der Umbrüche in den Beschäftigungsverhältnissen ein Risiko- und Problempotenzial aufgebaut hat, das sich negativ auf die Höhe der persönlichen Entgeltpunkte auswirkt. Bei einer größeren Zahl von Beschäftigten werden beim Renteneintritt Lücken in der Versicherungsbiografie und nur geringe Rentenanwartschaften zu verzeichnen sein.

Dazu kommt es vor allem dann, wenn es sich bei Arbeitslosigkeit nicht nur um eine kurzfristige und einmalige Episode handelt, sondern der Ausschluss aus dem Erwerbssystem für längere Zeit andauert und/oder die berufliche Wiedereingliederung nicht dauerhaft oder nur unterwertig gelingt. Dies ist auch deswegen besonders nachteilig, weil rund zwei Drittel aller Arbeitslosen in den Rechtskreis des SGB II fallen (siehe Abschnitt 6) und beim bedürftigkeitsgeprüften ALG II keine Rentenversicherungsbeiträge bezahlt werden. An Bedeutung gewonnen haben darüber hinaus diskontinuierliche Erwerbsverläufe, die durch mehrfache Wechsel zwischen Erwerbstätigkeit und Arbeitslosigkeit, regulärer und prekärer Beschäftigung sowie abhängiger und selbstständiger Arbeit charakterisiert sind.

Schließlich führt die Ausweitung des Niedriglohnsegments absehbar zu Niedrigrenten. Besonders problematisch ist es, wenn Niedriglöhne mit Arbeitszeiten einhergehen, die den Vollzeitstandard deutlich unterschreiten, denn eine Arbeitszeit im unteren Stundenbereich wird selbst bei Stundenlöhnen, die im mittleren Bereich liegen, nur zu einem geringen, unter dem Durchschnitt liegenden Monatseinkommen führen. Auf dem Arbeitsmarkt lassen sich weitere Veränderun- 
gen erkennen: Die Zahl der nicht obligatorisch abgesicherten Selbstständigen, insbesondere der Solo-Selbstständigen, dürfte vor dem Hintergrund von Digitalisierung und Dienstleistungsorientierung absehbar zunehmen. Zudem verschwimmen die Grenzen zwischen abhängiger und selbstständiger Beschäftigung.

Besonderen Absicherungsrisiken im Alter unterliegen schließlich all jene Zugewanderten, die in ihren Herkunftsländern keine Rentenanwartschaften erworben haben (bzw. deren Anwartschaften hier nicht anerkannt werden) und die in Deutschland erst langsam in den Arbeitsmarkt integriert werden und eine sozialversicherungspflichtige Beschäftigung aufnehmen. Hinsichtlich Versicherungsdauer und Einkommensposition werden sie kaum in der Lage sein, eine ausreichende Zahl von Entgeltpunkten zu erreichen.

Zwar lassen die steigende Frauenerwerbstätigkeit und die Verkürzung der erziehungsbedingten Unterbrechungszeiten erwarten, dass sich die Rentenanwartschaften von Frauen zukünftig erhöhen. Auch die rentenrechtliche Anrechnung von Pflegezeiten und Kindererziehungszeiten trägt dazu bei. Die Alterseinkommen von Ehepaaren könnten insofern stabilisiert werden, wenn den rückläufigen Anwartschaften der Männer steigende Anwartschaften der Frauen gegenüberstehen. Es ist aber schwierig abzuschätzen, ob und in welchem Maße die Entspannung auf dem Arbeitsmarkt bei den Rentenneuzugängen der nächsten Jahre zu einer Abschwächung des Trends zu niedrigen Entgeltpunkten und auf längere Sicht zu dessen Umkehr führen können.

Auch hat sich der Trend zum beruflichen Frühausstieg umgekehrt: Die Erwerbstätigenquoten bei älteren Arbeitnehmer:innen haben sich beträchtlich erhöht (vgl. Kaboth/Drescher/Brussig 2020). Die Beschättigten bleiben länger berufstätig, und das Renteneintrittsalter verschiebt sich nach oben - mit dem Ergebnis, dass mehr Entgeltpunkte erreicht werden können. Zwar betriff dies keineswegs alle Beschäftigten, denn gerade die Niedrigqualifizierten, die im Verlauf ihres Erwerbslebens unter belastenden Bedingungen gearbeitet haben und häufig durch Arbeitslosigkeit unterbrochene Berufsverläufe aufweisen, scheiden mehrheitlich vor Erreichen der Regelaltersgrenze aus dem Beruf aus. Gleichwohl ist der Trend zu einem späteren Altersübergang eindeutig.

Wenn es um die Abschätzung nicht nur der zukünftigen Höhe und Verteilung der Entgeltpunkte, sondern auch der zukünftigen Rentenzahlbeträge geht, spielt die Höhe und Dynamik des aktuellen Rentenwerts eine zentrale Rolle. Es kommt zu einem Doppeleffekt: Die Anwartschaften/Entgeltpunkte werden in vielen - aber eben nicht allen - Fällen zurückgehen, und zugleich verringert sich deren Wert durch das absinkende Rentenniveau.

Unter diesen Bedingungen müssen die Versicherten immer mehr Versicherungsjahre aufbringen, um eine Rente zu erhalten, die das Bedarfsniveau der 
Grundsicherung im Alter erreicht. Oder andersherum betrachtet: Selbst eine lange Versicherungsdauer führt dann bei Versicherten, deren Einkommensposition unterhalb des Durchschnitts liegt, nicht mehr dazu, dass ihre Rente die Grundsicherungsschwelle überschreitet (Blank/Türk 2018; Bäcker 2019b).

Dieses Problem einer Überschneidung von niedrigen Renten und Grundsicherungsniveau auch bei langjähriger Beitragszahlung wird durch die Einführung des ab 2021 geltenden Grundrentengesetzes gemildert: Langjährig Versicherte $^{7}$ erhalten durch die Hochwertung der Entgeltpunkte zwischen 0,3 und maximal 0,8 Entgeltpunkten pro Jahr Grundrentenbewertungszeit einen $\mathrm{Zu}$ schlag auf ihre Rente. ${ }^{8}$ Damit wird es leichter sein, eine den Grundsicherungsbedarf überschreitende individuelle Rentenhöhe zu erreichen.

Auf der anderen Seite führen die oben beschriebenen Freibeträge bei der Anrechnung des Einkommens dazu, dass mehr Rentner:innen berechtigt sind, eine aufstockende Grundsicherung zu erhalten. Das Gesamteinkommen für langjährig Versicherte liegt damit höher als das für Ältere, die diese Voraussetzung - aus welchen Gründen auch immer - nicht erfüllen.

Durch diese Gesetzgebung kommt es zu einer mehrfachen Paradoxie: Die Zahl der Grundsicherungsempfänger:innen wird zugleich begrenzt und erhöht, und die eigentlich vorleistungsunabhängige Grundsicherung entwickelt sich durch die Freibeträge zu einem vorleistungsabhängigen System. Außerdem wird in der Rentenversicherung die Zahlung eines Grundrentenzuschlags von einer Einkommensprüfung abhängig gemacht. ${ }^{9}$ Die eigentlich einkommensunabhängige Versicherungsleistung entwickelt sich zu einer einkommensgeprüf-

7 | Langjährig Versicherte sind in diesem Zusammenhang Versicherte mit einer Grundrentenzeit von mindestens 33 Jahren.

8 | Um die Komplexität der Regelung zu verdeutlichen: Die erworbenen Entgeltpunkte werden auf maximal 0,8 Entgeltpunkte pro Jahr und für maximal 35 Jahre verdoppelt. Der ermittelte Wert wird danach wieder um 12,5 Prozent gekürzt. Aus dem Zuschlag an Entgeltpunkten errechnet sich durch Multiplikation mit dem aktuellen Rentenwert der Grundrentenzuschlag in Euro.

9 | Auch hier die Details: Auf den Grundrentenzuschlag wird das "zu versteuernde Einkommen« des/der Versicherten und seiner Ehefrau/ihres Ehemanns bzw. bei eingetragenen Lebenspartnerschaften seines Partners/ihrer Partnerin angerechnet. Grundlage ist der Steuerbescheid des vorvergangenen Kalenderjahrs. Angerechnet werden bei alleinstehenden Personen alle Einkommen, die den Freibetrag vom 1.250 Euro übersteigen, zu 60 Prozent. Eine volle Anrechnung erfolgt bei einem Einkommen von über 1.600 Euro. Bei Ehepaaren bzw. Lebenspartnerschaften liegt der Freibetrag bei 1.950 Euro, eine volle Anrechnung erfolgt bei einem Einkommen von über 2.300 Euro. Die Freibeträge werden jährlich angepasst; eine Vermögensüberprüfung findet nicht statt. 
ten Leistung. Damit werden die Grundprinzipien von Versicherung und Grundsicherung verletzt, der Weg geht hin zu einer Verschmelzung von Renten und Grundsicherung (Steffen 2017).

Es ist zu befürchten, dass dieser Prozess dazu führen wird, eine Absenkung des Rentenniveaus nach 2025 zu rechtfertigen, da sich die Folgewirkungen für den Bereich niedriger Renten als weniger dramatisch erweisen. An den Rand gedrängt wird damit aber das Ziel der Lebensstandardsicherung im Bereich mittlerer und auch höherer Einkommen bzw. Renten; der Weg hin zu einer Ausdehnung der privaten und/oder betrieblichen Altersvorsorge, die die Versorgungslücken der gesetzlichen Rente auffüllt, ist vorgezeichnet (vgl. Bäcker 2020).

Die Grundrente macht deshalb die Notwendigkeit einer Stabilisierung des Rentenniveaus auch über 2025 hinaus keineswegs überflüssig, denn Alterssicherung ist mehr als die Vermeidung von Armut. Die Übertragung der Aufgabe einer Lebensstandardsicherung an kapitalbasierte Vorsorgesysteme hat sich als untauglich erwiesen und macht die Alterssicherung abhängig von den Turbulenzen auf den Kapital- und Aktienmärkten.

Die Höhe des zukünftigen Rentenniveaus ist zugleich entscheidend für das Ziel der Armutsvermeidung, da der Wert der durch die Grundrente aufgestockten niedrigen Entgeltpunkte vom Rentenniveau abhängt (vgl. Buslei/Fischer et al. 2019). Dies gilt gleichermaßen für die Forderung, Zeiten der Arbeitslosigkeit im SGBII-Bezug besser abzusichern; und auch die Perspektive einer Erwerbstätigenversicherung, die alle Selbstständigen miteinbezieht, kann nur überzeugen, wenn den Beitragszahlungen der Betroffenen auch angemessene Renten(anwartschaften) gegenüberstehen.

\section{Grundsicherung für Arbeitsuchende und Arbeitslosenversicherung}

Das SGB II ist ein umfassendes sozialpolitisches Gesetz, das für nahezu die gesamte Bevölkerung das Existenzminimum sicherstellen soll, nämlich für alle, die erwerbsfähig sind und die Regelaltersgrenze noch nicht erreicht haben. Damit wendet sich die Leistung nicht nur an Arbeitslose: Von den insgesamt 5,4 Millionen Regelleistungsberechtigten nach SGB II waren 2020 nur knapp 29 Prozent arbeitslos und 71 Prozent nicht arbeitslos (BA 2021). Darunter befinden sich in erster Linie Kinder und Personen in Ausbildung, in Maßnahmen der Arbeitsförderung und in Erwerbstätigkeit (Aufstocker:innen) sowie Alleinerziehende, denen eine Erwerbstätigkeit nicht zugemutet wird. Die Bezeichnung des Gesetzes »für Arbeitsuchende« führt insofern in die Irre. 
Betrachtet man nur die Arbeitslosen, so fielen im Jahr 2020 knapp 60 Prozent der registrierten Arbeitslosen in den Rechtskreis des SGB II und nur noch ein Drittel in den des SGB III (BA 2020). Die Arbeitslosenversicherung spielt hinsichtlich der sozialen Absicherung bei Arbeitslosigkeit also nur noch eine nachrangige Rolle. Der Tatbestand, dass eine Arbeitslosenversicherung nicht alle Arbeitslosen erfasst bzw. erfassen kann, ist nicht neu, denn gemäß dem Versicherungsprinzip ist der Leistungsbezug nicht nur vom Eintritt des Risikos, sondern auch von der Erfüllung von Anspruchsvoraussetzungen abhängig. Zudem ist die Leistungsdauer befristet.

Bei gegebenen Anspruchsvoraussetzungen (Wartezeit und Rahmenfrist) und bei gegebenen Regelungen der Höchstleistungsdauern kommt es bei anhaltender Arbeitslosigkeit und insbesondere bei einem hohen Sockel von Langzeitarbeitslosen dazu, dass ein wachsender Teil der Arbeitslosen die Leistungsvoraussetzungen nicht erfüllt oder die Leistungsdauer überschreitet. Wenn sich zudem auf dem Arbeitsmarkt Arbeitsverhältnisse ausbreiten, die nur von kurzer Dauer sind (Befristungen und Leiharbeit) oder die nicht der Versicherungspflicht unterliegen (so vor allem Minijobs), erhöht sich das Risiko, keine Leistungen aus der Arbeitslosenversicherung beanspruchen zu können.

Mit der Einführung des SGB II wurde 2005 die Arbeitslosenhilfe ersatzlos abgeschafft. Die gängige Formulierung, dass durch das Vierte Gesetz für moderne Dienstleistungen am Arbeitsmarkt (Hartz IV) die Arbeitslosenhilfe mit der Sozialhilfe zusammengeführt worden sei, legt nahe, dass zentrale Merkmale der Arbeitslosenhilfe - so vor allem Individualprinzip, Bemessung am vorherigen Arbeitsentgelt und eine Einkommens-, aber keine Bedürftigkeitsprüfung - beibehalten worden seien. Dies ist aber gerade nicht der Fall: Das neu eingeführte ALG II entsprach und entspricht vielmehr weitgehend den Bestimmungen der bisherigen Hilfe zum Lebensunterhalt nach dem BSHG.

Hinzu kommt, dass in einer Art Doppelstrategie zeitgleich mit dem Hartz-IVGesetz durch das Gesetz zu Reformen am Arbeitsmarkt auch die Leistungsvoraussetzungen und -bedingungen des ALG I erheblich verschlechtert wurden:

- Verkürzung der maximalen Leistungsdauer auf Arbeitslosengeldzahlung auf 12 Monate, wenn ein Versicherungspflichtverhältnis von 24 Monaten erfüllt ist. Der verlängerte Arbeitslosengeldbezug für Ältere (über 55 Jahre) wurde auf 18 Monate verkürzt. Vordem konnten Versicherte im Maximum - nach Vollendung des 57. Lebensjahrs - bis zu 32 Monate ALG I beziehen.

- Verkürzung der Rahmenfrist einer versicherungsplichtigen Beschäftigung von 36 Monaten auf mindestens 24 Monate. 
Im Ergebnis haben sich damit durch politische Entscheidungen Schutzwirkung und Reichweite der Arbeitslosenversicherung massiv verringert. Wesentliche Veränderungen im Bereich der Arbeitslosenversicherung hat es bis auf eine Ausnahme seitdem nicht mehr gegeben. Die Ausnahme betrifft die Verlängerung der maximalen Bezugsdauer von ALG I für Ältere (nach Vollendung des 58. Lebensjahrs) auf 24 Monate ab 2008.

Die Schutzwirkung der Arbeitslosenversicherung begrenzt sich insofern auf den besser gestellten, anteilig aber immer kleiner werdenden Kreis der Arbeitslosen, die aus einer versicherungspflichtigen Beschäftigung heraus arbeitslos werden, die Anwartschaftszeit und Rahmenfrist erfüllen und ihre Arbeitslosigkeit zügig beenden. Langzeitarbeitslose sind deshalb weit überwiegend dem SGB II zugeordnet.

Allerdings ist zu berücksichtigen, dass längst nicht alle Arbeitslosen, die in der BA-Statistik dem Rechtskreis des SGB III zugeordnet sind, auch die Versicherungsleistung ALG I erhalten. 2019 zählten rund 20 Prozent der SGB-IIIArbeitslosen zu den Nichtleistungsberechtigten; dies vor allem deswegen, weil wegen fehlender Bedürftigkeit kein Anspruch auf ALG II bestand. Insbesondere Ehefrauen sind davon betroffen.

Auf der anderen Seite kommt es dazu, dass aufgrund der niedrigen Ersatzrate beim ALG I ein ergänzender ALG-II-Anspruch besteht, weil das ALG I (im Kontext des Einkommens der Bedarfsgemeinschaft) nicht existenzsichernd ist. Die Daten der Bundesagentur für Arbeit zeigen, dass 26,6 Prozent der Frauen und 10,6 Prozent der Männer ein ALG I von weniger als 600 Euro erhalten (November 2020; Sozialpolitik-aktuell.de o.J.). Daher kann es nicht verwundern, dass rund 10 Prozent aller ALG-I-Empfänger:innen zu dieser Aufstockung veranlasst sind (Sozialpolitik-aktuell.de o.J.). ${ }^{10}$

Für jene Arbeitslosen, die trotz zum Teil langjähriger Beitragszahlung auf Hartz IV verwiesen und auf ein Grundsicherungsniveau herabgestuft werden, erwachsen daraus Gerechtigkeitsprobleme: Sie sehen sich mit denjenigen gleichgestellt, die noch überhaupt keine Beiträge gezahlt haben. Die Logik der Leistungsgerechtigkeit wird abrupt abgelöst durch die Logik einer Bedarfsgerechtigkeit auf unterem Niveau, nach der die frühere Erwerbstätigkeit, die

10 | Besondere Schnittstellenprobleme zwischen Versicherung (ALG-I-Bezug) und Grundsicherung (ALG-II-Bezug) ergeben sich immer dann, wenn sich im Verlauf der Arbeitslosigkeit die Einkommenslage des Haushalts/der Bedarfsgemeinschaft ändert, z. B. wenn ein anderes Haushaltsmitglied seine Stelle verliert oder im umgekehrten Fall wieder eine Beschäftigung findet. Dann kommt es zu einem »Hin und Her« zwischen SGB III und SGB II bzw. Arbeitsagentur und Jobcenter. 
Qualifikation und der erreichte berufliche Status keine Bedeutung mehr haben.

Die soziale Hierarchisierung zwischen den bessergestellten Arbeitslosen in der Arbeitslosenversicherung des SGB III und den schlechtergestellten Arbeitslosen im Fürsorgesystem SGB II bezieht sich allerdings nicht nur auf die soziale Absicherung, sondern auch auf den Zugang in arbeitsmarktpolitische Maßnahmen und die Chance auf eine nachhaltige Wiedereingliederung in den ersten Arbeitsmarkt (Arbeitskreis Arbeitmarktpolitik/Knuth 2018). Denn im Unterschied zum SGB III gilt nach dem SGB II jede Arbeit als zumutbar, die nicht gegen Gesetze oder gute Sitten verstößt, ungeachtet des vorherigen Einkommens oder der Qualifikation. Eine tarif- oder ortsübliche Entlohnung ist ebenfalls kein Maßstab für die Zumutbarkeit. Durch die erzwungene Aufnahme einer unterwertigen, nicht qualifikationsgerechten Beschäftigung droht die Gefahr eines beruflichen und sozialen Abstiegs. Das ALG II erweist sich weder als »Abfederung« noch als »Sprungbrett« nach oben.

Grundproblem ist, dass das ALG II keinen Bezug zum vormaligen Erwerbseinkommen aufweist und dass es kein Verbindungsglied zwischen Versicherung und Fürsorge gibt. Deshalb muss es darum gehen, die Schutzwirkung der Arbeitslosenversicherung auch "quantitativ« auszudehnen, sodass wieder mehr Arbeitslose, die jetzt trotz einer Beitragszahlung leer ausgehen, Anspruch auf eine Versicherungsleistung haben. Setzt sich hingegen der Bedeutungsverlust fort, verliert eine Pflichtversicherung mit einer nur noch geringen Schutzwirkung ihre Legitimation und Akzeptanz. Um dies zu verhindern, bieten sich mehrere Ansatzpunkte (vgl. Bäcker 2019a):

- Verlängerung der Rahmenfrist auf drei Jahre und Verkürzung der Anwartschaftszeit von zwölf Monaten auf sechs Monate: Arbeitnehmer:innen, die bereits nach einer kurzfristigen Beschäftigung ihren Arbeitsplatz verlieren, hätten dann zumindest einen Anspruch auf dreimonatigen Bezug von ALG I.

- Verlängerung der Bezugsdauer des ALG I: Vor allem Arbeitslose im mittleren und höheren Lebensalter sollten erst später aus der Arbeitslosenversicherung ausgesteuert werden. Belege für die These, dass eine Verlängerung des ALG-I-Bezugs die Motivation zur Arbeitsaufnahme und Wiedereingliederung in den Arbeitsmarkt schwäche, gibt es nicht. Durch eine verlängerte, nach Vorversicherungszeiten gestaffelte Bezugsdauer kann gerade für langjährige Beitragszahler:innen die Schutzwirkung der Versicherung ausgedehnt und die Grundsicherung entlastet werden. Ein Weg könnte sein, ein verlängertes »Arbeitslosengeld $\mathrm{Q}$ « einzuführen. Arbeitslosen, die nicht innerhalb von drei Monaten eine neue Stelle finden, sollte die Arbeitsagentur danach eine Qua- 
lifizierungsmaßnahme anbieten und für die Dauer dieser Weiterbildung ein verlängertes reguläres Arbeitslosengeld zahlen.

Das Gerechtigkeitsproblem, dass Arbeitslose, die langjährig Beiträge gezahlt haben, nach dem Auslaufen des Anspruchs auf ALG I auf dasselbe Leistungsniveau der Grundsicherung verwiesen werden wie Personen, die neu in den Arbeitsmarkt einsteigen, erweist sich bei einem Versicherungssystem mit begrenzter Bezugsdauer als systemimmanent. Es mindert sich, wenn die maximale Bezugsdauer des ALG I heraufgesetzt wird, löst sich aber nicht auf. Die vormalige Arbeitslosenhilfe war eine Antwort auf dieses Problem. Zwar geht es nicht darum, sie wiederherzustellen, aber es sollte geprüft werden, ob eine zeitlich befristete »Zwischenleistung« zwischen Versicherung und Fürsorge eingeführt werden könnte.

\section{Reformen: Schritte in die richtige oder falsche Richtung?}

Die in diesem Beitrag skizzierten Reformvorschläge sind kleinteilig, bewegen sich im Rahmen des Zusammenspiels von Sozialversicherung und Grundsicherung und stellen darauf ab, die Einkommenssicherung und den Sozialschutz der Bevölkerung durch die Veränderung mehrerer "Stellschrauben« zu verbessern. Sie unterscheiden sich damit grundlegend von der weitreichenden, gleichsam revolutionären Idee eines bedingungslosen Grundeinkommens (BGE; zu den Modellen vgl. Butterwegge/Rinke 2018).

Diese Forderung nach einem pauschalen Grundeinkommen für alle - ohne Voraussetzungen, ohne Anrechnung von Einkommen und Vermögen, ohne Bereitschaft zur Arbeitsaufnahme und ohne Sanktionen, und dies in einer Höhe des Maximalniveaus der Grundsicherung (Regelbedarfe, Kosten der Unterkunft, Mehrbedarfe, Einmalleistungen) - klingt attraktiv und findet großen Anklang. Betrachtet man die Modelle und deren Folgen allerdings näher, würde ein BGE tatsächlich eine Umorientierung der Gesellschaft in Richtung eines residualen Sozialstaats einleiten, denn die Finanzierung eines hohen BGE mit Kosten von mehr als 1 Billion Euro ist überhaupt nur möglich, wenn gleichzeitig die Steuern erhöht und die bisherigen Sozialleistungen zusammengestrichen würden. ${ }^{11}$

11 | Bei einem Pauschalbetrag von rund 1.100 Euro, der an die Gesamtbevölkerung (83 Millionen in 2019) ausgezahlt wird, summieren sich die Ausgaben auf rund 1 Billion Euro. Das entspricht in etwa dem Betrag aller Sozialleistungen (Sozialbudget). Das Volkseinkommen, das nach der Systematik der volkswirtschaftlichen Gesamtrechnung als Verteilungssumme zur Verfügung steht, erreicht einen Wert von etwa 2,6 Billionen 
In den Finanzierungsrechnungen eines BGE wird üblicherweise davon ausgegangen, dass sich die Förder- und Fürsorgesysteme wie ALG II, Sozialgeld, Kinderzuschlag, Sozialhilfe, Eingliederungshilfe, Teilhabeleistungen, Wohngeld, Kindergeld, Ausbildungsförderung und Elterngeld zu einem einzelnen Grundeinkommen zusammenfassen lassen. Das reicht aber bei Weitem nicht, denn damit könnten »nur« rund 180 Milliarden Euro eingespart werden.

Vernachlässigt wird bei dieser Vorstellung zudem, dass sich die Förder- und Fürsorgesysteme keineswegs auf die Zahlung von Geldleistungen beschränken. Denn so wichtig ein ausreichend hohes Einkommen auch ist, zur Vermeidung von Armut und sozialer Ausgrenzung sowie zur Sicherstellung von sozialer Teilhabe und gesellschaftlicher Integration bedarf es mehr als der Zahlung von Geldbeträgen. Deswegen spielen die Angebote an sozialen Dienstleistungen und Einrichtungen eine zentrale Rolle im Bereich der Kinder- und Jugendhilfe, der Sozialhilfe, der Grundsicherung für Arbeitsuchende sowie der Rehabilitation und Teilhabe von Menschen mit Behinderung.

Erforderlich ist ein breites, auf unterschiedliche Lebenslagen und -phasen abgestelltes Spektrum von Förderung, Beratung, Unterstützung, Vermittlung, Betreuung, Erziehung und Bildung. Die Kritik an der Ausrichtung des SGB II lautet ja gerade, dass die im Kontext des Ansatzes von »Fördern und Fordern« praktizierte Politik die Förderelemente vernachlässigt.

Da sich das zur Verteilung stehende Volkseinkommen nicht plötzlich durch ein BGE erhöht, müssen die erforderlichen Finanzierungsbeträge durch zusätzliche Steuern und auch durch Leistungskürzungen bei der Sozialversicherung aufgebracht werden. Im Ergebnis zeigt sich, dass von »Bedingungslosigkeit« im Sinne von Einkommensunabhängigkeit keine Rede sein kann. Diejenigen, die über ein ausreichendes Erwerbseinkommen verfügen, würden zwar zunächst das BGE erhalten, müssten aber im Gegenzug hohe Steuern abführen.

Insofern kann es nicht überraschen, dass neoliberale Modelle eines bedingungslosen Grundeinkommens von Leistungssätzen ausgehen, die noch deutlich unterhalb des aktuellen Bedarfsniveaus liegen. Die Menschen sind dadurch gehalten - nicht administrativ durch Zumutbarkeits- und Sanktionsregelungen, sondern durch ökonomischen Zwang - hinzuzuverdienen, um das sozial-kulturelle Existenzminimum zu erreichen. Hinzu kommt die Vorstellung, dass mit

Euro. Das gesamte Steueraufkommen liegt »nur« bei 800 Milliarden Euro (in 2019) und muss für alle Ausgaben von Bund, Ländern und Gemeinden eingesetzt werden - also für Bildung, innere und äußere Sicherheit, Verwaltung und Personal, Infrastruktur, Verkehr, Umwelt, Zuschüsse an die Sozialversicherungsträger etc. (zu den Berechnungen vgl. Bäcker 2017). 
der Zahlung eines pauschalen Geldbetrags auch das Sicherungsnetz der Sozialversicherung nahezu ersetzt werden soll (vgl. u. a. Straubhaar 2017). Vorgeschlagen wird, die Leistungen auf ein Mindest- oder Basisniveau zu senken, um den Angeboten der Privatversicherungen einen größeren Raum zu geben.

Einem solchen Szenario ist die Perspektive einer Stärkung der Sozialversicherung entgegenzustellen - hinsichtlich des erfassten Personenkreises in Richtung einer Erwerbstätigen- und Bürgerversicherung sowie bezüglich Anspruchsvoraussetzungen, -dauer und Höhe des Leistungsniveaus. Auf der Seite der Grundsicherung muss vor allem auf die Sicherstellung eines ausreichenden Bedarfsniveaus und eine die Menschenwürde achtenden Regelung von Sanktionen und Zumutbarkeitsanforderungen abgestellt werden. Ziel ist hier die Gewährleistung einer Teilhabe aller Menschen an Gesellschaft und Arbeitsmarkt aber eben nicht deren Daueralimentation.

\section{Literatur}

Arbeitskreis Arbeitsmarktpolitik (Hrsg.)/Knuth, M. (2018): Solidarische und sozialinvestive Arbeitsmarktpolitik. Vorschläge des Arbeitskreises Arbeitsmarktpolitik, Study Nr. 374 der Hans-Böckler-Stiftung, Düsseldorf.

BA = Bundesagentur für Arbeit (2020): Arbeitslose - Zeitreihe, Berichtsmonat Dezember 2020, Nürnberg.

BA = Bundesagentur für Arbeit (2021): Strukturen der Grundsicherung SGB II (Zeitreihe Monats- und Jahreszahlen ab 2005), Berichtsmonat März 2021, Nürnberg.

Bäcker, G. (2017): Das bedingungslose Grundeinkommen: Ein Traum und die harte Wirklichkeit, in: Soziale Sicherheit 12/2017, S. 452-460.

Bäcker, G. (2019a): Arbeitslosenversicherung stärken! Sozialgesetzbuch III und II harmonisieren!, in: Wirtschaftsdienst 4/2019, S. 252-255.

Bäcker, G. (2019b): Mindestsicherungselemente im Spannungsfeld von Versicherung, Versorgung und Fürsorge, in: Deutsche Rentenversicherung 3/2019, S. 206-221.

Bäcker, G. (2020): Rentenversicherung oder Kapitalmarkt?, in: Blank, F./Hofmann, M./Buntenbach, A. (Hrsg.): Neustart in der Rentenpolitik, Baden-Baden, S. 25-46.

Bäcker, G./Naegele, G./Bispinck, R. (2020): Sozialpolitik und soziale Lage in Deutschland, 6. Auflage, Wiesbaden.

Blank, F./Türk, E. (2018): Die Grundsicherung im Alter im Zusammenspiel mit der Rentenversicherung, in: Gesundheits- und Sozialpolitik 2/2018, S. 47-54. 
BMAS = Bundesministerium für Arbeit und Soziales (2020): Sozialbudget 2019, Berlin.

Buslei, H./Fischer, B./Geyer, J./Hammerschmid, A (2019): Das Rentenniveau spielt eine wesentliche Rolle für das Armutsrisiko im Alter, in: DIW Wochenbericht 21+22/2019, S. 375-383.

Buslei, H./Geyer, J./Haan, P./Harnisch, M. (2019): Starke Nichtinanspruchnahme von Grundsicherung deutet auf hohe verdeckte Altersarmut, in: DIW Wochenbericht 49/2019.

Butterwegge, C./Rinke, K. (Hrsg.) (2018): Grundeinkommen kontrovers, Weinheim.

DRV = Deutsche Rentenversicherung Bund (2020): Rentenversicherung in Zeitreihen, Berlin.

Geyer, J. (Projektleitung)/Buslei, H./ Gallego-Granados, P./Haan, P. (2019): Anstieg der Altersarmut in Deutschland: Wie wirken verschiedene Rentenreformen?, Bertelsmann Stiftung, Gütersloh.

Hönig, R. (2020): Der Abstand der Rente zur Grundsicherung, Münster u.a.

Kaboth, A./Drescher, S./Brussig, M. (2020): Erwerbstätigkeit im Alter und der Übergang in Rente - ein Überblick, in: Blank, F./Hofmann, M./Buntenbach, A. (Hrsg.): Neustart in der Rentenpolitik, Baden-Baden, S. 101-118.

Kaltenborn, B. (2019): Grundsicherung im Alter und bei Erwerbsminderung: ein statistisches Kompendium, DRV-Schriften, Band 118, Berlin.

Kohl, J. (2017): Akzeptanz des Wohlfahrtsstaates, Friedrich-Ebert-Stiftung, Bonn. Schäfer, I. (2014): Die Vollendung eines Paradigmenwechsels, in: Vierteljahreshefte zur Wirtschaftsforschung 2/2014, S. 21-32.

Sozialpolitik-aktuell.de (o.J.): Verteilung von Arbeitslosengeld I, Männer und Frauen 2020, www.sozialpolitik-aktuell.de/files/sozialpolitik-aktuell/_Politikfelder/ Arbeitsmarkt/Datensammlung/PDF-Dateien/abbIV53.pdf (Abruf am 23.7.2021).

Steffen, J. (2017): Rente und Grundsicherung. Prozess systemischer Verschmelzung, www.portal-sozialpolitik.de, 1/2017.

Steffen, J. (2020): Grundrente - Die drei Komponenten. Ein widersprüchliches Zusammenspiel, www.portal-sozialpolitik.de/index.php?page=grundrenten gesetz (Abruf am 18.6.2021).

Straubhaar, T. (2017): Radikal gerecht. Wie das bedingungslose Grundeinkommen den Sozialstaat revolutioniert, Hamburg. 



\title{
Sicherung des Existenzminimums mit Regelleistungen
}

\author{
Kritische Anmerkungen und Reformüberlegungen zu Hartz IV \\ und zum Familienlastenausgleich
}

Irene Becker

\section{Leistungen zur Sicherung des Existenzminimums - das letzte Auffangnetz im Sozialversicherungsstaat}

Deutschland wird bei wohlfahrtsstaatlichen Typisierungen häufig als Sozialversicherungsstaat bezeichnet, denn versicherungsorientierte Institutionen mit dem Ziel einer Lebensstandardsicherung dominieren gegenüber nachrangigen Maßnahmen der bedarfsorientierten Mindestsicherung. Die verschiedenen Zweige der deutschen Sozialversicherung werden weitgehend über Beiträge finanziert, dementsprechend sind Leistungsansprüche auf Mitglieder und teilweise deren Familienangehörige begrenzt und bei Geldleistungen - mit Ausnahme der Zahlungen der Pflegeversicherung - an die Höhe der Erwerbseinkommen bzw. der daraus erbrachten Beiträge gekoppelt.

Zudem ist die Sozialversicherung nicht als universelles System im Sinne einer Bürgerversicherung gestaltet. Insbesondere die gesetzliche Rentenversicherung bzw. die Beamtenversorgung und die Arbeitslosenversicherung sind auf unselbstständig Beschäftigte konzentriert.

Mit diesem selektiven und hinsichtlich vieler Leistungen erwerbszentrierten System werden weite Bevölkerungskreise nicht hinreichend für Lebensrisiken abgesichert. Darüber hinaus können mit einem Sozialversicherungskonzept die unserem Wirtschaftssystem immanenten Lücken bei der Existenzsicherung von Familien nicht geschlossen werden.

Diese sind die Folge des sogenannten Leistungsprinzips bei der Vergütung von Arbeit, ${ }^{1}$ das von Unterhaltspflichten losgelöst ist und Familien- und Sorge-

1 | Häufig spiegeln Entgeltunterschiede aber eher Knappheitsverhältnisse auf dem Arbeitsmarkt und Machtstrukturen als Leistungsunterschiede (Becker/Hauser 2009, S. 31-34). 
arbeit nicht berücksichtigt. Im Falle von geringen Einkommen infolge niedriger Stundenlöhne und/oder - familienbedingter - Teilzeittätigkeit kann möglicherweise zwar das Existenzminimum des bzw. der Erwerbstätigen, nicht aber das der Kinder und ggf. der Partnerin/des Partners gedeckt werden.

Vor diesem Hintergrund haben Mindestsicherung und Familienlastenausgleich (FLA), die aus Steuermitteln finanziert werden, eine zentrale Bedeutung im wohlfahrtsstaatlichen Regime. Zwar ist im Regelwerk der deutschen Sozialversicherung nicht ausschließlich das versicherungstechnische Äquivalenzprinzip $^{2}$, sondern auch Elemente des sozialen Ausgleichs implementiert - nomen est omen. Dennoch kann die Sicherung des soziokulturellen Existenzminimums damit allein nicht gewährleistet werden.

Es bedarf also eines dem besonderen Schutz der Familie entsprechenden Regelungswerks und eines letzten Auffangnetzes mit existenzsicherndem Leistungsniveau für alle, die durch das Raster der vorrangigen Institutionen fallen. Die diesbezüglichen Regelungen in Deutschland - einerseits FLA und andererseits Transfers nach dem Zweiten bzw. Zwölften Buch Sozialgesetzbuch (SGB II bzw. XII), also Hartz-IV-Leistungen bzw. Sozialhilfe ${ }^{3}-$, sowie die Schwächen und Reformmöglichkeiten dieser Regelungen stehen im Fokus dieses Beitrags.

Der FLA in Deutschland ist allerdings nicht am Ziel der Sicherung des Existenzminimums ausgerichtet. Er dient vielmehr der Umsetzung des verfassungsrechtlich gebotenen Grundsatzes, dass Einkommen insoweit nicht besteuert werden dürfen, als sie der Sicherung des Existenzminimums des bzw. der Steuerpflichtigen samt Familienangehörigen dienen.

Das Kindergeld ist lediglich als ergänzende soziale Komponente des FLA gestaltet, ohne an der Höhe des kindlichen Existenzminimums ausgerichtet zu sein. Angesichts dieser Sachlage im Status quo fungieren die nachrangigen Hartz-IV-Leistungen bzw. der diesen vorgeschaltete, aber ähnlich gestaltete Kinderzuschlag und die Sozialhilfe auch für Kinder als letztes Auffangnetz.

Die quantitative Bedeutung der Mindestsicherung - gemessen an der Zahl der Anspruchsberechtigten - ist also teilweise durch Schwächen des FLA bedingt. Laut Statistik der Bundesagentur für Arbeit (BA 2020 und 2021) waren im Juni 2020 knapp 9 Prozent der Gesamtbevölkerung bis zur Regelaltersgrenze,

2 | Damit ist die relative Gleichwertigkeit von Leistung (Versicherungsbeitrag) und Gegenleistung der Versicherung gemeint, wobei Letztere die Wahrscheinlichkeit des Eintritts des Risikofalls berücksichtigt; Versicherungen sind also nicht auf Äquivalenz auf der individuellen Ebene ausgerichtet.

3 | Die Besonderheiten des Asylbewerberleistungsgesetzes bleiben unberücksichtigt. 
aber etwa 13 Prozent der Kinder unter 18 Jahren - das entspricht etwa 1,8 Millionen Kindern - Leistungsberechtigte nach SGB II, d. h. sie lebten in Hartz-IVHaushalten. ${ }^{4}$ Dem könnte mit einer Koppelung des Kindergelds an das Existenzminimum von Kindern und Jugendlichen begegnet werden.

Damit sind die beiden institutionellen Regelungssysteme nicht nur infolge aktueller Sicherungslücken des FLA, sondern grundsätzlich bei Reformüberlegungen eng miteinander verwoben. Denn eine Neugestaltung des Kindergelds, die an Aspekten der Bedarfsgerechtigkeit orientiert ist, setzt ebenso wie eine zielführende Gestaltung des letzten Auffangnetzes eine sachgerechte Ermittlung des Existenzminimums voraus.

Hier ist somit eine Schnittstelle angelegt, die auch aktuell zwischen Steuerrecht und Regelleistungen nach SGB II bzw. XII - kurz: Hartz-IV-Niveau - besteht: Letztere fließen unmittelbar in die Berechnung von Grund- und Kinderfreibetrag ein und werden lediglich um Pauschalen für die Kosten für Unterkunft und Heizung (KdU) und für das Bildungs- und Teilhabepaket (BuT) erhöht.

Die Leistungssätze der nachrangigen Grundsicherung bestimmen also die Höhe zentraler Elemente vorrangiger Systeme - und zwar nicht nur des Steuerrechts, sondern beispielsweise auch des Unterhalts- und Pfändungsrechts. Entsprechend dieser Ausstrahlung des Mindestsicherungsniveaus auf andere gesetzliche Regelungen und Reformkonzepte wird im Weiteren zunächst die derzeitige Berechnung des gesetzlichen Existenzminimums kritisch hinterfragt und einem alternativen Ansatz gegenübergestellt (Abschnitt 2). Auf dieser Basis werden der im sozialen Sicherungssystem eigentlich vorrangige FLA und ein diesbezügliches Reformkonzept - die Kindergrundsicherung - in den Blick genommen (Abschnitt 3).

Die Darstellungen und Überlegungen sind auf Regelleistungen zur Sicherung des Existenzminimums beschränkt. Diese können lediglich pauschalierbare Bedarfe abdecken, wobei das Pauschalierbare im letzten Auffangnetz allerdings enger gefasst ist als im FLA. Sonderbedarfe werden im Weiteren nicht berücksichtigt - sie können nur einzelfallbezogen neben den Regelleistungen gedeckt werden. Die für die Existenzsicherung notwendige Infrastruktur wird in Teil III dieses Sammelbandes erörtert.

4 | Daneben gab es 124.000 Kinder unter 18 Jahren, die aufgrund eigener Einkommen - z.B. Unterhalt oder Erwerbseinkommen - keinen eigenen Leistungsanspruch hatten, aber in einer Hartz-IV-Familie lebten. 


\section{Ermittlung des soziokulturellen Existenzminimums}

\subsection{Anforderungen an eine zielgerechte Berechnung des Existenzminimums}

Das Existenzminimum bezieht sich - anders als nach dem Verständnis Anfang des vorigen Jahrhunderts (Becker 2013b) - nicht nur auf das kurzfristige Überleben. Es umfasst nach sozialwissenschaftlicher und politischer Definition die erforderlichen Mittel sowohl zur Sicherung der physischen Existenz als auch für ein Mindestmaß an zwischenmenschlichen Beziehungen und Teilhabe am Leben in der Gesellschaft.

Dabei sind beide existenziellen Bedarfsarten nicht als absolute Dimensionen, sondern grundsätzlich relativ zum gesellschaftlichen Umfeld zu definieren. Welche Ernährungsweise und Bekleidung, Hygieneartikel und Gesundheitspflege, Unterkunfts- und Heizungsart mindestens erforderlich sind, kann ebenso wenig losgelöst von Zeit, Raum und gesellschaftlichem Entwicklungsstand bestimmt werden wie der Grad der Teilhabe am gesellschaftlichen, kulturellen und politischen Leben, der Ausgrenzung verhindert (Becker 2017b).

Dementsprechend hat auch das Bundesverfassungsgericht (2010, Rn. 133) die Ausrichtung am Entwicklungsstand des Gemeinwesens und den bestehenden Lebensbedingungen gefordert. Es hat zudem die Gewährleistung der Mittel für das soziokulturelle Minimum dem Schutz der Menschenwürde zugeordnet - in Worten des UN-Sozialpakts geht es um nicht weniger als um ein Menschenrecht ${ }^{5}$-, woraus eine entsprechende objektive Verpflichtung des Staates und ein Leistungsanspruch der Bürger:innen folgt (Bundesverfassungsgericht 2010, Rn. 134).

Die allgemeine Beschreibung des soziokulturellen Existenzminimums ist zwar recht vage, impliziert aber eine grundlegende Anforderung an jegliche Methode zur Konkretisierung des Begriffs: Infolge der Relativität des Existenzminimums ist die Berücksichtigung gesellschaftlicher Ressourcen und Rahmenbedingungen zwingend. Berechnungsverfahren müssen also auf reale Verhältnisse und auf einen als üblich oder normal einzustufenden Lebensstandard Bezug nehmen.

Hinsichtlich dieser Vorgabe ist es naheliegend, Indikatoren zur materiellen Teilhabe verschiedener Schichten auf der Basis einer Bevölkerungsstichprobe zugrunde zu legen - die sogenannte empirisch-statistische Methode, kurz: Statistikmodell. Letztlich werden bei diesem Ermittlungsverfahren die durchschnittlichen Konsumausgaben einer unteren Einkommensgruppe in »bescheidenen"

5 | Internationaler Pakt über wirtschaftlich, soziale und kulturelle Rechte, Artikel 11. 
Verhältnissen (Referenzgruppe) als Indikator für das soziokulturelle Existenzminimum herangezogen, das ein Mindestmaß an gesellschaftlicher Teilhabe ermöglicht und Ausgrenzungsprozessen aufgrund materieller Armut entgegenwirkt.

Mit dem Statistikmodell wird ein Mindestbudget geschätzt, ohne Einzelpositionen im Sinne eines Mindestwarenkorbs festzulegen. Auch die Höhe der dem Mindestbudget zugrunde liegenden Durchschnittsausgaben für die diversen Güter und Dienstleistungen ist irrelevant, denn individuelle Ausgabenstrukturen fallen verschieden aus. Unterschiede zwischen Gruppendurchschnitt und Konsum im Einzelfall sind normal und spiegeln die jeweiligen persönlichen Umstände und Interessen.

Diese Abweichungen saldieren sich aber insgesamt - so die Grundannahme des Statistikmodells -, sodass der Gesamtbetrag eine Bedarfsdeckung ermöglicht. Die modellimmanente Annahme des internen Ausgleichs ist unter theoretischen Gesichtspunkten allerdings nur haltbar, wenn die Methode konsequent umgesetzt wird, d.h. ohne nachträgliche Vorschreibung eines begrenzten Warenkorbs etwa durch Herausnahme einzelner Produkte.

Die Zweckmäßigkeit des Statistikmodells für die Ermittlung des Existenzminimums ist an weitere Bedingungen geknüpft. Zum einen hat eine sorgfältige Auswahl des maßgeblichen unteren Einkommensbereichs zu erfolgen, damit die Annahme vertretbar ist, dass in der Referenzgruppe das soziokulturelle Existenzminimum gewährleistet ist. Andernfalls wären die Ergebnisse von Zirkelschlüssen - von den Ausgaben der Bedürttigen auf ihren Bedarf - geprägt.

Zum anderen muss eine realitätsgerechte Beschränkung der Schätzung auf pauschalierbare Bedarfe - kurz: Regelbedarfe - vorgenommen werden. Denn für Ausgaben, die unregelmäßig anfallen und meist nicht aus dem laufenden Einkommen finanziert werden (Anschaffung von Haushaltsgroßgeräten), die nicht grundsätzlich bei allen Mitgliedern der Referenzgruppe anfallen (Nachhilfeunterricht) oder denen systematische Preisunterschiede zugrunde liegen (Wohn- und Heizkosten), kann der interne Ausgleich über- und unterdurchschnittlicher Bedarfe nicht angenommen werden.

Die skizzierten Anforderungen an eine sachgerechte Ermittlung von Mindestbedarfen sind keineswegs hinreichend für »die« empirische Umsetzung. Vielmehr verbleiben etliche Freiheitsgrade, denn die Zielgröße ist ein theoretisch-normatives Konstrukt, das nicht rein wissenschaftlich quantifiziert werden kann, sondern gesellschaftspolitische Vorentscheidungen erfordert.

Dies hat auch das Bundesverfassungsgericht mehrfach betont, indem es auf den Gestaltungsspielraum des Gesetzgebers verwiesen hat (Bundesverfassungsgericht 2010, Rn. 138). Insbesondere über das Verhältnis von Existenzminimum und »Entwicklungsstand des Gemeinwesens« bzw. »bestehende Lebensbedin- 
gungen « (Bundesverfassungsgericht 2010, Rn. 133) ist letztlich politisch zu entscheiden. Allerdings sollte eine klare Trennung zwischen normativen Setzungen (auf politischer Ebene) und statistischen Berechnungen (auf wissenschaftlicher Ebene) erfolgen.

\subsection{Berechnung des soziokulturellen Existenzminimums - Status quo, Kritik und Alternativen}

\subsubsection{Hartz-IV-Regelbedarfe - Darstellung und Kritik des gesetzlichen Ermittlungsverfahrens}

Bei der Ermittlung von Regelbedarfen - den pauschalierbaren Elementen des soziokulturellen Existenzminimums - hat sich in Deutschland das Statistikmodell gegen die bis in die 1990er-Jahre angewandte Warenkorbmethode durchgesetzt. ${ }^{6}$ Datenquelle ist die jeweils aktuelle Einkommens- und Verbrauchsstichprobe (EVS), die allerdings nur in fünfjährigem Turnus durchgeführt wird - zuletzt 2018 -, sodass die Beträge für die dazwischen liegenden Jahre auf Basis der Volkswirtschaftlichen Gesamtrechnungen fortgeschrieben werden müssen.

Die zunächst mit Verordnungen festgelegte konkrete Vorgehensweise wurde mit dem Regelbedarfsermittlungsgesetz (RBEG) von 2011 reformiert, da das Bundesverfassungsgericht in seinem Urteil von 2010 die bis dahin geltenden Regelungen als nicht verfassungsgerecht gewertet hat (Bundesverfassungsgericht 2010). Die dem RBEG 2011 folgenden Gesetze von 2016 und $2020^{7}$ haben an der Grundstruktur des Ermittlungsverfahrens nichts geändert. Auf Details kann im Rahmen dieses Beitrags nicht eingegangen werden, sodass hier nur die wesentlichen Berechnungsvorgaben diskutiert werden, die für eine Gesamtbewertung unter den in Abschnitt 2.1 erläuterten Anforderungen zentral sind.

\section{a) Referenzeinkommensbereiche}

Laut Gesetz werden vor der Bildung der Referenzeinkommensbereiche - durchaus sachgerecht - die Haushalte ausgeschlossen, die Grundsicherungs- bzw. vergleichbare Leistungen ${ }^{8}$ und keine Erwerbseinkommen bezogen haben. Haus-

6 | Eine Gegenüberstellung beider Methoden findet sich in Becker 2010.

7 | Gesetz zur Ermittlung von Regelbedarfen und zur Änderung des Zweiten und Zwölften Buches Sozialgesetzbuch vom 24. März 2011; Gesetz zur Ermittlung der Regelbedarfe nach $\$ 28$ des Zwölften Buches Sozialgesetzbuch vom 22. Dezember 2016; Gesetz zur Ermittlung der Regelbedarfe und zur Änderung des Zwölften Buches Sozialgesetzbuch sowie weiterer Gesetze vom 9. Dezember 2020.

8 | Dazu zählen die Hilfe zum Lebensunterhalt bzw. Grundsicherung im Alter und bei Erwerbsminderung nach dem Dritten bzw. Vierten Kapitel des SGB XII, das Arbeits- 
halte mit aufstockendem Transferbezug verbleiben aber im Datensatz, obwohl die Anrechnungsfreiheit ihrer Erwerbseinkommen teilweise lediglich den mit der Erwerbstätigkeit verbundenen Mehrbedarf deckt, nicht aber zu einem Lebensstandard oberhalb des Grundsicherungsniveaus führt.

Zudem werden Anspruchsberechtigte in verdeckter Armut, die also ihren Leistungsanspruch nicht durchsetzen bzw. durchsetzen können, nicht aus dem Datensatz ausgeklammert. Insoweit ist mit Zirkelschlüssen zu rechnen. Nach einer vorsichtigen Schätzung auf Basis der EVS 2008 hätte allein die Herausnahme verdeckter Armut aus der Grundgesamtheit zu einem um etwa 12 Euro höheren Betrag der Regelbedarfsstufe 1 geführt (Becker 2015a, S. 30; Becker 2015b).

Aus der somit unvollständig bereinigten Grundgesamtheit der Stichprobenhaushalte werden verschiedene »untere Einkommensbereiche« definiert:

- Von den Alleinlebenden, deren Ausgaben für die Regelbedarfe von Erwachsenen maßgeblich sind, werden die unteren 15 Prozent als Referenzgruppe bestimmt.

- Von den drei - nach dem Kindesalter differenzierten - Familiengruppen, deren Ausgaben den Regelbedarfen von Kindern und Jugendlichen zugrunde liegen, bildet das jeweils unterste Quintil, also die unteren 20 Prozent, die Referenzgruppe.

Die Verwendung von Quantilen unterschiedlicher Breite (untere 15 Prozent und untere 20 Prozent) wird mit Verweis auf unterschiedliche relative Häufigkeiten der Leistungsbeziehenden an der jeweiligen Gesamtgruppe gerechtfertigt - die Quote ist bei Alleinlebenden mit 7,7 Prozent wesentlich höher als bei Paaren mit einem Kind (knapp 1 Prozent). Wenn die vorab ausgeschlossenen Haushalte mit den unteren 15 bzw. 20 Prozent zusammengefasst werden, ergibt sich bei den Alleinlebenden wie bei den Paaren mit einem Kind ein Anteil von etwa 21 Prozent der Haushalte des jeweiligen Typs.

Der jeweilige Anteil der Leistungsbeziehenden ist jedoch kein sinnvolles Kriterium im Kontext der Regelbedarfsbemessung. Von diesen Zahlen lässt sich keineswegs der Schluss ziehen, dass eine "möglichst ähnliche relative Einkommensabgrenzung aller Referenzgruppen in Bezug auf die zugrundeliegenden gesamten Haushalte« (BMAS 2020, S. 18) erreicht wäre. Denn die Verteilung

losengeld II bzw. Sozialgeld nach SGB II und Zahlungen nach dem Asylbewerberleistungsgesetz - Letztere wurden erstmals 2018 explizit erhoben. 
nach relativen Einkommenspositionen' innerhalb der unteren 21 Prozent der jeweiligen Gesamtgruppe (einschließlich aller Grundsicherungsbeziehenden) bzw. innerhalb der Referenzgruppen (untere 15 bzw. 20 Prozent nach Ausklammerung von Grundsicherungsbeziehenden) wird überhaupt nicht untersucht.

Letztlich impliziert die derzeitige Vorgehensweise, dass verschiedene Lebensstandards für die Regelbedarfe maßgeblich sind (Becker 2020, S. 354). ${ }^{10}$ Zudem ist die vonseiten des Bundesministeriums für Arbeit und Soziales vorgetragene Argumentation immanent zirkulär, weil die zwecks Vermeidung von Zirkelschlüssen zunächst ausgeklammerten Leistungsbeziehenden über den Umweg der Bestimmung der Breite der maßgeblichen unteren Einkommensbereiche indirekt wieder einbezogen werden.

Aber selbst wenn die Quantilsbreiten gleich wären - also beispielsweise einheitlich die unteren 20 Prozent zugrunde gelegt würden -, ist eine Vergleichbarkeit nicht gewährleistet, denn die tatsächlichen Lebensbedingungen der freihändig gewählten unteren Einkommensbereiche werden nicht in den Blick genommen. Ob die Konsumausgaben der Referenzgruppen auf Teilhabemöglichkeiten schließen lassen, wird nicht hinterfragt. Schließlich beziehen sich die derzeitigen Untergrenzen der Einkommensbereiche auf die Grundsicherungsschwelle, die aber Gegenstand der Prüfung ist, sodass die Vorgehensweise auch aus diesem Grund immanent zirkulär ist. ${ }^{11}$

9 | Haushaltsnettoeinkommen in Relation zum Medianeinkommen des jeweiligen Haushaltstyps.

10 | Folgendes Beispiel soll dies veranschaulichen: Vereinfachend wird von der Annahme ausgegangen, dass die derzeitigen - mit dem neuen RBEG zu überprüfenden - Regelleistungen zu einem vergleichbaren Lebensstandard von Alleinlebenden und Paaren mit einem Kind, die Grundsicherung beziehen, geführt haben. Wenn die Verteilung nach relativen Einkommenspositionen (in Prozent des Medianeinkommens des jeweiligen Haushaltstyps) innerhalb des Quintils oberhalb der Grundsicherungsschwelle bei beiden Haushaltstypen gleich ist, führt die Bezugnahme auf die unteren 15 Prozent der ersten Gruppe (Alleinlebende) zwangsläufig zu einem niedrigeren Ergebnis der Neubemessung der Regelbedarfe als die Bezugnahme auf die unteren 20 Prozent der zweiten Gruppe (Paare mit einem Kind). Falls die relativen Einkommenspositionen der Paare mit Kind vergleichsweise stärker im oberen Bereich des besagten Quintils konzentriert sind - darauf deuten Ergebnisse der Verteilungsforschung hin (Becker 2016, Tab. 13.9) -, fallen die Unterschiede zwischen den relativen Referenzeinkommen der beiden Haushaltstypen nochmals größer aus.

11 | Vgl. auch Dudel et al. (2013), S. 32 f.: »Eine [...] Abgrenzung mit Hilfe einer [...] Untergrenze, die sich an bestehenden Einkommensgrenzen für den Leistungsbezug orientiert, wird, gestützt auf statistische Verfahren zur Analyse des Verbrauchsverhal- 
Das seit Jahren praktizierte Verfahren zur Abgrenzung von unteren Einkommensbereichen ist also reformbedürftig, denn es impliziert Zirkelschlüsse mit unbekanntem Einfluss auf die Ergebnisse, und die Zielgerechtigkeit wird nicht kontrolliert - möglicherweise wird vom Mangel auf den Bedarf geschlossen.

\section{b) Bestimmung des pauschalierbaren und regelbedarfsrelevanten Konsums}

Der zweite - wegen der Annahme des internen Ausgleichs (siehe Abschnitt 2.1) - »neuralgische Punkt« des Statistikmodells liegt bei der Bestimmung der nicht pauschalierbaren, also der nicht mit dem Regelbedarf zu gewährleistenden Bedarfe. Diese werden mit dem RBEG sehr eng gefasst und sind im Wesentlichen auf den Wohnungsbedarf beschränkt, sodass Kosten der Unterkunft und Heizung (KdU) außerhalb des Regelbedarfs in ihrer tatsächlichen Höhe - soweit sie als »angemessen« eingestuft werden - erstattet werden.

Darüber hinaus nimmt der Gesetzgeber aber unter normativen Gesichtspunkten eine Eingrenzung der Ausgaben der Referenzgruppen auf als regelbedarfsrelevant erachtete Elemente vor. Ungeachtet der methodischen Anforderungen für die Eignung des Statistikmodells zur Bedarfsermittlung werden Kürzungen der Referenzausgaben vorgenommen, indem für zahlreiche Einzelpositionen - wie bei der Zusammenstellung eines Warenkorbs - behauptet wird, sie seien nicht Bestandteil des Existenzminimums. Dies betrifft die Ausgaben für die Nutzung eines Pkws ebenso wie die Kosten für eine Handtasche, Hundefutter, Tannenzweige zu Weihnachten, den Verzehr in Kantinen oder Eisdielen oder eine Flasche Wein - um nur einige Beispiele mit Bevormundungscharakter zu nennen. ${ }^{12}$

Die Summe der gestrichenen Beträge wird im RBEG nicht genannt, ist aber gravierend. Sie beläuft sich beim derzeitigen RBEG 2020 - ähnlich wie bei den Vorgänger-RBEG 2011 und 2016 - auf etwa ein Viertel der Konsumausgaben der jeweiligen Referenzgruppe (ohne Wohnkosten). ${ }^{13}$

Kürzungen in diesem Ausmaß bedeuten eine Aufweichung des Statistikmodells mit der Folge eines nicht zielführenden Methoden-Mix. Die Herausnahme einzelner Ausgabenpositionen führt zu einer generellen Bedarfsunterdeckung nicht nur bei den Grundsicherungsbeziehenden mit entsprechendem Bedarf

tens der jeweiligen Referenzgruppe, letztlich immer wieder in etwa diese Grenze reproduzieren.«

12 | Vgl. die ausführliche Erörterung in Becker 2011, S. 35-44. Mit dem RBEG 2020 werden nun immerhin die Kosten für Mobilfunk als regelbedarfsrelevant anerkannt.

13 | Becker 2011, S. 44 (Tab. 8); Becker 2016, S. 19 (Tab. 2); Becker 2020, S. 404 (Tab. 2). 
(z. B. wenn einem Kind der Familienhund nicht weggenommen werden kann), sondern auch bei denen, die die gestrichenen Güter nicht kaufen (z. B. wenn die Familie keinen Hund besitzt, das Kind aber am Fußballspiel interessiert ist).

Der Grund für die generelle Bedarfsunterdeckung liegt in der Basierung das Statistikmodells auf »unechten« Durchschnittsbeträgen, die von Nullfällen beeinflusst, also nach unten gedrückt sind. Dies steht dem Ziel der Bedarfsermittlung zwar nicht entgegen, sofern alle Einzelausgaben summiert werden, sodass sich im Einzelfall über- und unterdurchschnittliche Bedarfe kompensieren. Bei weitreichenden Streichungen von Konsumelementen ist aber die modellimmanente Annahme des internen Ausgleichs nicht haltbar und damit das Verfahren methodisch unzulänglich. Letztlich erfolgt damit eine Abkehr von realen Lebensweisen, was der Ermittlung eines relativen Existenzminimums zuwiderläuft.

\subsubsection{Ein Reformmodell}

Die Auseinandersetzungen um das $\mathrm{RBEG}^{14}$ und die einschlägigen Äußerungen des Bundesverfassungsgerichts (2010 und 2014) zeugen von dem in der derzeitigen Gestaltung des Statistikmodells angelegten Konflikt zwischen Gestaltungsspielraum des Gesetzgebers und methodischen Anforderungen an ein zweckmäßiges Verfahren. Derzeit fließen politische Setzungen unmittelbar, aber eher versteckt, an zwei Punkten in die Berechnungen ein: erstens durch die Wahl des Referenzeinkommensbereichs und zweitens durch Kürzungen der Referenzausgaben. Damit erfolgt eine Verquickung der normativen Ebene mit den statistischen Auswertungen, das Ergebnis dieses methodisch unzulänglichen Verfahrens bleibt intransparent.

Angesichts dieses Dilemmas ist es naheliegend, ein alternatives Konzept mit strikter Trennung zwischen normativer Ebene und methodischer Umsetzung zu verfolgen. Dieses erfordert in einem ersten Schritt eine explizite politische Entscheidung über die relative Höhe des Existenzminimums, die das Ergebnis eines gesellschaftspolitischen Diskurses sein sollte. Auf dieser Basis werden in einem zweiten Schritt methodisch stringente Berechnungen durchgeführt - ohne weitere normative Einflussnahme der Legislative.

Ein entsprechendes Konzept, das der Relativität des soziokulturellen Existenzminimums gerecht wird, ohne normativ festgelegt zu sein, wurde 2016 entwickelt und für verschiedene politische Setzungen - eher beispielhaft - um-

14 | Vgl. z. B. Münder 2011; Becker 2011; Lenze/Conradis 2015; Becker 2020; Lenze 2021 sowie den Aufruf "Soforthilfen für die Armen - jetzt!!« vom 25.1.2021 von 36 Gewerkschaften und Verbänden (DPWV et al. 2021). 
gesetzt (Becker/Tobsch 2016; Becker/Tobsch 2020; Becker/Held 2021). Mit dem Basiskonzept wird eine verfahrenstechnische und methodische Neuausrichtung vorgeschlagen, die gegenüber dem Status quo insbesondere eine alternative Regel zur Abgrenzung des Referenzeinkommensbereichs und eine Abkehr vom Methoden-Mix umfasst. Sie lässt sich mit folgenden zentralen Elementen skizzieren.

1. Normative Entscheidungen sind auf die Vorgabe eines akzeptablen maximalen Rückstands gegenüber der gesellschaftlichen Mitte beschränkt. So könnte beispielsweise vereinbart werden, dass soziokulturelle Teilhabe gerade noch gewährleistet ist, wenn die Ausgaben für physische Grundbedarfe (Ernährung, Bekleidung und Wohnen einschließlich Energie und Instandhaltung) um nicht mehr als 25 Prozent, die Ausgaben für sonstige Bedarfe um nicht mehr als 40 Prozent hinter den entsprechenden Ausgaben in der Mitte der Einkommensverteilung zurückbleiben. Nach diesem Vorschlag sollte also das relative Konsumniveau der Referenzgruppe bei etwa 75 Prozent (Grundbedarfe) bzw. 60 Prozent (sonstige Bedarfe) liegen.

2. Die Mitte fungiert demnach als Ankerpunkt des Konzepts. Dabei wäre die Bezugnahme auf das Medianeinkommen ungeeignet, denn die Ausgaben eines einzelnen Haushalts unterliegen zufälligen Schwankungen, die für die Regelbedarfsermittlung nicht maßgeblich sein sollten. Somit empfiehlt sich die Konkretisierung der Mitte als mittleres (drittes) Quintil der Einkommensverteilung: Bei der Durchschnittsbildung gleichen sich Ausreißer von individuellen Ausgaben nach oben (z. B. Kauf der ÖPNV-Jahreskarte) und nach unten (z. B. wegen Ausstattung mit notwendiger Bekleidung kurz vor der Befragung) aus.

Für die konkrete Abgrenzung des mittleren Quintils ist allerdings zwischen zwei Varianten zu entscheiden. Zum einen kann - ähnlich wie mit dem RBEG - auf die jeweilige Mitte der Referenzhaushaltstypen und damit auf die Konsumausgaben des mittleren Quintils der nach dem Haushaltsnettoeinkommen geordneten Alleinlebenden bzw. Paare mit einem Kind Bezug genommen werden (interne Mitten). ${ }^{15}$ Zum anderen kann das mittlere Quintil der Gesamtverteilung aller nach dem Nettoäquivalenzeinkommen geordneten Personen als Mitte definiert werden, aus der dann die Alleinlebenden und die Paare mit Kind als Ankerpunkte in den Fokus genommen werden. ${ }^{16}$

15 | Diese Vorgehensweise liegt den Studien von Becker/Tobsch 2016 und Becker/Held 2021 zugrunde.

16 | Dieser Ansatz wurde in Becker/Tobsch 2020 gewählt. 
Die erste Variante hat den Nachteil, dass die internen Mitten nicht unmittelbar vergleichbar sind - sie sind möglicherweise in einem jeweils anderen Bereich der Gesamtverteilung konzentriert. Abgesehen davon kann zur Sicherung des soziokulturellen Existenzminimums und der Zukunftschancen von Kindern allerdings die Anbindung an »übliche« Lebensverhältnisse speziell von Familien zweckmäßiger sein als die Bezugnahme auf die aus allen Haushaltstypen gebildete Mitte.

Bei der zweiten Variante sind die Ankerpunkte für die Referenzhaushaltstypen zwar definitionsgemäß weitgehend vergleichbar - aber nur sofern die Äquivalenzskala realitätsgerecht ist. Letzteres ist hinsichtlich der gängigen neuen OECD-Skala ${ }^{17}$ strittig mit der Folge einer Ungewissheit, die angesichts der verfassungsrechtlich strengen Vorgaben zur Bedarfsermittlung schwerer wiegt als bei allgemeinen Verteilungsanalysen. Bei der Umsetzung des Reformkonzepts ist zwischen dem Für und Wider der beiden potenziellen Abgrenzungen der Mitte abzuwägen.

3. Die für die Regelbedarfsermittlung letztlich maßgeblichen unteren Einkommensbereiche werden - nach Ausklammerung von Zirkelschluss-Haushal$\operatorname{ten}^{18}$ aus dem Datensatz - in Abhängigkeit der unter 1. genannten politischen Vorgaben iterativ gesucht. Sie werden also nicht freihändig oberhalb bestehender Einkommensgrenzen für den Leistungsbezug gewählt; vielmehr werden potenzielle Referenzbereiche, z. B. das unterste Quintil oder das zweite Dezil, daraufhin untersucht, ob die Ausgaben für Grundbedarfe einerseits und für sonstige Bedarfe andererseits ungefähr - um beim Beispiel unter 1 . zu bleiben - 75 Prozent bzw. 60 Prozent der Ausgaben des mittleren Quintils erreichen.

Falls keine Gruppe empirisch nachweisbar ist, die auf dem vorgegebenen Niveau lebt, müssten die politischen Setzungen geändert werden. Denn wenn Letztere hinter dem Konsumstandard aller getesteten unteren Bereiche zurückbleiben, fehlt der Bezug zur Realität. Insoweit impliziert das Alternativkonzept also eine untere Haltelinie.

4. Nachdem ein den Normen entsprechender Referenzeinkommensbereich statistisch nachgewiesen werden konnte, werden aus dessen Konsumausgaben die Regelbedarfe berechnet. Dabei erfolgen keine Streichungen wie aus einem Warenkorb, wohl aber Ausklammerungen von nicht pauschalierbaren Be-

17 | Die erste Person wird mit einem Bedarfsfaktor von 1 gewichtet, weiteren Personen ab 14 Jahren werden Bedarfsgewichte von 0,5 zugeordnet, Kindern unter 14 Jahren nur 0,3.

18 | Grundsicherungsbeziehende (auch Aufstocker:innen, zumindest die mit geringen Erwerbseinkommen) und Haushalte in verdeckter Armut. 
darfen, die neben den Regelsätzen individuell zu erstatten sind. Letztere umfassen - wie im Status quo - insbesondere die Wohn- und Heizkosten und beispielsweise Ausgaben für Nachhilfe. Empfehlenswert ist auch die Herausnahme der Kosten für Strom und langlebige Haushaltsgroßgeräte, wobei diese Details - wie auch weitere - von Politik und Wissenschaft gemeinsam erarbeitet werden sollten.

Obwohl also keine Kürzungen um (pauschalierbare) Referenzausgaben vorgesehen sind, wäre ein mit jeder Neuberechnung quasi automatisches "Hochschrauben« der Regelbedarfe, das häufig angenommen bzw. befürchtet wird, nicht die Folge. Denn nicht der Referenzeinkommensbereich, sondern das Grundsicherungsniveau in Relation zur gesellschaftlichen Mitte wäre konstant zu halten.

Nach der ersten Neuberechnung wäre bei Verfügbarkeit einer neuen Einkommens- und Verbrauchsstichprobe (EVS) zu prüfen, ob die fortgeschriebenen Regelbedarfe dem politisch gesetzten Niveau (in Relation zur Mitte) noch entsprechen und dem Lebensstandard eines empirisch nachweisbaren unteren Einkommensbereichs nahekommen. Bei deutlicher Annäherung der Regelbedarfe an die Mitte könnten die Neuberechnungen mit einem anderen Referenzeinkommensbereich durchgeführt werden.

Die unter 3. genannte untere Haltelinie wird mit dem gegenwärtigen Verfahren zur Regelbedarfsermittlung infolge der Streichungen aus den Referenzausgaben unterschritten. Beispielweise bleiben die regelbedarfsrelevanten Positionen für soziokulturelle Teilhabe bei der Berechnung des Erwachsenenbedarfs um 78 Prozent hinter der gesellschaftlichen Mitte zurück, während der Rückstand der Ausgaben für soziokulturelle Teilhabe einer restriktiv abgegrenzten Referenzgruppe - der untersten 5 Prozent der Alleinlebenden - »nur« 59 Prozent beträgt (Datengrundlage EVS 2013; vgl. Becker/Tobsch 2020, S. 19 f.). Selbst die untersten Einkommensbereiche, die statistisch nachweisbar sind, erreichen also einen Lebensstandard deutlich oberhalb des vom Gesetzgeber definierten Niveaus.

Vor diesem Hintergrund ist es nicht verwunderlich, dass alle bisher exemplarisch durchgeführten Berechnungen auf Basis des Reformmodells mit explizitem Bezug zum »Entwicklungsstand des Gemeinwesens« (Bundesverfassungsgericht 2010, Rn. 133) zu höheren Regelbedarfen als nach dem RBEG geführt haben. Dabei ergeben sich die größten Mehrbeträge für Erwachsene, da das RBEG hier eine Referenzgruppe zugrunde legt, die vergleichsweise stark im unteren Bereich der Einkommensverteilung konzentriert. Nach einer jüngst durchgeführten Studie würde die zugrunde liegende Reformvariante gegenüber dem Ergebnis laut RBEG 2020 zu einer um 46 Prozent höheren Regel- 
bedarfsstufe 1 führen (Becker/Held 2021). Für Kinder ab 6 Jahren und Jugendliche folgt aus der Reformvariante eine Erhöhung um etwa 20 Prozent; für die Jüngsten ergibt sich allerdings kaum ein Unterschied zum RBEG 2020 (Becker/ Held 2021).

Die zitierten Ergebnisse folgen nicht zwangsläufig aus dem Reformkonzept, weil andere politische Entscheidungen über die maximalen Rückstände gegenüber der Mitte denkbar sind und auch methodische Detailprobleme alternativ gelöst werden können. ${ }^{19}$ Letztlich kann Empirie politische Entscheidungen nicht ersetzen. Normen sollten aber transparent dargelegt werden, um einen sachlichen gesellschaftspolitischen Diskurs zu ermöglichen, und die politische Einflussnahme sollte auf die der Statistik vorgelagerte Ebene beschränkt sein und nicht zu Eingriffen in die Umsetzung führen, die der Methode zuwiderlaufen.

Dementsprechend werden mit dem Reformkonzept einerseits die Referenzbereiche unter Beachtung der normativen Vorgaben abgegrenzt, andererseits die darauf basierenden Ergebnisse nicht modifiziert - sprich: nicht gekürzt. Damit wäre die Gefahr einer unerkannten Bedarfsunterdeckung gebannt, die auch vom Bundesverfassungsgericht mehrfach, z. B. wie folgt, angesprochen wurde:

»Aus der statistischen Berechnung des Regelbedarfs in Orientierung an den auf der Grundlage einer Stichprobe berechneten Verbrauchsausgaben eines Teils der Bevölkerung folgt die Gefahr, dass [...] die Kosten für einzelne bedarfsrelevante Güter nicht durchgängig gedeckt sind. Dies gilt insbesondere, wenn [...] nachträglich einzelne Positionen wie aus einem Warenkorb herausgenommen werden.« (Bundesverfassungsgericht 2014, Rn. 115)

Die vorstehenden Überlegungen zur Bemessung des soziokulturellen Existenzminimums sind auch im Kontext der im Folgenden diskutierten Kindergrundsicherung relevant. Denn bei der Entwicklung dieser Reformoption zum gegenwärtigen Kinderlastenausgleich stellt sich die Herausforderung, wie ein Sicherungsniveau sinnvoll und widerspruchsfrei bestimmt werden kann, quasi als Vorfrage.

19 | So weichen die Ergebnisse einer 2020 publizierten Studie von denen des Forschungsberichts von $2021 \mathrm{ab}$, da andere normative Setzungen und methodische Details zugrunde gelegt wurden. Danach ergeben sich für Erwachsene Mehrbeträge von 26 bis 68 Prozent des gesetzlichen Niveaus (Becker/Tobsch 2020, S. 29), für Kinder und Jugendliche Erhöhungen um 23 bis 48 Prozent (Becker/Tobsch 2020, S. 34). 


\section{Familienlastenausgleich versus Kindergrundsicherung}

Das Kindergeld als wesentlicher Bestandteil des Familienlastenausgleichs (FLA) ist eine weitere Regelleistung im Sinne einer monetären Pauschale. Es ist aber grundsätzlich anders ausgerichtet als die Hartz-IV-Bedarfssätze. Als der Grundsicherung vorgelagertes Element ist das Kindergeld nicht nur pauschalisierend, sondern ein für alle Kinder gleicher Betrag, der

- einerseits ihr Existenzminimum nicht deckt und

- andererseits in oberen Einkommensschichten im Falle eines höheren Entlastungseffekts der kindbedingten Freibeträge automatisch vom Finanzamt in Form einer Steuerrückerstattung aufgestockt wird.

Diese Konstellation ist die Folge der steuerrechtlichen Fokussierung des FLA (siehe Abschnitt 1): Wegen des progressiven Steuertarifs nimmt die Entlastungswirkung der Freibeträge - Kinderfreibetrag und Freibetrag für Betreuung, Erziehung und Ausbildung (BEA), derzeit 8.388 Euro p.a. insgesamt - mit steigendem Einkommen zu. Dies wird in unteren und mittleren Schichten mit geringen Grenzsteuersätzen zwar teilweise kompensiert, solange der steuerliche Effekt der Freibeträge geringer als das Kindergeld ausfällt; ${ }^{20}$ der maximale Freibetragseffekt von 315 Euro monatlich ${ }^{21}$ wird aber nicht erreicht, weil das Kindergeld mit 219 Euro (erstes und zweites Kind), 225 Euro (drittes Kind) bzw. 250 Euro (weitere Kinder) deutlich darunter liegt. ${ }^{22}$

Da der FLA also keine Existenzsicherung für Kinder und Jugendliche gewährleistet, wurde 2005 parallel zum Hartz-IV-Gesetz der einkommensabhängige Kinderzuschlag eingeführt. Ziel ist es, das Existenzminimum von Kindern in Kombination mit dem Wohngeld außerhalb der Grundsicherung für Arbeitsuchende (SGB II) zu organisieren, sofern das elterliche Existenzminimum durch

20 | Beispiel Familien mit einem Kind: Bis zu einem Bruttolohn von ungefähr 78.000 Euro (zusammen veranlagte Elternpaare) bzw. 45.000 Euro (Alleinerziehende) übersteigt das Kindergeld den Freibetragseffekt (BMF 2020, S. 56). Bei höheren Einkommen ist es umgekehrt: Der Freibetragseffekt fällt - zunächst nur mäßig, dann zunehmend - höher aus als das Kindergeld (Erstattung der Differenz durch das Finanzamt). 21 | Dieser Betrag ergibt sich beim Reichensteuersatz von 45 Prozent, ohne Berücksichtigung des ab 2021 weitgehend abgeschafften Solidaritätszuschlags.

22 | Beträge ab 1.1.2021. Das Kindergeld insgesamt kann aber nicht als »Förderung« bezeichnet werden, da es sich aus einer Steuerrückerstattung - entsprechend dem individuellen Grenzsteuersatz und der Höhe der kindbedingten Freibeträge - und einem Förderanteil zusammensetzt; Letzterer sinkt mit steigendem Einkommen. 
das eigene Einkommen der Eltern gedeckt ist. Prüfung der Anspruchsvoraussetzungen und Höchstbetrag des Kinderzuschlags sind an die entsprechenden Regeln im SGB II angelehnt, die Einkommensanrechnung ist allerdings - insbesondere seit der Reform von 2019 - etwas moderater.

Neben dem Kinderzuschlag sieht das Sozialrecht weitere monetäre Leistungen für Kinder vor, insbesondere den Unterhaltsvorschuss und Leistungen nach dem Bundesausbildungsförderungsgesetz (BAföG). Ersterer soll im Falle von getrennt lebenden Eltern bei Zahlungsausfällen des bzw. der Barunterhaltspflichtigen greifen und ist vom Einkommen des erziehenden Elternteils unabhängig. Demgegenüber ist das BAföG als bedarfsorientiertes Instrument für Schüler:innen und Studierende gestaltet, wobei die Einkommensanrechnung allerdings anderen Regeln folgt als nach den allgemeinen Mindestsicherungsgesetzen.

Eltern mit geringem Einkommen sehen sich also einem komplexen »Leistungsdschungel « mit Schnittstellenproblemen und Verwerfungen durch hohe kumulierte Transferentzugsraten ${ }^{23}$ gegenüber. Vor diesem Hintergrund ist es nachvollziehbar, dass viele Haushalte die ihnen zustehenden Leistungen nicht in Anspruch nehmen. Die Nicht-Inanspruchnahmequote bei Hartz-IV-Leistungen wird auf etwa zwei Fünftel (Becker/Hauser 2005; Bruckmeier et al. 2013, S. 57 und 90), beim Kinderzuschlag und Wohngeld auf etwa zwei Drittel (Becker/ Hauser 2012, S. 62) geschätzt und dürfte hinsichtlich des Bildungs- und Teilhabepakets noch höher ausfallen (DPWV 2019).

Abgesehen von den komplizierten Vorschriften im Sozialrecht sind die Bemessungen des kindlichen Existenzminimums im letzten Auffangnetz einerseits und im vorgelagerten steuerlichen FLA andererseits inkonsistent. Das Sozialgeld laut SGB II bzw. XII wird - wie in Abschnitt 2 ausgeführt - mit einer unzulänglichen Anwendung des Statistikmodells berechnet, die zwar auch in den Kinderfreibetrag des Steuerrechts einfließt; dieser wird aber als Reaktion auf ein Urteil des Bundesverfassungsgerichts von 1998 über die Unzulänglichkeit dieses Freibetrags um den BEA-Freibetrag ergänzt. Demzufolge liegt das steuerliche Existenzminimum von Kindern - je nach Alter - um 76 bis 46 Prozent über dem sozialrechtlichen Vergleichsbetrag. ${ }^{24}$ Dies ist weder unter systematischen Aspekten noch normativ nachvollziehbar.

23 | Diese können 100 Prozent oder mehr erreichen, insbesondere wenn das Bildungsund Teilhabepaket (BuT) berücksichtigt wird; denn bei Wegfall von Hartz-IV-Transfers bzw. Kinderzuschlag und Wohngeld infolge gestiegenen Elterneinkommens entfällt auch der Anspruch auf BuT-Leistungen.

24 | Stand 2020. Den altersspezifischen Regelbedarfsstufen für Kinder wurden Wohnkosten von 100 Euro und eine Pauschale für das BuT von 19 Euro hinzugerechnet, 
Letztlich ist die anhaltend überdurchschnittliche Quote relativer Einkommensarmut von Kindern auf Schwächen des FLA und ergänzender Sozialleistungen für Kinder zurückzuführen. Nach Ergebnissen des Mikrozensus lebte 2019 gut ein Fünftel der Minderjährigen unter der Armutsschwelle gegenüber knapp 16 Prozent der Bevölkerung insgesamt (Statistische Ämter o.J.).

Angesichts der hier nur kurz skizzierten Probleme ist bereits 2009 das Konzept einer Kindergrundsicherung entwickelt worden. Die Existenzsicherung von Kindern soll - soweit pauschalierbar - mit einer Leistung außerhalb des SGB II bei niedrigschwelligem Zugang über eine Familienkasse oder das Finanzamt gewährleistet werden. Damit könnten Familien von aufwendigen Antragsverfahren entlastet und verdeckte Familienarmut sowie Stigmatisierungen vermieden werden.

Weiteres Ziel ist ein konsequenter vertikaler Ausgleich - der Transferbetrag soll mit steigendem Einkommen kontinuierlich bis zu einem Minimum abgeschmolzen werden. Dies soll mit einer moderaten Transferentzugsrate umgesetzt werden, sodass die Teilhabemöglichkeiten von Kindern im Niedrigeinkommensbereich generell verbessert werden und Erwerbsanreize verbleiben. Der steuerliche FLA über die kindbedingten Freibeträge muss und soll aus verfassungsrechtlichen Gründen durchaus beibehalten werden; er soll aber »leerlaufen« in dem Sinne, dass der Mindestbetrag der Kindergrundsicherung dem höchsten steuerlichen Entlastungsbetrag entspricht.

Das Reformmodell erfordert also eine enge Verzahnung mit den Freibetragsregelungen des Einkommensteuergesetzes und insbesondere eine einheitliche Pauschale für das Existenzminimum von Kindern in Steuer- und Sozialrecht. Dieser Schnittstelle muss eine sachgerechte Bemessung der Mindestbedarfe zugrunde liegen (siehe die Ausführungen in Abschnitt 2).

Die erste Spezifizierung der Kindergrundsicherung ist in zentralen Punkten an die relevanten Vorschriften des Einkommensteuergesetzes angelehnt (Becker/ Hauser 2012) und wurde bisher vom Bündnis Kindergrundsicherung (vgl. AWO 2021; Deutscher Kinderschutzbund 2021) vertreten: Das Existenzminimum und damit der maximale Zahlbetrag der Kindergrundsicherung entspricht der Summe aus Kinderfreibetrag und BEA-Freibetrag, die Abschmelzung erfolgt mit dem jeweiligen Grenzsteuersatz der Eltern. Damit sollten normative Parameter keineswegs festgelegt, diesbezügliche Fragen aber ausgeklammert werden, um die Grundidee herauszuarbeiten.

sodass sich 369, 427 bzw. 447 Euro im Sozialrecht gegenüber 651 Euro im Einkommensteuerrecht ergeben. 
Diese besteht in einem Ersatz von Kindergeld und Steuerrückerstattungen durch kindbedingte Freibeträge sowie von Kinderzuschlag, kindbedingten Wohngeldanteilen, Hartz-IV-Leistungen für Kinder, Unterhaltsvorschuss, Ausbildungsförderungsleistungen und einigen weiteren Regelungen (Becker/Hauser 2012, S. 41) durch einen einkommensabhängigen monetären Transfer. Nachgelagerte Systeme der Mindestsicherung - insbesondere das Wohngeld und das SGB II - wären für Kinder nur im Falle hoher Wohnkosten und bei Mehr- und Sonderbedarfen zuständig.

Die Anknüpfung an gegebene Parameter des Einkommensteuergesetzes ist zur Erreichung der Ziele der Kindergrundsicherung allerdings nicht zwingend, vielleicht sogar unangemessen. So ist die Abschmelzung mit dem Steuertarif zumindest fragwürdig, da damit auch die obere Mittelschicht und die Oberschicht in der Einkommensverteilung gegenüber dem Status quo begünstigt und kein Gestaltungsspielraum zur Begrenzung der fiskalischen Anforderungen bestehen würde. ${ }^{25}$ Zudem ist die Höhe der Kindergrundsicherung - also der maximale wie auch der Mindestzahlbetrag ${ }^{26}$ - strittig, da dem Kinderfreibetrag (2021: 479 Euro pro Monat) eine nicht sachgerechte Ermittlung des Existenzminimums zugrunde liegt (siehe Abschnitt 2.2) und der BEA-Freibetrag (220 Euro pro Monat) freihändig gesetzt ist.

Wenn die Idee der Kindergrundsicherung also hinsichtlich der genannten Parameter als normativ offen verstanden wird, sind entsprechende Konkretisierungen zu entwickeln und gesellschaftspolitisch zu diskutieren:

- Eine Abschmelzung der Kindergrundsicherung mit höheren Transferentzugsraten, als sie aus dem Einkommensteuertarif folgen, ist denkbar. Beispiele für einen progressiven Stufentarif wurden bereits vorgelegt (Becker 2013a), wobei allerdings das Ziel der Erwerbsanreize das Spektrum zweckdienlicher Tarife begrenzt.

- Eine sachgerechte Neuermittlung des kindlichen Existenzminimums kann zu einer Überwindung der derzeitigen kryptischen Trennung zwischen sächlichem Existenzminimum und BEA-Aufwand im Steuerrecht führen. Damit wäre der Weg für ein einheitliches soziokulturelles Existenzminimum

25 | Die Nettokosten der am Steuerrecht orientierten Variante der Kindergrundsicherung werden auf ca. 30 Milliarden Euro p. a. geschätzt (Becker 2017a).

26 | Bei gegebenem Steuertarif folgt aus dem Höchstbetrag unmittelbar der Mindestanspruch, da Letzterer dem maximalen steuerlichen Entlastungseffekt der weiterhin zu berücksichtigenden Freibetragsregelung entsprechen muss. 
in Steuer- und Sozialrecht geebnet. ${ }^{27}$ Die Neuregelung dieser Schnittstelle könnte z.B. mit einem methodisch stringenten Statistikmodell erfolgen (siehe Abschnitt 2.2).

Ob eine Kindergrundsicherung insbesondere Kinderarmut verringern kann, hängt entscheidend von der Gestaltung der normativen Parameter ab. Dementsprechend sind alle an das Basiskonzept anknüpfenden Reformmodelle von politischen Parteien, die mittlerweile in programmatische Aussagen einflieBen, ${ }^{28}$ und der Arbeitsgruppe Kindergrundsicherung der Arbeits- und Sozialministerkonferenz (Arbeitsgruppe Kindergrundsicherung der ASMK 2018) kritisch zu prüfen. ${ }^{29}$ Die unreflektierte Übernahme der Hartz-IV-Sätze für Kinder oder gar eine Absenkung dieser Beträge wäre nicht zielführend.

Neben der Höhe der Kindergrundsicherung und der Umsetzung der Einkommensabhängigkeit sind etliche weitere Gestaltungsaufgaben zu lösen. So ist zu entscheiden, ob es sich um einen Rechtsanspruch des Kindes oder der Eltern handeln soll und ob eine Differenzierung nach dem Kindesalter notwendig ist. Auch hinsichtlich der Berücksichtigung des elterlichen Einkommens sind nicht nur verwaltungstechnische, sondern auch grundsätzliche Fragen offen. Beispielsweise ist zu klären, ob zusammenlebende Eltern bei der Berechnung der Abschmelzrate gemeinsam veranlagt werden oder ob wie bei getrennt lebenden Elternteilen beide Individualeinkommen maßgeblich sein sollen.

Daneben sind Schnittstellen mit familienpolitischen Maßnahmen in anderen Rechtsgebieten sachgerecht zu regeln, was in einer jüngst erschienen Studie detailliert erörtert wird (Ott/Schürmann/Werding 2020). Das Autorenteam dieser aktuellen Analyse betont trotz der umfassenden Gestaltungsaufgaben aber das Potenzial des Reformkonzepts:

27 | Vgl. auch Ott/Schürmann/Werding 2011, S. 262: »Hinsichtlich des Mindestbedarfs von Kindern in verschiedenen Rechtsbereichen bietet sich als sinnvolle Grundidee an, dass es letztlich nur ein Existenzminimum von Kindern geben kann«.

28 | Vgl. die Übersicht in Becker 2019, S. 32-35, die allerdings nicht mehr ganz aktuell ist; zu den weiter entwickelten Modellen der SPD und von Bündnis 90/Die Grünen vgl. SPD (2019) und Bündnis 90/Die Grünen Bundestagsfraktion (2019).

29 | Am 26.11.2020 hat die ASMK auf ihrer 97. Sitzung mit großer Mehrheit beschlossen, »die Bundesregierung aufzufordern, gemeinsam mit den Ländern konkrete Umsetzungsschritte zur Einführung einer Kindergrundsicherung einzuleiten« (ASMK 2020). Damit sind aber entscheidende Eckpunkte noch völlig offen. 
»Unabhängig davon, wie sie genau ausgestaltet wird, kann eine Kindergrundsicherung, die einige zentrale familienbezogene Maßnahmen bündelt, sinnvoll sein, um für eine zielgerichtetere Verwendung der eingesetzten Mittel zu sorgen« (Ott/Schürmann/Werding 2020, S. 75).

\section{Zusammenfassung und Ausblick}

Regelleistungen der Mindestsicherung und des Familienlastenausgleichs sind von zentraler Bedeutung im wohlfahrtsstaatlichen Regime Deutschlands, da die vorgelagerten Sozialversicherungen keine generelle Gewährleistung des Existenzminimums umfassen. Die derzeitige Gestaltung der steuerfinanzierten Maßnahmen wird allerdings von Teilen der Wissenschaft und gesellschaftspolitischer Akteur:innen als reformbedürftig angesehen.

Im Rahmen des vorliegenden Beitrags wurden zwei zentrale Kritikpunkte aufgegriffen. Zum einen wurde das Verfahren der Ermittlung von Regelbedarfen nach SGB II, also der Hartz-IV-Sätze, analysiert und einem methodisch stringenten Konzept gegenübergestellt. Zum anderen wurde auf die fehlende Ausrichtung des Familienlastenausgleichs am Ziel der Sicherung des kindlichen Existenzminimums eingegangen und eine diesbezügliche Reform - die Kindergrundsicherung - diskutiert.

Beide Regelungssysteme sind miteinander verwoben, da eine an Aspekten der Bedarfsgerechtigkeit ausgerichtete Alternative zum Kindergeld die sachgerechte Berechnung des soziokulturellen Existenzminimums voraussetzt. Dabei geht es auch beim Familienlastenausgleich nicht nur um den kindlichen, sondern auch um den elterlichen Mindestbedarf.

Die empfohlenen Reformkonzepte sind bisher nur ansatzweise entwickelt, die normativen Parameter eher beispielhaft gesetzt. Es sind also sowohl weitere wissenschaftliche Grundlagenarbeit als auch gesellschaftspolitische Debatten erforderlich, wenn eine abgestimmte - hinsichtlich der Bestimmung des Existenzminimums kongruente - Umgestaltung des letzten Auffangnetzes und des vorgelagerten Familienlastenausgleichs gelingen soll. Zudem sind Analysen zu Möglichkeiten der fiskalischen Gegenfinanzierung in die Reformüberlegungen miteinzubeziehen. Letztlich wird der Gesamteffekt von politischen Maßnahmen nur dann als gerecht gewertet, wenn auch die Finanzierung solide und nach Aspekten sozialer Gerechtigkeit erfolgt. 


\section{Literatur}

Arbeitsgruppe Kindergrundsicherung der ASMK (2018): Wege zu einer Kindergrundsicherung. Grobkonzept, Länderoffene Arbeitsgruppe der Konferenz der Ministerinnen und Minister, Senatorinnen und Senatoren für Arbeit und Soziales (ASMK), Hannover.

ASMK = Arbeits- und Sozialministerkonferenz (2020): Ergebnisse der 97. Arbeits- und Sozialministerkonferenz, https://stm.baden-wuerttemberg.de/de/ service/presse/pressemitteilung/pid/ergebnisse-der-97-arbeits-und-sozialmi nisterkonferenz/ (Abruf am 21.6.2021).

AWO Bundesverband e.V. (2021): Bündnis Kindergrundsicherung, https://wir arbeitendran.awo.org/projekt/buendnis-kindergrundsicherung (Abruf am 21.6.2021).

BA = Bundesagentur für Arbeit (2020): Kinder in Bedarfsgemeinschaften (Monatszahlen), Berichtsmonat Juni 2020, Nürnberg.

BA = Bundesagentur für Arbeit (2021): Strukturen der Grundsicherung SGB II (Zeitreihe Monats- und Jahreszahlen ab 2005), Berichtsmonat März 2021, Nürnberg.

Becker, I. (2010): Bedarfsbemessung bei Hartz IV. Zur Ableitung von Regelleistungen auf der Basis des »Hartz-IV-Urteils« des Bundesverfassungsgerichts, WISO Diskurs, Friedrich-Ebert-Stiftung, Bonn, https://ibrary.fes.de/pdf-files/ wiso/07530.pdf (Abruf am 21.6.2021).

Becker, I. (2011): Bewertung der Neuregelungen des SGB II. Methodische Gesichtspunkte der Bedarfsbemessung vor dem Hintergrund des "Hartz-IV-Urteiles« des Bundesverfassungsgerichts, in: Soziale Sicherheit extra, Sonderheft, September 2011, S. 7-62.

Becker, I. (2013a): Abschied vom »dualen System«. Effekte einer Reform des Kindergeldes. Friedrich-Ebert-Stiftung, Berlin.

Becker, I. (2013b): Armut und Sozialhilfe in der Bundesrepublik, in: Justizministerium des Landes NRW (Hrsg.): Weichenstellungen im Arbeits- und Sozialrecht der Bundesrepublik Deutschland. Diktatorische Vergangenheit und demokratische Prägung, Juristische Zeitgeschichte Nordrhein-Westfalen, Band 20, Recklinghausen, S. 230-249.

Becker, I. (2015a): Der Einfluss verdeckter Armut auf das Grundsicherungsniveau, Arbeitspapier 309 der Hans-Böckler-Stiftung, Düsseldorf.

Becker, I. (2015b): Regelbedarfsermittlung: Die verdeckte Armut drückt das Ergebnis, in: Soziale Sicherheit, Zeitschrift für Arbeit und Soziales 64(4), S. $142-148$. 
Becker, I. (2016): Einkommen und Vermögen: Trend zu mehr Ungleichheit hält an, in: Forschungverbund Sozioökonomische Berichterstattung (Hrsg.): Berichterstattung zur sozioökonomischen Entwicklung in Deutschland. Exklusive Teilhabe - ungenutzte Chancen. Dritter Bericht, Bielefeld, www.wbv. de/download/shop/download/0/_0/0/listview/file/-direct\%406004498w013/ area/openaccess.html (Abruf am 21.6.2021).

Becker, I. (2017a): Aktualisierung der Kostenschätzung für eine Kindergrundsicherung. Kurzexpertise für das Bündnis Kindergrundsicherung, Riedstadt.

Becker, I. (2017b): Kritik am Konzept relativer Armut - berechtigt oder irreführend?, in: WSI-Mitteilungen 70(2), S. 98-107.

Becker, I. (2019): Kinderarmut in Deutschland - Bestandsaufnahme und Möglichkeiten der Gegensteuerung. Arbeitspapier im Auftrag der Evangelischen Kirche im Rheinland, Düsseldorf, www.kirche-gesellschaft-zusammenhalt. de/Downloads/LS2020_73-DS08-Kinderarmut-Arbeitspapier.pdf (Abruf am 21.6.2021).

Becker, I. (2020): Verfahren nach altem Muster. Das Regelbedarfsermittlungsgesetz 2020, in: Soziale Sicherheit, 69(10), S. 351-355 (Teil 1)/Soziale Sicherheit, 69(11), S. 402-408 (Teil 2).

Becker, I./Hauser, R. (2005): Dunkelziffer der Armut. Ausmaß und Ursachen der Nicht-Inanspruchnahme zustehender Sozialhilfeleistungen, Berlin.

Becker, I./Hauser, R. (2009): Soziale Gerechtigkeit - ein magisches Viereck. Zieldimensionen, Politikanalysen und empirische Befunde, Berlin.

Becker, I./Hauser, R. (2012): Kindergrundsicherung, Kindergeld und Kinderzuschlag: Eine vergleichende Analyse aktueller Reformvorschläge, WSI-Diskussionspapier 180, Wirtschafts- und Sozialwissenschaftliches Institut der Hans-Böckler-Stiftung, Düsseldorf.

Becker, I./Held, B. (2021): Regelbedarfsermittlung - eine Alternative zum gesetzlichen Verfahren. Berechnungen auf Basis der EVS 2018 unter Berücksichtigung von normativen Vorgaben der Diakonie Deutschland. Projektbericht im Auftrag der Diakonie Deutschland, Riedstadt/Heidelberg.

Becker, I./Tobsch, V. (2016): Regelbedarfsmessung - methodisch konsistente Berechnung auf Basis der EVS 2013 unter Berücksichtigung von normativen Vorgaben der Diakonie Deutschland. Projektbericht im Auftrag der Diakonie Deutschland - Evangelischer Bundesverband, Riedstadt/Berlin.

Becker, I./Tobsch, V. (2020): Ermittlung der »Grünen Garantiesicherungs-Regelbedarfe«. Bericht zum Gutachtensauftrag der Bundestagsfraktion Bündnis 90/Die Grünen (unveröffentlichtes Gutachten). 
BMAS = Bundesministerium für Arbeit und Soziales (2016): Gesetz zur Ermittlung von Regelbedarfen nach $\$ 28$ des Zwölften Buches Sozialgesetzbuch (Regelbedarfs-Ermittlungsgesetz - RBEG) vom 22.12.2016, Berlin.

BMAS = Bundesministerium für Arbeit und Soziales (2020): Entwurf eines Gesetzes zur Ermittlung von Regelbedarfen und zur Änderung des Zwölften Buches Sozialgesetzbuch sowie des Asylbewerberleistungsgesetzes (Bearbeitungsstand: 14.7.2020), Berlin.

BMF $=$ Bundesministerium der Finanzen (2020): Datensammlung zur Steuerpolitik 2019, Berlin.

Bruckmeier, K./Pauser, J./Riphahn, R. T./Walwei, U./Wiemers, J. (2013): Mikroanalytische Untersuchung zur Abgrenzung und Struktur von Referenzgruppen für die Ermittlung von Regelbedarfen auf Basis der Einkommens- und Verbrauchsstichprobe 2008. Simulationsrechnungen für das Bundesministerium für Arbeit und Soziales. Endbericht, Nürnberg.

Bundesverfassungsgericht (2010): Urteil vom 9.2.2010, Az. 1 BvL 1/09.

Bundesverfassungsgericht (2014): Urteil vom 23.7.2014, Az. 1 BVL 10/12 1 BVL 12/12 - 1 BVR 1691/13.

Bündnis 90/Die Grünen Bundestagsfraktion (2019): Fraktionsbeschluss. Faire Chance für jedes Kind - Grünes Konzept für eine Kindergrundsicherung, www.gruene-bundestag.de/files/beschluesse/Kindergrundsicherung.pdf (Abruf am 21.6.2021).

Deutscher Bundestag (2016): Gesetzentwurf der Fraktionen CDU/CSU und FDP. Entwurf eines Gesetzes zur Ermittlung von Regelbedarfen und zur Änderung des Zweiten und Zwölften Buches Sozialgesetzbuch, BundestagsDrucksache 17/3404.

Deutscher Kinderschutzbund Bundesverband e.V. (2021): Unser Vorschlag für eine Kindergrundsicherung, www.kinderarmut-hat-folgen.de/ (Abruf am 21.6.2021).

DPWV = Deutscher Paritätischer Wohlfahrtsverband - Gesamtverband e. V. (2019): Empirische Befunde zum Bildungs- und Teilhabepaket: Teilhabequoten im Fokus, Paritätische Forschungsstelle, Berlin.

DPWV et al. = Deutscher Paritätischer Wohlfahrtsverband - Gesamtverband e. V. et al. (2021): Soforthilfen für die Armen - jetzt!! (Aufruf von 36 Gewerkschaften und Verbänden vom 25.1.2021), www.der-paritaetische.de/filead $\mathrm{min} /$ user_upload/Seiten/Presse/docs/Soforthilfen_fuer_die_Armen_Aufruf. pdf (Abruf am 21.6.2021).

Dudel, C./Garbuszus, M./Ott, N./Werding, M. (2013): Überprüfung der bestehenden und Entwicklung neuer Verteilungsschlüssel zur Ermittlung von Regelbedarfen auf Basis der Einkommens- und Verbrauchsstichprobe 2008. 
Endbericht für das Bundesministerium für Arbeit und Soziales, Ruhr Universität Bochum, Fakultät für Sozialwissenschaft.

Lenze, A. (2021): Regelbedarf zur Sicherung des Existenzminimums, in: Münder, J./Geiger U. (Hrsg.): Sozialgesetzbuch II. Grundsicherung für Arbeitsuchende. Lehr- und Praxiskommentar (LPK-SGB II), 7. Auflage, Baden-Baden.

Lenze, A./Conradis, W. (2015): Die Entscheidungen des Bundesverfassungsgerichts und des Bundessozialgerichts vom 23.7.2014 zu den Regelbedarfen und die Folgen für die Praxis, in: Informationen zum Arbeitslosenrecht und Sozialhilferecht (info also), H. 3/2015, S. 99-105.

Münder, J. (2011): Verfassungsrechtliche Bewertung des Gesetzes zur Ermittlung von Regelbedarfen und zur Änderung des Zweiten und Zwölften Buches Sozialgesetzbuch vom 24.03.2011 - BGBl. I, S. 453. In: Soziale Sicherheit extra, Sonderheft, September 2011, S. 63-94.

Ott, N./Schürmann, H./Werding, M. (2011): Schnittstellen im Sozial-, Steuerund Unterhaltsrecht. Endbericht im Auftrag der Geschäftsstelle für die Gesamtevaluation ehe- und familienbezogener Maßnahmen, Ruhr-Universität Bochum.

Ott, N./Schürmann, H./Werding, M. (2020): Rechtliche Schnittstellen bei der Einführung einer Kindergrundsicherung. Endbericht im Auftrag des Ministeriums für Arbeit, Gesundheit und Soziales NRW (MAGS NRW), RuhrUniversität Bochum.

SPD (2019): Bei uns kommen die Kleinen groß raus. SPD-Konzept für Kindergrundsicherung, www.spd.de/aktuelles/kindergrundsicherung-news/ (Abruf am 21.6.2021).

Statistische Ämter des Bundes und der Länder (o.J.): Armutsgefährdungsquoten, Bundesländer nach soziodemografischen Merkmalen (Bundesmedian), www.statistikportal.de/de/sbe/ergebnisse/einkommensarmut-und-verteilung/ armutsgefaehrdung-0 (Abruf am 23.7.2021). 


\section{Grundsicherung für alle Bedürftigen?}

Die Stellung von Ausländer:innen im System der Grundsicherung

\section{Florian Blank}

\section{Einleitung'}

Die Grundsicherung im deutschen Sozialstaat umfasst Leistungen, die im Falle von Bedürftigkeit gewährt werden. Sie sollen Notlagen durch Existenzsicherung mildern. Einkommensarmut wird durch diese Leistungen nicht bekämpft, aber gemildert (siehe den Beitrag von Dorothee Spannagel in diesem Band).

Aus dieser Anlage ergeben sich neben der Notwendigkeit zur Prüfung der Bedürftigkeit und der Orientierung der Leistungen an einem politisch definierten Existenzminimum zwei Eigenschaften: Zum einen werden Grundsicherungsleistungen in der Regel temporär gewährt, bis die existenzielle Notlage behoben ist. $^{2}$ Zum anderen können sie auf die individuellen Bedürfnisse der Leistungsempfänger:innen abgestimmt werden.

Diese individuelle Anpassung betrifft die Maßnahmen zur Arbeitsmarktintegration in der Grundsicherung für Arbeitsuchende (SGB II), aber auch Transfers wie etwa die Übernahme der Kosten von Unterkunft und Heizung bis hin zum Auftrag der Sozialhilfe, Leistungen »nach der Besonderheit des Einzelfalls" (SGB XII, \$9) zu erbringen. Vom Gedanken der Bedürftigkeit ausgehend, bestimmt sich die Leistungshöhe nicht nach Vorleistungen.

1 | Der Autor dankt Robert Nazarek und Gerhard Bäcker für hilfreiche Anmerkungen.

2 | Eine Ausnahme stellt die Grundsicherung im Alter und bei Erwerbsminderung dar. Deren Leistungen können zwar aufgrund einer Verbesserung der Einkommens- und Vermögensverhältnisse von Bezieher:innen gekürzt werden oder ganz entfallen. Da aber keine Erwartung - und häufig genug auch keine Möglichkeit - besteht, durch Erwerbsarbeit ein Einkommen zu erzielen, weicht die Grundsicherung hier vom Prinzip der aktiven Mitarbeit an der Beendigung der Hilfebedürftigkeit ab. Die Leistungen sind damit auf Dauer angelegt. 
Außerdem kann die Leistung durch Sanktionen verringert werden. Diese Sanktionen werden aus dem Ziel abgeleitet, dass die Notlage durch die Mitarbeit des bzw. der Bedürftigen überwunden werden soll. ${ }^{3}$

Im Falle von Ausländer:innen - also Menschen ohne deutsche Staatsangehörigkeit - folgt die Grundsicherung diesen Prinzipien nur teilweise. Wie in vielen anderen europäischen Ländern ist die Grundsicherung bestimmten Bezugsgruppen entsprechend in Teilsysteme gegliedert (vgl. Hubl/Pfeifer 2013 sowie die Beiträge von Gerhard Bäcker und Thomas Bahle in diesem Band), die nicht nur nebeneinanderstehen, sondern teils auch als Hierarchie aufgefasst werden müssen.

Neben den Regelsystemen des Sozialgesetzbuches (SGB II - Grundsicherung für Arbeitsuchende, SGB XII - Sozialhilfe) sind mit Blick auf Ausländer:innen die Leistungen des Asylbewerberleistungsgesetzes (AsylbLG) relevant, das 1993 im Rahmen des »Asylkompromisses« beschlossen wurde. ${ }^{4}$ Die Leistungen für Asylbewerber:innen, denen ein nur temporärer Aufenthalt in Deutschland unterstellt wurde (und bis heute wird), wurden von der Sozialhilfe abgegrenzt. Der Gesetzentwurf zum AsylbLG zielte auf im Vergleich zur damaligen Sozialhilfe abgesenkte Leistungen und einen Vorrang von Sach- gegenüber Geldleistungen (vgl. Deutscher Bundestag 1993).

Der Zugang zu Leistungen des Sozialgesetzbuchs bzw. des AsylbLG richtet sich für Ausländer:innen neben der Grundvoraussetzung der Bedürftigkeit im Wesentlichen nach dem Aufenthaltsstatus einerseits - also der rechtlichen Grundlage, auf der sich ihre Anwesenheit in Deutschland gründet - und nach ihrem Status als Arbeitnehmer:innen oder Selbstständige andererseits. Es existieren Aufenthaltskonstellationen, in denen Menschen keinerlei Anspruch auf Unterstützung durch Grundsicherung haben. Diese Konstellationen umfassen

3 | Neben der Leistungshöhe waren und sind die Sanktionen ein zentraler Kritikpunkt speziell an der Grundsicherung für Arbeitsuchende (»Hartz IV«). Sie waren auch Gegenstand einer Entscheidung des Bundesverfassungsgerichts im November 2019, in dem zwar die Möglichkeit der Sanktionen bestätigt wurde, diese aber in ihrem Umfang beschränkt wurden und darauf hingewiesen wurde, dass Sanktionen strengen Anforderungen der Verhältnismäßigkeit unterlägen, wobei sich der Gesetzgeber auf fundierte Einschätzungen zur Erreichung der Ziele von Sanktionen zu stützen habe (Bundesverfassungsgericht 2019b).

4 | Relevant sind im Kontext der Grundsicherung zudem BAföG, Kindergeld und Wohngeld; insbesondere um den Bezug von Kindergeld durch Ausländer:innen gab es politische Debatten. Aus Platzgründen kann hier nicht weiter auf diese Leistungen und Diskussionen eingegangen werden. Zur Diskussion um den Kindergeldbezug vgl. Funk/ Starzmann 2018 und Sell 2018, zu den Zugangsbestimmungen des BAföG Knuth 2020. 
nicht nur beispielsweise Tourist:innen, sondern auch EU-Bürger:innen, die sich zum Zweck der Arbeitsuche in Deutschland aufhalten, in den ersten Jahren ihres Aufenthalts.

Die Leistungen und Zugangskriterien des SGB II und XII sowie des AsylbLG sind im Laufe der Zeit mehrfach geändert worden, häufig auch im Kontext aufgeregter gesellschaftlicher und medial begleiteter Debatten. Sie waren außerdem wiederholt Gegenstand von Gerichtsverfahren, in denen unter anderem geprüft werden sollte, ob die Gesetzgebung dem deutschen Verfassungsrecht, aber auch europäischem Recht entspricht. ${ }^{5}$

Die Änderungen der gesetzlichen Vorgaben ergaben sich nicht nur aus sozialpolitischen Gesichtspunkten - beispielsweise der Neuentwicklung von Instrumenten, die zu einer gesellschaftlichen Integration beitragen sollen -, sondern auch aus fiskalischen und migrationspolitischen Impulsen. Während von der einen Seite zwar immer wieder Zweifel an der Angemessenheit von Leistungen und Sanktionen angemeldet werden, sind die Regelungen andererseits unter Druck, weil ihre (vermeintliche) Großzügigkeit argumentativ mit »Sozialtourismus" (Unwort des Jahres 2013; vgl. auch Menkens 2016) verbunden wird. Dieser Debattenkontext hat häufig zu Einschränkungen von Leistungen geführt; schon die Einführung des AsylblG zielte darauf ab, den deutschen Sozialstaat weniger attraktiv zu machen.

Verglichen mit der politischen Relevanz, die dem Thema Migration und soziale (Grund-)Sicherung teils zukommt, ist die Rolle der Leistungen im deutschen Sozialstaat allerdings eher randständig. Zugleich zeigen sich an diesem System die Problematiken des deutschen Grundsicherungssystems in deutlich verschärfter Form.

Was sich am Umgang des Gesetzgebers mit Ausländer:innen zeigen lässt, ist einerseits, dass die Frage nach einem menschenwürdigen Existenzminimum vor dem Hintergrund von Finanzierungsfragen und der Sorge um eine als illegitim empfundene Nutzung beantwortet wird. Außerdem stellt sich die im Kontext dieses Bandes nur am Rande behandelte Frage nach Sanktionen und Mitwir-

5 | Zur Auseinandersetzung um den unten in Abschnitt 2.1 behandelten Ausschluss von arbeitsuchenden Ausländer:innen von Leistungen nach dem SGB II in den ersten Jahren ihres Aufenthalts vgl. Absenger/Blank 2017 und die späteren Urteile des Bundesverfassungsgerichts aus dem Dezember 2019 (Bundesverfassungsgericht 2019a) sowie des Europäischen Gerichtshofs vom Oktober 2020 (EuGH 2020). Die in Abschnitt 2.2 behandelte Neuordnung der Regelbedarfe für Asylbewerber:innen ist Gegenstand eines Normenkontrollantrags, den das Sozialgericht Düsseldorf am 13. April 2021 beim Bundesverfassungsgericht eingereicht hat (Mitzkat/Stepputat 2021). 
kungspflichten in der Grundsicherung unter anderen Vorzeichen in schärferer Form.

Dieser Beitrag stellt zunächst das Asylbewerberleistungsgesetz (AsylbLG), seine Leistung und Kriterien in Abgrenzung zum Sozialgesetzbuch (SGB) vor und skizziert Besonderheiten des SGB in Hinblick auf Ausländer:innen (Abschnitt 2). Daran anschließend werden empirische Daten zu aktuellen Entwicklungen präsentiert (Abschnitt 3). Abschließend werden Kritikpunkte genannt und Hinweise zu einer Weiterentwicklung dieser Grundsicherungssysteme gegeben (Abschnitt 4).

\section{Asylbewerberleistungsgesetz und Grundsicherung im Sozialgesetzbuch: Zugangskriterien und Leistungen}

Migration nach Deutschland und der folgende Aufenthalt in Deutschland finden aus unterschiedlichen Motiven statt. In Deutschland werden verschiedene Personengruppen rechtlich unterschiedlich behandelt, in Abhängigkeit sowohl vom Herkunftsland als auch von den Motiven für Einreise und Aufenthalt. ${ }^{6}$ Für den Zweck dieses Beitrags ist es ausreichend, anstelle einer Darstellung und Diskussion der einzelnen Aufenthaltstitel eine grobe Unterteilung vorzunehmen in Ausländer:innen mit Zugang zum SGB II und XII, Ausländer:innen mit Zugang zum AsylbLG und weiteren Gruppen ohne Zugang zur Grundsicherung.

\subsection{Zugang zu den Leistungen}

Die Leistungen des SGB II (Grundsicherung für Arbeitsuchende) und SGB XII (Sozialhilfe) haben als Grundvoraussetzung neben der materiellen Bedürftigkeit den gewöhnlichen - also längerfristigen und nicht nur vorübergehenden - Aufenthalt in der Bundesrepublik Deutschland. Hinzu kommen im SGB II Altersgrenzen und die Erwerbsfähigkeit als weitere Bedingungen. Ausgeschlossen von den Leistungen des SGB II und des SGB XII sind

- Ausländer:innen ohne Arbeitnehmer- bzw. Selbstständigenstatus und ihre Familienangehörigen für die ersten drei Monate ihres Aufenthalts. Nach dieser Frist greift allerdings das Aufenthaltsrecht, d.h. nach drei Monaten kann die Aufenthaltserlaubnis entfallen.

6 | Einen Einblick in die Vielzahl der Möglichkeiten des Aufenthalts nach dem Aufenthaltsgesetz und deren Bezüge zu SGB und Arbeitsmarkt bietet IQ Netzwerk Niedersachsen (2020). 
- Ausländer:innen ohne Aufenthaltsrecht (das umfasst auch Nichterwerbstätige ohne ausreichende Existenzmittel),

- Ausländer:innen mit Aufenthaltsrecht allein zur Arbeitssuche und

- Leistungsberechtigte nach dem Asylbewerberleistungsgesetz.

Ausländer:innen muss als Voraussetzung für den originären Leistungsbezug die Arbeit grundsätzlich erlaubt sein $(\$ 8$ SGB II). Ein Ausschluss von Leistungen des SGB XII ist zudem möglich, wenn die Einreise nur mit dem Ziel erfolgte, Sozialhilfe zu erlangen. Anerkannte Asylbewerber:innen und Flüchtlinge im Besitz einer Aufenthaltserlaubnis haben nach Ablauf des Anspruchs auf Leistungen nach dem AsylbLG Anspruch auf Leistungen nach dem SGB II und XII.

Im Regelfall wird der Zugang zum SGB II und XII Ausländer:innen erst nach fünf Jahren gewöhnlichem Aufenthalt in Deutschland gewährt. In Hinblick auf die Frist von fünf Jahren gilt eine Ausnahme für EU-Bürger:innen, wenn diese durch Beschäftigung in Deutschland den Selbstständigen- oder Arbeitnehmer:innenstatus erworben haben: Dieser Status hat für Unionsbürger:innen zur Folge, dass sie bei unfreiwilliger Arbeitslosigkeit oder Aufgabe einer selbstständigen Tätigkeit von mindestens einem Jahr und der Meldung als arbeitsuchend schon vor Ablauf der Fünfjahresfrist nicht vom SGB II ausgeschlossen werden. ${ }^{7}$

Das gilt auch bei Tätigkeit von mindestens sechs Monaten und weniger als einem Jahr - dann ist ein Leistungsbezug für sechs Monate möglich und auch darüber hinaus, wenn ein Nachweis der Arbeitssuche verbunden mit begründeter Aussicht, eingestellt zu werden, erbracht wird. Die erfolgreiche und mit einiger Gewissheit auch zukünftig wieder erfolgreiche Erwerbsintegration führt damit für Unionsbürger zur Integration in die Grundsicherungssysteme.

Das Asylbewerberleistungsgesetz gilt für Asylbewerber:innen und weitere Konstellationen im Kontext von Flucht und Migration. Dazu zählen einerseits Menschen, die individuell Asyl im Rahmen des Asylrechts beantragt haben (bzw. beantragen wollen; dabei gelten auch Folge- oder Zweitantrag) und während des Verfahrens eine Aufenthaltserlaubnis besitzen.

Andererseits zählen dazu eine Reihe von Flüchtlingsgruppen, etwa Menschen, in deren Heimatland Krieg herrscht, Menschen mit dem Status der Duldung und vollziehbar Ausreisepflichtige, »wenn die Abschiebedrohung noch nicht oder nicht mehr vollziehbar ist (Wissenschaftliche Dienste 2020, S. 6). Auch Ehegatten, Lebenspartner:innen oder minderjährige Kinder der Genannten sind leistungsberechtigt.

7 | Vorher greift nach sozialversicherungspflichtiger Beschäftigung ggf. das Arbeitslosengeld nach SGB III. 
Schließlich gibt es Guppen von Menschen, die keinen Zugang zur Grundsicherung haben. Dies betriff nicht nur Personen ohne festen Aufenthalt in Deutschland und solche, die nicht bedürftig sind. Wie oben beschrieben, sind auch EU-Bürger:innen ohne Selbstständigen- oder Arbeitnehmerstatus in den ersten fünf Jahren ihres Aufenthalts von der Grundsicherung ausgeschlossen. Außerdem haben Personen, für deren Schutz ein anderer EU-Mitgliedsstaat oder ein spezieller Drittstaat zuständig ist (»Dublin-III-Verordnung«; vgl. Wissenschaftliche Dienste 2020, S. 6f.), „vollziehbar Ausreisepflichtige« (Personen ohne oder mit erloschenem Aufenthaltstitel, Personen, die unerlaubt eingereist sind) sowie »Illegale« keine Ansprüche. ${ }^{8}$

\subsection{Die Leistungen}

Die Leistungen des SGB II und XII werden im Beitrag von Irene Becker in diesem Band kritisch beleuchtet. Das AsylbLG als Sondersystem der Sozialhilfe unterscheidet sich von der Grundsicherung im SGB in Hinblick auf die Leistungshöhe, den Modus der Leistungserbringung und auch die damit verbundene soziale Zielsetzung.

Im AsylbLG wird zwischen den Leistungen zur Deckung des Bedarfs an Ernährung, Unterkunft, Heizung, Kleidung, Gesundheitspflege und Gebrauchsund Verbrauchsgütern des Haushalts einerseits und Leistungen zur Deckung des notwendigen persönlichen Bedarfs andererseits unterschieden. Im Unterschied zum SGB spielen Sachleistungen eine deutlich größere Rolle: Bei Unterbringung in Aufnahmeeinrichtungen werden die Leistungen zur Deckung des notwendigen Bedarfs als Sachleistungen gewährt. Kleidung kann durch Gutscheine gewährt werden; Gebrauchsgüter können leihweise zur Verfügung gestellt werden. Bei Unterbringung außerhalb von Einrichtungen können die Leistungen als Sachleistungen gewährt werden, hier gilt aber der Vorrang von Geldleistungen (Wissenschaftliche Dienste 2020, S. 5 und 9f.).

Der notwendige Bedarf mit Ausnahme der Bedarfe für Unterkunft, Heizung, Hausrat, Wohnungsinstandhaltung und Haushaltsenergie ist - wenn er als Geldleistung gewährt wird - in Abhängigkeit von Alter, Familiensituation und Unterbringung auf 143 bis 202 Euro festgelegt. Der notwendige persönliche Bedarf ist - wenn er als Geldleistung gewährt wird - in Abhängigkeit von Alter, Familiensituation und Unterbringung auf 104 bis 162 Euro festgelegt. Tabelle 1 fasst die Höhe der Leistungen zusammen und stellt sie den Leistungen

8 | In manchen Fällen können hier wiederum Überbrückungsleistungen nach SGB XII bis zur Ausreise gewährt werden. 
des SGB II und XII gegenüber, die nur in Ausnahmefällen als Sachleistungen gewährt werden können.

Tab. 1: Leistungssätze im Asylbewerberleistungsgesetz und Regelsätze im SGB II und XII (2021)

\begin{tabular}{|c|c|c|c|c|}
\hline & $\begin{array}{l}\text { Not- } \\
\text { wendiger } \\
\text { persönlicher } \\
\text { Bedarf }\end{array}$ & $\begin{array}{l}\text { Notwendiger Bedarf } \\
\text { (ohne Unterkunft, } \\
\text { Heizung, Hausrat Woh- } \\
\text { nungsinstandhaltung } \\
\text { und Haushaltsenergie) }\end{array}$ & Gesamt & $\begin{array}{c}\text { Regel- } \\
\text { bedarf } \\
\text { SGB II/ } \\
\text { XII }\end{array}$ \\
\hline $\begin{array}{l}\text { alleinstehende Erwachsene } \\
\text { oder Jugendliche ohne } \\
\text { Elternteil }\end{array}$ & $162 €$ & $202 €$ & $364 €$ & $446 €$ \\
\hline $\begin{array}{l}\text { Erwachsene, die mit Gatte/ } \\
\text { Gattin oder Partner:in zusam- } \\
\text { menleben, oder die in einer } \\
\text { Aufnahmeeinrichtung oder } \\
\text { Gemeinschaftsunterkunft } \\
\text { untergebracht sind }\end{array}$ & $146 €$ & $182 €$ & $328 €$ & $401 €$ \\
\hline $\begin{array}{l}\text { Erwachsene unter } 25 \text { Jahren, } \\
\text { die mit einem Elternteil zu- } \\
\text { sammenleben, oder Erwach- } \\
\text { sene in einer stationären } \\
\text { Einrichtung }\end{array}$ & $130 €$ & $162 €$ & $292 €$ & $357 €$ \\
\hline $\begin{array}{l}\text { Jugendliche vom 15. bis } \\
\text { 18. Lebensjahr }\end{array}$ & $110 €$ & $213 €$ & $323 €$ & $373 €$ \\
\hline $\begin{array}{l}\text { Kinder vom 7. bis } 14 \text {. Lebens- } \\
\text { jahr }\end{array}$ & $108 €$ & $174 €$ & $282 €$ & $309 €$ \\
\hline Kinder bis zum 6. Lebensjahr & $104 €$ & $143 €$ & $247 €$ & $283 €$ \\
\hline
\end{tabular}

Quelle: eigene Darstellung

Seit 2014 orientieren sich diese Leistungen am SGB XII und sollen (wie die des SGB II) entsprechend der im SGB XII festgelegten Änderungsrate fortgeschrieben werden. Die gegenüber den Sozialgesetzbüchern niedrigere Leistungssumme wird vom Gesetzgeber mit dem Hinweis darauf begründet, dass Menschen, die eine nur temporäre Bleibeperspektive hätten, bestimmte in den Regelbedarfen der Sozialgesetzbücher berücksichtigte Produkte und Dienstleistungen nicht bräuchten oder dass diese Leistungen an anderer Stelle gewährt würden. Dies betrifft Leistungen für den Hausrat, teilweise Ausgaben für die Gesund- 
heitspflege und die Kosten für die Beschaffung eines Personalausweises (sic!; vgl. Deutscher Bundestag 2014, S. 21).

Nach Inkrafttreten des AsylbLG 1993 waren die Leistungen zunächst bis 2013 nicht mehr angepasst und erst aufgrund eines Urteils des Bundesverfassungsgerichts (2012) angehoben worden. 2015 wurden Leistungen für manche Gruppen von Ausreisepflichtigen eingeschränkt.

2019 wurden die Bedarfsstufen zwar angepasst und die Sätze des notwendigen persönlichen Bedarfs angehoben, letztlich wurde diese Anhebung jedoch durch die neue Eingruppierung von Personen, die in einer Aufnahmeeinrichtung leben, von der ersten in die zweite Regelbedarfsstufe konterkariert. Zudem wurden Kostenpunkte (Wohnungsinstandhaltung und Strom) aus den notwendigen Leistungen ausgegliedert, die nun gesondert als Geld- oder Sachleistung gewährt werden. In der Summe sind die Leistungen damit gekürzt worden. ${ }^{9} 2020$ und 2021 wiederum wurden die Bedarfssätze entsprechend der Fortschreibung der Regelbedarfe im SGB XII auf die in Tabelle 1 angegebenen Werte angepasst.

Entsprechend der Setzung, dass nur eine temporäre Bleibeperspektive besteht, werden nach 18 Monaten (bis 2019 nach 15 Monaten) die Regeln des SGB XII angewandt, die sogenannte Analogleistung (mit Ausnahme für Personen in einer »dem Grunde nach förderfähigen Ausbildung«). In der Gesetzesbegründung von 2014 heißt es dazu, dass Leistungsberechtigte vorher »noch keine Perspektive auf einen Daueraufenthalt haben« (Deutscher Bundestag 2014, S. 19). ${ }^{10}$ Rechtlich bleiben die Bezieher:innen der Analogleistungen allerdings leistungsberechtigt nach dem AsylbLG. ${ }^{11}$

Der Zugang zum Arbeitsmarkt ist für Asylbewerber:innen und Geflüchtete zunächst eingeschränkt. Grundsätzlich besteht ein Beschäftigungsverbot wäh-

9 | Vgl. hierzu auch die Stellungnahme des DGB (2019) und den Entwurf eines Dritten Gesetzes zur Änderung des Asylbewerberleistungsgesetzes (Deutscher Bundestag 2019). 10 | Zu den Verfahrensdauern und damit zur Relevanz der Analogleistungen vgl. Deutscher Bundestag 2020.

11 | Die Grundleistungen werden noch ergänzt durch Leistungen, die an den individualisierten Charakter der Sozialhilfe erinnern, indem sie auf spezielle Lebensumstände reagieren: Leistungen zur Bildung und Teilhabe für Kinder, Jugendliche und junge Erwachsene, Krankenleistungen, Leistungen für besondere Bedarfe, Arbeitsgelegenheiten und Integrationsleistungen. Krankenleistungen ( $\$ 4$ AsylbLG) sind laut Gesetz auf akute Behandlung, Schutzimpfungen und Vorsorgeuntersuchungen beschränkt. Mütter und Wöchnerinnen erhalten Hilfe durch Ärzt:innen; Pfleger:innen und Hebammen sowie Arznei-, Verbands- und Hilfsmittel. 
rend der dreimonatigen Wartefrist ${ }^{12}$, während der Pflicht, in einer Aufnahmeeinrichtung zu wohnen, oder nach Einreise aus einem sicheren Herkunftsland. Ansonsten ist in vielen Fällen eine Genehmigung zur Arbeitsaufnahme notwendig (Bundesagentur für Arbeit o.J.).

Die genannte Einschränkung ist nicht der einzige Bezugspunkt von Sozialleistungen und Arbeit für Asylbewerber:innen - wie in anderen Bereichen der Grundsicherung auch spielt die Verpflichtung zur Arbeit eine Rolle im Leistungsrecht. Schammann (2017, S. 748) weist sogar auf eine »punktuelle Liberalisierung durch meritokratische Elemente des Asylrechts« im Zuge gesetzlicher Änderungen in den Jahren 2015/2016 hin, also auf eine Stärkung des Leistungsgedankens auch im Umgang mit Geflüchteten. ${ }^{13}$

Konkret können in Aufnahmeeinrichtungen Arbeitsgelegenheiten geschaffen werden, die »insbesondere zur Aufrechterhaltung und Betreibung der Einrichtung " dienen, sowie "soweit wie möglich" Arbeitsgelegenheiten bei staatlichen, kommunalen und gemeinnützigen Trägern, »sofern die zu leistende Arbeit sonst nicht, nicht in diesem Umfang oder nicht zu diesem Zeitpunkt verrichtet werden würde« ( $\$ 5$ AsylbLG). Die Aufwandsentschädigung beträgt 80 Cent pro Stunde.

In diesem Fall sind arbeitsfähige, nicht erwerbstätige Leistungsberechtigte zur Wahrnehmung der Arbeitsgelegenheit verpflichtet, ansonsten erfolgt eine Leistungskürzung bis auf notwendige Leistungen zur Ernährung, Unterkunft, Körper- und Gesundheitspflege. Es handelt sich hier nicht um ein arbeits- oder sozialversicherungspflichtiges Arbeitsverhältnis. Außerdem kann eine Aktivierung durch Teilnahme an einer "Flüchtlingsintegrationsmaßnahme" - Arbeitsgelegenheiten der Bundesagentur für Arbeit - erfolgen oder den Leistungsberechtigten die Teilnahme an Integrationskursen zur Pflicht gemacht werden.

Entsprechend dem Grundgedanken, dass Grundsicherungsleistungen und damit auch die Leistungen des AsylbLG im Falle von Bedürftigkeit zu gewähren sind, sind vorhandenes Einkommen und Vermögen aufzubrauchen (Freibeträge). Hier unterscheiden sich die Grenzen von denen des SGB II. Im Rahmen des AsylbLG existiert bei Erwerbseinkommen ein Selbstbehalt von 25 Prozent, höchstens die Hälfte des maßgeblichen Satzes zur Deckung aller notwendigen

12 | Ab Ausstellung eines Ankunftsnachweises, Asylantragstellung oder Erteilung einer Duldung - diese Frist betrug bis 2014 noch neun Monate.

13 | Dieser Gedanke findet sich auch bei Scherschel (2016, S. 261), die darauf hinweist, dass Erwerbsarbeit »in mancher Hinsicht zur Voraussetzung [wird], um Aufenthaltschancen zu verbessern«. Der humanitäre Aufenthalt werde - so Scherschel (2016, S. 262) - politisch im Hinblick auf Arbeitsmarktbelange konditioniert. 
persönlichen und notwendigen Bedarfe. Alternativ bleibt bei entsprechender ehrenamtlicher Tätigkeit eine sogenannte Übungsleiterpauschale von höchstens 250 Euro anrechnungsfrei. Im Unterschied dazu sind im SGB II Erwerbseinkünfte bis 100 Euro (alternativ 250 Euro Übungsleiterpauschale), weitere 20 Prozent des Einkommens zwischen 100 und 1.000 Euro und 10 Prozent des darüber liegenden Einkommens bis 1.200 Euro anrechnungsfrei. ${ }^{14}$

Ähnlich wie im Bereich des SGB II können auch die Leistungen des AsylbLG gekürzt werden, und zwar bis auf die Leistungen für Ernährung und Unterkunft einschließlich Heizung sowie Körper- und Gesundheitspflege. Verglichen mit dem SGB II bezwecken die Sanktionen aber nicht die Überwindung von Hilfsbedürftigkeit, sondern die Herstellung von Rechtstreue:

»Die Erfüllung aufenthaltsrechtlicher Mitwirkungsplichten dient jedoch nicht der Überwindung der eigenen Hilfebedürftigkeit, sondern dem Vollzug aufenthaltsbeendender Maßnahmen. Mit der Abschiebung endet lediglich die Leistungsberechtigung nach $\$ 1$ AsylbLG, nicht jedoch die Hilfebedürftigkeit der betroffenen Person.« (Seidl 2020, S. 215).

Das stelle sich nur bei den Arbeitsgelegenheiten und Maßnahmen zur Integration anders dar (Seidl 2020, S. 217). Zudem existieren Regelungen, die dazu führen, dass in bestimmten Fällen Leistungskürzung nicht durch eigenes Verhalten abgewendet werden kann, etwa wenn der Asylantrag als unzulässig abgelehnt wurde (Seidl 2020, S. 215 f.).

Der Vergleich mit den Regelungen der Sozialgesetzbücher legt damit zunächst eine zentrale Gemeinsamkeit nahe: Zielsetzung des AsylbLG ist in einem ersten Schritt die Gewährleistung des soziokulturellen Existenzminimums wie auch das Bundesverfassungsgericht in seinem Urteil vom 18. Juli 2012 betont:

»Auch eine kurze Aufenthaltsdauer oder Aufenthaltsperspektive in Deutschland rechtfertigte es im Übrigen nicht, den Anspruch auf Gewährleistung eines menschenwürdigen Existenzminimums auf die Sicherung der physischen Existenz zu beschränken. [...] Art. 1 Abs. 1 GG garantiert ein menschenwürdiges Existenzminimum, das durch im Sozialstaat des Art. 20 Abs. 1 GG auszugestaltende Leistungen zu sichern ist, als einheitliches, das physische und soziokulturelle Minimum umfassendes Grundrecht. Ausländische Staatsangehörige verlieren den Geltungsanspruch als soziale Individuen nicht dadurch, dass sie ihre Heimat verlassen und sich in der Bundesrepublik Deutsch-

14 | Für Leistungsberechtigte, die zusammen ein minderjähriges Kind haben oder mit einem solchen zusammenleben, beträgt die Grenze nicht 1.200 Euro, sondern 1.500 Euro. 
land nicht auf Dauer aufhalten [...]. Die einheitlich zu verstehende menschenwürdige Existenz muss daher ab Beginn des Aufenthalts in der Bundesrepublik Deutschland realisiert werden.« (Bundesverfassungsgericht 2012)

Diese Gemeinsamkeit bedeutet auch, dass die grundlegende Kritik an der Berechnung der Regelbedarfe, mithin der politischen Interpretation des Existenzminimums für beide Bereiche gilt. Darüber hinaus zeigen sich aber deutliche Unterschiede, nicht nur bei der Leistungsbemessung, sondern auch beim Umgang mit Arbeit.

Die Anrechnungsregeln sind im AsylbLG bei Erwerbstätigkeit härter als im SGB II, hinzu kommen Einschränkungen bei der Arbeitsmarktbeteiligung. Allerdings sind Tendenzen zu einer Stärkung des Leistungsgedankens auch in der Asyl- und Flüchtlingspolitik auszumachen (Scherschel 2016; Schammann 2017). Dennoch scheint der temporäre Charakter der Hilfe im AsylbLG nach wie vor weniger von der - idealen - Emanzipation von der Hilfe durch gesellschaftliche Integration her gedacht (Selbsthilfe durch Erwerbsarbeit und Pflicht zur Selbsthilfe), sondern eher von der - angenommenen - Begrenztheit des Rechtstatus bzw. der Leistungen her, dem ein weiterer Status folgt (Anerkennung und längerer Aufenthalt, Abschiebung oder Analogleistung).

Dies führt zu einer problematischen Nutzung der Sanktionen im AsylbLG, wenn davon ausgegangen wird, dass eine Kürzung von existenzsichernden Leistungen nur durch das Ziel gerechtfertigt werden kann, Leistungsempfänger:innen zu veranlassen, ihre Hilfebedürftigkeit durch Nutzung einer bestehenden Möglichkeit zur Arbeitsaufnahme zu überwinden. ${ }^{15}$ In diesem Sinne erscheint die Kürzung von Leistungen im Rahmen des AsylbLG zur Erzwingung von Mitarbeit im Verwaltungsprozess bei gleichzeitiger Einschränkung der Arbeitsmarktbeteiligung als äußerst fragwürdig.

\section{Empirische Entwicklungen}

Wie relevant sind die Leistungen des AsylbLG und die Nutzung der Grundsicherungsleistungen der Sozialgesetzbücher durch Ausländer:innen? Hier geht es nicht nur darum, ob die in diesem Beitrag behandelte Problematik mehr als ein Randproblem ist; diese Frage ist auch vor dem Hintergrund wiederkehrender Debatten um Leistungsmissbrauch von politischer Relevanz.

15 | Und auch dieses Ziel müsste in Hinblick auf gute und angemessen entlohnte Arbeit noch weiter qualifiziert werden. 
Zur Einordnung der im Folgenden dargestellten Entwicklungen seien hier noch die Zahlen zu In- und Ausländer:innen insgesamt genannt: Zum 31.12.2019 lebten in Deutschland rund 10,4 Millionen Ausländer:innen bei einer Gesamtbevölkerung von 83,2 Millionen (Destatis 2021b). Abweichend davon werden auf Grundlage des Ausländerzentralregisters zum gleichen Stichtag 11,2 Millionen Personen genannt (Destatis 2021a). ${ }^{16}$ Rund ein Achtel der Bevölkerung hatte damit nicht die deutsche Staatsangehörigkeit.

Die Zahl der Asylbewerber:innen ist seit dem Höchststand 2016 (745.545 Personen mit Erst- und Folgeanträgen) auf 122.170 Personen im Jahr 2020 gefallen (BAMF 2021, S. 5). ${ }^{17}$ Die Daten zum Bezug von Sozialleistungen müssen vor dem Hintergrund dieser Größenverhältnisse gesehen werden.

Die Nutzung der Grundsicherung ist in der Summe von der Entwicklung der Bedürftigkeit in der deutschen Gesellschaft geprägt - ausschlaggebend ist hier die Entwicklung des Arbeitsmarktes. Im Falle der Ausländer:innen wird die Nutzung zusätzlich durch die Migration geprägt; die Anzahl der Leistungsberechtigten bzw. Bedürftigen ist also nicht nur von ökonomischen Entwicklungen innerhalb Deutschlands abhängig, sondern auch von internationalen Wanderungsbewegungen. Anders formuliert: Nicht nur Werte wie die Quoten der Leistungsbezieher:innen nach SGB II ändern sich im Zeitverlauf, sondern auch die absoluten Zahlen, auf die sie sich beziehen.

\subsection{Nutzung von Leistungen des SGB II und XII durch Ausländer:innen}

Dieses Zusammenspiel von Migration und Arbeitsmarkt zeigt sich in den Quoten des SGB-II-Bezugs, die in der Statistik der Bundesagentur für Arbeit (2021) teilweise ${ }^{18}$ nach Nationalität ausgewiesen werden.

Ein Blick auf die Statistiken zeigt dabei erstens, dass im Dezember 2020 Deutsche die absolut größte Gruppe unter den Bezieher:innen gestellt haben (3,3 Millionen deutsche Regelbedarfsberechtigte gegenüber 2,0 Millionen ausländischen im Dezember 2020), dass aber die SGB-II-Quoten unter Ausländer:innen

16 | "Sowohl das AZR [Ausländerzentralregister] als auch die Bevölkerungsfortschreibung tendieren aufgrund der Untererfassung von Fortzügen ins Ausland zur Ermittlung überhöhter Ausländerinnen- und Ausländerzahlen. Ein Großteil der Differenz zwischen AZR und Bevölkerungsfortschreibung erklärt sich durch die regelmäßige Korrektur der Bevölkerungsfortschreibung durch den Zensus« (Destatis 2021g, S. 5).

17 | In diesen Zahlen sind überwiegend Erstantragsteller:innen enthalten. Die Anzahl der Erstanträge lag 2016 bei 722.370 und 2020 bei 102.581 (BAMF 2021, S. 6).

18 | Nicht alle Nationalitäten werden einzeln aufgeführt. 
deutlich höher sind (5,9 Prozent gegenüber 19,1 Prozent, jeweils bezogen auf die Bevölkerung, siehe Abbildung 1). ${ }^{19}$

Abb. 1: SGB-II-Quote bezogen auf die jeweilige Bevölkerungsgruppe (1/2012-12/2020; in Prozent)

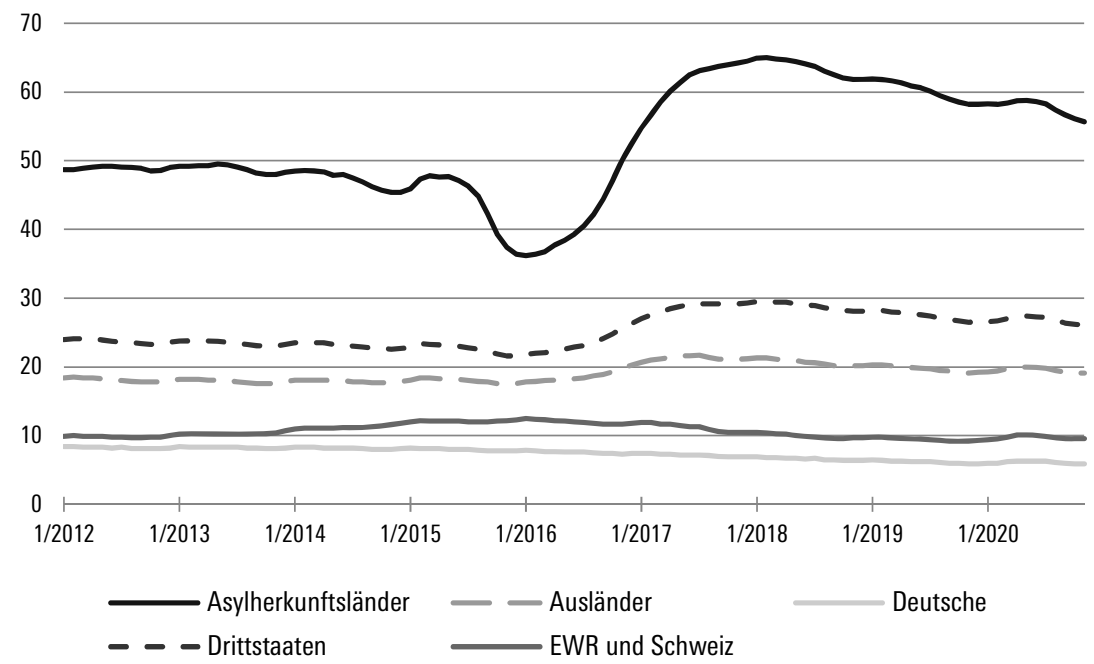

Quelle: Bundesagentur für Arbeit 2021 (eigene Darstellung)

Zugleich wird zweitens deutlich, dass die Kategorie "Ausländer:innen « für eine in sich stark differenzierte Gruppe steht: Bei Personen aus dem Europäischen Wirtschaftsraum (EWR) und der Schweiz lag die SGB-II-Quote bei 9,8 Prozent, bei Personen aus den von der Bundesagentur als "Asylherkunftsländern« bezeichneten Ländern ${ }^{20}$ bei 55,7 Prozent. In den unterschiedlichen Bezugsquoten zeigen sich Arbeitsmarktnähe bzw. -ferne; hier spiegeln sich auch die Gründe für Migration.

Unterschiede zeigen sich auch in den Zahlen zu Erwerbsintegration und Arbeitslosigkeit. Die Beschäftigungsquoten von Rumän:innen beispielsweise

19 | Die Relation der deutschen zu den ausländischen Bezieher:innen bei der Hilfe zum Lebensunterhalt (HLU) nach SGB XII weicht deutlich von der Grundsicherung für Arbeitsuchende ab: Von insgesamt 344.841 Bezieher:innen am Stichtag 31.12.2019 waren mit 29.046 Personen weniger als ein Zehntel Ausländer:innen (Destatis 2021d). 20 In dieser Kategorie sind Afghanistan, Eritrea, Irak, Iran, Nigeria, Pakistan, Somalia und Syrien zusammengefasst. 
erreichten zuletzt (Dezember 2020) fast die von Deutschen (inklusive wie auch exklusive geringfügiger Beschäftigung $)^{21}$, zugleich lagen ihre Arbeitslosenquoten wie auch die SGB II- und ELB-Quote ${ }^{22}$ merklich über denen der Deutschen (Bundesagentur für Arbeit 2021). Hier dürften unter anderem geringere Löhne der Rumän:innen dazu führen, dass trotz Erwerbsintegration aufstockende Sozialleistungen bezogen werden.

Insgesamt deuten diese Ergebnisse sowohl auf eine qualitativ unterschiedliche Arbeitsmarktintegration der Angehörigen verschiedener Nationalitäten hin, auch in Bezug auf die erzielten Löhne, als auch auf eine unterschiedliche personelle Zusammensetzung der jeweiligen Grundgesamtheit, etwa mit Blick auf die Empfängerhaushalte hinsichtlich der Anzahl der Kinder.

Drittens unterstreicht der Blick in die Statistiken auch die zwei Logiken, die auf die Nutzung der Grundsicherung durch Ausländer:innen Einfluss haben, wie sich auch an Abbildung 1 zeigt: In Zeiten geringerer Migration laufen die Linien für Deutsche und Ausländer:innen annähernd parallel (bis 2015, ab 2018); gröBere Abweichungen in der Entwicklung werden durch das Migrationsgeschehen bestimmt, durch das sich die Zusammensetzung der Gruppe der Ausländer:innen verändert.

Aus diesen Beobachtungen und Entwicklungen lässt sich zunächst ableiten, dass die über den Arbeitsmarkt vermittelte gesellschaftliche Integration und ökonomische Unabhängigkeit für bestimmte Gruppen der Bevölkerung ein Problem darstellt. Hinzu kommen weitere soziale Unterschiede zwischen Bevölkerungsgruppen, die Rückwirkungen auf die (nachgewiesene) Bedürftigkeit haben. Für die höhere Nutzung der Leistungen durch bzw. für die Bedürftigkeit von Ausländer:innen lassen sich verschiedene Gründe anführen (vgl. Sozialpolitik-aktuell.de o.J. a). Dies betrifft den Zugang zu Arbeit bzw. das Problem der Arbeitslosigkeit, den Zugang zu Arbeit mit angemessener Bezahlung (vgl. Sozialpolitik-aktuell. de o.J. b), ${ }^{23}$ die Arbeitsteilung im Haushalt bzw. Erwerbstätigkeit der Geschlechter und die Anzahl der Kinder, die Rückwirkungen auf die materielle Bedürftigkeit hat.

21 | Quote der sozialversicherungspflichtigen Beschäftigung: Deutsche 63,4 Prozent, Rumän:innen 59,8 Prozent. Beschäftigungsquote inklusive ausschließlich geringfügig Beschäftigter: Deutsche 69,2 Prozent, Rumän:innen 64,2 Prozent.

22 | Quote der erwerbsfähigen Leistungsberechtigten.

23 | Hier spiegeln sich auch unterschiedliche Qualifikations- und Tätigkeitsprofile wider; vgl. Bundesagentur für Arbeit 2021, Tabelle 2.5. 


\subsection{Nutzung der Leistungen des AsylbLG}

Deutlicher zeigt sich die Migration naturgemäß in den Zahlen zum Bezug von Leistungen nach dem AsylbLG, die die Fluchtmigration widerspiegeln (Destatis 2021e). Die Zahlen stiegen auf rund 975.000 zum Stichtag 31.12.2015 und sanken in der Folge auf 385.000 zum 31.12.2019. Zur Interpretation dieser Daten und zum Zusammenhang mit den oben skizzierten Entwicklungen im SGB II ist der Hinweis notwendig, dass nur ein Teil der Asylbewerber:innen später Leistungen nach dem SGB II bezieht, was in der Anerkennung bzw. Ablehnung der Asylanträge begründet liegt. 2020 lag die Gesamtschutzquote - d.h. die Anerkennung als Flüchtling, Gewährung von subsidiärem Schutz oder Abschiebeverbot - bei 43,1 Prozent der Anträge (bpb 2021).

In den Zahlen zeigt sich zudem die politische Steuerung der Unterbringung, die für die Gewährung von Geld- oder Sachleistungen einen Unterschied macht (siehe Abbildung 2).

Abb. 2: Empfänger:innen von Regelleistungen nach dem Asylbewerberleistungsgesetz nach Art der Unterbringung (1994-2019)

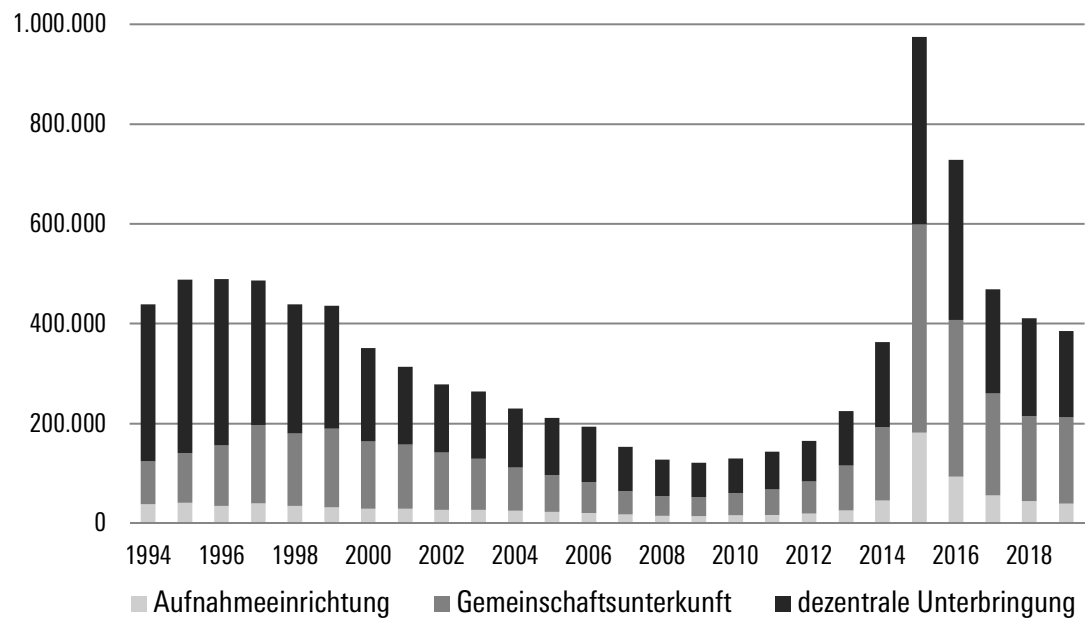

Anmerkung: Stichtag jeweils 31. Dezember

Quelle: Destatis 2021e (siehe dort für methodische Hinweise; eigene Darstellung)

Die vom Statistischen Bundesamt (Destatis) vorgehaltenen Daten erlauben darüber hinaus eine Analyse nach Erwerbsstatus. Die Zahlen machen deutlich, dass eine Arbeitsmarktintegration von Bezieher:innen von Leistungen nach dem 
AsylbLG so gut wie nicht stattfindet (Destatis 2021f), was den oben skizzierten rechtlichen Einschränkungen der Erwerbsbeteiligung entspricht.

In der gesellschaftlichen Debatte um Migration wird teils auch auf die Kosten der Sozialleistungen verwiesen. In der Tat spiegeln - wenig überraschend die Ausgaben für Leistungen nach dem AsylbLG die Zahl der Empfänger:innen. Im Jahr 2016 lagen die Ausgaben bei 9,4 Milliarden Euro; in der Folge sanken sie bis 2019 wieder auf 4,4 Milliarden Euro (Destatis 2021c).

Es ist sinnvoll, die Kosten, die speziell für diese Grundsicherungsleistung entstehen, in Relation zum Sozialstaat insgesamt zu sehen. Im Jahr 2016 standen laut Sozialbudget den genannten Ausgaben für Leistungen nach dem AsylbLG Ausgaben für die Förder- und Fürsorgesysteme in Höhe von 180,2 Milliarden Euro gegenüber, bei einem Sozialbudget von insgesamt 928,3 Milliarden Euro (BMAS 2020).

Anders formuliert: Beim Höchststand der Zahl der Leistungsempfänger:innen machten die Ausgaben für Leistungen nach dem AsylbLG etwa 1 Prozent der Sozialausgaben aus. Problematisch bleiben allerdings die Fragen nach der Planbarkeit und nach den Kosten einer stark ansteigenden Fluchtmigration ähnlich wie die Leistungen bei Arbeitslosigkeit sind diese Ausgaben nur schwer direkt steuerbar bzw. prognostizierbar.

\section{Fazit}

Bei Personen mit dauerhaftem Aufenthalt in Deutschland und Arbeitsmarktintegration wird beim Bezug von Sozialleistungen - und das heißt auch beim Bezug von Leistungen der Grundsicherung - kein Unterschied zwischen Deutschen und Ausländer:innen gemacht. Deutlich wird auch die Rolle des Arbeitsmarktes, da eine Erwerbsintegration für EU-Bürger:innen einen Anspruch auf Leistungen schon vor Ablauf der Fünfjahresfrist ermöglicht. Dennoch zeigen sich erhebliche Probleme, die über die Grundfrage der angemessenen Leistungshöhe und Regelbedarfsberechnung sowie die generelle Kritik am System des "Förderns und Forderns« hinausgehen.

In Hinblick auf den zeitlich begrenzten Ausschluss von Ausländer:innen von Leistungen des SGB II und XII ist sowohl auf verfassungs- und europarechtliche als auch auf internationale Normen zu verweisen und zu kritisieren, dass die Gewährung eines menschenwürdigen Existenzminimums in bestimmten Konstellationen trotz längerfristigen Aufenthalts in Deutschland offensichtlich verweigert wird. Der Ausschluss von Arbeitsuchenden steht zudem im Widerspruch zur europäischen Arbeitskräftemobilität und setzt eine Interpretation 
der Grundsicherung für Arbeitsuchende voraus, die den Grundsicherungscharakter gegenüber der arbeitsmarktpolitischen Funktion überbetont.

Mit Blick auf das Asylbewerberleistungsgesetz (AsylbLG) stellt sich zunächst die Frage, ob und inwiefern ein spezielles System überhaupt gerechtfertigt ist (vgl. hierzu DGB 2019). Da die ursprüngliche migrationspolitische Intention rechtlich keinen Bestand hat, muss sich das System entsprechend an ähnlichen Maßstäben messen lassen wie das SGB II. Dann ist zwar eine Verrechnung von Sachleistungen und Geldleistungen denkbar, die abgesenkte Leistungshöhe allerdings scheint kaum begründbar, insbesondere wenn das soziokulturelle Existenzminimum durch ein Statistikmodell und nicht durch ein Warenkorbmodell bestimmt wird (siehe den Beitrag von Irene Becker in diesem Band).

$\mathrm{Zu}$ kritisieren ist zudem, dass genau zu dem Zeitpunkt, als die Leistungen des AsylbLG hätten angehoben werden müssen, eine Neuberechnung der Bedarfe mit dem Ergebnis einer Absenkung der Leistungen für bestimmte Personenkonstellationen stattfand. Damit muss festgehalten werden, dass nicht nur bestimmte Gruppen von Ausländer:innen vollständig vom Leistungsbezug in der Grundsicherung ausgeschlossen sind, sondern dass darüber hinaus das Existenzminimum nicht als Menschenrecht verstanden und realisiert wird, sondern als eine Hierarchie von Leistungen.

Die Sanktionen sind im SGB II ein wiederholt diskutiertes Problem und mussten letztlich wegen fehlender nachgewiesener Effektivität korrigiert werden - so das Urteil des Bundesverfassungsgerichts (2019b). Bei den Sanktionen im AsylbLG fehlt genau dieser Nachweis der Effektivität; vor allem aber stößt hier die Sanktionslogik insgesamt an ihre Grenzen.

Im SGB II werden Sanktionen als grundsätzlich sinnvoll erachtet, weil es deren Zielsetzung ist, die Hilfebedürftigkeit zu überwinden. In diesem Sinne ist aus Sicht des Bundesverfassungsgerichts (2019b) die Ablehnung einer Arbeitsmöglichkeit ein legitimer Sanktionsgrund, da bei Vorliegen einer Arbeitsmöglichkeit das Existenzminimum auf andere Art und Weise sichergestellt werden könne. ${ }^{24}$ Genau diese Logik greift aber im AsylbLG kaum. Ganter zieht entsprechend die Schlussfolgerung:

»Aus diesem Grund ist die Verfassungsmäßigkeit der Verknüpfung von ausländerrechtlichen Mitwirkungspflichten ohne Bezug zur ökonomischen Selbsthilfe mit sozialrechtlichen Sanktionen insgesamt in Frage zu stellen.« (Ganter 2020, S. 116)

24 | Wobei die Kürzung um 60 bzw. 100 Prozent als nicht verfassungsgemäß eingestuft wurde, da die Wirksamkeit dieser Sanktionen auf die Arbeitsmarktintegration nicht erwiesen sei; vgl. Bundesverfassungsgericht 2019b. 
Letztlich müsste also - im Unterschied auch zur bisherigen Rechtsprechung aus diesen systematischen Überlegungen eine deutliche Lockerung der Sanktionsmöglichkeiten im AsylbLG folgen.

In der Gesamtschau zeigt sich damit nicht nur das Grundsicherungssystem als ein wie in anderen Ländern nach Sachbereichen ausdifferenziertes Programm, sondern als eine Hierarchie von Leistungen und Beziehergruppen. Sichtbar wird auch der Bezug von sozialer Sicherung und Arbeit, der über das Sozialversicherungssystem herausgeht, nämlich wenn vorherige Beschäftigung und Arbeitssuche zum SGB-II-Bezug berechtigen.

Bei einer Weiterentwicklung der Grundsicherung in einem erweiterten Sinne steht damit nicht nur die Verbesserung der Leistungen und Berechnungsmethoden auf dem Programm. Vor dem Hintergrund der Ziele gesellschaftlicher und wirtschaftlicher Teilhabe sowie der Gewährung eines Existenzminimums, das als Menschenrecht verstanden werden muss, ist über eine Entdifferenzierung der Grundsicherung nachzudenken. In diesem Zusammenhang sollten Sanktionen auf die vom Bundesverfassungsgericht skizzierte Logik zurückgeführt werden, nämlich auf Leistungskürzung in den Fällen, in denen die Bedürftigkeit zu angemessenen Bedingungen selbstständig überwunden werden kann.

\section{Literatur}

Absenger, N./Blank, F. (2017): From Social Myths to Legal Reality: Limiting the Freedom of Movement of EU Citizens, in: Politiche Sociali/Social Policies 3/2017, S. 496-488.

BAMF = Bundesamt für Migration und Flüchtlinge (2021): Aktuelle Zahlen, Mai 2021, www.bamf.de/SharedDocs/Anlagen/DE/Statistik/AsylinZahlen/ aktuelle-zahlen-mai-2021.html (Abruf am 18.6.2021).

BMAS $=$ Bundesministerium für Arbeit und Soziales (2020): Sozialbudget 2020, Bonn.

$\mathrm{bpb}=$ Bundeszentrale für politische Bildung (2021): Asylentscheidungen und Klagen, www.bpb.de/gesellschaft/migration/flucht/zahlen-zu-asyl/265711/ent scheidungen-und-klagen (Abruf am 18.6.2021).

Bundesagentur für Arbeit (o.J.): Aufenthaltsstatus und Arbeitsmarktzulassung, www.arbeitsagentur.de/unternehmen/arbeitskraefte/aufenthaltsstatus (Abruf am 18.6.2021).

Bundesagentur für Arbeit (2021): Migrationsmonitor (Monatszahlen), Mai 2021, Nürnberg. 
Bundesverfassungsgericht (2012): Urteil vom 18. Juli 2012, Az. 1 BvL 10/10, www. bundesverfassungsgericht.de/SharedDocs/Entscheidungen/DE/2012/07/ls20 120718_1bvl001010.html (Abruf am 29.6.2021).

Bundesverfassungsgericht (2019a): Beschluss vom 04. Dezember 2019, Az. 1 BvL 4/16, www.bundesverfassungsgericht.de/SharedDocs/Entscheidungen/DE/20 19/12/lk20191204_1bvl000416.html (Abruf am 29.6.2021).

Bundesverfassungsgericht (2019b): Urteil vom 05. November 2019 - 1 BvL 7/16, www.bundesverfassungsgericht.de/SharedDocs/Entscheidungen/DE/2019/ 11/ls20191105_1bvl000716.html (Abruf am 24.6.2021).

Deutscher Bundestag (1993): Gesetzentwurf der Fraktionen der CDU/CSU und F.D.P. Entwurf eines Gesetzes zur Neuregelung der Leistungen an Asylbewerber, Drucksache 12/4451, https://dserver.bundestag.de/btd/12/044/1204451.pdf (Abruf am 24.6.2021).

Deutscher Bundestag (2014): Entwurf eines Gesetzes zur Änderung des Asylbewerberleistungsgesetzes und des Sozialgerichtsgesetzes, Drucksache 18/2592, https://dserver.bundestag.de/btd/18/025/1802592.pdf (Abruf am 29.6.2021).

Deutscher Bundestag (2019): Entwurf eines Dritten Gesetzes zur Änderung des Asylbewerberleistungsgesetzes, Drucksache 19/10052, https://dserver.bundes tag.de/btd/19/100/1910052.pdf (Abruf am 29.6.2021).

Deutscher Bundestag (2020): Antwort der Bundesregierung auf die Kleine Anfrage der Abgeordneten Ulla Jelpke, Dr. André Hahn, Gökay Akbulut, weiterer Abgeordneter und der Fraktion DIE LINKE, Drucksache 19/23630, https://dserver.bundestag.de/btd/19/236/1923630.pdf (Abruf am 29.6.2021).

Destatis $=$ Statistisches Bundesamt (2021a): Ausländer: Deutschland, Stichtag, Geschlecht/Altersjahre/Familienstand, GENESIS-Online, Code 12521-0001, www-genesis.destatis.de/genesis/online (Abruf am 18.6.2021).

Destatis = Statistisches Bundesamt (2021b): Bevölkerung: Deutschland, Stichtag, Nationalität, GENESIS-Online, Code 12411-0002, www-genesis.destatis.de/ genesis/online (Abruf am 18.6.2021).

Destatis $=$ Statistisches Bundesamt (2021c): Bruttoausgaben nach dem Asylbewerberleistungsgesetz: Deutschland, Jahre, Leistungsart, GENESIS-Online, Code 22211-0002, www-genesis.destatis.de/genesis/online (Abruf am 18.6.2021).

Destatis $=$ Statistisches Bundesamt (2021d): Empfänger von Hilfe zum Lebensunterhalt: Deutschland, Stichtag, Ort der Leistungserbringung, Geschlecht, Nationalität, GENESIS-Online, Code 22121-0002, www-genesis.destatis.de/ genesis/online (Abruf am 18.6.2021).

Destatis = Statistisches Bundesamt (2021e): Empfänger von Regelleistungen nach dem Asylbewerberleistungsgesetz: Deutschland, Stichtag, Art der Unterbrin- 
gung, GENESIS-Online, Code 22221-0003, www-genesis.destatis.de/genesis/ online (Abruf am 18.6.2021).

Destatis = Statistisches Bundesamt (2021f): Empfänger von Regelleistungen nach dem Asylbewerberleistungsgesetz: Deutschland, Stichtag, Erwerbsstatus, GENESIS-Online, Code 22221-0006, www-genesis.destatis.de/genesis/ online (Abruf am 18.6.2021).

Destatis = Statistisches Bundesamt (2021g): Qualitätsbericht Ausländerstatistik. Ergebnisse des Ausländerzentralregisters, Wiesbaden.

DGB (2019): Schriftliche Stellungnahme zur öffentlichen Anhörung von Sachverständigen in Berlin am 03. Juni 2019, Drucksache des Ausschusses für Arbeit und Soziales 19(11)343, Berlin.

EuGH = Europäischer Gerichtshof (2020): Urteil vom 6. Oktober 2020, Rechtssache C-181/19, https://curia.europa.eu/juris/document/document.jsf?text=\&doc id=232081\&pageIndex=0\&doclang=DE\&mode=req\&dir=\&occ=first\&part=1 (Abruf am 29.6.2021).

Funk, A./Starzmann, P. (2018): Kindergeld für EU-Ausländer. Die meisten Kinder leben in Deutschland, www.tagesspiegel.de/politik/kindergeld-fuer-euauslaender-die-meisten-kinder-leben-in-deutschland/22899518.html (Abruf am 18.6.2021).

Ganter, J. (2020): Das menschenwürdige Existenzminimum zwischen Karlsruhe und Luxemburg, in: Zeitschrift für europäisches Sozial- und Arbeitsrecht (ZESAR) 3/2020, S. 113-119.

Hubl, V./Pfeifer M. (2013): Categorical differentiation in the light of deservingness perceptions: institutional structures of minimum income protection for immigrants and for the disabled, in: Marx, Y./Nelson K. (Hrsg.): Minimum Income Protection in Flux, Houndsmills/Basingstoke, S. 161-189.

IQ Netzwerk Niedersachsen (2020): Übersicht - Zugang zum SGB II und zur Erwerbstätigkeit für drittstaatsangehörige Ausländerinnen und Ausländer. Stand: 26.8.2020, www.einwanderer.net/fileadmin/downloads/tabellen_und_ uebersichten/Zugang_SGB_II_und_Arbeitsmarkt.pdf (Abruf am 18.6.2021).

Knuth, M. (2020): Das Bundesausbildungsförderungsgesetz in der Einwanderungsgesellschaft, in: Soziale Sicherheit 69(5), S. 193-198.

Menkens, S. (2016): Leistungskürzung. Regierung zeigt Härte gegen europäischen »Sozialtourismus«, www.welt.de/politik/deutschland/article158697044/ Regierung-zeigt-Haerte-gegen-europaeischen-Sozialtourismus.html (Abruf am 18.6.2021).

Mitzkat, N./Stepputat, H. (2021): Alleinstehende Geflüchtete. Reichen 328 Euro zum Leben?, www.tagesschau.de/investigativ/panorama/alleinstehende-ge fluechtete-sozialleistungen-101.html (Abruf am 18.6.2021). 
Schammann, H. (2017): Eine meritokratische Wende? Arbeit und Leistung als neue Strukturprinzipien der deutschen Flüchtlingspolitik, in: Sozialer Fortschritt 66(11), S. 741-757.

Scherschel, K. (2016): Citizenship by work? Arbeitsmarktpolitik im Flüchtlingsschutz zwischen Öffnung und Selektion, in: Prokla 46(2), S. 245-266.

Sell, S. (2018): Wenn »unser« Kindergeld ins Ausland fließt - dann kann es sich nur um »EU-Irrsinn« handeln. Oder ist es wieder einmal komplizierter?, https://aktuelle-sozialpolitik.de/2018/03/27/wenn-unser-kindergeld-ins-aus land-fliesst/ (Abruf am 18.6.2021).

Seidl, J. (2020): Die Leistungskürzungen des AsylbLG unter dem Damoklesschwert der Verfassungswidrigkeit. Eine Untersuchung von $₫ 1$ a AsylbLG im Lichte der Vorgaben des BVerfG-Urteils vom 5.11.2019 und der AufnahmeRichtlinie, in: Zeitschrift für europäisches Sozial- und Arbeitsrecht (ZESAR) 5-6/2020, S. 213-217.

Sozialpolitik-aktuell.de (o. J. a): Empfängerquoten Leistungen der Grundsicherung für Arbeitsuchende (SGB II), 2005-2020, www.sozialpolitik-aktuell.de/ files/sozialpolitik-aktuell/_Politikfelder/Sozialstaat/Datensammlung/PDFDateien/abbIII63b.pdf (Abruf am 18.6.2021).

Sozialpolitik-aktuell.de (o.J. b): Struktur der Niedriglohnbeschäftigten in Deutschland 2018, www.sozialpolitik-aktuell.de/files/sozialpolitik-aktuell/_Politikfel der/Einkommen-Armut/Datensammlung/PDF-Dateien/abbIII33.pdf (Abruf am 18.6.2021).

Wissenschaftliche Dienste Deutscher Bundestag (2020): Sachstand. Überblick zu Leistungen nach dem Asylbewerberleistungsgesetz. Ansprüche und Anspruchseinschränkungen, WD 6 - 3000 - 137/19, www.bundestag.de/resource/ blob/681254/724f14ae6f257a528be9e7d9ab124a9c/WD-6-137-19-pdf-data.pdf (Abruf am 29.6.2021). 



\section{Teil II}

Grundsicherung für Teilhabe durch Arbeit 



\title{
Grundsicherung "für Arbeitsuchende»
}

\author{
Arbeitsförderung, Teilhabe und Sozialer Arbeitsmarkt
}

Matthias Knuth

\section{Einleitung}

Wie verhält sich eine Grundsicherung, die laut Untertitel "für Arbeitsuchende» geschaffen wurde, zu Arbeitslosigkeit, Arbeitslosenversicherung und Arbeitsförderung? In der diskursiven Rahmung der Hartz-Reformen diente die Einführung des SGB II im Jahr 2005 der "Zusammenführung von Arbeitslosen- und Sozialhilfe«, die mit der Absicht begründet wurde, die bisherigen Beziehenden von Sozialhilfe - soweit sie als erwerbsfähig gelten - in die aktive Arbeitsförderung einzubeziehen.

Mindestens im ersten Jahrzehnt der Geltung des SGB II waren öffentliche Wahrnehmung und politische Debatte von der Vorstellung geprägt, die »Arbeitslosengeld II« genannte Leistung sei - so wie zuvor die Arbeitslosenhilfe - eine Anschlussleistung für "Langzeitarbeitslose«, deren Ansprüche an die Arbeitslosenversicherung aufgrund der Dauer ihrer Arbeitslosigkeit erschöptt seien. SGB III und SGB II waren in dieser Vorstellung die beiden »Rechtskreise« der Arbeitsmarktpolitik, die zwar durch die Art der Leistung voneinander unterschieden, aber durch gemeinsame Ziele, ein gemeinsames Instrumentarium der Förderung und die Tätigkeit der Bundesagentur für Arbeit in beiden Rechtskreisen miteinander verbunden seien. Die Grundsicherung für Arbeitsuchende war in diesem Verständnis »Arbeitsmarktpolitik für nicht bzw. nicht ausreichend versicherte Arbeitslose«.

Doch schon bei der Einführung der Grundsicherung für Arbeitsuchende hatte dieses Bild Risse bekommen: Die Absicht des ursprünglichen Gesetzentwurfs, die Grundsicherung nach einer Übergangsphase der alleinigen Zuständigkeit der Bundesagentur für Arbeit zuzuweisen, ließ sich nicht durchsetzen. Der konfliktreiche Weg bis zur heutigen fragmentierten Mischform der Trägerschaft ${ }^{1}$ soll hier

1 | Siehe Fußnote 11 dieses Beitrags. 
nicht nochmals nachgezeichnet werden $;{ }^{2}$ jedoch kann argumentiert werden, dass schon hier nicht nur zufällige politische Mehrheitsverhältnisse, sondern Pfadabhängigkeiten am Werk waren, die aus den unterschiedlichen Regimelogiken von Arbeitslosenversicherung und Grundsicherung bzw. Sozialhilfe resultierten (Knuth 2009) und eine Drift in Richtung einer zunehmenden »Kommunalisierung« erzeugten (Knuth/Larsen 2010).

Ist also die Grundsicherung von vornherein organisational und hinsichtlich ihrer Governance ein eigenständiges Gebilde, so trifft auch empirisch die Vorstellung nicht zu, dass die insgesamt sehr heterogene Gruppe der Beziehenden von Leistungen nach dem SGB II von »Langzeitarbeitslosen« dominiert würde, die ihre Anspruchsdauer auf Arbeitslosengeld erschöpft haben (Abschnitt 2.1). Die vorherrschenden Lebenslagen und Lebensverläufe entsprechen eher denen in der einstmaligen Sozialhilfe, die insofern - aufgrund von strukturellen Veränderungen sowohl des Arbeitsmarktes als auch der Bevölkerung - vom Minderheitszum Mehrheitsregime geworden ist.

Auch normativ gibt es entgegen dem Gründungsdiskurs keinen Gleichklang von SGB II und SGB III, und dieser wurde auch nie angestrebt (Abschnitt 2.2). Stattdessen haben Urteile des Bundesverfassungsgerichts die 2005 teilweise gekappten normativen Wurzeln des SGB II im einstigen Bundessozialhilfegesetz wiederbelebt (Abschnitt 4.1).

Die Erfahrung, dass trotz Beschäftigungsrekorden, Fachkräfteengpässen und vielfältiger arbeitsmarktpolitischer Instrumentierung ein »harter Kern" von Leistungsbeziehenden nicht in den Arbeitsmarkt integriert werden konnte (Abschnitt 3.4), führte - teilweise über den Umweg von Bundesprogrammen - zu einer zunehmend eigenständigen Instrumentierung des SGB II, die nicht mehr ausschließlich arbeitsmarktpolitischen Zielen dient und nicht allein an arbeitsmarktpolitischen Erfolgskriterien gemessen werden kann. Sozialwissenschaftliche Diskurse lieferten hierzu Begrifflichkeiten, die zumindest terminologisch mit der Sprache der Verfassungsrichter:innen konvergieren.

Die sozialwissenschaftliche Evaluationsforschung entwickelte darüber hinaus Verfahren zur Messung von »sozialer Teilhabe « (Abschnitt 4.2), und durch das "Teilhabechancengesetz " wurde ab 2019 unter diesem Begriff ein Instrument geförderter Beschäftigung eingeführt, das Entwicklungslinien der Beschäftigungsförderung im SGB II fortführt, aber auch Ergebnis der jahrelangen Debatte über einen "Sozialen Arbeitsmarkt« ist (Abschnitt 5). Inwieweit mit diesem Instrument die Forderungen nach einem »Sozialen Arbeitsmarkt« erfüllt sind, ist die Fragestellung des abschließenden Ausblicks (Abschnitt 6).

2 | Vgl. hierzu Knuth 2012. 


\section{Grundsicherung und Arbeitsmarkt}

\subsection{Der hybride Charakter der Grundsicherung "für Arbeitsuchende" im Hinblick auf Arbeitslosigkeit und Arbeitsmarkt}

Seit der Einführung der »Grundsicherung für Arbeitsuchende« (SGB II) im Jahr 2005 befindet sich die Mehrheit der im amtlich-statistischen Sinne Arbeitslosen - die per definitionem zugleich Arbeitsuchende sind ( $\$ 16$ Abs. 1 SGB III) in diesem Leistungssystem. Die aktuelle, durch die Coronapandemie ausgelöste Krise relativiert allerdings diesen Befund: Sechs Millionen Kurzarbeitende im April 2020 waren zwar nicht arbeitslos, erhielten aber für die ausgefallenen Arbeitsstunden Lohnersatzleistungen aus der Arbeitslosenversicherung. Dies demonstriert deren potenzielle Reichweite zumindest im kurzfristigen Kriseneinsatz, der aber bereits Ende 2020 durch Bundesdarlehen gestützt werden musste.

Letztlich hängt die Verteilung der Arbeitslosen zwischen den beiden »Rechtskreisen « sowohl davon ab, wie viele sozialversicherungspflichtig Beschäftigte ihren Arbeitsplatz verlieren und mit »frischen " Ansprüchen an die Arbeitslosenversicherung in Arbeitslosigkeit einmünden, als auch davon, mit welcher Dauer diese Ansprüche ausgestattet sind. Unter den Vorzeichen der Deckelung von Sozialversicherungsbeiträgen insgesamt und des bisher immer erfolgreichen Drucks auf Senkung der Beiträge zur Arbeitslosenversicherung in Phasen guter Beschäftigungslage (und damit der Begrenzung der Rücklagenbildung im Haushalt der Bundesagentur für Arbeit) wird man davon ausgehen können, dass die Zuordnung der Mehrheit der Arbeitslosen zur Grundsicherung den im deutschen System der sozialen Sicherung bei Arbeitslosigkeit angelegten »Normalfall« darstellt, der sich allenfalls in akuten Beschäftigungskrisen vorübergehend umkehren könnte.

Andererseits ist die Mehrheit der Leistungsberechtigten nach dem SGB II weder arbeitslos noch arbeitsuchend (vgl. Knuth 2016). Auch wenn man die »nicht erwerbsfähigen Leistungsberechtigten « - also vor allem Kinder und Jugendliche bis 15 Jahre - aus der Betrachtung ausschließt, so ist die Mehrheit der Menschen, die ein sogenanntes »Arbeitslosengeld II« (ALG II) beziehen, nicht arbeitslos: Sie gehen zur Schule oder absolvieren eine Ausbildung und sind mit Vollendung des 15. Lebensjahrs vom Sozialgeld in den Bezug von ALG II gewechselt; andere sind wegen Kindererziehung oder Pflege nicht für den Arbeitsmarkt verfügbar, sind langzeitig erkrankt oder wurden von den Jobcentern wegen ihres Lebensalters zu »hoffnungslosen Fällen« erklärt und damit aus der Arbeitslosenstatistik herausdefiniert ( $\$ 53 \mathrm{a}$ Abs. 2 SGB II). 
Bei der Aufnahme einer sozialversicherungspflichtigen Beschäftigung aus dem SGB-II-Leistungsbezug heraus führt nur etwa die Hälfte zu dessen Beendigung (Bruckmeier/Hohmeyer 2018), die andere Hälfte zu einem nicht bedarfsdeckenden Erwerbseinkommen (siehe den Beitrag von Dorothee Spannagel in diesem Band) und damit - bei einer Wochenarbeitszeit von mindestens 15 Stunden - in einen Status fortgesetzten Leistungsbezugs ohne Arbeitslosigkeit.

Dieser Beschäftigungsstatus ist häufig nicht stabil. Fast die Hälfte der aus dem SGB-II-Leistungsbezug heraus aufgenommenen Beschäftigungen dauerte weniger als sechs Monate, und ebenfalls für fast die Hälfte handelte es sich um mindestens die fünfte Beschäftigungsaufnahme in den letzten fünf Jahren (Bruckmeier/Hohmeyer 2018).

Die Prekaritätszone am unteren Rand des Arbeitsmarktes führt zusammen mit statistischen Unterbrechungen von Arbeitslosigkeitsdauern durch Teilnahme an Maßnahmen, Langzeiterkrankung oder sonstige Zeiten längerer Nichtverfügbarkeit dazu, dass auch die verbreitete Kennzeichnung der Beziehenden von ALG II als »Langzeitarbeitslose « falsch ist: Eine knappe Mehrheit ist nicht langzeitarbeitslos im Sinne der amtlichen Definition. Unterbeschäftigung, diskontinuierliche Erwerbstätigkeit und Phasen der gesundheitlich oder familiär bedingten Nichtverfügbarkeit bilden Lebenslagen, die mit der überkommenen arbeitsmarktpolitischen Dichotomie von Beschäftigung und Arbeitslosigkeit nicht zu erfassen sind.

Insofern war es nur folgerichtig, dass das SGB II in seiner ursprünglichen Fassung - dem Bundessozialhilfegesetz folgend - Begriffe wie »arbeitslos« oder "Arbeitslosigkeit« mit Ausnahme der Bezeichnung der Leistungen als "Arbeitslosengeld« und »Arbeitslosengeld II« überhaupt nicht kannte.

\subsection{Bedürftigkeit statt Arbeitslosigkeit als Schlüsselbegriff}

Während Beschättigung und Arbeitslosigkeit gewissermaßen die kategorialen Eckpfeiler der Arbeitslosenversicherung darstellen, ist der Dreh- und Angelpunkt der Grundsicherung die Hilfebedürftigkeit. Damit steht die Grundsicherung voll in der Tradition des Bundessozialhilfegesetzes.

Der entscheidende Unterschied zur Arbeitslosenbilfe - dem anderen Leistungssystem, das von der Grundsicherung abgelöst wurde - besteht darin, dass die Bedürttigkeit dort lediglich anspruchsbegrenzend, aber nicht anspruchsbegründend wirkte (Knuth 2006). Anspruchsbegründend für die Arbeitslosenhilfe ebenso wie für das Arbeitslosengeld war die Arbeitslosigkeit. Die Arbeitslosenhilfe war ein steuerfinanziertes Anschlusssystem der Arbeitslosenversicherung, während die Grundsicherung lediglich ein Auffang-, aber kein Anschlusssystem 
ist. In der Bevölkerung wird die Grundsicherung eher als »Aufprallsystem« nach zu tiefem Fall wahrgenommen - nicht als Sicherungsversprechen, sondern als Zumutung.

Aufgabe und Ziel des SGB II bestehen in der Vermeidung, Beseitigung oder Verringerung der Hilfebedürftigkeit. Die angestrebte Stärkung der Eigenverantwortung dient unmittelbar dem Ziel, dass erwerbsfähige Leistungsberechtigte ihren Lebensunterhalt unabhängig von der Grundsicherung aus eigenen Mitteln und Kräften bestreiten können; Eigenverantwortung stellt insofern kein eigenständiges Ziel menschlicher Entfaltung dar, sondern ist Mittel zum Zweck. Erwerbstätigkeit dient im SGB II dazu, von staatlichen Leistungen unabhängig zu werden, und ist nicht mit Teilhabe- oder Aufstiegsversprechen verbunden.

Im Gegensatz zum SGB III enthält das SGB II keinerlei positive Leitbilder zur Qualität von Beschäftigung und Arbeitsmarkt. Während das - nach betroffenen Personen in der Regel kleinere - System des SGB III beispielsweise unterwertiger Beschäftigung entgegenwirken will ( $\mathbb{1} 1$ Abs. 2 Satz 1 Nr. 2 SGB III), erklärt das größere System des SGB II die individuelle Unterwertigkeit einer Beschäftigung gegenüber der erworbenen Qualifikation ausdrücklich als zumutbar ( $\$ 10 \mathrm{Abs} .2$ Nr. 1 und 2 SGB II) und setzt bei der Eingliederung in Arbeit vorrangig auf Maßnahmen, die die unmittelbare Aufnahme (irgend)einer Erwerbstätigkeit ermöglichen ( $\$ 3$ Abs. 1 Satz 2 SGB II).

Da der Arbeitsmarkt nicht nach Rechtskreisen unterteilt ist, besteht hier eine arbeitsmarktpolitische Zielinkonsistenz zwischen den beiden »Rechtskreisen ${ }^{3}$. Auch wenn das SGB II mehr ist als Arbeitsmarktpolitik, wie noch weiter auszuführen sein wird, so ist es doch auch Arbeitsmarktpolitik und über $\mathbb{} 16$ SGB II an die arbeitsmarktpolitischen Instrumente des SGB III, aber eben nicht an dessen Zielsetzungen angebunden.

\section{Der lange Weg der geförderten Beschäftigung in Richtung "Sozialer Arbeitsmarkt"}

\subsection{Geförderte Beschäftigung in der Urform des SGB II}

Das SGB II startete im Jahr 2005 mit zwei aus dem Bundessozialhilfegesetz "geerbten« Formen der geförderten Beschäftigung, nämlich mit »Arbeitsgelegenheiten« entweder als sozialrechtliche Beschäftigungsverhältnisse ohne Arbeitsentgelt und nur mit Mehraufwandsentschädigung (in der Folge bald »Ein-Euro-Jobs« genannt) oder aber als als sozialversicherungspflichtige Arbeitsverhältnisse (die

3 | Vgl. ausführlicher Arbeitskreis Arbeitsmarktpolitik/Knuth 2018. 
sogenannte Entgeltvariante). Über die Verknüpfung mit der aktiven Arbeitsförderung des SGB III durch $\$ 16$ SGB II war außerdem die Beschäftigung in Arbeitsbeschaffungsmaßnahmen möglich; aber die Nutzung dieses Instruments blieb in der Grundsicherung marginal. ${ }^{4}$

Außerdem ermöglichte die ursprüngliche Formulierung von $\$ 16$ SGB II durch eine Art Generalklausel die Förderung von gesetzlich hinsichtlich ihrer Ausgestaltung nicht näher definierten Maßnahmen. ${ }^{5}$ Unter den insgesamt fast 140.000 Teilnehmenden dieser Kategorie in 2007 dürften sich weitere Förderungen befunden haben, die eine Beschäftigung beinhalteten, und zwar oft in Kombination mit Qualifizierungs- oder Aktivierungsmaßnahmen, weil solche Kombinationen mit den gesetzlich im Detail definierten Instrumenten nur ungleich schwieriger oder überhaupt nicht darzustellen waren.

\section{2 "JobPerspektive» und Beschäftigungszuschuss}

Bereits der Koalitionsvertrag der »GroKo I« von 2005 enthielt einen Prüfauftrag zur Suche nach einer »Perspektive« für Personen, »die keine Arbeit auf dem regulären Arbeitsmarkt finden können« (CDU/CSU/SPD 2005). In Abgrenzung zu den immer schon von vornherein befristeten Angeboten des "zweiten Arbeitsmarktes " und als Alternativbegriff zum eher abwertend klingenden »dritten Arbeitsmarkt« kam bereits damals kurzzeitig der Begriff "Sozialer Arbeitsmarkt« auf (vgl. Cremer 2007).

In Anknüpfung an die Formulierung im Koalitionsvertrag setzte sich jedoch auf der politischen Ebene der vom damaligen Parlamentarischen Staatssekretär im Bundesarbeitsministerium, Klaus Brandner, und dem nordrhein-westfälischen Arbeitsminister Karl-Josef Laumann geprägte Begriff "JobPerspektive« durch. Gesetzgeberisch umgesetzt wurde diese durch das Zweite SGB-II-Änderungsgesetz vom Oktober 2007 als »Leistungen zur Beschäftigungsförderung« ( $\$ 16 a$ SGB II), die in den gesetzlichen Einzelbestimmungen und in der Statistik der Bundesagentur für Arbeit als »Beschäftigungszuschuss« (BeZ) bezeichnet wur-

4 | Laut Eingliederungsbilanzen waren dies zu Spitzenzeiten (2006) jahresdurchschnittlich gut 35.000 Teilnehmende gegenüber fast 300.000 in Arbeitsgelegenheiten (ohne Daten der zugelassenen kommunalen Träger).

5 | Die heute in $\$ 16$ a SGB II zusammengefassten »kommunalen Eingliederungsleistungen« wurden eingeleitet mit »über die in Absatz 1 genannten Leistungen hinaus können weitere Leistungen erbracht werden [...]. Dazu gehören insbesondere [...]«. Aus dieser nicht abschließenden Aufzählung ergab sich die Förderung »sonstiger weiterer Leistungen«. Diese gingen später in der »freien Förderung« (heute $\$ 16 f$ SGB II) auf, die aber quantitativ nie eine vergleichbare Bedeutung erlangte. 
den. Damit erhielt das SGB II erstmals ein speziell für diesen Rechtskreis konzipiertes Beschäftigungsinstrument, das in der aktiven Arbeitsförderung nach dem SGB III nicht verfügbar war.

Der »Beschäftigungszuschuss" beinhaltete gegenüber den vorherigen Formen geförderter sozialversicherungspflichtiger Beschäftigung zwei grundlegende Neuerungen:

- Während die zulässigen Tätigkeitsfelder bis dahin entweder (bei ABM und Arbeitsgelegenheiten) durch die ordnungspolitischen Schranken der "Zusätzlichkeit« und des "öffentlichen Interesses« oder (bei den »Strukturanpassungsmaßnahmen« in den damals noch neuen Bundesländern) durch Aufzählung (Umwelt, Soziale Dienste, Jugendhilfe, Breitensport, Kulturarbeit etc.) beschränkt worden waren, wurde erstmals auf eine solche Einhegung verzichtet. Der Beschäftigungszuschuss war eine Lohnkostensubvention von bis zu 75 Prozent, die bei Einstellung einer förderungsfähigen, ALG II beziehenden Person von jedem Arbeitgeber für Tätigkeiten jeglicher Art in Anspruch genommen werden konnte.

Damit war gewissermaßen eine "soziale Ausstrahlung « auf den gesamten Arbeitsmarkt möglich und laut Aussage von Karl-Josef Laumann in der Bundestagsdebatte zum Zweiten SGB-II-Änderungsgesetz auch ausdrücklich beabsichtigt - die geförderten Arbeitsplätze sollten nicht ausschließlich bei der öffentlichen Hand oder bei Beschäftigungsträgern entstehen (Deutscher Bundestag 2007, S. 11278). Die Tragweite dieser Neuerung muss seinerzeit vielen politischen Akteur:innen entgangen sein, denn in der späteren Debatte unter dem Schlagwort "Sozialer Arbeitsmarkt" wurde immer wieder der Verzicht auf die einengenden ordnungspolitischen Kriterien gefordert, obwohl das längst gesetzlich verwirklicht war (Einzelnachweise bei Knuth 2020).

- Die individuelle Förderungsdauer war zwar zunächst auf 24 Monate begrenzt; wenn nach dieser Zeit jedoch weiterhin eine ungeförderte Erwerbstätigkeit voraussichtlich nicht möglich war, "sollte« die Förderung anschließend nahtlos in eine unbefristete übergehen. Für die unbefristete Förderung gab es bei deren Einführung einen gesonderten Haushaltsposten, der aber in nachfolgenden Haushaltsgesetzen nicht fortgeführt wurde. Die unbefristeten Förderzusagen schlugen deshalb voll auf die Mittelbindung der Jobcenter durch, was zu erheblichen Vorbehalten der Jobcenter gegenüber diesem Instrument und auch den Nachfolgeinstrumenten führte.

Der Bestand an »mit BeZ« Geförderten betrug in den Spitzenjahren 2009 und 2010 jeweils etwa 35.000; die Anzahl der unbefristet Geförderten lässt sich nur 
indirekt auf der Grundlage des Bestands an Teilnehmenden abschätzen, der in den Jahren nach der Schließung des Instruments für Neuzugänge (am 31.3.2012) noch vorhanden war: gut 4.000 im Jahr 2014, noch knapp 2.000 im Jahr 2019. Die ursprünglich angekündigte Zielgröße von 100.000 Teilnehmenden wurde nicht erreicht, selbst wenn man diese Zahl als Summe der Eintritte über die Jahre hinweg interpretiert, denn diese betrug weniger als 65.000 (Statistik der Bundesagentur für Arbeit 2020).

Bei anziehender Beschäftigungskonjunktur und abnehmenden Eingliederungsmitteln setzten die Jobcenter andere Prioritäten und vermieden dadurch, nach zwei Jahren Förderung in Begründungsnöte gegenüber der lokalen Politikarena zu kommen, weshalb sie entgegen der gesetzlichen Sollbestimmung die Förderung nicht unbefristet fortsetzten.

\section{2 "Förderung von Arbeitsverhältnissen«}

Zum 1.4.2012 wurde der »BeZ« durch das Instrument der »Förderung von Arbeitsverhältnissen« (FAV) abgelöst, das im Rahmen des »Gesetzes zur Verbesserung der Eingliederungschancen am Arbeitsmarkt« (auch bekannt als »Instrumentenreform«) eingeführt wurde. Dieses Instrument sah keine unbefristete Fördermöglichkeit mehr vor. Im Gesetzgebungsverfahren war auch die andere Neuerung von 2007 infrage gestellt worden: Im Referentenentwurf hieß das Instrument »Förderung zusätzlicher Arbeitsverhältnisse« und sollte unter die altbekannten Schranken von Zusätzlichkeit und öffentlichem Interesse fallen sowie unter eine dritte, die im Zuge dieses Gesetzgebungsverfahrens für die Arbeitsgelegenheiten dann auch tatsächlich eingeführt wurde, nämlich die der »Wettbewerbsneutralität« ${ }^{6}$

Beim Instrument »Förderung von Arbeitsverhältnissen« dagegen blieb die Offenheit für jegliche Art von Tätigkeit und Arbeitgeber formal erhalten. Die Finanzierung des Instruments war gedeckelt, denn die Jobcenter durften für dieses Instrument und die »freie Förderung zusammen maximal 20 Prozent

6 | »Arbeiten sind wettbewerbsneutral, wenn durch sie eine Beeinträchtigung der Wirtschaft infolge der Förderung nicht zu befürchten ist und Erwerbstätigkeit auf dem allgemeinen Arbeitsmarkt weder verdrängt noch in ihrer Entstehung verhindert wird" ( $\$ 16 \mathrm{~d}$ Abs. 4 SGB II). Später wurde $\ 18 \mathrm{~d}$ über die örtlichen Beiräte um folgende Bestimmung ergänzt: "Stellungnahmen des Beirats, insbesondere diejenigen der Vertreter der Arbeitgeber und Arbeitnehmer, hat die gemeinsame Einrichtung zu berücksichtigen." Auch wenn dies im Gesetzestext nicht ausdrücklich gesagt wird, geht es hier um eine Art Vetorecht der Sozialpartner bei Arbeitsgelegenheiten, die sie für nicht wettbewerbsneutral halten. 
ihrer Eingliederungsmittel verausgaben. Tatsächlich wurde diese Marge nicht ausgeschöptt, und der Bestand an Geförderten erreichte im Spitzenjahr 2014 gerade einmal gut 9.000 .

Die wissenschaftliche Auseinandersetzung mit diesem Instrument konzentrierte sich auf Förderprogramme von Bundesländern, die das Instrument ergänzten; ${ }^{7}$ eine bundesweite Evaluation oder Implementationsanalyse fand nicht statt. Laut Bundesrechnungshof nutzte ein Drittel der Jobcenter das Instrument in den Jahren 2012 und 2013 überhaupt nicht, und nur etwa ein Fünftel der Jobcenter sprach mehr als 50 Förderzusagen aus.

"Die Jobcenter gaben zur Begründung an, dass sie teilweise andere arbeitsmarktpolitische Schwerpunkte setzten oder dass die FAV wegen regionaler Besonderheiten am Arbeitsmarkt nicht nachgefragt wurde.« (Bundesrechnungshof 2015, S. 9)

\subsection{Schätzungen des Bedarfs öffentlich geförderter Beschäftigung}

Wohl nicht zuletzt die extrem geringe Nutzung des Instruments »Förderung von Arbeitsverhältnissen« beflügelte die Diskussion über Notwendigkeit und künftige Möglichkeiten öffentlich geförderter Beschäftigung. Zahlreiche Untersuchungen waren darauf gerichtet, einerseits auf das Ausmaß faktischer Ausschlüsse von Erwerbschancen aufmerksam zu machen und andererseits Kriterien zur Bestimmung der Zielgruppe von Beschäftigungsförderungsmaßnahmen jenseits von amtlich gemessenen Arbeitslosigkeitsdauern zu entwickeln.

Mit den Daten das Panels "Arbeitsmarkt und Soziale Sicherung« (PASS) fanden Obermeier, Sell und Tiedemann (2014), dass im Jahr 2012 fast 500.000 Personen mindestens vier Vermittlungshemmnisse aufwiesen, aktuell nicht beschäftigt waren und in den letzten 36 Monaten mehr als 90 Prozent der Zeit keine Beschäftigung hatten. Ebenfalls für 2012 (Dezember) ermittelte Lietzmann (2016) auf Basis administrativer Daten, dass fast 40 Prozent der erwerbsfähigen Leistungsberechtigten - umgerechnet mehr als 1,6 Millionen - vier Jahre und länger im Leistungsbezug waren, ohne beschäftigt gewesen zu sein oder an einer Fördermaßnahmen teilgenommen zu haben.

Allein für Nordrhein-Westfalen ermittelte Bauer (2018) zum Stichtag 31.12.2014 rund 100.000 »abgekoppelte Langzeitarbeitslose«, die in den zurückliegenden fünf Jahren maximal einen Monat in Beschäftigung gewesen waren und mindestens 48 Monate Arbeitslosigkeit aufsummierten. All diese Versuche, ergänzende oder alternative Kriterien zu dem der Arbeitslosigkeit zu

7 | Vgl. Bauer et al. 2018; Knuth et al. 2013; ISG/IAB/Regionales Forschungsnetz Standort Baden-Württemberg 2016. 
finden, können als Vorarbeiten für die heute beim Instrument "Teilhabe am Arbeitsmarkt« angewandte Zielgruppendefinition verstanden werden (siehe Abschnitt 5).

\section{5 "Passiv-Aktiv-Transfer»}

Wie auch immer man die Zielgruppe abgrenzen und beziffern mochte - die Diskrepanz zur Anzahl der Arbeitsplätze, die mit dem budgetär gedeckelten Instrument »Förderung von Arbeitsverhältnissen « gefördert werden konnte, war in jedem Fall immens. Dies führte zur Konkretisierung einer alten Debatte: Die Forderung "Arbeit statt Arbeitslosigkeit finanzieren « war zwar in erster Linie auf eine aktivere Ausrichtung der Arbeitsmarktpolitik gemünzt gewesen, enthielt aber auch schon den Gedanken, dass geförderte Beschäftigung sich zum Teil selbst finanziert, indem sie Lohnersatz- oder Unterhaltsleistungen einspart, die bei fortgesetzter Arbeitslosigkeit angefallen wären, und Sozialversicherungsbeiträge und Lohnsteuer generiert.

Die nunmehr haushaltstechnisch zugespitzte Forderung nach einem "Passiv-Aktiv-Transfer« oder »-Tausch« (in jedem Fall »PAT« abgekürzt) zielte darauf ab, die bei Aufnahme einer geförderten Beschäftigung durch Verlassen oder Verringerung des Leistungsbezugs eingesparten Unterhaltsmittel unmittelbar für die Förderung weiterer Beschäftigungen verfügbar zu machen. Bei gesamtfiskalischer Betrachtungsweise erschien die geförderte Beschäftigung mit PAT geradezu als selbstfinanzierendes Perpetuum mobile (vgl. z. B. Aktion Arbeit im Bistum Trier 2017).

Aber Renten- und Krankenversicherungsbeiträge sowie etwaige Lohnsteuerzahlungen können ja nicht in den Eingliederungshaushalt der Jobcenter zurückgeführt werden. Bei rein SGB-II-immanenten Modellrechnungen kommt man deshalb zu moderateren Refinanzierungsquoten (vgl. Knuth/Kirsch/Zink 2013), die aber gleichwohl nicht zu vernachlässigen sind.

Vonseiten der Ministerialverwaltung wurde lange Zeit argumentiert, dass eine solche Umbuchung von Mitteln haushaltsrechtlich unzulässig sei. Schließlich handle es sich bei den "passiven" Unterhaltsleistungen um Anspruchsleistungen, die im Falle zunehmender Zahlen von Leistungsbeziehenden unabhängig von Haushaltsansätzen automatisch ansteigen; folglich dürfe man nicht etwaige Einsparungen abzweigen und umleiten. Dass zusammen mit dem »Teilhabechancengesetz" seit 2019 ein PAT in begrenzter Form ermöglicht wurde (siehe Abschnitt 5), zeigt, dass diese haushälterischen Grundsätze nicht auf ewig in Stein gemeißelt sind. 


\section{Gesellschaftliche Teilhabe - der neue Leitbegriff des SGB II?}

\subsection{Zusammentreffen von sozialwissenschaftlichen Diskursen und Verfassungsrechtsprechung}

Die Rezeption des französischen Diskurses von Exklusion versus Inklusion, der zeitweilig die Terminologie der sozialpolitischen Bemühungen der Europäischen Kommission dominierte (Mayes/Berghman/Salais 2001), und des »Capabilities-Approach« (Nussbaum 2000; Sen 2008) in der deutschen sozialpolitischen Debatte (Brettschneider 2007; Bartelheimer 2007; Kronauer 2010) führten dazu, dass der bis dahin nur mit Sozialpolitik für Menschen mit Behinderungen konnotierte Begriff der »Teilhabe« nunmehr zum Universalbegriff der Sozialpolitik und auch der Armutsforschung (Arndt/Volkert 2006) avancierte.

Der diskurspraktische Grund dafür dürfte ganz einfach darin liegen, dass »Inklusion« oder »Capabilities« nicht unmittelbar verständliche Fremdwörter sind; Übertragungsversuche wie »Verwirklichungschancen« für »Capabilities« wirken reichlich künstlich, während »Einschluss« für »Inklusion« etwas Beklemmendes hat. »Teilhabe« steht semantisch zwischen der passiv klingenden "Einbindung« (der Mensch wird eingebunden) und den hohen Anforderungen an Eigenaktivität, die mit »Partizipation« oder »Beteiligung« verbunden sind: Sowohl ein eher passives "Anteilhaben « als auch ein eher aktives »Teilnehmen« und »Einflussnehmen « sind durch »Teilhabe« abgedeckt. $^{8}$

Auch das Bundesverfassungsgericht griff den Terminus der »Teilhabe« auf und tenorierte 2010 in seinem Urteil zur Gewährleistung eines existenzwürdigen Existenzminimums:

»Das Grundrecht auf Gewährleistung eines menschenwürdigen Existenzminimums [...] in Verbindung mit dem Sozialstaatsprinzip [...] sichert jedem Hilfebedürftigen diejenigen materiellen Voraussetzungen zu, die für seine physische Existenz und für ein Mindestmaß an Teilhabe am gesellschaftlichen, kulturellen und politischen Leben unerlässlich sind.» (Bundesverfassungsgericht 2010)

8 | Dass sich dann wenig später die Terminologie rund um Menschen mit Behinderungen davon wiederum absetzte und verstärkt für »Inklusion« optierte (das Bundesteilhabegesetz von 2016 führte diesen Begriff in das SGB IX ein), lässt sich wohl nur mit dem Distinktionsbedürfnis der betreffenden Fachkreise erklären. Denn der Auslöser für die grundlegende Novellierung des SGB IX, die UN-Behindertenrechtskonvention, kommt in der amtlichen deutschen Übersetzung mit "Teilhabe und "Einbeziehung" sehr gut zurecht, wo die englische Urfassung »inclusion « oder "participation « benutzt; vgl. United Nations 2006 und Beauftragter der Bundesregierung für die Belange von Menschen mit Behinderungen 2018. 
Im Urteil selbst ist dann ausgeführt, dass der Gesetzgeber bei der Konkretisierung des Leistungsanspruchs die soziale Wirklichkeit zeit- und realitätsgerecht im Hinblick auf die Gewährleistung des menschenwürdigen Existenzminimums zu erfassen habe und dass dieses sich in einer technisierten Informationsgesellschaft anders darstelle als früher, also historisch veränderlich sei. Der Gestaltungsspielraum des Gesetzgebers sei dabei enger, soweit es um die Konkretisierung des zur Sicherung der physischen Existenz Notwendigen gehe, und weiter, wo es »um Art und Umfang der Möglichkeit zur Teilhabe am gesellschaftlichen Leben geht« (Bundesverfassungsgericht 2010).

Im Übrigen bleibe es dem Gesetzgeber grundsätzlich überlassen, ob er das Existenzminimum durch Geld-, Sach- oder Dienstleistungen sichere. Die Teilhabe am gesellschaftlichen Leben - so heißt es nicht explizit, aber so könnte man schlussfolgern - muss nicht ausschließlich durch Geldleistungen gesichert werden, mit denen man sich ein Mindestmaß an Zugang beispielsweise zu Kulturveranstaltungen kaufen kann, sondern kann auch durch staatlich organisierte Gelegenheitsstrukturen verwirklicht werden.

In Reaktion auf dieses Urteil wurde 2011 den ansonsten unveränderten Formulierungen zu Aufgabe und Ziel der Grundsicherung der Absatz vorangestellt: »Die Grundsicherung für Arbeitsuchende soll es Leistungsberechtigten ermöglichen, ein Leben zu führen, das der Würde des Menschen entspricht« (SGB II $\$ 1$ Satz 1). Diese Formulierung, die sich sinngemäß schon im Bundessozialhilfegesetz fand, war bei dessen Ablösung durch die Hartz-Reformen zunächst nur in das SGB XII, aber nicht in das SGB II übernommen worden. ${ }^{9}$

Auch wenn der Begriff der Teilhabe bei der Umsetzung des zitierten Urteils zunächst nur in der Gestalt des »Bildungs- und Teilhabepakets« für Kinder und Jugendliche explizit Eingang in das SGB II fand, markiert das Urteil dennoch bedeutsame Diskursverschiebungen. Zum einen wurde die Traditionslinie zum Bundessozialhilfegesetz explizit wiederhergestellt, zum anderen ist damit klar, dass das SGB II nicht einfach nur »Arbeitsförderung für Arbeitslose ohne Versicherungsanspruch « beinhaltet.

Der bis heute gültige Untertitel »Grundsicherung für Arbeitsuchende« ist nicht nur sachlich viel zu eng, wie bereits in Abschnitt 2.1 gezeigt wurde - die Mehrheit der Beziehenden von Leistungen nach diesem Gesetz ist nicht arbeit-

9 | Das Bundessozialhilfegesetz postulierte zwar die Ermöglichung eines Lebens, »das der Würde des Menschen entspricht« ( $\$ 1$ Abs. 2 Satz 1 BSHG), verwendete aber den Begriff der Teilhabe ausschließlich im Kontext von Leistungen für Menschen mit Behinderungen und stellte keine Verbindung zwischen »Menschenwürde« und »Teilhabe« her. Diese Verknüpfung erfolgte erst durch das Urteil des Bundesverfassungsgerichts. 
suchend -, sondern er stellt auch das Gesetz einseitig in einen ausschließlich arbeitsmarktpolitischen Kontext. Doch wenn die Überwindung von Hilfebedürttigkeit mit Mitteln der Arbeitsmarktpolitik nicht gelingt, kommt das latent sozialpflegerische Erbe des Bundessozialhilfegesetzes im SGB II wieder auf die Tagesordnung.

\subsection{Teilhabe als Erfolgsmaßstab von Interventionen}

Während bei der umfassenden Evaluation von arbeitsmarktpolitischen Instrumenten, die durch die Hartz-Reformen angestoßen wurde, noch niemand daran dachte, im Zusammenhang mit Arbeitsbeschaffungsmaßnahmen nach Teilhabeeffekten zu fragen (vgl. COMPASS et al. 2006), wurde dies wenig später bei der Evaluation von geförderter Beschäftigung zum Standard (ISG/IAB/RWI 2011; Christoph/Hohmeyer 2012; Hirseland/Ramos Lobato/Ritter 2012; BMAS 2015; Fuchs/Hamann 2017). Schritt für Schritt bildete sich ein Kanon von Variablen und ihnen zuzuordnenden Befragungs-Items heraus, mit denen versucht wird, soziale Teilhabe zu messen (IAQ et al. 2019).

Es konnte empirisch gesichert werden, dass sich geförderte Beschäftigung positiv unter anderem auf die allgemeine Lebenszufriedenheit, die Konsummöglichkeiten, den Gesundheitszustand, das Selbstvertrauen und die wahrgenommene gesellschaftliche Anerkennung auswirkt (Aurich-Beerheide et al. 2020). Dabei nehmen die Wirkungen auf die einzelnen Indikatoren mit zunehmender Dauer der Beschäftigung in unterschiedlich starkem Maße ab, was einerseits auf Gewöhnungseffekte, andererseits - bei absehbarem Ende einer befristeten Maßnahme - auf erneute Verunsicherung der Lebensperspektive zurückzuführen sein dürfte. Einige Effekte hielten jedoch auch ein Jahr nach dem Ende des Programms an, und zwar bei Selbstvertrauen, Soft Skills und dem persönlichen sozialen Netzwerk sogar bei Personen, für die das Programmende zu erneuter Erwerbslosigkeit führte (Aurich-Beerheide et al. 2020).

Mit den oben in Abschnitt 4.1 skizzierten Diskursverschiebungen und den dadurch angestoßenen Untersuchungen veränderte sich der Legitimationsrahmen geförderter Beschäftigung. War diese Kategorie arbeitsmarktpolitischer Maßnahmen traditionell nach dem gleichen Outcome-Kriterium bewertet worden wie alle anderen arbeitsmarktpolitischen Maßnahmen, nämlich dem späteren Übergang in ungeförderte Beschäftigung und deren Dauerhaftigkeit (mit notorisch ernüchternden Ergebnissen; vgl. Koch et al. 2011), so gibt es nun ein komplementäres Erfolgskriterium, nämlich die gesellschaftliche Teilhabe.

Wenn die Zielsetzungen der "Grundsicherung für Arbeitsuchende« nicht auf Arbeitsförderung im Sinne des SGB III beschränkt sind und die gesellschaft- 
liche Teilhabe eine zur Integration in ungeförderte Erwerbsarbeit gleichwertige Zielsetzung darstellt, ist geförderte Beschäftigung auch dann gerechtfertigt, wenn sie die Wahrscheinlichkeit des Übergangs in ungeförderte Erwerbsarbeit nicht erhöht - solange sie die gesellschaftliche Teilhabe der geförderten Personen verbessert und ihnen damit ein menschenwürdigeres Leben ermöglicht. In der Bundestagsdebatte zum Teilhabechancengesetz (Deutscher Bundestag 2018a) brachten drei Abgeordnete diesen Paradigmenwechsel in Reaktion auf Kritik von der AfD- und der FDP-Fraktion auf den Punkt:

»Hier steht die Integration in den Arbeitsmarkt nicht im Vordergrund, sondern die Möglichkeiten der Teilhabe durch Arbeit.«(Dr. Matthias Zimmer, Fraktion der CDU/ CSU)

»Es geht in erster Linie um soziale Teilhabe.«(Beate Müller-Gemmeke, Fraktion Bündnis 90 - DIE GRÜNEN)

"Dabei geht es in erster Linie um Teilhabe durch Arbeit. Das steht im Vordergrund.» (Dr. Martin Rosemann, Fraktion der SPD)

Man kann nur hoffen, dass sich die arbeitsmarktpolitischen Akteur:innen noch an diese Festlegung erinnern, wenn die Evaluationsergebnisse zum Teilhabechancengesetz vorliegen.

\section{Das Instrument "Teilhabe am Arbeitsmarkt»}

Der Gesetzentwurf zum »Teilhabechancengesetz« erfuhr eine weitaus intensivere parlamentarische Bearbeitung als die Vorläuferinstrumente "Beschäftigungszuschuss« und »Förderung von Arbeitsverhältnissen « zu ihrer jeweiligen Zeit (vgl. Knuth 2020). Im Ergebnis kam ein ${ }^{10}$ Förderinstrument heraus, das sich durch folgende Charakteristika auszeichnet:

- Die in der Gesetzesbegründung betonte Offenheit des Instruments für alle Arten von Arbeitgebern blieb erhalten; der 2008 eingeleitete Paradigmenwechsel von einem eingehegten "zweiten Arbeitsmarkt « zu einem sozialen Mechanismus inmitten des gesamten Arbeitsmarktes wurde nicht revidiert. Aber anders als bei dem aus dem Europäischen Sozialfonds (ESF) kofinan-

10 | Eigentlich zwei, aber der Eingliederungszuschuss für Langzeitarbeitslose nach $\$ 16 \mathrm{e}$ ist nicht dem Sozialen Arbeitsmarkt im engeren Sinne zuzurechnen und wird daher hier nicht behandelt. 
zierten »ESF-Langzeitarbeitslosenprogramm« (vgl. ISG/IAW/SOKO 2019) oder bei dem auf der »Förderung von Arbeitsverhältnissen« aufbauenden badenwürttembergischen Landesprogramm »Gute und sichere Arbeit« (vgl. die Literaturangaben in Fußnote 7) gibt es keine »Betriebsakquisiteure« oder sonstigen Vorkehrungen, um privatwirtschaftlich orientierte Arbeitgeber für die Beteiligung zu gewinnen - man vertraut ausschließlich auf die Attraktivität hoher Lohnkostenzuschüsse.

- Es liegt in der Logik eines hohen Lohnkostenzuschusses, der auch für erwerbswirtschaftlich orientierte Betriebe zugänglich ist, dass man die Zielgruppe auf diejenigen beschränken muss, deren Produktivität auf längere Sicht voraussichtlich stark eingeschränkt ist. Bei den beiden Vorläuferinstrumente war dies - zusätzlich zu den Voraussetzungen der Langzeitarbeitslosigkeit und vergeblicher Eingliederungsbemühungen - durch die Anforderung zweier »in der Person liegender« Vermittlungshemmnisse und einer Negativprognose bezüglich der Eingliederungsmöglichkeiten für die Dauer der Förderung erfolgt.

Diese Kriterien waren einerseits als potenziell stigmatisierend und andererseits als durch die Fachkräfte in den Jobcentern schwer anwendbar kritisiert worden. Nicht zuletzt aufgrund der nunmehr vorliegenden differenzierteren Forschungsergebnisse zu Leistungsbezugsverläufen und der persistenten Abkoppelung eines Teils der Leistungsbeziehenden von jeglicher Beschäftigung (siehe Abschnitt 3.4) wurden die Zugangskriterien nun auf objektiv vorliegende Tatbestände der Erwerbs- und Leistungsbiografie umgestellt.

Die im Gesetzentwurf vorgesehene Regelung »sieben Jahre Leistungsbezug in den letzten acht Jahren und keine nennenswerte Erwerbstätigkeit in diesem Zeitraum« wurde im Zuge der Gesetzesberatungen geringfügig auf »sechs Jahre in sieben « gelockert. Ergänzend und angelehnt an die Regelungen im Bundesprogramm »Soziale Teilhabe am Arbeitsmarkt« wurde für schwerbehinderte Personen und Eltern minderjähriger Kinder eine niedrigere Schwelle von fünf Jahren Leistungsbezug eingefügt.

- Zwar wurde davon abgesehen, wieder wie beim Beschäftigungszuschuss eine für einzelne Teilnehmende unbefristete Fördermöglichkeit vorzusehen; dafür kann die Förderung jetzt aber bis zu fünf Jahre andauern, wobei der Lohnkostenzuschuss in den ersten beiden Jahren 100 Prozent beträgt und in den Folgejahren um jeweils 10 Prozentpunkte abgesenkt wird.

- Die zu bezuschussende Entlohnung richtet sich nach dem Mindestlohn bzw. bei Tarifbindung des Arbeitgebers nach Tarifvertrag. Letzteres war im Gesetzentwurf - dem Koalitionsvertrag folgend - zunächst nicht vorgesehen und wurde aufgrund der Bundestagsdebatte und der Ausschussempfehlun- 
gen des Bundesrats (2018) eingefügt. Entgegen den Empfehlungen aus dem Bundesratsausschuss blieb es beim Ausschluss aus der Arbeitslosenversicherung, einer im Zuge der Hartz-Reformen begründeten Tradition für seitherige Formen öffentlich geförderter Beschäftigung.

- Weiterbildungskosten können auf Antrag des Arbeitgebers mit bis zu 3.000 Euro je Förderfall bezuschusst werden. Entgegen der großen Bedeutung, die in der öffentlichen Debatte, in Landes- und Bundesprogrammen der Begleitung und dem Coaching beigemessen wurde (vgl. Brussig/Kotlenga 2019; Bauer 2021), ist eine »erforderliche ganzheitliche beschäftigungsbegleitende Betreuung" lediglich als Soll-Vorschrift ausgestaltet.

Je nachdem, ob die Jobcenter diese Aufgabe selbst wahrnehmen oder von beauftragten Dritten wahrnehmen lassen, gehen die Kosten voll zulasten des Verwaltungshaushalts oder zulasten der Eingliederungsmittel, da die Refinanzierung durch »Passiv-Aktiv-Transfer" (s. u.) ausschließlich für den Lohnkostenzuschuss eingesetzt werden darf. An der Umsetzung des Programms beteiligte Beschäftigungsträger können nicht mit der Betreuung der eigenen Beschäftigten beauftragt werden.

- In der Bundestagsdebatte kritisierte ein weites politisches Spektrum von der Linken (Katja Kipping) bis zur CDU (Matthias Zimmer), dass die Aufnahme einer Beschäftigung im »Sozialen Arbeitsmarkt« nicht explizit freiwillig gestellt wurde. Allerdings führte möglicherweise ein Redaktionsversehen dazu, dass das Instrument auch nicht explizit in die Sanktionsgründe nach $\$ 31$ Abs. 1 Satz 1 Nr. 2 SGB II aufgenommen wurde.

- Der »Passiv-Aktiv-Transfer«(PAT) findet sich im Gesetz nicht, weil es sich hier um ein haushaltstechnisches Verfahren handelt, das nicht im SGB II zu regeln ist. Er wird gleichwohl umgesetzt, wenn auch auf eine sehr kompliziert anmutende Weise aufgrund von Pauschalierungen für drei sehr konservativ kalkulierte Fallkonstellationen (vgl. Bundesagentur für Arbeit 2020; BMAS/ BA 2019). Nach den Abrechnungsergebnissen der Gemeinsamen Einrichtungen ${ }^{11}$ deckte der PAT im Jahr 2019 immerhin 24 Prozent der Ausgaben für »Teilhabe am Arbeitsmarkt« (Knuth 2020, S. 815). ${ }^{12}$

11 | „Gemeinsame Einrichtungen« heißen die drei Viertel der Jobcenter, die von der Bundesagentur für Arbeit und den jeweiligen Kommunen gemeinsam betrieben werden. Die übrigen sind die »zugelassenen kommunalen Träger«, von denen manche statistischen Kennzahlen nur mit größerer Verzögerung oder überhaupt nicht verfügbar werden.

12 | Die dort zitierte Quelle war bei der Abfassung dieses Manuskripts nicht mehr auffindbar. Generell ist zu beklagen, dass seit der Umstellung des Internetangebots der 
- Entgegen den weitverbreiteten Forderungen nach Verstetigung der öffentlich geförderten Beschäftigung, aber auch entgegen dem Gesetzentwurf ist das Instrument »Teilhabe am Arbeitsmarkt« nach $\mathbb{1} 16 \mathrm{i}$ bis Ende 2024 befristet, wobei bis dahin begonnene Förderungen ausfinanziert werden können. Im parlamentarischen Aushandlungsprozess war die Befristung der Preis für tarifliche Entlohnung und die leichte Lockerung der Zugangskriterien gegenüber dem Regierungsentwurf.

\section{Sind wir im "Sozialen Arbeitsmarkt» angekommen?}

Vor allem gegenüber dem unmittelbaren Vorläuferinstrument »Förderung von Arbeitsverhältnissen« stellt die »Teilhabe am Arbeitsmarkt" einen deutlichen Fortschritt dar. Hinter Ansprüchen und Erwartungen, die in jahrelangen Debatten zum »Sozialen Arbeitsmarkt« entwickelt wurden, bleibt der $\$ 16$ S SGB II jedoch ebenso deutlich zurück.

Wenn man »sozial« nicht im Sinne einer randständigen Veranstaltung für "sozial Benachteiligte", sondern im Sinne einer sozialen Qualität des Arbeitsmarktgeschehens insgesamt verstehen will, müsste die Sicherung der Teilhabe von andernfalls Ausgeschlossenen in ähnlichem Maße zu einem gesamtgesellschaftlichen Anliegen und einer Verantwortung der Arbeitsmarktparteien und -institutionen werden wie die Sicherung des Ausbildungszugangs für die nachwachsenden Generationen. Von der Größenordnung her sind beide Aufgaben durchaus vergleichbar: Rund 500.000 jährlich abgeschlossenen betrieblichen Ausbildungsverträgen standen im Juni 2018 laut Bundesregierung rund 450.000 Personen über 25 Jahre gegenüber, die sieben Jahre und mehr ohne Einkommen aus Erwerbstätigkeit im Regelleistungsbezug standen (Deutscher Bundestag 2018b).

Aber während die Sicherung des Ausbildungszugangs trotz pandemiebedingter Absage von Orientierungsveranstaltungen im Sommer/Herbst 2020 Gegenstand fast täglicher Berichterstattung war, nahm niemand öffentlich Notiz davon, dass die Umsetzung des Sozialen Arbeitsmarktes durch die Pandemie empfindlich gebremst wurde (vgl. Hofmann 2020; Beckmann/Schad 2021). Die Sozialpartner wurden im Hinblick auf das neue Instrument auf eine Rolle als Wächter der Wettbewerbsordnung festgelegt ( $\$ 16 \mathrm{i}$ Abs. 9 SGB II) und nicht in Verantwortung für das Gelingen des Vorhabens gestellt. Für die Gewinnung

Statistik der Bundesagentur für Arbeit ein verlässliches wissenschaftliches Arbeiten mit BA-Statistiken nicht mehr möglich ist. 
von erwerbswirtschaftlich orientierten Betrieben als Arbeitgeber im Sozialen Arbeitsmarkt wurden keine besonderen Vorkehrungen getroffen, und auch der Umstand, dass die Geltung des neuen Instruments befristet ist, macht es nicht zu einer Größe, mit der Arbeitgeber mittelfristig rechnen werden.

Diese perspektivische Unsicherheit betrifft auch das schmale Segment erwerbswirtschaftlich orientierter Beschäftigungsinitiativen, also Initiativen von Beschättigungsträgern, die eine Beteiligung an Güter- und Dienstleistungsmärkten nicht in Gewinnabsicht suchen, sondern zwecks Kostendeckung und Vermittlung realistischer und von Kunden wertgeschätzter Arbeitserfahrungen für andernfalls vom Arbeitsmarkt Ausgeschlossene (vgl. Schulze-Böing 2021). Diese »sozialen Unternehmen« brauchen stabile Rahmenbedingungen über die Entfristung des Förderinstruments hinaus, z.B. einen anerkannten und definierten Status im Wirtschaftsgeschehen analog zu den Inklusionsbetrieben für Menschen mit Behinderungen ( $\$ 215$ ff. SGB IX; Knuth/Tenambergen 2015).

Gesetzlich definierte Kriterien z. B. hinsichtlich eines Prozentsatzes von Beschäftigten, die zwecks »Teilhabe am Arbeitsmarkt« gefördert werden, würden es erleichtern, dieses Merkmal als soziales Zuschlagskriterium in kommunalen Vergabeverfahren zu verwenden. Für einen stabilen und wachsenden Sozialen Arbeitsmarkt braucht man soziale Mechanismen zumindest in den von öffentlicher Nachfrage bestimmten Güter- und Dienstleistungsmärkten.

Die Vergaberechtsreform von 2016 hat die Spielräume hierfür erweitert, aber ihre rechtssichere Umsetzung ist sehr arbeitsaufwendig. Kommunen, die eine solche Strategie verfolgen wollen, sollten für eine Anlaufzeit von einigen Jahren über Förderprogramme der Länder Personalaufstockungen in ihren Vergabestellen vornehmen können, um den Mehraufwand abzufangen, der mit der Implementierung sozialer Vergabekriterien verbunden ist. Wenn die öffentlichen Kunden vorangehen, könnte der Soziale Arbeitsmarkt auch über den öffentlich finanzierten Bereich hinaus zu einem Label analog »Fair Trade«, »Frei von Kinderarbeit« oder »Dieser Betrieb bildet aus« werden (vgl. Knuth 2017).

$\mathrm{Zu}$ einem solchermaßen gesellschaftlich integrierten Sozialen Arbeitsmarkt passt der Ausschluss der Beschäftigten von der Arbeitslosenversicherung nicht. Wenn die individuelle Förderung befristet ist und wenn sich Arbeitgeber beteiligen, für die ein Insolvenzrisiko nicht ausgeschlossen ist, gibt es keine Rechtfertigung dafür, dass man nach mehrjähriger wertschöpfender Tätigkeit keine Lohnersatzleistungen der Arbeitslosenversicherung bekommen soll, nur weil die Beschäftigung öffentlich bezuschusst wurde.

In der aktuellen Covid-19-Krise führt der Ausschluss von der Arbeitslosenversicherung dazu, dass das Instrument der Kurzarbeit für die im Sozialen Arbeitsmarkt Beschäftigten nicht anwendbar ist, obwohl gerade Einsatzfelder 
wie Tafeln, Schul-Catering, Sozialkaufhäuser oder Café-Treffpunkte vom Lockdown betroffen sind. Zwar wurden Sonderregelungen gefunden, aber damit ist eine Gleichbehandlung gegenüber den ohne Förderung Beschäftigten gerade nicht möglich (Beckmann/Schad 2021); auch die Besserstellung (Freistellung bei voller Bezahlung) demonstriert einen »Exotenstatus«, der dem Ziel der Teilhabesicherung durch »simulierte Erwerbsnormalität« (Bauer et al. 2016) zuwiderläuft.

Vieles spricht für die Annahme, dass die derzeitigen pauschalen Gegenbuchungen im Rahmen des Passiv-Aktiv-Transfers zu konservativ gerechnet sind. Der Stand der Datenverarbeitung bei der Bundesagentur für Arbeit sollte es eigentlich ermöglichen, für Teilnehmende des Sozialen Arbeitsmarktes oder eine repräsentative Stichprobe fiktive Parallelkonten zu führen, mit denen man ermitteln kann, was die betreffende Bedarfsgemeinschaft ohne diese Beschäftigung an Leistungen bekommen hätte. So lassen sich empirisch gesicherte Erkenntnisse über die Refinanzierungseffekte geförderter Beschäftigung gewinnen, was den Finanzierungsspielraum zugunsten höherer Teilnehmerzahlen oder längerer Förderungszeiträume für Ältere erweitern würde.

In den grundlegenden Zielsetzungen des $₫ 1$ SGB II sollte die Gelegenheit zu sozialer Teilhabe als Verwirklichungsform der Menschenwürde verankert werden. Daraus würde zwingend folgen, dass die Annahme einer Beschäftigung im Sozialen Arbeitsmarkt sanktionsfrei gestellt werden muss, da eine durch Sanktionen erzwingbare »Verpflichtung zur Teilhabe« einen Widerspruch in sich darstellt.

\section{Literatur}

Aktion Arbeit im Bistum Trier (2017): Integrierter Arbeitsmarkt. Ein Weg zur Auflösung der verfestigten Langzeitarbeitslosigkeit. Stand: Februar 2017, www.o-ton-arbeitsmarkt.de/wp-content/uploads/AKTIO_Integrierter_Ar beitsmarkt-WEB.pdf (Abruf am 5.7.2021).

Arbeitskreis Arbeitsmarktpolitik (Hrsg.)/Knuth, M. (2018): Solidarische und sozialinvestive Arbeitsmarktpolitik. Vorschläge des Arbeitskreises Arbeitsmarktpolitik, Study Nr. 374 der Hans-Böckler-Stiftung, Düsseldorf.

Arndt, C./Volkert, J. (2006): Amartya Sens Capability-Approach. Ein neues Konzept der deutschen Armuts- und Reichtumsberichterstattung, in: Vierteljahrshefte zur Wirtschaftsforschung 75(1), S. 7-29.

Aurich-Beerheide, P./Brussig, M./Gabler,A./Ivanov, B./Kirsch, J./Kotlenga, S., et al. (2020): Öffentlich geförderte Beschäftigung zur Förderung der Teilhabe von Langzeitarbeitslosen, ZEW Policy Brief Nr. 4. 
Bartelheimer, P. (2007): Politik der Teilhabe. Ein soziologischer Beipackzettel, Fachforum. Analysen \& Kommentare 1/2007, Friedrich-Ebert-Stiftung, Forum Berlin.

Bauer, F. (2018): Zur Verringerung der Langzeitarbeitslosigkeit in NordrheinWestfalen. Anhörung des Ausschusses für Arbeit, Gesundheit und Soziales des Landtags Nordrhein-Westfalen am 14. März 2018, IAB-Stellungnahme $2 / 2018$.

Bauer, F. (2021): „Coaching« im Rahmen öffentlich geförderter Beschäftigung. Begriff, Funktionen und Situationslogik, in: Sozialer Fortschritt 70(1), S. 19-39.

Bauer, F./Bruckmeier, K./Kupka, P./Lietzmann, T./Promberger, M./Ramos Lobato, P./Wolff, J. (2018): Integration und Teilhabe durch öffentlich geförderte Beschäftigung - auf die Zielgruppe kommt es an, Öffentliche Anhörung von Sachverständigen vor dem Ausschuss für Arbeit und Soziales des Deutschen Bundestages am 5. November 2018, IAB-Stellungnahme 10/2018.

Bauer, F./Franzmann, M./Fuchs, P./Jung, M. (2016): Simulierte Normalität in (dauerhaft) geförderter Arbeit, in: Garstenauer, T./Hübel, T./Löffler K. (Hrsg.): Arbeit im Lebenslauf. Verhandlungen von (erwerbs-)biographischer Normalität, Bielefeld, S. 155-269.

Beauftragter der Bundesregierung für die Belange von Menschen mit Behinderungen (2018): Die UN-Behindertenrechtskonvention. Übereinkommen über die Rechte von Menschen mit Behinderungen. Die amtliche, gemeinsame Übersetzung von Deutschland, Österreich, Schweiz und Lichtenstein, Berlin.

Beckmann, F./Schad, D. (2021): Der soziale Arbeitsmarkt in Zeiten der CoronaKrise: Auslauf- oder Zukunftsmodell, in: Sozialer Fortschritt 70(1), S. 3-18.

BMAS = Bundesministerium für Arbeit und Soziales (Hrsg.) (2015): Evaluation der Modellprojekte »Bürgerarbeit«. Endbericht, vorgelegt von IAW und ISG, BMAS Forschungsbericht 458, Berlin.

BMAS/BA = Bundesministerium für Arbeit und Soziales/Bundesagentur für Arbeit (2019): Fragen und Antworten zur Bewirtschaftung des Passiv-AktivTransfers in der Grundsicherung für Arbeitsuchende, http://biaj.de/images/ BMAS-BA_PAT-FAQ-Bewirtschaftung_Juni_2019.pdf (Abruf am 5.7.2021).

Brettschneider, A. (2007): Jenseits von Leistung und Bedarf. Zur Systematisierung sozialpolitischer Gerechtigkeitsdiskurse, in: Zeitschrift für Sozialreform 53(4), S. 365-389.

Bruckmeier, K./Hohmeyer, K. (2018): Arbeitsaufnahmen von Arbeitslosengeld-IIEmpfängern. Nachhaltige Integration bleibt schwierig, IAB-Kurzbericht 2/2018. Brussig, M./Kotlenga, S. (2019): Sozialintegrative und beschäftigungsbegleitende Aktivitäten: Erfahrungen mit einer neuen Instrumentengruppe der Arbeitsförderung, in: Sozialer Fortschritt 68(12), S. 947-972. 
Bundesagentur für Arbeit (2020): $\$ 16$ i SGB II »Teilhabe am Arbeitsmarkt«. Förderung sozialversicherungspflichtiger Beschäftigung am sozialen Arbeitsmarkt, www.arbeitsagentur.de/datei/dok_ba040168.pdf (Abruf am 5.7.2021).

Bundesrat (2018): Drucksache 366/18 vom 10. August 2018, www.bundesrat.de/ SharedDocs/drucksachen/2018/0301-0400/366-18.pdf?_blob=publicationFile $\& v=1$ (Abruf am 5.7.2021).

Bundesrechnungshof (2015): Förderung von Arbeitsverhältnissen nach $\mathbb{1 6 \mathrm { e }}$ SGB II. Abschließende Mitteilung an das Bundesministerium für Arbeit und Soziales, Bonn.

Bundesverfassungsgericht (2010): Urteil vom 9. Februar 2010, Az. 1 BvL 1/09, 1-220, www.bundesverfassungsgericht.de/SharedDocs/Entscheidungen/DE/ 2010/02/1s20100209_1bvl000109.html (Abruf am 5.7.2021).

CDU/CSU/SPD (2005): Gemeinsam für Deutschland - mit Mut und Menschlichkeit. Koalitionsvertrag zwischen CDU, CSU und SPD, 11.11.2005, Berlin.

Christoph, B./Hohmeyer, K. (2012): Ein-Euro-Jobs aus Sicht der Betroffenen: Zur Binnenwahrnehmung eines kontroversen Instruments, in: Sozialer Fortschritt 61(6), S. 118-126.

COMPASS/IMU/SÖSTRA/PIW/Universität Hamburg (2006): Evaluation der Maßnahmen zur Umsetzung der Vorschläge der Hartz-Kommission - Modul 1c: Arbeitsbeschaffungsmaßnahmen. Endbericht, Berlin/Bremen/Hamburg, BMAS Forschungsbericht F356c.

Cremer, G. (2007): »Sozialer Arbeitsmarkt«: Sinnvoll bei enger Zielgruppendefinition. Ist eine langfristig orientierte öffentliche Beschäftigung für Personen ohne Beschäftigungschancen sinnvoll?, in: ifo-Schnelldienst 60(10), S. 27-32. Deutscher Bundestag (2007): Plenarprotokoll 16/109. Stenografischer Bericht. 109. Sitzung. Berlin, Freitag, den 6. Juli 2007, https://dserver.bundestag.de/ btp/16/16109.pdf (Abruf am 26.7.2021).

Deutscher Bundestag (2018a): Plenarprotokoll 19/55. Stenografischer Bericht. 55. Sitzung. Berlin, Donnerstag, den 11. Oktober 2018, https://dserver.bun destag.de/btp/19/19055.pdf (Abruf am 5.7.2021).

Deutscher Bundestag (2018b): Sozialer Arbeitsmarkt - Teilhabechancen für Langzeitarbeitslose ermöglichen. Antwort der Bundesregierung auf die Kleine Anfrage der Abgeordneten Beate Müller-Gemmeke, Markus Kurth, Sven Lehmann, weiterer Abgeordneter und der Fraktion Bündnis 90/Die Grünen, Bundestagsdrucksache 19/5482.

Fuchs, P./Hamann, S. (2017): Öffentlich geförderte Beschäftigung von Langzeitarbeitslosen: Betreuung, Wohlfahrtseffekte und Beschäftigungsperspektiven bei privaten und gemeinnützigen Arbeitgebern, in: Sozialer Fortschritt 66(9), S. 611-631. 
Hirseland, A./Ramos Lobato, P./Ritter, T. (2012): Soziale Teilhabe durch geförderte Beschäftigung? Das Beispiel des Beschäftigungszuschusses, in: WSIMitteilungen 65(2), S. 94-102.

Hofmann, T. (2020): Zu gut, um befristet zu sein. Zur Förderung von langzeiterwerbslosen Menschen mit der neuen »Teilhabe am Arbeitsmarkt«, in: Soziale Sicherheit 69(4), S. 140-144.

IAQ/ZEW/zoom/SOKO-Institut Bielefeld (2019): Evaluation des Bundesprogramms »Soziale Teilhabe am Arbeitsmarkt«. Endbericht, Berlin, BMAS Forschungsbericht 535.

ISG/IAB/Regionales Forschungsnetz - Standort Baden-Württemberg (2016): Wissenschaftliche Begleitung und Evaluation des Programms »Modellhafte Entwicklung eines sozialen Arbeitsmarktes >Passiv-Aktiv-Tausch (PAT)«. Endbericht. 30. September 2016, https://wm.baden-wuerttemberg.de/filead $\mathrm{min} /$ redaktion/m-wm/intern/Publikationen/Arbeit/Endbericht_PAT_ISG_ IAB.pdf (Abruf am 26.7.2021).

ISG/IAB/RWI (2011): Endbericht der Evaluation der Leistungen zur Beschäftigungsförderung nach $\$ 16 \mathrm{e}$ Absatz 10 des Zweiten Buches Sozialgesetzbuch. Unterrichtung durch die Bundesregierung, Bundestagsdrucksache 17/6880.

ISG/IAW/SOKO (2019): Evaluation des ESF-Bundesprogramms zur Eingliederung langzeitarbeitsloser Leistungsberechtigter nach dem SGB II auf dem allgemeinen Arbeitsmarkt. Zwischenbericht, Berlin, BMAS Forschungsbericht 533.

Knuth, M. (2006): »Hartz IV « - die unbegriffene Reform. Wandel der Erwerbsordnung durch Verallgemeinerung des Fürsorge-Regimes, in: Sozialer Fortschritt 55(7), S. 160-168.

Knuth, M. (2009): Path shifting and path dependence: Labour market policy reforms under German federalism, in: International Journal of Public Administration 32(12), S. 1048-1069.

Knuth, M. (2012): Grundsicherung "für Arbeitsuchende«: ein hybrides Regime sozialer Sicherung auf der Suche nach stabiler Governance, in: Bothfeld, S./ Sesselmeier, W./Bogedan, C. (Hrsg.): Arbeitsmarktpolitik in der Sozialen Marktwirtschaft. Vom Arbeitsförderungsgesetz zum Sozialgesetzbuch II und III, Wiesbaden, S. 70-90.

Knuth, M. (2016): Arbeitslosigkeit unter dem »Aktivierungsparadigma«: die Entwicklung seit den Hartz-Reformen 2005, in: Deutscher Verein für öffentliche und private Fürsorge e. V. (Hrsg.): Langzeitarbeitslosigkeit: Auswege aus der Sackgasse, Archiv für Wissenschaft und Praxis der sozialen Arbeit 47(4), Berlin/Freiburg, S. 16-31. 
Knuth, M. (2017): Kernpunkte eines dauerhaften Sozialen Arbeitsmarktes. Fachbeitrag für die Gesellschaft für innovative Beschäftigungsförderung $\mathrm{mbH}$ NRW, April 2017 (unveröffentlichtes Manuskript).

Knuth, M. (2020): Per Achterbahn zum Sozialen Arbeitsmarkt. Zur Vorgeschichte des »Teilhabechancengesetzes«, in: Sozialer Fortschritt 69(12), S. 797-824.

Knuth, M./Kirsch, J./Zink, L. (2013): Ansätze zur Entwicklung eines »Sozialen Arbeitsmarktes«. Studie im Auftrag des Deutschen Paritätischen Wohlfahrtsverbandes, Landesverband Baden-Württemberg, Duisburg/Essen, www.iatev. de/downloads/2013_kirsch_knuth_zink.pdf (Abruf am 5.7.2021).

Knuth, M./Larsen, F. (2010): Increasing roles for municipalities in delivering public employment services. The cases of Germany and Denmark, in European Journal of Social Security 12(3), S. 174-199.

Knuth, M./Tenambergen, T. (2015): »Inklusiver Arbeitsmarkt«. Vereinheitlichung der öffentlich geförderten Beschäftigung für behinderte und nicht behinderte Menschen? Gutachten für die Fraktion BÜNDNIS 90/DIE GRÜNEN im Landtag NRW, Düsseldorf, www.iatev.de/downloads/2015_knuth_ ten.pdf (Abruf am 5.7.2021).

Koch, S./Spies, C./Stephan, G./Wolff, J. (2011): Arbeitsmarktinstrumente auf dem Prüfstand, IAB-Kurzbericht 11.

Kronauer, M. (2010): Inklusion - Exklusion. Eine historische und begriffliche Annäherung an die soziale Frage der Gegenwart, in: Kronauer, M. (Hrsg.): Inklusion und Weiterbildung. Reflexionen zur gesellschaftlichen Teilhabe in der Gegenwart, Bielefeld, S. 24-58.

Lietzmann, T. (2016): Langzeitleistungsbezug und Langzeitarbeitslosigkeit: Definitionen, Quantitäten, Strukturen, in: WSI-Mitteilungen 69(5), S. 334-343.

Mayes, D. G./Berghman, J./Salais, R. (2001): Social exclusion and European policy, Cheltenham.

Nussbaum, M.C. (2000): Women and human development. The capabilities approach, Cambridge.

Obermeier, T./Sell, S./Tiedemann, B. (2014): Es werden mehr. Aktualisierte Abschätzung der Zielgruppe für eine öffentlich geförderte Beschäftigung aus der sich verfestigenden Langzeitarbeitslosigkeit, Remagen.

Schulze-Böing, M. (2021): Sozialwirtschaft - Chance für den sozialen Arbeitsmarkt? Neue Perspektiven durch das neue Förderinstrument im SGB II, in: Sozialer Fortschritt 70(1), S. 41-51.

Sen, A. (2008): Commodities and capabilities, New Delhi.

Statistik der Bundesagentur für Arbeit (2020): Arbeitsmarktpolitische Instrumente (Zeitreihe Jahreszahlen). Deutschland, West- und Ostdeutschland, Regionaldirektionen, Bundesländer, https://statistik.arbeitsagentur.de/Statis 
tikdaten/Detail/Aktuell/iiia5/amp-zr-amp-zr-jz/amp-zr-jz-dlrd-0-xlsx.xlsx? $\mathrm{blob}=$ publicationFile\&v=1 (Abruf am 5.7.2021).

United Nations (2006): Convention on the Rights of Persons with Disabilities and Optional Protocol, www.un.org/disabilities/documents/convention/con voptprot-e.pdf (Abruf am 5.7.2021). 


\title{
Grundsicherung trotz Arbeit statt Integration durch Arbeit
}

\author{
Erwerbsarmut und das Problem der Aufstocker:innen
}

Dorothee Spannagel

\section{Einleitung'}

Erwerbstätigkeit spielt in der deutschen Gesellschaft und im Sozialstaat eine zentrale Rolle. Sie ist wesentlich für die materielle und gesellschaftliche Teilhabe von Menschen; zugleich ist sie der wichtigste Zugangsmechanismus zur Sozialversicherung und ihren Leistungen.

In der Grundsicherung für Arbeitsuchende nach SGB II ist Erwerbsbeteiligung zentral für die Überwindung von Hilfebedürftigkeit. Entsprechend dem weiten Verständnis von Grundsicherung, das diesem Band zugrunde liegt, kann auch der Zugang zu guter Arbeit selbst als ein Teil bzw. eine Aufgabe von Grundsicherung gesehen werden.

Es ist allerdings offensichtlich, dass nicht alle Menschen gleichermaßen an Arbeit und durch Arbeit am gesellschaftlichen Leben teilhaben. Dies zeigt sich an dem nach wie vor bestehenden Problem der Arbeitslosigkeit, aber auch daran, dass Erwerbstätigkeit nicht zwangsläufig gesellschaftliche und materielle Teilhabe gewährleistet.

Zum einen gibt es zahlreiche Tätigkeiten, die die gesellschaftliche Teilhabe einschränken können, etwa bei sehr unregelmäßigen Arbeitszeiten oder Schichtdiensten; zum anderen gibt es Fälle, in denen Arbeit die materielle Teilhabe nicht gewährleistet, etwa aufgrund von Niedriglöhnen oder zu geringem Arbeitsumfang. Statt einer Unabhängigkeit von der staatlichen Grundsicherung durch Arbeit müssen in vielen Fällen Grundsicherungsleistungen trotz Arbeit bezogen werden.

1 | Die Autorin dankt Florian Blank und Claus Schäfer herzlich für ihre Unterstützung sowie Karin Schulze Buschoff für ihre Kommentare. 
Die Beobachtung, dass es in Deutschland Erwerbsarmut und sogenannte Aufstocker:innen gibt - also Bezieher:innen von Grundsicherungsleistungen, die diese Leistungen zusätzlich zum Erwerbseinkommen erhalten -, steht im Mittelpunkt dieses Beitrags. Es geht somit um die Einschränkung der ökonomischen Teilhabe trotz Erwerbstätigkeit.

Erwerbsarmut und »Aufstocken" sind allerdings nicht identisch. Sogenannte Erwerbsarmut liegt vor, wenn in einem Haushalt mindestens eine Person erwerbstätig ist, der Haushalt aber dennoch arm ist. Häufig werden in diesen Haushalten dann aufstockende Sozialleistungen bezogen. Wenn die vorgelagerten Leistungen Wohngeld und Kinderzuschlag nicht ausreichen, fallen diese Erwerbsarmen in das System der Grundsicherung für Arbeitsuchende (Arbeitslosengeld II; es handelt sich damit um »erwerbstätige ALG-II-Empfänger:innen«). Für die Bestimmung der Bedürftigkeit sowie für die gewährten Leistungen sind die Leistungshöhen des ALG II (Regelsatz und Kosten für Unterkunft und Heizung sowie ggf. Mehrbedarfe) maßgeblich.

Erwerbseinkommen wird auf die Leistungen nach dem SGB II angerechnet, wobei Freibeträge gelten. Insgesamt haben die Aufstocker:innen durch diese Freibeträge mehr Einkommen als arbeitslose ALG-II-Empfänger:innen, die keinerlei Beschäftigung nachgehen.

Laut Bundesagentur für Arbeit verfügt rund ein Viertel der erwerbsfähigen Leistungsbezieher:innen über ein Erwerbseinkommen, die überwiegende Mehrheit davon aus abhängiger Beschäftigung, was auch geringfügige Beschäftigung mit einschließt (Statistik der Bundesagentur für Arbeit 2021). Grundsätzlich ist das ALG II als Grundsicherung für Arbeitsuchende allerdings darauf ausgerichtet, Menschen möglichst schnell in den Arbeitsmarkt zu vermitteln und sie so aus dem Bezug der Grundsicherung herauszubringen. Für Aufstocker:innen kann dieses Ziel so nicht gelten, sodass sich die Frage nach der angemessenen Verortung im System der Arbeitsmarktpolitik stellt.

An den Problemen der Erwerbsarmut und des Aufstockens zeigt sich erstens, dass eine »einfache" Lösung für mangelnde materielle und gesellschaftliche Teilhabe, nämlich Erwerbsintegration - sowohl im Sinne des»Förderns und Forderns« als auch im Sinne eines »Rechts auf Arbeit« - an der Realität vorbeigeht. Zweitens wird im Folgenden argumentiert, dass auch die institutionelle Bearbeitung des Problems, nämlich die Zahlung der aufstockenden Leistungen im Rahmen der »Grundsicherung für Arbeitsuchende« nach SGB II, das Problem nicht trifft.

Die Grundsicherung für Arbeitsuchende hat eine Zwitterstellung zwischen Arbeitsmarktpolitik und Grundsicherung: Sie zielt auf eine Erwerbsintegration $\mathrm{ab}$ und gewährt materielle Leistungen, wenn Hilfebedürftigkeit vorhanden ist - 
die aber durch die Hilfebedürftigen überwunden werden soll. Aber weder müssen die Aufstocker:innen bzw. Erwerbsarmen aktiviert werden noch ist klar, ob sie mehr dazu beitragen können, ihre Hilfebedürftigkeit zu überwinden.

Damit stellt sich die Frage nach den arbeitsmarkt- und sozialpolitischen Konsequenzen: Sollen erwerbsarme Haushalte durch die Grundsicherung lediglich finanziell (besser) alimentiert werden? Oder muss nicht vielmehr dafür Sorge getragen werden, dass solche Haushalte durch ihre Erwerbstätigkeit (wieder) materiell an der Gesellschaft teilhaben können und vor Armut geschützt sind, und zwar ohne unnötigen Druck etwa hinsichtlich der Teilnahme an arbeitsmarktpolitischen Maßnahmen auszuüben?

Entsprechend ist die Frage zu analysieren, warum der Lohn nicht zum Leben reicht: Liegt es an niedrigen Löhnen, hohen Bedarfen im Haushaltskontext oder an zu geringem Umfang der Arbeitsmarktintegration? Damit verbunden sind die politisch-praktischen Fragen, durch welche Maßnahmen der Arbeitsmarkt so gestaltet werden kann, dass der Lohn zum Leben reicht und keine aufstockenden staatlichen Transferzahlungen notwendig sind, ${ }^{2}$ und ob Erwerbsarme überhaupt im ALG-II-System richtig untergebracht sind bzw. wie eine Alternative aussehen könnte.

In diesem Beitrag gehen wir in einem ersten Schritt den Zusammenhängen zwischen Grundsicherung und Arbeit nach (Abschnitt 2). Darauf aufbauend werden die Gruppen der Erwerbsarmen und der Aufstocker:innen in den Blick genommen (Abschnitt 3). Damit wird gezeigt, dass Erwerbsintegration allein nicht unbedingt zu voller materieller und gesellschaftlicher Teilhabe führt. Abschließend werden Vorschläge unterbreitet, wie Rahmenbedingungen für gute, armutsfeste Arbeit aussehen können (Abschnitte 4 und 5).

\section{Grundsicherung durch Arbeit?}

In der deutschen Gesellschaft ist Erwerbsarbeit der zentrale Mechanismus, um materielle und gesellschaftliche Teilhabe zu gewährleisten. Im Sinne des in diesem Band verfolgten erweiterten Konzepts von Grundsicherung kann daher sogar von Grundsicherung durch Arbeit bzw. durch Teilhabe am Arbeitsmarkt gesprochen werden. Eine gelungene Teilhabe am Arbeitsmarkt setzt nicht nur ein

2 | Dies wiederum führt zu den im Rahmen dieses Beitrags nicht zu bearbeitenden Fragen nach einem angemessenen Erwerbsumfang und der Haushaltszusammensetzung, die zur Beurteilung der Angemessenheit eines Erwerbslohns mitgedacht werden müssten. 
Erwerbsverhältnis aus abhängiger oder selbstständiger Tätigkeit voraus, sondern auch eine Höhe des Erwerbseinkommens, die ökonomische, soziale, kulturelle und politische Exklusion vermeidet.

Die erste Voraussetzung, Teilhabe an Erwerbsarbeit in einer Erwerbsgesellschaft, ist lange als Recht auf Arbeit $^{3}$ diskutiert worden. Aber in einer überwiegend marktwirtschaftlich verfassten Wirtschaftsordnung ist dieses Recht schwer umzusetzen.

Ein zum ersten Arbeitsmarkt paralleler "zweiter", sozialstaatlich organisierter Arbeitsmarkt könnte eine wichtige Ergänzung sein, gerade für Arbeitsuchende mit besonderen persönlichen Handicaps - allerdings bleiben schon bisherige Ansätze zur Verwirklichung eines »sozialen« Arbeitsmarkt hinter den in arbeitsmarktpolitische Debatten geäußerten Vorstellungen zurück (siehe hierzu den Beitrag von Matthias Knuth in diesem Band).

Ein Recht auf Arbeit wird in der deutschen Arbeitsmarktpolitik, wenn überhaupt, allenfalls indirekt durch den Versuch verfolgt, das »Unrecht Arbeitslosigkeit« möglichst a priori zu vermeiden oder zumindest a posteriori zu lindern: durch eine gute Schul- und Berufsausbildung, die die Beschäftigungsmöglichkeit erhöht und das Arbeitslosigkeitsrisiko vermindert, durch Ausbildungsplatzgarantien, gesetzliche Mindestquoten für Arbeitgeber zur Einstellung körperlich beeinträchtigter Beschäftigungswilliger, öffentliche Leistungen zur Entlastung von Familien mit Kindern zugunsten der Erwerbsaufnahme insbesondere von Frauen - und schließlich vor allem durch die Arbeitslosenversicherung im Verbund mit der nachgelagerten Grundsicherung für Arbeitsuchende. Beide Systeme sollen beschäftigungslose Personen aktiv vermitteln und Arbeitgeber, die Arbeitskräfte suchen, durch diverse Anreize einstellungswillig machen.

Der wichtigste Beleg für das Fehlen eines echten Rechts auf Arbeit ist eine auch heute nach vielen Jahren des konjunkturellen Aufschwungs noch hohe Zahl an offener und verdeckter Arbeitslosigkeit - Letztere durch Menschen, die sich vom Arbeitsmarkt zurückgezogen haben -, und vor allem die immer noch vielen Langzeitarbeitslosen. Das Institut für Arbeitsmarkt- und Berufsforschung (IAB) beziffert zuletzt für 2020 die Zahl der offenen Arbeitslosen auf 2,7 Millionen und die der verdeckten Arbeitslosigkeit - der »stillen Reserve«-auf 0,9 Millionen Personen (Sozialpolitik-aktuell 2021b), wobei die »stille Reserve« in Abgrenzung von IAB und Bundesagentur für Arbeit nur eine ge-

3 | Das Recht auf Arbeit wurde beispielsweise von Kronauer (2020) in Auseinandersetzung mit Vorschlägen für ein bedingungsloses Grundeinkommen wieder in die Diskussion eingebracht. 
schätzte Teilmenge derjenigen umfasst, die sich vom Arbeitsmarkt zurückgezogen haben.

Diesem Problembereich fehlender Arbeit und entsprechend fehlender Teilhabe geht der vorliegende Beitrag nicht weiter nach; er wird teilweise in anderen Beiträgen dieses Bandes behandelt. Die zweite Voraussetzung für Teilhabe an und durch Arbeit soll in diesem Beitrag dagegen im Mittelpunkt stehen: Arbeit mit einem Erwerbseinkommen in ausreichender Höhe. Auch diese Voraussetzung wird in Deutschland zu einem erheblichen Teil nicht erfüllt, obwohl eigentlich vorhandene sozialstaatliche Instrumente dafür sorgen sollten.

Teilhabe an und durch Arbeit ist aber nicht nur bei Aufstocker:innen nicht erfüllt; es gibt auch eine Art »verhinderte Aufstockung«. Gemeint sind damit Erwerbstätige, die nur deshalb kein Recht auf aufstockende ALG-II-Leistungen haben, weil sie Wohngeld bzw. Kinderzuschlag empfangen. ${ }^{4}$ Sie sind keine Aufstocker:innen im eigentlichen Sinne, aber es gelingt ihnen ebenfalls nicht, sich kraft ihrer Erwerbstätigkeit ein Leben ohne Notwendigkeit des Sozialleistungsbezugs zu sichern.

Eine dritte Gruppe besteht aus der Dunkelziffer derjenigen, die zwar faktisch Anspruch auf die eben erwähnten Leistungen haben, aber diesen Anspruch aus Unkenntnis, Scham vor sozialer Stigmatisierung oder Aversion gegen die HartzIV-Zugangsbedingungen nicht durch einen entsprechenden Antrag einlösen. Darunter dürfte es auch Personen und Haushalte geben, die einen Antrag lediglich auf Wohngeld und Kinderzuschlag vorziehen, obwohl sie einen Anspruch auf Grundsicherung hätten.

Der vorliegende Beitrag konzentriert sich im Folgenden auf die Aufstocker:innen.

4 | Das Wohngeld soll eine angemessene Wohnung ermöglichen; der Kinderzuschlag wiederum wurde explizit mit dem Ziel eingeführt, Familien mit mindestens einer erwerbstätigen Person vor dem ALG-II-Bezug zu bewahren. Beide Leistungen sind sozialrechtlich dem Bezug von ALG II vorgelagert, können also nicht gleichzeitig mit ALG-II-Leistungen bezogen werden. Erwerbseinkommen werden sowohl auf Wohngeld als auch auf Kinderzuschlag angerechnet, die Transferentzugsraten liegen mit jeweils 50 Prozent allerdings etwas niedriger als beim ALG II.

Wohngeld, Kinderzuschlag und ALG II greifen eng ineinander und werden in sehr komplexen Strukturen wechselseitig aufeinander angerechnet. Wohngeld wird im unteren Einkommensbereich als Zuschuss zu den Wohnkosten gezahlt, umfasst aber im Unterschied zu den Kosten der Unterkunft, die ALG-Bezieher:innen erhalten, keine Heizkosten (vgl. hierzu ausführlicher Bruckmeier/Mühlhan/Wiemers 2018, S. $14 \mathrm{f}$.$) .$ 


\section{Aktuelle Entwicklungen}

Gesellschaftliche Teilhabe wird in diesem Beitrag als materielle Teilhabe verstanden. Der Arbeitsmarkt schafft eine materielle Lücke (Armut trotz Arbeit); ob die Erwerbstätigen möglicherweise - neben dem unzureichenden Lohn - auch andere Arbeitsbedingungen haben, die ihre gesellschaftliche Teilhabe einschränken, kann hier nicht untersucht werden. Dieser Fokus ist allerdings insofern gerechtfertigt, als das Grundsicherungssystem dieses Problem ebenfalls vorrangig materiell bearbeitet, nämlich durch finanzielle Zuschüsse. Was ist unter »materieller Teilhabe« zu verstehen? Es gibt zwei unterschiedliche Perspektiven zur Beantwortung dieser Frage.

Messlatte für ein Mindestmaß an materieller Teilhabe ist zum einen das soziokulturelle Existenzminimum als politisch-rechtlicher Anspruch. Dieser Anspruch ist in $\$ 1$ Absatz 2 SGB XII definiert als »die Führung eines Lebens [...], das der Würde des Menschen entspricht«. Die materielle Höhe dieses Existenzminimums ist im Sozialgesetzbuch (SGB) festgelegt $t^{5}$ und untergliedert die Leistungen nach Lebensjahren und unterschiedlichen Haushaltskonstellationen (Regelbedarfsstufen). ${ }^{6}$ Aus dieser sozialrechtlichen Perspektive ist materielle Teilhabe nicht erfüllt, wenn eine Bedarfsgemeinschaft über weniger Einkommen verfügt, als ihr nach dem Sozialhilfesatz zustünde.

Die zweite Perspektive auf materielle Teilhabe zieht Armut als Messlatte heran. Hier gilt: Eine volle materielle Teilhabe ist nicht gegeben, wenn eine Person bzw. ein Haushalt von Armut betroffen ist. Armut wird in diesem Beitrag, der gängigsten Armutsdefinition folgend, als relative Einkommensarmut verstanden. ${ }^{7}$ Als arm gilt eine Person demnach, wenn sie in einem Haushalt lebt, dessen

5 | Und zwar in SGB XII (Sozialhilfe); das SGB II nimmt auf die entsprechende Stelle Bezug.

6 | Die Berechnung der Regelsätze und damit der angemessene Geldwert des soziokulturellen Existenzminimums sind in Politik und Wissenschaft umstritten; siehe hierzu den Beitrag von Irene Becker in diesem Band.

7 | Die Frage, was Armut ist und wie sie zu messen ist, ist seit Beginn der Armutsforschung äußerst umstritten; entsprechend viel Literatur gibt es dazu (vgl. u. a. Spannagel 2017). Oft wird, gerade in den Medien, der Bezug von ALG II mit Armut gleichgesetzt. Ungeachtet der Tatsache, dass das Einkommen solcher Haushalte oftmals tatsächlich unterhalb der Armutsgrenze liegt, ist diese Beschreibung äußerst problematisch; schließlich wird ALG II ja gezahlt, um ein soziokulturelles Existenzminimum zu sichern. Um dieses Dilemma deutlich zu machen, wird die ALG-II-Empfängerquote deshalb auch als »bekämpfte Armut« bezeichnet. 
verfügbares Haushaltsnettoeinkommen weniger als 60 Prozent des Medianeinkommens beträgt. ${ }^{8}$

Gemeinsam ist beiden Betrachtungsweisen, dass immer alle Mitglieder eines Haushalts arm sind oder eben nicht - ein Aspekt, der später auch im Hinblick auf Erwerbsarmut von Bedeutung sein wird. Beide Ansätze nehmen unterschiedliche Bevölkerungsgruppen in den Blick. Bei den Erwerbstätigen sind dies im zuerst genannten Fall die Aufstocker:innen, im zweiten die Erwerbsarmen.

\subsection{Erwerbsarmut}

Erwerbsarmut verbindet ein individuelles Merkmal (Erwerbstätigkeit einer Person) mit einem Haushaltsmerkmal (Haushaltseinkommen unterhalb der Armutsgrenze). ${ }^{9}$ Dabei gilt, dass immer alle Mitglieder eines Haushalts denselben Status haben, also erwerbsarm oder nicht erwerbsarm.

Daten zur Entwicklung der Erwerbsarmut werden von der EU regelmäßig erhoben. Für Deutschland wird der Anteil der erwerbsarmen Haushalte für das Jahr 2019 - das letzte Jahr, für das Zahlen ausgewiesen werden - mit 8,0 Prozent angegeben (siehe Abbildung 1). Der Anteil war in den letzten Jahren zwar rückläufig, hat aber nach einem deutlichen Anstieg zwischen 2009 und 2014 (Höchststand von 9,9 Prozent) das Niveau der zweiten Hälfte der Nullerjahre noch nicht wieder erreicht.

8 | Das verfügbare Haushaltsnettoeinkommen umfasst die Einkommen aller Haushaltsmitglieder nach Abzug von Steuern und Sozialabgaben inklusive aller staatlichen Transferzahlungen wie etwa ALG II oder Renten. Es ist das Einkommen, das einem Haushalt tatsächlich zur Verfügung steht.

Der Einkommensmedian, der der relative Bezugspunkt für die Armutsgrenze ist, ist der Wert, der alle Haushalte, wenn man sie der Einkommenshöhe nach anordnet, in eine obere und eine untere Hälfte teilt. 2019 lag das Medianeinkommen bei einem verfügbaren Jahreseinkommen von 22.693 Euro, was eine Armutsgrenze von 13.616 Euro ergibt. Ein Einpersonenhaushalt, dessen Einkommen darunter liegt, ist arm. Für einen Paarhaushalt mit zwei Kindern unter 14 Jahren liegt der Schwellenwert bei 28.594 Euro im Jahr (Eurostat 2021b auf der Datenbasis des European Survey on Income and Living Conditions [EU-SILC]).

9 | In den hier verwendeten Daten des EU-SILC wird Erwerbstätigkeit als Selbsteinschätzung folgendermaßen definiert: Die Befragten geben an, im Jahr vor der Befragung mindestens sechs Monate lang erwerbstätig gewesen zu sein. 
Abb. 1: Arbeits-Armutsgefährdungsquote, Erwerbstätige, 18 Jabre und älter (20072019; in Prozent der Erwerbstätigen)

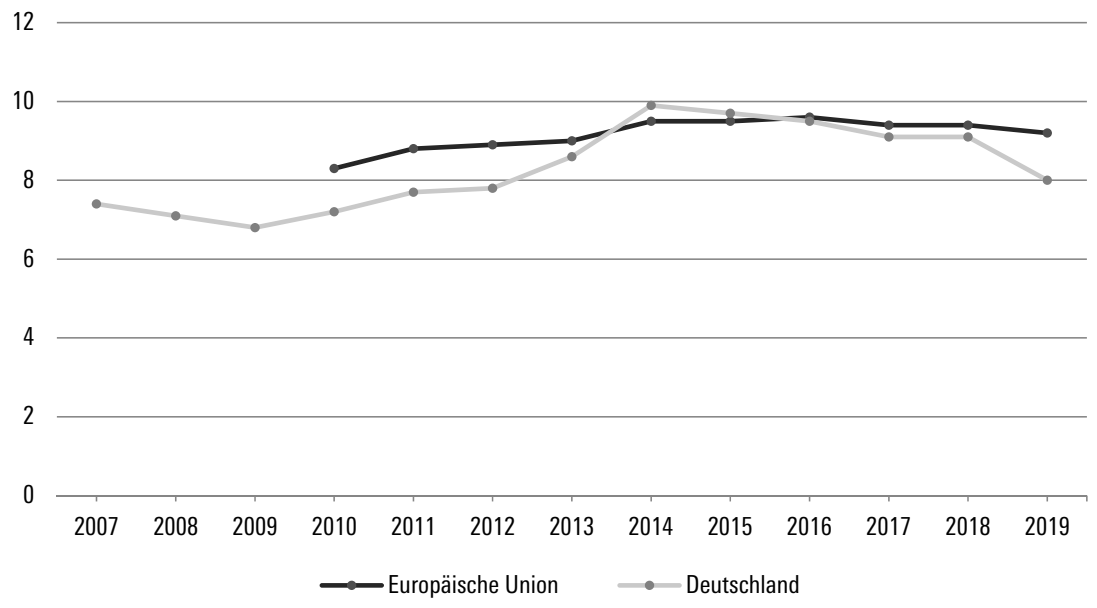

Anmerkung: Wert für EU 2019 geschätzt

Quelle: Eurostat 2021a auf der Datenbasis EU-SILC (eigene Darstellung)

Im Vergleich zum Durchschnitt der EU-28 lag der Wert für Deutschland zunächst etwas darunter, holte dann aber auf und ist seitdem ähnlich hoch. Anfang der 2010er-Jahre weist Deutschland im EU-Vergleich den höchsten Zuwachs an Erwerbsarmut auf (Spannagel et al. 2017, S. 6). Dies hat politische Ursachen, denn die wichtigsten Treiber dieser Entwicklung waren der politisch geförderte Ausbau der atypischen Beschäftigung und des Niedriglohnsektors (vgl. Spannagel et al. 2017, S. 9).

Einige Bevölkerungsgruppen sind besonders von Erwerbsarmut betroffen (vgl. u. a. European Commission 2011; Goerne 2011; Ponthieux 2010; Spannagel 2013). Dazu gehören Haushalte mit einfachen beruflichen Tätigkeiten, niedrigem Bildungsniveau, geringer Erwerbsbeteiligung (wenn etwa nur eine Person erwerbstätig ist oder wenn alle Erwerbstätigen nur in Teilzeit arbeiten) sowie Migrantenhaushalte und Alleinerziehende. Diese Aufzählung weist auf zwei Hauptmechanismen hin, die zu Erwerbsarmut führen: niedrige Erwerbseinkommen der Individuen - die auch im Haushalt in der Summe nicht zu einem Leben ohne Armut führen - und unzureichende Transferleistungen auf der Haushaltsebene, die nicht ausreichen, um dem Haushalt aus der Armut zu bringen.

Hinter niedrigen Erwerbseinkommen stehen zumeist Niedriglöhne und/ oder eine geringe Erwerbsbeteiligung. So sinkt das Risiko der Erwerbsarmut, 
je höher der Anteil an Haushaltsmitgliedern ist, die unbefristet in Vollzeit beschäftigt sind (Nolan/Whelan/Maître 2010). Ein niedriges Einkommen führt aber nicht zwangsläufig zu Erwerbsarmut, denn diese ist immer ein Haushaltsmerkmal. Es ist oft der Fall, dass das niedrige Einkommen einer Person durch die Einkommen weiterer Haushaltsmitglieder so weit ausgeglichen wird, dass der Haushalt insgesamt oberhalb der Armutsgrenze liegt.

Dieser Mechanismus spielt gerade bei Frauen eine wichtige Rolle, denn diese sind oftmals nur teilzeiterwerbstätig und/oder arbeiten im Niedriglohnsektor. Wäre dies das einzige Einkommen, wären sie erwerbsarm; das Einkommen des Mannes reicht aber in vielen Fällen aus, um den Haushalt vor Erwerbsarmut zu bewahren. Wenn dieses zweite Einkommen fehlt, wie das bei Singlehaushalten der Fall ist, steigt das Erwerbsarmutsrisiko deutlich an. Das gilt insbesondere für Alleinerziehende, da in diesem Fall von dem einen Einkommen auch ein oder mehrere Kinder zu ernähren sind.

Niedrige Erwerbseinkommen können auf der Haushaltsebene auch durch staatliche Transferzahlungen ausgeglichen werden. Hier spielen vor allem die Höhe des Arbeitslosengeldes und der Sozialhilfe eine Rolle (European Commission 2011; Spannagel et al. 2017). Damit sind wir beim ALG II und den Aufstocker:innen.

\subsection{Die Aufstocker:innen}

Ursprünglich wurde der Begriff »Aufstocker« in der Verwaltungssprache der Bundesagentur für Arbeit für Personen verwendet, deren ALG I so niedrig ist, dass sie es mit ALG-II-Leistungen aufstocken müssen (Bundesagentur für Arbeit 2010, S. 5). Inzwischen wird der Begriff in Politik und Öffentlichkeit üblicherweise für Haushalte gebraucht, die ihr niedriges Einkommen mit ALG II aufstocken (müssen). Die Bundesagentur spricht in ihren Publikationen von »erwerbstätigen Leistungsbeziehern in der Grundsicherung für Arbeitsuchende« und definiert sie als »erwerbsfähige Hilfebedürftige, die Leistungen aus der Grundsicherung für Arbeitsuchende erhalten und gleichzeitig Brutto-Monatseinkommen aus abhängiger oder selbständiger Erwerbstätigkeit beziehen« (Bundesagentur für Arbeit 2010, S. 5).

Aufstocker:innen beziehen zwar Grundsicherung für Arbeitsuchende, sind aber nicht arbeitslos. Beispielsweise gehören zu den Aufstocker:innen auch Pflegende oder Alleinerziehende mit Kindern, die einer geringfügigen Beschäftigung nachgehen, aber nicht zu (weiterer) Erwerbstätigkeit angehalten werden (vgl. Sozialpolitik-aktuell 2021a). 
Das Erwerbseinkommen, das solche Haushalte erzielen, mindert ihren ALG-II-Anspruch, da von einigen Ausnahmen abgesehen alle Einkommensarten auf das ALG II angerechnet werden (vgl. hierzu ausführlicher Bundesagentur für Arbeit 2010, S. 5 f.). Auch Transfereinkommen wie etwa Kindergeld und ALG I werden auf den ALG-II-Anspruch angerechnet.

Aktuell sind für erwerbsfähige Leistungsberechtigte, die erwerbstätig sind, nach Berücksichtigung von Abgaben 100 Euro im Monat anrechnungsfrei; ab 100 Euro werden 80 Prozent des Einkommens auf das ALG II angerechnet. Dies gilt auch für sozialversicherungspflichtige Mini- und Midijobs. Von 1.000 bis 1.200 Euro im Monat steigt der Abzug auf 90 Prozent für die Einkommensteile, die 1.000 Euro übersteigen. Für erwerbsfähige Leistungsberechtigte, die mit mindestens einem minderjährigen Kind zusammen in einer Bedarfsgemeinschaft leben oder mindestens ein minderjähriges Kind haben, beträgt die Grenze nicht 1.200, sondern 1.500 Euro.

Wie in Abbildung 2 zu sehen, ist die Zahl der Aufstocker:innen seit rund zehn Jahren kontinuierlich rückläufig (Statistik der Bundesagentur für Arbeit 2021). Für das Jahr 2020 weist die Bundesagentur knapp 933.000 erwerbsfähige Leistungsberechtigte aus, die zugleich erwerbstätig waren. Das sind über 400.000 weniger als im Jahr 2010, dem Jahr mit dem höchsten Wert. Unter diesen Erwerbstätigen bezog die große Mehrheit ein Einkommen aus abhängiger Erwerbstätigkeit (863.965 Personen). 2020 waren insgesamt 24 Prozent aller erwerbsfähigen ALG-II-Berechtigten erwerbstätig. ${ }^{10}$ Dieser Anteil ist von rund 23 Prozent im Jahr 2007 auf 30 Prozent in den Jahren 2011-2014 gestiegen und sinkt seither wieder (2020: 24 Prozent; Statistik der Bundesagentur für Arbeit 2021).

Interessant ist ein Blick auf die Einkommenshöhe der Aufstocker:innen (siehe Abbildung 3): Vor allem um das Jahr 2010 herum hatte die überwiegende Mehrheit der Aufstocker:innen ein Erwerbseinkommen von 450 Euro oder weniger, also einen Minijob. Seitdem ist ihr Anteil deutlich gesunken, was auch den Rückgang der Gesamtzahl der Aufstocker:innen erklärt. Die Gruppe der Leistungsberechtigten, deren Einkommen zwischen 450 und 1.300 Euro liegt, ist über die Jahre hinweg recht stabil bei etwa 400.000 . Haushalte mit einem Einkommen über 1.300 Euro gibt es unter den Aufstocker:innen vergleichsweise wenige; es sind in keinem Jahr mehr als 140.000 .

Was ist darüber bekannt, welche Haushalte aufstocken? Es gibt einige typische Merkmale (vgl. u. a. Bruckmeier/Becker 2018, S. 20; Bruckmeier et al. 2015,

10 | 2020 betrug die Zahl der erwerbsfähigen Leistungsberechtigten 3.889.188 Personen, die der Leistungsberechtigten insgesamt 5.485.738 Personen (Statistik der Bundesagentur für Arbeit 2021). 
Abb. 2: Erwerbsfähige Leistungsberechtigte nach SGB II insgesamt und erwerbstätige Leistungsberechtigte (2007-2020)

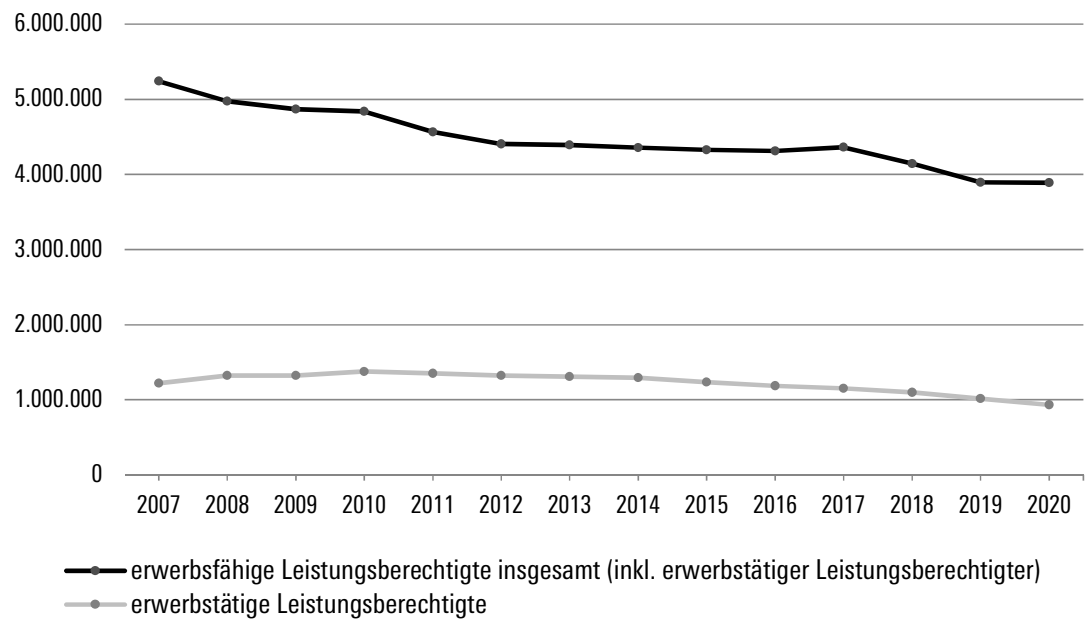

Quelle: Statistik der Bundesagentur für Arbeit 2021 (eigene Darstellung)

Abb. 3: Einkommenshöhe der abhängig beschäftigten erwerbstätigen Leistungsbezieber:innen (2007-2020)

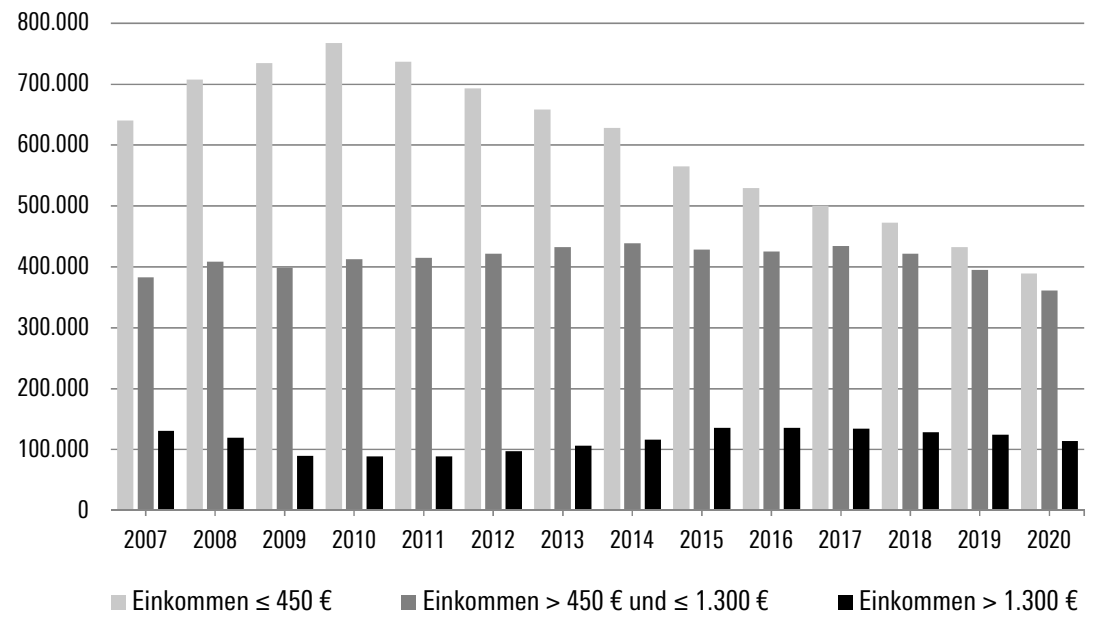

Quelle: Statistik der Bundesagentur für Arbeit 2021 (eigene Darstellung) 
S. 3; Bundesagentur für Arbeit 2010, S. 13 ff.; Schröter 2012, S. 7): Die überwiegende Mehrheit der Aufstocker:innen arbeitet im Dienstleistungssektor; Personen ohne Berufsabschluss sind überrepräsentiert; erwerbstätige Migrant:innen sind deutlich häufiger auf aufstockende Leistungen angewiesen als Deutsche. Die Mehrheit der Aufstocker:innen lebt in Paarhaushalten, darunter doppelt so viele Haushalte mit Kindern wie ohne Kinder. Etwa ein Drittel sind Singlehaushalte und rund 20 Prozent Alleinerziehende.

Aufstocker:innen arbeiten überwiegend in Teilzeit (etwa zwei Drittel arbeiten weniger als 22 Wochenstunden). Dies gilt insbesondere für Frauen und für Ältere über 50 Jahre. Zudem haben Aufstocker:innen oftmals einen unterdurchschnittlichen Stundenlohn. Auch geringfügige Beschäftigung ist weit verbreitet, hat allerdings im Zeitverlauf abgenommen. Ähnlich wie bei den Erwerbsarmen trifft bei den Aufstocker:innen eine besondere Erwerbskonstellation auf bestimmte Haushaltsmerkmale.

Die Bundesagentur für Arbeit (2010, S. 9) hat die Erwerbskonstellationen der Aufstocker:innen in drei Gruppen unterteilt: Teilzeiterwerbstätige mit geringfügiger Beschäftigung, die die größte Gruppe ausmachten und vor allem Frauen umfassten, Teilzeiterwerbstätige mit sozialversicherungspflichtiger Beschäftigung sowie Vollzeiterwerbstätige mit sozialversicherungspflichtiger Beschäftigung. Bei der letzten Gruppe handelt es sich üblicherweise um Haushalte mit nur einem Verdiener oder einer Verdienerin. Auch Auszubildende fallen oft in diese Gruppe.

Studien zeigen, dass bei kleineren Haushalten (vor allem bei Singlehaushalten) meist eine zu geringe Stundenzahl der Grund für das Aufstocken ist. Je größer der Haushalt ist, desto wahrscheinlicher ist es, dass selbst eine Vollzeittätigkeit nicht ausreicht, um über die ALG-II-Schwelle zu kommen (Bruckmeier et al. 2015, S. 2). Viele Merkmale kennzeichnen sowohl Erwerbsarme als auch Aufstocker:innen, allerdings sind diese Gruppen nicht deckungsgleich. Zum einen hat nicht jeder erwerbsarme Haushalt einen Anspruch auf aufstockende ALG-II-Leistungen, zum anderen nimmt nicht jeder erwerbsarme Haushalt, der einen Anspruch hätte, diesen auch wahr - etwa aus Unkenntnis oder aus Scham (Bruckmeier/Mühlhan/Wiemers 2018, S. 15 ff.).

Zusammenfassend kann man folgende Merkmale festhalten, die für aufstockende Haushalte typisch sind: ein niedriger Lohn - entweder durch niedrige Stundenlöhne oder einen geringen Stundenumfang aufgrund von geringfügiger Beschäftigung -, Teilzeiterwerbstätigkeit und/oder ein hoher Bedarf auf der Haushaltsebene durch viele Haushaltsmitglieder, die nicht oder nur geringfügig erwerbstätig sind. Diese Merkmale stehen für unterschiedliche Wege, die einen Haushalt mit einer erwerbstätigen Person in den ALG-II-Bezug führen. 


\section{Erwerbsarmut: Verschiedene Problemlagen - verschiedene Ansatzpunkte zur Bekämpfung}

Erste Hinweise auf Maßnahmen, die aus der Aufstockung herausführen, finden sich in Untersuchungen zu den Merkmalen, die die Chance erhöhen, aus einer solchen Situation herauszukommen. Zwar ist im Falle der Aufstocker:innen das Problem Armut aus politisch-administrativer Sicht »behoben«, indem das soziokulturelle Existenzminimum durch die aufstockenden Leistungen erreicht wird. Das tieferliegende Problem ist aber, dass die Grundsicherungsleistungen nicht armutssicher sind und dass vor allem durch Erwerbsarmut und Aufstockung von Erwerbseinkommen sichtbar wird, dass Arbeit als Integrationsmechanismus - also Grundsicherung durch Arbeit - nicht funktioniert.

Damit stößt auch die Logik des Sozialgesetzbuchs an ihre Grenzen, wenn nämlich Personen und Haushalte »aktiviert« sind und dennoch nicht vom Leistungsbezugs unabhängig werden. Die Überwindung der Hilfebedürftigkeit als Ziel, auf das das Sozialgesetzbuch ausgerichtet ist, wird bei Aufstocker:innen nicht erreicht, und das oft dauerhaft: Viele Aufstocker:innen sind über Jahre hinweg in atypischer Beschäftigung gefangen und schaffen den Schritt in eine reguläre Beschäftigung nicht (Bruckmeier/Mühlhan/Wiemers 2018, S. 12).

Die analysierten Daten legen nahe, dass es sich bei Erwerbsarmut nicht um eine Art »selbstbestimmte« Arbeitsmarktferne handelt, sondern um strukturelle Probleme bestimmter Erwerbstätigengruppen. Die beste Chance auf Überwindung von Erwerbsarmut haben Haushalte mit höherem Bildungsgrad und beruflicher Ausbildung oder mit Meister- oder Hochschulabschluss ohne gesundheitliche Einschränkungen (Bruckmeier et al. 2013, S. 5 f.). Zieht ein Kind aus einem solchen Haushalt aus oder wird eine weitere Person erwerbstätig, führt das zumeist auch in ein Leben ohne aufstockendes ALG II.

Haushalte, die besonders lange im Bezug von aufstockendem ALG II bleiben, zeichnen sich typischerweise durch eines oder mehrere der folgenden Merkmale aus (Bruckmeier et al. 2013, S. 5): alleinerziehend, hohes Alter, gesundheitliche Einschränkungen, Leiharbeiter:in, geringfügige Beschäftigung und/oder Job mit einem Verdienst von unter 100 Euro im Monat. Außerdem gilt: Je höher das Erwerbseinkommen, desto schneller gelingt der Ausstieg aus dem Leistungsbezug (Bundesagentur für Arbeit 2010, S. 17).

Wie müssen also Rahmenbedingungen für gute und armutsfeste Arbeit aussehen? Diese umfassen Maßnahmen aus Lohn-, Arbeitsmarkt- und Familienpolitik, die zu einer besseren Arbeitsmarktintegration und zu einem auskömmlichen Einkommen erwerbsarmer Haushalte beitragen. Im Sinne der Bekämpfung von Erwerbsarmut sind dabei zwei Zielsetzungen relevant: erstens die Stärkung der 
bestehenden Erwerbstätigkeit durch Steigerung des bestehenden Erwerbseinkommens, zweitens die Ermöglichung der Ausweitung der Erwerbstätigkeit auf Haushaltsebene, also die Förderung von Doppelverdienerhaushalten und/oder die Umwandlung von Teilzeit- in Vollzeiterwerbstätigkeit. Die wichtigsten Ansatzpunkte hierfür werden im Folgenden skizziert.

\section{Lohnpolitische Ansätze zur Bekämpfung von Erwerbsarmut}

Höhere Löhne im unteren Lohnsegment wirken gezielt gegen Erwerbsarmut. Ein wesentliches Instrument hier ist der gesetzliche Mindestlohn. Eine Kernforderung an den Mindestlohn war immer, dass dieser ein Einkommen sichern soll, das keiner weiteren Aufstockungsleistungen bedarf (Herzog-Stein et al. 2020, S. 12). Seit dem 1.1.2021 liegt der Mindestlohn bei 10,50 Euro pro Stunde.

Berechnungen zeigen, dass ein Stundenlohn zwischen 8,69 und 12,05 Euro ${ }^{11}$ nötig ist, damit kein Anspruch auf Aufstockungsleistungen nach SGB II besteht (Bezugsgröße: Singlehaushalt, Vollzeiterwerbstätigkeit, 40-Stunde-Woche; Herzog-Stein et al. 2020, S. 12). ${ }^{12}$ Demnach liegen aktuell zahlreiche Mindestlohnbezieher:innen selbst bei Vollzeitbeschäftigung immer noch unterhalb der Aufstockungsschwelle.

Eine Erhöhung des Mindestlohns auf 12 Euro wäre in den allermeisten Fällen armutsfest und würde damit zahlreiche Haushalte dazu bringen, finanziell auf eigenen Füßen zu stehen (Schulten/Pusch 2019). Darüber hinaus ist eine angemessene Tarifpolitik, die auskömmliche Löhne und Beschäftigungsbedingungen sichert, ein wichtiger Baustein (Schulten/WSI-Tarifarchiv 2021).

\section{Arbeitsmarktpolitische Ansätze zur Bekämpfung von Erwerbsarmut}

Derzeit sind viele Erwerbsarme prekär beschäftigt, haben einen hohen Anteil an Teilzeitbeschäftigung oder sind Paarhaushalte, in denen nur eine Person er-

11 | Ein eindeutiger Wert lässt sich nicht bestimmen, weil die Aufstockungsschwelle vor allem aufgrund der Kosten der Unterkunft regional stark variiert.

12 | Hier stellt sich die Frage, auf welche Lebenssituation die Forderung nach Mindestlöhnen geeicht werden soll: Welche Haushaltskonstellation soll durch einen Vollzeitlohn armutsfest sein? Diese Frage knüpft an die Diskussionen um einen »Living Wage« an, die hier nur angerissen werden kann: Soll und kann ein Living Wage - egal ob auf gesetzlicher oder tariflicher Ebene - so hoch ausfallen, dass wie zu früheren Zeiten des Alleinverdiener-Arbeitsmarkts auch Kinder vor Einkommensarmut und sozialem Ausschluss bewahrt werden (Familienlohn)? Oder ist dieser Anspruch unrealistisch hoch, weil die meisten Frauen heutzutage erwerbstätig sind und damit das männliche Alleinverdienermodell größtenteils ausgedient hat? (vgl. hierzu WSI 2013 und Schulten/ Müller 2017). 
werbstätig ist - zumeist der Mann. Hier stehen deshalb vor allem zwei Ansatzpunkte im Vordergrund: zum einen Maßnahmen, die einer Prekarisierung von Arbeit entgegenwirken, und zum anderen Maßnahmen, die auf eine Ausweitung von Arbeitszeit zielen. Damit gilt es, Erwerbsarme in sichere (Normal-)Arbeitsverhältnisse zu bringen.

Dies ist vor allem über eine passgenaue (Weiter-)Qualifizierung der Erwerbsarmen zu erreichen. Daher sollten zertifizierte Weiterbildungsmöglichkeiten auch für atypisch Beschäftigte bzw. im Niedriglohnbereich Beschäftigte eröffnet werden. Qualifizierungsmaßnahmen sind auch hilfreich, um eine Ausweitung des Erwerbsumfangs auf Haushaltsebene zu erreichen - vor allem, wenn sie sich an (Ehe-)Frauen richten, die bislang nicht oder allenfalls geringfügig erwerbstätig waren. Durch Qualifikation und Weiterbildung könnte auch die Aufwärtsmobilität von atypisch Beschäftigten unterstützt werden.

Entsprechend wäre auch an die Arbeitsvermittlung die Forderung zu formulieren, Aufstocker:innen und Personen mit geringem Einkommen besser zu unterstützen bzw. dafür zu sorgen, dass Personen gar nicht erst in die Lage geraten, aufstocken zu müssen:

"Kritisch ist, dass die Vermittlung in den Arbeitsmarkt häufig nicht mit einer existenzsichernden Beschäftigung einhergeht, die die Betroffenen in die Lage versetzt, ohne Lohnzuschüsse auszukommen, und sich so Armutsfallen verfestigen.« (Breuer 2019, S. 83)

Im Rahmen der Arbeitsvermittlung gilt es also, auf eine langfristige wirtschaftliche Integration anstelle von kurzfristigen »Erfolgen« umzustellen, also die Vermittlung in niedrig entlohnte und kurzfristige Beschäftigungsmöglichkeiten einzuschränken und stattdessen eine nachhaltige, qualifikationsgerechte und auf das Individuum abgestimmte Vermittlung zu gewährleisten.

Schließlich sind im Kontext der arbeitsmarktpolitischen Maßnahmen auch höhere Freibeträge zu diskutieren. Aus Perspektive der Grundsicherung geht es dabei schlicht um eine Verbesserung der materiellen Lebenslage der Leistungsberechtigten und weniger um das Argument, dass dadurch ein stärkerer "Anreiz« zur Arbeitsaufnahme gesetzt wird. Auch das Argument, Aufstocker:innen hätten sich mit ihrer Situation arrangiert und würden nicht oder nur halbherzig nach einer besser entlohnten Stelle suchen oder sich nicht um eine Ausweitung ihrer Arbeitszeit bemühen (vgl. exemplarisch Bruckmeier et al. 2015, S. 5), sollte nicht als Hindernis für verbesserte Vermittlungsleistungen und Rahmenbedingungen gelten. 


\section{Familienpolitische Ansätze zur Bekämpfung von Erwerbsarmut}

Hier geht es in erster Linie um Maßnahmen, die der besseren Vereinbarkeit von Familie und Beruf dienen, um so Doppelverdienerhaushalte und die Ausweitung der Arbeitszeit zu fördern. Noch immer entscheiden sich Beschäftigte für Teilzeitarbeit oder Minijobs, weil ihnen nur so die Vereinbarkeit von Familie und Beruf möglich erscheint (Absenger et al. 2014, S. 10). Ein wesentlicher Ansatzpunkt ist mithin der Zugang zu verlässlicher, guter und kostengünstiger oder am besten kostenloser ganztägiger Kinderbetreuung.

\section{Bessere Verortung von Erwerbsarmen und Aufstocker:innen in der Arbeitsmarktpolitik}

Die im vorausgegangenen Abschnitt 4 umrissenen Maßnahmen können dazu beitragen, Erwerbsarmut vorzubeugen oder diese zu mildern. Unter dem Stichwort »arbeitsmarktpolitische Ansätze« wurden dabei auch Hinweise auf Vermittlung und Freibetragsregelungen gegeben. Abschließend möchten wir den Blick auf das Problem der rechtlichen Verortung der Aufstocker:innen in der Sozial- und Arbeitsmarktpolitik lenken.

Aufstocker:innen sind nicht die eigentliche Zielgruppe des SGB II, da sie bereits erwerbstätig sind. Die meisten Maßnahmen der Jobcenter richten sich an klassische ALG-II-Empfänger:innen und zielen darauf ab, diese möglichst schnell in den Arbeitsmarkt zu bringen, um so die Hilfebedürftigkeit zu überwinden. Das ALG-II-System ist mithin nicht ausreichend geeignet, Aufstocker:innen nachhaltig aus ihrer prekären Lage zu helfen. Eine andere Verortung im Sozialgesetzbuch könnte zugleich das Problem lösen helfen, dass Leistungen nicht abgerufen werden, da sie mit dem ungeliebten »Hartz-System« in Verbindung gebracht werden.

Wie könnten die Grundzüge eines alternativen, besser passenden Systems für solche Haushalte aussehen? Kurzgefasst folgendermaßen: Die aktive Arbeitsförderung entspricht den Regeln des SGB III, und finanzielle Leistungen werden entsprechend einem (verbesserten) ALG-II-Regelsatz und weiteren Bedarfen gewährt (vgl. hierzu Arbeitskreis Arbeitsmarktpolitik/Knuth 2018, S. 53 f. und den Beitrag von Matthias Knuth in diesem Band). ${ }^{13}$

Im SGB III hat traditionell das Fördern gegenüber dem Fordern einen deutlich höheren Stellenwert. So werden etwa deutlich weniger Jobs für zumutbar

13 | Dabei sollte zudem berücksichtigt werden, dass das SGB II bezüglich der Zielsetzung "gute Arbeit« in Richtung des SGB III weiterentwickelt wird (vgl. Arbeitskreis Arbeitsmarktpolitik/Knuth 2018, S. 46-47). 
erachtet als beim SGB II, und es gibt kein Sanktionsregime wie im SGB II. Die Sanktionen im SGB III beschränken sich auf eine Sperrfrist, wenn die Arbeitsuchendmeldung nicht rechtzeitig erfolgt, nämlich drei Monate vor dem absehbaren Ende einer Beschäftigung.

Nur eine Kombination der Rechtskreise SGB II und III wird der Stellung der Aufstocker:innen gerecht. Die Zielgruppe einer Neuordnung sind nicht nur diejenigen, welche derzeit aufstockendes ALG II empfangen, sondern alle von Erwerbsarmut Betroffene - also alle Haushalte bzw. Bedarfsgemeinschaften, in denen mindestens eine Person erwerbstätig ist und die dennoch von Armut betroffen sind. Dies umfasst auch jene Bedarfsgemeinschaften, die einen Anspruch auf ALG II haben, diesen aber derzeit nicht geltend machen.

Geldleistungen können sich grundsätzlich an den Leistungen für ALG-IIBezieher:innen orientieren - solange sie ein Leben oberhalb der Armutsgrenze sichern. Allerdings muss dazu das Existenzminimum, d.h. die Sozialhilfesätze, armutsfest gemacht werden, also so weit angehoben werden, dass kein Haushalt mehr, der diese Leistungen bezieht, unterhalb der 60-Prozent-Armutsgrenze liegt (siehe hierzu Beitrag von Irene Becker in diesem Band).

Grundlegendes Ziel muss aber sein, Erwerbsarmut zu verhindern. Transferzahlungen sind zur Überbrückung der Armutslage nur der erste Baustein; übergeordnetes Ziel bleibt der Zugang zu Erwerbstätigkeit, und zwar im Falle der Erwerbsarmen zu besserer, auskömmlicherer oder umfangreicherer Erwerbstätigkeit. Entsprechend sind sozial- und arbeitsmarktpolitische Dienstleistungen notwendig, die den Betroffenen helfen, materiell dauerhaft auf eigenen Beinen zu stehen.

Damit muss im Sinne eines erweiterten Verständnisses von Grundsicherung dafür Sorge getragen werden, dass Sozialleistungsbezug vermieden werden kann und gesellschaftliche Teilhabe durch gute Arbeit ermöglicht wird, dass aber zugleich Sozialleistungen - auch die Grundsicherung im engeren Sinne großzügig, unterstützend und leicht zugänglich gestaltet werden.

\section{Literatur}

Absenger, N./Ahlers, A./Bispinck, R./Kleinknecht, A./Klenner, C./Lott, Y./ Pusch, T./Seifert S. (2014): Arbeitszeiten in Deutschland. Entwicklungstendenzen und Herausforderungen für eine moderne Arbeitszeitpolitik, WSIReport Nr. 19, Düsseldorf.

Arbeitskreis Arbeitsmarktpolitik (Hrsg.)/Knuth, M. (2018): Solidarische und sozialinvestive Arbeitsmarktpolitik. Vorschläge des Arbeitskreises Arbeitsmarktpolitik, Study Nr. 374 der Hans-Böckler-Stiftung, Düsseldorf. 
Breuer, C. (2019): Dilemma Hartz IV: Geringverdiener entlasten, in: Wirtschaftsdienst 99(2), S. 82 f.

Bruckmeier, K./Becker, S. (2018): Auswirkung des gesetzlichen Mindestlohns auf die Armutsgefährdung und die Lage von erwerbstätigen Arbeitslosengeld II-Bezieherinnen und -Beziehern. Endbericht. Studie im Auftrag der Mindestlohnkommission, Nürnberg.

Bruckmeier, K./Eggs, J./Sperber, C./Trappmann, M./Walwei, U. (2013): Aufstocker im SGB II. Steinig und lang - der Weg aus dem Leistungsbezug, IAB-Kurzbericht 14/2013, Nürnberg.

Bruckmeier, K./Eggs, J./Sperber, C./Trappmann, M./Walwei, U. (2015): Arbeitsmarktsituation von Aufstockern: Vor allem Minijobber suchen nach einer anderen Arbeit, IAB-Kurzbericht 19/2015, Nürnberg.

Bruckmeier, K./Mühlhan, J./Wiemers, J. (2018): Erwerbstätige im unteren Einkommensbereich stärken. Ansätze zur Reform von Arbeitslosengeld II, Wohngeld und Kinderzuschlag, IAB-Forschungsbericht 9/2018, Nürnberg.

Bundesagentur für Arbeit (2010): Grundsicherung für Arbeitsuchende. Erwerbstätige Arbeitslosengeld II-Bezieher: Begriff, Messung, Struktur und Entwicklung, Nürnberg.

European Commission (2011): Is Working enough to avoid Poverty? In-work Poverty Mechanisms and Policies in the EU, in: European Commission (Hrsg.): Employment and Social Developments in Europe 2011, Luxemburg. Eurostat (2021a): Arbeits-Armutsgefährdungsquote auf Basis des Alters und des Geschlechts - EU-SILC Erhebung (Datencode: ilc_iw01), https://appsso.euro stat.ec.europa.eu/nui/show.do?dataset=ilc_iw01\&lang=de (Abruf am 6.7.2021).

Eurostat (2021b): Armutsgefährdungsschwelle - EU-SILC Erhebung (Datencode: TESSI014), https://ec.europa.eu/eurostat/databrowser/view/tessi014/default/ta ble?lang=de (Abruf am 6.7.2021).

Herzog-Stein, A./Lübker, L./Pusch, T./Schulten, T./Watt, A./Zwiener, R. (2020): Fünf Jahre Mindestlohn - Erfahrungen und Perspektiven. Gemeinsame Stellungnahme von IMK und WSI anlässlich der schriftlichen Anhörung der Mindestlohnkommission 2020, WSI Policy Brief Nr. 42, Düsseldorf.

Goerne, A. (2011): A Comparative Analysis of In-Work Poverty in the European Union, in: Fraser, N./Gutierrez, R./Pena-Casas, R. (Hrsg.): Working Poverty in Europe. A Comparative Approach, Basingstoke.

Kronauer, M. (2020): Bedingungsloses Grundeinkommen oder besser ein Recht auf Arbeit?, in: WSI-Mitteilungen 73(2), S. 143-145.

Nolan, B./Whelan, C./Maître, B. (2010): Low Pay, In-Work Poverty and Economic Vulnerability. A Comparative Analysis Using EU-SILC, Dublin. 
Ponthieux, S. (2010): Assessing and analysing in-work poverty risks, in: Atkinson, T. B./Eric, M. (Hrsg.): Income and living conditions in Europe, Luxemburg.

Schulten, T./Müller, T. (2017): Living wages - normative und ökonomische Gründe für einen angemessenen Mindestlohn, in: WSI-Mitteilungen 50(7), S. 507-514. Schulten, T./Pusch, T. (2019): Auswirkungen und Perspektiven eines Mindestlohns von 12 Euro, in: Wirtschaftsdienst 99(5), S. 335-339.

Schulten, T./WSI-Tarifarchiv (2021): Tarifpolitischer Jahresbericht 2020: Tarifpolitik unter den Bedingungen der Corona-Pandemie, in: WSI-Mitteilungen 74(2), S. 140-150.

Schröter, A. (2012): Zur Bedürftigkeit von Aufstocker-Familien. Wie das traditionelle Familienmodell und ambivalente sozialstaatliche Instrumente zur Falle werden können, Schriftenreihe Institut Arbeit und Wirtschaft, Nr. 13/2012, Bremen.

Sozialpolitik-aktuell (2021a): Erwerbstätige ALG II-Empfänger*innen 2007-2020, www.sozialpolitik-aktuell.de/files/sozialpolitik-aktuell/_Politikfelder/Arbeits markt/Datensammlung/PDF-Dateien/abbIV81b.pdf (Abruf am 6.7.2021).

Sozialpolitik-aktuell (2021b): Registrierte Arbeitslose und Stille Reserve 2000-2020, www.sozialpolitik-aktuell.de/files/sozialpolitik-aktuell/_Politikfelder/Arbeits markt/Datensammlung/PDF-Dateien/abbIV34.pdf (Abruf am 6.7.2021).

Spannagel, D. (2013): In-work Poverty in Europe - Extent, Structure and Causal Mechanisms, Oldenburg, www.cope-research.eu/wp-content/uploads/2013/ 03/In-work_Poverty_in_Europe.pdf (Abruf am 6.7.2021).

Spannagel, D. (2017): Menschen mit niedrigem sozioökonomischen Status - Armut und Teilhabe, in: Diehl, E. (Hrsg.): Teilhabe für alle?! Lebensrealitäten zwischen Diskriminierung und Partizipation, Bonn, S. 77-102.

Spannagel, D./Seikel, D./Schulze Buschoff, K./Baumann, H. (2017): Aktivierungspolitik und Erwerbsarmut, WSI-Report Nr. 36, Düsseldorf.

Statistik der Bundesagentur für Arbeit (2021): Strukturen der Grundsicherung SGB II (Zeitreihe Monats- und Jahreszahlen ab 2005), Berichtsmonat Februar 2021, erstellt am 1.6.2021, Nürnberg.

WSI = Wirtschafts- und Sozialwissenschaftliches Institut der Hans-Böckler-Stiftung (Hrsg.) (2013): Vom Ernährerlohn zum Familieneinkommen? Schwerpunktheft der WSI-Mitteilungen 3/2013, Düsseldorf. 



\section{Teil III}

\section{Zum Konzept einer erweiterten Grundsicherung}





\section{Digitale Daseinsvorsorge}

Voraussetzung für soziale und kulturelle Teilhabe im 21. Jahrhundert

Tanja Klenk

\section{Einleitung}

Arbeiten von zu Hause, sich mit Vorgesetzen und Kolleg:innen via Online-Chat und Videotelefonie koordinieren, Mathe- und Deutschaufgaben aus der digitalen Lernplattform herunterladen und sie im virtuellen Klassenzimmer von den Lehrer:innen erklärt bekommen, Arztbesuch per Videosprechstunde, der Diskussion der gestreamten Gemeinderatssitzung zuhören und Beschlüsse bei der virtuellen Mitgliederversammlung des Sportvereins fassen: Die Coronapandemie, die die Welt seit Januar 2020 in Atem hält, hat für viele - ganz unabhängig von der konkreten Betroffenheit durch die Viruserkrankung - das alltägliche Leben einschneidend verändert.

Arbeit, Familie, Freizeit, politisches und ehrenamtliches Engagement - nahezu alle Lebensbereiche haben einen Digitalisierungsschub erhalten. In Deutschland, das im internationalen Vergleich Anfang 2020 nur wenig digitalisiert war und wo es nach wie vor große Ungleichheiten zwischen urbanen und ländlichen Bereichen beim Zugang zu digitaler Infrastruktur gibt, war dieser Wandel besonders deutlich zu spüren.

Wäre der vorliegende Band zur Grundsicherung in Deutschland im Jahr 2019 erschienen, hätten vermutlich viele Leser:innen einen Beitrag über die digitale Daseinsvorsorge als fehl am Platze, zumindest jedoch als irritierend wahrgenommen. Der Begriff »Grundsicherung « löst oft Assoziationen an finanzielle Transfers aus und ist nicht mit der Vorstellung von Infrastrukturleistungen verbunden. Zudem wurden eine digitale Infrastruktur und digitale Leistungen bis vor Kurzem immer noch als Add-on betrachtet, als ein Zusatzangebot, das nicht zwingend für die gleichberechtigte Teilhabe am gesellschaftlichen, wirtschaftlichen und kulturellen Leben erforderlich ist. 
Nach den Krisenerfahrungen der Pandemie dürfte sich diese Haltung bei vielen verändert haben. Auch wenn wir gegenwärtig noch nicht wissen, welche Aktivitäten sich nach dem Ende der Pandemie wieder in den physischen und analogen Bereich zurückverlagern, so besteht doch bereits jetzt Einigkeit darüber, dass sich durch die Digitalisierungserfahrungen während der Coronazeit vieles verändert hat.

In diesem Sammelband - der sich das Ziel gesetzt hat, die Grundsicherung weiterzudenken - über digitale Daseinsvorsorge zu sprechen ist auch deshalb notwendig, weil der Zugang zu digitaler Infrastruktur in Deutschland mit großen Ungleichheiten verbunden ist. Die sozialen Spaltungslinien verlaufen auch hier zwischen Arm und Reich, noch viel mehr aber zwischen urbanen Ballungszentren und dünn besiedelten strukturschwachen Regionen.

Das zunehmende Auseinanderdriften von Umfang und Qualität der Daseinsvorsorge zwischen städtischen und ländlichen Gebieten wird insbesondere vor dem Hintergrund eines zunehmenden Erfolgs rechtspopulistischer Parteien kritisch diskutiert. Wenngleich der Erfolg solcher Parteien nicht monokausal erklärt werden kann, so legt die Forschung zur Geografie der politischen Unzufriedenheit in Deutschland doch nahe, dass eine weniger intensive Versorgung mit öffentlichen Gütern insbesondere im ländlichen Raum mit der Wahrnehmung des »Abgehängtseins" und einer schleichenden Entfremdung der Bevölkerung von etablierten Parteien einhergehen kann (Deppisch 2020; Diermeier 2020).

Kurzum: Es gibt genügend sozial- und gesellschaftspolitische Anlässe, die Grundsicherung auch in Richtung digitaler Daseinsvorsorge weiterzudenken. Vor diesem Hintergrund klärt der folgende Beitrag zunächst den Begriff der Daseinsvorsorge und fragt, was Gegenstand einer digitalen Daseinsvorsorge sein soll und wie diese organisiert werden kann (Abschnitt 2). Im Anschluss daran wird nach dem Stand und den Perspektiven der digitalen Daseinsvorsorge gefragt (Abschnitt 3). Besondere Aufmerksamkeit gilt dabei der Frage, wie sich die Debatte über ein Recht auf digitale Daseinsvorsorge gegenwärtig gestaltet.

\section{Daseinsvorsorge als Voraussetzung für gesellschaftliche Teilhabe}

\subsection{Daseinsvorsorge - ein dynamischer und veränderungsoffener Begriff}

Daseinsvorsorge wird als die Pflicht des Staates verstanden, eine angemessene, d.h. den jeweiligen zeitlichen, örtlichen, wirtschaftlichen und technischen Gegebenheiten angepasste Befriedigung der Bedürfnisse der Bürger:innen zu garantieren (Luch/Schulz 2011, S. 105). Der Begriff zählt zu den prägenden Be- 
griffen des Verwaltungsrechts, ohne jedoch in rechtlicher Hinsicht abschließend definiert zu sein. Zumeist wird nur in einer offenen Aufzählung umschrieben, was als Bestandteil einer solchen angemessenen Bedürfnisbefriedigung verstanden wird: Versorgung mit Energie und Wasser, Entsorgung von Abwasser und Abfall, ein öffentlicher Personennahverkehr, Post- und Telekommunikationsdienstleistungen, ein Schul- und Bildungssystem.

Der Vergleich der verschiedenen Aufzählungen zeigt, dass zwischen einem weiten und einem engen Verständnis der Daseinsvorsorge unterschieden werden kann: Während Daseinsvorsorge für die einen vor allem in der Gewährleistung von netzgebundener Infrastruktur besteht (Energie, Wasser, Verkehr; vgl. z. B. Leisner 2011), haben andere ein umfassendes Verständnis von Bedürfnisbefriedigung, das beispielsweise auch öffentliche Bibliotheken und kulturelle Einrichtungen umfasst (Luch/Schulz 2011; Schulz 2019).

Daseinsvorsorge ist damit nicht nur ein rechtlicher, sondern vor allem ein politischer Begriff, dessen Bedeutungsgehalt immer wieder neu in politischen Kontexten auszuhandeln und zu definieren ist. Wie dynamisch und wandlungsfähig der Begriff ist, zeigt auch ein Blick in die Geschichte. Der Begriff Daseinsvorsorge wird insbesondere auf Ernst Forsthoff (1938) zurückgeführt, der ihn in den 1920er Jahren als Neologismus in das Verwaltungsrecht einbrachte.

Die wirtschaftlichen und sozialen Transformationen seiner Zeit vor Augen, konstatiert Forsthoff einen Wandel der Aufgaben des Staates. Aufgrund von Industrialisierung und zunehmender Urbanisierung wird der bzw. die Einzelne von notwendigen Lebensgütern getrennt. Grundbedürfnisse wie Essen, Zugang zu sauberem Wasser und Wohnen können in städtischen Kontexten nicht mehr vom Individuum und auch nicht im Familienverbund organisiert werden. Die Industrialisierung und Urbanisierung macht den Menschen sozial bedürftig, und aus der Verletzbarkeit und Abhängigkeit des bzw. der Einzelnen in modernen Massengesellschaften resultiert für Forsthoff die Verantwortung - aber auch die Legitimation! - des Staates, Daseinsvorsorge für den Einzelnen bzw. die Einzelne zu leisten.

Das Begriffspaar Eingriffs- und Leistungsverwaltung, das ebenfalls von Forsthoff geprägt wurde, bringt den Wandel der Staatsaufgaben zum Ausdruck. Neben die Verwaltung als Herrschaftsapparat, die den Bürger:innen als befehlende, nehmende und ordnungsstiftende Institution gegenübertritt, tritt eine Verwaltung, die Bürger:innen Dienste und Leistungen zur Verfügung stellt (Gröttrup 1984, S. 94).

Forsthoffs Buch »Die Verwaltung als Leistungsträger« erschien 1938 und ist, wie er in späteren Arbeiten selbstkritisch feststellt, "auf die Situation des Jahres 1938 hin formuliert« (Forsthoff 1959). Forsthoff distanziert sich in seinem frühen 
Werk nicht vom nationalsozialistischen Denken des totalitären Staats, in dem die Unterscheidung zwischen Gesellschaft und Staat aufgegeben wird und die Bürger:innen den politischen Zielen des Staates unterworfen sind, im Gegenteil: Die Daseinsvorsorge als staatliche Aktivität ist für Forsthoff Mittel zur Inklusion des bzw. der Einzelnen in den totalitären Aufbau der Gesellschaft. Sie erhält damit einen sozialdisziplinierenden Charakter und dient nicht der Wiederherstellung verloren gegangener Autonomie (Kersten 2005, S. 547).

Forsthoff distanzierte sich jedoch bereits in der zweiten Hälfte der 1930erJahre von der nationalsozialistischen Ideologie. Er bleibt allerdings auch nach dem Ende der NS-Diktatur und der Gründung der Bundesrepublik Deutschland ein Konservativer, der der Realität der Massengesellschaft kulturpessimistisch gegenüberstand. Akademisch wurde Forsthoff trotz seiner anfänglich fehlenden Distanzierung von der nationalsozialistischen Ideologie in der Nachkriegszeit rehabilitiert, und sein Denken prägt die (verwaltungs-)rechtlichen Debatten der 1950er- und 1960er-Jahre. Seine Arbeit zeigt auch in der freiheitlichen Ordnung der Bundesrepublik Wirkung: Daseinsvorsorge setzt sich als Begriff durch und findet breite Verwendung.

Auch wenn die Daseinsvorsorge nicht explizit als Prinzip im Grundgesetz verankert ist, so lässt sich die staatliche Verantwortung für die Gewährleistung von Leistungen der Daseinsvorsorge implizit aus den Grundrechten und dem Sozialstaatsgebot ableiten. Damit die Bürger:innen von ihren Freiheiten Gebrauch machen können, muss zunächst der Staat tätig werden, denn die »Freiheit durch den Staat" (Isensee 2006, Rn. 14) manifestiert sich nicht nur in der Abwesenheit materieller Not, sondern soll für den Einzelnen bzw. die Einzelne eine effektive Teilhabe an der Gesellschaft gewährleisten (Kingreen 2002, S. 99). Eine solche effektive gesellschaftliche Teilhabe wiederum kann nicht nur durch Finanztransfers gewährleistet werden, sondern verlangt auch und gerade die Infrastrukturleistungen der Daseinsvorsorge.

Schon früh lässt sich dieser Gedanke in der Rechtsprechung des Bundesverfassungsgerichts wiederfinden. So hat das Gericht bereits 1959 im Kontext eines Beschlusses über die Ausgestaltung des sozialrechtlichen Verfahrens das »Recht der Daseinsvorsorge [...] zu den Fundamenten unserer sozialen Ordnung « gezählt (Bundesverfassungsgericht 1959). Diese Einschätzung wird auch in späteren Jahren bestätigt, z. B. im »Garzweiler-Urteil«, das sich im Kontext der Energieversorgung mit Fragen der Enteignung für gemeinwohlorientierte Zwecke befasst (Bundesverfassungsgericht 2013).

Der Begriff der Daseinsvorsorge hat auch Eingang in die Gesetzgebung gefunden: Das Raumordnungsgesetz benennt in $\$ 2(2)$ die Sicherung einer nachhaltigen Daseinsvorsorge als gesetzliche Aufgabe. Der Landtag Brandenburg 
wiederum hat 2011 ein »Gesetz zur Stärkung der kommunalen Daseinsvorsorge« beschlossen, das vor allem die Verbesserung der Stellung von kommunalen Anbietern zum Ziel hat. In Bayern schließlich wird der öffentliche Personennahverkehr als Aufgabe der Daseinsvorsorge verstanden (Art. 2,1 BayÖPNVG).

In allen genannten Beispielen bleibt die Daseinsvorsorge jedoch ein unbestimmter Rechtsbegriff, der allenfalls umschrieben, jedoch nicht eindeutig definiert wird. Die begriffliche Offenheitsoll hier jedoch nicht als grundsätzliches Problem verstanden werden (Dümke 2015, S. 46), im Gegenteil: In einer sozial, wirtschaftlich und kulturell dynamischen Umwelt sind die Versorgungsbedürfnisse der Bürger:innen nicht konstant, sondern verändern sich. Die Unbestimmtheit von Daseinsvorsorge als Rechtsbegriff bietet die Möglichkeit, den Begriff immer wieder neu auf den Prüfstand zu stellen und hinsichtlich seines Gehalts zu prüfen.

\subsection{Daseinsvorsorge 4.0: Konturen der Daseinsvorsorge im 21. Jahrhundert}

Wenn nun aber die Daseinsvorsorge nach Forsthoff (1938) alle für ein sinnvolles menschliches Dasein notwendigen oder nützlichen Leistungen und Güter umfasst und wenn hierzu in den 20er-Jahren des 21. Jahrhunderts auch digitale Leistungen und Güter gehören - was bedeutet dies dann konkret? Ebenso wie der Daseinsvorsorgebegriff generell ist auch der Begriff der »digitalen Daseinsvorsorge" nicht eindeutig definiert (Schallbruch 2020, S. 157). Einigkeit besteht darüber, dass die digitale Daseinsvorsorge drei Gestaltungsfelder umfasst: die physische Infrastruktur, neue Dienstleistungen, die auf der digitalen Infrastruktur aufbauen, und digitale Inhalte (Schulz 2019, S. 4).

Was die physische Infrastruktur betrifft, so setzt die Bereitstellung von schnellem Internet - nach dem gegenwärtigen Stand der Technologie - den Ausbau von Glasfasernetzen voraus. Darüber hinaus sind Kabel- und Mobilfunknetze, die bislang als unterschiedliche Übertragungswege getrennt behandelt wurden, künftig integriert zu betrachten und zu einem einheitlichen Netz auszubauen (Ilgmann/Störr 2020).

Neue Dienste, die Städte und Kommunen als Teil einer digitalen Daseinsvorsorge anbieten könnten, wären beispielsweise gänzlich neu gedachte ÖPNV-Modelle, bei denen unterschiedliche öffentliche Mobilitätsangebote (Bahn, Bus, Leihräder, E-Bikes und Ridepooling-Dienste) gebündelt und integriert über ein digitales Portal angeboten werden, ergänzt um Services wie Fahrplanauskunft, Ticket- oder Parkscheinkauf etc. Die Digitalisierung der kommunalen Verkehrssysteme könnte einen entscheidenden Beitrag liefern, um Mobilität auch in ländlichen Regionen weniger abhängig vom Vorhandenseins eines individuellen PKW zu machen. 
Auch im Bereich Energieversorgung, dem zweiten wichtigen Handlungsfeld der kommunalen Daseinsvorsorge, ermöglicht die Digitalisierung neue Dienste und Leistungen. Die Einsatzmöglichkeiten reichen hier von Maßnahmen zur Verbesserung der Energieeffizienz und Netzstabilität, die ihrerseits wiederum Voraussetzung für die Mobilitätswende in Richtung Elektromobilität sind, über die vorausschauende Instandhaltung von Netzen und Erzeugungsanlagen durch digital ausgewertete Daten zum Zustand von Materialressourcen, Reparaturund Wartungsarbeiten (predictive maintenance) bis hin zu digitalen Modellen der Kundenbetreuung, die gerade vor dem Hintergrund der räumlichen Distanzen im ländlichen Raum sinnvoll erscheinen (Roth 2018).

Weitere Handlungsfelder im Bereich der sozialen Daseinsvorsorge sind denkbar. Zum Beispiel kann die Digitalisierung helfen, dem Problem des Landarztmangels zu begegnen und durch telemedizinische Angebote und eine bessere Vernetzung des ambulanten und des Pflegesektors die Gesundheitsversorgung in ländlichen Regionen weiterzuentwickeln.

Schließlich kann digitale Daseinsvorsorge auch die Bildungs- und Arbeitsbedingungen der lokalen Bevölkerung verbessern, indem freies WLAN im Stadtkern, Co-Working-Spaces mit digitalem Anschluss und digitale Multifunktionshäuser geschaffen werden, die verschiedene Angebote der Daseinsvorsorge bündeln (z. B. Bildung und Weiterbildung, Kita- und sonstige Betreuung, Beratung, Mütter- und Jugendzentren und Dorfgemeinschaftsräume). Solche öffentlichen Räume mit digitalem Anschluss werden insbesondere für den ländlichen Raum als Möglichkeit gesehen, die Chancen der Digitalisierung möglichst vielen Bürger:innen zukommen zu lassen und sozial-, wirtschafts- und strukturpolitische sowie umweltpolitische Ziele gleichermaßen zu realisieren.

Öffentliche Räume mit Digitalanschluss sind kein Ersatz für den individuellen Zugang zum Netz, aber sie können helfen, gegenwärtig vorhandene Engpässe zu überwinden und Homeoffice auch in Gegenden zu praktizieren, in denen die Versorgung mit schnellem Internet immer noch prekär ist. Sie können dazu beitragen, die Zahl der Pendler:innen zu reduzieren, die Vereinbarkeit von Familie und Beruf zu erleichtern und abgelegene Gegenden zu revitalisieren.

Durch freies WLAN und digitale Multifunktionshäuser kann die einkommensbedingte Kluft zwischen On- und Offlinern überbrückt und beispielsweise auch Kindern aus einkommensschwächeren Schichten der Zugang zum digitalen Leben ermöglicht werden. Einen Nukleus für solche Multifunktionshäuser können öffentliche Bibliotheken bilden, die dann nicht mehr nur Bücher ausleihen, sondern zu Orten der Begegnung, Zusammenarbeit und Kommunikation werden (Thiele 2020). 
Was die Inhalte der digitalen Daseinsvorsorge betrifft, so wird unter anderem über das Streaming der digitalen Ratsarbeit und von Veranstaltungen, die für das Gemeinwesen von Relevanz sind, nachgedacht (z. B. Mitgliederversammlungen der örtlichen Vereine und Verbände). Darüber beinhaltet die Diskussion über die Inhalte der digitalen Daseinsvorsorge insbesondere Open Data, d.h. (tendenziell) unentgeltlich bereitgestellte Daten der öffentlichen Verwaltungen, die beliebig weiterverarbeitet werden können. Open Data werden sowohl von zivilgesellschaftlichen Organisationen als auch von Internetunternehmen gefordert, die sich davon ein Mehr an Transparenz und Partizipation, neue bürgerrelevante digitale Verwaltungsdienste, aber auch neue Geschäftsmodelle versprechen (Kubicek/Jarke 2019).

Mit der ersten Änderung des E-Government-Gesetzes im Juli 2017 wurden für die Bereitstellung von Open Public Data wichtige Weichen gestellt: Bundesbehörden haben nun die Aufgabe, ihre Daten als Open Data zu veröffentlichen (vgl. \$12a EGovG); ein beim Bundesverwaltungsamt neu eingerichtetes "Kompetenzzentrum Open Data (CCOP) unterstützt sie dabei. Allerdings betreffen diese Vorgaben nur Behörden der unmittelbaren Bundesverwaltung. Auf kommunaler Ebene - wo Daseinsvorsorge faktisch stattfindet - ist der Umsetzungsstand noch sehr gering: Im Juni 2020 haben von den ca. 11.000 deutschen Kommunen nur etwa 90 Open-Data-Portale zur Verfügung gestellt (Schweigel et al. 2020).

\section{Stand und Perspektiven der digitalen Daseinsvorsorge}

\subsection{Status quo - von löchrigen Netzen und weißen Flecken}

Dass gesellschaftliche Teilhabe im 21. Jahrhundert Zugang zu digitaler Infrastruktur und ein Angebot an digital erbrachten Leistungen voraussetzt, dürfte - insbesondere nach den Erfahrungen der Coronapandemie - unstrittig sein. Allerdings klaffen gesellschaftlicher Anspruch und Realität weit auseinander. Gemäß dem Index für die digitale Wirtschaft und Gesellschaft der EU (DESI; vgl. European Commission 2019) stand Deutschland 2019, im Jahr vor der Coronapandemie, auf Platz 20 von 28 Ländern, was den Bereich der Digitalisierung der öffentlichen Verwaltung angeht. Im Bereich der Konnektivität schneidet Deutschland mit Platz 12 etwas besser ab - wobei diese mittlere Position auch erst in den vorangehenden Jahren erarbeitet wurde.

Eine vom Deutschen Städte- und Gemeindebund und dem Branchenverband Bitkom gemeinsam durchgeführte Umfrage aus dem Herbst 2020 zeigt, 
dass trotz - oder gerade wegen - der Coronapandemie Digitalisierung eines der drängendsten Themen der Städte und Gemeinden bleibt und sich 61 Prozent der 623 befragten Kommunen als Nachzügler im Bereich der Digitalisierung begreifen (Landsberg/Rohleder 2020).

Über die Gründe für die im internationalen Vergleich schleppende Digitalisierung von Staat und Verwaltung wurde in jüngster Zeit viel diskutiert (Lühr 2020). Als einer der hemmenden Faktoren gilt dabei die Governance und Organisation von Digitalisierungsprojekten im öffentlichen Sektor. So hat die stark vertikal über die verschiedenen Politikebenen und horizontal über Politikfelder segmentierte Verwaltungsstruktur im deutschen Exekutivföderalismus bislang die Digitalisierung von Staat und öffentlicher Verwaltung eher gebremst denn vorangetrieben (Kuhlmann/Kühn/Danken 2020, S. 131). Fehlende zentrale Koordination, unterschiedliche Innovationsdynamiken und ungleiche Ressourcenausstattung haben den digital divide zwischen wirtschaftlich prosperierenden und strukturschwachen Bundesländern bzw. zwischen urbanen Ballungszentren und ländlichen Regionen weiter verschärft und Insellösungen mit Interoperabilitätsproblemen erzeugt.

Dass Aspekte der Governance und der Organisation rückständige und gleiche Entwicklungen maßgeblich erklären können, gilt auch und gerade für den Ausbau der digitalen Daseinsvorsorge (Ilgmann/Störr 2020, S. 617; Kersting/ Graubner 2020, S. 233). Galt bis weit in die 1980er-Jahre hinein der Bau und Betrieb von leitungsgebundenen Infrastrukturen in Deutschland als eine staatliche Kernaufgabe, so änderte sich dies im Verlauf der 1990er-Jahre grundlegend. Vor dem Hintergrund einer öffentlichen Debatte, die immer stärker die Grenzen staatlicher Ressourcen betonte, vollzog sich ein doppelter Paradigmenwechsel, der bis heute die Strukturen der (digitalen) Daseinsvorsorge nachhaltig prägt.

Zum einen wurde die Aufgabenteilung zwischen öffentlichen und privaten Akteuren neu gestaltet: In einer Privatisierungswelle wurden im Verlauf der 1990erJahre zahlreiche öffentliche Aufgabenfelder an private Akteure überantwortet, so auch der Telekommunikationssektor. Die Auflösung des Post- und Telekommunikationsministeriums fiel jedoch 1997 zeitlich in eine Phase, in der auch wichtige Grundsatzentscheidungen über den Ausbau der digitalen Infrastruktur zu treffen waren, etwa Investitionen in Glasfaser- und Mobilfunknetze, für die nun aber private Akteure verantwortlich waren (Kersting/Graubner 2020, S. 235).

Zwar weist der im Zug der Post- und Telekommunikationsprivatisierung 1992 neu geschaffene Artikel 87f des Grundgesetzes dem Bund eindeutig eine Gewährleistungsverantwortung für Telekommunikationsleistungen zu, in den vergangenen drei Dekaden wurde diese allerdings sehr restriktiv ausgelegt. So 
gibt es in Deutschland heute zwar einen individuellen Rechtsanspruch auf einen Telekommunikationsanschluss, ein Zugang zum Glasfasernetz für schnelles Internet ist in der Grundversorgung jedoch nicht enthalten (Hofmann 2019; Ilgmann/Störr 2020). Der Ausbau des Glasfasernetzes ist - ebenso wie das Mobilfunknetz - wettbewerblich organisiert; den Zugang der Bürger:innen zum schnellen Internet soll also der Markt richten.

Ein zweiter Paradigmenwechsel, der sich im Laufe der 1990er-Jahre vollzog, betriff das grundgesetzlich verankerte Gleichheitsversprechen (Hofmann 2019): Statt »einheitlicher Lebensverhältnisse" - jahrzehntelang das übergeordnete Prinzip der Infrastrukturpolitik - wurden ab 1994 nur noch "gleichwertige Lebensverhältnisse« angestrebt. Der doppelte Paradigmenwechsel hatte zur Folge, dass Infrastruktur vor allem dort ausgebaut wurde, wo es für private Akteure ökonomisch rational erschien.

So kommt es, dass in Ballungszentren oftmals Doppelstrukturen aufgebaut wurden und werden: Unternehmen setzen auf eigene Übertragungswege, um sich strategische Wettbewerbsvorsteile zu sichern, während die Erschließung von dünn besiedelten Gebieten der Gewährleistungsverantwortung des Staates anheimfällt (Ilgmann/Störr 2020, S. 617). So muss auch das Bundesverkehrsministerium in seinem Breitbandatlas konstatieren, dass »bei der Breitbandverfügbarkeit [...] sich weiterhin ein Stadt-Land-Gefälle erkennen« lässt (Bundesministerium für Verkehr und digitale Infrastruktur 2020, S. 6).

Die Verfügbarkeit von Gigabitanschlüssen ist mit 74,6 Prozent in städtischen Gebieten besonders hoch; in ländlichen Regionen lag sie Mitte 2020 bei 16,7 Prozent der Haushalte (Bundesministerium für Verkehr und digitale Infrastruktur 2020, S. 56). Ein ähnliches Bild zeigt sich beim Mobilfunkausbau, wie sich aus der Funklochkarte der Bundesnetzagentur (o.J.) erkennen lässt.

\subsection{Perspektiven: Auf dem Weg zur Daseinsvorsorge 4.0?}

Schon im Koalitionsvertrag von 2013 (!) wurde die Sorge über ein zunehmendes Auseinanderdriften von smarten Städten und digital abgehängten ländlichen Gegenden geäußert, dem man durch eine detaillierte Breitbandstrategie entgegenwirken wollte (CDU/CSU/SPD 2013, S. 34-36). Auch im Koalitionsvertrag von 2018 nahm die digitale Daseinsvorsorge einen wichtigen Stellenwert ein. So setzte sich die schwarz-rote Koalitionsregierung das Ziel, im Jahr 2025 in der Gigabitgesellschaft angekommen zu sein, in der ein flächendeckendes Glasfasernetz möglichst direkt bis zu jedem Haus besteht, bestehende Funklöcher beseitigt sind und es freies WLAN in allen öffentlichen Einrichtungen, Zügen der Deutschen Bahn und Bahnhöfen gibt (CDU/CSU/SPD 2018, S. 12). 
In keiner der beiden Regierungsperioden wurden jedoch die Privatisierungsentscheidungen der 1990er-Jahre kritisch überdacht und neue Governance-Modelle entwickelt; stattdessen wurde am Grundprinzip der wettbewerblichen Organisation der digitalen Daseinsvorsorge festgehalten. Maßnahmen, die zu deren Förderung bereitgestellt wurden, wurden in die wettbewerbliche Logik eingefügt. So werden Fördermittel des Bundes wie etwa aus dem Breitbandförderprogramm über den Weg der wettbewerblichen Ausschreibung auf Ebene der Kommunen organisiert und als Zuwendungen an Unternehmen vergeben.

Die Transaktionskosten dieser Vorgehensweise sind hoch; zudem überfordert diese Vorgehensweise regelmäßig die Kommunen, denen es an Personal und fachlichem bzw. technischem Know-how fehlt, um das Ausschreibungsverfahren adäquat zu organisieren. Berichte über nicht abgerufene Fördermittel sind daher regelmäßig in den öffentlichen Medien zu lesen.

Die zum Zeitpunkt des Verfassens dieses Beitrags jüngste Regierungsaktivität in Sachen digitale Daseinsvorsorge ist die Verabschiedung eines Referentenentwurfs des Bundesministeriums für Wirtschaft und Energie und des Bundesministeriums für Verkehr und digitale Infrastruktur für ein Telekommunikationsmodernisierungsgesetz. Buchstäblich in letzter Sekunde wurde damit dem Europäischen Kodex für die elektronische Kommunikation (Europäische Union 2018) entsprochen, der am 20. Dezember 2018 in Kraft getreten ist und bis Ende 2020 in nationales Recht zu übersetzen war. Der Gesetzentwurf sieht unter anderem die bereits im Koalitionsvertrag 2018 angekündigte Gründung einer Mobilfunkinfrastrukturgesellschaft vor, die vor allem bei der Suche nach neuen Standorten für Mobilfunkmasten unterstützen und für die Verteilung der Fördermittel verantwortlich sein soll.

Die Idee der Gründung einer Mobilfunkinfrastrukturgesellschaft stößt aber auf Kritik beim Bundesrechnungshof, den Oppositionsparteien und selbst beim Koalitionspartner SPD, weil die geplante Gesellschaft die Ursache des Problems nicht löst, nämlich die wettbewerbliche Organisation des Netzausbaus und fehlende Anreize für die Erschließung dünn besiedelter Gegenden. Zudem überschneiden sich ihre Aufgabenbereiche mit anderen Gesellschaften des Bundes, z. B. dem Gigabitbüro, der Autobahn GmbH und der Deutschen Bahn AG, was hinsichtlich der Effizienz der Aufgabenerfüllung als fragwürdig erscheint (Bundesrechnungshof 2020).

Wie sehen also politische Gestaltungsalternativen aus, um der Problematik der Parallelität von zeitgleicher Über- und Unterversorgung zu begegnen? Im politischen und wissenschaftlichen Diskurs werden unter anderem drei Handlungsmöglichkeiten genannt, die im Folgenden skizziert werden. 


\section{Märkte der digitalen Daseinsvorsorge besser regulieren}

Grundsätzlich können Leistungen der digitalen Daseinsvorsorge sowohl von öffentlichen, privat-gemeinnützigen oder privat-gewinnorientierten Akteuren erbracht werden, sofern Finanzierungsfragen und Zugangsrechte so gestaltet sind, dass alle Bürger:innen die Dienste und Leistungen tatsächlich nutzen können. Eine marktliche Erbringung von Gütern und Diensten der Daseinsvorsorge muss also entsprechenden reguliert werden.

Dass ein gleicher Zugang zur technischen Infrastruktur der digitalen Daseinsvorsorge (Glasfaser- und Mobilfunknetz) gegenwärtig nicht gegeben ist, liegt auch daran, dass Mechanismen zur Marktregulation nicht genutzt bzw. nicht effektiv durchgesetzt werden. Um ein Beispiel zu nennen: Um Unterversorgung zu vermeiden (und keine Überversorgung entstehen zu lassen), könnten Mobilfunkanbieter beispielsweise zum Roaming verpflichtet werden, also zur Möglichkeit für Mobilfunkteilnehmer:innen, auf ein anderes Netz ausweichen, wenn das Heimnetz nicht zur Verfügung steht. Bei der Versteigerung der Frequenzen für den Mobilfunkstandard $5 \mathrm{G}$ wurde es aber versäumt, eine verpflichtende Roaming-Regelung als Auflage durchzusetzen. Auch das 2021 verabschiedete Telekommunikationsmodernisierungsgesetz enthält Roaming nur als schwache Kann-Regelung ( $\$ 106$ Telekommunikationsmodernisierungsgesetz).

\section{Recht auf digitale Daseinsvorsorge stärken}

Die regionalen Disparitäten beim Zugang zur digitalen Infrastruktur liegen zudem auch darin begründet, dass es bislang kein umfassendes Recht auf schnelles Internet gibt. Mit dem Europäischen Kodex für die elektronische Kommunikation wurde der Breitband-Internetzugang zwar 2018 in die Universaldienstverpflichtung aufgenommen. Die EU-Richtlinie ist positiv zu bewerten, weil sie das individuelle Recht auf digitale Daseinsvorsorge stärkt; zugleich greift der Kodex aber zu kurz und bietet nur eine Teillösung, weil er nur eine stationäre und keine mobile Breitbandversorgung umfasst.

So können Bürger:innen im ländlichen Raum zwar darauf hoffen, dass sie in ihrer Wohnung künftig auf schnelles Internet zugreifen können; sobald sie die Wohnung jedoch verlassen, stehen sie mit hoher Wahrscheinlichkeit wieder im Funkloch. Damit verschafft die Umsetzung des Europäischen Kodex Linderung, aber keine Heilung der diagnostizierten Ungleichheiten in der digitalen Daseinsvorsorge. Der Entwurf für ein Telekommunikationsmodernisierungsgesetz wiederum begnügt sich mit den europäischen Mindestvorgaben, kann damit aber das im Koalitionsvertrag 2018 gegebene Versprechen, der Gigabitgesellschaft einen größeren Schritt näherzukommen, nicht einlösen. 


\section{Öffentlicher statt privater Ausbau der technischen Infrastruktur}

Schließlich wird als Alternative zum kompetitiv organisierten Netzausbau die Errichtung der physischen Infrastruktur durch eine öffentliche Organisation diskutiert, die vom Bund bereitgestellte Fördermittel erhält.

Während die ersten beiden Handlungsalternativen die bestehende Governance des Netzausbaus inkrementell weiterentwickeln würden, kommt diese dritte Reformoption einem erneuten Paradigmenwechsel gleich und stellt die vor gut dreißig Jahren getroffenen Privatisierungsentscheidungen infrage. Der Netzausbau und -betrieb würde wieder in die Verantwortung der öffentlichen Hand fallen, die Inhalte und Dienste hingegen könnten weiterhin wettbewerblich angeboten werden.

Es ist umstritten, ob eine solches Modell im Einklang mit den wettbewerbsrechtlichen Vorgaben der Europäischen Union stünde (Ilgmann/Störr 2020, S. 620). Zudem müsste durch eine agile und responsive Organisationsgestaltung sichergestellt werden, dass der Netzausbau und -betrieb für Innovationen offenbleibt und nicht bürokratisch wie zu Zeiten der Deutschen Bundespost erfolgt. In Anbetracht der Chance auf Realisierung eines gleichwertigen Zugangs zum schnellen Internet, die dieses Modell mit sich brächte, würde sich jedoch eine intensivere politische Auseinandersetzung lohnen.

Der Vorteil des Modells liegt zum einen in der Ressourcenallokation: Gewinne, die aus der Infrastrukturnutzung in Ballungsgebieten resultieren, flieBen nicht privaten Akteuren zu, sondern können direkt für den Ausbau der Infrastruktur in ländlichen Räumen genutzt werden. Zum anderen entfallen die hohen Transaktionskosten für die Vergabe von Fördermitteln für den strukturschwachen Raum. Insgesamt könnte also mit vorhandenen Mitteln das Ziel einer gleichwertigen digitalen Daseinsvorsorge effektiver erreicht werden.

Ist der Ausbau der physischen Infrastruktur für einen gleichwertigen Zugang zum schnellen Internet gesichert, können neue Dienste und digitale Inhalte entwickelt werden. Auch hier ist kritisch darüber nachzudenken, wie viel Markt bei der Erbringung digitaler Dienste und Inhalte dem Gemeinwohl zuträglich bzw. an welcher Stelle Regulation notwendig ist.

Damit sich Bürger:innen im virtuellen Raum frei entfalten können, darf der virtuelle Marktplatz nicht durch ein Oligopol großer privater Anbieter beherrscht werden, die jeweils mit einer eigenen technischen Infrastruktur arbeiten und deren Dienste nicht interoperabel sind, weil dies kleinere, gerade auch von öffentlichen Organisationen entwickelte Online-Service-Plattformen verschwinden ließe (Bizer 2020, S. 144). 
Schließlich ist auch darüber nachzudenken, wie viel Zentralität und Dezentralität bei der Entwicklung von Diensten und Inhalten der digitalen Daseinsvorsorge sinnvoll ist. Auch wenn deren Dienste und Inhalte auf Ebene der Kommunen erbracht werden, ergeben sich die Vorteile der digitalen Daseinsvorsorge insbesondere dann, wenn sie quer zu bestehenden territorialen und funktionalen Verwaltungsstrukturen gedacht werden. Eine App für den ÖPNV beispielsweise ist für die Nutzer:innen am attraktivsten, wenn sie die Dienstleitungen mehrerer Anbieter (Bahn, Bus, Car- und Bikesharing) über mehrere Verwaltungsbezirke hinweg integriert.

\section{Fazit}

Im digitalen Zeitalter muss sich die Staatstätigkeit verändern: Die Gewährleistung des Zugangs zur digitalen Infrastruktur und zu digitalen Diensten und Inhalten gehört zur Kernaufgabe eines Staates, der gleichwertige Lebensverhältnisse als Staatsziel anerkennt. Gegenwärtig wird dieses Ziel nicht erreicht, und eine Ursache hierfür liegt in der wettbewerblichen Organisation der digitalen Daseinsvorsorge, durch die Ungleichheiten perpetuiert statt abgebaut werden.

Um diesen Ungleichheitsstrukturen zu begegnen, bedarf es einer stärkeren Regulation des Marktes für die digitale Daseinsvorsorge, einer Stärkung des Rechts auf digitale Daseinsvorsorge und der Überlegung, ob eine grundsätzliche Reform der Governance der digitalen Daseinsvorsorge - z. B. Ausbau der physischen Infrastruktur durch öffentliche Organisationen - nicht effektiver zum Staatsziel beiträgt. Mit der durch das Coronavirus ausgelösten Pandemie hat sich ein politisches Gelegenheitsfenster für grundlegende Reformen geöffnet. Auch das Superwahljahr 2021 bietet viele Anlässe, um öffentlich zu debattieren, wie ein gleichwertiger Zugang zur digitalen Daseinsvorsorge realisiert werden kann.

Es bleibt zu hoffen, dass diese Gelegenheitsfenster genutzt werden, um darüber nachzudenken, welche Güter der Daseinsvorsorge marktförmig erbracht und welche durch die öffentliche Hand garantiert werden müssen, damit sich das Potenzial einer sozialen Marktwirtschaft entfalten kann, die Innovationen schafft und freien Austausch ermöglicht. 


\section{Literatur}

Bizer, J. (2020): Digitale Daseinsvorsorge - Aufgabenfelder, in: Lühr, H. (Hrsg.): Digitale Daseinsvorsorge. Bremer Gespräche zur digitalen Staatskunst, Bremen, S. 141-147.

Bundesministerium für Verkehr und digitale Infrastruktur (2020): Breitbandatlas Langbericht (Stand Mitte 2020), Berlin, www.bmvi.de/SharedDocs/ DE/Artikel/ZukunftBreitband/breitbandatlas-langbericht.html (Abruf am 7.7.2021).

Bundesnetzagentur (o.J.): Breitbandmessung/Funkloch-App, https://breitband messung.de/kartenansicht-funkloch (Abruf am 26.7.2021).

Bundesrechnungshof (2020): Bereitstellen einer Mobilfunkinfrastruktur des Bundes Gründung einer bundeseigenen Mobilfunkinfrastrukturgesellschaft (MIG). Bericht an den Haushaltsausschuss des Deutschen Bundestages nach $\$ 88$ Abs. 2 BHO, Bonn, www.bundesrechnungshof.de/de/veroeffentlichungen/ produkte/beratungsberichte/2020/bereitstellen-einer-mobilfunkinfrastruk tur-des-bundes-gruendung-einer-bundeseigenen-mobilfunkinfrastrukturge sellschaft-mig (Abruf am 7.7.2021).

Bundesverfassungsgericht (1959): Beschluss vom 22. Januar 1959, Az. 1 BvR 154/55, in: BVerfGE 9, 124.

Bundesverfassungsgericht (2013): Urteil vom 17. Dezember 2013, Az. 1 BvR 3139/08 und 1 BvR 3386/08, in: BVerfGE 134, 242.

CDU/CSU/SPD (2013): Deutschlands Zukunft gestalten. Koalitionsvertrag zwischen CDU, CSU und SPD. 18. Legislaturperiode, Rheinbach.

CDU/CSU/SPD (2018): Ein neuer Aufbruch für Europa. Eine neue Dynamik für Deutschland. Ein neuer Zusammenhalt für unser Land. Koalitionsvertrag zwischen CDU, CSU und SPD. 19. Legislaturperiode, Berlin.

Deppisch, L. (2020): `Gefühle des Abgehängtseins` - ein Angstdiskurs, in: Martin, S./Linpinsel, T. (Hrsg.): Angst in Kultur und Politik der Gegenwart, Wiesbaden, S. 179-203.

Diermeier, M. (2020): Ist mehr besser? Politische Implikationen der disparaten Daseinsvorsorge in Deutschland, in: Zeitschrift für Politikwissenschaft 30(4), S. 539-568, DOI: 10.1007/s41358-020-00239-y.

Dümke, C. (2015): Daseinsvorsorge, Wettbewerb und kommunale Selbstverwaltung im Bereich der liberalisierten Energiewirtschaft, KWI-Gutachten 10, Potsdam.

Europäische Union (2018): Richtlinie (EU) 2018/1972 des Europäischen Parlaments und des Europäischen Rates vom 11. Dezember 2018 über den Euro- 
päischen Kodex für die elektronische Kommunikation (Neufassung), Amtsblatt der Europäischen Union vom 17.12.2018.

European Commission (2019): The Digital Economy and Society Index (DESI), https://ec.europa.eu/digital-single-market/en/digital-economy-and-socie ty-index-desi (Abruf am 7.7.2021).

Forsthoff, E. (1938): Die Verwaltung als Leistungsträger, Stuttgart.

Forsthoff, E. (1959): Rechtsfragen der leistenden Verwaltung. Res Publica 1, Stuttgart.

Gröttrup H. (1984): Daseinsvorsorge (DVS), in: Voigt, R. (Hrsg.): Handwörterbuch zur Kommunalpolitik. Studienbücher zur Sozialwissenschaft, Wiesbaden, S. 94-97.

Hofmann, J. (2019): Digitale Kommunikationsinfrastrukturen, in: Klenk, T./ Nullmeier, F./Wewer G. (Hrsg.): Handbuch Digitalisierung in Staat und Verwaltung, Wiesbaden, https://link.springer.com/referencework/10.1007/9783-658-23669-4 (Abruf am 7.7.2021).

Ilgmann, C./Störr, A. (2020): Telekommunikationsnetze in Deutschland - mit einem öffentlichen Unternehmen ausbauen, in: Wirtschaftsdienst 100(8), S. 614-621, DOI: 10.1007/s10273-020-2720-5.

Isensee, J. (2006): Staatsaufgaben, in: Isensee, J./Kirchhof, P. (Hrsg.): Handbuch des Staatsrechts, Band IV: Aufgaben des Staates, Heidelberg, $\$ 73$.

Kersten, J. (2005): Die Entwicklung der Daseinsvorsorge im Werk von Ernst Forsthoff, in: Der Staat 44(4), S. 543-569.

Kersting, N./Graubner, D. (2020): Die digitale Transformation der deutschen Verwaltung. Analysen zu Marktversagen und Daseinsvorsorge in Zeiten der Covid-19-Pandemie, in: Wolfgang, R./Gräf, H./Wollmann, H. (Hrsg.): Zukunft denken und verantworten, Wiesbaden, S. 231-252.

Kingreen, T. (2002): Die sozialen Sicherungssysteme als Elemente der Daseinsvorsorge in Europa, in: Hrbek, R./Nettesheim, M. (Hrsg.): Europäische Union und mitgliedstaatliche Daseinsvorsorge, Baden-Baden, S. 96-117.

Kubicek, H./Jarke, J. (2019): Offene Daten (Open Data), in:Klenk, T./Nullmeier, F./ Wewer, G. (Hrsg.): Handbuch Digitalisierung in Staat und Verwaltung, Wiesbaden, https://link.springer.com/referencework/10.1007/978-3-658-23669-4 (Abruf am 7.7.2021).

Kuhlmann, S./Kühn, H./Danken, T. (2020): Digitale Governance im Bundesstaat: föderale Architektur, kommunale Umsetzung und Perspektiven einer neuen Staatskunst, in: Lühr, H. (Hrsg.): Digitale Daseinsvorsorge. Bremer Gespräche zur digitalen Staatskunst, Bremen, S. 131-140.

Landsberg, G./Rohleder, B. (2020): Kommunen und Corona - digitaler nach der Pandemie?, www.bitkom.org/sites/default/files/2020-12/prasentation-bit 
kom-dstgb-pk-kommunen-corona-digitalisierung-02-12-2020_final.pdf (Abruf am 7.7.2021).

Leisner, W.G. (2011): Daseinsvorsorge: Begriff, Bedeutung, Grenzen: Von existenziellen Leistungen zu infrastruktureller Gewährleistung, in: Wirtschaft und Verwaltung 57(2), S. 55-77.

Luch, A.D./Schulz, S.E. (2011): Die E-Daseinsvorsorge als Grundlage der Online-Handlungsfreiheit und »Eintrittskarte« zur Digitalen Agora, in: Verwaltung und Management 17(2), S. 104-112, DOI: 10.5771/0947-9856-2011-2-104.

Lühr, H. (Hrsg.) (2020): Digitale Daseinsvorsorge. Bremer Gespräche zur digitalen Staatskunst, Bremen.

Roth, I. (2018): Digitalisierung in der Energiewirtschaft. Technologische Trends und ihre Auswirkungen auf Arbeit und Qualifizierung, Working Paper Forschungsförderung 73 der Hans-Böckler-Stiftung, Düsseldorf, www.boeckler. de/pdf/p_fofoe_WP_073_2018.pdf (Abruf am 7.7.2021).

Schallbruch, M. (2020): Wir brauchen eine Plattformökonomie, die zum Gemeinwohl beiträgt, in: Lühr, H. (Hrsg.): Digitale Daseinsvorsorge. Bremer Gespräche zur digitalen Staatskunst, Bremen, S. 156-167.

Schulz, S.E. (2019): Digitale Daseinsvorsorge, in: Klenk, T./Nullmeier, F./Wewer G. (Hrsg.): Handbuch Digitalisierung in Staat und Verwaltung, Wiesbaden, https://link.springer.com/referencework/10.1007/978-3-658-23669-4 (Abruf am 7.7.2021).

Schweigel, H./Lindner, M./Mock, J./Straub, S./Zinke, G. (2020): Open Public Data in Deutschland. Rahmenbedingungen und Potenziale der Bereitstellung und Nutzung von Daten des öffentlichen Sektors, Berlin.

Thiele, K. (2020): Daseinsvorsorge in Gefahr: öffentliche Bibliotheken zwischen Digitalisierung und Austerität, in: Geographica Helvetica 75(2), S. 107-122, DOI: $10.5194 / \mathrm{gh}-75-107-2020$. 


\section{Öffentlicher Wohnungsbau als Element einer breit verstandenen Grundsicherung}

Sebastian Dullien, Tom Krebs

\section{Einleitung}

Das Recht auf Wohnen ist im UN-Sozialpakt von 1966 als Teil eines angemessenen Lebensstandards für jeden Menschen verankert. Das deutsche Grundgesetz erwähnt ein Recht auf Wohnen zwar nicht explizit, es lässt sich aber aus der Garantie der Menschenwürde und dem Sozialstaatsgebot ableiten. Damit gilt dieses Recht nicht nur für Empfänger:innen der Grundsicherung, für die in SGB II $\$ 1$ das Ziel festgelegt ist, "Leistungsberechtigten [zu] ermöglichen, ein Leben zu führen, das der Würde des Menschen entspricht«, sondern für alle Bürger:innen.

So versucht der Staat in Deutschland durch eine Vielzahl von Instrumenten - von der Übernahme der Kosten der Unterkunft für Haushalte in der Grundsicherung über die Zahlung von Wohngeld bis hin zur Förderung von Wohnungserwerb durch Baukindergeld und Wohnriester -, die finanzielle Belastung von Haushalten mit geringen und mittleren Einkommen durch die Wohnkosten zu senken.

Anders als bei einigen anderen Voraussetzungen eines angemessenen Lebensstandards kann "guter Wohnraum« nicht immer nur dadurch bereitgestellt werden, dass den Wohnungssuchenden ein bestimmter Geldbetrag zur Verfügung gestellt wird. Oftmals fehlt es objektiv an ausreichendem bezahlbaren Wohnraum in akzeptabler Qualität und Lage. In anderen Fällen ist bezahlbarer Wohnraum so knapp, dass Vermieter:innen zwischen Mieter:innen diskriminieren, und oft bleiben dabei Randgruppen oder Haushalte mit geringem Einkommen und insbesondere die Bezieher:innen von Grundsicherung außen vor. Besonders in nachgefragten Ballungsgebieten bleibt bezahlbarer Wohnraum extrem knapp.

So ist es wenig verwunderlich, dass vom ärmsten Fünftel der Bevölkerung in Deutschland 2018 laut OECD-Zahlen rund 15 Prozent in zu kleinen Wohnun- 
gen lebten (OECD 2021). Dies entspricht zwar ungefähr dem Schnitt der OECDLänder, ist aber ein etwa doppelt so großer Anteil wie in den Niederlanden, Großbritannien, Portugal oder Spanien. Zugleich ist der Anteil des Bestands an Sozialwohnungen in Deutschland kontinuierlich zurückgegangen. Laut OECDZahlen waren 2018 lediglich 3 Prozent der Wohnungen in Deutschland Sozialwohnungen. Im OECD-Schnitt waren es rund doppelt so viele, in Österreich siebenmal und in den Niederlanden sogar dreizehnmal so viele (OECD 2021).

Dieser Beitrag betrachtet deshalb die Frage, inwieweit ein verstärkter öffentlicher Wohnungsbau helfen könnte, den Grundbedarf nach angemessenem gutem Wohnraum von Grundsicherungsempfänger:innen ebenso zu decken wie den von Menschen, die knapp über der relevanten Einkommensgrenze leben. Hierzu wird zunächst beschrieben, welche Rolle bezahlbarer, gut gelegener Wohnraum für die soziale Inklusion hat (Abschnitt 2). Danach wird die aktuelle Lage am Wohnungsmarkt analysiert (Abschnitt 3) und es wird beschrieben, welche Rolle staatliche Eingriffe in die Miethöhe bei der Lösung des Wohnungsmangels spielen (Abschnitt 4). In einem letzten Schritt wird die Rolle des privaten und öffentlichen Wohnungsbaus beleuchtet (Abschnitt 5) und für die Bundesebene eine Initiative "Zukunft Wohnen« zur Stärkung des öffentlichen Wohnungsbaus vorgeschlagen (Abschnitt 6). ${ }^{1}$

\section{Die Rolle von bezahlbarem Wohnraum als Element einer effektiven Grundsicherung}

Angemessener Wohnraum ist Voraussetzung für ein Leben in Würde und deshalb ein wichtiger Baustein einer effektiven Grundsicherung. Dabei bedeutet "angemessen" nicht nur eine entsprechende Mindestqualität und Mindestgröße der Wohnung, sondern auch eine Versorgung mit den grundlegenden öffentlichen Diensten im Verkehrs- und Bildungsbereich, denn nur so kann die notwendige Teilhabe am gesellschaftlichen Leben sichergestellt werden. Darüber hinaus hilft eine solche umfassende Daseinsvorsorge den betroffenen Menschen, ihre Lebenssituation zu verbessern, z. B. durch Arbeitsaufnahme im Falle eines arbeitslosen Leistungsempfängers.

In diesem erweiterten Sinne setzt die Forderung nach angemessenem Wohnraum voraus, dass es ein hinreichendes Angebot an bezahlbaren Wohnungen in sozial durchmischten Nachbarschaften mit gutem Verkehrs- und Bildungsan-

1 | Die folgenden Ausführungen wurden in Teilen schon publiziert (vgl. Dullien/Krebs 2020) und für die Veröffentlichung in diesem Sammelband angepasst und bearbeitet. 
gebot gibt, denn der Wohnort bestimmt nicht nur die Lebensqualität in Bezug auf die Konsummöglichkeiten, sondern auch den Zugang zum Arbeitsmarkt erwachsener Leistungsempfänger:innen und den Bildungserfolg von Kindern.

Neuere Studien für die USA belegen, dass die soziale Mischung der Wohngegend, in der ein Kind aufwächst, einen starken Einfluss auf sein künftiges Arbeitseinkommen als erwachsene Erwerbsperson hat (Chetty/Hendren 2018). Diese sogenannten "Nachbarschaftseffekte« haben zur Folge, dass die unsichtbare Hand des Marktes ohne staatliche Eingriffe eine erhebliche Ungleichheit der Bildungschancen erzeugt. Daher stärkt eine sozial ausgerichtete Wohnpolitik immer auch die Chancengleichheit und ist mithin zwingender Teil einer Grundsicherung, wenn diese breit verstanden werden soll und gesellschaftliche Teilhabe mit impliziert.

Aus ökonomischer Sicht bestimmt Wohnraum die Produktivität von Erwerbspersonen und beeinflusst so das wirtschaftliche Wachstum. Dabei führen Agglomerationseffekte (Externalitäten) zu einer klassischen Situation des Marktversagens und rechtfertigen, dass der Staat durch Instrumente wie z. B. den öffentlichen Wohnungsbau in städtischen Ballungsräumen ein angemessenes Wohnraumangebot für alle sozialen Gruppen schafft. Ohne staatliche Eingriffe würde es zu Wachstumsverlusten kommen, wenn Erwerbspersonen in Grundsicherung wegen zu hoher Pendler- oder Betreuungskosten keinen oder einen nur schlecht bezahlten Job finden können. Solche und ähnliche Agglomerationseffekte (Externalitäten) wurden ausgiebig in der Literatur zur Stadtökonomik untersucht (Ahlfeldt et al. 2015; Südekum 2010).

\section{Die aktuelle Lage am Wohnungsmarkt}

Nachdem in den Jahren 2012 bis 2018 die Wohnbevölkerung in Deutschland um rund 2,5 Millionen Personen gestiegen ist und zudem die größten Städte des Landes einen weiteren Zuzug aus dem Rest des Landes erlebt haben (Destatis 2019), herrscht in vielen Ballungsgebieten ein Mangel an Wohnraum. In einer aktuellen Studie schätzen Baldenius, Kohl und Schularick (2019) den Bedarf an Neubauten deutschlandweit auf mindestens 330.000 Wohnungen pro Jahr bis 2030.

Zum künftigen Bedarf aufgrund des demografischen Wandels und absehbarer Einkommenszuwächse kommt hinzu, dass bereits in den Jahren 2009 bis 2015 die Nachfrage schneller gestiegen ist als das Angebot, sodass die Prognos AG (2017) in einem Gutachten von einer aufgelaufenen Wohnungsbaulücke von rund 1 Million Wohnungen ausgeht. Dieser Nachfrage nach zusätzli- 
chem Wohnraum stand in den Jahren 2017 und 2018 ein Angebot von 285.000 bzw. 287.000 fertiggestellten Wohnungen gegenüber. Der Wohnungsneubau in Deutschland muss also weiter gesteigert werden, um die langfristige Nachfrage zu befriedigen.

Diese Entwicklungen auf den Wohnungsmärkten der städtischen Ballungsräume haben regional zu sehr starken Mietpreiszuwächsen geführt, und besonders bezahlbarer Wohnraum ist vielerorts zur Mangelware geworden. Dies sollte eigentlich für eine massive Ausweitung der öffentlichen Wohnraumförderung sprechen, doch bis vor Kurzem war das Gegenteil der Fall: Während der Bestand an Sozialwohnungen zu Beginn der 1980er-Jahre noch 4 Millionen umfasste, ist er bis 2006 auf 2,1 Millionen zurückgegangen; im Jahr 2019 gab es weniger als 1,2 Millionen Sozialwohnungen (siehe Abbildung 1). Jährlich fallen weiter etwa 80.000 Wohnungen aus der Förderung heraus, während nur circa 25.000 neue Sozialwohnungen gebaut werden (Pestel-Institut 2019). Das Pestel-Institut (2019) empfiehlt aufgrund einer detaillierten Bedarfsanalyse die Schaffung von jährlich 155.000 Wohnungen mit Sozialbindungen, um bis 2030 das Ziel von 2 Millionen Sozialwohnungen im Bestand zu erreichen. Dabei sollten 80.000 durch Neubau und 75.000 durch Modernisierungsförderung geschaffen werden.

Abb. 1: Gesamtbestand an gebundenen Mietwohnungen (2006-2019)

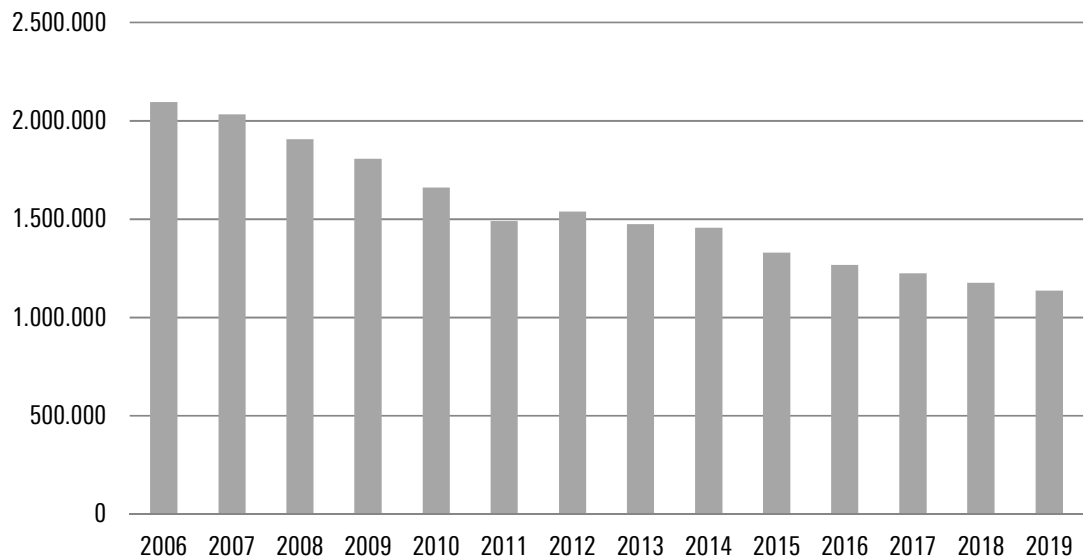

Quelle: Deutscher Bundestag 2019 (eigene Darstellung)

Eine ähnlich große Lücke zwischen Angebot und Bedarf besteht im mittleren Preissegment der städtischen Wohngebiete, wo auch Haushalte mit mittleren Einkommen häufig die Marktmieten nicht bezahlen können. Holm et al. (2018) 
beziffern den ungedeckten Bedarf an bezahlbaren Wohnungen in den Großstädten auf 1,9 Millionen.

Als Reaktion auf die angespannte Lage auf dem Wohnungsmarkt hat das Bundeskabinett im März 2016 das »Bündnis für bezahlbares Wohnen und Bauen « beschlossen und so eine erste Trendwende im geförderten Wohnungsbau eingeleitet - im Jahr 2016 ist zum ersten Mal seit langer Zeit die Anzahl der neu gebauten Sozialwohnungen wieder gestiegen. Doch die anfängliche Dynamik scheint erlahmt und die erhoffe Beschleunigung ist ausgeblieben, obwohl die Bundesregierung im Koalitionsvertrag 2018 und auf dem Wohnungsgipfel im Herbst 2018 die Bereitstellung zusätzlicher Mittel für den geförderten Wohnungsbau beschlossen hat.

Die von der Bundesregierung eingeleiteten Maßnahmen sind ein Schritt in die richtige Richtung, aber das Angebot an bezahlbarem Wohnraum in den wachsenden Städten und Regionen wird auf absehbare Zeit weit hinter der Nachfrage zurückbleiben. Darüber hinaus besteht die Gefahr, dass die von der Bundesregierung gesteckten Ziele zur Steigerung des Wohnneubaus aufgrund von Problemen in der Umsetzung nicht erreicht werden. Deshalb erscheinen weitere Politikmaßnahmen angebracht.

\section{Die Rolle von Mietpreisregulierung für die Wohnungsversorgung}

Ein in den vergangenen Jahren breit und kontrovers diskutierter Ansatz, um die Bezahlbarkeit von Wohnraum für die breite Bevölkerung bereitzustellen, ist die gesetzliche Begrenzung von Wohnungsmieten. Während die Bundesregierung zuletzt 2019 die bundesrechtlich verankerte Mietpreisbremse verschärft und in

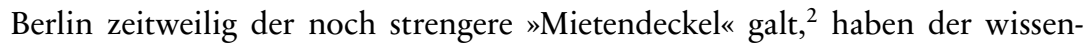
schaftliche Beirat beim Bundesministerium für Wirtschaft und Energie (2018) und der Sachverständigenrat zur Begutachtung der gesamtwirtschaftlichen Entwicklung (2018) vor der gesetzlichen Begrenzung von Mieten gewarnt.

Befürworter:innen in der politischen Sphäre argumentieren dabei oft, dass eine gesetzliche Begrenzung von Mieten verhindert, dass Vermieter:innen ihre Machtposition ausnutzen, und Wohnraum so durch die Dämpfung des Mietanstiegs oder die tatsächliche Absenkung von Mieten bezahlbarer wird. Die Kritiker:innen von Mietregulierungen argumentieren dagegen, dass eine Be-

2 | Der »Mietendeckel « wurde durch ein Urteil des Bundesverfassungsgerichts im März 2021 als unvereinbar mit dem Grundgesetz erklärt, da solch eine Regelung nicht in den Kompetenzbereich der Länder fällt (Bundesverfassungsgericht 2021). 
grenzung der Mieten das Wohnungsangebot verknappe, weil zum einen Neubau unterlassen werde, zum anderen bestehende Mietwohnungen in möblierte Wohnungen oder Eigentumswohnungen zur Selbstnutzung umgewandelt würden. Außerdem führe eine Begrenzung der Mieten zu einer übermäßigen Nachfrage nach Wohnraum, was wiederum den Wohnungsmangel weiter verschärfe.

Tatsächlich ist die Ökonomie der Mietpreiseingriffe komplexer als von vielen Kritiker:innen dargestellt. Üblicherweise unterscheidet die moderne Literatur zwischen Mietregulierungen der ersten und der zweiten Generation; Letztere zeichnen sich dadurch aus, dass sie zwischen Altbestand und Neubauten unterscheiden (Arnott 1995). Preise werden üblicherweise nur im Altbestand begrenzt. Dadurch bleibt der Anreiz für Neubauten bestehen und wird möglicherweise sogar verstärkt, weil Wohnungssuchende aus dem Markt für Bestandswohnungen in den Markt für Neubauten gedrängt werden, wodurch der Preis dort möglicherweise noch stärker steigt.

Gegner:innen der Mietpreisbremse verweisen bei diesem Argument gerne darauf, dass eine Mietpreiskontrolle nur für Bestandsgebäude zu einem gespaltenen Wohnungsmarkt führe. Da die Mieten pro Quadratmeter im Bestandsmarkt niedriger lägen als im Markt für Neubauten, werde dort zu viel Wohnfläche nachgefragt, was zu einem Mangel im Bestandsmarkt führe. Dieses Argument ist prinzipiell richtig, vernachlässigt aber die Interaktion der Mietpreisbremse (die für Neuvermietungen von Bestandsgebäuden gilt) mit allgemeinen Regeln zur Erhöhung von Bestandsmieten.

In Deutschland ist die Erhöhung von Bestandsmieten gesetzlich gedeckelt. Bei massivem Anstieg des allgemeinen Mietniveaus, wie wir ihn in den vergangenen Jahren in einigen deutschen Großstädten erlebt haben, steigt dabei der Anreiz für Menschen, in zu großen Wohnungen wohnen zu bleiben. Viele ältere Ehepaare in Großstädten, deren Kinder inzwischen ausgezogen sind, belegen weiter Familienwohnungen. Weil aber eine neu gemietete 50-QuadratmeterWohnung inzwischen oft teurer ist als eine 120-Quadratmeter-Wohnung mit Altvertrag, ist ein Umzug wenig attraktiv.

Die Mietpreisbremse entspannt diesen Lock-in-Effekt tendenziell, weil der Mietpreisanstieg zumindest auf dem Markt für Bestandsbauten begrenzt ist und damit eine kleinere Wohnung günstiger gemietet werden kann. Somit wird zum Teil die Nachfrage nach Wohnraum wieder gesenkt. Ob insgesamt der nachfragesteigernde Effekt niedriger Quadratmeterpreise oder der nachfragesenkende Effekt durch erhöhte Mobilität von Haushalten größer ist, ist eine empirische Frage, für die es für Deutschland keine belastbaren Erkenntnisse gibt.

Beachtet werden sollte in der Debatte auch, dass nicht jeder Preisanstieg als Marktsignal ökonomisch sinnvoll ist. Gerade weil Angebotsanpassungen im 
Wohnungsmarkt durch lange Plan- und Bauprozesse, kombiniert mit möglichen Kapazitätsengpässen in der Bauwirtschaft und beim Boden, extrem lange dauern (relativ unelastisches Angebot in der kurzen Frist) und zugleich die Nutzung von Wohnraum (und damit die Nachfrage) schlecht über die Zeit verschoben werden kann, kommt es immer wieder zum Überschießen von Neuvertragsmieten wie auch Kaufpreisen.

Weil die meisten Haushalte einen signifikanten Anteil ihres verfügbaren Einkommens fürs Wohnen ausgeben, führt dies zu einer massiven Umverteilung von jenen, die mieten müssen, zu jenen, die Eigentum besitzen. Insbesondere im Zusammenhang mit der Debatte um eine Garantie angemessenen Wohnraums im Rahmen einer breit verstanden Grundsicherung ist eine solche Umverteilung problematisch, weil sie die finanziellen Lebensgrundlagen insbesondere des einkommensschwächeren Teils der Bevölkerung angreift. Wenn die Beschränkungen zur weiteren Ausweitung des Wohnungsangebots jenseits des Wohnungsmarkts liegen (etwa in begrenzter Planungskapazität oder begrenzten Kapazitäten der Bauindustrie), hat ein weiterer Preisanstieg keinen positiven Einfluss auf den Wohnungsbau und der Verteilungseffekt dominiert.

Eingriffe in die Mietpreisbildung - sei es durch Regeln für Bestandsmieten, aber auch durch Regeln für Neuvermietungen wie im Fall der deutschen Mietpreisbremse - haben hier eine sinnvolle Funktion: Sie begrenzen bei unerwarteten Nachfrageverschiebungen die Umverteilung von Mieter:innen zu Immobilieneigentümer:innen. Damit begrenzen sie die Vermögensverwerfungen, die sich etwa aus einer unerwarteten Wanderungsbewegung in die Städte oder plötzlich höherer Immigration ergeben, und können so helfen, die finanziellen Lebensgrundlagen der Mieter:innen zu schützen, und damit zu einer breiter verstandenen Grundsicherung beitragen.

Allerdings darf bei der Regulierung von Mieten auch das Argument nicht vernachlässigt werden, dass eine unbedachte Begrenzung von Mieten tatsächlich das Angebot an frei vermieteten Wohnungen beeinträchtigen kann. Eine Deckelung von Mieten auf zu niedrigem Niveau etwa erhöht den Anreiz für private Eigentümer:innen, insbesondere kleinere Wohnungen gar nicht zu vermieten und als Zweitwohnung zu halten.

Wenn Eingriffe in den Mietmarkt die Rentabilität von Wohnungsbauunternehmen gefährden und dort zu Liquiditätsproblemen führen, kann auch dies negative Effekte auf den Wohnungsbau haben. Einen ähnlichen Effekt könnte es geben, wenn Bauherr:innen nicht mehr glauben, dass die Regulierung auf den Altbestand beschränkt bleibt, und deshalb Neubauaktivitäten einschränken.

Darüber hinaus muss berücksichtigt werden, dass eine Mietpreisregulierung auch unerwünschte Verteilungseffekte haben kann. Zum Beispiel besteht bei 
einer Obergrenze für den Mietpreis immer die Gefahr, dass die einkommensstarken Haushalte, die im Durchschnitt auch die qualitativ hochwertigeren Wohnungen anmieten, besonders stark von der Regulierung profitieren.

Die deutsche Mietpreisbremse scheint nach bisheriger Evaluation so konstruiert zu sein, dass schädliche Nebenwirkungen minimiert werden und eine Dämpfung des Mietenanstiegs in angespannten Wohnungsmärkten gelingt (Kholodilin/Mense/Michelsen 2018). Inwieweit dies auch für den kurzlebigen Berliner Mietendeckel galt, muss sich erst noch zeigen. Insbesondere bei den Regeln zur Absenkung von Mieten im Altbestand, aber auch bei der Umlagefähigkeit von durchaus sinnvollen Modernisierungen war der Mietendeckel deutlich restriktiver als die Mietpreisbremse, sodass bei einem längeren Bestand der Regeln negative Nebenwirkungen auf das Wohnungsangebot wahrscheinlicher gewesen wären. Diese wären dann auch dem Ziel der Wohnungspolitik entgegengelaufen, allen Menschen eine Versorgung mit qualitativ hochwertigem Wohnraum zu garantieren.

Grundsätzlich sollte nicht vergessen werden, dass Mietpreiseingriffe vor allem das Symptom der Probleme am Wohnungsmarkt bekämpfen. Diese Symptomtherapie kann durchaus sinnvoll sein, doch sollte sie in der Regel nur zeitlich begrenzt eingesetzt werden. Für eine langfristige Entspannung ist eine Ausweitung des Angebots unerlässlich.

\section{Aktuelle Probleme im Wohnungsbau und die Rolle des öffentlichen Wohnungsbaus}

Angesichts der massiv gestiegenen Mieten und der niedrigen Leerstandsquoten bei geringer Verzinsung alternativer Anlagen stellt sich die Frage, warum nicht schneller mehr Wohnungen gebaut werden. Gemeinhin werden eine Reihe unterschiedlicher Ursachen genannt:

- Mangelnde Planungskapazitäten auf kommunaler Ebene: Oftmals sind viele Kommunen nach Jahren des Personalabbaus nicht in der Lage, zügig Bebauungspläne zu erstellen oder Baugenehmigungen zu erteilen.

- Kapazitätsengpässe im Baugewerbe: Angesichts der gestiegenen Nachfrage nach Bauleistungen sind viele Unternehmen an ihren Kapazitätsgrenzen. Die angespannte Lage auf dem Arbeitsmarkt erschwert es den Unternehmen zudem, neues Personal einzustellen.

- Mangelnde Finanzierung komplementärer lokaler Infrastruktur: Insbesondere der Ausweis größerer Neubauprojekte und Neubauflächen bringt für die Kom- 
munen oft Folgekosten in Form von erhöhtem Bedarf an Infrastruktur (Straßen, Kitas, Schulen) mit sich. Viele Kommunen können oder wollen diese Infrastruktur nicht finanzieren.

- Mangel an Bauland: Insbesondere in den größten Städten wird zum Teil das Bauland für größere Bauprojekte knapp.

Bei dieser Problemaufstellung muss allerdings beachtet werden, dass nicht all diese Hindernisse als gegeben hingenommen werden müssen. So war der Wohnungsbau als Anteil des Bruttoinlandsprodukts sowohl in anderen Ländern des Euroraums als auch historisch in Deutschland schon einmal größer als heute (siehe Abbildung 2).

Abb. 2: Wohnungsbau in Prozent des Bruttoinlandsprodukts (1960-2019)

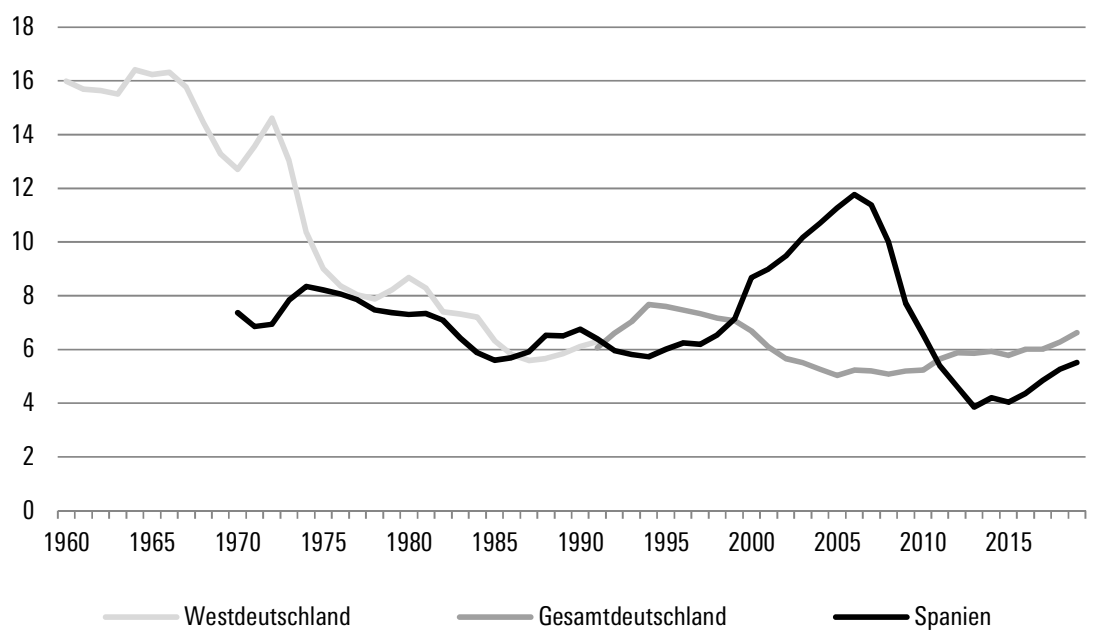

Quelle: Ameco, IMK-Berechnungen (eigene Darstellung)

Mittelfristig müsste es möglich sein, bei anhaltenden Personalengpässen am Bau mehr Arbeitskräfte aus dem Inland oder aus anderen EU-Staaten anzuwerben. Eine verlässliche Perspektive für mittelfristig stabile Wohnbaunachfrage könnte zudem die Unternehmen anregen, verstärkt in die benötigten Ausrüstungen zu investieren. Auch der Mangel an Bauland ist nicht immer eine starre objektive Beschränkung für den Neubau von Wohnungen. So besitzt die öffentliche Hand in den größten Städten zum Teil noch ungenutzte Flächen, die prinzipiell für den Wohnungsbau geeignet wären. Zudem gibt es oftmals noch Gewerbeflächen, die zu Wohnraum umgewandelt oder verdichtet werden können. 
Gerne wird argumentiert, dass die Probleme des Wohnungsmangels sich über privatwirtschaftliche Bereitstellung von Wohnraum und durch verstärkte bzw. höhere Zahlung von Wohngeld lösen ließen (SVR 2018; Wissenschaftlicher Beirat 2018). Dieser Schluss ist allerdings verfehlt. Es gibt verschiedene ökonomische Gründe, warum die reine Marktlösung zu Fehlallokationen führt und somit aus gesamtgesellschaftlicher Perspektive suboptimal ist. Der Wohnungsmarkt weicht deutlich von dem theoretischen Idealbild eines vollkommenen Marktes ab, sodass die Analyse dieses Marktes etwas komplexer ist und zu anderen Schlussfolgerungen kommt.

Zum einen sind auf dem Wohnungsmarkt Agglomerations- und Nachbarschaftseffekte wichtig. Der Nutzen einer Wohnung hängt auch davon ab, wer die Grundstücke in unmittelbarer Nähe nutzt. Bei rein privaten Entscheidungen über die Nutzung von vorhandenem Bauland werden diese Externalitäten oft nicht beachtet, sodass vorhandenes Bauland nicht optimal verwendet wird. Insbesondere führen diese Mechanismen dazu, dass bei privater Entscheidung auf vorhandenem Bauland üblicherweise zu wenig Wohneinheiten insgesamt und insbesondere zu wenig Wohneinheiten für Haushalte mit niedrigen und mittleren Einkommen geschaffen werden. Dies verursacht in Wechselwirkung mit bestehenden Einkommens- und Vermögensungleichheiten eine unerwünschte Segregation der Bevölkerung.

Zum anderen ist Wohnraum nicht nur ein Konsumgut, sondern beeinflusst auch Produktivität und Bildungschancen. Fehlallokationen auf dem Wohnungsmarkt wirken sich daher negativ auf die Chancengleichheit und das wirtschaftliche Wachstum aus. Konkret würden private Investoren in den attraktiven Stadtlagen überwiegend teure Luxuswohnungen bauen, weil diese Wohnungen die höchsten privaten Renditen erwirtschaften. Aus gesamtwirtschaftlicher und gesellschaftlicher Perspektive wäre es jedoch besser, wenn ein gewisser Anteil der Wohnungen auch in den attraktiven Stadtlagen für untere und mittlere Einkommen bezahlbar bliebe.

Öffentlicher Wohnungsbau (Objektförderung) kann solche Fehlentwicklungen vermeiden. Ein gut organisierter öffentlicher Wohnungsbau fördert sozial ausgewogene Wohnquartiere und begrenzt so die Segregation. Er schließt den Bau der notwendigen Infrastruktur (Freizeit, Kitas, Schulen) mit ein und zielt darauf ab, die Städte für alle Menschen lebenswert zu gestalten. Wie man in Österreich sieht, erzeugt ein solcher öffentlicher Wohnungsbau keine sozialen Gettos, wie sie im sozialen Wohnungsbau der 1970er-Jahre aufgetreten sind (Kunnert/ Baumgartner 2012).

Neben dem öffentlichen Wohnungsbau können auch Wohnbaugenossenschaften einen Beitrag zur Linderung der Wohnungsnot leisten. Deshalb sollten 
Genossenschaften ebenfalls von der öffentlichen Hand gefördert werden, etwa durch bevorzugte Zuteilung von Baugrundstücken gegenüber gewinnorientierten Investoren und öffentliche Kreditgarantien oder Kredite öffentlicher Banken.

Wohnungsbau in Form eines klug organisierten öffentlichen Wohnungsbaus und eines klug geförderten Genossenschaftsbaus hat fünf Vorteile im Vergleich zur reinen Marktlösung:

1. Er schafft mehr bezahlbare Wohnungen für die gleiche Menge an Bauland, da er einen Schwerpunkt auf Wohnungen unterhalb des Luxussegments legen kann.

2. Er ist ein effizientes Mittel, spekulative Leerstände zu vermeiden und Mietpreisexplosionen zu begrenzen, indem zum einen Leerstände in den von der öffentlichen Hand gehaltenen Wohnungen klein gehalten werden und zum anderen Mieter:innen mit einem öffentlichen Mietwohnungsangebot eine Option jenseits des privaten Wohnungsmarktes gegeben wird.

3. Öffentlicher Wohnungsbau senkt die ökonomische Ungleichheit und steigert zugleich die gesamtwirtschaftliche Produktion, indem er Segregation entgegenwirkt und damit Niedrigeinkommenshaushalte stärker von positiven Agglomerations- und Wachstumseffekten profitieren lässt.

4. Der induzierte Wachstumseffekt generiert wiederum Steuermehreinnahmen, die einen Beitrag zur Tragfähigkeit der öffentlichen Finanzen leisten.

5. Es gibt Indizien, dass ein ausreichend großes Gewicht des öffentlichen Sektors im Wohnungsmarkt Immobilienpreisblasen begrenzen kann, weil potenzielle Käufer:innen dann die Option haben, in bezahlbare Mietwohnungen auszuweichen und nicht gezwungen sind, überteuerte Immobilien zu kaufen (Dullien/Joebges/Márquez-Velázquez 2016).

In der Praxis hat sich die Einsicht des Nutzens öffentlichen Wohnungsbaus teilweise schon durchgesetzt; in den vergangenen Jahren sind in deutschen Städten neue Wohnquartiere mit ausgewogener sozialer Mischung entstanden oder in Planung. Doch es gibt Probleme in der Umsetzung, die dazu führen, dass immer noch zu wenig und teilweise auch "falsch" gebaut wird.

Verfechter:innen privatwirtschaftlicher Lösungen beim Wohnungsbau verweisen gern darauf, dass eine bessere Allokation des Baulands auch durch Auflagen an private Bauherr:innen gelöst werden könnte. Tatsächlich haben in den vergangenen Jahren die meisten Städte versucht, in die Planungen privater Bauherr:innen einzugreifen. Dabei ist der übliche Lösungsansatz, Projekte unter Auflagen (z. B. 30 Prozent der Wohnungen mit Mietpreisbindung) und mit Vergünstigungen (zinsgünstige Kredite) an private Bauherr:innen zu vergeben. Die 
Auflagen werden in einem Vertrag zwischen Stadt bzw. Kommune und privaten Bauherr:innen festgehalten.

Dieser Ansatz hat Vorteile, er birgt jedoch zwei fundamentale Risiken, die immer wieder auftreten und zu ineffizienten Ergebnissen führen:

- Hold-up-Problem: Der Bauträger erfüllt nach Baubeginn die Auflagen nicht, aber die Kosten einer Vertragsauflösung sind für die Kommune sehr hoch.

- Unvollständige Verträge und asymmetrische Information: Der Bauträger erfüllt den Vertrag de jure, versucht aber, durch Lücken im Vertrag und seinen Informationsvorteil die Auflagen »aufzuweichen«.

Ein typisches Beispiel für das erste Problem ist der Fall des Investors Christoph Gröner, der am Postscheckamt in Berlin-Kreuzberg ein gemischtes Projekt (Wohnen, Büros, Gewerbe) mit 623 geplanten Wohnungen umsetzen wollte. Im Sommer 2018 änderte der Investor einseitig die Vertragsbedingungen und reduzierte den Anteil des Wohnraums am Gesamtprojekt um 7 Prozentpunkte, was zu einem spürbaren Rückgang des Anteils bezahlbaren Wohnraums geführt hätte (Backes 2018). Zudem lieferte er sich einen medienwirksamen Schlagabtausch mit dem Berliner Baustadtrat Florian Schmidt.

Die Stadt (der Bezirk) kann in diesem Fall der Genehmigung für den von dem Investor eingebrachten Änderungsvorschlag verweigern (und hat das auch getan), was aber in der Regel zum Baustopp führt. Die ökonomische Lösung für dieses Problem wäre, die Kosten bzw. die Strafe für vertragswidriges Verhalten des Investors bzw. der Investorin zu erhöhen, aber eine solche ökonomische Lösung steht häufig im Widerspruch zum geltenden Rechtsrahmen oder ist in der juristischen Praxis schwierig durchzusetzen, weil sie zu langjährigen Prozessen mit unbestimmtem Ausgang führt.

Ein typisches Beispiel für das zweite Problem ist der Fall der Groth-Gruppe, die in Berlin am Mauerpark und in der Lehrter Straße zwei größere Wohnprojekte entwickelt (Wiedemeier 2018). In beiden Projekten werden die geplanten Sozialwohnungen, die von der städtischen Wohnungsbaugesellschaft Gewobag gebaut und vermietet werden, als Lärmschutzriegel für die teureren Wohnungen ohne Mietpreisbindungen verwendet. Diese Vorgehensweise ist rechtlich einwandfrei, weil die Berliner Lärmschutzregeln eingehalten werden.

Wie dieses Beispiel zeigt, nutzen private Bauherr:innen fast immer den vorhandenen vertraglichen Spielraum aus, um bei den geförderten Wohnungen Kosten zu reduzieren und die Qualität zu senken. Zudem zeigt sich, dass auch die öffentlichen Wohnungsbaugesellschaften gelegentlich aus betriebswirtschaftlichen Gründen eine Lösung wählen, die aus gesamtgesellschaftlicher Perspektive fragwürdig ist. 


\section{Den öffentlichen Wohnungsbau stärken}

Basierend auf diesen Überlegungen wäre es zielführend, durch Neubau so schnell wie möglich den Bestand an Wohnungen im öffentlichen Eigentum in Deutschland zu erhöhen. Dabei sollte der Neubau möglichst so organisiert werden, dass folgende gesamtgesellschaftlichen Ziele erreicht werden:

- Soziale Durchmischung: Der Anteil an geförderten Wohnungen in attraktiven Stadtlagen sollte erhöht und gezielt eine bessere Durchmischung in allen Stadtquartieren gefördert werden. Hierzu gehört die Stärkung der Kommunen als Eigentümern von Grund und Boden (Vergabe kommunaler Grundstücke über Erbbaurecht), um soziale Zielvorgaben zeitlich unbegrenzt zu erfüllen.

- Ökologische Nachhaltigkeit: Die öffentliche Hand sollte ambitionierte Klimastandards im Neubau und den Ausbau klimafreundlicher Infrastruktur fördern, einschließlich einer Signal- und Schrittmacherfunktion für den privaten Wohnungsbau.

- Schaffung zusätzlichen Wohnraums: Verdichtungsmöglichkeiten in den Städten sollten genutzt (z. B. Bauen auf einstöckigen Supermärkten), neue Quartiere in städtischen Ballungsräumen entwickelt (z. B. HafenCity Hamburg) und eine bessere Anbindung und Nutzung der städtischen Randgebiete (z. B. Oberbillwerder in Hamburg-Bergedorf) geschaffen werden.

- Senkung der Baukosten: Durch Weiterentwicklung und Anwendung des seriellen Bauens mit Fokus auf qualitativ hochwertigen Produkten sollten die Baukosten im Wohnungsbau gesenkt werden.

Die genannten Vorgaben sollen gewährleisten, dass der öffentliche Wohnungsbau Personen in Grundsicherung mit einem angemessenen Wohnungsangebot versorgt. Dabei bedeutet "angemessen« nicht nur eine entsprechende Mindestqualität und -größe der Wohnung, sondern auch ein Leben in sozial durchmischten Nachbarschaften und eine Versorgung mit den grundlegenden öffentlichen Diensten im Verkehrs- und Bildungsbereich, denn nur so kann eine angemessene Teilhabe am gesellschaftlichen Leben sichergestellt und die Chancengleichheit gestärkt werden - beides wichtige Elemente einer breit verstandenen Grundsicherung (siehe Abschnitt 2).

Diese Ziele lassen sich sinnvoll nur mit einer gewissen Zentralisierung bestimmter Elemente des Wohnungsbaus erreichen. So könnte man auf übergeordneter Ebene stärker von den Effizienzgewinnen seriellen Bauens, moderner Logistik- und Beschaffungsmaßnahmen oder anderer Skaleneffekte profitieren als auf kommunaler Ebene, die üblicherweise für den Wohnungsbau zuständig ist. 
Auch würde eine gewisse Zentralisierung gerade solchen Gemeinden helfen, die selbst nicht das Know-how oder Personal für effiziente Planungsprozesse haben oder die aufgrund historischer Entwicklungen Finanzierungsprobleme haben. Viele der positiven Effekte eines stärkeren öffentlichen Wohnungsbaus hätten zudem positive externe Effekte über Kommunal- oder Landesgrenzen hinaus, sodass politökonomisch eine zumindest teilweise Finanzierung durch übergeordnete Ebenen sinnvoll wären, um eine unzureichende Bereitstellung öffentlicher Wohnungen zu vermeiden.

Zugleich hat allerdings auch eine Dezentralisierung des öffentlichen Wohnungsbaus Vorteile, weil der konkrete Bedarf und die lokalen Gegebenheiten oft auf Ebene der Kommunen am besten zu beurteilen ist. Außerdem wurde die Kompetenz für viele Bereiche des Wohnungsbaus mit der Föderalismusreform II im Jahr 2006 ausschließlich den Ländern zugewiesen.

Gefragt sind deshalb Instrumente, die den öffentlichen Wohnungsbau bedarfsgerichtet, aber bundesweit fördern, Skalen- und Synergieeffekte heben und zugleich die Ortskenntnis lokaler Verwaltungen und Entscheidungsträger:innen nutzen. Konkret könnte dies durch eine Initiative "Zukunft Wohnen" geschehen, in deren Rahmen eine Beratungsgesellschaft und zwei Investitionsfonds auf Bundesebene die Länder und Kommunen in den drei Bereichen Planung, Finanzierung und Steuerung unterstützen:

- Beratungsgesellschaft "Zukunft Wohnen«: Beratungsgesellschaft zur Unterstützung der kommunalen Verwaltungen bei der Planung und Entwicklung von Wohn- und Stadtteilprojekten. Größere Wohnungsbau- und Stadtentwicklungsprojekte sind häufig hochkomplex und erfordern die Bündelung verschiedener Ideen und Konzepte. Integrierte Stadtentwicklung benötigt eine sehr breite fachliche Aufstellung: Ingenieur:innen, Stadtplaner:innen, Grundstücksentwickler:innen, Ökonom:innen, Kulturwissenschaftler:innen, Geistes- und Sozialwissenschaftler:innen, Geograf:innen und Landschaftsarchitekt:innen müssen eng zusammenarbeiten. Die Beratungsgesellschaft würde Kommunen bei diesen Aufgaben helfen.

- Bodenfonds »Zukunft Wohnen«: Investitionsfonds zur Förderung des Aufbaus kommunaler und regionaler Boden- und Infrastrukturfonds. Er soll die Kommunen dabei unterstützen, das öffentliche Eigentum von Grund und Boden auszuweiten. Eine effektive Liegenschaftspolitik ist häufig nur möglich, wenn Grund und Boden im Eigentum der öffentlichen Hand verbleiben, doch vielen Kommunen fehlen die finanziellen und personellen Ressourcen für eine effektive Liegenschaftspolitik. Hier kann der Bundesfonds mit Expertise, Finanzmitteln und Grundstücken aus Bundeseigentum helfen. 
- Beteiligungsfonds »Zukunft Wohnen«: Beteiligungsgesellschaft zur Stärkung des Eigenkapitals existierender kommunaler Wohnungsbaugesellschaften und zur Unterstützung bei der Gründung neuer kommunaler oder landeseigener Wohnungsbaugesellschaften.

Um die oben beschriebenen Aufgaben in enger Zusammenarbeit mit den kommunalen Verwaltungen, den kommunalen Wohnungsbaugesellschaften und der Bauwirtschaft umzusetzen, bietet sich folgende Organisationsstruktur für die drei Bundesgesellschaften an:

- Die Beratungsgesellschaft "Zukunft Wohnen" wird als Projektentwicklungsgesellschaft gegründet, die die Kommunen bei der Planung, Entwicklung und Umsetzung von Wohnungsbau- und größeren Stadtteilprojekten unterstützt. Diese Projektentwicklungsgesellschaft könnte zusammen mit der jeweiligen Kommune eine Beteiligungsgesellschaft gründen. Auf Bundesebene gibt es bereits die Gesellschaft »Partnerschaft Deutschland«, die ähnliche Aufgaben wie die hier angedachte Beratungsgesellschaft erfüllt, jedoch nicht mit dem Fokus Wohnen. ${ }^{3}$ Daher könnte die Beratungsgesellschaft »Zukunft Wohnen « in einem ersten Schritt als eigenständige Abteilung der »Partnerschaft Deutschland « realisiert werden.

- Der Investitionsfonds "Zukunft Wohnen " wäre eine Beteiligungsgesellschaft des Bundes, die die existierenden kommunalen Wohnbaugesellschaften bei der Stärkung der Eigenkapitaldecke unterstützt. Zudem soll diese Gesellschaft die Gründung neuer kommunaler oder landeseigener Wohnungsbaugesellschaften finanziell und organisatorisch begleiten.

In einem ersten Schritt könnten die Ziele dieses Bundesfonds durch ein Beteiligungsprogramm der KfW umgesetzt werden. Die KfW hat in ihrem »Instrumentenkasten « bereits Eigenkapitalprogramme (z. B. das ERP-Beteiligungsprogramm), über die sich Beteiligungsgesellschaften die für Beteiligungen notwendigen Mittel bei der KfW leihen können. Das hier vorgeschlagene Programm - eine öffentlich-öffentliche Partnerschaft - hätte große Ähnlichkeit mit den schon existierenden Beteiligungsprogrammen, aber der Fokus läge auf dem öffentlichen Wohnungsbau. Um einen zielgerichteten Einsatz der Mittel zu gewährleisten, würde das Programm nur für solche regionalen Gebiete gelten, in denen derzeit Mangel an bezahlbarem Wohnraum herrscht.

3 | Die in öffentlicher Hand befindliche »Partnerschaft Deutschland « berät unter anderem Bund, Länder und Kommunen bei Planung, Bau und Bewirtschaftung von komplexeren Infrastruktur- und Immobilienvorhaben. 
- Der Bodenfonds »Zukunft Wohnen « wäre ein Investitionsfonds des Bundes, der die Kommunen finanziell und personell in ihrer Liegenschaftspolitik unterstützt. Konkret soll der Bodenfonds des Bundes den Kommunen durch finanzielle Beiträge, Sacheinlagen (Bundesliegenschaften) und organisatorisches Know-how helfen, neue kommunale Boden- und Infrastrukturfonds zu gründen. In einem ersten Schritt könnten die Ziele dieses Bundesfonds durch ein spezielles Programm der Bundesanstalt für Immobilienaufgaben verfolgt werden.

Die drei Bundesgesellschaften sollten als rechtlich selbstständige Einheiten realisiert werden, deren Eigentümer zu 100 Prozent die öffentliche Hand ist. Dabei sollte es neben den üblichen Aufsichtsgremien auch einen wissenschaftlichen Beirat als beratendes Fachgremium geben, der mit Vertreter:innen der Fachverbände und der Wissenschaft besetzt wird. Eine regelmäßige wissenschaftliche Evaluierung würde einen effizienten Mitteleinsatz gewährleisten.

Das Grundkapital der drei Bundesgesellschaften würde sich im Wesentlichen aus Finanzmitteln des Bundes speisen. Dabei ist angedacht, dass der Bund Kredite aufnimmt, um damit das Eigenkapital der drei Bundesgesellschaften aufzustocken. Eine Aufstockung des Eigenkapitals dieser Unternehmen wäre dabei eine »finanzielle Transaktion« für den Bund und würde daher nicht unter die Regeln der Schuldenbremse fallen. Die Bundes-AGs könnten zudem Finanzmittel durch Fremdfinanzierung aufnehmen, was hauptsächlich durch Ausgabe eigener Anleihen oder Aufnahme zinsgünstiger Darlehen erfolgen sollte.

Die Einnahmen der Beratungsgesellschaft "Zukunft Wohnen« würden im Wesentlichen aus den Beiträgen der Länder und Kommunen und den Beratungsgebühren (wie bereits heute bei der »Partnerschaft Deutschland «) bestehen. Der Beteiligungsfonds "Zukunft Wohnen« würde eine Kompensation für die Eigenkapitalbeteiligung von der jeweiligen kommunalen Wohnungsbaugesellschaft erhalten. Die Einnahmen des Bodenfonds »Zukunft Wohnen« bestünden aus einer eventuellen Kompensation der jeweiligen Kommunen für die geleistete Unterstützung bei dem Erwerb von Grund und Boden. Da Wohnungsbauinvestitionen in der langen Frist stabile und gute Renditen erwirtschaften und das Risiko von dauerhaften Forderungsausfällen gering ist, können diese Gebühren so günstig ausfallen, dass es den kommunalen Wohnungsbauunternehmen ermöglicht wird, preisgünstige Wohnungen anzubieten.

\section{Schlussfolgerungen}

Wohnen ist ein Grundrecht und ein essenzielles Element eines menschenwürdigen Lebens. Zugleich gibt es genügend theoretische und empirische Gründe, 
warum ein freier Markt nicht ausreichend Wohnraum insbesondere für ärmere Haushalte zur Verfügung stellt. Deshalb ist es nicht ausreichend, einfach die Einkommen jener aufzustocken, die mit hohen Wohnkosten im Verhältnis zu ihren Einkommen konfrontiert sind. Eine solche Politik mag zwar individuelle finanzielle Nöte lindern, dürfte aber nicht dazu beitragen, dass wirklich eine ausreichende Menge bezahlbaren Wohnraums in attraktiven Lagen entsteht. Hier sollte vielmehr direkt an der Angebotsseite angesetzt werden.

Viel spricht dafür, dass ein stärkerer öffentlicher Wohnungsbau helfen könnte, die angespannte Situation auf dem Wohnungsmarkt zu entspannen. Eine Bundesinitative wie hier vorgeschlagen mit starker Einbindung der Kommunen hätte das Potenzial, den öffentlichen Wohnungsbau in angespannten Wohnungsmärkten bundesweit zu beflügeln und günstig und effizient Wohnraum bereitzustellen.

\section{Literatur}

Ahlfeldt, G. M./Redding, S.J./Sturm, D.M./Wolf, N. (2015): The Economics of Density: Evidence from the Berlin Wall, in: Econometrica 83(6), S. 2127-2189.

Arnott, R.J. (1995): Time for Revisionism on Rent Control?, in: Journal of Economic Perspectives 9(1), S. 99-120.

Backes, J. (2018): Bauprojekt in Kreuzberg auf Eis. Investor attackiert Berliner Wohnungspolitik mit Riesen-Plakat, in: Berliner Zeitung vom 22.8.2018, www.bz-berlin.de/berlin/friedrichshain-kreuzberg/investor-attackiert-berli ner-wohnungspolitik-mit-riesen-plakat (Abruf am 9.7.2021).

Baldenius, T./Kohl, S./Schularick, M. (2019): Die neue Wohnungsfrage. Gewinner und Verlierer des deutschen Immobilienbooms, Bonn, www.researchgate. net/publication/333856266_Die_neue_Wohnungsfrage_Gewinner_und_Ver lierer_des_deutschen_Immobilienbooms (Abruf am 9.7.2021).

Bundesverfassungsgericht (2021): Beschluss vom 25. März 2021, Az. 2 BvF 1/20 2 BvL 4/20 - 2 BvL 5/20, www.bundesverfassungsgericht.de/SharedDocs/Ent scheidungen/DE/2021/03/fs20210325a_2bvf000120.html (Abruf am 29.7.2021).

Chetty, R./Hendren, N. (2018): The Impacts of Neighborhoods on Intergenerational Mobility I: Childhood Exposure Effects, in: Quarterly Journal of Economics 113(3), S. 1163-1228.

Destatis (2019): Städte-Boom und Baustau: Entwicklungen auf dem deutschen Wohnungsmarkt 2008-2018, Pressemitteilung Nr. N 012 vom 4. Dezember 2019, Wiesbaden.

Deutscher Bundestag (2019): Antwort der Bundesregierung auf die Kleine Anfrage der Abgeordneten Caren Lay, Dr. Gesine Lötzsch, Lorenz Gösta Beutin, weite- 
rer Abgeordneter und der Fraktion DIE LINKE, Drucksache 19/12786, Berlin, https://dserver.bundestag.de/btd/19/127/1912786.pdf (Abruf am 9.7.2021).

Dullien, S./Joebges, H./Márquez-Velázquez, A. (2016): Wann verursachen Niedrigzinsen Hauspreisblasen? Lehren aus einem systematischen Fallstudienansatz, in: Vierteljahreshefte zur Wirtschaftsforschung 85(1), S. 125-138.

Dullien, S./Krebs, T. (2020): Wege aus der Wohnungskrise. Vorschlag für eine Bundesinitiative "Zukunft Wohnen«, IMK Report 156, Düsseldorf.

Holm, A./Lebuhn, H./Junker, S./Neitzel, K. (2018): Wie viele und welche Wohnungen fehlen in deutschen Großstädten?, Working Paper Forschungsförderung Nr. 63 der Hans-Böckler-Stiftung, Düsseldorf.

Kholodilin, K./Mense, A./Michelsen, C. (2018): Mietpreisbremse ist besser als ihr Ruf, aber nicht die Lösung des Wohnungsproblems, in: DIW-Wochenbericht 7/2018, S. 107-117.

Kunnert, A./Baumgartner, J. (2012): Instrumente und Wirkungen der österreichischen Wohnungspolitik, Österreichisches Institut für Wirtschaftsforschung (WIFO), Wien.

OECD (2021): OECD Affordable Housing Database, www.oecd.org/housing/ data/affordable-housing-database/ (Abruf am 24.3.2021).

Pestel-Institut = Eduard Pestel Institut e. V. (2019): Kurzstudie. Leitfaden für soziales und bezahlbares Wohnen in Deutschland, Hannover, www.impulse-fuerden-wohnungsbau.de/fileadmin/images/Pressekonferenzen/STUDIE_Akut plan_soziales_und_bezahlbares_Wohnen_in_Deutschland_-_Pestel-Institut. pdf (Abruf am 9.7.2021).

Prognos AG (2017): Studie Wohnungsbautag 2017. Wohnraumbedarf in Deutschland und den regionalen Wohnungsmärkten, Stuttgart/Freiburg.

SVR = Sachverständigenrat zur Begutachtung der gesamtwirtschaftlichen Entwicklung (2018): Vor wichtigen wirtschaftspolitischen Weichenstellungen. Jahresgutachten 2018/19, Wiesbaden.

Südekum, J. (2010): Human Capital Externalities and Growth of High- and LowSkilled Jobs, in: Journal of Economics and Statistics 230(1), S. 92-114.

Wiedemeier, J. (2018): Wohnraum in Berlin. Sozialwohnungsquote - eine Regel voller Ausnahmen, in: Der Tagesspiegel vom 27.1.2018, www.tagesspiegel. de/berlin/wohnraum-in-berlin-sozialwohnungsquote-eine-regel-voller-aus nahmen/20892018.html (Abruf am 9.7.2021).

Wissenschaftlicher Beirat $=$ Wissenschaftlicher Beirat beim Bundesministerium für Wirtschaft und Energie (2018): Soziale Wohnungspolitik, Berlin. 


\section{Daseinsvorsorge in der Gesundheitspolitik}

Thomas Gerlinger

\section{Einleitung}

Der Begriff der Daseinsvorsorge beschreibt die Verantwortung des Staates für die Sicherstellung jener Leistungen und Strukturen, auf die Einzelne für ihre Existenzsicherung angewiesen sind, die sie aber aufgrund der fortschreitenden Arbeitsteilung und des wissenschaftlich-technischen Fortschritts gesellschaftlicher Verflechtungen nicht mehr allein gewährleisten können (Forsthoff 1938 und 1958). Die Gesellschaftsmitglieder müssen davon ausgehen können, dass diese Leistungen und Strukturen ohne Wenn und Aber zur Verfügung stehen, weshalb ihre Bereitstellung nicht von den Regeln des Marktes abhängig gemacht werden kann, sondern in der Verantwortung des Staates liegen muss.

Dabei sind nicht nur Bund und Länder, sondern in besonderer Weise auch die Kommunen gefordert. Zur staatlichen Daseinsvorsorge zählen bekanntlich Wasser- und Energieversorgung, Abwasserbeseitigung und Müllentsorgung, öffentlicher Personenverkehr und Bildung, aber auch die Gesundheits- und Krankenversorgung und die Vorhaltung einer entsprechenden Infrastruktur (Dahme/Wohlfahrt 2011; Klenk/Reiter 2012). Die öffentlich bereitgestellten Leistungen der Daseinsvorsorge repräsentieren, so Wolfgang Streeck, einen »alltäglichen Kommunismus, der unserem alltäglichen Kapitalismus unterliegt und ihn überhaupt erst ermöglicht« (Streeck 2019, S. 7).

Gesundheits- bzw. krankheitsbezogene Leistungen beschreiben wiederum ein weites Feld, das von der Gesundheitsförderung und Prävention über die Krankenversorgung bis hin zur Pflege und Palliativversorgung reicht. Im Folgenden soll es um einen Kernbereich dieses Spektrums gehen, nämlich die Vorhaltung einer angemessenen Infrastruktur für die stationäre und ambulante Krankenversorgung. Dieser Beitrag geht der Frage nach, welche Leistungen und Defizite auf diesem Feld anzutreffen sind, wodurch sich insbesondere die Defizite erklären und welche Möglichkeiten zur Problemlösung existieren. 
Zunächst wird dargelegt, welche grundlegenden Anforderungen im Sinne einer Daseinsvorsorge an die Vorhaltung von Versorgungseinrichtungen zu stellen sind (Abschnitt 2). Sodann wird zunächst für die Krankenhausversorgung (Abschnitt 3), anschließend für die ambulante Versorgung (Abschnitt 4) der IstZustand beschrieben und die Gründe für die existierenden Defizite erläutert. Anschließend werden Lösungsansätze vorgestellt und Hindernisse bei deren Umsetzung skizziert (Abschnitt 5).

\section{Daseinsvorsorge in der Krankenversorgung}

Ein gutes Krankenversorgungssystem muss gewährleisten, dass jede erkrankte Person in angemessener Zeit und so gut wie möglich versorgt wird. Im Mittelpunkt steht damit der Begriff der Bedarfsgerechtigkeit. Das Fünfte Buch Sozialgesetzbuch (SGB V) kann man als Kompendium für die Anforderungen verstehen, die daraus erwachsen. So verlangt es - im Hinblick auf die gesetzliche Krankenversicherung - »eine bedarfsgerechte und gleichmäßige, dem allgemein anerkannten Stand der medizinischen Erkenntnisse entsprechende Versorgung der Versicherten« ( $\$ 70$ Abs. 1 Satz 1 SGB V). Und weiter:

„Die Versorgung der Versicherten muss ausreichend und zweckmäßig sein, darf das $\mathrm{Maß}$ des Notwendigen nicht überschreiten und muss in der fachlich gebotenen Qualität sowie wirtschaftlich erbracht werden.«( $\$ 70$ Abs. 1 Satz 2 SGB V)

Diese Anforderungen, die sich primär auf die Leistungserbringung selbst beziehen, haben Implikationen für die Vorhaltung von Einrichtungen der Krankenversorgung: Diese müssen nach Anzahl, räumlicher Verteilung, fachlicher Ausrichtung und personeller wie technischer Ausstattung so bemessen sein, dass ein adäquater Zugang der Bevölkerung möglich ist. Dabei sind auch regionale Unterschiede des Versorgungsbedarfs (z. B. Alter und Morbidität) und der Siedlungsstruktur zu berücksichtigen.

Daseinsvorsorge beinhaltet also die Kombination verschiedener Eigenschaften: schnelle Erreichbarkeit und damit Wohnortnähe der Versorgungseinrichtungen insbesondere in Notfällen, aber auch eine personell-technische Ausstattung von Versorgungseinrichtungen, die es erlaubt, Leistungen in hoher Qualität nach dem Stand der medizinischen Erkenntnisse zu erbringen. Zwischen diesen unterschiedlichen Gesichtspunkten müssen nicht zwangsläufig, können aber durchaus Widersprüche und Trade-offs auftreten. Zudem müssen die Leistungserbringer:innen unter Bedingungen handeln können, die es ihnen erlauben, ihr Handeln am Wohl der Patient:innen auszurichten. 
Schließlich erstreckt sich Daseinsvorsorge noch auf einen weiteren, über die reine Krankenversorgung hinausweisenden und das soziale Sicherungssystem einschließenden Aspekt: Vorausgesetzt ist stets, dass der Zugang zu Versorgungseinrichtungen nicht durch soziale Hürden beschränkt werden darf.

Im Folgenden stehen zwei Aspekte im Mittelpunkt, die für die Daseinsvorsorge im Gesundheitssystem besonders problematisch sind: das Nebeneinander von Über- und Unterversorgung bei der Vorhaltung von Einrichtungen der Krankenversorgung und der Bedeutungszuwachs privater Akteure, vor allem großer Finanzinvestoren, als Träger dieser Einrichtungen. Zunächst wird der Krankenhaussektor behandelt (Abschnitt 3), daran anschließend der Bereich der ambulanten Versorgung (Abschnitt 4).

\section{Daseinsvorsorge im Krankenhaussektor}

Der Krankenhaussektor ist der wohl am stärksten vom Staat geprägte Sektor des deutschen Gesundheitswesens. Insbesondere die Länder spielen hier eine wichtige Rolle, denn sie haben den sogenannten Sicherstellungsauftrag inne, also die Verantwortung, eine bedarfsgerechte Krankenhausinfrastruktur sicherzustellen. Sie nehmen diese Aufgabe wahr, indem sie

- einen Krankenhausbedarfsplan erstellen ( $\$ 6$ des Krankenhausfinanzierungsgesetzes/KHG) und damit über die in ihrem Zuständigkeitsbereich erforderlichen Krankenhäuser und Betten entscheiden und

- die für die Versorgung erforderlichen Investitionsmittel bereitstellen.

Die konkrete Durchführung der Krankenhausplanung wird von Bundesland zu Bundesland unterschiedlich gehandhabt: Während einige Länder sich auf Rahmenvorgaben wie die Standorte der Krankenhäuser beschränken und alles Weitere den Verhandlungen von Krankenkassen und Krankenhausträgern überlassen, arbeiten andere konkrete Vorgaben aus, die bisweilen sogar die Gliederung einzelner Abteilungen beinhalten (Deutsche Krankenhausgesellschaft 2019).

Deutschland weist im internationalen Vergleich trotz eines jahrzehntelangen kräftigen Bettenabbaus (Statistisches Bundesamt 2020) eine sehr hohe Bettendichte auf (OECD 2020; Eurostat 2021). Zahlreiche Expert:innen verbinden dies mit der Schlussfolgerung, dass in der Krankenhausversorgung - insgesamt betrachtet - erhebliche Überkapazitäten existieren (z. B. Loos/Albrecht/Zich 2019), und erhalten darin Unterstützung durch die Krankenkassen. Daher ist 
die Forderung nach einem deutlichen Bettenabbau und einer Reduzierung der Krankenhausstandorte weit verbreitet.

Zwar ist einzuwenden, dass der internationale Vergleich und die hohe Bettendichte allein noch nichts über etwaige Überkapazitäten aussagen, denn die geringere Bettendichte in anderen Ländern kann auch ein Hinweis auf eine dortige Unterversorgung sein, die in einigen Ländern - wie z. B. Großbritannien, den Niederlanden oder Schweden - in der Tat anzutreffen ist und dort zu teilweise langen Wartelisten bei bestimmten Eingriffen führt (Tikkanen et al. 2020; Schölkopf/Grimmeisen 2021).

Darüber hinaus wäre darauf hinzuweisen, dass angesichts des demografischen Wandels in den kommenden Jahrzehnten ein Anstieg der Behandlungsfallzahlen in Krankenhäusern zu erwarten ist. Ob dieser Trend noch durch den medizinisch-technischen Fortschritt verstärkt wird oder ob eine weitere Verkürzung der Verweildauer je Behandlungsfall und die von der Medizintechnik getriebene Ambulantisierung der Krankenversorgung diesen Trend abschwächen oder gar überkompensieren werden, lässt sich kaum vorhersagen.

Schließlich hat sich während der Covid-19-Pandemie die hohe Bettendichte einschließlich der Intensivbetten als ein Vorzug des deutschen Gesundheitssystems erwiesen. Dennoch traten auch hier im Zuge der Pandemie Engpässe auf. Mahnen allein schon diese Erfahrungen zur Vorsicht bei der Diskussion über einen Bettenabbau, so wird man die Pandemieerfahrungen allerdings auch nicht als Legitimation für eine dauerhafte Aufrechterhaltung erheblicher Überkapazitäten heranziehen können.

Selbstverständlich erfordert eine auf Daseinsvorsorge gerichtete Krankenhausplanung auch die Vorhaltung von Kapazitäten für die adäquate Versorgung etwaiger Bedarfsspitzen. Ungeachtet dessen ist insbesondere in Ballungsräumen für bestimmte Fachabteilungen die Zahl der aufgestellten Betten aber derart hoch, dass man von einer Überversorgung ausgehen muss, auch wenn man in Rechnung stellt, dass die dort vorgehaltenen Krankenhäuser eine Versorgungsfunktion für das nähere oder weitere Umland wahrnehmen. Es geht also nicht nur um die Anzahl der Betten, sondern auch um deren räumliche Verteilung.

Die Debatte über die Krankenhausstrukturen schließt aber noch einen weiteren Aspekt ein, nämlich die Qualität des Leistungsgeschehens (z. B. Loos/Albrecht/Zich 2019), denn es gibt für eine Reihe von Leistungen - insbesondere für komplexe Eingriffe, die nicht zur Routine eines Fachgebiets gehören - Hinweise auf oder Belege für einen Zusammenhang zwischen der Häufigkeit, in der eine Behandlung durchgeführt wird, und deren Qualität. Dennoch werden derartige Leistungen oft auch in kleineren Häusern erbracht, oft zum Nachteil der Patient:innen. 
Stattdessen sei, so die Kritik an den bestehenden Strukturen, bei zahlreichen planbaren Behandlungen eine Zentrenbildung und Spezialisierung erforderlich. Eine solche Konzentration könne zwar die Wege zwischen Wohnort und Krankenhausstandort verlängern, verbessere aber im Gegenzug die Versorgungsqualität. Auch wenn es kleine Häuser gibt, die bei den Qualitätsindikatoren für derartige Leistungen nicht schlechter abschneiden als manche größere, so gibt es doch eine Reihe von empirischen Hinweisen, die diese Kritik im Grundsatz stützen.

Neben der Krankenhausplanung erfordert die »bedarfsgerechte Versorgung der Bevölkerung mit leistungsfähigen, eigenverantwortlich wirtschaftenden Krankenhäusern « ( $\$ 1$ Abs. 1 KHG) eine wirtschaftliche Sicherung der betreffenden Einrichtungen. Die sich daraus ergebende Pflicht der Länder zur Finanzierung der Krankenhausinvestitionen betrifft den Krankenhausbau, die Erstausstattung der Krankenhäuser und die Neubeschaffung von Anlagegütern. Diese Verantwortung des Staates für die Krankenhausinvestitionen ist Ausdruck der verbreiteten Auffassung, dass die Gewährleistung ausreichender Versorgungskapazitäten Gegenstand der öffentlichen Daseinsvorsorge ist.

Die in den Landeskrankenhausplan aufgenommenen Krankenhäuser (»Plankrankenhäuser«) haben einen Rechtsanspruch auf Investitionsfinanzierung durch das Land. Im Unterschied zu den Investitionen werden die laufenden Betriebskosten eines Krankenhauses von den Krankenkassen getragen (»duale Finanzierung«; $\$ 4$ KHG). In der Praxis sind aber auch die Krankenhausträger an der wirtschaftlichen Sicherung von Krankenhäusern beteiligt, indem sie etwaige Defizite durch Zuschüsse ausgleichen. Dies trifft besonders häufig öffentliche Träger, vor allem Kommunen.

Die meisten Länder haben ihre Pflicht zur Krankenhausfinanzierung in der Vergangenheit nicht oder nur unzureichend erfüllt (z. B. Augurzky et al. 2011; Deutsche Krankenhausgesellschaft 2019). Allein zwischen 1991 und 2018 sank der Anteil der gemäß dem KHG bewilligten Investitionsmittel am Bruttoinlandsprodukt (BIP) von 0,24 Prozent auf 0,09 Prozent (Deutsche Krankenhausgesellschaft 2019, S. 77), obwohl im gleichen Zeitraum der Anteil der Krankenhausausgaben am BIP deutlich anstieg. Selbst nominal gingen die Investitionsmittel während dieses Zeitraums um 16 Prozent zurück. Im Jahr 2015 beliefen sich die Investitionen durch KHG-Fördermittel auf 3,5 Prozent des Krankenhausumsatzes (RWI 2017, S. 21).

Der GKV-Spitzenverband, der Verband der Privaten Krankenversicherung und die Deutsche Krankenhausgesellschaft (2019) bezifferten für das Jahr 2019 den bestandserhaltenden Investitionsbedarf für die Krankenhäuser auf deutlich mehr als 6 Milliarden Euro, wohingegen sich die Summe der KHG-Fördermittel 
im Jahr 2018 gerade einmal auf 3 Milliarden Euro belief (Deutsche Krankenhausgesellschaft 2019).

Angesichts der Zurückhaltung der Länder setzen die Krankenhäuser in beträchtlichem Umfang Eigenmittel für ihre Investitionen ein (Augurzky et al. 2011, S. 36 ff.; Deutsche Krankenhausgesellschaft 2019) - eine vierte Quelle der Krankenhausfinanzierung. Dies setzt natürlich voraus, dass die Krankenhäuser tatsächlich Gewinne erwirtschaften, was auf viele von ihnen - insbesondere in öffentlicher Trägerschaft - nicht zutriff.

Im Ergebnis führt die unzureichende Finanzierung von Krankenhausinvestitionen durch die Länder dazu, dass zahlreiche Krankenhäuser für die Wahrnehmung ihrer Versorgungsaufgaben nicht angemessen ausgestattet sind. Dieser Effekt ist mit dem Anliegen der Daseinsvorsorge nicht vereinbar.

Zur Krankenhausplanung und Krankenhausfinanzierung tritt ein weiterer Aspekt, der für die Daseinsvorsorge in der Krankenhausversorgung von Bedeutung ist. Traditionell weist Deutschland eine gemischtwirtschaftliche Trägerstruktur auf, geprägt von öffentlichen, privaten und freigemeinnützigen Trägern. Dies ist auch heute noch der Fall, auch wenn seit Jahrzehnten private Träger auf dem Vormarsch sind.

Waren 1991 lediglich 14,8 Prozent aller Krankenhäuser (4,0 Prozent der Krankenhausbetten) in privater Trägerschaft, so war dieser Anteil im Jahr 2018 auf 37,6 Prozent (19,1 Prozent) gestiegen. Insbesondere hat der Anteil von groBen Kapitalgesellschaften, vor allem von Aktiengesellschaften im Vergleich zu Ärzt:innen als privaten Trägern deutlich zugenommen. Der Zuwachs privater Krankenhäuser geht vor allem zulasten der öffentlichen Häuser, deren Anteil in diesem Zeitraum von 46,0 auf 28,7 Prozent zurückging. Allerdings stand 2018 noch fast jedes zweite Bett (48,0 Prozent) in einem öffentlichen Krankenhaus (Statistisches Bundesamt 2020).

Privatisierung kommt aber nicht allein im rückläufigen Anteil öffentlicher Träger zum Ausdruck, sondern auch im Wandel der Rechtsformen öffentlicher Krankenhäuser. Während 2002 nur 28,3 Prozent von ihnen in privatrechtlicher Form (z. B. als GmbH) geführt wurden, waren es 2017 bereits 59,8 Prozent (Statistisches Bundesamt 2018, S. 8). Unter den in öffentlich-rechtlicher Form betriebenen Krankenhäusern nahm der Anteil der rechtlich unselbstständigen Einrichtungen (z. B. Eigenbetriebe oder Regiebetriebe) gegenüber den rechtlich selbstständigen (z. B. Zweckverband, Anstalt oder Stiftung) stark ab. Ihr Anteil an allen öffentlichen Krankenhäusern sank in diesem Zeitraum von 56,9 Prozent auf 15,0 Prozent (Statistisches Bundesamt 2018, S. 8). Private Rechtsformen und ein rechtlich selbstständiger Status gestatten dem Krankenhaus eine größere Unabhängigkeit vom jeweiligen Träger. 
Die Privatisierung von Krankenhäusern bringt unter dem Gesichtspunkt der Daseinsvorsorge erhebliche Probleme mit sich. Private Träger treffen betriebswirtschaftliche Entscheidungen unter Gewinnaspekten. Steht bei öffentlichen Krankenhäusern - durchaus auch unter Verfolgung von Gewinninteressen - die Versorgungsfunktion im Mittelpunkt, so ist es bei privaten Trägern die Gewinnmaximierung. Dieses Ziel bestimmt das Bettenangebot und die Leistungserbringung ebenso wie die technische und personelle Ausstattung ihrer Häuser. So setzen viele private Krankenhäuser darauf, durch eine Spezialisierung Skaleneffekte zu erzielen und auf diesem Weg ihre Gewinne zu steigern. Derartige Entscheidungen mögen nicht immer mit dem Versorgungsbedarf der Bevölkerung übereinstimmen.

Das Nebeneinander von öffentlichen und freigemeinnützigen Häusern einerseits und privaten Einrichtungen andererseits bringt eine Reihe von Problemen mit sich. Aufgrund der Spezialisierung und der höheren Behandlungsfallzahlen können private Einrichtungen kostengünstiger - und zum Teil auch in einer besseren Qualität - Leistungen erbringen. Dadurch verschlechtert sich die Kalkulationsbasis derjenigen - zumeist öffentlichen - Krankenhäuser, die aufgrund ihres Versorgungsauftrags nach wie vor die gesamte Palette an Leistungen und die dafür notwendige Infrastruktur vorhalten müssen, ohne an der Kostenersparnis bei standardisierbaren Leistungen teilhaben zu können (Böhlke et al. 2009).

Die Privatisierung von Krankenhäusern folgte und folgt keinem übergreifenden politischen Plan. Gleichwohl ist sie insbesondere aus wirtschaftsliberaler Perspektive ein durchaus erwünschter Trend, der die Folge einer Vielzahl von Einzelentscheidungen vor allem kommunaler Träger ist. Kommunen waren und sind angesichts ihrer prekären Haushaltslage häufig bemüht, sich ihrer nicht selten defizitären Krankenhäuser zu entledigen. Zudem folgten manche Kommunen in den 1990er- und 2000er-Jahren auch dem neoliberalen Zeitgeist, der in der Privatisierung kommunaler Aufgaben generell ein geeignetes Instrument für Kostensenkung und Qualitätsverbesserung sah. Konzepte des New Public Management drangen auch in die Krankenversorgung vor. Die seit 2011 verbindliche Schuldenbremse erhöhte den Druck zum Verkauf von Krankenhäusern weiter.

Es sank also sowohl die Fähigkeit als auch die Bereitschaft von Kommunen, Defizite ihrer Krankenhäuser zu decken. Häufig traten und treten in solchen Fällen private Kapitalgesellschaften auf, um potenziell profitable Häuser zu übernehmen und nach eigenen Vorstellungen umzugestalten. Zumeist gelingt es ihnen dann, die Krankenhäuser profitabel zu machen (Böhlke et al. 2009; Simon 2019a). Die Privatisierung des Krankenhaussektors schreitet auch aktuell weiter voran, auch wenn sich die Dynamik in den letzten Jahren etwas abgeschwächt hat (Simon 2019b). 


\section{Ambulante (vertragsärztliche) Versorgung}

Ebenso wie die Krankenhäuser unterliegen auch die zur ambulanten Versorgung von Kassenpatient:innen zugelassenen Ärzt:innen (Vertragsärzt:innen) einer Bedarfsplanung. Den Sicherstellungsauftrag für die vertragsärztliche Versorgung haben aber nicht die Länder, sondern die Kassenärztlichen Vereinigungen inne.

Dieses Verfahren ist eingebunden in das für die gesetzliche Krankenversicherung charakteristische System der gemeinsamen Selbstverwaltung der Ärzt:innen und Krankenkassen: Der Gesetzgeber legt einen Rechtsrahmen fest, der durch Verhandlungen der Verbände der Ärzt:innen und Krankenkassen ausgefüllt wird, wobei die dabei getroffenen Entscheidungen und vereinbarten Verträge der Genehmigung durch die zuständige Exekutive unterliegen (Gerlinger/ Rosenbrock 2021). In diesem Rahmen legt der Gemeinsame Bundesausschuss (G-BA), das oberste Gremium der gemeinsamen Selbstverwaltung der Ärzt:innen und Krankenkassen, nach gesetzlichen Rahmenvorgaben Sollwerte für das Verhältnis von Bevölkerung und Vertragsärzt:innen fest, und zwar für einzelne Ärztegruppen und in den verschiedenen Raumordnungskategorien.

Auf der regionalen Ebene können die Landesausschüsse der Ärzt:innen und Krankenkassen von den bundesweiten Rahmenvorgaben abweichen, z. B. durch die Feststellung von alters- oder morbiditätsbedingten Sonderbedarfen. Bei einer Überversorgung, die beim Erreichen von 110 Prozent des Sollwerts vorliegt, muss der Zulassungsbezirk für die Zulassung weiterer Ärzt:innen geschlossen werden.

Eine Unterversorgung liegt dann vor, wenn die Ärztedichte in der hausärztlichen Versorgung weniger als 75 Prozent und in der fachärztlichen Versorgung weniger als 50 Prozent des Sollwerts beträgt. Dieses Planungsverfahren betrifft nur die Vertragsärzt:innen - Ärzt:innen, die ausschließlich Privatleistungen erbringen, werden dadurch nicht erfasst. Für sie gibt es somit keine Niederlassungsbeschränkungen.

Zwar sind die Verhältniszahlen, die der G-BA nach Arztgruppen und Gebietstypen zugrunde gelegt hat, nicht unbedingt ein valider Indikator für eine bedarfsgerechte Versorgung, denn sie beruhen im Hinblick auf den Versorgungsbedarf auf den Ist-Verhältnissen Anfang der 1990er-Jahre und nicht auf einer aktuellen objektiven Ermittlung. Zudem berücksichtigen sie nicht die in der Zwischenzeit in der ambulanten Versorgung eingetretene Veränderung der Versorgungssituation (geprägt vor allem durch medizinischen Fortschritt, demografischen Wandel und Verkürzung der stationären Verweildauer). Ungeachtet dessen gibt der Vergleich zwischen Bedarfsplanungsdaten und tatsächlichen Verhältniszahlen einen Hinweis auf die Versorgungslage bei einzelnen Arztgruppen und in einzelnen Regionen. 
Der Blick auf die aggregierten Daten zeigt zunächst, dass die Gesamtzahl der Ärzt:innen in Deutschland - bei einer nur geringfügig steigenden Bevölkerungszahl - kontinuierlich ansteigt, auch in der vertragsärztlichen Versorgung (Kassenärztliche Bundesvereinigung 2021). Ebenso wie die Bettendichte ist auch die Arztdichte in der ambulanten Versorgung im internationalen Vergleich außerordentlich hoch (OECD 2020; Eurostat 2021).

Der Vergleich zwischen Bedarfsplanungsdaten und tatsächlichen Verhältniszahlen verdeutlicht, dass bei allen Ärztegruppen, die der Bedarfsplanung unterliegen, die Versorgungsgrade im bundesweiten Durchschnitt den Sollwert überschreiten, der sich aus den Bedarfsplanungsrichtlinien ergibt. Besonders deutlich fiel diese Überschreitung bei den Fachärzt:innen aus, aber auch bei Hausärzt:innen lag der Gesamtversorgungsgrad im Jahr 2016 immerhin noch bei 108 Prozent (Klose/Rehbein 2017, S. 8). Von den Hausärzt:innen abgesehen, waren bei allen Ärztegruppen fast sämtliche Planungsbereiche überversorgt; nur bei den Hausärzt:innen gab es in erheblichem Umfang noch freie Planungsbereiche.

Auf Landesebene aggregiert, gab es bei den Hausärzt:innen in keinem Bundesland eine Unterversorgung. Rund 40 Prozent aller Planungsbereiche waren überversorgt, legt man die vom G-BA vorgegebenen Verhältniszahlen unter Einschluss der Optionen zur regionalen Abweichung zugrunde (Klose/Rehbein 2017, S. 8). Insgesamt gab es bundesweit selbst in der hausärztlichen Versorgung einen Überschuss von insgesamt knapp 3.900 Ärzt:innen (Klose/Rehbein 2017, S. 9); in manchen Planungsbezirken werden die Soll-Werte in nachgerade eklatanter Weise überschritten.

Im Hinblick auf die Verteilung der Ärzt:innen wird aber deutlich, dass den erheblichen Überkapazitäten eine drohende oder bereits eingetretene Unterversorgung in einigen strukturschwachen Räumen gegenübersteht. 2016 existierte in der hausärztlichen Versorgung in insgesamt 8 der bundesweit 957 Planungsbereiche eine Unterversorgung. In weiteren 86 Planungsbereichen lag der Versorgungsgrad zwischen 75 und unter 90 Prozent, in 169 Planungsbereichen zwischen 90 und unter 100 Prozent (Klose/Rehbein 2017, S. 30). In vielen Disziplinen reicht bereits die Aufgabe eines einzigen nicht wieder besetzten Vertragsarztsitzes aus, um eine Unterversorgung herbeizuführen.

Das Nebeneinander von Überversorgung und drohender oder bereits eingetretener Unterversorgung lässt sich im Verhältnis zwischen Ballungsräumen sowie einigen aus anderen Gründen attraktiven Regionen, z. B. der Insel Sylt oder dem Landkreis Starnberg - einerseits und strukturschwachen Landstrichen andererseits erkennen. Darüber hinaus treten aber auch innerhalb von Ballungsräumen erhebliche Unterschiede in den Versorgungsgraden auf. Insbesondere Stadtteile, in denen der Bevölkerungsanteil von Arbeitslosen oder von Sozial- 
hilfeempfänger:innen hoch und der von Privatpatient:innen besonders niedrig ist, weisen eine erheblich geringere Ärztedichte auf als wohlhabendere Bezirke und sind auf manchen Fachgebieten sogar von einer Unterversorgung betroffen.

Die Gründe für das Nebeneinander von Über- und Unterversorgung sind vielschichtig. Für viele Ärzt:innen ist die Niederlassung in ländlichen, strukturschwachen Regionen wegen der dortigen Lebensbedingungen unattraktiv. Ferner haben sie dort und in sozial benachteiligten Stadtteilen meist geringere Möglichkeiten als in Kernstädten, ihre Einnahmen durch privatärztliche Tätigkeit zu erhöhen, denn sowohl der Anteil von Privatpatient:innen als auch der von zahlungskräftigen GKV-Patient:innen ist dort gering.

Im Hinblick auf die hausärztliche Versorgung kommt hinzu, dass die Zahl der altersbedingt aus der Berufstätigkeit ausscheidenden Ärzt:innen die Zahl der Nachrücker:innen deutlich übersteigt und die Vergütung von Hausärzt:innen hinter vielen Facharztgruppen zurückbleibt (Gerlinger/Rosenbrock 2021). Ohne wirksame Interventionen dürften sich die Ungleichheiten bei der ärztlichen Versorgung weiter verschärfen, denn dass die skizzierten Trends zum Stillstand kämen geschweige denn sich umkehren würden, ist nicht zu erwarten.

Neben dem Problem der gleichzeitigen Über- und Unterversorgung ist die Privatisierung von Versorgungseinrichtungen für die Daseinsvorsorge in der vertragsärztlichen Versorgung von wachsender Bedeutung, auch wenn dies von der Öffentlichkeit nicht so stark wahrgenommen wird wie im Falle der stationären Versorgung. Große Kapitalgesellschaften dringen nicht nur in die stationäre, sondern auch in die ambulante Versorgung ein. Diese ist nach wie die Domäne frei niedergelassener Ärzt:innen, ob in Einzel- oder Gemeinschaftspraxen. Insofern basiert sie auf der Investition privaten Kapitals, aber eben nicht auf der Investition großer Kapitalgesellschaften. Genau dies ist im Begriff sich zu verändern. Der Weg, auf dem dies geschieht, ist die Investition in Medizinische Versorgungszentren (MVZ).

MVZ wurden erstmals 2004 zur ambulanten Versorgung von Kassenpatient:innen (»vertragsärztliche Versorgung«) zugelassen. Bei ihnen handelt es sich um ambulante Versorgungseinrichtungen, in denen Angehörige verschiedener ärztlicher Fachgruppen (als Selbstständige oder Angestellte) die Versorgung übernehmen. Träger eines MVZ können alle zugelassenen Leistungserbringer nach SGB V sein; insbesondere Ärztegemeinschaften und Krankenhäuser kommen dafür infrage. Krankenkassen, Wohlfahrtsverbände, aber auch pharmazeutische Unternehmen sind von der Trägerschaft ausgeschlossen, weil sie keine Leistungen unmittelbar am Patienten bzw. der Patientin erbringen.

MVZ sollen die fächer- und berufsübergreifende Kooperation im Gesundheitswesen erleichtern und so die ambulante Versorgungsqualität verbessern. 
Idealerweise arbeiten dort Ärzt:innen unterschiedlicher Disziplinen und nichtärztliche Gesundheitsberufe (z. B. Pflegekräfte, Physiotherapeut:innen, Psychotherapeut:innen etc.) unter einem Dach zusammen. Die Zulassung von MVZ hatte zum Ziel, die Integration von Versorgungseinrichtungen und -verläufen zu beschleunigen und die Kooperation an den Schnittstellen zur Krankenhausversorgung zu verbessern.

Für Ärzt:innen kann ein MVZ durch die gemeinsame Nutzung von Räumen, Personal und medizinisch-technischen Geräten neben den fachlichen Vorteilen einer intensiveren Kooperation auch ökonomische Vorteile bieten. Zudem ermöglicht ein MVZ mit wachsender Größe auch eine stärkere Arbeitsteilung und begründet damit für die beteiligten Leistungserbringer:innen auch die Aussicht auf geregelte Arbeitszeiten und eine Entlastung von bürokratischen Tätigkeiten (z. B. Leistungsabrechnung). Patient:innen wiederum können bei einer Versorgung im MVZ auf eine stärker interdisziplinär ausgerichtete Versorgung hoffen und mit kürzeren Wegen rechnen. Ärzt:innen können in einem MVZ auch als Angestellte arbeiten, seit 2007 sogar in unbegrenzter Zahl (Gerlinger/Rosenbrock 2021).

MVZ haben seit ihrer Zulassung zur vertragsärztlichen Versorgung eine rasante Entwicklung durchlaufen. Ende 2019 waren mehr als $3.500 \mathrm{MVZ}$ zugelassen, in denen knapp 22.000 Ärzt:innen tätig waren, davon gut 20.000 als Angestellte. Damit arbeiteten mehr als 12 Prozent der an der vertragsärztlichen Versorgung beteiligten Ärzt:innen in einem MVZ (Kassenärztliche Bundesvereinigung 2021).

Immerhin 43,1 Prozent der MVZ, in absoluten Zahlen knapp 1.600, befanden sich Ende 2019 in der Trägerschaft von Krankenhäusern - mit steigender Tendenz. Krankenhäuser sind aus verschiedenen Gründen an der Gründung von MVZ interessiert: Zum einen verbessert sich mit einem hauseigenen MVZ die Chance, Verweildauern in der stationären Versorgung durch frühzeitige Entlassung und ambulante Nachsorge zu verkürzen. Zum anderen verbessert ein MVZ auch die Chance, die Zahl der Krankenhauseinweisungen zu erhöhen und so den Krankenhausstandort langfristig zu sichern (Kassenärztliche Bundesvereinigung 2016, S. 21 und 26).

Allerdings birgt die einheitliche Trägerschaft von ambulanten und stationären Versorgungseinrichtungen auch Risiken. So können krankenhauseigene MVZ missbraucht werden, um Patient:innen zur stationären Versorgung in das angeschlossene Krankenhaus des Trägers einzuweisen - und zwar auch dann, wenn dieses Krankenhaus nicht das am besten geeignete ist. Eine Umfrage des Sachverständigenrats zur Begutachtung der Entwicklung im Gesundheitswesen (2012, Ziffer 330) zeigt die hohe Bereitschaft bzw. die Pläne von Krankenhäu- 
sern, ein MVZ zu gründen, bestehende MVZ zu vergrößern oder weitere MVZ zu errichten.

Das Eindringen großer Kapitalgesellschaften in die vertragsärztliche Versorgung vollzieht sich zum einen über die Trägerschaft von Krankenhäusern, denn unter denjenigen Krankenhäusern, die ein MVZ betreiben, befinden sich auch private Krankenhäuser. Zum anderen versuchten Private-Equity-Gesellschaften bereits kurz nach der Zulassung von MVZ im Jahr 2004, MVZ zu aufzukaufen oder Anteile an ihnen zu erwerben. Der Gesetzgeber schob dieser Praxis einen Riegel vor, indem er mit dem Versorgungsstrukturgesetz 2011 (BGBl. I, S. 2983) den Betrieb von MVZ auf Ärzt:innen, Krankenhäuser sowie Dialysedienstleister beschränkte.

Private-Equity-Gesellschaften umgehen diese Regelung, indem sie Krankenhäuser aufkaufen, auch in der Absicht, auf diesem Wege MVZ gründen zu können (Deutscher Bundestag 2019; Bobsin 2019; Scheuplein/Evans/Merkel 2019), oder indem sie Anteile an MVZ erwerben, die es ihnen gestatten, Einfluss auf die betreffenden Einrichtungen auszuüben (z. B. Korzilius 2018; Scheuplein/ Evans/Merkel 2019). Vor diesem Hintergrund ist die Vermutung begründet, dass solche Gesellschaften über ihre Trägerschaft von oder ihre Beteiligung an MVZ ungeachtet des tatsächlichen Versorgungsbedarfs die kurzfristigen Gewinninteressen ihrer Investor:innen verfolgen und die Unterordnung der medizinischen Versorgung unter ökonomische Verwertungsinteressen weiter verstärkt wird.

\section{Lösungsansätze}

Die Schaffung einer bedarfsgerechten Versorgungsinfrastruktur ist wie eingangs dargelegt eine zentrale Aufgabe der Daseinsvorsorge im Gesundheitswesen. Die skizzierten Probleme bei der Sicherstellung einer bedarfsgerechten Versorgungsinfrastruktur haben vielschichtige Ursachen. Daher gibt es auch nicht das eine Instrument oder die eine Maßnahme, die diese Probleme mit einem Schlag lösen würde.

Die Aufmerksamkeit bei der Daseinsvorsorge in der Krankenversorgung richtet sich traditionell - wie auch in anderen Bereichen - eher auf die Vermeidung einer Unterversorgung als einer Überversorgung, denn mit Unterversorgung geht eine Nichterfüllung eines als geboten oder zwingend zu erfüllenden Versorgungbedarfs einher. Es leuchtet unmittelbar ein, dass dies mit wohlfahrtsstaatlichen Zielen und Grundsätzen nicht vereinbar ist.

Es ist aber auch darauf hinzuweisen, dass eine Überversorgung im Sinne einer Daseinsvorsorge nicht hinnehmbar ist, denn sie verursacht nicht nur un- 
nötige Ausgaben für die Vorhaltung von Kapazitäten, sondern schafft für die Leistungsanbieter:innen auch Gelegenheit und Anreiz zur Durchführung medizinisch nicht notwendiger Behandlungen, die ihrerseits mit unnötigen Risiken, Belastungen oder gar Schäden für Patient:innen verbunden sein können. Inakzeptabel ist Überversorgung besonders dann, wenn ihr eine Unterversorgung in anderen Regionen gegenübersteht - aber auch, wenn Leistungsanbieter:innen ihre Leistungen an ihrem finanziellen Partikularinteresse und nicht am Versorgungsbedarf orientieren.

Im Hinblick auf die Krankenhausversorgung ist eine stärkere Zentralisierung und Spezialisierung auf jene Leistungen, bei denen sich ein Zusammenhang zwischen Leistungsfrequenz und Leistungsqualität nachweisen lässt, zwar sinnvoll. Der Entzug von Behandlungskompetenzen für Fachabteilungen in kleineren Krankenhäusern könnte in vielen Fällen aber die wirtschaftliche Existenz ganzer Häuser gefährden.

Daraus könnte jenseits überversorgter Ballungsräume gerade in ländlichen Räumen ein Problem für die Vorhaltung bedarfsgerechter Krankenhauskapazitäten erwachsen. Zugleich wird sich vor allem in ländlichen Regionen eine wohnortnahe fachärztliche Versorgung im Rahmen der traditionellen Strukturen (Einzel- und Gemeinschaftspraxen) kaum gewährleistet lassen, weil sich Ärzt:innen wegen der wirtschaftlichen Risiken nicht in ausreichender Zahl zu einer Niederlassung bereitfinden. Auch Kommunen, die mittlerweile Eigeneinrichtungen der ambulanten Versorgung betreiben dürfen, können - selbst wenn sie sich zur Wahrnehmung dieser Option entscheiden - nicht ohne Weiteres die Schwierigkeiten bei der Gewinnung von Ärzt:innen überwinden.

Hier kann den Krankenhäusern eine Rolle als zentralen Akteuren in der ambulanten fachärztlichen Versorgung und bei der Koordination der Leistungserbringer:innen in einem regionalen Versorgungsnetz zukommen (Hildebrandt et al. 2017). Die Zukunft der Versorgungsorganisation und damit auch die Rolle der Krankenhäuser kann also regional höchst unterschiedlich ausfallen. Dies kann neben der stationären Versorgung auch eine - möglicherweise durchgängige - ambulante Versorgung im Krankenhaus oder in angeschlossenen Medizinischen Versorgungszentren (MVZ) einschließen (Sachverständigenrat 2014). Die Wahrnehmung solcher Optionen setzt voraus, dass der Gesetzgeber die für eine derart flexible Aufgabenwahrnehmung erforderlichen gesetzlichen Regelungen zur Öffnung der Krankenhäuser trifft.

Von herausragender Bedeutung für die Schaffung einer bedarfsgerechten Versorgungsinfrastruktur ist aber ein anderer Ansatzpunkt: die Durchsetzung einer sektorenübergreifenden, also die ambulante und die stationäre Versorgung integrierenden Bedarfsplanung. In Deutschland sind die Systeme der vertrags- 
ärztlichen (ambulanten) und der stationären Bedarfsplanung, wie oben in Abschnitt 3 und Abschnitt 4 ausgeführt, traditionell strikt voneinander getrennt. Die Doppelzuständigkeit der Länder einerseits und der Kassenärztlichen Vereinigungen andererseits hat erheblich zu dem skizzierten Nebeneinander von Über- und Unterversorgung beigetragen.

Eine sektorenübergreifende Planung bedeutet nicht nur, die Planung für zwei nach wie vor getrennte Sektoren aufeinander abzustimmen, sondern auch, die Grenzen zwischen den Einrichtungen durchlässiger zu machen. Angesichts der unerwünschten Wirkungen einer sektoral getrennten Bedarfsplanung hat der Gesetzgeber in der jüngeren Vergangenheit eine Reihe von Maßnahmen für eine stärkere Abstimmung beider Planungssysteme ergriffen. Dabei geht es vor allem um eine stärkere Einflussnahme der Länder auf die vertragsärztliche Versorgung.

Allerdings sind die einschlägigen Gestaltungsrechte nach wie vor nur schwach ausgeprägt. So können die Länder zwar ein Gremium für eine sektorenübergreifende Versorgungsplanung einberufen, das aber nur Empfehlungen abgeben und keine verbindlichen Entscheidungen treffen kann ( $(90 \mathrm{a}$ SGB V). Die Mitwirkung der Länder an der vertragsärztlichen Bedarfsplanung ist auf ein Mitberatungsrecht zur Bedarfsplanungsrichtlinie im Gemeinsamen Bundesausschuss (G-BA) und auf ein Recht zur Beanstandung der Beschlüsse des Landesausschusses der Ärzte und Krankenkassen und des von diesem aufgestellten Bedarfsplans begrenzt ( $\$ 90$ Abs. 6 SGB V; $\$ 99$ Abs. 1 SGB V).

Die Planung einer bedarfsgerechten Versorgungsinfrastruktur muss als Kernaufgabe der öffentlichen Daseinsvorsorge unter staatlicher Verantwortung durchgeführt werden. In einem demokratischen Gemeinwesen muss ein solcher Planungsprozess auch in demokratischer Partizipation der Betroffenen - ob nun in ihrer Eigenschaft als Nutzer:innen, Patient:innen, Versicherten oder Bürger:innen - erfolgen.

Damit ist auch die soziale Selbstverwaltung und sind insbesondere die Versichertenvertreter:innen gefordert. $\mathrm{Zu}$ den verbliebenen Zuständigkeiten der Selbstverwaltung zählen Aufgaben von grundsätzlicher Bedeutung und die Kontrolle des Vorstands ( $\$ 197$ Abs. 1 SGB V). Zwar lässt diese gesetzliche Vorgabe Interpretationsspielräume zu und wenden die einzelnen Kassen diese Rahmenbestimmung auch in unterschiedlicher Weise an, aber oft genug entzieht sich das operative Geschäft, zu dem auch Verhandlungen mit Leistungsanbieter:innen über die Vorhaltung von Kapazitäten gehören, eben doch dem Zugriff der sozialen Selbstverwaltung.

Den Versichertenvertretungen in den Krankenkassen(verbänden) stellen sich in diesem Zusammenhang vor allem zwei Herausforderungen: Erstens sollten 
sie die Kapazitäts- bzw. Strukturplanung als ein essenzielles Anliegen der Versichertengemeinschaft und als ihr eigenes Handlungsfeld verstehen. Zweitens sollten sie, gerade wegen des Bedeutungszuwachses regional differenzierter Versorgungskonzepte, die enge Zusammenarbeit mit regionalen und kommunalen Akteuren suchen, die für die Entwicklung von Versorgungsnetzwerken relevant sind. Dies ist für die Selbstverwaltung eine große Herausforderung, insbesondere in jenen Kassenarten, die in der Tradition einer stark zentralisierten Verwaltung stehen oder zu klein sind, um angemessene regionale Bezüge in ihrer Selbstverwaltungstätigkeit herzustellen.

Die Widerstände gegen eine grundlegende Reform des Planungssystems und der Versorgungsstrukturen sind allerdings groß. So halten Kassenärztliche Vereinigungen und Länder entschlossen an ihren jeweiligen Bedarfsplanungsrechten fest. Die Organisationen der Ärzteschaft haben einen erheblichen Einfluss auf Politik und öffentliche Meinung, weshalb man sie als »informelle Veto-Spieler« bezeichnen kann, während die Länder sogar eine Rolle als »institutionelle Veto-Spieler« wahrnehmen.

Gegen den Abbau einer Überversorgung gibt es auf beiden Seiten starke Widerstände. Die Kassenärztlichen Vereinigungen müssten dafür Mittel aufwenden oder nachrückenden Ärzt:innen den Zugang zu begehrten Planungsbezirken sperren. Eine stärkere Öffnung der Krankenhäuser für die ambulante Behandlung lehnen sie entschieden ab, weil sie darin eine Gefahr für das (weitgehende) ambulante Versorgungsmonopol ihrer Mitglieder sehen.

Durch die Unterversorgung in benachteiligten Regionen und Quartieren sind weniger die Kassenärztlichen Vereinigungen betroffen, weil ihre Existenz und ihr Wirken weithin unbekannt sind und sie wegen der Nichterfüllung ihres Sicherstellungsauftrags auch sonst kaum Nachteile zu befürchten haben. Weit stärker bekommen die Verwaltungen der betreffenden Gebietskörperschaften die Auswirkungen von Unterversorgung zu spüren. Der Rückgriff auf punktuelle Ermächtigung von Krankenhäusern für die ambulante Behandlung ist ein bequemes Mittel, um der Unterversorgung entgegenzuwirken.

Die Länder fürchten bei Krankenhausschließungen den Protest der betroffenen Bevölkerung und damit verbundene politische Legitimationsverluste. Überdies sind Krankenhäuser ein wichtiger Wirtschaftsfaktor; ihre laufenden Kosten werden überwiegend von den Krankenkassen getragen. Die eigenen finanziellen Aufwendungen für die Vorhaltung einer Krankenhausinfrastruktur halten die Länder ohnehin in engen Grenzen, weil sie ganz überwiegend ihrer Pflicht zur Übernahme der Krankenhausinvestitionen nicht nachkommen.

Auch der Unterversorgung in benachteiligten Regionen und Quartieren lässt sich nur durch ein Bündel verschiedener Maßnahmen entgegenwirken. 
Die Gesetzgebung der vergangenen Jahre beinhaltete eine Vielzahl solcher Initiativen. Diese reichten von der Flexibilisierung der Bedarfsplanung und der ärztlichen Vergütung in unterversorgten oder von Unterversorgung bedrohten Gebieten über die Stärkung der allgemeinmedizinischen Ausbildung und finanzielle Anreize für die Aufnahme einer Landarzttätigkeit bis hin zum Einsatz mobiler Versorgungskonzepte und der Entwicklung telemedizinischer Angebote (Gerlinger/Rosenbrock 2021) - ohne bisher allerdings durchschlagenden Erfolg verzeichnen zu können.

Möglicherweise liegt dies auch daran, dass bisher eine ordnungspolitische Maßnahme unterblieben ist, die den finanziellen Anreiz zur Entscheidung für die Niederlassung in Ballungsräumen verringern würde: die Einführung einer solidarischen Bürgerversicherung. Sie würde neben anderen Effekten auch die Beseitigung der bisherigen Unterschiede zwischen vertragsärztlicher und privatärztlicher Versorgung mit sich bringen. Damit würde sie Ärzt:innen einen wichtigen Grund für die Entscheidung gegen eine Landarzttätigkeit nehmen.

Allerdings wäre auch dieser Schritt allein bei Weitem nicht ausreichend und müsste zudem hohe politische Hürden überwinden. Es bleibt darauf hinzuweisen, dass die geringe Neigung zur Aufnahme des Arztberufs in strukturschwachen Gebieten auch mit gesellschaftlichen Entwicklungen zusammenhängt, die sich der direkten Einflussnahme der Gesundheitspolitik entziehen.

Ein gegenüber den erörterten Planungs- und Finanzierungsfragen eigenständiges Problem stellt das Vordringen großer Finanzinvestoren in die Krankenhaus- und ambulante Versorgung und der damit verbundene Bedeutungszuwachs (kurzfristiger) Gewinninteressen zulasten der Bedarfsorientierung dar. Eine radikale Option wäre die Verstaatlichung privater Versorgungseinrichtungen. Freilich würde ein solcher Schritt erhebliche Kosten mit sich bringen, und es wäre abzuwarten, wie das Bundesverfassungsgericht in einem Verfahren, das ein solcher Schritt sicherlich nach sich zöge, entscheiden würde.

Eine andere Option wäre das Verbot der weiteren Privatisierung staatlicher Häuser, wie es z. B. in Frankreich existiert (Klenk/Reiter 2012). Damit ließe sich zwar die vollzogene Privatisierung nicht rückgängig machen, aber immerhin ein Voranschreiten auf diesem Weg verhindern. Allerdings würde dies auch voraussetzen, dass der Staat seine Verantwortung für die wirtschaftliche Sicherung der Krankenhäuser tatsächlich in vollem Umfang wahrnimmt.

Im Hinblick auf die wachsende Rolle von Private-Equity-Gesellschaften in der ambulanten Versorgung bestünde eine Möglichkeit darin, privaten Finanzinvestoren die Beteiligung an ambulanten Versorgungseinrichtungen zu untersagen. Eine entsprechende gesetzliche Regelung wurde bereits $2011 \mathrm{mit}$ dem Versorgungsstrukturgesetz (BGBl. I, S. 2983) verabschiedet, hat aber Schlupf- 
löcher gelassen, die Finanzinvestoren auszunutzen wissen (Bobsin 2019). Eine konsequentere Regelung auf diesem Feld dürfte zu eher geringeren Widerständen führen, als sie auf anderen Feldern zu erwarten wären.

Die Hürden, vor denen Maßnahmen zur Sicherstellung einer bedarfsgerechten Versorgungsinfrastruktur stehen, sind also hoch. Wichtige Gründe dafür liegen in der traditionell starken Fragmentierung des deutschen Gesundheitssystems. Diese hängt mit verschiedenen Faktoren zusammen:

- mit dem föderalen Staatsaufbau und den Politikverflechtungen zwischen Bund und Ländern, die nicht nur auf dem Gebiet der Gesundheitsförderung und Prävention, sondern auch auf dem Gebiet der Krankenversorgung anzutreffen sind (Rudzio 2015),

- mit der strukturellen Einbindung von Verbänden in die gesundheitspolitische Entscheidungsfindung des deutschen Krankenversicherungsstaates (»Selbstverwaltung«) und einer darin begründeten eigenständigen Rolle von Interessenverbänden (Mayntz 1992) und

- mit den unterschiedlichen Zuständigkeiten der Akteure der gesetzlichen Krankenversicherung (Krankenkassen, Kassenärztliche Vereinigungen) und der Länder auf einigen Feldern der Gesundheitsversorgung, die Folge gesundheitspolitischer Weichenstellungen am Ende des 19. und im ersten Drittel des 20. Jahrhunderts sind (Tennstedt 1977; Kießling 2016).

Das Zusammenwirken dieser Faktoren hat eine stattliche Anzahl von Akteuren hervorgebracht, die Träger starker Partikularinteressen und zugleich mit erheblichen Machtressourcen ausgestattet sind. Damit können sie als informelle oder gar formelle Veto-Spieler (Tsebelis 2002) in Erscheinung treten und wünschenswerte Reformen erschweren oder auch verhindern. Diese Merkmale des politischen Systems tragen bekanntlich häufig dazu bei, dass flexible Reaktionen auf neue Herausforderungen, auch im Sinne einer Daseinsvorsorge, erschwert werden.

\section{Literatur}

Augurzky, B./Gülker, R./Krolop, S./Schmidt, C.M./Schmidt, H./Schmitz, H./ Terkatz, S. (2011): Krankenhaus Rating Report 2011. Die fetten Jahre sind vorbei, RWI Materialien 67, Essen.

Bobsin, R. (2019): Finanzinvestoren in der Gesundheitsversorgung in Deutschland. 20 Jahre Private Equity - Eine Bestandsaufnahme, 4. Auflage, Hannover. 
Böhlke, N./Gerlinger, T./Mosebach, K./Schmucker, R./Schulten, T. (Hrsg.) (2009): Privatisierung von Krankenhäusern. Erfahrungen und Perspektiven aus Sicht der Beschäftigten, Hamburg.

Dahme, H.-J./Wohlfahrt, N. (2011): Handbuch Kommunale Sozialpolitik, Wiesbaden.

Deutsche Krankenhausgesellschaft (DKG) (2019): Bestandsaufnahme zur Krankenhausplanung und Investitionsfinanzierung in den Bundesländern. Stand: Dezember 2019, www.dkgev.de/themen/versorgung-struktur/planung/ (Abruf am 9.7.2021).

Deutscher Bundestag (2019): Wissenschaftliche Dienste - Dokumentation: Private Equity im deutschen Gesundheitssektor, Berlin.

Dieterich, A./Braun, B./Gerlinger, T./Simon, M. (Hrsg.) (2019): Geld im Krankenhaus. Eine kritische Bestandsaufnahme des DRG-Systems, Wiesbaden.

Eurostat (2021): Krankenhausbetten (Tabellen-Code tps00046), https://ec.euro pa.eu/eurostat/databrowser/view/tps00046/default/table?lang=de (Abruf am 28.4.2021).

Forsthoff, E. (1938): Die Verwaltung als Leistungsträger, Stuttgart.

Forsthoff, E. (1958): Die Daseinsvorsorge und die Kommunen, Stuttgart.

Gerlinger, T./Rosenbrock, R. (2021): Gesundheitspolitik. Eine systematische Einführung, 4. Aufl., Bern (im Erscheinen).

Hildebrandt, H./Gröne, O./Pimperl, A./Werner, U./Huber, B. (2017): Das vernetzte Krankenhaus der Zukunft ist primär ein regionales Versorgungssystem - eine Skizze, in: Klauber, J./Geraedts, M./Friedrich, J./Wasem, J. (Hrsg.): Krankenhaus-Report 2017. Schwerpunkt: Zukunft gestalten, Stuttgart, S. 167-183.

Kassenärztliche Bundesvereinigung (o.J.): Entwicklungen der Medizinischen Versorgungszentren. Statistische Informationen zum Stichtag 31.12.2019, www.kbv.de/media/sp/mvz_entwicklungen.pdf (Abruf am 9.7.2021).

Kassenärztliche Bundesvereinigung (2016): KBV 2020. Versorgung gemeinsam gestalten. Ein Konzept der KBV und der Kassenärztlichen Vereinigungen. Stand: 20. Mai 2016, www.kbv.de/media/sp/2016-05-20_Konzept_KBV-2020. pdf (Abruf am 4.10.2021).

Kassenärztliche Bundesvereinigung (2021): Gesundheitsdaten. Mehr Ärzte, aber kürzere Arbeitszeiten, https:/gesundheitsdaten.kbv.de/cms/html/16393.php (Abruf am 28.4.2021).

Kießling, A. (2016): Der deutsche Sozialstaat als Sozialversicherungsstaat und seine Auswirkungen auf das Präventionsrecht, in: Rechtswissenschaft 7(4), S. 597-624. 
Klenk, T./Reiter, R. (2012): Öffentliche Daseinsvorsorge, privat organisiert? Ein deutsch-französischer Vergleich der Bereitstellung der Krankenhausinfrastruktur, in: Zeitschrift für Sozialreform 58(4), S. 401-425.

Klose, J./Rehbein, I. (Hrsg.) (2017): Ärzteatlas 2017. Daten zur Versorgungsdichte von Vertragsärzten, Berlin.

Korzilius, H. (2018): Investoren auf Einkaufstour, in: Deutsches Ärzteblatt (Ausgabe A) 115(39), S. 1688-1692.

Loos, S./Albrecht, M./Zich, K. (2019): Simulation und Analyse einer Neustrukturierung der Krankenhausversorgung am Beispiel einer Versorgungsregion in Nordrhein-Westfalen, Gütersloh.

Mayntz, R. (Hrsg.) (1992): Verbände zwischen Mitgliederinteressen und Gemeinwohl, Gütersloh.

OECD (2020): Gesundheitsdaten (Health Care Resources: Hospital Beds), www. oecd.org/els/health-systems/health-data.htm (Abruf am 28.4.2021).

Rudzio, W. (2015): Das politische System der Bundesrepublik Deutschland, Wiesbaden.

RWI = RWI - Leibniz-Institut für Wirtschaftsforschung e. V. (2017): Stand und Weiterentwicklung der Investitionsförderung im Krankenhausbereich. Endbericht. Gutachten im Auftrag des Bundesministeriums für Gesundheit. Stand: 6. Dezember 2017, Essen.

Sachverständigenrat zur Begutachtung der Entwicklung im Gesundheitswesen (2012): Sondergutachten 2012 Wettbewerb an der Schnittstelle zwischen ambulanter und stationärer Gesundheitsversorgung, Deutscher Bundestag, Drucksache 17/10323 vom 10.7.2012.

Sachverständigenrat zur Begutachtung der Entwicklung im Gesundheitswesen (2014): Gutachten 2014. Bedarfsgerechte Versorgung - Perspektiven für ländliche Regionen und ausgewählte Leistungsbereiche, Deutscher Bundestag, Drucksache 18/1940 vom 26.6.2014.

Sachverständigenrat zur Begutachtung der Entwicklung im Gesundheitswesen (2018): Gutachten 2018. Bedarfsgerechte Steuerung der Gesundheitsversorgung, Deutscher Bundestag, Drucksache 19/3180 vom 4.7.2018.

Scheuplein, C./Evans, M./Merkel, S. (2019): Übernahme durch Private Equity im deutschen Gesundheitssektor. Eine Zwischenbilanz für die Jahre 2013 bis 2018, Gelsenkirchen.

Schölkopf, M./Grimmeisen, S. (2021): Das Gesundheitswesen im internationalen Vergleich. Gesundheitssystemvergleich, Länderberichte und europäische Gesundheitspolitik, Berlin.

Simon, M. (2019a): Das deutsche DRG-System: Vorgeschichte und Entwicklung seit seiner Einführung, in: Dieterich, A./Braun, B./Gerlinger, T./Simon, M. 
(Hrsg.): Geld im Krankenhaus. Eine kritische Bestandsaufnahme, Wiesbaden, S. 3-27.

Simon, M. (2019b): Das deutsche DRG-System: Weder Erfolgsgeschichte noch leistungsgerecht, in: Dieterich, A./Braun, B./Gerlinger, T./Simon, M. (Hrsg.): Geld im Krankenhaus. Eine kritische Bestandsaufnahme, Wiesbaden, S. 295-324.

Statistisches Bundesamt (2018): Grunddaten der Krankenhäuser. Fachserie 12, Reihe 6.1.1, Wiesbaden.

Statistisches Bundesamt (2020): Grunddaten der Krankenhäuser. Fachserie 12, Reihe 6.1.1, Wiesbaden.

Streeck, W. (2019): Vorwort zur deutschen Ausgabe, in: Foundational Economy Collective: Die Ökonomie des Alltagslebens. Für eine neue Infrastrukturpolitik, Berlin, S. 7-30.

Tennstedt, F. (1977): Geschichte der Selbstverwaltung in der Krankenversicherung von der Mitte des 19. Jahrhunderts bis zur Gründung der Bundesrepublik Deutschland, Bonn.

Tikkanen, R./Osborn, R./Mossialos, E./Djordjevic, A./Wharton, G. (Hrsg.) (2020): International Profiles of Health Care Systems 2020, www.common wealthfund.org/sites/default/files/2020-12/International_Profiles_of_Health_ Care_Systems_Dec2020.pdf (Abruf am 9.7.2021).

Tsebelis, G. (2002): Veto Players. How Political Institutions Work, Princeton. 


\section{Grundsicherung und rechtliche Beratung}

Matthias Meißner

\section{Einleitung}

Dieser Beitrag nähert sich dem Themengebiet der Beratungsleistungen und -pflichten in der Grundsicherung aus sozialrechtlicher Sicht. Dabei stehen die im Sozialgesetzbuch (SGB) verankerten Auskunfts- und Beratungspflichten im Mittelpunkt, speziell jene, die die Grundsicherungsleistungen betreffen. Hierbei geht es um die Vermittlung von Informationen, die Personen mit einem tatsächlichen oder möglichen Anspruch auf Grundsicherung benötigen, um ihre sozialen Rechte wahrzunehmen bzw. durchzusetzen, aber auch, um ihren gesetzlichen Pflichten nachzukommen.

Trotz der großen Relevanz von Sozialleistungen und der Vielzahl von Sozialleistungsträgern gibt es keine systematische bzw. umfassende Forschung zu Nutzen und Nutzer:innen der Sozialrechtsberatung. Einzelne Studien lassen gleichwohl den Schluss zu, dass Beratungspraxis und Herausforderungen bei den Sozialleistungsträgern sehr unterschiedlich sind.

So werden beispielsweise Effizienzsteigerungen und Kostendruck als Grund für die Verschlankung von Strukturen und die Zusammenlegung von Einheiten bei den Trägern der Sozialversicherung angeführt (Vitols/Gabriel 2020, S. 9). Beratungs- und Unterstützungsleistungen würden hierdurch teilweise in den Hintergrund treten, was insbesondere Beschäftigte der Kranken- und Pflegekassen kritisierten - eine Ausnahme hierzu bilde die Bundesagentur für Arbeit, wo gerade der Bereich Beratung in den letzten Jahren eine Aufwertung erfahren habe (Vitols/Gabriel 2020, S. 9).

Für die Beratung im Bereich der Grundsicherung für Arbeitsuchende gibt es besondere Herausforderungen. Die Beratung gleiche einem Balanceakt, in dem Beratende dem Prinzip des Förderns und Forderns entsprechend einerseits Beziehungsarbeit leisten und ein konstruktives Arbeitsbündnis aufbauen, andererseits aber auch kontrollieren und sanktionieren müssen (vgl. Nixdorf 2020, 
S. 5). Hieraus können Konflikte entstehen (Bähr et al. 2018, S. 87). Beratende müssen dementsprechend vielfältige Kenntnisse aufweisen, z. B. interkulturelle Kompetenz, Kenntnisse in Gesprächsführung, Deeskalationsfähigkeit, juristische Kenntnisse und Wissen über Berufe und den Arbeitsmarkt (vgl. Nixdorf 2020, S. 5).

Dieser Beitrag fokussiert die Regelungen im Sozialgesetzbuch, also die dort festgelegten allgemeinen und spezifischen Auskunfts- und Beratungspflichten der Sozialleistungsträger, die mit der Grundsicherung befasst sind (Abschnitt 2). Anschließend geht es um die im Grundgesetz verankerte Gleichheit der Rechtswahrnehmung, die durch das Beratungshilfegesetz sichergestellt wird (Abschnitt 3). Die Sozialrechtsberatung durch weitere Akteur:innen findet beispielhaft Erwähnung (Abschnitt 4).

Der Beitrag schließt mit Beispielen für Herausforderungen (Abschnitt 5) und Perspektiven für Beratungsleistungen in der Grundsicherung (Abschnitt 6), insbesondere im Hinblick auf Ratsuchende mit psychischen Erkrankungen, den Zugang zu Leistungen für Bildung und Teilhabe und die Beratung von Jugendlichen mit Behinderung. Hierbei wird deutlich, dass der Anspruch des Gesetzgebers an Beratungsleistungen auf vielfältige Herausforderungen in der Praxis triff.

\section{Vorgaben für die Beratung durch Sozialleistungsträger}

Das Recht des Sozialgesetzbuchs dient nach $₫ 1$ Abs. 1 Satz 1 SGB I der Verwirklichung sozialer Gerechtigkeit und sozialer Sicherheit, wodurch der Gesetzgeber das in der Verfassung verankerte Sozialstaatsprinzip aus Art. 20 Abs. 1 Satz 1 GG und Art. 28 Abs. 1 Satz 1 GG konkretisiert. Die Sozialleistungsträger haben hierbei den Auftrag und das Ziel, die sozialen Rechte der Bürger:innen möglichst weitgehend zu verwirklichen (vgl. $\$ 2$ Abs. 2 SGB I) und erbringen hierzu Dienst-, Sach- und Geldleistungen ( $\$ 11$ Satz 1 SGB I).

Information und Beratung gehören zu den Dienstleistungen der Sozialleistungsträger und haben eine zentrale Bedeutung für das Funktionieren des sozialen Leistungssystems (BSG 28.2.1984 - 12 RK 31/83 = SGb 1985, S. 295 f.). Der Zweck von Sozialleistungen, Wechselfälle des Lebens bzw. Lebensrisiken abzufedern, kann nur erreicht werden, wenn die Sozialleistungsträger durch ihre Informations- und Beratungstätigkeit auf eine möglichst umfassende Information aller Beteiligten hinwirken (a.a.O.). Grundlage hierfür sind die allgemeinen und besonderen Vorschriften zur Beratung im Sozialgesetzbuch. 
Die Grundlage für die Beratung durch die Sozialleistungsträger wiederum bildet der sozialrechtliche Beratungsbegriff, bei dem es um die mündliche oder schriftliche Vermittlung von Informationen geht, die Ratsuchende zur Kenntnis und zur Wahrnehmung ihrer Rechte und Pflichten nach dem Sozialgesetzbuch benötigen (Trenk-Hinterberger 2020, $\$ 14$ Rn. 7). Die Beratung erfolgt auf Basis einer genauen Erfassung der Bedarfe und sozialen Situation der zu Beratenden; über die Information und Aufklärung hinaus kommen eine Bewertung unterschiedlicher Alternativen und konkrete Handlungsempfehlungen bzw. -anleitungen zur Sprache (vgl. Knickrehm 2021, $\$ 4$ Rn. 15 mit weiteren Nachweisen).

Hiervon abzugrenzen sind andere Arten bzw. Gebiete der Beratung wie z. B. psychologische bzw. psychosoziale Beratung, Lebensberatung oder Familienberatung, bei denen persönliche Entwicklungsprozesse der Ratsuchenden im Mittelpunkt stehen, nicht jedoch die individuellen Rechte und Pflichten nach dem Sozialgesetzbuch. Während die Sozialrechtsberatung oftmals von den Sozialleistungsträgern selbst erbracht wird, gibt es viele sonstige Akteur:innen, die auch weitere Arten bzw. Gebiete der Beratung abdecken (siehe Abschnitt 4).

\subsection{Allgemeine Aufklärung, Beratung und Auskunft nach SGB I}

Der in $₫ 14$ SGB I verankerte allgemeine Beratungsanspruch ${ }^{1}$ ist von großer praktischer Relevanz, weil daraus ein Individualanspruch für Bürger:innen und dementsprechend eine Beratungspflicht der Sozialbehörden auf fachlich richtige Beratung im Einzelfall folgt. Dabei muss die Sozialbehörde auch auf zweckmäBige Verhaltensweisen und naheliegende Gestaltungsmöglichkeiten hinweisen (BSG 27.9.1983 - 12 RK 44/82 = SGb 1984, S. 108). Ziel der Beratung ist es, Bürger:innen zu einer möglichst weitgehenden Verwirklichung ihrer Rechte bzw. Erfüllung ihrer Ansprüche zu befähigen, weshalb eine möglichst umfassende Information aller Beteiligten erforderlich ist (BSG 28.2.1984 - 12 RK 31/83 = SGb 1985, S. 295 f.).

Auskünfte und Belehrungen der Sozialbehörden müssen richtig, klar, unmissverständlich, eindeutig und vollständig sein (Spellbrink 2020, \$14 Rn. 47). Hierbei kommt es auf den Empfängerhorizont an. Anlass und Umfang der Information bestimmen sich nach dem »erkennbaren Interesse« des bzw. der Auskunftsuchenden und deren »Fragestellung« (vgl. BSG 24.3.1988 - 5/5b RJ 84/86=

1 | »eder hat Anspruch auf Beratung über seine Rechte und Pflichten nach diesem Gesetzbuch. Zuständig für die Beratung sind die Leistungsträger, denen gegenüber die Rechte geltend zu machen oder die Pflichten zu erfüllen sind« (\$14 SGB I). 
BSGE 63, S. 112 ff.). Zuständig für die Beratung ist derjenige Leistungsträger, der aufgrund seiner Sachkenntnis für die Aufgabe sachlich und örtlich zuständig und damit am besten geeignet ist (Trenk-Hinterberger 2020, $\$ 14$ Rn. 2).

Der Leistungsträger darf sich aber nicht nur auf die Beantwortung konkreter Fragen oder abgegrenzter Bitten beschränken, sondern muss sich bemühen, das konkrete Anliegen des bzw. der Ratsuchenden zu ermitteln und - unter dem Gesichtspunkt einer verständnisvollen Förderung - zu prüfen, ob über die konkrete Fragestellung hinaus Anlass besteht, auf Gestaltungsmöglichkeiten, Voroder Nachteile hinzuweisen, die sich mit dem Anliegen verbinden. Besondere Lagen und Verhältnisse können zusätzliche (Fürsorge-)Pflichten begründen, z. B. die Pflicht, Ratsuchende über die zur Erreichung ihrer Ziele notwendigen Maßnahmen belehrend aufzuklären oder in anderer Weise helfend tätig zu werden, wenn Betroffene ihre Lage in tatsächlicher oder rechtlicher Hinsicht nicht richtig zu beurteilen vermögen.

Hierzu gehört es auch, Schäden des Bürgers bzw. der Bürgerin durch einen kurzen Hinweis, eine Belehrung mit wenigen Worten oder eine entsprechende Aufklärung über die Sach- und Rechtslage zu vermeiden. Dies betrifft Fallkonstellationen, in denen sich die notwendige Hilfe oder eine andere gebotene Verhaltensweise situationsbedingt aufdrängen (BGH, Urteil vom 2.8.2018 III ZR 466/16 = NJW 2019, S. 68 f.).

Die gesetzliche Beratungspflicht aus $\$ 14$ SGB I besteht nach Wortlaut sowie Sinn und Zweck der Vorschrift unabhängig von einem sogenannten Sozialrechtsverhältnis (Spellbrink 2020, $\$ 14$ Rn. 23). Die Verpflichtung zur Spontanberatung besteht auch ohne Antrag auf Beratung, weshalb Sozialleistungsträger gleichsam »von Amts wegen « zur Beratung verpflichtet sind, wenn ein Verwaltungsverfahren läuft oder eine irgendwie geartete Beziehung zum Bürger bzw. zur Bürgerin besteht. Dementsprechend müssen Sozialleistungsträger auch ohne einen konkreten Antrag auf Beratung auf naheliegende Gestaltungsmöglichkeiten hinweisen (Spellbrink 2020, $\$ 14$ Rn. 22 ff.).

Die Beratungspflicht aus $₫ 14$ SGB I trifft dabei nicht nur diejenigen Leistungsträger, denen gegenüber Rechte geltend zu machen oder Pflichten zu erfüllen sind, sondern durchaus auch andere Behörden. Dementsprechend hat der Bundesgerichtshof entschieden, dass ein:e Sachbearbeiter:in, die bzw. der mit Fragen der Grundsicherung befasst ist, auf die Notwendigkeit einer Beratung durch den zuständigen Rentenversicherungsträger hinweisen muss, wenn bei Beantragung von laufenden Leistungen der Grundsicherung wegen Erwerbsminderung ein dringender rentenversicherungsrechtlicher Beratungsbedarf erkennbar ist - auch ohne ein entsprechendes Beratungsbegehren (vgl. BGH, Urteil vom 2.8.2018 - III ZR 466/16 = NJW 2019, S. 68 f.). 
In $₫ 13$ SGB I ist zudem die Verpflichtung der Leistungsträger verankert, im Rahmen ihrer Zuständigkeit die Bevölkerung über die Rechte und Pflichten nach dem Sozialgesetzbuch aufzuklären. Dieser Aufklärungsanspruch ist aber allgemeiner Natur und wird bereits durch allgemeine Veröffentlichungen der Leistungsträger (Broschüren, Merkblätter, Internetseiten etc.) erfüllt - ein Anspruch auf individuelle bzw. persönliche Beratung des konkreten Falls folgt daraus nicht (vgl. Spellbrink 2020, $\$ 13$ Rn. 2 und 4).

Ein eingeschränkter individueller Anspruch auf Auskunftserteilung - insbesondere zur Benennung des zuständigen Leistungsträgers und zur Beantwortung weiterer Sach- bzw. Rechtsfragen - folgt aus $₫ 15$ SGB I. Der Auskunft kommt eine Wegweiserfunktion im System der Sozialberatung nach dem SGB I zu, da sie zur Beratung gemäß $\$ 14$ SGB I durch den zuständigen Sozialleistungs-

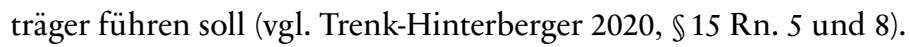

Der Verwirklichung des Ziels, die sozialen Rechte der Bürger:innen möglichst weitgehend zu verwirklichen (vgl. $\$ 2$ Abs. 2 SGB I), dienen weitere Regelungen im SGB I, die die Ansprüche auf Aufklärung, Beratung und Information ( \$\$ 13-15 SGB I) ergänzen: Die Leistungsträger sind verpflichtet, Anträge, die bei einem unzuständigen Leistungsträger gestellt werden, unverzüglich an den zuständigen Leistungsträger weiterzuleiten ( $\mathbb{1 6}$ Abs. 2 SGB I). Darüber hinaus müssen sie darauf hinwirken, dass unverzüglich klare und sachdienliche Anträge gestellt und unvollständige Angaben ergänzt werden ( $\$ 16$ Abs. 3 SGB I). Letztendlich sind sie verpflichtet, darauf hinzuwirken, dass jeder und jede Berechtigte die ihm bzw. ihr zustehenden Sozialleistungen in zeitgemäßer Weise, umfassend und zügig erhält ( $\$ 17$ Abs. 1 Nr. 1 SGB I).

\subsection{Spezielle Beratungs- und Unterstützungspflichten nach SGB II und SGB XII}

Der Gesetzgeber hat die Beratungs- und Unterstützungspflichten der Leistungsträger durch $\$ 14$ SGB II konkretisiert. Der Fokus der umfassenden Unterstützung liegt dabei ausdrücklich auf der Eingliederung in Arbeit (vgl. $\$ 14$ Abs. 1 SGB II). Aufgabe der Beratung der Jobcenter ist demensprechend insbesondere die Erteilung von Auskunft und Rat zu Selbsthilfeobliegenheiten und Mitwirkungspflichten, zur Berechnung der Leistungen zur Sicherung des Lebensunterhalts und zur Auswahl der Leistungen im Rahmen des Eingliederungsprozesses.

Art und Umfang der Beratung sollen sich zwar nach dem Beratungsbedarf der leistungsberechtigten Person richten. Sofern der Leistungsträger einen weitergehenden Beratungsbedarf erkennt, z. B. nach Schuldnerberatung, psycho- 
sozialer Betreuung oder Suchtberatung, erbringt er diese Beratung aber nicht selbst, sondern greift auf geeignete Einrichtungen und Dienste Dritter zurück (vgl. $\$ 16 a$ und 17 Abs. 1 SGB II.).

Zur Verwirklichung von Bildung und Teilhabe von Kindern und Jugendlichen hat der Gesetzgeber in $\$ 4$ Abs. 2 Satz 2-4 SGB II weitere besondere Anforderungen an die Leistungsträger gestellt. ${ }^{2}$ Das Gebot der »Hinwirkung « bezweckt, dass Kinder und Jugendliche Zugang zu geeigneten vorhandenen Angeboten der gesellschaftlichen Teilhabe erhalten.

Hierzu zählt auch die Information, Beratung und Unterstützung der Eltern. Zwar sollen Eltern ausweislich der Gesetzesbegründung gemeinsam mit den Trägern möglichst »verbindliche Absprachen« über die Inanspruchnahme von Angeboten zur Deckung der Bedarfe für Bildung und Teilhabe ihrer Kinder treffen (BT-Drucksache 17/3404, 91). Hiermit ist allerdings kein umfassender Sicherstellungsauftrag der Träger verbunden; diese haben lediglich den Auftrag, eine Struktur zu schaffen, die den Zugang zu vorhandenen Angeboten der gesellschaftlichen Teilhabe im sozialen und kulturellen Bereich ermöglicht (Knickrehm 2021, $\$ 4$ Rn. 33 f.).

Der Verstoß gegen die Absprache kann mangels ausdrücklicher gesetzlicher Regelung nicht zu einer Sanktionierung der Eltern führen; Eltern können überdies nicht durch eine Nebenbestimmung im Leistungsbescheid oder in der Eingliederungsvereinbarung verpflichtet werden, dafür zu sorgen, dass ihre Kinder an den Angeboten teilnehmen (Knickrehm 2021, $\$ 4$ Rn. 33 f.).

Im Gegensatz zur Beratung der Jobcenter nach SGB II haben die Sozialhilfeträger im SGB XII durch den Gesetzgeber einen speziellen und weitergehenden Auftrag erhalten, die Leistungsberechtigten zu beraten und, soweit erforderlich, auch zu unterstützen (siehe Kasten). Außer der Beratung zu persönlicher Situation, Bedarf und eigenen Kräften und Mitteln gehört dazu auch, Leistungsberechtigten Möglichkeiten der Teilnahme am Leben in der Gemeinschaft aufzuzeigen und sie für den Erhalt von Sozialleistungen zu befähigen. Die Beratungs- und Unterstützungspflicht aus $\$ 11$ SGB XII geht damit über die der $₫ 14$ SGB II bzw. $\$ 14$ SGB I hinaus. Letztlich spiegelt sich damit auch in den Beratungs- und Unterstützungspflichten der traditionelle fürsorgerechtliche Ansatz der Sozialhilfe.

$2 \mid$ |Die [...] zuständigen Träger wirken auch darauf hin, dass Kinder und Jugendliche Zugang zu geeigneten vorhandenen Angeboten der gesellschaftlichen Teilhabe erhalten« ( $\ 4$ Abs. 2 Satz 2 SGB II). 


\section{SGB XII: $\$ 11$ Beratung und Unterstützung, Aktivierung}

(1) Zur Erfüllung der Aufgaben dieses Buches werden die Leistungsberechtigten beraten und, soweit erforderlich, unterstützt.

(2) Die Beratung betrifft die persönliche Situation, den Bedarf sowie die eigenen Kräfte und Mittel sowie die mögliche Stärkung der Selbsthilfe zur aktiven Teilnahme am Leben in der Gemeinschaft und zur Überwindung der Notlage. Die aktive Teilnahme am Leben in der Gemeinschaft umfasst auch ein gesellschaftliches Engagement. Zur Überwindung der Notlage gehört auch, die Leistungsberechtigten für den Erhalt von Sozialleistungen zu befähigen. Die Beratung umfasst auch eine gebotene Budgetberatung.

(3) Die Unterstützung umfasst Hinweise und, soweit erforderlich, die Vorbereitung von Kontakten und die Begleitung zu sozialen Diensten sowie zu Möglichkeiten der aktiven Teilnahme am Leben in der Gemeinschaft unter Einschluss des gesellschaftlichen Engagements. Soweit Leistungsberechtigte zumutbar einer Tätigkeit nachgehen können, umfasst die Unterstützung auch das Angebot einer Tätigkeit sowie die Vorbereitung und Begleitung der Leistungsberechtigten. Auf die Wahrnehmung von Unterstützungsangeboten ist hinzuwirken. [...]

(5) Auf die Beratung und Unterstützung von Verbänden der freien Wohlfahrtspflege, von Angehörigen der rechtsberatenden Berufe und von sonstigen Stellen ist zunächst hinzuweisen. Ist die weitere Beratung durch eine

Schuldnerberatungsstelle oder andere Fachberatungsstellen geboten, ist auf ihre Inanspruchnahme hinzuwirken. [...]

\section{Gleichheit der Rechtswahrnehmung durch das Beratungshilfegesetz}

Während die (Geld-)Leistung der Grundsicherung und das Grundrecht auf Gewährleistung eines menschenwürdigen Existenzminimums unmittelbar aus Art. 1 Abs. 1 GG in Verbindung mit dem Sozialstaatsprinzip des Art. 20 Abs. 1 GG folgt, entspringt der Anspruch auf Beratungshilfe nach dem Beratungshilfegesetz (BerHG) aus dem allgemeinen Gleichbehandlungsgebot des Art. 3 Abs. 1 GG in Verbindung mit dem Rechtsstaatsprinzip (Art. 20 Abs. 1 und 3 GG).

Die Beratung nach dem Beratungshilfegesetz kann durch Anwält:innen, weitere Beratungspersonen oder auch das Amtsgericht erfolgen (vgl. $\$ 3$ Abs. 1 
und 2 BerHG). Der Kreis der Berechtigten ist nach $\$ 1$ Abs. 1 Nr. 1 BerHG auf diejenigen beschränkt, die die erforderlichen finanziellen Mittel für eine Rechtsberatung nicht aufbringen können. Gerade für diese Personen ist die Chancengleichheit bei der Rechtswahrung und Rechtsverfolgung erst durch die Gewährung einer vorprozessualen Beratungsmöglichkeit gegeben; sie können in der Regel erst nach einer Beratung beurteilen, ob und welche Rechte ihnen zustehen und wie sie diese effektiv wahrnehmen können (vgl. Graßhof 2020, \$34a Rn. 15; Wache 2020, $\$ 114$ Rn. 13).

Derselbe Maßstab - nämlich Art. 3 Abs. 1 GG in Verbindung mit Art. 20 Abs. 3 GG - gilt für die verfassungsrechtliche Kontrolle gerichtlicher Entscheidungen über Prozess- bzw. Verfahrenskostenhilfeanträge. Hierdurch soll eine weitgehende Angleichung der Situation von Bemittelten und Unbemittelten bei der Verwirklichung des Rechtsschutzes erfolgen (BVerfG, Beschluss vom 29.9.2015 - 1 BvR 1125/14 Rn. 13 = BeckRS 2016, 40279). Die Beratungshilfe greift allerdings erst, wenn eine ablehnende Entscheidung eines Sozialleistungsträgers vorliegt.

So hat das Bundesverfassungsgericht Verfassungsbeschwerden gegen die Versagung von Beratungshilfe stattgegeben, die die Begründung von Widersprüchen zum Inhalt hatte, die gegen Bescheide zu Leistungen zur Sicherung des Lebensunterhalts nach SGB II gerichtet waren. Das Bundesverfassungsgericht sah durch die Versagung der Beratungshilfe den Anspruch auf Rechtswahrnehmungsgleichheit (Art. 3 Abs. 1 GG in Verbindung mit Art. 20 Abs. 1 und Abs. 3 GG) verletzt. Die vom Amtsgericht befürwortete Auslegung des Beratungshilfegesetzes, dass es Rechtsuchenden zumutbar sei, selbst kostenlos Widerspruch einzulegen und dabei die Beratung derjenigen Behörde in Anspruch zu nehmen, die zuvor den Ausgangsverwaltungsakt erlassen hat, wurde den verfassungsrechtlichen Anforderungen nicht gerecht und damit verworfen (BVerfG, Beschluss vom 6.8.2009 - 1 BvR 1549, BeckRS 2009, 39171).

Im Ergebnis haben Grundsicherungsempfänger:innen damit bereits im Widerspruchsverfahren einen Anspruch auf Beratung und Vertretung nach dem Beratungshilfegesetz, das Hilfe für die Wahrnehmung von Rechten außerhalb eines gerichtlichen Verfahrens vorsieht (vgl. $\$ 1$ Abs. 1, $\$ 2$ Abs. 1 Satz 1 und $\$ 3$ Abs. 1 Satz 1 BerHG). Vor einem Widerspruchsverfahren - also bei Antragstellung oder im Rahmen einer (Erst-)Beratung vor Antragstellung - müssen sie allerdings die Beratung der Sozialbehörden oder sonstiger Beratungsstellen in Anspruch nehmen, die regelmäßig als zumutbare andere Hilfsmöglichkeit gilt (vgl. $\$ 1$ Abs. 1 Nr. 2 BerHG). 


\section{Beratung durch sonstige Akteur:innen}

Die Sozialleistungsträger haben kein gesetzliches Beratungsmonopol, weshalb Sozialrechtsberatung auch durch sonstige bzw. private Akteur:innen ausgeführt werden darf (Spellbrink 2020, \$14 Rn. 40). Dies gilt vorbehaltlich der Beachtung der Vorschriften des Rechtsdienstleistungsgesetzes (vgl. \$\$2, 3 und 6 RDG). In der Praxis gibt es vielfältige Akteur:innen, nämlich Gewerkschaften, Sozialverbände (z. B. VdK und SoVD), Selbsthilfegruppen (z. B. Erwerbsloseninitiativen), Träger der freien Wohlfahrtspflege (z. B. Arbeiterwohlfahrt, Deutscher Caritasverband, Diakonie Deutschland) sowie Rechtsanwält:innen und Rentenberater:innen, die Sozialrechtsberatung leisten ${ }^{3}$ (vgl. Trenk-Hinterberger 2020, $\$ 14$ Rn. 23). Diese sonstigen bzw. privaten Akteur:innen können auch - je nach Institution, Satzung und Auftrag - auf anderen Gebieten Beratung erbringen, z. B. psychosoziale, Schuldner- oder Familienberatung.

Die »Ergänzende unabhängige Teilhabeberatung« (EuTB) für Menschen mit Behinderung bzw. von Behinderung bedrohter Menschen ist gesetzlich als »ergänzende« Beratung im Vorfeld der Beantragung konkreter Leistungen ausgestaltet (vgl. $\$ 32$ Abs. 1 Satz 1 und 2 SGB IX). Die EuTB soll eine qualifizierte neutrale, aber parteiliche Beratung gewährleisten (vgl. Meißner 2019, S. 6 ff.). Ein besonderer Stellenwert kommt dabei nach $₫ 32$ Abs. 3 SGB IX der Beratung von Betroffenen für Betroffene zu (»Peer-Counseling«).

\section{Herausforderungen für Beratungsleistungen im Rahmen der Grundsicherung}

Die Herausforderungen in der Sozialrechtsberatung sind vielfältig: Schon die Zielsetzung einer umfassenden und individuellen Beratung (siehe Abschnitt 2) im »komplizierten, unübersichtlichen und vielfach auch unverständlichen « Sozialleistungssystem (Trenk-Hinterberger 2020, $\$ 14$ Rn. 2) ist groß, gerade angesichts der Vielzahl von sozialen Rechten (vgl. \$\$2-10 SGB I), Sozialleistungen und Sozialleistungsträgern (vgl. $\$ \mathbb{S} 18-29$ SGB I) in Deutschland. Dem steht in der Praxis ein regional, städtisch bzw. kommunal unterschiedliches und teilweise auch unübersichtliches Geflecht von öffentlichen, freigemeinnützigen und

3 | Die Träger der freien Wohlfahrtspflege erhalten hierfür staatliche Zuwendungen, da sie oftmals unentgeltliche Beratung für Bedürftige leisten. Private Vereine (Selbsthilfegruppen etc.) oder Gesellschaften werden nicht direkt gefördert, genießen aber steuerliche Privilegien, wenn sie als gemeinnützig anerkannt sind. 
privaten Beratungseinrichtungen und Berater:innen gegenüber, das vielen Ratsuchenden unbekannt ist und sie auch tatsächlich überfordert.

In diesem Geflecht verfügen die einzelnen Beratungseinrichtungen und Berater:innen oftmals nur über begrenzte personelle und fachliche Kompetenzen, teils auch über unterschiedliche Organisationsmodelle. Hinzu kommen besondere Bedarfe vonseiten der Ratsuchenden, z. B. sprachliche Probleme bei der Verständigung, psychische Erkrankungen oder komplexe Lebenssituationen mit vielfältigen gesundheitlichen, familiären und finanziellen Problemlagen. Nachfolgend soll beispielhaft auf drei aktuelle Herausforderungen eingegangen werden.

\subsection{Psychische Erkrankungen in der Beratung der Jobcenter}

Im Rahmen des Forschungsprojekts »Psychisch Kranke im SGB II: Situation und Betreuung" hat sich herausgestellt, dass mit 37 Prozent mehr als ein Drittel der Versicherten, die Arbeitslosengeld II (ALG II) bezogen, mindestens eine psychiatrische Diagnose aufwiesen (Schubert et al. 2013, S. 7). Die Daten zu Versicherten der Allgemeinen Ortskrankenkassen (AOK) zeigten dabei, dass der Anteil an Personen mit psychiatrischer Diagnose in fast allen Diagnosegruppen merklich gestiegen war und ALG-II-Bezieher:innen wesentlich häufiger psychiatrische Diagnosen aufwiesen als Berufstätige (Gühne/Riedel-Heller/Kupka 2020, S. 7).

Bei mehr als ein Fünftel der Leistungsbeziehenden in der Grundsicherung wurde dabei eine Erkrankung aus dem Bereich »Neurotische, Belastungs- und somatoforme Störungen" diagnostiziert, bei etwa einem Sechstel der Betroffenen eine affektive Störung oder Suchterkrankung (Gühne/Riedel-Heller/Kupka 2020, S. 7).

Fachkräfte der Jobcenter wiesen in einer Fallstudie darauf hin, dass die Besprechung von Gesundheitsfragen ein ausreichendes Vertrauen zwischen der Fachkraft und den Betroffenen voraussetzt (vgl. Oschmiansky/Popp 2020, S. 6). Tatsächlich wird das sogenannte Profiling in den Jobcentern regelmäßig bereits im Erstgespräch vorgenommen; zum Aufbau eines Vertrauensverhältnisses seien jedoch häufig mehrere Beratungstermine nötig (Oschmiansky/ Popp 2020, S. 5).

An dieser Stelle kollidiert das Interesse der Sozialverwaltung an der effizienten Bearbeitung und Erledigung von Fällen mit den Bedarfen der betroffenen Menschen. Zum Zeitpunkt der Fallstudie mangelte es zudem an Arbeitsvorlagen und Konzepten im Jobcenter, wie mit sensiblen Themen wie psychische Erkrankung etc. im Rahmen der Beratung umgegangen werden kann (Oschmiansky/ 
Popp 2020, S. 6). Dies lässt den Schluss zu, dass viele Fachkräfte in der Beratung nicht angemessen auf die Bedarfe von Menschen mit psychischen Erkrankungen reagieren.

Schon das Versorgungssystem für psychisch Kranke führt aufgrund des "Dschungels« der Zuständigkeiten und Einrichtungen zur Überforderung psychisch Kranker (vgl. Rademaker 2020). Dies gilt umso mehr, wenn psychisch Kranke in der Beratung mit dem weitaus komplexeren Bild der Sozialleistungen, Sozialleistungsträger und Sozialleistungserbringer konfrontiert werden.

\subsection{Bildung und Teilhabe}

Mit dem sogenannten Starke-Familien-Gesetz zur Neugestaltung des Kinderzuschlags und des Bildungs- und Teilhabepakets wurde das spezifische soziokulturelle Existenzminimum von Kindern, Jugendlichen und jungen Erwachsenen durch Bildungs- und Teilhabeleistungen ab dem 1.8.2019 verbessert (vgl. $\$ 28$ SGB II; BT-Drucksache 19/7504, 19/8036 und 19/8613). Die Evaluation der bundesweiten Inanspruchnahme und Umsetzung der Leistungen für Bildung und Teilhabe (BuT) hatte zuvor auch Hinweise auf Beratungsprobleme (in allen Rechtskreisen) gegeben. So erfuhren Leistungsberechtigte etwa erst nach einer längeren Zeit des Leistungsbezugs zum ersten Mal von den BuT-Leistungen oder kannten nur bestimmte Leistungsarten.

Haushalte mit geringen Deutschkenntnissen und Haushalte ohne Schulkinder waren schlechter informiert als andere Leistungsberechtigte. Auch das Wissen darum, dass in bestimmten Einkommenssituationen allein die BuT-Leistung anspruchsbegründend wirken kann, war - teilweise auch in den Leistungsstellen - nur wenig verbreitet. Ebenso wussten auch manche Wohngeld beziehende Haushalte nicht, dass allein der Wohngeldbezug zu BuT-Leistungen berechtigt (Bartelheimer et al. 2016, S. 229).

Der Grund hierfür lag auch darin, dass nur ein kleiner Teil der untersuchten Kommunen die in $₫ 4$ Abs. 2 Satz 2-4 SGB II verankerte Hinwirkung der Jobcenter - Information, Beratung und Unterstützung der Leistungsberechtigten einerseits, Förderung der Information und Beratung unterstützender Dritter andererseits (siehe Abschnitt 2.2) - als fortlaufende Aufgabe gestaltete. Viele Kommunen hatten ihre Aktivitäten zur Information von Leistungsberechtigten, aber auch Dritten, nach der Anfangsphase weitgehend eingestellt (Bartelheimer et al. 2016, S. 229).

Trotz dieser Herausforderungen bei der Inanspruchnahme und Umsetzung der BuT-Leistungen hat der Gesetzgeber die Vorgaben für die Hinwirkung, Unterstützung und Zusammenarbeit (vgl. \$4 Abs. 2 Satz 2-4 SGB II) im Rah- 
men des Starke-Familien-Gesetzes nicht fortentwickelt. Nach wie vor enthalten die Vorgaben kein einklagbares Recht, und konkrete Vereinbarungen der Jobcenter mit den Eltern - zur Inanspruchnahme von BuT-Leistungen - können nicht Gegenstand einer Eingliederungsvereinbarung nach $\$ 15$ SGB II sein.

\subsection{Frühzeitige Beratung von Jugendlichen mit Behinderung}

Eine weitere Herausforderung stellte die frühzeitige Beratung von Jugendlichen mit Behinderung dar. Sowohl für Schüler:innen als auch für Berater:innen ist es eine Herausforderung, die Komplexität der Bildungsangebote und Unterstützungsstrukturen zu überblicken (vgl. Arndt 2018, S. 40). Schätzungsweise nur weniger als ein Zehntel der Schulabgänger:innen mit Förderbedarf fängt eine betriebliche Ausbildung in einem anerkannten Ausbildungsberuf an (Jochmaring/Rathmann 2018, S. 7). Rund 80 bis 90 Prozent der Jugendlichen mit Förderbedarf beginnen nach der Schule eine Maßnahme zur Berufsvorbereitung (Jochmaring 2019, S. 344).

Bereits in der sogenannten Berufsorientierungsphase an den Schulen müssen verbesserte Bedingungen geschaffen werden, insbesondere durch Information und Unterstützung der Jugendlichen sowie fachlich kompetente und frühzeitig einsetzende Beratung (Deutsches Institut für Menschenrechte 2020, S. 53 f.). Verantwortlich dafür sind die Schulbehörden der Länder in Zusammenarbeit mit der Bundesagentur für Arbeit.

Letztere ist als Rehabilitationsträger für Leistungen zur Teilhabe am Arbeitsleben zuständig (vgl. $\$ 19$ und $\$ 112$ Abs. 1 SGB III in Verbindung mit $\$ 6$ Abs. 1 Nr. 2, $\$ 49$ SGB IX). Dies betrifft auch Jugendliche, die nicht erwerbsfähig sind und als Sozialgeldempfänger:innen nach SGB II mit erwerbsfähigen Leistungsberechtigten in einer Bedarfsgemeinschaft leben (vgl. $\$ 19$ Abs. 1 Satz 2 SGB II). Mit Vollendung des 18. Lebensjahres können sie nach $\$ 41$ Abs. 3 SGB XII Grundsicherung wegen einer dauerhaften vollen Erwerbsminderung beziehen, was gegenüber dem Sozialgeld vorrangig ist (vgl. \$5 Abs. 2 Satz 2 SGB II).

\section{Perspektiven für Beratungsleistungen}

Die besonderen Beratungs- und Unterstützungsansprüche in den Sozialgesetzbüchern dienen der Umsetzung des in der Verfassung verankerten Sozialstaatsprinzips sowie der Verwirklichung sozialer Gerechtigkeit und sozialer Sicherheit (vgl. \$1 SGB I). Die sozialen Rechte der Bürger:innen sollen hierbei möglichst weitgehend verwirklicht werden. 
Die in Abschnitt 5 genannten Beispiele zeigen, dass Berater:innen in der Praxis aufgrund des hohen gesetzlichen Anspruchs oftmals an ihre Grenzen stoßen, etwa aufgrund der Rahmenbedingungen (Kostendruck), gesetzlichen Anforderungen (Fördern und Fordern) oder besonderen Bedarfe der Ratsuchenden (z. B. Probleme bei der Verständigung, psychische Erkrankungen oder komplexe Lebenssituationen mit vielfältigen Problemlagen). Es bedarf daher umfassender Forschung, Konzeptentwicklung und Kapazitätsentwicklung, um die vielfältigen Herausforderungen der Praxis noch besser zu bewältigen. Hierbei sollte an das bestehende System aus öffentlichen, freigemeinnützigen und privaten Beratungsstellen angeknüpft werden.

Die im Folgenden dargestellten Ansatzpunkte erscheinen essenziell, um bessere Beratungsleistungen durch die Sozialleistungsträger zu ermöglichen und damit die sozialen Rechte der Menschen noch wirkungsvoller verwirklichen zu können.

\section{Frühzeitige und umfassende Beratung}

Menschen, die von längerer bzw. chronischer Erkrankung betroffen und damit zugleich von Behinderung bedroht sind, benötigen möglichst frühzeitig umfassende Beratung, um ihre sozialen Rechte durch Dienst-, Sach- und Geldleistungen sowie ihre Teilhabe am Leben in der Gesellschaft zu verwirklichen. Im besten Fall kann hierdurch eine Verbesserung ihrer gesundheitlichen Situation oder ihrer Teilhabe erzielt oder aber eine Verschlimmerung verhindert werden. Die durch die Rechtsprechung entwickelten Grundsätze der sogenannten Spontanberatung - auch des unzuständigen Leistungsträgers - sollten daher gestärkt und ausdrücklich in das Sozialgesetzbuch aufgenommen werden.

Das beratende Personal der Leistungsträger müsste zugleich gezielt darin geschult werden, noch besser auf die Verzahnung unterschiedlicher Dienst-, Sachund Geldleistungen der Leistungsträger hinzuweisen. So haben beispielsweise bereits von Behinderung bedrohte Menschen nach $₫ 42$ Abs. 3 bzw. $\$ 49$ Abs. 6 SGB IX Anspruch auf medizinische, psychologische und pädagogische Hilfen gegenüber den Rehabilitationsträgern, wozu unter anderem auch Hilfen zur Aktivierung von Selbsthilfepotenzialen, Hilfen zur seelischen Stabilisierung sowie Anleitung und Motivation zur Inanspruchnahme von Leistungen gehören. Betroffene sollten dahingehend beraten werden, unabhängig davon, welcher Leistungsträger von einer länger andauernden bzw. chronischen Erkrankung Kenntnis erlangt. 


\section{Beratungskompetenz und Fallmanagement}

Die angeführten Studien bzw. die Herausforderungen legen nahe, dass das Konzept der Beratung und teilweisen Unterstützung in der Grundsicherung für Arbeitsuchende nicht bei allen Leistungsberechtigten hinreichende Wirkung entfaltet. Daher werden mehr Fachkräfte in den Jobcentern als notwendig erachtet, die besser im psychosozialen und im Betreuungsbereich ausgebildet sind (vgl. Kupka, zit. in Rademaker 2020; ähnlich Oschmiansky/Popp 2020, S. 5).

Dies bedeutet nicht, dass das gesetzgeberische Konzept der Beratung und teilweisen Unterstützung in der Grundsicherung für Arbeitsuchende gescheitert wäre. Für einen nicht unerheblichen Teil der Leistungsberechtigten - z. B. auch Personen mit psychischen Erkrankungen - genügt allerdings die Beratung mit Fokussierung auf Arbeitsmarktintegration nicht. Diese Leistungsberechtigten haben besondere persönliche Bedarfe, die eine Fortentwicklung der Handlungskompetenzen und Handlungsinstrumente der Jobcenter erfordern.

Es bedarf daher auch der Entwicklung konkreter Arbeitsvorlagen und systematischer Konzepte, wie mit psychischen Erkrankungen im Rahmen der Beratung umgegangen werden kann, einer verbesserten Schulung der Fachkräfte, auch hinsichtlich der Umsetzung einer zugewandten, Vertrauen schaffenden Gesprächsführung, eines verbesserten internen Austauschs im Jobcenter, passgenauer Unterstützungsangebote und ausreichend Zeit für die Leistungsberechtigten (Oschmiansky/Popp 2020, S. 5 und 6).

\section{Betreuungs- und Unterstützungsmöglichkeiten}

Zusätzliche Betreuungs- und Unterstützungsmöglichkeiten - ähnlich wie dies im Rechtskreis des SGB XII generell vorgesehen ist (siehe Abschnitt 2.2; $\$ 11$ SGB XII) - gibt es im SGB II nur punktuell, wie z. B. im Rahmen der Förderung schwer zu erreichender junger Menschen nach $\$ 16 \mathrm{~h}$ SGB II. Die Förderung umfasst hier zusätzliche Betreuungs- und Unterstützungsleistungen mit dem Ziel, dass Leistungen der Grundsicherung für Arbeitsuchende in Anspruch genommen und erforderliche therapeutische Behandlungen eingeleitet werden und an die Regelangebote zur Aktivierung und Stabilisierung nach SGB II sowie eine frühzeitige intensive berufsorientierte Förderung herangeführt wird $(\$ 16 \mathrm{~h}$ Abs. 1 Satz 2 SGB II).

Eine Ausweitung dieses besonderen Förderungstatbestands auf weitere Leistungsberechtigte, auch nach Vollendung des 25. Lebensjahres, erscheint angezeigt, gerade angesichts des großen Anteils von Leistungsberechtigten mit psychischen oder Suchterkrankungen. Um den besonderen Bedarfen von Leistungsberechtigten mit psychischen Erkrankungen gerecht zu werden, wird teilweise sogar gefordert, dass psychosoziales Coaching direkt im Jobcenter statt- 
finden soll, unter Mitwirkung von klinisch geschulten Psycholog:innen, die im besten Fall an eine Klinik vor Ort angebunden sind. Die Coachs sollen hierbei als Lotsen für Arbeitslose im Dschungel der Möglichkeiten fungieren (Rademaker 2020).

\section{Verbessertes Fallmanagement}

Die Herausforderungen bei der Inanspruchnahme und Umsetzung von Leistungen für Bildung und Teilhabe legen nahe, dass die vom Gesetzgeber intendierte "Hinwirkung « der Jobcenter (damit Kinder und Jugendliche Zugang zu geeigneten vorhandenen Angeboten der gesellschaftlichen Teilhabe erhalten) als fortlaufende Aufgabe verstanden werden sollte - durch Information, Beratung und Unterstützung der Leistungsberechtigten einerseits und Förderung der Information und Beratung unterstützender Dritter andererseits (vgl. Bartelheimer et al. 2016, S. 229). Hieraus folgt, dass Beratungsleistungen künftig noch stärker in ein Gesamtkonzept im Rahmen des Fallmanagements eingebunden werden sollten, um dem Auftrag und Ziel noch besser gerecht zu werden, die sozialen Rechte der Bürger:innen möglichst weitgehend zu verwirklichen (vgl. \2 Abs. 2 SGB I).

\section{Literatur}

Arndt, I. (2018): Verbesserung der Übergänge von Jugendlichen von der Schule in Ausbildung und Beruf durch die Umsetzung von Inklusion im Bildungssystem, in: Arndt, I./Neises, F./Weber, K. (Hrsg.): Inklusion im Übergang von der Schule in Ausbildung und Beruf. Hintergründe, Herausforderungen und Beispiele aus der Praxis, Bonn/Leverkusen, S. 38-54.

Bähr, H./Dietz, M./Kupka, P./Ramos Lobato, P./Stobbe, H. (2018): Grundsicherung und Arbeitsmarkt in Deutschland, Lebenslagen - Instrumente - Wirkungen, IAB-Bibliothek, Band 370, Bielefeld.

Bartelheimer, P./Henke, J./Kaps, P./Kotlenga, S./Marquardsen, K./Nägele, B./ Wagner, A./Söhn, N./Achatz, J./Wenzig, C. (2016): Evaluation der bundesweiten Inanspruchnahme und Umsetzung der Leistungen für Bildung und Teilhabe, Schlussbericht, Göttingen/Nürnberg.

Deutsches Institut für Menschenrechte (2020): Entwicklung der Menschenrechtssituation in Deutschland. Juli 2019-Juni 2020. Bericht an den Deutschen Bundestag gemäß $₫ 2$ Absatz 5 DIMRG, Berlin.

Graßhof, K. (2020), in: Maunz, T./Schmidt-Bleibtreu, B./Klein, F./Bethge, H. (Hrsg.): Bundesverfassungsgerichtsgesetz. Kommentar, Stand: 60. Ergänzung, Juli 2020, München. 
Gühne, U./Riedel-Heller, S./Kupka, P. (2020): Wie sich psychische Erkrankungen auf die Teilhabe am Arbeitsmarkt auswirken - ein Überblick, in: IAB-Forum, 25. August 2020, www.iab-forum.de/wie-sich-psychische-erkrankungen-aufdie-teilhabe-am-arbeitsmarkt-auswirken-ein-ueberblick (Abruf am 12.7.2021).

Jochmaring, J. (2019): Übergänge von Schüler/innen mit sonderpädagogischem Förderbedarf in die Berufsausbildung. Eine Auswertung von Sekundärstatistiken, in: Zeitschrift für Pädagogik 65(3), S. 335-354.

Jochmaring, J./Rathmann, K. (2018): Präferenzen und Barrieren beim Übergang von der Förderschule in die berufliche Bildung. Ergebnisse einer qualitativen Regionalstudie, in: Impulse. Magazin der Bundesarbeitsgemeinschaft für Unterstützte Beschäftigung, 2018(85), S. 6-10.

Knickrehm, S. (2021), in: Gagel, A. (Hrsg.): SGB II/SGB III. Grundsicherung und Arbeitsförderung, Stand: 80. Ergänzung, 1. Februar 2021, München, $\mathbb{} 4$ SGB II.

Meißner, M. (2019): Die ergänzende unabhängige Teilhabeberatung (EUTB) im System der Sozialberatung, in: Lemme, S./Lüngen, H./Pilcher, J. (Hrsg.): Rehabilitation und Nachsorge nach Schädelhirnverletzung. Bundesteilhabegesetz-Umsetzung: Impulse aus dem Labyrinth?, ZNS - Zentrales Nervensystem, Band 13, Bad Honnef, S. 6-15.

Nixdorf, C.P. (2020): Kompetentes Handeln im Jobcenter: Zusammenfassung der Befunde der Studie »Kompetentes Handeln im Jobcenter - Band I: Kompetenz aus Sicht der Grundsicherungsträger und Integrationsfachkräfte«, Hannover, https://nbn-resolving.org/urn:nbn:de:0168-ssoar-70532-8 (Abruf am 12.7.2021).

Oschmiansky, F./Popp, S. (2020): Psychische Probleme von Menschen im SGB II: Was Fachkräfte im Jobcenter tun, um diese zu erkennen, in: IAB-Forum, 14. September 2020, www.iab-forum.de/psychische-probleme-von-menschenim-sgb-ii-was-fachkraefte-im-jobcenter-tun-um-diese-zu-erkennen/ (Abruf am 12.7.2021).

Rademaker, M. (2020): Psychisch Kranke im Hartz-IV-System. Im Dschungel der Zuständigkeiten, www.deutschlandfunk.de/psychisch-kranke-im-hartziv-system-im-dschungel-der.724.de.html?dram:article_id=483359 (Abruf am 12.7.2021).

Schubert, M./Parthier, K./Kupka, P./Krüger, U./Holke, J./Fuchs, P. (2013): Menschen mit psychischen Störungen im SGB II, IAB-Forschungsbericht 12/2013, Nürnberg.

Spellbrink, W. (2020), in: Körner, A./Leitherer, S./Mutschler, B./Rolfs, C (Hrsg.): Kasseler Kommentar Sozialversicherungsrecht, Band 1, Stand: 111. Ergänzung, 1. September 2020, München. 
Trenk-Hinterberger, P. (2020), in: Krahmer, U./Trenk-Hinterberger, P. (Hrsg.): Sozialgesetzbuch I. Allgemeiner Teil. Lehr- und Praxiskommentar, 4. Auflage, Baden-Baden.

Vitols, K./Gabriel, S. (2020): Branchenanalyse Sozialversicherungsträger. Veränderungen auf Arbeit, Beschäftigung und Leistungsanforderungen durch Digitalisierung und Organisationsstrategien, Working Paper Forschungsförderung Nr. 195 der Hans-Böckler-Stiftung, Düsseldorf.

Wache, D. (2020), in: Rauscher, T./Krüger, W. (Hrsg.): Münchener Kommentar zur Zivilprozessordnung, 6. Auflage, München. 



\title{
Gesellschaftliche Teilhabe durch Bildung?
}

\author{
Ein sozial inklusives Bildungssystem braucht Alternativen \\ zum Prinzip der Meritokratie
}

Ulf Banscherus

\section{Einleitung}

Bildung gilt als wesentliche Voraussetzung für die individuelle gesellschaftliche Teilhabe, die nicht nur die Ausübung einer Erwerbstätigkeit umfasst, sondern soziale, kulturelle und demokratische Aspekte ausdrücklich einschließt. Das Verständnis von gesellschaftlicher Teilhabe durch Bildung ist somit anschlussfähig an erweiterte Konzepte der sozialen Grundsicherung, die ebenfalls nicht nur auf eine Integration in den Arbeitsmarkt abzielen, sondern auch soziale, kulturelle und demokratische Prozesse berücksichtigen.

Im Unterschied zu diesem programmatischen Konzept dominiert allerdings in Deutschland sowohl im bestehenden Bildungssystem als auch im System der sozialen Sicherung das grundlegende Prinzip der Leistungsgerechtigkeit, dem zufolge der Erwerb von Ansprüchen immer spezifische individuelle (Vor-)Leistungen voraussetzt. Dies bedeutet beispielsweise, dass die Höhe des Arbeitslosengeldes an erfolgte Beitragsleistungen gekoppelt ist oder die Zulassung zum Studium vielfach über die Note des Zertifikats der Hochschulzugangsberechtigung geregelt wird.

Eine weitere korrespondierende Gemeinsamkeit zwischen dem Bildungssystem und dem System der sozialen Sicherung ist die hohe Relevanz der individuellen Eigenverantwortung. Diese drückt sich unter anderem darin aus, dass Scheitern - sowohl in der Schullaufbahn als auch in der beruflichen Karriere im bestehenden Wirtschafts- und Gesellschaftsmodell in erster Linie als individuelles Versagen betrachtet wird und strukturelle Aspekte als deutlich weniger relevant betrachtet werden (Kaps et al. 2017; Quenzel/Hurrelmann 2019; Hopf 2011). 
Das Prinzip der Bedarfsgerechtigkeit ist in Deutschland - bei der sozialen Sicherung ebenso wie im Bildungswesen - den Prinzipien der Leistungsgerechtigkeit und der Eigenverantwortlichkeit klar untergeordnet. Dies zeigt sich z. B. darin, dass die Höhe der Grundsicherung für Arbeitslose wie auch der Bezug von BAföG-Leistungen für Studierende familiären Unterhaltsverpflichtungen nachgelagert ist und entsprechende Unterstützungszahlungen eine umfassende Bedürttigkeitsprüfung voraussetzen. Hinzu kommt, dass sowohl die Grundsicherung als auch das BAföG in erster Linie an der materiellen Absicherung des soziokulturellen Existenzminimums orientiert ist - wobei aufgrund der festgelegten Höhe der Sätze hochgradig umstritten ist, inwieweit dieses Ziel in der Praxis tatsächlich erreicht wird.

Aktive Unterstützungsmaßnahmen wie Coachings, Trainings oder weitere individuell abgestimmte Eingliederungsmaßnahmen in das Erwerbsleben oder den Studienalltag stehen hingegen im Bildungssystem erst in Ansätzen (z.B. Schulsozialarbeit, Mentoringprogramme von privaten Initiativen, individuelle Bildungsberatung) und im Rahmen der Grundsicherung nur in geringem Umfang (z. B. Weiterbildungsangebote) oder mit Einschränkungen (z. B. Nachholen einer Berufsausbildung) zur Verfügung (Kuhlmann 2018; Knuth 2018; Kaps et al. 2017).

Vor diesem Hintergrund hinterfragt dieser Beitrag - bezogen auf das Bildungssystem in Deutschland und im Kontext der Diskussion um ein erweitertes Verständnis von sozialer Grundsicherung - das traditionelle Prinzip der Leistungsgerechtigkeit, indem das normative Modell der Meritokratie (Abschnitt 2) mit Befunden aus der empirischen Bildungsforschung (Abschnitt 3) konfrontiert wird. Anschließend werden in Abschnitt 4 mögliche Ansätze umrissen, die eher dem Prinzip der Bedarfsgerechtigkeit folgen und dabei helfen könnten, dass der Zugang zu Bildung für einen größeren Teil der Bevölkerung im weiteren Lebensverlauf zu einer gesicherten gesellschaftlichen Teilhabe beitragen kann.

\section{Meritokratie als Grundprinzip von Bildungssystem und Bildungspolitik}

Meritokratie als Grundprinzip des Bildungssystems knüpft an das Versprechen an, dass »die individuelle Leistung den Status und den Erfolg einer Person bestimmt" (El-Mafaalani 2014, S. 11; Hadjar 2008; Becker/Hadjar 2017). Dieses Verständnis ist insgesamt kennzeichnend für marktwirtschaftlich verfasste (Leistungs-)Gesellschaften, in denen individuelle Chancen unmittelbar an individuelle Begabungen und Leistungen geknüptt sein sollen. 
Dieser Ansatz spiegelt sich unter anderem in den Vereinbarungen der Regierungschefs von Bund und Ländern im Rahmen des "Dresdner Bildungsgipfels" im Oktober 2008. Darin heißt es zum einen: »Jede und jeder soll die Chance zum Aufstieg durch Bildung haben." Auch wird betont, dass »[j]eder und jede, die oder der beruflich weiter lernen und aufsteigen will, [...] eine Chance auf Unterstützung haben« soll (Bundesregierung/Regierungschefs der Länder 2008, S. 11). Zum anderen wird diese Chance jedoch an spezifische Leistungsbedingungen zurückgebunden.

Die gesellschaftlich tief verankerte Vorstellung, dass ein sozialer Aufstieg durch Bildung zwar strukturell möglich, jedoch immer an die individuellen Leistungen rückgebunden sein soll, für die jede:r Einzelne selbst die Verantwortung hat, legitimiert allerdings de facto die (Re-)Produktion sozialer Ungleichheiten, weil sie den immensen Einfluss der sozialen Herkunft auf die Bildungsbiografien konzeptionell ausblendet.

Beispielsweise wurden schon vor dem Ersten Weltkrieg vor allem von sozialdemokratischen und liberalen Parteien verstärkt Forderungen nach der Möglichkeit für einen »Aufstieg der Begabten« unabhängig von der Herkunftsfamilie erhoben, aber auch diese waren programmatisch an die individuelle Leistung und »Tüchtigkeit" gekoppelt (Banscherus/Wolter 2016; Wollersheim 2014). Die strukturellen - und natürlich auch habituellen - Selektionsmechanismen beim Zugang zu höherer Bildung blieben trotz anderslautender Reforminitiativen sowohl in der Weimarer Republik als auch nach dem Zweiten Weltkrieg weitgehend stabil (Keim 2000).

Wirkliche Öffnungstendenzen bestanden in (West-)Deutschland nur in einer kurzen Phase bildungspolitischer Reformen in den 1960er- und 1970er-Jahren. Neben sozialen Erwägungen (Chancengleichheit und Durchlässigkeit) trugen hierzu allerdings vorrangig ökonomische Motive bei. Vor dem Hintergrund des internationalen Trends zum Ausbau der Bildungssysteme, der Ergebnisse erster internationaler Leistungsvergleiche und eines befürchteten Rückstands vor allem im naturwissenschaftlich-technischen Bereich breitete sich - insbesondere infolge des »Sputnik-Schocks« von 1957 - die Sorge aus, aufgrund veralteter Strukturen des Bildungswesens gegenüber der Sowjetunion und ihren Satellitenstaaten ökonomisch ins Hintertreffen zu geraten (Massing 1984).

Dieses Modernisierungspostulat, das nicht nur auf die Schulen, sondern auch auf die Hochschulen abzielte, sollte nicht zuletzt erweiterte Spielräume für den individuellen Aufstieg durch Bildung schaffen - wenngleich auch hier stets eine enge Bindung an das individuelle Begabungskonzept impliziert war (Friedeburg 1997; Massing 1984). Im Zentrum stand - basierend auf der Annahme, dass viele Begabungen insbesondere in den Reihen der Industriearbeiterschaft 
und der Landbevölkerung noch nicht entdeckt und ausgeschöpft würden - die Frage, wie eine Erschließung der bestehenden »Begabungsreserven« in der Bevölkerung möglich sein könnte.

Bildung wurde in dieser Zeit parteiübergreifend als umfassende gesellschaftspolitische Aufgabe verstanden, in deren Rahmen vor allem weiterführende Bildungswege für breitere Bevölkerungsschichten geöffnet werden sollten (Rudloff 2007 und 2014; Massing 1984). Außerdem sollten Schulorganisation und Didaktik so verändert werden, dass sie die optimale individuelle Förderung aller Kinder und Jugendlichen ermöglichen und zugleich Lernwege möglichst lange offenhalten (Keim 2000).

In der zweiten Hälfte der 1970er-Jahre wurden die ideologischen Konflikte in bildungspolitischen Fragen vor allem zwischen konservativen und sozialdemokratischen Politiker:innen erneut virulent. Auch stießen bildungspolitische Motive, die wieder stärker die Unterschiede zwischen den Individuen betonten und weniger auf umfassende Förderung als vielmehr auf Mechanismen wie Wettbewerb und Differenzierung setzten, in der Bevölkerung wieder auf eine höhere Zustimmung.

Wurde die Bildungsexpansion in den 1960er- und frühen 1970er-Jahren noch stark von arbeitsmarktpolitischen Zielen begleitet, die zumindest auf der programmatischen Ebene für viele Menschen die Chance auf einen individuellen Aufstieg durch Bildung in sich bargen, wich dies nun zunehmend einer insbesondere an meritokratischen Argumentationsmustern orientierten Steuerung der Bildungsnachfrage (z. B. Stärkung der beruflichen Bildung anstelle akademischer Bildung, neue Fokussierung auf »Hochbegabung«; Rudloff 2014; Wollersheim 2014).

Das zähe politische Ringen um die Ausgestaltung des Bildungssystems zwischen den Polen Chancengleichheit und Förderung individueller Begabungen in stark ausdifferenzierten Strukturen sollte die Bildungspolitik der 1980er- und 1990er-Jahre, die in der Praxis allerdings recht weitgehend von gegenseitigen Blockadeversuchen dominiert war, in besonderer Weise prägen.

Eine breite öffentliche Diskussion über die unterschiedlichen Bildungschancen und die nach wie vor beträchtlichen Hürden für einen Bildungsaufstieg innerhalb des deutschen Bildungssystems wurde erst wieder durch die mediale Rezeption der internationalen PISA-Studien ab dem Jahr 2001 angestoßen, wobei allerdings auch der internationalen Wettbewerbsfähigkeit zwischen den Bildungssystemen eine wichtige Rolle zukam (Büchner 2003).

Die praktischen Konsequenzen bleiben jedoch auch zwei Jahrzehnte später überschaubar. Zwar lassen sich in den Bundesländern teilweise Tendenzen zu einer Entdifferenzierung des Schulsystems im Sinne einer Zweigliedrigkeit beobachten, weitergehende Schritte wurden allerdings dadurch verhindert, dass 
meritokratische Prinzipien bis weit in die Sozialdemokratie hinein tief verankert sind und somit ein Anspruch auf individuelle Förderung hin zu einem Aufstieg durch Bildung zumindest eine positive Prognose über die Leistungsfähigkeit der einzelnen (Weiter-)Bildungsteilnehmer:innen voraussetzt.

Aus theoretischer Perspektive lässt sich das deutsche Bildungssystem - wenig überraschend - als historisch gewachsene und gesamtgesellschaftlich relevante Institution mit einem Geflecht aus formellen und informellen Regeln begreifen, die zum Teil durch ihre Verankerung in Gesetzen ein hohes Maß an Verbindlichkeit aufweisen, zum Teil aber auch in der Praxis der beteiligten Organisationen sowie der kollektiven Alltagspraxis tief verankert sind. Das Bildungswesen kann somit als gesellschaftliche Selektions- und Klassifikationsinstitution verstanden werden, als ein auf Dauer gestelltes Gefüge sozialer Regeln und Normen, das aus den in einer Gesellschaft bestehenden Machtverhältnissen resultiert (Meyer 1977).

Die verschiedenen Einrichtungen des Bildungswesens wie Schulen oder Hochschulen sind diesem Verständnis nach einerseits gesellschaftlich legitimierte Instanzen zur formalen Anerkennung individueller Leistungen und spielen andererseits über die Verleihung von Bildungstiteln als institutionalisierten Formen kulturellen Kapitals eine wichtige Rolle bei der Statuszuweisung im sozialen Raum (vgl. Bourdieu 2003). Das deutsche Bildungswesen ist somit strukturell in erster Linie auf die Bewertung individueller Leistungen (Klassifikation) ausgerichtet, die ungleiche gesellschaftliche Positionierungen legitimiert (Selektion).

Die Regeln des Bildungssystems stellen aber zugleich auch eine Orientierungsmarke für die Entscheidungen und Aktivitäten der einzelnen Personen dar. So legen diese Regeln beispielsweise fest, wer die Kriterien für den Erwerb eines bestimmten Abschlusses oder für die Zulassung zu einem spezifischen Bildungsgang erfüllt.

Die institutionellen Regeln des Bildungswesens gehen also mit erheblichen Distinktionsprozessen einher, die - vermittelt über Habitusmuster und soziale Praktiken - die Wahrnehmung der eigenen Möglichkeiten beeinflussen. Dies liegt vor allem darin begründet, dass die Wahrnehmungs-, Beurteilungs- und Handlungsmuster einer Person in hohem Maße durch ihre Einbindung in soziale Strukturen und eine in diesen geübte soziale Praxis geprägt sind (Bourdieu 1993, 2001 und 2003; Bourdieu/Passeron 1973; vgl. Meyer/Rowan 1977).

Heike Solga (2005) führt dies auf die historisch gewachsene und gesellschaftlich tief verankerte Prämisse zurück, nach der das deutsche Bildungssystem meritokratisch ausgestaltet sei und in der kollektiven Vorstellung somit nicht die soziale Herkunft, sondern die individuelle Leistungsfähigkeit die Grundlage für die unterschiedlichen Selektions- und Klassifikationsprozesse bilde. Auch 
wenn vielfältige erziehungs- und sozialwissenschaftliche Forschungsergebnisse die vorgebliche Rationalität meritokratischer Selektion und Rekrutierung eindrucksvoll widerlegt und so das Konzept der Meritokratie als Illusion enthüllt haben, konnte die Relevanz der Meritokratie als Legitimationsmuster sozialer Ungleichheit bislang nicht gebrochen werden - zu fest ist die Ausgestaltung des Bildungssystems als prägender Bestandteil der Sozialstruktur in Deutschland etabliert (Hadjar 2008; Becker/Hadjar 2017).

Insgesamt bleibt das politische Versprechen eines sozialen Aufstiegs durch Bildung ein Lippenbekenntnis, wenn die Verantwortung hierfür in meritokratischer Tradition in erster Linie bei jedem einzelnen Menschen liegen soll. Dies blendet herkunftsbedingte Ungleichheiten bei den »Startchancen" aus und legitimiert zugleich die Reproduktion bestehender Ungleichheiten, indem denjenigen, die von höherer Bildung ausgeschlossen werden, einerseits vermittelt wird, dass sie selbst nicht "gut genug" gewesen seien, und bei den Angehörigen der unteren sozialen Milieus zugleich der Eindruck entsteht, dass Aufstiegsambitionen »nichts für uns« sein können.

Daran ändern auch die zahlreichen Einzelfälle eines erfolgreichen sozialen Aufstiegs durch Bildung nichts, sondern diese stützen im Gegenteil die in Politik und Gesellschaft dominante Haltung, dass soziale Durchlässigkeit auch in den gegebenen Strukturen möglich sei, sofern die individuellen Begabungen hierfür vorhanden seien.

\section{Soziale Ungleichheit im Bildungswesen aus empirischer Sicht}

Die Literaturlage zur sozialen Ungleichheit im Bildungswesen ist ungeheuer vielfältig. Zudem werden bereits seit Längerem neben der sozialen Herkunft, die häufig durch die Bildungsabschlüsse der Eltern operationalisiert wird, weitere Diversitätsdimensionen wie Migrationshintergrund, Geschlecht oder Behinderung vertiefend analysiert.

In hohem Maße ausdifferenziert sind außerdem die untersuchten Personengruppen bzw. Organisationen des Bildungssystems, die von der frühen Kindheit bis ins hohe Alter reichen. Gleiches gilt für den räumlichen Fokus der Untersuchungen. Dieser reicht von der lokalen Ebene bis hin zu weltweiten Entwicklungen. Nicht zuletzt weisen Studien zur sozialen Ungleichheit im Bildungswesen und möglichen gesellschaftlichen Folgewirkungen sowohl hinsichtlich ihrer theoretischen Basis als auch in methodischer Perspektive eine außerordentlich große Bandbreite auf (z. B. Becker/Lauterbach 2016; Baader/Freytag 2017; Quenzel/ Hurrelmann 2019). 
Ein besonders anschauliches Beispiel für die Entwicklung der Bildungsbeteiligung abhängig vom Bildungshintergrund der Eltern ist der sogenannte Bildungstrichter, der die abnehmende Bildungsbeteiligung im allgemeinbildenden Zweig des deutschen Bildungssystems über die verschiedenen Stufen des formalen Bildungswegs dargestellt. Für die Wochenzeitung »DIE ZEIT« haben Astrid Herbold, Louisa Reichstetter und Anna-Lena Scholz (2017) unter Rückgriff auf die Daten des Hochschul-Bildungs-Reports (Stifterverband 2017) die Unterschiede bei der herkunftsbedingten Bildungsbeteiligung von der Grundschule bis zur Promotion dargestellt (siehe Abbildung 1).

Abb. 1: Bildungsbeteiligung und Übertrittsquoten nach Bildungsherkunft

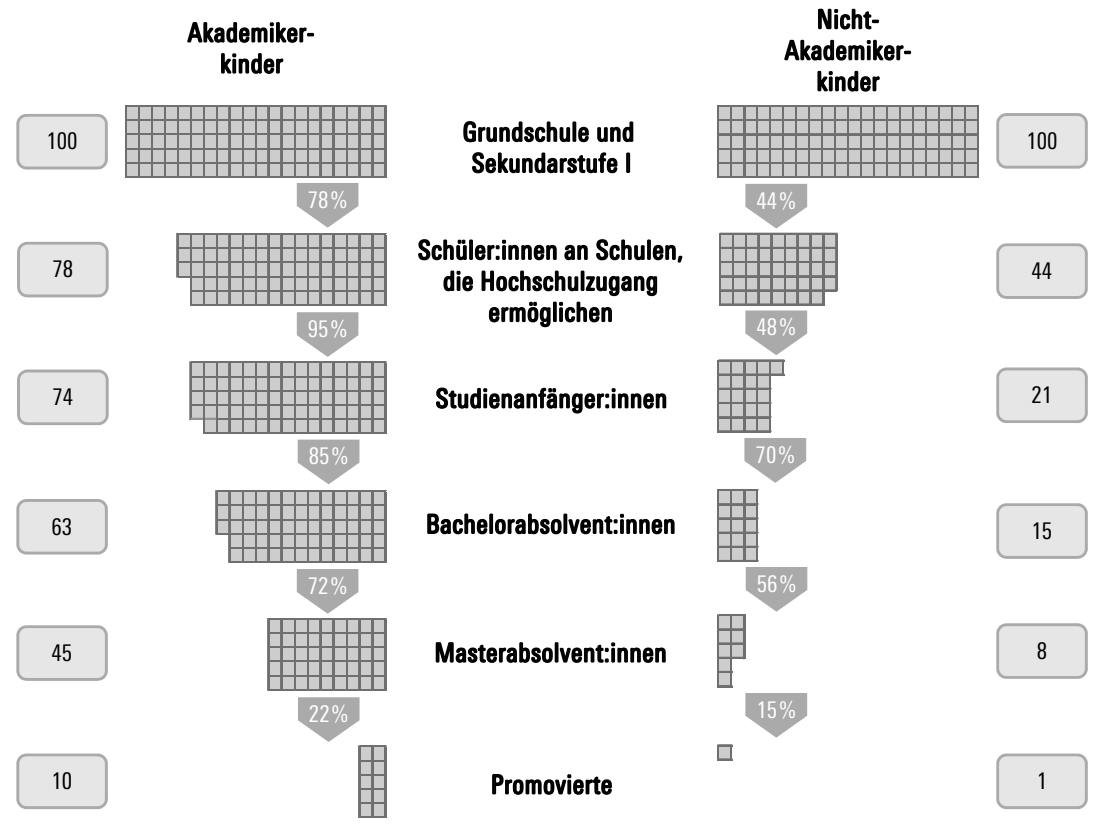

Quelle: Herbold/Reichstetter/Scholz 2017 (eigene Darstellung)

Die in dieser Darstellung erfasste herkunftsbedingte Selektion von Schüler:innen beginnt bereits beim Übergang von der Grundschule in die verschiedenen Formen weiterführender Schulen, die über unterschiedliche Profile verfügen und zu unterschiedlichen Abschlüssen führen. Hier zeigt sich, dass von 100 Schüler:innen aus einem nicht-akademischen Elternhaus nur 44 in eine Schulform wechseln, die zu einer Hochschulzugangsberechtigung führt, während es 
bei Kindern, deren Eltern über einen Hochschulabschluss verfügen, mit 78 fast doppelt so viele sind.

Von der Studienberechtigung macht nur rund die Hälfte (48 Prozent) der Studienberechtigten ohne akademische Bildungsherkunft Gebrauch, während es bei denjenigen mit akademischer Bildungsherkunft fast alle (95 Prozent) sind. Von 100 Kindern aus einem nicht-akademischen Elternhaus beginnen also nur 21 ein Hochschulstudium, demgegenüber sind es bei Kindern aus einem akademischen Elternhaus mit 74 rund dreieinhalb Mal so viel. ${ }^{1}$

Von den Studienanfänger:innen, die als Erste in ihrer Familie die Hochschule besuchen, schließen 70 Prozent ein Bachelor-Studium ab, was 15 von ursprünglich 100 Kindern entspricht. Bei denjenigen mit akademischer Bildungsherkunft erreichen 85 Prozent einen Bachelorabschluss, somit 63 von 100 Kindern. Einen Masterabschluss erreichen 45 von 100 Kindern, deren Eltern bereits über einen Hochschulabschluss verfügen, während es bei denjenigen mit nicht-akademischer Bildungsherkunft nur 8 sind. Auch beim Abschluss einer Promotion setzen sich die herkunftsbedingten Unterschiede fort. Einen Doktortitel erwerben 10 von 100 Kindern aus einem akademisch gebildeten Elternhaus, aber nur eines mit einer nicht-akademischen Bildungsherkunft.

Trotz der zweifellos bestehenden methodischen Schwächen des Konzepts des Bildungstrichters, die sich aus der Kombination unterschiedlicher Datensätze und der Verknüpfung unterschiedlicher Erhebungszeiträume ergeben, geht diese Darstellung bestehender sozialer Ungleichheiten eindeutig konform mit unzähligen Befunden zur (Selbst-)Selektion von Personen aus niedrigeren sozialen Milieus im Bildungssystem auf seinen verschiedenen Stufen und veranschaulicht auch quantitativ die bestehenden herkunftsbedingten Exklusionsrisiken.

\section{Mögliche Ansätze für mehr gesellschaftliche Teilhabe durch Bildung}

Das auf Selektion und Klassifikation angelegte institutionelle Gefüge des deutschen Bildungssystems, das die Beteiligungschancen relevanter Teile der Bevölkerung systematisch einschränkt - insbesondere bei von Exklusion und Bildungsarmut bedrohten Personengruppen (Quenzel/Hurrelmann 2019) - und

1 | Zu etwas höheren Anteilswerten kommen Nancy Kracke, Daniel Buck und Elke Middendorf (2018) auf Basis von Daten der amtlichen Statistik und des Deutschen Zentrums für Hochschul- und Wissenschaftsforschung (DZHW). Zu Übergängen ins, im und aus dem Hochschulsystem vgl. z. B. Banscherus et al. 2014. 
nicht zuletzt Ungleichheiten legitimiert, hat einen erheblichen Einfluss auf individuelle Biografien und gesellschaftliche Teilhabechancen. Beispielsweise gibt es nachgewiesenermaßen Wechselwirkungen zwischen der Bildungsbeteiligung und gesundheitlicher Situation, Einbindung in soziale Netzwerke oder Risiko für deviantes Verhalten bis hin zur Kriminalität.

Hinzu kommen deutliche Unterschiede hinsichtlich der individuellen Erwerbschancen, für die der Bildungsgrad ebenfalls eine erhebliche Bedeutung hat (z. B. Quenzel/Hurrelmann 2019; Baader/Freytag 2017). Die drohende Exklusion aus dem Erwerbsleben verstärkt wiederum gesellschaftliche Exklusionsrisiken, die negative Folgen für den sozialen und kulturellen Zusammenhalt der Gesellschaft haben können (Kronauer 2009 und 2015).

Insgesamt sprechen nicht nur ökonomische Gründe (z. B. Fachkräftebedarf, Wettbewerbsfähigkeit; Hanushek/Wößmann 2019) und die genannten individuellen wie sozialen Gründe für eine deutliche Ausweitung der Beteiligungschancen an Bildung, sondern auch grundlegende Erwägungen der Sozialphilosophie: Aus demokratietheoretischer Sicht setzt die gleichberechtigte Teilhabe aller Bürger:innen im Sinne einer aktiven Mitgestaltung von Staat und Gesellschaft die Befähigung jedes und jeder Einzelnen zur kritischen Beurteilung politischer, sozialer und ökonomischer sowie durchaus auch technischer und ökologischer Herausforderungen und Gestaltungsmöglichkeiten voraus (Gerhardt 2018). Es gilt also:

„Eine gute Erziehung und eine republikanische Staatsordnung sind deswegen komplementär aufeinander angewiesen, weil jene Form eines allgemeinen und öffentlichen Unterrichts im Einzelnen erst die kulturellen und moralischen Befähigungen hervorbringt, mit deren Hilfe diese dann derart existieren und gedeihen kann, dass die Bürgerschaft an der politischen Emanzipation auch des niederen Volks noch Anteil nimmt.« (Honneth 2012, S. 430)

Greift man diese Erwägungen auf, lässt sich weitergehend sagen,

»dass unter den Aufgaben des demokratischen Rechtsstaates jene an vorderster Stelle zu stehen hat, jedem seiner zukünftigen Mitglieder durch angemessene Bildungseinrichtungen gleichermaßen die Möglichkeit an die Hand zu geben, an der öffentlichen Legitimierung seiner eigenen Entscheidungen >ohne Angst und Scham (Honneth 2012, S. 435)

Realisierbare Beteiligungschancen an einem öffentlich verantworteten Bildungssystem sind nach diesem Verständnis also mehr als das von Ralf Dahrendorf postulierte »Bürgerrecht«, sie sind nicht weniger als eine zentrale Voraussetzung für die Zukunftsfähigkeit der Demokratie. Gemessen an den demokratietheore- 
tischen Anforderungen - die immer auch kompensatorische Elemente im Sinne einer Förderung der Chancengleichheit ausdrücklich mit einschließen, da in der humanistischen Tradition »[a]lle Urteile über den Staat, den Frieden und [...] die Freiheit [...] aus der Perspektive der Öffentlichkeit gefällt [werden], zu der ausdrücklich alle, und gerade auch die Ärmsten, gehören« (Gerhardt 2018, S. 524) können die bestehenden Beteiligungschancen an Bildung auf Basis der vorliegenden empirischen Befunde nur als unzureichend betrachtet werden.

Es liegen bereits zahlreiche Vorschläge für vielfältige Maßnahmen und ebenso zahlreiche Beispiele für entsprechende Projekte und Initiativen vor, die darauf abzielen, durch individuelle Förderung die Exklusionsrisiken aus dem Bildungssystem - innerhalb der bestehenden Funktionslogik - zumindest zu reduzieren und somit (perspektivisch) die gesellschaftlichen Inklusionschancen zu verbessern. Hierzu gehören unter anderem Angebote zur frühkindlichen Sprach- und Bewegungsförderung, gezielte kompensatorische Förderangebote im Kindergartenalter wie beispielsweise Hilfen für den Spracherwerb bei Kindern, deren primäre Sprache nicht deutsch ist, lernförderliche Unterrichtskonzepte, sozialpädagogische Angebote im Rahmen der Schulsozialarbeit, flexiblere Formate der beruflichen Weiterbildung oder auch zielgruppenspezifische Informations-, Beratungs- und Unterstützungsangebote beim Übergang in ein Hochschulstudium (z. B. Kuhlmann 2018; Knuth 2018; Banscherus/Kamm/Otto 2016).

Trotz des großen Engagements des ganz überwiegenden Teils der Erzieher:innen, Lehrer:innen und Dozent:innen bei der individuellen Bildungsförderung ist allerdings nach wie vor ein relevanter Teil der Personen, die das Bildungssystem verlassen, von Exklusion bedroht. Dies gilt insbesondere für diejenigen 6,8 Prozent der Schulabsolvent:innen, die im Jahr 2018 keinen Abschluss erworben haben (Autorengruppe Bildungsberichterstattung 2020, Tab. D8-1web), sowie diejenigen Personen, die bis zum Alter von 35 Jahren keine Ausbildung abgeschlossen haben; der entsprechende Anteilswert lag 2018 bei 17,8 Prozent (Autorengruppe Bildungsberichterstattung 2020, Tab. E5-7web).

Um langfristig gesellschaftliche Exklusion durch fehlende Bildung zu vermeiden, ist die strukturelle Orientierung des Bildungssystems an den Prinzipien der Leistungsgerechtigkeit und der Eigenverantwortlichkeit insgesamt nicht ausreichend. Daran können auch die skizzierten Einzelmaßnahmen strukturell nichts ändern, wenngleich sie auf der individuellen Ebene vielfach Erfolge zeitigen. Notwendig scheint deshalb eine Stärkung des Prinzips der Bedarfsgerechtigkeit im Sinne einer staatlichen Gewährleistung des Erwerbs von Grundkompetenzen für alle Menschen.

Mit Blick primär auf Länder des globalen Südens haben Eric Hanushek und Ludger Wößmann vorgeschlagen, darunter das Erreichen der ersten PISA-Kom- 
petenzstufe zu verstehen, was dem entspreche, "was man heute als moderne funktionale Alphabetisierung bezeichnen könnte« (Hanushek/Wößmann 2019, S. 548; Wrase 2020). Darüber hinaus sollte Grundbildung - im Sinne einer »Art zzivilisatorische[r] Mindestausstattung für gesellschaftliche Teilhabe« - unter Berücksichtigung des im internationalen Vergleich insgesamt hohen Bildungsniveaus in Deutschland allerdings auch grundlegende Fremdsprachenkenntnisse, politische und ästhetische Aspekte sowie übergreifende Fähigkeiten beispielsweise in den Bereichen Problemlösung, Kommunikation und Kooperation umfassen (Hopf 2011, Zitat S. 197).

Die Sicherung von kontinuierlichen, am individuellen Bedarf der Lernenden unterschiedlicher Altersstufen orientierten Aktivitäten von Schulen, Betrieben und Einrichtungen der Erwachsenen- und Weiterbildung mit dem Ziel, flächendeckend die Vermittlung eines gesellschaftlichen Standards der Grundbildung zu garantieren, wäre nicht weniger als ein Paradigmenwechsel auf dem Weg zu individuellen und kollektiven Chancen auf gesellschaftliche Teilhabe - weg von der individuellen Verpflichtung zur Teilnahme am Unterricht (Schulpflicht) hin zu einem durch die Institutionen des Bildungswesens zu gewährleistenden Rechts auf den Erwerb von grundlegenden Kenntnissen und Fähigkeiten mindestens auf dem definierten Niveau der Grundbildung. ${ }^{2}$

Eine entsprechende Modifikation der grundlegenden Strukturen des deutschen Bildungswesens wäre jedoch zweifellos nicht ohne die Schaffung eines verbindlichen - und nicht zuletzt prinzipiell einklagbaren - sozialen Rechts auf Bildung, einen Anstieg der Bildungsausgaben in relevanter Größenordnung und den flächendeckenden Aufbau geeigneter Förderangebote in erheblichem Umfang denkbar (Kronauer 2009 und 2015; Spieß/Zambre 2016). Dies ist aus völkerrechtlicher Sicht allerdings ohnehin geboten, um die Vereinbarungen im Rahmen des »Internationalen Paktes über wirtschaftliche, soziale und kulturelle Rechte« sowie weiterer Abkommen umzusetzen (Wrase 2020).

Dabei sollte zudem die mögliche Reichweite eines verbrieften Rechts auf Grundbildung nicht unterschätzt werden, denn vom Aufbau der für die Umsetzung notwendigen Test- und Beratungsinfrastruktur sowie der Entwicklung und Implementation geeigneter Bildungskonzepte und Unterstützungsangebo-

2 | Weitergehend könnte sich hieran die gerade in gewerkschaftlichen Kontexten verbreitete Forderung nach einem Recht auf den Abschluss einer Berufsausbildung bzw. eines Erststudiums anschließen. Mit Blick auf die faktisch bestehende soziale Strukturierung in Deutschland und die damit verbundenen Teilhabechancen würde dieses Ziel allerdings über den Ansatz einer Erweiterung der sozialen Grundsicherung um bildungspolitische Ansätze hinausgehen. 
te würden über die unmittelbare Adressatengruppe hinaus zahlreiche weitere Bildungsteilnehmer:innen, mitunter sogar das Bildungssystem insgesamt profitieren.

\section{Fazit}

Insgesamt konnte in diesem Beitrag gezeigt werden, dass es sich bei den persistenten sozialen Ungleichheiten im Bildungssystem nicht um einen bedauerlichen »Betriebsunfall« handelt und dass der im politischen Raum nahezu unablässig beschworene »Aufstieg durch Bildung « keineswegs die Regel, sondern vielmehr eine von günstigen Umständen abhängige Ausnahme ist. $Z u$ sehr sind im bestehenden institutionellen Gefüge des deutschen Bildungswesens die Prinzipien der Selektion und Klassifikation systematisch angelegt.

Die normative Idee der Leistungsgerechtigkeit oder Meritokratie erweist sich deshalb im Spiegel der überwältigenden Zahl empirischer Befunde als notwendige Illusion zur Legitimation (sozialer) Ungleichheiten, was wiederum die individuellen gesellschaftlichen Teilhabechancen gefährdet und vielfach mit einem erhöhten Exklusionsrisiko verbunden ist. Ein erster Schritt zur Verbesserung gesellschaftlicher Teilhabechancen durch Bildung im Sinne einer konzeptionellen Einbeziehung des Bildungssystems in ein erweitertes Konzept der sozialen Grundsicherung könnte die gesetzliche Garantie einer realistischen Möglichkeit zum Erwerb eines gesellschaftlichen (Mindest-)Standards an individuellen Kenntnissen und Fähigkeiten im Sinne einer definierten Grundbildung sein.

Dieses Recht auf Bildung könnte zugleich der Ausgangspunkt für eine deutliche Stärkung des Prinzips der Bedarfsgerechtigkeit im deutschen Bildungswesen bedeuten, was längerfristig auch positive Auswirkungen auf die berufliche Aus- und Fortbildung, die hochschulische Bildung sowie die Erwachsenen- und Weiterbildung haben dürfte.

\section{Literatur}

Autorengruppe Bildungsberichterstattung (2020): Bildung in Deutschland 2020. Ein indikatorengestützter Bericht mit einer Analyse zu Bildung in einer digitalisierten Welt, Bielefeld.

Baader, M. S./Freytag, T. (Hrsg.) (2017): Bildung und Ungleichheit in Deutschland, Wiesbaden.

Banscherus, U. (2020): Lebenslanges Lernen an Hochschulen. Eine institutionentheoretische Analyse internationaler Reformdiskussionen, in: Jütte, W./Kon- 
dratjuk, M./Schulze, M. (Hrsg.): Hochschulweiterbildung als Forschungsfeld. Kritische Bestandsaufnahme und Perspektiven, Bielefeld, S. 113-134.

Banscherus, U./Bülow-Schramm, M./Himpele, K./Staack, S./Winter, S. (Hrsg.) (2014): Übergänge im Spannungsfeld von Expansion und Exklusion. Eine Analyse der Schnittstellen im deutschen Hochschulsystem, Bielefeld.

Banscherus, U./Kamm, C./Otto, A. (2016): Gestaltung von Zu- und Übergängen zum Hochschulstudium für nicht-traditionelle Studierende. Empirische Befunde und Praxisbeispiele, in: Wolter, A./Banscherus, U./Kamm, C. (Hrsg.): Zielgruppen Lebenslangen Lernens an Hochschulen. Ergebnisse der wissenschaftlichen Begleitung des Bund-Länder-Wettbewerbs »Aufstieg durch Bildung: offene Hochschulen«, Band 1, Münster, S. 295-319.

Banscherus, U./Wolter, A. (2016): Aufstieg durch Bildung: offene Hochschulen Annäherungen an ein Begriffspaar, in: Wolter, A./Banscherus, U./Kamm, C. (Hrsg.): Zielgruppen Lebenslangen Lernens an Hochschulen. Ergebnisse der wissenschaftlichen Begleitung des Bund-Länder-Wettbewerbs "Aufstieg durch Bildung: offene Hochschulen«, Band 1, Münster, S. 31-52.

Becker, R./Hadjar, A. (2017): Meritokratie - Zur gesellschaftlichen Legitimation ungleicher Bildungs-, Erwerbs- und Einkommenschancen in modernen Gesellschaften, in: Becker, R. (Hrsg.): Lehrbuch der Bildungssoziologie, Wiesbaden, S. 33-62.

Becker, R./Lauterbach, W. (Hrsg.) (2016): Bildung als Privileg. Erklärungen und Befunde zu den Ursachen der Bildungsungleichheit, Wiesbaden.

Bourdieu, P. (1993): Sozialer Sinn. Kritik der theoretischen Vernunft, Frankfurt am Main.

Bourdieu, P. (2001): Unterrichtssysteme und Denksysteme, in: Steinrücke, M. (Hrsg.): Pierre Bourdieu. Wie die Kultur zum Bauern kommt. Über Bildung, Schule und Politik, Hamburg, S. 84-110.

Bourdieu, P. (2003): Die feinen Unterschiede. Kritik der gesellschaftlichen Urteilskraft, Frankfurt am Main.

Bourdieu, P./Passeron, J.-C. (1973): Grundlagen einer Theorie der symbolischen Gewalt, Frankfurt am Main.

Büchner, P. (2003): Stichwort: Bildung und soziale Ungleichheit, in: Zeitschrift für Erziehungswissenschaft 6(1), S. 5-24.

Bundesregierung/Regierungschefs der Länder (2008): Aufstieg durch Bildung. Die Qualifizierungsinitiative für Deutschland, Dresden.

El-Mafaalani, A. (2014): Vom Arbeiterkind zum Akademiker. Über die Mühen des Aufstiegs durch Bildung, Sankt Augustin.

Friedeburg, L. von (1997): Bildungsreform in Deutschland. Geschichte und gesellschaftlicher Widerspruch, Frankfurt am Main. 
Gerhardt, V. (2018): Öffentlichkeit, in: Voigt, R. (Hrsg.): Handbuch Staat, Wiesbaden, S. 519-529.

Hadjar, A. (2008): Meritokratie als Legitimationsprinzip. Die Entwicklung der Akzeptanz sozialer Ungleichheit im Zuge der Bildungsexpansion, Wiesbaden.

Hanushek, E.A./Wößmann, L. (2019): Volkswirtschaftliche Folgen von Bildungsarmut: Was ein Entwicklungsziel »Grundkompetenzen für alle« erreichen könnte, in: Quenzel, G./Hurrelmann, K. (Hrsg.): Handbuch Bildungsarmut, Wiesbaden, S. 547-554.

Herbold, A./Reichstetter, L./Scholz, A.-L. (2017): Soziale Herkunft. Mehr Luft für den Aufstieg, www.zeit.de/2017/22/soziale-herkunft-eltern-bildung-studi um/komplettansicht (Abruf am 1.7.2021).

Honneth, A. (2012): Erziehung und demokratische Öffentlichkeit. Ein vernachlässigtes Kapitel der politischen Philosophie, in: Zeitschrift für Erziehungswissenschaft 15(3), S. 429-442.

Hopf, W. (2011): Bildung, chancengleiche Konkurrenz und gleiche gesellschaftliche Teilhabe, in: WSI-Mitteilungen 64 (4), S. 195-201.

Kaps, P./Bothfeld, S./Brussig, M./Hofmann, T./Knuth, M. (2017): Normen und Strukturen einer solidarischen und investiven Arbeitsmarktpolitik, Working Paper Forschungsförderung der Hans-Böckler-Stiftung, Nr. 47, Düsseldorf.

Keim, W. (2000): Die uneingelöste Gleichheit - Rückblick auf 50 Jahre bundesdeutscher Bildungspolitik, in: Himmelstein, K./Keim, W. (Hrsg.): Gleichheit und Ungleichheit in der Pädagogik, Jahrbuch für Pädagogik 2000, Frankfurt am Main, S. 125-147.

Knuth, M. (2018): Arbeitsmarktpolitik als Inklusionsprojekt?, in: WSI-Mitteilungen 71(6), S. 456-467.

Kracke, N./Buck, D./Middendorf, E. (2018): Beteiligung an Hochschulbildung. Chancen(un)gleichheit in Deutschland, DZHW-Brief 03/2018, Hannover.

Kronauer, M. (2009): Inklusion - Exklusion: eine historische und begriffliche Annäherung an die soziale Frage der Gegenwart, in: Kronauer, M. (Hrsg.): Inklusion und Weiterbildung. Reflexionen zur gesellschaftlichen Teilhabe in der Gegenwart, Bielefeld, S. 24-58.

Kronauer, M. (2015): Wer Inklusion möchte, darf über Exklusion nicht schweigen. Plädoyer für eine Erweiterung der Debatte, in: Kluge, S./Liesner, A./ Weiß, E. (Hrsg.): Inklusion als Ideologie, Jahrbuch für Pädagogik 2015, Frankfurt am Main, S. 147-158.

Kuhlmann, C. (2018): Soziale Sicherung durch Bildung, in: Kaiser, L. C. (Hrsg.): Soziale Sicherung im Umbruch, Wiesbaden, S. 127-143. 
Massing, P. (1984): Die Bildungspolitik, in: Glaeßner, G.-J./Holz, J./Schlüter, T. (Hrsg.): Die Bundesrepublik in den siebziger Jahren. Versuch einer Bilanz, Opladen, S. 197-216.

Meyer, J. (1977): The Effects of Education as an Institution, in: American Journal of Sociology 83(1), S. 55-77.

Meyer, J./Rowan, B. (1977): Institutionalized Organizations: Formal Structure as Myth and Ceremony, in: American Journal of Sociology 83(2), S. 340-363.

Quenzel, G./Hurrelmann, K. (Hrsg.) (2019): Handbuch Bildungsarmut, Wiesbaden.

Rudloff, W. (2007): Bildungspolitik als Sozial- und Gesellschaftspolitik. Die Bundesrepublik in den 1960er- und 1970er Jahren im internationalen Vergleich, in: Archiv für Sozialgeschichte, Band 47, S. 237-277.

Rudloff, W. (2014): Ungleiche Bildungschancen, Begabung und Auslese. Die Entdeckung der sozialen Ungleichheit in der bundesdeutschen Bildungspolitik und die Konjunktur des »dynamischen Bildungsbegriffs« (1950 bis 1980), in: Archiv für Sozialgeschichte, Band 54, S. 193-244.

Solga, H. (2005): Meritokratie - die moderne Legitimation ungleicher Bildungschancen, in: Berger, P.A./Kahlert, H. (Hrsg.): Institutionalisierte Ungleichheiten. Wie das Bildungswesen Chancen blockiert, Weinheim, S. 19-38.

Spieß, C.K./Zambre, V. (2016): Bildungsinvestitionen zielgerichtet ausbauen!, in: Wirtschaftsdienst 96(7), S. 455-459.

Stifterverband (Hrsg.) (2017): Hochschul-Bildungs-Report 2020. Höhere Chancen durch höhere Bildung? Jahresbericht 2017/18 - Halbzeitbilanz 2010 bis 2015, Essen.

Wollersheim, H.-W. (2014): Traditionslinien der Begabungsdiskussion im zwanzigsten Jahrhundert. Hintergründe und Anmerkungen aus bildungshistorischer Sicht, in: Hackl, A./Imhof, C./Steenbuck, O./Weigand, G. (Hrsg.): Begabung und Traditionen, Karg Hefte, H. 6, Frankfurt am Main, S. 30-38.

Wrase, M. (2020): Das Recht auf Grundbildung und die Pflicht des Staates zur Sicherung des bildungsrechtlichen Existenzminimums. Expertise mit Blick auf das Problem geringer Literalität, Berlin (Rechtsgutachten im Auftrag der Max-Traeger-Stiftung). 



\section{Teil IV}

\section{Zur Grundsicherung in Europa}





\section{Mindestsicherung im europäischen Vergleich}

\section{Thomas Bable}

\section{Einleitung}

Dieser Beitrag analysiert die Funktion der Mindestsicherung für die sozialen Sicherungssysteme in EU- und OECD-Ländern. Dafür wird in Abschnitt 2 Mindestsicherung für den Zweck des internationalen Vergleichs definiert. Anschließend werden die wichtigsten Unterschiede zwischen verschiedenen Ländergruppen bzw. Wohlfahrtsregimen dargestellt (Abschnitt 3). Der darauffolgende Abschnitt 4 behandelt zentrale Entwicklungstendenzen der Mindestsicherung in EU- und OECD-Ländern. Der Fokus ist dabei auf die Entwicklung der Mindestsicherung und ihre Stellung im weiteren sozialen Sicherungssystem gerichtet. Gibt es in dieser Hinsicht ähnliche Entwicklungen in verschiedenen Wohlfahrtsregimen, vielleicht sogar eine Konvergenz?

Der abschließende Abschnitt 5 bündelt die Ergebnisse im Hinblick darauf, welche Rolle die Mindestsicherung in einem effektiven sozialen Sicherungssystem spielen kann und ob sie vielleicht durch ein bedingungsloses Grundeinkommen ersetzbar wäre. Der Beitrag kommt zu dem Schluss, dass Mindestsicherung nur im Rahmen eines insgesamt inklusiven sozialen Sicherungssystems effektiv funktionieren kann. Mindestsicherung kann kein Ersatz für ein ausgebautes Sicherungssystem sein, und auch ein bedingungsloses Grundeinkommen wäre keine gute Alternative. Sozial schwache Bevölkerungsgruppen können auf Dauer nur durch eine bessere allgemeine soziale Sicherung »oberhalb« der Mindestsicherung effektiv geschützt werden.

\section{Definition und Funktion der Mindestsicherung im Wohlfahrtsstaat}

Soziale Mindestsicherung wird in diesem Beitrag als bedürftigkeitsgeprüfte und bedarfsorientierte Leistung an Privatpersonen oder Haushalte definiert, die der 
Sicherung eines sozialen Existenzminimums dient (Bahle/Pfeifer/Wendt 2010; Cantillon/van Mechelen/Schulte 2008; ILO 1942).

Bedürftigkeitsgeprüft bedeutet, dass nur dann Anspruch auf Leistungen besteht, wenn die Eigenmittel der Empfänger:innen (Einkommen oder Vermögen) unterhalb einer definierten Schwelle liegen und es darüber hinaus keine Ansprüche gegenüber Dritten gibt, die den Bedarf decken könnten. Aus der Bedürttigkeitsprüfung folgt, dass die Leistungen der Mindestsicherung residual sind, d.h. nach Berücksichtigung anderer Ansprüche gewährt werden.

Bedarfsorientiert bedeutet, dass die Leistungen die persönlichen Lebensumstände der Empfänger:innen, insbesondere die Haushalts- und Familienkonstellation berücksichtigen. Die Leistungen sind in der Regel gestaffelt nach der Größe und Zusammensetzung der Haushalte und Familien der Empfänger:innen. Sie werden regelmäßig gewährt, d.h. solange der Status der Bedürftigkeit anhält. Punktuelle oder einmalige Leistungen zur Überbrückung bestimmter Notlagen werden hier nicht betrachtet.

Ziel der Leistungen ist es, das soziale Existenzminimum der Empfänger:innen zu sichern. Darunter kann jedoch je nach Kontext und Land Unterschiedliches verstanden werden. Das soziale Existenzminimum umfasst in der Regel die Ausgaben für die notwendigen Dinge des Lebens wie z.B. Nahrung, Kleidung oder Unterkunft. In vielen Ländern werden darüber hinaus Bedürfnisse berücksichtigt, die eine soziale Teilhabe in der jeweiligen Gesellschaft ermöglichen, z.B. Mobilität, Kommunikation oder Teilnahme an kulturellen und Freizeitaktivitäten (vgl. Deeming 2020).

Zur sozialen Mindestsicherung gehört in den meisten Ländern auch der $\mathrm{Zu}-$ gang zu sozialen Diensten wie Gesundheit, Bildung, Kinderbetreuung und Pflege. Diese wichtigen Teilhaberechte variieren zwischen den Ländern und werden in diesem Beitrag nicht betrachtet (vgl. aber die Beiträge von Thomas Gerlinger und Ulf Banscherus in diesem Band).

Im entwickelten Wohlfahrtsstaat hat die soziale Mindestsicherung zwei zentrale Funktionen: Zum einen spannt sie ein letztes Auffangnetz im sozialen Sicherungssystem, zum andern bildet sie das Fundament sozialer Bürgerrechte. Mindestsicherung hat also einen genuin ambivalenten Charakter; sie ist residual und essenziell zugleich (Bahle 2019). Zwischen diesen beiden Polen hat sich die Mindestsicherung historisch entwickelt und bewegt.

$\mathrm{Zu}$ Beginn der staatlichen Sozialpolitik bis zum Ende des 19. Jahrhunderts war die Mindestsicherung in Form der Armenfürsorge von großer Bedeutung. Mit dem Ausbau der sozialen Sicherungssysteme im 20. Jahrhundert gewann der residuale Charakter der Mindestsicherung in Form von Sozialhilfe die Oberhand. Mit der Krise des Wohlfahrtsstaates und dem Anstieg neuer sozialer Risi- 
ken seit den 1980er-Jahren gewann wiederum die essenzielle Funktion der Mindestsicherung als Instrument zur Sicherung fundamentaler sozialer (Bürger-) Rechte an Bedeutung. Allerdings verlief diese Entwicklung in den verschiedenen Wohlfahrtsregimen und Ländern sehr unterschiedlich.

\section{Mindestsicherung in verschiedenen Wohlfahrtsregimen}

Aufgrund der beiden zentralen Merkmale der Mindestsicherung, ihrem residualen und zugleich essenziellen Charakter hinsichtlich sozialer Rechte, ist sie eng mit dem Aufbau der anderen sozialen Sicherungssysteme verknüpft (Bahle/ Hubl/Pfeifer 2011). Zum einen hängt ihre Bedeutung und Funktion davon ab, wie stark soziale Rechte in einer Gesellschaft überhaupt verankert sind. Diese Dimension der sozialen Mindestsicherung betrifft die Schnittstellen zwischen (Sozial-)Staat und Familie sowie zwischen Staat und Markt. Zum andern hängt ihre Funktion davon ab, wie stark die anderen sozialen Sicherungssysteme wie Alterssicherung und Arbeitslosenschutz ausgebaut sind.

Hinsichtlich der zweiten Schnittstelle kann man von einem System kommunizierender Röhren sprechen: Bei gleichbleibenden sozialen Risiken sinkt die Bedeutung der sozialen Mindestsicherung in dem Maße, in dem die Bedeutung der anderen Sicherungssysteme wächst, und umgekehrt. Je mehr soziale Risiken durch die anderen Systeme aufgefangen oder vermindert werden, desto geringer ist die faktische Bedeutung der Mindestsicherung für die Absicherung der Bevölkerung. Daraus sollte jedoch nicht auf eine geringe institutionelle Bedeutung der Mindestsicherung für die Architektur des Wohlfahrtsstaates geschlossen werden, denn ein gutes und festes Fundament ist eine wesentliche Bedingung für die Stabilität des ganzen Hauses.

Auf den ersten Blick gibt es im Ländervergleich eine große und teilweise unübersichtliche Vielfalt an sozialen Mindestsicherungssystemen (vgl. die exzellente Analyse bei Neumann 2016). Tatsächlich existieren in fast allen europäischen Ländern mehrere unterschiedliche Leistungen, die im Sinne der hier verwendeten Definition zur sozialen Mindestsicherung gehören (Bahle/Hubl/ Pfeifer 2011).

In Deutschland z.B. zählen dazu die Grundsicherung für Arbeitsuchende, die Grundsicherung für Ältere und Erwerbsgeminderte, die Sozialhilfe, die Asylbewerberleistungen und die Kriegsopferfürsorge. All diese Leistungen sind bedürttigkeitsgeprüft und bedarfsbezogen und zielen auf die Absicherung eines sozialen Existenzminimums, auch wenn sie sich in der Strenge des Bedürftigkeitstests und beim Inhalt des sozialen Minimums teilweise erheblich voneinander 
unterscheiden. In Deutschland ist die soziale Mindestsicherung also kategorial differenziert, d.h. es gibt unterschiedliche essenzielle soziale Rechte für verschiedene Bevölkerungsgruppen. Dies ist in fast allen europäischen Ländern der Fall.

Trotz dieser Unübersichtlichkeit lassen sich im internationalen Vergleich klare Grundmuster erkennen, die sich aus dem Charakter der jeweiligen Wohlfahrtsregime einerseits und dem Ausbau der sozialen Sicherungssysteme »oberhalb« der Mindestsicherung andererseits ergeben (Bahle 2019; Eardley et al. 1996). Wohlfahrtsregime sind durch einen jeweils typischen Zusammenhang zwischen Staat, Markt und Familie charakterisiert, der für den Ausbau grundlegender sozialer Rechte entscheidend ist. Esping-Andersen (1990) unterscheidet drei klassische Wohlfahrtsregimetypen:

- den sozialdemokratischen Typus mit einer zentralen Rolle des Staates und starken sozialen Rechten (hoher Dekommodifizierung),

- den liberalen Typus mit einer zentralen Rolle des Marktes und schwachen sozialen Rechten (niedriger Dekommodifizierung) und

- den konservativen Typus, der zwischen den beiden erstgenannten liegt.

Leibfried (1992) und Ferrera (2005) identifizieren einen vierten Typus mit schwachen sozialen Rechten und einer großen Bedeutung der (erweiterten) Familie. Für diesen Typus hat sich eine geografische Kennzeichnung eingebürgert: Südeuropa. ${ }^{1}$ Dies gilt auch für einen fünften Typus, der nach dem Zusammenbruch des sozialistischen Systems in Osteuropa entstanden ist und sich von den anderen Typen vor allem durch eine deutlich geringere Entwicklung unterscheidet (Cerami 2006; Nelson 2010).

\section{Südeuropa}

Aus der Perspektive der Mindestsicherung betrachtet, ist zunächst das Verhältnis zwischen Staat und Familie von zentraler Bedeutung. Hierbei sticht vor allem der Unterschied zwischen Südeuropa und den anderen Regimetypen hervor: In den südeuropäischen Ländern übernimmt die Familie nach wie vor eine

1 | In der vergleichenden Forschung werden die Länder üblicherweise wie folgt den verschiedenen Typen zugeordnet:

- liberal: Irland, Kanada, Großbritannien, USA;

- sozialdemokratisch: Dänemark, Finnland, Norwegen, Schweden;

- konservativ: Belgien, Deutschland, Frankreich, Niederlande, Österreich, Schweiz;

- Südeuropa: Griechenland, Italien, Portugal, Spanien.

In einigen Studien werden Finnland dem konservativen, die Schweiz dem liberalen und die Niederlande dem sozialdemokratischen Typus zugeordnet. 
starke Sicherungsfunktion; entsprechend rudimentär sind grundlegende soziale Rechte verankert.

Bis zur Finanz- und Wirtschaftskrise 2008/2009 war Portugal das einzige dieser Länder, das überhaupt ein auf nationaler Ebene institutionalisiertes Mindestsicherungssystem besaß. In Spanien existierten äußerst begrenzte Systeme auf regionaler Ebene, während es in Italien und Griechenland nur sehr wenige lokale oder stark eingeschränkte kategoriale Systeme gab. Nach der Wirtschaftskrise haben diese Länder allerdings Anläufe zu einer auf nationaler Ebene verankerten sozialen Mindestsicherung unternommen. Der Zugang zu diesen Leistungen ist jedoch nach wie vor restriktiv.

\section{Liberale Wohlfahrtsregime}

Im liberalen Wohlfahrtsregime ist die soziale Mindestsicherung hingegen stark verankert, obwohl der Markt hier die Rolle des Staates erheblich einschränkt. Diese starke Verankerung der Mindestsicherung findet sich zumindest in Großbritannien und Irland, den europäischen »Vertretern« dieses Typus. Beide Länder haben soziale Mindestsicherungen, die leicht zugänglich sind und im internationalen Vergleich auch relativ hohe Leistungen gewähren.

Der Grund für diese große Bedeutung der sozialen Mindestsicherung ist, dass die anderen Systeme der sozialen Sicherung hier weniger entwickelt sind. Die Mindestsicherung übernimmt also wesentliche Teile der wohlfahrtsstaatlichen Funktion. Da es sich um zentrale Elemente der sozialen Sicherung handelt, sind die Mindestleistungen auch entsprechend institutionalisiert und bilden ein festes Fundament sozialer Rechte.

Die beherrschende Rolle des Marktes wirkt sich bei diesem Typus also erst auf der zweiten Ebene aus. Diese betrifft nicht die grundsätzliche Frage wohlfahrtsstaatlicher Verantwortung, sondern die Frage der Aufteilung sozialer Risiken im sozialen Sicherungssystem zwischen Sicherung des Lebensstandards einerseits und Grund- und Mindestsicherung andererseits. Der Schwerpunkt im liberalen Typus liegt klar auf den letztgenannten Elementen. Dies gilt jedoch nicht für die USA, wo es bis heute kein starkes Fundament für soziale Bürgerrechte gibt.

\section{Sozialdemokratische Wohlfahrtsregime}

Im sozialdemokratischen Typus hat der Staat die beherrschende Rolle. Dies führt zu einer faktisch rudimentären Funktion der Mindestsicherung im Wohlfahrtsstaat, denn die anderen Systeme der sozialen Sicherung sind hoch entwickelt. Der Zugang zu diesen allgemeinen Systemen ist inklusiv, viele davon sind universal auf die ganze Bevölkerung gerichtet, und die Leistungen liegen deutlich 
über dem Existenzminimum. Aus diesem Grund fallen nur wenige Menschen, hauptsächlich solche mit persönlicher Migrationserfahrung, ins letzte Auffangnetz der sozialen Mindestsicherung.

Deshalb ist der Bezug von Mindestsicherung in diesen Ländern tatsächlich häufig ein klares Indiz für soziale Exklusion. Ein weiterer Indikator dafür sind auch die im internationalen Vergleich niedrigen Leistungen der Mindestsicherung. Nur die dänischen und finnischen Leistungen liegen im oberen Drittel der europäischen Länder, allerdings nicht in den Systemen für zugewanderte Menschen mit kürzerem Aufenthalt. In Schweden und Norwegen sind die Leistungen eher unterdurchschnittlich (Frazer/Marlier 2016; OECD 2021).

\section{Konservative Wohlfahrtsregime}

Im konservativen Regimetyp ist die Situation in den verschiedenen Ländern deutlich heterogener. Grundsätzlich liegt dieser Typus zwischen den drei anderen. Im Vergleich zu den südeuropäischen Ländern sind soziale Risiken weit stärker durch den Wohlfahrtsstaat als durch die Familie abgesichert, d.h. soziale Rechte sind stärker verankert. In allen Ländern des konservativen Wohlfahrtsregimetypus existieren insofern Mindestsicherungssysteme, die aber wie im deutschen Fall zumeist kategorial differenziert sind (Hubl/Pfeifer 2013). Es gibt also keine einheitliche Mindestsicherung für alle Bürger:innen oder Bewohner:innen eines Landes.

Nicht nur werden verschiedene Gruppen unterschiedlich behandelt, einige Bevölkerungsgruppen sind de facto von der Mindestsicherung ausgeschlossen, in Deutschland z. B. Studierende. Für diese Gruppe ist nach wie vor primär die Herkunftsfamilie zuständig, erst in zweiter Linie greift das BAföG, das man jedoch aufgrund seiner spezifischen Merkmale nicht zu den Mindestsicherungsleistungen zählen kann.

Hinsichtlich des Ausbaus anderer Systeme der sozialen Sicherung liegen die Länder des konservativen Typus zwischen dem liberalen und dem sozialdemokratischen Typus. Die Systeme der Alters- und Arbeitslosenversicherung sind z. B. stärker ausgebaut als in den liberalen Ländern, aber weniger als in den sozialdemokratischen. Auch die Familienpolitik bewegt sich etwa im Mittelfeld zwischen diesen Typen. Ältere Menschen, Arbeitslose und Alleinerziehende sind in fast allen Ländern die Hauptgruppen, die auf Mindestsicherung angewiesen sind. Da sich die konservativen Länder teilweise stark in den sozialen Sicherungssystemen für diese drei Gruppen unterscheiden, weisen auch ihre Mindestsicherungssysteme unterschiedliche Profile auf (vgl. für ältere Menschen Goedemé und Marchal 2016). 
In den Niederlanden z. B. gibt es aufgrund der hohen staatlichen Grundrenten für alle Wohnbürger:innen kaum ältere Menschen unter den Bezieher:innen von Mindestsicherung. In Deutschland wiederum sind aufgrund der Hartz-Reformen überdurchschnittlich viele Arbeitslose auf Mindestsicherung angewiesen. Hierzulande ist auch der Anteil der Alleinerziehenden, die von Mindestsicherung leben, sehr hoch, unter anderem weil die Familienpolitik das Problem der Vereinbarkeit von Familie und Beruf nicht effektiv gelöst hat.

Trotz dieser Variationen innerhalb der Gruppe der konservativen Länder spielt die Mindestsicherung in allen eine deutlich geringere Rolle als in den liberalen Ländern (weil die sozialen Sicherungssysteme stärker entwickelt sind), aber eine größere als in den sozialdemokratischen Ländern (weil die Systeme weniger inklusiv sind). In allen Ländern des konservativen Typus zeigen sich Lücken im sozialen Sicherungssystem, wenn auch in unterschiedlichen Bereichen, die vom letzten Netz der Mindestsicherung aufgefangen werden müssen.

\section{Osteuropa}

Die osteuropäischen Länder zeigen wiederum ein eigenes Profil. Dort sind die Mindestsicherungen in der Regel auf einem sehr niedrigen Niveau verankert, weshalb nur die extrem Bedürftigen überhaupt Leistungen bekommen (Bahle/Hubl/ Pfeifer 2011; Frazer/Marlier 2016). Auf der anderen Seite haben viele dieser Länder relativ umfassende, wenngleich ebenfalls auf niedrigem Niveau angesiedelte soziale Sicherungssysteme. Strukturell ähneln die meisten osteuropäischen Länder den konservativen Wohlfahrtsstaaten, aber auf deutlich niedrigerem Niveau. In den südosteuropäischen Ländern spielt außerdem ähnlich wie in Südeuropa die Familie noch eine große Rolle. Insgesamt haben diese Länder also eher rudimentäre Mindestsicherungssysteme, die nur wenige Bedürttige tatsächlich erreichen.

Die bisherige Analyse konzentrierte sich auf die Rolle der Mindestsicherung im Gesamtgefüge der sozialen Sicherung in verschiedenen Wohlfahrtsregimen. Dabei stand der Aspekt der Inklusion und des Ausbaus sozialer Rechte im Mittelpunkt. Eine weitere zentrale Frage ist, wie hoch die Leistungen der Mindestsicherung sind und ob sie Armut effektiv bekämpfen können. Die Antwort darauf fällt in fast allen Ländern eindeutig negativ aus: Die Höhe der Leistungen reicht in der Regel nicht aus, um die betroffenen Menschen aus der relativen Einkommensarmut zu befreien, zumindest dann nicht, wenn man die Armutsschwelle bei 60 Prozent des verfügbaren äquivalenzgewichteten Medianeinkommens im jeweiligen Land festlegt (siehe Abbildung 1; vgl. auch Bahle 2019; Frazer/Marlier 2016). 
Abb. 1: Garantiertes Mindesteinkommen* in Relation zum nationalen äquivalenzgewichtetem Medianeinkommen (alleinstehende Person ohne Beschäftigung 2020; in Prozent)

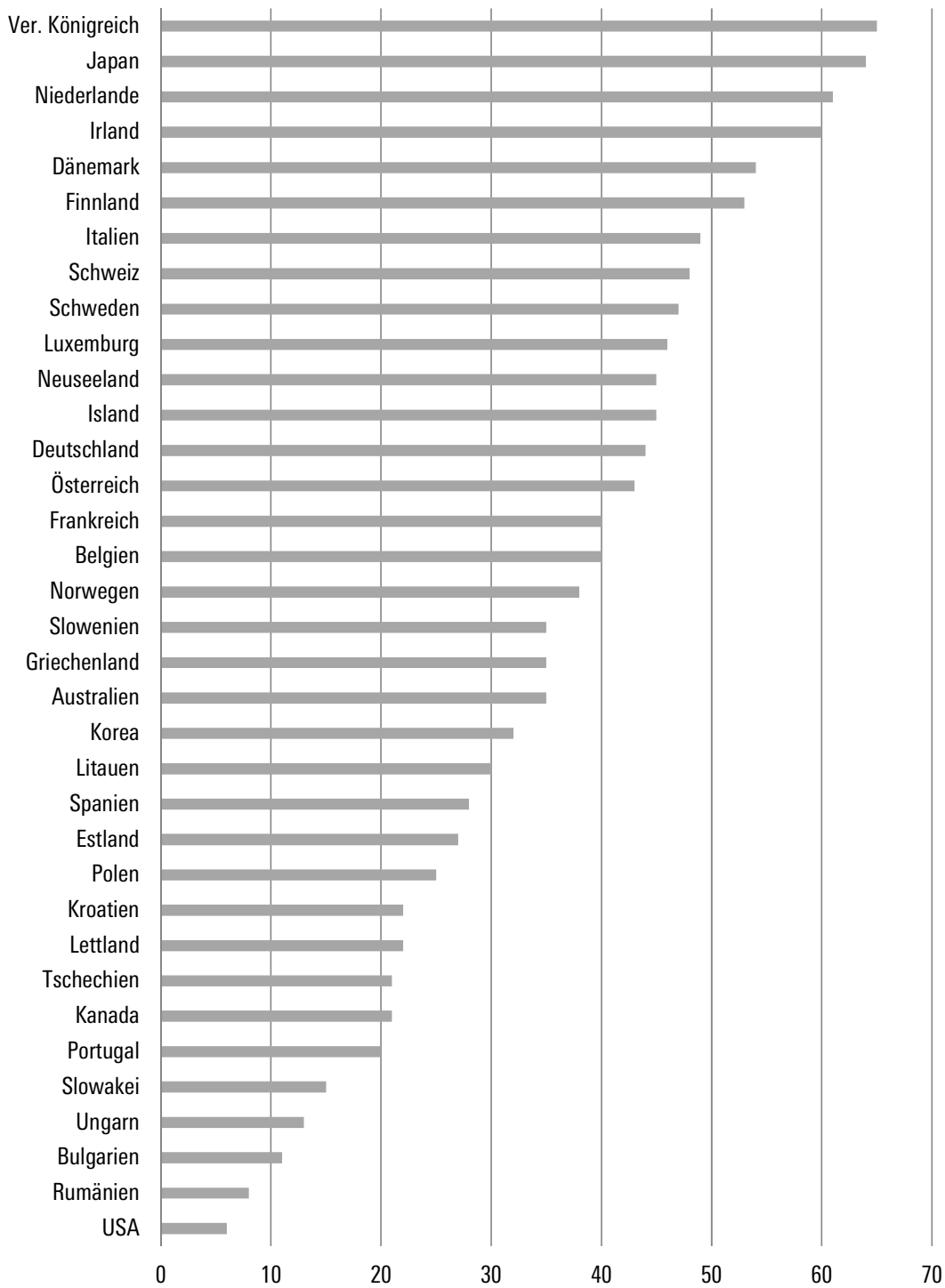

*Anmerkung: garantiertes Mindesteinkommen einschließlich Wohnleistungen Quelle: OECD 2021 (eigene Darstellung). 
Selbst bei einer strengeren Schwelle von 50 Prozent des Medianeinkommens liegen die Leistungen der Mindestsicherung in den meisten Ländern darunter. Nur in Finnland, Dänemark, den Niederlanden und Japan sowie - vielleicht überraschenderweise - in Irland und Großbritannien liegen die Leistungen darüber.

Die Werte schwanken je nach Familienkonstellation; in Deutschland und Polen z. B. erhalten Paare mit Kindern relativ zum jeweiligen äquivalenzgewichteten Medianeinkommen deutlich höhere Leistungen. Die Zahlen für Großbritannien schwanken außerdem stark, je nachdem welche Wohnkosten in den Modellanalysen veranschlagt werden (vgl. die Analysen von Bradshaw und Marchal in Frazer/Marlier 2016).

Die fünf europäischen Länder, in denen die Werte über 50 Prozent liegen, sind auch diejenigen, in denen die Idee der Grundsicherung generell eine wichtige Rolle spielt. Mindestsicherung ist also offensichtlich vor allem dann mit höheren Leistungen verbunden, wenn soziale Grundrechte insgesamt stark in der Gesellschaft verankert sind, sei es auf eher »liberalem« Weg (wie z. B. in Großbritannien oder Irland) oder sozialdemokratisch gefärbt (wie in Dänemark).

Dennoch ist der klare Befund aus dem internationalen Vergleich, dass Mindestsicherung in kaum einem Land relative Armut tatsächlich effektiv vermindert (van Mechelen/Marchal 2013). Unbestritten ist hingegen, dass sie Armutslagen lindert und Deprivation relativ erfolgreich verhindern kann (Nelson 2010).

Ob die Mindestsicherung den betroffenen Menschen auch Chancen vermitteln kann, um sich aus eigenen Kräften aus dieser prekären Lage zu befreien, ist eine weitere zentrale Frage, die hier nicht systematisch analysiert werden kann. Es kommt dabei entscheidend auf die Verknüpfung der Mindestsicherung mit anderen gesellschaftlichen Bereichen an, vor allem mit dem Arbeitsmarkt, der Familienpolitik, dem Gesundheits- und dem Bildungssystem.

In den liberalen Ländern, vor allem auch in den USA, sind die Mindestsicherungssysteme sehr stark auf die Aufnahme einer Erwerbstätigkeit gerichtet. In den USA z. B. ist der Earned Income Tax Credit (EITC), eine Steuergutschrift für Niedrigverdiener:innen, seit vielen Jahren das größte Wohlfahrtsprogramm überhaupt. In Großbritannien ist die Jobseeker Allowance ebenfalls mit starken Anreizen zur Aufnahme einer Erwerbsarbeit verbunden, auch im Niedriglohnbereich.

In Deutschland zielt die Grundsicherung für Erwerbsfähige in die gleiche Richtung. Ob damit jedoch nur eine Beschäftigung im Niedriglohnbereich erzielt wird oder es darüber hinaus einen »Fahrstuhleffekt « nach oben gibt, ist umstritten und hängt sicher auch von der konjunkturellen Lage auf dem Arbeitsmarkt ab. 
Der Zusammenhang mit dem Gesundheitssystem ist ebenfalls wesentlich. In Deutschland beispielsweise sind viele Menschen, die Arbeitslosengeld II beziehen, de facto aus gesundheitlichen Gründen nicht voll erwerbsfähig; umso wichtiger ist der Zugang zu medizinischen und sozialen Diensten für die Betroffenen. In den USA sind jedoch viele ärmere Menschen trotz der Reform von 2010 immer noch ohne Krankenversicherungsschutz, was ihre ohnehin prekäre Lage, die ja häufig mit starken gesundheitlichen Einschränkungen einhergeht, zusätzlich verschärft (vgl. Case/Deaton 2020). Bildung schließlich ist vor allem in der Generationenfolge ein Schlüssel zum Ausstieg aus Armutslagen.

Der internationale Vergleich zeigt somit erhebliche Unterschiede in Ausgestaltung und Bedeutung der Mindestsicherung zwischen den Ländern. Es lassen sich einerseits Zusammenhänge mit den verschiedenen Wohlfahrtsregimen erkennen, andererseits spielt auch die Entwicklung der sozialen Sicherungssysteme in den verschiedenen Bereichen eine Rolle, die zum Teil auch innerhalb der Typen variiert. Mindestsicherung korrespondiert eng mit den Systemen der Alterssicherung, des Arbeitslosenschutzes und der Familienpolitik, um nur die wichtigsten Bereiche und Risikogruppen zu nennen.

Auch die Frage, wie Migrant:innen und Flüchtlinge in den jeweiligen Systemen der verschiedenen Länder behandelt werden, wird zunehmend wichtiger, weil der Anteil dieser Bevölkerungsgruppe in allen Ländern zunimmt. Hierbei wird deutlich, dass Flüchtlinge und Asylbewerber:innen in fast allen Ländern deutlich weniger soziale Rechte haben als andere Gruppen (Hubl/Pfeifer 2013). Dies gilt gerade auch in den ansonsten generösen nordischen Wohlfahrtsstaaten.

Die Mindestsicherung allein kann also kein effektives Mittel zur Bekämpfung von Armut sein. Dafür sind die Systeme zu heterogen und lückenhaft, in manchen Ländern generell zu wenig ausgebaut. Darüber hinaus sind die Leistungen deutlich zu niedrig, um die Menschen aus Armut zu befreien. Damit Betroffene die Chance erhalten, sich selbst aus einer solchen Lage befreien zu können, ist vor allem der Zugang zu anderen Systemen wie Arbeit, Gesundheit und Bildung zentral. Das letzte Auffangnetz allein kann somit kein wirkliches Fundament für den Wohlfahrtsstaat sein; es ist aber Teil einer Gesamtarchitektur, die für ein stabiles Haus nötig ist.

\section{Wandel der Mindestsicherung}

Im vorangehenden Abschnitt 3 standen die Unterschiede zwischen den Mindestsicherungssystemen der verschiedenen Wohlfahrtsregimetypen und Länder im Mittelpunkt. In diesem Abschnitt geht es um den Wandel der Mindestsiche- 
rung über die Zeit: Welche Trends lassen sich hierbei beobachten? Gibt es große internationale Unterschiede oder überwiegen gemeinsame Entwicklungen?

An dieser Stelle ist eine systematische Analyse vieler Länder über die Zeit hinweg nicht möglich; auch gibt es dazu kaum umfassende Langzeitstudien. Die meisten vergleichenden Analysen betrachten einen bestimmten Zeitpunkt (Querschnitt), einen bestimmten Aspekt über die Zeit hinweg (z. B. die Höhe der Leistungen; vgl. Marchal/Marx/van Mechelen 2014; Nelson 2010) oder nur wenige oder einzelne Länder im Zeitverlauf. Dennoch lassen sich hier einige wichtige Befunde festhalten.

Insgesamt haben sich die Mindestsicherungssysteme in den verschiedenen Ländern und Wohlfahrtsregimen einander etwas angenähert, aber die grundlegenden Unterschiede blieben erhalten. Es fand also keine Konvergenz statt. In den südeuropäischen Ländern erfuhr die Mindestsicherung seit der Finanz- und Wirtschaftskrise 2008/2009 einen Ausbauschub (vgl. Matsaganis 2017); diese Länder holten ihren Rückstand in dieser Hinsicht also auf. In vielen - vor allem konservativen - Ländern fand ein Umbau der sozialen Sicherungssysteme mit teilweise massiven Folgen für die Mindestsicherung statt.

Dies war vor allem in Deutschland der Fall. Hierdurch gewann die Mindestsicherung für die soziale Sicherung insgesamt an Bedeutung. In fast allen Ländern konnten die Leistungen der Mindestsicherung jedoch nicht mit dem allgemeinen Einkommenszuwachs Schritt halten (Marchal/Marx/van Mechelen 2014). Dieser Befund ist von zentraler Bedeutung für die Frage, welche Rolle die Mindestsicherung in Zukunft innerhalb der sozialen Sicherung spielen sollte (vgl. Marx/Nelson 2013).

Die Folgen der Finanz- und Wirtschaftskrise waren vor allem in den südeuropäischen Ländern gravierend. Der Anstieg der Arbeitslosigkeit bei gleichzeitig wachsendem Druck auf die Staatsfinanzen stürzte viele Menschen in eine äußerst prekäre Lage. Davon betroffen waren vor allem junge Menschen und Erwerbstätige, während die Älteren zunächst relativ gut abgesichert waren. In vielen Ländern fehlte ein adäquates soziales Sicherungssystem für diese Gruppen, und die Familien waren mit dieser Aufgabe hoffnungslos überfordert.

Die Einführung einer effektiven sozialen Mindestsicherung erschien somit als Gebot der Stunde, darüber hinaus als eine kostengünstige Alternative zu anderen Instrumenten. Dennoch dauerte es einige Jahre, bis Griechenland und Italien zum ersten Mal tatsächlich Mindestsicherungssysteme auf nationaler Ebene einführten. Dies geschah erst, als die schlimmsten Folgen der Krise schon abgeebbt waren.

Dafür gab es zum einen politische Gründe - im Falle Griechenlands auch im Zusammenhang mit dem nationalen Widerstand gegen die Politik der Troika 
aus EU, EZB und IWF -, zum anderen aber auch gewichtige systemische Ursachen. Mindestsicherungssysteme sind mit erheblichem Informationsbedarf und Verwaltungsaufwand verbunden. Nicht zuletzt hängt ihre Wirksamkeit vom Vertrauen der Menschen in den Staat und vom Vertrauen des Staates in die Bürger:innen ab. Insbesondere die Bedürftigkeitsprüfung und die Bedarfsfeststellung erfordern effektive administrative Strukturen und Handlungskapazitäten des Staates sowohl auf nationaler als auch auf lokaler Ebene.

Diese Voraussetzungen waren und sind in einigen südeuropäischen Ländern nicht gegeben. Weder funktioniert die Kooperation zwischen Zentralstaat, Regionen und Kommunen noch sind Finanz-, Arbeits- und Sozialverwaltung miteinander koordiniert. Selbst in Deutschland mit seiner grundsätzlich gut funktionierenden öffentlichen Verwaltung hat die Zusammenlegung von Arbeits- und Sozialbehörden in den Jobcentern größere Verwerfungen hervorgerufen, die bis heute nachwirken. Gerade eine effektive und einheitliche Mindestsicherung ist organisatorisch und kulturell höchst voraussetzungsvoll. Erst langsam wurden in den betroffenen südeuropäischen Ländern die dafür notwendigen administrativen Bedingungen geschaffen.

Die kulturellen Grundlagen für Vertrauen und für das Sozialkapital einer Gesellschaft lassen sich ohnehin nur schwer beeinflussen. Es ist deshalb kein $\mathrm{Zu}-$ fall, dass die Bewegung Cinque Stelle in Italien massiv für ein staatlich garantiertes Grundeinkommen anstelle einer Mindestsicherung in klassischer Form eintrat, denn ein solches Grundeinkommen wäre weit weniger anspruchsvoll. Dies zeigen auch Erfahrungen aus ärmeren Schwellen- und Entwicklungsländern, wo statt einer allgemeinen Mindestsicherung meistens kategoriale Programme für bestimmte Bevölkerungsgruppen wie Ältere oder Kinder (sogenannte »Demogranten«) eingeführt wurden, weil diese Programme leichter zu administrieren und mit weniger Missbrauchsmöglichkeiten verbunden sind (Leisering 2019).

Italien und Griechenland haben mit der Einführung ihrer Programme in den 2010er-Jahren teilweise zum europäischen Standard aufgeschlossen. In anderen europäischen Ländern wurden die sozialen Sicherungssystem und mit ihnen die Mindestsicherung zum Teil gründlich umgebaut. Dieser Prozess setzte in den westeuropäischen Ländern in den 1990er-Jahren ein, in den osteuropäischen Ländern mussten die Systeme nach dem Fall des Kommunismus überhaupt erst neu aufgebaut werden, denn in der sozialistischen Gesellschaft gab es (angeblich) weder Armut noch Arbeitslosigkeit.

In den osteuropäischen Ländern wurden im Zuge der Transformation tatsächlich in der Regel umfassende (universelle) Mindestsicherungen eingeführt, wenngleich auf sehr niedrigem Sicherungsniveau. In den westeuropäischen Län- 
dern hingegen überwog ein Trend zur Differenzierung, also die kategoriale Aufsplitterung ehemals einheitlicher Mindestsicherungen.

Paradigmatisch dafür ist der deutsche Fall. Aus der 1962 eingeführten umfassenden Sozialhilfe wurden im Laufe der Zeit vier verschiedene Systeme mit unterschiedlichen Regeln und Zielen: Leistungen für Asylbewerber:innen und Flüchtlinge (1993), Grundsicherung für Ältere und Erwerbsgeminderte (2003) und Grundsicherung für Erwerbsfähige (2005); daneben fungiert die »alte« Sozialhilfe weiter als »Residuum der Residuen «. In der Diskussion ist derzeit eine Grundsicherung für Kinder (allerdings meistens nicht bedürftigkeitsgeprüft!). Diese würde dem Tableau ein weiteres Feld hinzufügen, hätte darüber hinaus aber auch Rückwirkungen auf die bereits existierenden Systeme, insbesondere auf dasjenige für Erwerbsfähige.

Wie auch immer, diese Entwicklung folgt einer klaren Logik der zunehmenden Bearbeitung sozialer Risiken durch Elemente der Mindestsicherung. Mindestsicherung wird auf diese Weise zu einem Massenprogramm, zu einem zentralen Element der sozialen Sicherung in allen Bereichen (siehe hierzu auch den Beitrag von Gerhard Bäcker in diesem Band). Etwas pointiert könnte man sagen, dass wir damit in Deutschland auf dem Weg in eine durchgehend zweistufige Landschaft der sozialen Sicherung in allen Feldern sind: Alterssicherung (plus Grundsicherung für Ältere), Arbeitslosensicherung (plus Grundsicherung für Erwerbsgeminderte) und Familienpolitik (plus Grundsicherung für Kinder?).

Die zentrale Frage ist, ob sich dadurch tatsächlich der Schutz benachteiligter Gruppen in den einzelnen Bereichen verbessern lässt oder ob diese Entwicklung nicht letztlich in einen Sozialstaat für zwei Klassen mündet, wobei die benachteiligten Gruppen weiter zurückfallen.

Zeigt sich in verschiedenen Ländern zum Teil ein Ausbau, zum Teil ein Umbau der Mindestsicherungssysteme, so lässt sich generell für fast alle Länder sagen, dass die Leistungen der Mindestsicherung über die Zeit hinweg bestenfalls stagnieren, in weiten Teilen sogar im Vergleich zu anderen Maßzahlen wie Durchschnittseinkommen oder Preisniveau sinken. Überhaupt bringen sie die Empfänger:innen nur in wenigen Ländern knapp über die Armutsschwelle (siehe Abschnitt 3).

Diese Entwicklung offenbart, dass Mindestsicherung kein sonderlich leistungsfähiges oder inklusives Instrument der sozialen Sicherung ist. Sie mildert Armutslagen, schafft aber kein gutes Fundament für den Wohlfahrtsstaat. Alarmieren müssen in diesem Zusammenhang Überlegungen und Ideen, die Grundsicherung quasi zum herrschenden Prinzip für den Sozialstaat machen zu wollen. 
Im Ländervergleich lässt sich zur Entwicklung der Mindestsicherung also folgendes Fazit festhalten: Beginnend mit der britischen Social Assistance von 1945 haben mit Griechenland (2017) und Italien (2019) nach über siebzig Jahren nunmehr alle europäischen Länder eine Form der Mindestsicherung auf nationaler Ebene eingeführt (vgl. Neumann 2016). Der größte Schub in diese Richtung ergab sich durch die um 1990 geschaffenen Systeme im Zuge der Transformation in Osteuropa.

Trotz dieser allgemeinen Entwicklung bleiben große Unterschiede in der Ausgestaltung der Mindestsicherung zwischen den Ländern erhalten, die in groben Zügen den verschiedenen Wohlfahrtsregimen entsprechen. Mindestsicherung ist Teil der Gesamtarchitektur des Wohlfahrtsstaates und nur im Zusammenhang mit den anderen Systemen der sozialen Sicherung zu verstehen. Wollte man zentrale gemeinsame Entwicklungstrends ausmachen, gäbe es dafür zwei Punkte:

- In fast allen Ländern wurden die Mindestsicherungen stärker kategorial differenziert.

- In fast allen Ländern hinken die Leistungen weiter hinter der allgemeinen Einkommensentwicklung her.

Mindestsicherung wird also weniger universal und weniger effektiv.

\section{Von der Mindestsicherung zum bedingungslosen Grundeinkommen?}

Die Analyse ergab große Unterschiede, aber auch einige Gemeinsamkeiten in den Mindestsicherungssystemen europäischer Länder. In dieser abschließenden Diskussion und Bewertung der zentralen Ergebnisse möchte ich auf zwei Fragen eingehen:

- Ist die Einführung von Mindestsicherungen in verschiedenen Bereichen ein adäquates Instrument sozialer Sicherung?

- Wäre ein bedingungsloses Grundeinkommen eine gute Alternative zur Mindestsicherung?

An dieser Stelle kann und soll keine ausführliche Diskussion über das Für und Wider eines bedingungslosen Grundeinkommens erfolgen, vielmehr geht es um die Frage, ob ein solches Instrument dazu geeignet wäre, die dargelegten Defizite der Mindestsicherung zu beheben. 


\section{Ist die Einführung von Mindestsicherungen in verschiedenen Bereichen ein adäquates Instrument sozialer Sicherung?}

Auf den ersten Blick erscheinen Mindestsicherungen in den verschiedenen Bereichen des Wohlfahrtsstaates als ein geeignetes Instrument, um die Position sozial schwacher Gruppen im Gefüge der sozialen Sicherung zu verbessern. Die Grundsicherung für Ältere ist ein Schutz für diejenigen, deren Einkommen im Alter nicht zum Leben ausreicht.

Doch wir sprechen hier von einer Mindestsicherung, d.h. die Leistungen sind bedürftigkeitsgeprüft und bedarfsorientiert. Das Problem ist hierbei vor allem das erste Merkmal. De facto wurde durch die Einführung einer solchen kategorialen Mindestsicherung für Ältere ein zweistufiges System der öffentlichen Alterssicherung geschaffen - ohne hier auf die privaten oder betrieblichen Formen »oberhalb« der öffentlichen Säulen eingehen zu wollen. Diese Dualität führt zu zwei großen Gefahren:

- Erstens entsteht eine dauerhafte Spaltung zwischen den Sicherungssystemen und Interessen der Durchschnittsverdiener:innen einerseits und der sozial Benachteiligten andererseits. Dadurch verlieren die Leistungen im bedürftigkeitsgeprüften System über die Zeit an Akzeptanz, Legitimität und Wert. Dieses »Paradoxon der Umverteilung« (Korpi/Palme 1998) ist ein zentraler Befund der vergleichenden Wohlfahrtsstaatsforschung: Je stärker sich soziale Programme auf ärmere Schichten konzentrieren, desto schwächer wird deren politische und soziale Unterstützungsbasis.

- Die zweite Gefahr schließt unmittelbar daran an: Zwar wird die Mittelschicht »ihr« einkommensbezogenes und nicht bedürftigkeitsgeprüftes Alterssicherungssystem verteidigen, aber die Existenz eines darunterliegenden letzten Auffangnetzes der Mindestsicherung erleichtert ein Absenken des Sicherungsniveaus im allgemeinen Rentensystem - denn sollte das Niveau im Einzelfall unter eine existenzbedrohende Schwelle absinken, gibt es ja für die Betroffenen einen Schutz. Potenziell ist also in einem zweistufigen System die »untere« Schicht im »oberen« System immer der Gefahr des Abgleitens ins »untere« System ausgesetzt.

Das System kann dadurch leicht ins Rutschen kommen. Da im unteren System eine Bedürftigkeitsprüfung greift, müssen die Betroffenen aber vor Leistungsbeginn aus diesem System noch ihre eigenen Reserven liquidieren. Tatsächlich schreckt diese Aussicht - und andere Gründe wie Scham oder Informationsmangel - schon heute viele Leistungsberechtigte davon ab, Mindestsicherung zu beantragen. Aus der Perspektive des Risikoschutzes für sozial schwächere Gruppen wäre deshalb ein einstufiges System der Ab- 
sicherung mit dortiger Einführung eines Mindestniveaus die weit effektivere Lösung, also beispielsweise eine Mindestrente innerhalb der allgemeinen Alterssicherung.

Dieselben Argumente lassen sich ebenso für alle anderen Bereiche des Wohlfahrtsstaates anführen. Inklusion ist für sozial Schwächere immer besser als Exklusion und "Sonderbehandlung« in spezifischen Programmen und Einrichtungen. Alleinerziehende profitieren z.B. mehr von einer hoch entwickelten Familienpolitik als von speziell für sie entworfenen Programmen. Dies belegt der Vergleich zwischen den skandinavischen und den angelsächsischen Ländern eindrücklich. Ärmere Menschen profitieren mehr von einer guten inklusiven Gesundheitsversorgung als von speziellen Programmen, wie das Beispiel der USA zeigt. Kinder aus sozial benachteiligten Schichten haben größere Chancen in Kindergärten und Schulen, die Kindern aller Gesellschaftsschichten offenstehen, als in speziellen Einrichtungen.

Jede Form der Mindestsicherung birgt die Gefahr einer wachsenden sozialen Spaltung. Lücken in der sozialen Absicherung sollten deshalb bevorzugt innerhalb und nicht unterhalb bestehender allgemeiner Systeme geschlossen werden.

\section{Wäre ein bedingungsloses Grundeinkommen eine gute Alternative zur Mindestsicherung?}

Daher stellt sich die Frage, ob die Defizite der Mindestsicherung nicht auf einen Schlag durch ein allgemeines bedingungsloses Grundeinkommen beseitigt werden könnten. Tatsächlich hat diese Idee auf den ersten Blick Charme, würde ein solches Instrument doch vieles vereinfachen und dazu noch den ungeliebten Bedürftigkeitstest überflüssig machen. Blickt man nur auf die Seite der Mindestsicherung, wird man dem »alten« System wahrscheinlich tatsächlich keine Träne nachweinen.

Doch beim bedingungslosen Grundeinkommen besteht die Gefahr auf der anderen Seite der Ungleichung, denn durch dieses könnten die etablierten sozialen Sicherungssysteme insgesamt unter Druck geraten bzw. sogar scheinbar überflüssig werden. In einigen radikalen Vorschlägen zum bedingungslosen Grundeinkommen geht es tatsächlich um nichts anderes als um die Abschaffung des Sozialstaats. Hiermit sind aber zwei große Gefahren verbunden:

- Erstens gilt zumindest mittel- bis langfristig auch für das bedingungslose Grundeinkommen das Paradoxon der Umverteilung. Die Mehrheit der Bevölkerung, deren Einkommen über dem Grundeinkommen liegt, hat keinerlei Interesse daran, dieses Grundeinkommen langfristig abzusichern oder gar 
weiterzuentwickeln. Es würde also auch hier de facto eine klare und noch dazu tiefe Spaltung in der Gesellschaft entstehen, wobei die tatsächlich Bedürftigen die Verlierer:innen sein dürften.

- Zweitens würde der bisher stärkste Pfeiler der sozialen Sicherung zerstört, der für die Stabilität und Legitimität des Wohlfahrtsstaates bisher von entscheidender Bedeutung war: die Bindung und Begründung sozialer Leistungen durch klar identifizierte und anerkannte soziale Risiken. Dieser Zusammenhang ist geradezu der Beginn der modernen staatlichen Sozialpolitik, als bestimmte Lebensrisiken, die mit dem Aufkommen der Industrialisierung einhergingen, als soziale Risiken anerkannt wurden, für die spezifische Sicherungsinstitutionen wie Alters- und Krankenversicherung geschaffen wurden - gerade die Abkehr vom Fürsorgeprinzip hat den Aufstieg des Wohlfahrtsstaates ermöglicht.

Ein bedingungsloses Grundeinkommen wäre ein radikaler Schritt zurück und ein Bruch mit dem Grundprinzip der kollektiven Absicherung sozialer Risiken zugunsten einer radikal individualistischen Sichtweise von Selbstverantwortung des Individuums. Dies erscheint aufgrund der offensichtlichen Zunahme sozialer Risiken, auch bedingt durch Migration und die Digitalisierung der Arbeitswelt, als gefährlicher Weg, bei dem die sozial Schwachen nur verlieren können.

Der große Vorteil, den ein bedingungsloses Grundeinkommen aus Sicht einer effektiven sozialen Sicherung tatsächlich haben könnte, wäre, dass sich die Lücken im System sozusagen auflösen würden. Dass diese Lücken bestehen und zum Teil wachsen, ist offensichtlich. Doch ein besserer, wenngleich mühsamerer Weg zu dem Ziel, diese Lücken zu schließen, liegt innerhalb der bestehenden Systeme der sozialen Sicherung.

In Deutschland würde dies z. B. bedeuten, dass Selbstständige und Niedrigverdiener:innen in die allgemeinen Systeme integriert werden. Alleinerziehende sollten durch besser ausgebaute Maßnahmen zur Vereinbarung von Familie und Beruf unterstützt werden. Der Weg zu einer effektiveren sozialen Sicherung führt nicht über Exklusion, sondern über Inklusion. Die individualistisch inspirierte Idee eines bedingungslosen Grundeinkommens ist ein Irrweg - soziale Mindestsicherung kann ihre essenzielle Funktion als Fundament sozialer Bürgerrechte nur dann effektiv erfüllen, wenn sie zugleich residual innerhalb eines entwickelten sozialen Sicherungssystems ist.

Insofern löst sich die zu Beginn dieses Beitrags festgestellte konzeptionelle Ambivalenz der Mindestsicherung als Fundament des Wohlfahrtsstaates und letztem Auffangnetz der sozialen Sicherung in der Praxis auf: In einer differen- 
zierten und sich ständig wandelnden Gesellschaft können grundlegende soziale Rechte nur im Rahmen differenzierter Sicherungssysteme für alle Gruppen der Bevölkerung nachhaltig gesichert werden.

\section{Literatur}

Bahle, T. (2019): Soziale Mindestsicherung, in: Obinger, H./Schmidt, M.G. (Hrsg.): Handbuch Sozialpolitik, Heidelberg, S. 761-782.

Bahle, T./Hubl, V./Pfeifer, M. (2011): The Last Safety Net. A Handbook of Minimum Income Protection in Europe, Bristol.

Bahle, T./Pfeifer, M./Wendt, C. (2010): Social assistance, in: Castles, F./Leibfried, S./Lewis, J./Obinger, H./Pierson, C. (Hrsg.): The Oxford Handbook of the Welfare State, Oxford, S. 448-461 (eine aktualisierte Ausgabe erscheint 2021).

Cantillon, B./van Mechelen, N./Schulte, B. (2008): Minimum income policies in old and new member states, in: Alber, J./Fahey, T./Saraceno, C. (Hrsg.): Handbook of Quality of Life in the Enlarged European Union, London, S. 218-234.

Case, A./Deaton, A. (2020): Deaths of Despair and the Future of Capitalism, Princeton.

Cerami, A. (2006): Social Policy in Central and Eastern Europe. The Emergence of a New European Welfare Regime, Berlin.

Deeming, C. (Hrsg.) (2020): Minimum Income Standards and Reference Budgets. International and Comparative Policy Perspectives, Bristol.

Eardley, T./Bradshaw, T./Ditch, J./Gough, I./Whiteford, P. (1996): Social Assistance in OECD Countries. Synthesis Report, Department of Social Security, Research Report No. 46, London.

Esping-Andersen, G. (1990): The Three Worlds of Welfare Capitalism, Cambridge.

Ferrera, M. (2005): Welfare states and social safety nets in Southern Europe: an introduction, in: Ferrera, M. (Hrsg.): Welfare State Reform in Southern Europe. Fighting Poverty and Exclusion in Italy, Spain, Portugal and Greece, London, S. 1-32.

Frazer, H./Marlier, E. (2016): Minimum Income Schemes in Europe. A Study of National Policies 2015, European Commission. European Social Policy Network (ESPN), Brüssel.

Goedemé, T./Marchal, S. (2016): Exploring a blind spot in comparative pension reform research: long-term trends in non-contributory pensions in Europe, in: International Journal of Social Welfare 25(2), S. 161-175. 
Hubl, V./Pfeifer, M. (2013): Categorical differentiation in the light of deservingness perceptions: institutional structures of minimum income protection for immigrants and for the disabled, in: Marx, I./Nelson, K. (Hrsg.): Minimum Income Protection in Flux, Houndmills/Basingstoke, S. 108-136.

ILO = International Labour Organization (1942): Approaches to social security: an international survey, Studies and Reports, Series M, No. 1, Geneva.

Korpi, W./Palme, J. (1998): The paradox of redistribution and the strategy of equality: Welfare state institutions, inequality and poverty in the Western countries, in: American Sociological Review 63(5), S. 661-87.

Leibfried, S. (1992): Towards a European welfare state? On integrating poverty regimes into the European Community, in: Ferge, Z./Kolberg, J. E. (Hrsg.): Social Policy in a Changing Europe, Frankfurt am Main, S. 245-279.

Leisering, L. (2019): The Global Rise of Social Cash Transfers. How States and International Organizations Constructed a New Instrument for Combating Poverty, Oxford.

Marchal, S./Marx, I./van Mechelen, N. (2014): The great wake-up call? Social citizenship and minimum income provisions in Europe in times of crisis, in: Journal of Social Policy 43(2), S. 247-267.

Marx, I./Nelson, K. (2013): A new dawn for minimum income protection?, in: Marx, I./Nelson, K. (Hrsg.): Minimum Income Protection in Flux, Houndmills/Basingstoke, S. 1-27.

Matsaganis, M. (2017): The impact of the great recession on child poverty in Greece, in: Cantillon, B./Chzhen, Y./Handa, S./Nolan, B. (Hrsg.): Children of Austerity. Impact of the Great Recession on Child Poverty in Rich Countries, Oxford, S. 94-117.

Nelson, K. (2010): Social assistance and minimum income benefits in old and new EU democracies, in: International Journal of Social Welfare 19(4), S. 367-78.

Nelson, K. (2012): Counteracting material deprivation: the role of social assistance in Europe, in: Journal of European Social Policy 22(2), S. 148-163.

Neumann, F. (2016): Soziale Mindestsicherung in Europa. Leistungsprofile im Vergleich, Berlin.

OECD (2021): Adequacy of Minimum Income Benefits (table), www.oecd.org/ els/benefits-and-wages-statistics.htm (Abruf am 19.3.2021).

van Mechelen, N./Marchal, S. (2013): Struggles for life: social assistance benefits. 1992-2009, in: Marx, I./Nelson, K. (Hrsg.): Minimum Income Protection in Flux, Houndmills/Basingstoke, S. 28-53. 



\section{Grundsicherung und europäische Regulierung}

Benjamin Benz

\section{Einleitung}

Asylbewerberleistungsgesetz, Mindesteinkommen, Mindestsicherung, Sozialhilfe - unter all diesen und weiteren Begriffen werden lokale, regionale und nationale Grundsicherungssysteme in Deutschland und auch auf europäischer und internationaler Ebene thematisiert und mit politischen Initiativen "gerahmt«. Nachfolgend liegt der Fokus auf der EU-Ebene und regulierender, d.h. Regeln setzender Politik (in Abgrenzung etwa zu Geldmittel umverteilender Politik).

Die EU hat in diesen Fragen bislang wenig Handlungskompetenz. Grundsicherungspolitische Zuständigkeiten sind in den europäischen Verträgen nicht eindeutig enthalten; entsprechende sozialpolitische Initiativen mittels EURichtlinien oder erst recht EU-Verordnungen gibt es im Gegensatz zu anderen Politikfeldern nur wenige, und selbst die sind juristisch und politisch umstritten. Deshalb ist es nicht verwunderlich, dass die Aktivitäten der EU in Sachen Grundsicherung bisher rar sind und sich dabei meist juristischer und politischer Umwege bedienen. Auch zukünftig scheinen diese Umwege für vermehrte Aktivitäten in dieser Hinsicht naheliegend, aber eben auch langwieriger und weniger effektiv zu sein als Regulierungen.

Vor diesem schwierigen Hintergrund werden in diesem Beitrag die bisherigen und denkbare neue Aktivitäten samt ihren Grundlagen diskutiert. Dies geschieht zu einer Zeit, zu der im Rahmen der deutschen (2. Halbjahr 2020) und portugiesischen EU-Ratspräsidentschaft (1. Halbjahr 2021) intensiv um die künftige Qualität europäischer Regulierung der Grundsicherung gerungen wird: Wie gestaltet sich der rechtliche und sozialpolitische Kontext? Welche Hinweise zum Inhalt der derzeitigen und ggf. künftigen Regulierung und welche Alternativen und Grenzen für eine Regulierung lassen sich derzeit erkennen? 
Zur Beantwortung dieser Fragen werden zunächst der sozialpolitische Problemdruck und Kontext skizziert (Abschnitt 2), darauf folgend die alternativen politischen Interventionsmöglichkeiten und deren realpolitische Nutzung (Abschnitt 3). Dies mündet in die Frage, welche Inhalte weitergehende Schritte EU-europäischer Regulierung zu mitgliedstaatlichen Grundsicherungen haben können und wie die politischen und juristischen Erfolgsaussichten einzuschätzen sind.

\section{Was ist der Kontext, was der Problemdruck?}

Die Unterschiede in den Grundsicherungen der Mitgliedstaaten (siehe hierzu den Beitrag von Thomas Bahle in diesem Band) belegen zunächst ein beachtliches »Reich des Möglichen « - von Regelleistungen oberhalb der Armuts(risiko)grenze und verbunden mit Zugang etwa zu qualifizierter Schuldnerberatung bis hin zur erst ab dem 30. Lebensjahr und nur unter Gegenleistungsverpflichtungen gewährten Sozialhilfe.

Warum ist diese Vielfalt in Europa auch ein Thema für europäische Politik? Als sachlichen und politischen Anlass für EU-europäische Regulierungen in der Grundsicherung lassen sich vor diesem Hintergrund acht Phänomene differenzieren und auch miteinander verschränken.

- Erstens werden Grundsicherungen, die häufig nicht armutsfest sind und nur zum Teil durch soziale Dienste und den Zugang zu armutsfester Arbeit Wege aus Armutslagen eröffnen, zu einem Thema aufgrund der Ziele und mangelnden Zielerreichung der EU. Denn nach den europäischen Verträgen hat die EU eine Aufwärtskonvergenz der Lebensbedingungen, die Überwindung von Armutslagen und den Zugang zu ausreichenden Mitteln für ein menschenwürdiges Leben zum Ziel. EU-weit ist es jedoch in den letzten zwei Dekaden nicht gelungen, die jeweils formulierten Armutsbekämpfungsziele zu erreichen.

- Zweitens finden die Grundsicherungspolitiken der Kommunen, Regionen und Staaten in einem EU-Europa statt, dessen Integration ökonomisch, politisch und sozial sehr ungleichzeitig (politikfeldspezifisch früh oder spät) und unterschiedlich intensiv ist (von loser Koordinierung mitgliedstaatlicher Politiken bis hin zu EU-weiter Standardisierung). Kompetenzordnung und EU-Regelungen sind vor allem auf die Schaffung eines Binnenmarktes ausgerichtet und üben einen strukturellen Sozialdumping-Druck auf die Lohn-, Steuer- und Sozialpolitiken aus, die nach der Abschaffung von Binnenzöllen 
und Möglichkeiten zur Währungsabwertung als Konkurrenzinstrumente zwischen den EU-Staaten verbleiben. Dies wird politikwissenschaftlich als "Spillover« von einem Politikfeld in ein anderes diskutiert.

- Durchaus mit dem zweiten Punkt in Verbindung steht drittens seit den 1990erJahren ein Paradigmenwechsel vom sogenannten Welfare- zum WorkfareState, also vom schützenden zum aktivierenden Sozialstaat, der europaweit zu beobachten ist. Dabei werden sogenannte "passive« Geldleistungen eher problematisiert und unter anderem über Sanktionsdrohungen verschärfte "Arbeitsanreize«favorisiert. Diese Ausrichtung ist als mitgliedstaatliches und EU-weites Leitbild einerseits »wählbar« und lässt sich damit auch korrigieren oder überwinden, andererseits liegt sie wegen der damit verbundenen Hoffnung auf Einsparung sozialpolitischer Ausgaben unter »Wettbewerbstaaten" im europäischen Binnenmarkt und auf weltweiten Märkten nahe. Für Anhänger:innen des jeweiligen Leitbilds ist es attraktiv, seine Durchsetzung und Festigung auch auf übernationaler Ebene zu betreiben.

- Mit den ersten drei Punkten in Verbindung stehend, findet viertens innereuropäische Armutsmigration aufgrund zum Teil völlig unzureichender Grundsicherungen und Lebensperspektiven in manchen Staaten statt, worauf andere Staaten zum Teil ebenfalls mit sozialer Ausgrenzung reagieren. Dies führt zu einer »kalten Integration«, in deren Rahmen EU-Bürger:innen weder im Heimatland noch in anderem EU-Ländern einen substanziellen Grundsicherungszugang erhalten. Damit hilfreich (problemlösend) umzugehen überfordert den lokalen Sozialstaat (Schmidt 2019; MAGS 2020, S. 490 ff.) und ließe sich erst über Grundsicherungsgarantien erreichen, die zwischen den EU-Staaten abgestimmt sind. Dies beschreibt im mit personaler Freizügigkeit ausgestatteten EU-Europa einen zweiten Spillover-Effekt.

- Ferner besteht fünftens ein weiterer auf die Grundsicherungspolitik wirkender Spillover-Effekt durch die Asyl-, Flucht- und Außen(handels)politiken der Mitgliedstaaten, durch die die Migration aus Drittstaaten beeinflusst wird oder beeinflusst werden soll. Auch hier werden teils restriktive Grundsicherungsregelungen gefordert und durchzusetzen versucht, um neben sogenannten Push-Faktoren (etwa verlorenen Lebensgrundlagen und Perspektiven im Herkunftsland der Flucht) die Pull-Faktoren mit unangemessen restriktiven Grundsicherungsansprüchen in möglichen Zielländern zu minimieren. Dies führt inzwischen allerdings zur Problematisierung allzu restriktiver Grundsicherungspolitiken vor Gericht (siehe den siebten Punkt unten).

- Einen sechsten Anlass zu EU-europäischer Regulierung mitgliedstaatlicher Grundsicherungssysteme liefert das Faktum, dass die EU inzwischen $e x-$ territoriale Grundsicherungspolitik betreibt, etwa seit Mitte des vergangenen 
Jahrzehnts für aus Syrien in die Türkei geflüchtete Menschen, deren Not in der Türkei gelindert werden soll, um diesen Push-Faktor zu verringern und zu verhindern, dass sie in die EU weiterziehen. Dies wirft zumindest politische Akzeptanzfragen im Abgleich mit EU-europäischem Nichthandeln gegenüber innereuropäischer Armutsmigration auf (siehe den vierten Punkt oben).

- In dieser insgesamt vielschichtigen Lage kommt es siebtens vermehrt zu juristischen Einzelfallentscheidungen zur Grundsicherungspolitik vor dem Europäischen Gerichtshof (EuGH) der EU in Luxemburg und dem Europäischen Gerichtshof für Menschenrechte (ECHR) des Europarats in Straßburg. Per Richterrecht entstehen zwar noch keine konsistenten EU-Regulierungen zur Grundsicherung, aber der politische Druck, sich auf diese zu verständigen, nimmt damit zu.

- Aktuell zeigt sich achtens schließlich angesichts der Coronapandemie und in Rückschau auf die Austeritäts- und Kürzungspolitiken in Reaktion auf die Finanzkrise der Jahre 2008 ff., dass Grundsicherungen auch eine Funktion als sautomatische Stabilisatoren " gerade in Krisenzeiten haben, indem sie die Kaufkraft unterer und unterster Einkommensbezieher:innen stützen und diese vor dem Fall in Lebenslagen absoluter Armut bewahren. Grundsicherung hilft sowohl individuelle Lebenslagen als auch gesellschaftliche (ökonomische, politische und soziale) Verhältnisse zu stabilisieren. Dies lässt Grundsicherungspolitik als Element und Ansatzpunkt zur EU-weit abgestimmten Krisenbewältigung attraktiv erscheinen.

Die genannten Punkte zeigen, dass es vielfältige Anlässe gibt, Grundsicherungspolitik auch auf EU-europäischer Ebene weiterzuentwickeln - oder eher: die Grundsicherung überhaupt erst substanziell zu einem europäischen Projekt zu machen. Schlüssig wäre dies, da andere Politikbereiche eine sozialpolitische Begleitung notwendig machen, aber auch aufgrund des Selbstverständnisses der EU und institutioneller Impulse zu dieser Frage.

Bevor in Abschnitt 4 auf diese Initiativen und ihre mögliche Ausgestaltung eingegangen wird, sollen zunächst potenzielle politische Handlungsmöglichkeiten benannt und hieran orientiert bereits auf EU-Ebene bestehende Realpolitiken skizziert werden. Denn nationalstaatliche, regionale und lokale Politiken über EU-europäische "Regulierungen" untereinander abzustimmen und einander anzunähern ist nur eine unter mehreren denkbaren Handlungsmöglichkeiten auf EU-Ebene (Regeln setzen, Einkommen umverteilen, Infrastruktur bereitstellen etc.). 


\section{Zum möglichen und tatsächlichen Bestand an EU-europäischer Regulierung und ihren Ergänzungen}

Hinweise auf mögliche (grundsicherungs)politische Alternativen lassen sich anhand von Kategorisierungen politischer Eingriffsmöglichkeiten identifizieren, die politische Ordnungen (»polities«), politische Inhalte (»policies») oder politische Prozesse (»politics«) näher in den Blick nehmen:

- Hier geht es um regulative Politik, die Ge- und Verbote, persönliche Rechtsstellungen und politische Kompetenzordnungen, Verfahrensgrundsätze etc. regelt (vgl. hierzu etwa Kaufmann 1982; Hartlapp 2019).

- Hiervon lässt sich appellative Politik unterscheiden, mit der den politischen Steuerungsmodi »Geld « und »Gesetz - mit Blick auf bedeutende Reden, feierliche Erklärungen und Appelle an die Bevölkerung - der Modus der "guten Worte« zur Seite gestellt wird. Deren Wirkmächtigkeit ist begrenzt, sollte jedoch auch nicht unterschätzt werden. So ist etwa die Rede des ehemaligen Kommissionspräsidenten Jean-Claude Juncker vom Anspruch eines sozialen Triple-A-Ratings für die EU (Juncker 2014) zum geflügelten Wort in diesbezüglichen Debatten geworden.

- Polity- und Politics-bezogen lassen sich ferner informationsbezogene und zielvereinbarende Politiken von regulativer Politik unterscheiden: Diese versuchen ihre Wirkungen nicht über Bekundungen, Gesetze oder Geldmittel zu erreichen, sondern über wechselseitiges Lernen (etwa an Informationen zu unterschiedlichen Effekten verschiedener Grundsicherungspolitiken der Mitgliedstaaten) und die Formulierung gemeinsamer Ziele (etwa zur Reduktion der Anzahl von Armut betroffener EU-Bürger:innen), die dann dezentral und mit unterschiedlichen Mitteln in den Mitgliedstaaten umzusetzen sind (vgl. hierzu Benz 2004, S. 202 f.).

- Ebenfalls stärker Policy-orientiert lassen sich regulative Politiken einordnen und ergänzen, etwa durch redistributive Politik. Hier werden Regeln setzende (beispielsweise den gesetzlichen Kündigungsschutz verändernde) und Geldmittel umverteilend einsetzende Politikansätze (etwa Steuermittel für Grundsicherungsleistungen) voneinander unterschieden.

- Als weitere Möglichkeit, den Inhalt eines politischen Programms auszugestalten, werden hiervon zum Teil infrastrukturelle Politik bzw. ökologische Interventionen und dienstleistungsbezogene Politik bzw. pädagogische Interventionen abgegrenzt (vgl. Kaufmann 1982, S. 85; Bäcker/Naegele/Bispinck 2020, S. 5 f.). Denn hier geht es nicht um »Berechtigungen« oder die ökonomischen Grundlagen etwa eines Privathaushalts im Grundsicherungsbezug, sondern 
um den Zugang unter anderem der Haushaltsmitglieder zu Orten (etwa einem Bürgerhaus oder Spielplätzen) und Bildungsgelegenheiten (etwa zur Haushaltsführungskompetenz oder Beratung zu Grundsicherungsansprüchen und deren Durchsetzung).

Alle skizzierten politischen Handlungsmöglichkeiten sind grundsicherungspolitisch relevant, wurden aber auf EU-Ebene bislang in ganz unterschiedlicher Intensität und Extensität kultiviert.

\subsection{Europäische Regulierungen der Grundsicherung}

So steht eine rechtlich bindende und systematische europäische Regulierung der Grundsicherung durch EU-Gesetzgebung bislang aus. Allerdings legen die europäischen Verträge und einschlägige Gerichtsurteile durchaus Grundlagen für ein stärkeres europäisches Engagement in diesem Bereich. Zuständigkeitsbezogen ("polity«) und politikprozessbezogen (»politics«) kann eine europäische Regulierung von Inhalten der Grundsicherungspolitik (»policy«) an folgende Grundentscheidungen anknüpfen: Nach Artikel 3 des Vertrags über die Europäische Union (EUV)

»bekämpft [die EU] soziale Ausgrenzung und Diskriminierungen und fördert soziale Gerechtigkeit und sozialen Schutz [...]. Sie fördert den wirtschaftlichen, sozialen und territorialen Zusammenhalt und die Solidarität zwischen den Mitgliedstaaten« (Art. 3 EUV).

Nach Artikel 5 EUV sind dabei »der Grundsatz der begrenzten Einzelermächtigung« und die »Grundsätze der Subsidiarität und der Verhältnismäßigkeit« für EU-Maßnahmen zu beachten, ferner - nach Artikel 6 EUV - dass die »Rechte, Freiheiten und Grundsätze«, die in der Charta der Grundrechte der EU enthalten sind, und die "Verträge (EUV und AEUV = Vertrag über die Arbeitsweise der Europäischen Union) »rechtlich gleichrangig« sind.

Nach Artikel 151 AEUV verfolgen die Union und die Mitgliedstaaten gemeinsam unter anderem das Ziel eines mangemessenen sozialen Schutz[es]« zur »Bekämpfung von Ausgrenzungen«. Daher »unterstützt und ergänzt die Union die Tätigkeit der Mitgliedstaaten " nach Artikel 153 AEUV unter anderem auf den Gebieten "soziale Sicherheit und sozialer Schutz der Arbeitnehmer" und »berufliche Eingliederung der aus dem Arbeitsmarkt ausgegrenzten Personen«. Hierzu kann die EU mithilfe von schrittweise verbindlich in den Mitgliedstaaten umzusetzenden »Richtlinien " (also nicht mit unmittelbar in allen Mitgliedstaaten geltenden "Verordnungen«) Mindeststandards für die Politik in den Mitgliedstaaten setzen (Art. 153 AEUV). 
Eine solche EU-Richtlinie, die von dieser durchaus umstrittenen (siehe Abschnitt 4) EU-Kompetenz zur Vereinbarung von Mindeststandards für die Grundsicherung Gebrauch machen würde, gibt es wie gesagt bislang nicht. Auch vom Geltungsbereich der EU-Verordnung 883/2004 zur Koordinierung der Systeme der sozialen Sicherheit sind Sozialhilfesysteme laut deren Artikel 3 bislang ausgenommen.

Allerdings hat der Europäische Gerichtshof etwa im November 2018 in einer Klage gegen Österreich geurteilt, dass auch geflüchteten Menschen ein »Kernbereich" des Fürsorgerechts zu garantieren sei (EuGH 2018). Im März 2019 folgte ein Urteil zu Deutschland und Italien, dass nicht in EU-Länder abgeschoben werden darf, wenn dort das Recht auf Fürsorge derart eingeschränkt werde, dass die Wahrung der menschlichen Würde ernsthaft in Gefahr gerate (EuGH 2019). Im Juli 2020 folgte ein Urteil des Europäischen Gerichtshofs für Menschenrechte gegen Frankreich, dass asylsuchende Menschen nicht zu einem Leben auf der Straße gezwungen werden und ihnen nötige Mittel zum Lebensunterhalt nicht vorenthalten werden dürfen (ECHR 2020).

Einen entscheidenden präventiven Beitrag, dass trotz Erwerbsarbeit einkommensarme Menschen in den Mitgliedstaaten nicht auf Grundsicherung angewiesen sind, könnte derzeit die Annahme des Vorschlags der EU-Kommission für eine EU-Richtlinie zu Mindeststandards für Mindestlöhne leisten (EU-Kommission 2020).

\section{2 Über Regulierung hinausgehende Aktivitäten, die den politischen Rahmen ("polity») und Prozess ("politics") gestalten}

Für eine regulative EU-Grundsicherungspolitik bestehen demnach zwar Grundlagen und Anknüpfungspunkte, diese werden aber bisher nicht genutzt. Das bedeutet allerdings nicht, dass auf europäischer Ebene keine Aktivitäten festzuhalten wären, durch die die Politik angeregt, gestaltet und in Ansätzen auch koordiniert wird. Dabei geht es etwa um Anregung politischen Lernens mittels Informationsaustausch und die Abstimmung von jeweils innerstaatlich umzusetzenden Zielen.

\section{Appellierend, postulierend und empfehlend}

"Jeder Mensch hat ein Recht auf Grundsicherung, wenn er keine ausreichenden Mittel hat.« Dies bekennen mit Abweichungen im Wortlaut und Detail alle heutigen Mitgliedstaaten der Europäischen Union unter anderem in der Allgemeinen Erklärung der Menschenrechte der Vereinten Nationen von 1948, in der Charta der Grundrechte der Europäischen Union aus dem Jahr 2000 
sowie zuletzt in der 2017 unterzeichneten Europäischen Säule sozialer Rechte der EU.

Bereits 1992 haben sich alle damaligen Arbeits- und Sozialminister:innen wechselseitig einen detaillierten Katalog von Mindeststandards für die Grundsicherung ihrer Bürger:innen empfohlen (Rat 1992), auf deren Verabschiedung als rechtsverbindliche Richtlinie man sich nicht hatte verständigen können (PeñaCasas et al. 2013, S. 2). Individuell einklagbar sind all diese "Garantien« bislang nach keiner der genannten staatlichen Selbstverpflichtungen und Empfehlungen.

So behält etwa Artikel 34 Absatz 3 der EU-Grundrechtecharta seinen appellativen Charakter, wenn die Union nach dieser Bestimmung zur Bekämpfung von Armut und sozialer Ausgrenzung zwar »anerkennt und achtet«, dass ein »Recht auf eine soziale Unterstützung und eine Unterstützung für die Wohnung « bestehe, »die allen, die nicht über ausreichende Mittel verfügen, ein menschenwürdiges Dasein sicherstellen sollen « - allerdings nur unter der Einschränkung "nach Maßgabe des Unionsrechts und der einzelstaatlichen Rechtsvorschriften und Gepflogenheiten«. Insoweit diese Normen und Praktiken nicht bestehen, bleibt hier materiell nichts zu achten und anzuerkennen, und von "garantieren" ist nicht die Rede.

Konkret enthalten inzwischen die "länderspezifischen Empfehlungen" im Rahmen des sogenannten Europäischen Semesters mitunter Aufforderungen, in der Grundsicherungspolitik für besseren Zugang zu Leistungen, angemessenere Leistungshöhen oder integrativere Hilfen zu sorgen (vgl. etwa Republic of Bulgaria 2019, S. 20 ff.; Bontout/Szatmari 2020, S. 75).

\section{Informiert wechselseitig lernend}

Beispiel für die armuts- und grundsicherungspolitische Nutzung von Informationsgenerierung, -diskussion und -verbreitung sind zunächst mehrere europäische Armutsprogramme zwischen 1975 und 1994 (Benz 2004, S. 164 ff.). Seit den späten 1990er-Jahren wurde dann in der Beschäftigungs-, Armuts- und Sozialschutzpolitik die »Offene Methode der Koordinierung« (OMK) entwickelt, über die ebenfalls Informationsgewinnung und Konsensbildung sowie die Identifizierung von Best- und Worst-Praktiken betrieben wird.

Das Statistische Amt der EU (Eurostat) informiert zu den mitgliedstaatlichen Grundsicherungssystemen mittels der systematischen Übersichten des "Mutual Information System on Social Protection in the EU Member States and the EEA« (MISSOC) und mittels statistischer Übersichten des »European System of integrated Social Protection Statistics«(ESSPROS). Hinzu kommt mittlerweile ein Benchmarking-Rahmen zum Mindesteinkommen des Ausschusses für Sozialschutz beim Rat der nationalen Fachminister:innen (Bontout/Szatmari 2020, S. 75). 


\section{Gemeinsame Ziele vereinbarend}

Neben wechselseitigem Lernen setzt die »Offene Methode der Koordinierung (OMK) auch auf die Vereinbarung von gemeinsamen Zielen. Statt mittels Regulierung wird versucht, über konsensuale und dabei möglichst konkrete Ziele die Beschäftigungs-, Armuts- und Sozialpolitik der Mitgliedstaaten zu beeinflussen. In der Dekade der 2000er-Jahre firmierte dies unter dem Begriff der "LissabonStrategie«, in den 2010er-Jahren als »EU-2020-Strategie«, bei denen die Grundsicherungspolitik jeweils mittelbar über formulierte - und am Ende unionsweit nicht erreichte - Armutsreduktionsziele berührt war.

Die tatsächliche Verfolgung von Zielen blieb und bleibt dabei von der Bereitschaft der einzelnen Mitgliedstaaten abhängig. Zur mangelnden Zielerreichung wird unter anderem auf die Finanz- und Staatsschuldenkrise der Jahre $2008 \mathrm{ff}$. und die Fluchtbewegungen in EU-Länder in den 2010er-Jahren verwiesen. Für die laufende Dekade - die "2030-Ziele» sehen unter anderem 15 Millionen weniger armutsgefährdete Personen vor - wird bereits vorsorglich auf die schwer abzuschätzenden Folgen der Coronapandemie hingewiesen.

Teil der europäischen Armutsprogramme und OMK-Prozesse war und ist es, die Vernetzung von Wissenschaftler:innen, die Durchführung von Forschungsund Evaluationsprojekten und die Organisierung von ansonsten politisch chronisch schwachen Interessenträger:innen (Winter 2019) finanziell zu fördern, damit wechselseitiges Lernen sowie die Erarbeitung, Verfolgung und Evaluation gemeinsamer Politikziele auch unter Einbezug des Wissens und der Interessen von "Expert:innen aus Erfahrung« (etwa Bezieher:innen von Grundsicherung) stattfinden kann. Hierfür unterstützt die EU-Kommission europäische Zusammenschlüsse - etwa in der Wohnungslosenhilfe sowie das »European Anti Poverty Network« - und regt hierüber auch die Etablierung nationaler Armutskonferenzen an.

\section{3 Über Regulierung hinausgehende Aktivitäten, die Politikinhalte gestalten ("policy»)}

Auch wenn bindende EU-europäische Regulierungen zur Grundsicherung bislang nicht bestehen, gibt es neben den skizzierten proklamatorischen, informierenden und zielvereinbarenden Instrumenten auch Programme, über die Finanzmittel eingesetzt werden, die grundsicherungspolitisch wirken und teils sogar wirken sollen.

\section{Ökonomisch umverteilend}

Eine EU-europäische Kofinanzierung regulärer mitgliedstaatlicher Sozialschutzsysteme sieht das Kapitel des AEUV zur Sozialpolitik (Artikel 151 ff.) nicht vor. 
Erstmals ist jedoch jenseits einer dortigen Fundierung eine Arbeitslosenrückversicherung in fortgeschrittener Diskussion, über die in Krisenzeiten mitgliedstaatliche Versicherungssysteme, die in Bedrängnis geraten sind, durch rückzuzahlende europäische Hilfsgelder künftig stabilisiert werden könnten.

Im Bereich der Grundsicherung plädiert etwa Martin Höpner dafür, »statt auf sanktionsbewehrte Vorgaben auf ein faires Angebot« über Strukturfondshilfen zu setzen, »das von den ärmeren Ländern abgerufen werden kann [...], aber nicht abgerufen werden muss", wenn sie mit dieser Hilfe Standards der Grundsicherung im eigenen Land verbessern möchten (Höpner 2019, S. 2).

Auch wenn die EU keine regulären Sozialschutzsysteme in den Mitgliedstaaten kofinanziert, so unterstützt sie gleichwohl sehr begrenzt und punktuell Maßnahmen, etwa im Kontext der gemeinschaftlichen Agrarpolitik seit 1987 die Verteilung von überschüssigen Erzeugnissen an bedürftige Personen in Mitgliedstaaten, die sich an diesem Nahrungsmittelnotprogramm beteiligen.

Exterritorial finanziert die EU seit 2016 zwei Grundsicherungsprogramme für eine fast 4 Millionen Menschen umfassende Zielgruppe jenseits ihrer Außengrenze, nämlich geflüchtete Menschen in der Türkei, die meist aus Syrien stammen:

»The 2 main EU's humanitarian flagship programmes in Turkey are the Emergency Social Safety Net (ESSN) and the Conditional Cash Transfers for Education (CCTE). The ESSN is a social assistance scheme that helps the most vulnerable among the refugee population meet their daily needs through cash assistance. [...] To help vulnerable refugees during the coronavirus pandemic, the $\mathrm{EU}$ has further adapted its [...] response to meet new challenging needs. For example, the EU provided an additional on-off ESSN cash payment to help refugees with the devasting economic impact of coronavirus." (European Commission 2020)

Hier sollen Grundsicherungen helfen, verlässlich »negative coping mechanisms« (European Commission 2020) in Reaktion auf soziale Not und Perspektivlosigkeit zu verhindern, die EU-Interessen berühren.

Erst 2021 von den Mitgliedstaaten gebilligt wurden die Wiederaufbau- und Resilienzmittel, die vom Europäischen Rat der Staats- und Regierungschefs im Juli 2020 zur Bewältigung der Folgen der Coronapandemie in den Mitgliedstaaten vereinbart wurden und die teils als Kredite, teils als nicht rückzahlbare Zuschüsse für konkret von den Mitgliedstaaten vorzuschlagende Maßnahmen vorgesehen sind. Die für die Bewilligung vereinbarten Kriterien sehen grundsicherungsrelevant auch die »Übereinstimmung mit den länderspezifischen Empfehlungen« (siehe hierzu Abschnitt 3.2) und die »soziale Resilienz des Mitgliedstaates« vor (Europäischer Rat 2020, S. 6). 


\section{Infrastrukturell gestaltend}

Während einzelne EU-Staaten am Mittelmeer zumindest zeitweise Schiffe als Rettungsorte und Anlandungen zu Zufluchtsorten ermöglichen, konnte man sich auf EU-Ebene bislang weder auf eine diesbezügliche Fluchtpolitik verständigen noch für alle Einwohner:innen der EU die Garantie des Rechts auf Wohnen in allen Mitgliedstaaten durchsetzen.

Die EU betreibt jedoch auch infrastrukturell zumindest punktuell über Projektförderungen grundsicherungsrelevante Politiken. So fördert sie seit 2014 über den »Europäischen Hilfsfonds für die am stärksten benachteiligten Personen (EHAP; gestützt auf Artikel 175 AEUV) insbesondere lokale Projekte für Personen, die keinen Zugang zu hinreichenden Grundsicherungen haben (EP/ Rat 2014). In Deutschland sind dies vor allem wohnungslose Personen und neu zugewanderte Unionsbürger:innen, die über die EHAP-Projekte ausdrücklich keine materiellen Leistungen erhalten, sondern etwa mittels der Förderung aufsuchender Sozialarbeit (Streetwork) leichteren Zugang zu sozialen Hilfseinrichtungen und Diensten (BMAS o.J.).

\section{Pädagogisch wirkend}

Mit EHAP-Projektmitteln werden auch Beratungsdienstleistungen zu Angeboten etwa der Sprachförderung oder der medizinischen Beratung und zu Freizeitangeboten für Kinder gefördert (BMAS o.J.). Unter anderem im Internet klärt die EU Bürger:innen über ihre Grundsicherungs- und weitergehenden Sozialschutzansprüche bei Beschäftigungsaufnahme in anderen EU-Staaten oder Wohnortverlagerung auf (EU o.J.). Stärker auf die Gesamtbevölkerung und das nationale wie internationale politische Agenda-Setting sind etwa die »Europäischen Jahre " gerichtet, so im Jahr 2010 zur Bekämpfung von Armut und sozialer Ausgrenzung.

Zum Status quo lässt sich damit festhalten: Es gibt auf EU-Ebene zahlreiche appellative Erklärungen und Empfehlungen, insuläre ökonomische, ökologische und pädagogische Interventionen sowie vereinzelt verbindliche juristische Einzelfallentscheidungen. Was es bislang nicht gibt, ist die politische Verständigung über regulative verbindliche Mindeststandards zur Ausgestaltung der Grundsicherungssysteme und darüber, was diesbezüglich aus den Kriterien der Menschenwürde und der »nicht ausreichenden Mittel« folgt. Damit bauen wir bislang an einem europäischen Haus und Sozialmodell weitgehend ohne grundsicherungspolitisches Fundament, ohne »letztes Netz«(Benz 2019).

Dabei gibt es inzwischen neben verbindlichen Neuverschuldungs- und Defizitkriterien im Euroraum und EU-weit geltenden Mindestmehrwertsteuersätzen 
auch Beispiele für die europäisch verbindliche Verständigung auf Mindestleistungsstandards im sozialpolitischen Bereich. So wurde 2019 per Richtlinie ein EU-weiter Rechtsanspruch auf einen mindestens zehntätigen Vaterschaftsurlaub geschaffen, für den eine Geldleistung mindestens in Höhe des jeweils nationalen Krankengelds zu zahlen ist (EP/Rat 2019). Grundsicherungspolitisch ist EUEuropa hingegen regulativ noch auf dem Stand, zwar den Zugang zu einem Basiskonto auch für obdachlose Menschen zu garantieren, nicht aber einen Zahlungseingang darauf.

\section{Europäische Regulierung der Grundsicherung über verbindliche Mindeststandards?}

Eine tatsächlich europaweit abgestimmte garantierte Grundsicherung in den Mitgliedstaaten müsste Gegenstand von EU-Verordnungen oder EU-Richtlinien sein. Zur Grundsicherung wurden inhaltliche Aspekte bereits in der Empfehlung von 1992 (siehe Abschnitt 3.2) in rechtlich unverbindlicher Weise detailliert ausformuliert, etwa zum individuellen Rechtsanspruch und Widerspruchsmöglichkeiten, zur Differenzierung der Leistungshöhe nach mitgliedstaatlich unterschiedlichem Lebensstandard und Anzahl der Haushaltsmitglieder, zur regelmäßigen Anpassung der Leistungshöhe nach transparenten Kriterien und zur Verknüpfung mit sozialen Diensten und Eingliederungshilfen in den Arbeitsmarkt. Umgekehrt kann eine solche Regulierung den Mitgliedstaaten weitreichende Spielräume garantierend belassen, etwa zur Verortung der Finanzierungs- und Verwaltungsverantwortung auf lokaler, regionaler oder nationaler Ebene (zu zahlreichen weiteren Regulierungsgegenständen und -vorschlägen verschiedener Autor:innen vgl. Benz 2019, S. 27).

Inzwischen sprechen sich etwa das Europäische Parlament, der Europäische Wirtschafts- und Sozialausschuss und verschiedene Parteien - in Deutschland etwa Die Linke, die SPD und Bündnis 90/Die Grünen - für rechtsverbindliche europäische Mindeststandards in der Grundsicherung aus (Benz 2019, S. 20 ff.). Es gibt also Gegenbewegungen zum Sozialdumping-Druck auf die Mindestsicherungssysteme und ihre Berechtigten und damit zu Interessenträger:innen, die das »Europäische Sozialmodell« im Binnenmarkt grundsicherungspolitisch möglichst unkonkret und faktisch allein in nationaler Zuständigkeit belassen wollen.

Unter Fürsprecher:innen und Gegner:innen europäischer Grundsicherungsregulierung ist die nötige Rechtsgrundlage, an die für eine Richtlinie anzuknüpfen wäre, durchaus umstritten: 
- Fokussiert man Grundsicherungssysteme als Sozialschutzsysteme (so etwa Kingreen 2017), bietet sich Artikel 153, Absatz 1, Buchstabe c im Vertrag über die Arbeitsweise der EU an. Hiernach sind Mindeststandards auf Vorschlag der EU-Kommission per Richtlinie möglich, allerdings mit der hohen Hürde eines einstimmigen Beschlusses des Rates aller 27 nationalen Arbeits- und Sozialminister:innen. Das Europäische Parlament, der Europäische Wirtschafts- und Sozialausschuss und der Ausschuss der Regionen sind vor Beschlussfassung lediglich anzuhören. Gegebenenfalls könnten auch einzelne Zweige der Sozialhilfe und Systeme wie das Asylbewerberleistungsgesetz von den Mindeststandards der im AEUV verankerten Richtlinie erfasst sein (Kingreen 2017, S. 25 f.).

- Sieht man Grundsicherungssysteme hingegen (so etwa Gorjão-Henriques/ Sousa Ferro/Brito Camacho 2010; van Lancker/Aranguiz/Verschueren 2020) primär als wesentliches Element einer Politik zur Integration von Personen, die vom Arbeitsmarkt ausgeschlossen sind (Art. 153 Abs. 1 lit. h AEUV), kann der Rat die Richtlinie - wiederum nur auf Vorschlag der EU-Kommission mit mindestens 15 hinreichend bevölkerungsstarken seiner 27 Mitglieder (Art. 16 Abs. 4 EUV) und nur zusammen mit dem Europäischen Parlament (Art. 294 AEUV) erlassen. Der Europäische Wirtschafts- und Sozialausschuss und der Ausschuss der Regionen sind auch hier anzuhören.

- Keine regulierenden, sondern nur wechselseitiges Lernen fördernde und zielvereinbarende Maßnahmen sind möglich, so man die beiden oben genannten Buchstaben in Artikel 153 Absatz 1 AEUV für nicht tragfähig hält, um eine Mindeststandards setzende EU-Richtlinie zu legitimieren, sondern nur die Buchstaben j (Bekämpfung der sozialen Ausgrenzung) und/oder k (Modernisierung der Sozialschutzsysteme). Diese Ansicht vertritt etwa die Gruppe der Arbeitgeber:innen im Europäischen Wirtschafts- und Sozialausschuss (vgl. EWSA 2019, S. 4 und 15).

Nachdem der Rat die EU-Kommission auf Initiative der deutschen EU-Ratspräsidentschaft im Oktober 2020 aufgerufen hat, den bestehenden Rahmen an EU-Aktivitäten zur Grundsicherung in den Mitgliedstaaten weiterzuentwickeln (Rat 2020), hat die Kommission im Frühjahr 2021 den Vorschlag einer erneuten Ratsempfehlung für 2022 angekündigt. Bemerkenswert hierbei ist, dass sich Nicolas Schmit, der amtierende EU-Kommissar für Beschäftigung und Soziales, für die Verständigung auf eine Richtlinie ausgesprochen hatte (Schmit 2020, 12:52-15:13), nachdem seine Vorgängerin im Amt, Marianne Thyssen, noch 2016 vor dem EU-Parlament bekundet hatte, sie sehe keine Kompetenzgrundlage für rechtlich bindende EU-Maßnahmen zur Grundsicherung (EP 2017, S. 41). 
Neben der EU-Kommission - und je nach Gesetzgebungsverfahren auch dem EU-Parlament - sind auf jeden Fall die im Rat der Arbeits- und Sozialminister:innen versammelten Mitgliedstaaten zentrale Akteure, die einen appellativen Empfehlungs- oder regulierenden Richtlinienvorschlag der EU-Kommission auch scheitern lassen könnten. Die Erzielung von einstimmiger Zustimmung ist hier auch nach dem erfolgten Austritt des Vereinigten Königreichs kaum zu erwarten. Für eine Mehrheitsentscheidung müssten immer noch hinreichend viele Nationalregierungen gewonnen werden.

Hier deutet sich eine unsichere und vielschichtige Interessenlage an. Zwischen dem Vereinigten Königreich einerseits, das bislang meist verlässlich europäische Sozialstandards abgelehnt hat, und den Sozialminister:innen von Italien, Portugal und Spanien andererseits, die öffentlich eine Grundsicherungsrichtlinie fordern (Mendes Godinho/Iglesias/Catalfo 2020), finden sich ungeachtet der Zustimmung aller Nationalregierungen zur Grundrechtecharta und zur Säule sozialer Rechte verschiedene Positionen:

- EU-skeptische, konservative und rechtsliberale Parteien und Regierungen, die EU-europäische Sozialstandards eher ablehnen;

- skandinavische Länder, die EU-Sozialstandards gegenüber ebenfalls skeptisch sind, so sie früher oder später einheimisch höhere Standards infrage stellen könnten;

- Koalitionsregierungen mit unterschiedlichen Positionen, etwa in Österreich, wo die Grünen europäische Rechtsvorschriften für »angemessene Mindesteinkommenssysteme in allen europäischen Ländern « fordern (Die Grünen 2019, S. 19). Wird sich das grün geführte Sozialministerium aber im Rahmen einer konservativ geführten Regierung in Brüssel tatsächlich für bindende Mindeststandards einsetzen?

\section{Hinzu kommen}

- eher wenig am Thema interessierte Länder, weil dort kein hinreichender Problemdruck gesehen wird oder es als politisch heikel erscheint - etwa mit seinen Bezügen zur EU-Binnenwanderung aus Armutsgründen und zur Flucht-/ Asylpolitik -;

- wegen Neuwahlen zeitweise kaum sprachfähige Regierungen, etwa während der zuletzt über einjährigen Bemühungen um eine Koalition in Belgien;

- Länder wie Bulgarien, Griechenland und Rumänien, für die schrittweise erhöhte EU-weite Mindeststandards erhebliche Mehraufwendungen, umge- 
kehrt aber ansonsten nicht zu erwartende finanzielle Umsetzungshilfen bedeuten könnten;

- Länder bzw. Regierungen, in denen sich einflussreiche Politiker:innen schon häufiger für europäische Sozialstandards und die Solidarität zwischen Mitgliedstaaten in sozialen Fragen eingesetzt haben - so etwa in Luxemburg der langjährige christdemokratische Regierungschef und spätere EU-Kommissionspräsident Jean-Claude Juncker und der sozialdemokratische Außenminister Jean Asselborn -, freilich ohne dass damit bereits Gewissheit über die Position des Landes im konkreten Fall bestünde.

Erfahrungen aus früheren Prozessen und mit dem komplexen Entscheidungssystem der EU sprechen auch hier eher für nur zum Teil verhärtete Pro- und Kontra-Positionen, teils aber auch für fragile, irritierbare Interessenbalancen bei einzelnen Akteur:innen (vgl. hierzu Eichener 2000), aber nicht für ein Vorhaben, dessen Scheitern oder Gelingen bereits vorab vorhersagbar ist.

Falls die nötige Einstimmigkeit oder Mehrheit im Rat nicht herstellbar sein sollte, aber ein Teil der Mitgliedstaaten sich gleichwohl wechselseitig Mindeststandards in der Grundsicherung garantieren will, bliebe im Rahmen der EU noch die sogenannte »Verstärkte Zusammenarbeit« (siehe etwa den "Schengenraum «) und jenseits der EU entweder das Instrument bi- oder multilateraler Verträge oder eine an das Europäische Fürsorgeabkommen von 1953 anknüpfende Initiative im Europarat. In bestimmten Fallkonstellationen können juristische Klagen weiterhin eine Option zur Klärung und Durchsetzung von grundsichernden Mindeststandards für Einwohner:innen der Mitgliedstaaten sein.

\section{Fazit}

Einer Lesart der Kompetenzgrundlagen für EU-europäische Politik zufolge kann diese lediglich gegenseitiges Lernen mittels Peer-Reviews und Kennzahlen sowie unverbindliche gemeinsame Zielsetzungen anregen. Dies gilt, so man glaubt oder behauptet, sich grundsicherungspolitisch allein auf Buchstabe $\mathrm{j}$ und k des für sozialpolitische Fragen einschlägigen Artikels 153 des Vertrags über die Arbeitsweise der EU berufen zu können. Unter der Bedingung einstimmiger Entscheidungen im Rat der nationalen Arbeits- und Sozialminister:innen sind hingegen schrittweise einzuführende Mindeststandards für die Grundsicherung von Personen möglich, die die sozialrechtliche Eigenschaft als (ehemalige) Arbeitnehmer:innen erfüllen (Art. 153 lit. c AEUV; vgl. Kingreen 2017). 
Per Mehrheitsentscheidung erscheint eine europäische Regulierung über Mindeststandards für die Grundsicherung bei Anknüpfung an Buchstabe $h$ auch für weitere klassische Bereiche der Sozialhilfe möglich. Dies gilt, so man auch deren Leistungsberechtigte (sachlich korrekt!) als Personen begreift, die aus dem Arbeitsmarkt teilweise (»working poor«) oder bei Nichterwerbsfähigkeit vollständig ausgegrenzt sind, und Sozialhilfe- oder Asylbewerberleistungen ferner (ebenfalls sachlich korrekt!) als Instrumente ansieht, die nicht nur die Existenzsicherung zum Ziel haben, sondern auch auf eine (Re-)Integration in die Gesellschaft und - wo immer möglich und politisch gewollt - berufliche (Wieder-)Eingliederung zielen (Benz 2019).

Auch das in Abschnitt 2 angesprochene europäische Richterrecht und die wiederholt auf EU-Ebene von Rat, Kommission und Parlament "proklamierten Grundsicherungsgarantien«- nicht nur beschränkt auf Arbeitnehmer:innen sprechen für die Plausibilität dieser Lesart. Eine solche Fundierung EU-europäischer Regulierung der mitgliedstaatlichen Grundsicherungspolitiken könnte mit redistributiven Programmatiken (Höpner 2019) und solchen zum Einbezug von Personen, die auch im engeren Sinne nicht vom Arbeitsmarkt ausgeschlossen sind (van Lancker/Aranguiz/Verschueren 2020), verbunden werden.

Der wissenschaftliche, juristisch-praktische und politische Kampf liegt darin begründet, dass die europäischen Verträge zumindest keine eindeutigen Aussagen zur Frage grundsicherungspolitischer Kompetenzen auf EU-Ebene enthalten. Andere Regulierungs- und Redistributionsfragen zeigen allerdings, dass diese »Offenheit« der Verträge keineswegs grundsicherungsspezifisch ist. So deuten auch die Ablehnung gemeinsamer Schuldenaufnahme bei der "Griechenland-Rettung" und ihre Annahme beim "Corona-Wiederaufbauprogramm" oder die Fundierung der bezahlten Vaterschaftsmonate über Art. 153 Abs. 1 lit. i AEUV statt lit. c (siehe Abschnitt 3.3) eher auf den Modus »Wer nicht will, findet Gründe - wer will, findet Wege« hin.

Fraglich bleibt nach den Erfahrungen der letzten drei Dekaden, ob und wie Europa mittels alternativer Handlungsansätze und ohne eine auch auf EUeuropäischer Ebene regulierende Grundsicherungspolitik - mit den begrenzten Effekten etwa der appellierenden Empfehlung von 1992 bis 2030 - zu einer Weltregion werden soll, die die Ziele der Vereinten Nationen für nachhaltige Entwicklung bis 2030 tatsächlich und verlässlich erreicht. Wie soll sich Europa ohne eigenes soziales Fundament glaubhaft für einen sozialen Basisschutz auch in anderen Weltregionen einsetzen, und was will Europa grundsicherungslosen Menschen und marktradikalen rechts- und linksextremen Kritiker:innen von Sozialstaatlichkeit und europäischer Integration glaubwürdig antworten (Benz 2021)? 


\section{Literatur}

Bäcker, G./Naegele, G./Bispinck, R. (2020): Sozialpolitik und soziale Lage in Deutschland, Band 1, 6. Auflage, Wiesbaden.

Benz, B. (2004): Nationale Mindestsicherungssysteme und europäische Integration, Wiesbaden.

Benz, B. (2019): Ohne Fundament? Perspektiven verbindlicher EU-Mindeststandards für die Mindestsicherung, Friedrich-Ebert-Stiftung, Berlin.

Benz, B. (2021): Wohlfahrtsstaatlichkeit und Soziale Arbeit in machtressourcentheoretischer Perspektive, in: Kraus, B./Krieger, W. (Hrsg.): Macht in der Sozialen Arbeit. Interaktionsverhältnisse zwischen Kontrolle, Partizipation und Freisetzung, 5. Auflage, Detmold, S. 225-252.

BMAS = Bundesministerium für Arbeit und Soziales (o. J.): Europäischer Hilfsfonds für die am stärksten benachteiligten Personen in Deutschland (EHAP), www.bmas.de/DE/Themen/Soziales-Europa-und-Internationales/Europae ische-Fonds/EHAP/ehap.html (Abruf am 30.10.2020).

Bontout, O./Szatmari, K. (2020): Benchmarking von Mindesteinkommen zur Förderung der Aufwärtskonvergenz, in: Bundesministerium für Arbeit und Soziales (Hrsg.): Deutschlands Vorsitz im Rat der Europäischen Union JuliDezember 2020. Begleitband des Bundesministeriums für Arbeit und Soziales, Berlin, S. 72-77.

Die Grünen (2019): Mutig für Europa! Wahlprogramm zur Europawahl 2019, Wien. ECHR = European Court of Human Rights (2020): Inhuman and degrading living conditions of homeless asylum-seekers without any means of subsistence: violation of Convention, Press Release ECHR 202 (2020), 2 July 2020, Straßburg.

Eichener, V. (2000): Das Entscheidungssystem der Europäischen Union. Institutionelle Analyse und demokratietheoretische Bewertung, Opladen.

EP = European Parliament, Directorate-General for Internal Policies, Policy Department A: Economic and Scientific Policy (Hrsg.) (2017): Minimum Income Policies in EU Member States, Study for the European Parliament's Committee on Employment and Social Affairs, Brüssel.

EP/Rat = Europäisches Parlament/Rat der Europäischen Union (2014): Verordnung (EU) Nr. 223/2014 des Europäischen Parlaments und des Rates vom 11. März 2014 zum Europäischen Hilfsfonds für die am stärksten benachteiligten Personen, Abl. Nr. L 72 vom 12.3.2014, S. 1-41.

EP/Rat = Europäisches Parlament/Rat der Europäischen Union (2019): Richtlinie (EU) 2019/1158 des Europäischen Parlaments und des Rates vom 20. Juni 
2019 zur Vereinbarkeit von Beruf und Privatleben für Eltern und pflegende Angehörige und zur Aufhebung der Richtlinie 2010/18/EU des Rates, Abl. Nr. L 188 vom 12.7.2019, S. 79-93.

EU = Europäische Union (o.J.): Arbeiten im Ausland, https://europa.eu/youreur ope/citizens/work/work-abroad/index_de.htm (Abruf am 30.10.2020).

EuGH = Gerichtshof der Europäischen Union (2018): Urteil vom 21. November 2018 in der Rechtssache C-713/17, Verfahren Ahmad Shah Ayubi gegen Bezirkshauptmannschaft Linz-Land, Luxemburg.

EuGH = Gerichtshof der Europäischen Union (2019): Pressemitteilung Nr. 33/19 vom 19. März 2019, Luxemburg.

EU-Kommission = Europäische Kommission (2020): Vorschlag für eine Richtlinie des Europäischen Parlaments und des Rates über angemessene Mindestlöhne in der Europäischen Union, COM(2020) 682 final, Brüssel.

Europäischer Rat (2020): Außerordentliche Tagung des Europäischen Rates (17., 18., 19., 20. und 21. Juli 2020) - Schlussfolgerungen, EUCO 10/20, Brüssel.

European Commission (2020): European Civil Protection and Humanitarian Aid Operations. Turkey. Factsheet (last updated 26/08/2020), Brüssel.

EWSA = Europäischer Wirtschafts- und Sozialausschuss (2019): Für eine europäische Rahmenrichtlinie zum Mindesteinkommen. Initiativstellungnahme, angenommen auf der 541. Plenartagung am 20. Februar 2019, Brüssel.

Gorjão-Henriques, M./Sousa Ferro, M./Brito Camacho, A. (2010): An EU Directive on Adequate Minimum Income: a legal assessment, Lissabon.

Hartlapp, M. (2019): Revisiting patterns in EU regulatory social policy: (still) supporting the market or social goals in their own right?, in: Zeitschrift für Sozialreform 65(1), S. 59-82.

Höpner, M. (2019): Eine Strategie für das "Soziale Europa«, www.mpifg.de/peop le/mh/paper/2019\%20Hoepner\%20Strategie\%20fuer\%20das\%20Soziale $\% 20$ Europa.pdf (Abruf am 14.7.2021).

Juncker, J.-C. (2014): Zeit zum Handeln - Erklärung in der Plenarsitzung des Europäischen Parlaments vor der Abstimmung über die neue Kommission, Straßburg, 22. Oktober 2014, https://ec.europa.eu/commission/presscorner/ detail/de/SPEECH_14_1525 (Abruf am 14.7.2021).

Kaufmann, F.X. (1982): Elemente einer soziologischen Theorie sozialpolitischer Intervention, in: Kaufmann, F.X. (Hrsg.): Staatliche Sozialpolitik und Familie, München/Wien, S. 49-86.

Kingreen, T. (2017): Ein verbindlicher EU-Rechtsrahmen für soziale Grundsicherungssysteme in den Mitgliedstaaten, Rechtsgutachten für das Bundesministerium für Arbeit und Soziales, Forschungsbericht 491, Regensburg. 
MAGS = Ministerium für Arbeit, Gesundheit und Soziales des Landes Nordrhein-Westfalen (Hrsg.) (2020): Sozialbericht NRW 2020. Armuts- und Reichtumsbericht, Düsseldorf.

Mendes Godinho, A./Iglesias, P./Catalfo, N. (2020): Rumo a um rendimento mínimo europeu, in: Público, 8. Mai 2020.

Peña-Casas, R./Ghailani, D./Sabato, S./Nicaise, I. (2013): Towards a European minimum income, Final Report, EESC, Contribution Workers' Group, Brüssel.

Rat = Rat der Europäischen Gemeinschaften (1992): Empfehlung 92/441/EWG des Rates vom 24.6.1992 über gemeinsame Kriterien für ausreichende $\mathrm{Zu}$ wendungen und Leistungen im Rahmen der Systeme der sozialen Sicherung, Abl. Nr. L 245 vom 26.8.1992, S. 46-48.

Rat = Rat der Europäischen Union (2020): Stärkung der Mindestsicherung zur Bekämpfung von Armut und sozialer Ausgrenzung in der COVID-19-Pandemie und darüber hinaus, Schlussfolgerung des Rates vom 9. Oktober 2020, Brüssel.

Republic of Bulgaria, Ministry of Finance (2019): Europe 2020: National Reform Programme. 2019 Update, Sofia.

Schmidt, S. K. (2019): Ein Kampf der Staatsgewalten? Die schwierige soziale Absicherung des europäischen Freizügigkeitsregimes, in: Zeitschrift für Sozialreform 65(1), S. 29-57.

Schmit, N. (2020): mündlicher Beitrag, in: Alexandru, V./Mendes Godinho, A./ Heil, H./Schmit, N./Walker, N. (Moderation): Minister:innen und Stakeholder im Dialog: Das soziale Europa in der Diskussion, Dokumentation der Konferenz »Soziales Europa - Starker Zusammenhalt«, veranstaltet vom Bundesministerium für Arbeit und Soziales, Berlin, 16. September 2020, www. bmas.de/DE/EU-Ratspraesidentschaft/SozialesEuropa-Konferenz/panel-arti kel-4.html (Abruf am 29.10.2020).

van Lancker, A./Aranguiz, A./Verschueren, H. (2020): Making the case for an EU framework directive on minimum income. Expert study, commissioned by EAPN, www.eapn.eu/wp-content/uploads/2020/10/EAPN-european-mini mum-income-eu-framework-expert-study_October-2020-4734.pdf (Abruf am 14.7.2021).

Winter, T. von (2019): Schwache Interessen in Gesellschaft und Staat, in: Toens, K./Benz, B. (Hrsg.): Schwache Interessen? Politische Beteiligung in der Sozialen Arbeit, Weinheim/Basel, S. 26-35. 



\section{Europäische Kindergarantie und Grundsicherung}

Walter Hanesch

\section{Einleitung}

Vor dem Hintergrund der Tatsache, dass mehr als ein Viertel der Kinder und Jugendlichen in der Europäischen Union (EU) in Armut oder sozialer Ausgrenzung leben, ${ }^{1}$ verabschiedete das Europäische Parlament 2015 eine Resolution, in der es die Einführung einer »Kindergarantie« forderte, mit der sichergestellt werden sollte, dass alle von Armut und Ausgrenzung bedrohten Kinder in den EUMitgliedstaaten Zugang zu freier Gesundheitsversorgung, freier frühkindlicher Bildung und Betreuung, freier Bildung, einer menschenwürdigen Unterkunft und angemessener Ernährung haben sollten (Europäisches Parlament 2015). ${ }^{2}$ Die Garantie des Zugangs zu qualitativ hochwertigen sozialen Diensten in den fünf genannten Handlungsfeldern sollte Bestandteil einer integrierten europäischen Strategie zur Vermeidung bzw. Überwindung von Kinderarmut sein.

In einem weiteren Schritt forderte das Europäische Parlament die Europäische Kommission 2017 auf, vorbereitende Maßnahmen für die Umsetzung eines möglichen Kindergarantieprogramms zu ergreifen. Zu diesem Zweck gab die Kommission zunächst eine zweistufige Machbarkeitsstudie für eine Kindergarantie für armutsgefährdete Kinder in Auftrag (siehe Abschnitt 3.1). Als dritte Phase der vorbereitenden Maßnahmen gab die Kommission ein experimentelles Projekt zur Umsetzung in ausgewählten Mitgliedstaaten in Auftrag, das 2021

1 | Laut Eurostat (2021) waren das in der EU ohne Großbritannien auf Basis von EUSILC-Daten im Jahr 2015 26,1 Prozent der unter 16-Jährigen. Bis 2019 hat sich diese Quote allerdings kontinuierlich auf 21,8 Prozent verringert.

2 | Dabei stützte sich das Europäische Parlament unter anderem auf die UN-Kinderrechtskonvention von 1989, laut der allen Kindern das Recht auf Bildung, Gesundheitsleistungen, Wohnraum, Schutz, Beteiligung an Entscheidungen, die sie selbst betreffen, Freizeit und Freizeitbeschäftigung, ausgewogene Ernährung und Fürsorge im familiären Umfeld garantiert werden muss. 
angelaufen ist (Abschnitt 3.2). Im März 2021 legte die Kommission schließlich den Vorschlag für eine Ratsempfehlung zur Einführung einer Kindergarantie in den Mitgliedstaaten vor (Abschnitt 3.3).

Im vorliegenden Beitrag soll die europäische Initiative dargestellt und diskutiert werden. Dazu wird in Abschnitt 2 der Hintergrund der europäischen Parlamentsinitiative skizziert. In Abschnitt 3 wird der aktuelle Stand zur Etablierung der Kindergarantie vorgestellt. Anschließend werden Probleme der Operationalisierung und Umsetzung der Kindergarantie in der EU (Abschnitt 4) und mögliche Auswirkungen der Kindergarantie in Deutschland (Abschnitt 5) diskutiert.

\section{Hintergrund der europäischen Kindergarantie}

Die Initiative des Europäischen Parlaments folgte zum einen dem Beispiel der Ratsempfehlung zur Einführung einer Jugendgarantie von 2013, einem innovativen arbeitsmarkpolitischen Ansatz, mit dem allen Jugendlichen und jungen Erwachsenen unter 25 Jahren zugesichert werden sollte, innerhalb von vier Monaten nach Schulabschluss oder Eintritt von Arbeitslosigkeit ein hochwertiges Angebot für einen Ausbildungsplatz, einen Arbeitsplatz, eine Weiterbildungsmöglichkeit oder ein Praktikum zu erhalten. Alle Mitgliedstaaten haben sich seither zur Umsetzung dieser Garantie verpflichtet und sie - aus Sicht der Kommission - erfolgreich umgesetzt (European Commission 2020). ${ }^{3}$

Die Parlamentsinitiative knüpfte darüber hinaus an zwei weitere EU-Initiativen an, die Initiative »Investitionen in Kinder« und die Initiative »Europäische Säule sozialer Grundrechte«.

\subsection{Die EU-Initiative »Investitionen in Kinder»}

Bereits 2013 hat die Europäische Kommission im Rahmen des Pakts für Sozialinvestitionen eine Empfehlung »Investitionen in Kinder: Den Kreislauf der Benachteiligung durchbrechen" verabschiedet (Europäische Kommission 2013). ${ }^{4}$ Darin wurden die Mitgliedstaaten aufgefordert, eine integrierte Strategie zur Vermeidung bzw. Überwindung von Armut und Benachteiligungen von Kin-

3 | Wissenschaftliche Studien kommen allerdings zu eher ernüchternden Befunden (vgl. z. B. Felgueroso/Jansen 2015; Eichhorst/Rinne 2017).

4 | Die Empfehlung stützte sich unter anderem auf eine Serie von Länderberichten des EU-Netzwerks unabhängiger Expertinnen und Experten zur sozialen Eingliederung, in denen die Lage der Kinder und der Stand der Politik gegen Kinderarmut in den Mitgliedstaaten dargestellt wurde (vgl. zu Deutschland Hanesch 2013). 
dern zu entwickeln und dabei möglichst frühzeitig einzugreifen und präventiv tätig zu werden. In der Empfehlung heißt es, dass sich die integrierten Strategien der Mitgliedstaaten auf drei Grundpfeiler stützen sollten:

- den Zugang zu angemessenen Ressourcen,

- den Zugang zu erschwinglichen hochwertigen Dienstleistungen und

- das Recht auf soziale Teilhabe.

Die EU-Länder wurden aufgefordert,

- den Zugang der Eltern zum Arbeitsmarkt zu unterstützen und sicherzustellen, dass sich die Berufstätigkeit lohnt;

- den Zugang zu erschwinglicher frühkindlicher Bildung und Betreuung, schulischer Bildung, gesundheitlicher Versorgung und angemessenen Wohnbedingungen zu sichern;

- angemessene Einkommensbeihilfen wie Kinder- und Familiengeld bereitzustellen - diese Beihilfen sollten so gestaltet sein, dass keine Anreize für Erwerbslosigkeit geschaffen werden und Stigmatisierung vermieden wird;

- die Teilnahme von Kindern an Spiel-, Erholungs-, Sport- und kulturellen Aktivitäten zu fördern und ihnen Möglichkeiten der Beteiligung in relevanten Lebensbereichen zu bieten.

2017 wurde der Stand der Umsetzung der Initiative »Investitionen in Kinder" in den Mitgliedstaaten vom Europäischen Netzwerk für Sozialpolitik (ESPN) untersucht und dokumentiert Der Synthesebericht (Frazer/Marlier 2017) ${ }^{5} \mathrm{kam}$ zu dem ernüchternden Ergebnis, dass zwar eine Reihe von Ländern ihre Anstrengungen verstärkt haben, die Situation der Kinder zu verbessern, aber eine Umsetzung der Empfehlung bisher nur in begrenztem Umfang stattgefunden hat. Auch der auf dieser Basis vorgelegte Fortschrittsbericht der Kommission (European Commission 2017) bestätigte diesen Befund und kündigte weitere Initiativen an.

\subsection{Die "Europäische Säule sozialer Rechte»}

2017 wurde gemeinsam vom Europäischen Parlament, dem Rat der Europäischen Union und der Europäischen Kommission die »Europäische Säule sozialer Rechte« (ESSR) proklamiert (Europäisches Parlament/Rat der Europäischen

5 | Vgl. dazu auch den Bericht für Deutschland (Hanesch 2017). 
Union/Europäische Kommission 2017). Ziel der ESSR ist die Gewährleistung sozialer Bürgerrechte, die auf insgesamt zwanzig Grundsätzen aufbauen. Der elfte Grundsatz der ESSR sieht für Kinder das Recht auf

- hochwertige und bezahlbare frühkindliche Bildung und Betreuung,

- den Schutz vor Armut und

- besondere Maßnahmen zur Förderung der Chancengleichheit vor.

Die Kommission hat sich verpflichtet, die Umsetzung der ESSR zu überwachen und zu unterstützen. Zur Umsetzung der Strategie »Investitionen in Kinder « und zur Stärkung der genannten Kinderrechte wurden bisher folgende Maßnahmen entwickelt:

- Wichtige Politikbereiche werden im Rahmen des "Europäischen Semesters" anhand eines Social Scoreboard mit sozialen Indikatoren beobachtet.

- Die Kommission berät die Mitgliedstaaten, wie sie die Mittel aus dem »Hilfsfonds für die am stärksten von Armut betroffenen Personen« oder dem »Europäischen Sozialfonds « am besten für Investitionen in Kinder nutzen können.

- Auf der "Europäischen Plattform für Investitionen in Kinder" (EPIC) werden Befunde zu innovativen und erfolgreichen Verfahren sowie weitere Informationen und Forschungsergebnisse in Bezug auf Kinder und Familien verbreitet, um regionsübergreifende Lernprozesse zu ermöglichen (vgl. z.B. Bruckmayer/Picken/Janta 2020).

Im März 2021 hat die Kommission einen Aktionsplan zur ESSR veröffentlicht (European Commission 2021c), der unter anderem ein revidiertes Social Scoreboard sowie eine Liste geplanter Maßnahmen bis 2025 enthält, zu denen auch die Kindergarantie gehört. Bis heute besteht allerdings eine große Diskrepanz zwischen Anspruch und Wirklichkeit der in der ESSR enthaltenen sozialen Rechte. Expert:innen sind sich einig, dass die ESSR ihre volle Wirkung erst entfalten kann, wenn die darin beschriebenen Rechte für die Bürger:innen einklagbar werden. Das ist bis heute nicht der Fall (vgl. z. B. Hacker 2018).

\section{Die Kindergarantie in Europa - Stand der Dinge}

\subsection{Ergebnisse der Machbarkeitsstudien}

Die beiden in Abschnitt 1 erwähnten Machbarkeitsstudien zur Kindergarantie (Frazer/Guio/Marlier 2020; Guio/Frazer/Marlier 2021) sollten unter anderem 
die Stärken und Schwächen der jeweiligen Versorgungsstrukturen in den fünf Dienstleistungsbereichen Gesundheitsversorgung, frühkindliche Bildung und Betreuung, Bildung, Unterkunft und Ernährung untersuchen und dabei als Bewertungskriterien insbesondere Verfügbarkeit, Zugänglichkeit, Erschwinglichkeit, Anpassungsfähigkeit und Akzeptanz heranziehen.

Die Studien sollten das mögliche Design sowie die Steuerung und Umsetzung der in den Mitgliedstaaten bereits existierenden Programme untersuchen und mit dem zusätzlichen Nutzen einer Kindergarantie vergleichen. Dabei sollten sie sich auf vier Zielgruppen konzentrieren, bei denen ein hoher akuter Bedarf beim Zugang zu Diensten angenommen wurde: Kinder in prekären Familiensituationen, institutionell untergebrachte Kinder, Migranten- bzw. Flüchtlingskinder und Kinder mit Behinderungen oder anderen speziellen Bedarfslagen. Die Studien sollten zugleich Hinweise liefern, inwieweit die Schlussfolgerungen und Lösungen für die beispielhaften Gruppen von gefährdeten Kindern Vorbild für das Design einer Kindergarantie für alle Kinder in der EU sein könnten.

Die Studien wurde auf der Grundlage von 28 Länderstudien zu jedem Mitgliedsland sowie Studien zu jeder Zielgruppe und jedem Handlungsfeld erstellt (vgl. dazu auch die Länderstudie Deutschland: Hanesch 2019). Im Folgenden werden zentrale Ergebnisse der Studien zusammengefasst.

Auch wenn die Rahmenbedingungen und der Versorgungsgrad in den untersuchten Dienstleistungsbereichen stark variieren, kam die Studie zu dem Ergebnis, dass in allen Mitgliedstaaten viele Kinder Zugangsprobleme bei einem oder mehreren Dienstleistungsbereichen aufweisen. Insofern besteht ein Bedarf, diesen Zugang zu verbessern, was insbesondere für den Zugang zu qualitativ hochwertigen und integrativen Diensten gilt. Dieser Bedarf ist umso größer, als Zugangs- bzw. Versorgungsmängel mittel- und langfristig negative Folgen für die betroffenen Kinder haben.

Da die Zugangs- bzw. Versorgungsmängel der untersuchten Zielgruppen oft für Defizite bei allen Kindern standen, sollte eine europäische Strategie auf einen zweigleisigen Ansatz setzen: Zum einen sollte der Zugang für alle Kinder garantiert werden; zum anderen sollte der Zugang für benachteiligte Kinder durch besondere Programme gezielt unterstützt und gefördert werden. Dabei sollten die Dienste benachteiligte Gruppen gezielt ansprechen und einbeziehen.

Die Kindergarantie sollte in den Mitgliedstaaten im Rahmen eines umfassenden Ansatzes umgesetzt werden, der von Kinderrechten ausgeht und alle Aspekte der Lebenslage von Kindern mit einbezieht, da diese in einem engen Wechselverhältnis stehen. Wichtig ist dabei eine Koordinierung zwischen den verschiedenen Ministerien bzw. Fachressorts auf horizontaler Ebene und eine enge Zusammenarbeit zwischen den verschiedenen staatlichen Ebenen. 
Die Politik der Mitgliedstaaten im Hinblick auf den Zugang von Kindern zu sozialen Diensten war bisher zu wenig priorisiert, koordiniert und effektiv. Daher sollte die EU ihre diesbezüglichen Anstrengungen verstärken. Die Zuständigkeit für die Sicherung des Zugangs zu sozialen Diensten liegt zwar nach wie vor bei den Mitgliedstaaten; die EU kann diese dabei lediglich fördern und unterstützen. Dazu können insbesondere die Instrumente der politischen Koordinierung und der politischen Beratung (Forschung, Innovation, Wissensaustausch) sowie der finanziellen Unterstützung genutzt werden.

Die Kindergarantie würde die Bemühungen der EU, die Situation von Kindern im Bereich der sozialen Dienste zu verbessern, deutlich stärken, da diesem Thema in den Mitgliedstaaten durch die europäische Initiative eine höhere politische Priorität zukommen würde. Vor allem die Europäischen Fonds könnten eine wichtige Rolle dabei spielen, die Entwicklung und Umsetzung geeigneter Maßnahmen in den Mitgliedstaaten insbesondere für benachteiligte Kinder zu unterstützen. Dazu müssten die Mitgliedstaaten aufgefordert werden, eine Strategie zur Bekämpfung von Kinderarmut und zur Sicherung des Zugangs von Kindern zu sozialen Diensten zu entwickeln und umzusetzen.

Die Hoffnung und Erwartung des Europäischen Parlaments, mit der Kindergarantie die anhaltende Kinderarmut in der EU senken zu können, wurde allerdings relativiert. Wie schon 2013 in der Kommissionsempfehlung zu »Investitionen in Kinder « hervorgehoben (Europäische Kommission 2013), wird hierbei nur ein Handlungsfeld einer notwendigen Strategie gegen Kinderarmut thematisiert. Ohne die anderen Handlungsfelder - insbesondere die Sicherung des Zugangs zu auskömmlicher Beschäftigung und des Zugangs zu ausreichenden materiellen Ressourcen für Familien - kann eine Kindergarantie nur begrenzte Wirkung haben, da alle drei Handlungsfelder als komplementäre Elemente einer Erfolg versprechenden Strategie anzusehen sind.

\subsection{Die testweise Umsetzung der Kindergarantie}

Im Oktober 2020 gab UNICEF bekannt, dass die Europäische Kommission UNICEF damit beauftragt hat, in Anknüpfung an die beiden Machbarkeitsstudien im Rahmen des zweijährigen Programms »Testing the Child Guarantee in the EU Member States « die testweise Umsetzung der Kindergarantie in ausgewählten Mitgliedstaaten zu begleiten (UNICEF 2020a). Mit Bulgarien, Deutschland, Griechenland, Italien, Kroatien, Litauen und Spanien haben sich sieben Länder bereit erklärt, innovative Ansätze zu erproben, um nationale Aktionspläne zur Verringerung der Kinderarmut zu entwickeln. 
Gleichzeitig soll sichergestellt werden, dass besonders gefährdete Gruppen von Kindern Zugang zu qualitativ hochwertigen Diensten haben, um die Auswirkungen der Armut für hilfe- und schutzbedürftige Kinder abzumildern. Dies ist umso dringlicher, als zu befürchten ist, dass die Armutsrisiken - nicht zuletzt als Folge der Covid-19-Pandemie - aktuell und in den kommenden Jahren weiter zunehmen werden.

In Zusammenarbeit mit den nationalen und lokalen öffentlichen Trägern, mit Vertreter:innen der Zivilgesellschaft, Familien und Kindern sollen in vier Ländern (Bulgarien, Griechenland, Italien und Kroatien) konkrete Pilotprojekte zu Diensten und Interventionsformen entwickelt und umgesetzt werden, die dazu beitragen, Kinderarmut und soziale Ausgrenzung wirksam zu überwinden. Zugleich soll die Fähigkeit der nationalen und lokalen Akteur:innen gestärkt werden, qualitativ hochwertige Dienste für Kinder und Familien bereitzustellen und insbesondere den Zugang zu Bildung, Gesundheit, frühkindlicher Bildung und Betreuung, menschenwürdiger Unterkunft und ausgewogener Ernährung sicherzustellen. In Deutschland, Litauen und Spanien werden dagegen die politischen Rahmenbedingungen zur Überwindung von Armut und sozialer Ausgrenzung von Kindern untersucht.

Gleichzeitig soll die Testphase dazu genutzt werden, die Berichterstattung zu Armut und Ausgrenzung von Kindern auf Basis von sozialen Indikatoren zu verbessern (UNICEF 2020b).

\subsection{Die Kommissionsempfehlung an den Rat zur Kindergarantie}

Am 24. März 2021 hat die Europäische Kommission eine umfassende EU-Kinderrechtsstrategie vorgestellt. In einer Empfehlung an das Europäische Parlament und den Europäischen Rat schlug sie eine Strategie vor, die in den kommenden Jahren gezielte Aktionen in sechs Handlungsfeldern vorsieht, mit denen die Rechte von Kindern in der EU gestärkt werden sollen. Ausgehend von dem Grundsatz, dass jedes Kind Anspruch auf einen angemessenen Lebensstandard und auf gleiche Lebenschancen hat, soll die EU die Armut und soziale Ausgrenzung von Kindern bekämpfen und eine integrative, kinderfreundliche Gesellschaft fördern (European Commission 2021a).

Ergänzend dazu legte die Kommission eine Empfehlung an den Europäischen Rat zur Einführung einer europäischen Kindergarantie vor. Demnach sollen die Mitgliedstaaten ihren von Armut und Ausgrenzung bedrohten Kindern und Jugendlichen den Zugang zu einer Reihe zentraler Dienste garantieren, um die negativen Folgen des Aufwachsens in Armut abzumildern, den intergenerationalen Kreislauf der Benachteiligung zu verhindern und gleiche Chancen für 
alle Kinder zu sichern (European Commission 2021b). Freier und effektiver Zugang soll insbesondere zu folgenden Diensten garantiert werden:

- frühkindliche Bildung und Betreuung,

- schulische Bildung und schulbasierte Aktivitäten,

- mindestens eine gesunde Mahlzeit pro Schultag,

- medizinische Versorgung, z.B. medizinische Untersuchungen und Gesundheitsvorsorge.

Diese Dienste sollten den Kindern kostenfrei und leicht zugänglich zur Verfügung stehen. Darüber hinaus sollten die Mitgliedstaaten eine gesunde Verpflegung auch an anderen Tagen und die Vermeidung von Wohnungslosigkeit sicherstellen. Dabei sollten zugleich die besonderen Bedarfe von Kindern in benachteiligten Lebenslagen berücksichtigt werden, insbesondere Wohnungslosigkeit, Behinderung, prekäre Familiensituation, Migrationshintergrund, Minoritätenstatus, alternative Betreuung etc.

Zugleich sollten die Mitgliedstaaten integrierte und fördernde Rahmenbedingungen schaffen, durch die Armut und soziale Ausgrenzung von Kindern und Jugendlichen überwunden wird und die negativen Auswirkungen der Pandemie auf die Lebenslagen von Kindern und Jugendlichen beseitigt werden. $\mathrm{Zu}$ diesem Zweck sollten die Mitgliedstaaten

- ihre Bildungs-, Gesundheits-, Ernährungs-, Wohnungs- und Sozialpolitik auf nationaler, regionaler und lokaler Ebene in Übereinstimmung mit den Empfehlungen weiterentwickeln und dabei nach Möglichkeit ihre Kinder in integrierter Weise unterstützen;

- ihre Investitionen in Bildung, Gesundheit und soziale Sicherung aufstocken (sowohl in Infrastrukturmaßnahmen als auch in Fachkräfte), um dem Bedarf insbesondere von armuts- und ausgrenzungsgefährdeten Kindern und Jugendlichen und ihren Familien in angemessener Weise gerecht zu werden;

- angemessene Politikprogramme und Maßnahmen bereitstellen, um - insbesondere durch Arbeitsmarktintegrationsprogramme für Eltern und Erziehungsberechtigte und durch materielle Hilfen für Familien und Haushalte - zu verhindern, dass materielle Barrieren den Zugang zu qualitativ hochwertigen Diensten verhindern.

Bei den ergriffenen Maßnahmen sollten regionale Aspekte der Benachteiligung mit berücksichtigt werden; ebenso sollten Diskriminierung und Stigmatisierung vermieden werden. Schließlich sollte eine enge Kooperation zwischen den 
verschiedenen staatlichen Ebenen, aber auch zwischen staatlichen und privaten Akteur:innen bei der Planung, Umsetzung und Auswertung der Politiken und Dienste sichergestellt werden.

Zur Umsetzung der Maßnahmen sollten ausreichende Finanzmittel zur Verfügung gestellt werden. Neben den nationalen Mitteln sollten auch die Mittel der Europäischen Strukturfonds dafür eingesetzt werden, insbesondere der Europäische Sozialfonds plus und der EU-Regionalfonds, aber z.B. auch das Programm InvestEU und der europäische Wiederaufbaufonds.

Für die neue Förderperiode 2021-2027 ist vorgesehen, dass Mitgliedstaaten, die in den Jahren 2017-2019 eine höhere Quote an Kindern und Jugendlichen in Armut oder sozialer Ausgrenzung als der Durchschnitt aller Mitgliedstaaten hatten, 5 Prozent ihrer Mittel aus dem Europäischen Sozialfonds plus für die Bekämpfung von Kinderarmut einsetzen müssen. Die übrigen Mitgliedstaaten sollen diese Mittel lediglich in angemessenem Umfang dafür einsetzen. Die vom Europäischen Parlament geforderte und von der Kommission unterstützte Mindestquote an Mitteln aus dem Europäischen Sozialfonds plus für die Kindergarantie ist - als Folge des Widerstands der Mitgliedstaaten im Europäischen Rat - in der Kommissionsstrategie nicht mehr enthalten.

Zur Umsetzung der vorgesehenen Ratsempfehlung sollten die Mitgliedstaaten einen nationalen Koordinator bzw. eine nationale Koordinatorin für die Kindergarantie benennen. Diese:r sollte mit dem Mandat und den Ressourcen ausgestattet sein, die Umsetzung der Empfehlungen effektiv zu koordinieren und zu überwachen. Weiterhin sollte die Gruppe der von Armut und Ausgrenzung bedrohten Kinder und Jugendlichen im nationalen Kontext festgelegt werden, wobei nationale, regionale und lokale Organisationen bei der Festlegung der Zielgruppen, der Identifikation von Barrieren und der Auswahl der wesentlichen Dienste im Hinblick auf die Ratsempfehlung einbezogen werden sollten.

Sechs Monate nach Verabschiedung der Ratsempfehlung sollten die Mitgliedstaaten der Kommission einen Aktionsplan vorlegen, der für den Zeitraum bis 2030 die wesentlichen Maßnahmen auf nationaler, regionaler und lokaler Ebene beinhalten sollte. In den Aktionsplänen sollten aufsuchende Maßnahmen enthalten sein, mit denen bedürftige Kinder und Familien insbesondere auf regionaler und lokaler Ebene angesprochen und erreicht werden können. Dabei sollten die vorhandenen Bildungseinrichtungen, Kinder-, Jugend- und Familiendienste, ausgebildete Mediator:innen, Akteur:innen der Zivilgesellschaft und andere mehr einbezogen werden.

Schließlich sollten die Mitgliedsstaaten im Zweijahresturnus über die Umsetzung der nationalen Aktionspläne an die Kommission berichten. Die Kommission wird die Fortschritte im Rahmen des Europäischen Semesters über- 
wachen und ggf. länderspezifische Empfehlungen aussprechen. Dazu wird die Kommission einen Überwachungs- und Berichtsrahmen mit geeigneten Indikatoren entwickeln.

\section{Probleme der Operationalisierung und Umsetzung der Kindergarantie in der EU}

Untersuchungen zu (kinderbezogenen) sozialen Diensten haben gezeigt, dass die EU-Mitgliedstaaten in diesem Bereich eine große Heterogenität aufweisen. Dies gilt für die Berichte im Rahmen der EU-Initiative »Investing in Children«, auf die bereits hingewiesen wurde; dies gilt aber auch für EU-Berichte zu sozialen Diensten von allgemeinem Interesse und für unabhängige wissenschaftliche Studien zu dieser Thematik (vgl. z. B. Alber 1995; Bahle/Pfenning 2001; Munday 2003; European Commission 2011).

Die Unterschiede liegen zum einen darin, dass die Bereitstellung sozialer Dienstleistungen in unterschiedlichem Ausmaß durch professionalisierte Dienste erfolgt. Zum anderen unterscheiden sich die Länder darin, in welcher Weise bzw. auf welcher (staatlichen) Ebene die Dienstleistungsproduktion reguliert wird und in welchem Verhältnis öffentliche, privat-gemeinnützige und privatgewinnorientierte Anbieter an der Bereitstellung sozialer Dienste beteiligt sind. Schließlich liegen Unterschiede aber auch in dem Umfang, in dem der Dienstleistungskonsum privat oder kollektiv (über den staatlichen Sozialhaushalt) finanziert wird.

Diese und weitere Kriterien prägen die unterschiedlichen Dienstleistungsregime, die in den Mitgliedstaaten anzutreffen sind. Allerdings variieren die Regime auch innerhalb des einzelnen Landes von Dienstleistungsbereich zu Dienstleistungsbereich, was generelle länderbezogene Regimevergleiche erschwert.

Stark vereinfacht lässt sich feststellen, dass die west- und nordeuropäischen Länder eine breite Palette zumeist qualitativ hoch entwickelter sozialer Dienste aufweisen, während die Versorgung in den süd- und mittel- bzw. osteuropäischen Staaten durch zum Teil erhebliche Lücken und Defizite gekennzeichnet ist. Insbesondere in diesen Ländern könnte die Umsetzung der EU-Initiative zur Kindergarantie einen wichtigen Anstoß zur bedarfsgerechten Weiterentwicklung und qualitativen Verbesserung der Versorgungslage darstellen.

Die Operationalisierung einer solchen Strategie wirft allerdings viele Fragen auf: Welche Unterstützungs- oder Schutzbedarfe und damit welche Dienstleistungsbereiche werden als notwendig angesehen? Welche konkreten Leistungen sollen dabei im Vordergrund stehen? Wie lassen sich Quantität und Qualität die- 
ser Dienstleistungen erfassen? Wie sollen Schwellenwerte definiert und festgesetzt werden, mit deren Hilfe eine angemessene Versorgung gemessen werden kann? Ein besonderes Problem liegt schließlich darin, dass die Wirksamkeit sozialer Dienste umso größer ist, je besser sie - unter Beteiligung und Mitwirkung der Adressat:innen - passgenau auf die jeweils individuellen Bedarfe zugeschnitten sind.

In der Machbarkeitsstudie wurde mit den fünf Qualitätskriterien Verfügbarkeit, Zugänglichkeit, Erschwinglichkeit, Anpassungsfähigkeit und Akzeptanz versucht, diesen besonderen Anforderungen Rechnung zu tragen (vgl. z. B. auch The Social Protection Committee 2010).

In der zweiten Machbarkeitsstudie wurde für jeden Dienstleistungsbereich eine Maßnahme ausgewählt, anhand derer die möglichen Operationalisierungen einschließlich Kosten- und Nutzenschätzungen vorgenommen wurden. Auch wenn die Angaben zu diesen Maßnahmen interessante Einblicke in einzelne Aspekte der Versorgungslage in den untersuchten Dienstleistungsbereichen liefern, wird sich daraus kaum ein gesichertes Bild der jeweils gesamten Versorgungslage in den einzelnen oder allen Mitgliedsstaaten ableiten lassen.

Daraus wird erkennbar, wie schwierig es in der Praxis sein wird, aussagekräftige Indikatoren für eine angemessene Versorgung in sozialen Diensten zu entwickeln. Und diese Aufgabe wird umso größer, je anspruchsvoller und umfassender die Standards der Versorgung bzw. der Bedarfsdeckung definiert werden.

Vieles spricht daher für ein Pilotprojekt - wie es jetzt als Phase 3 der Kindergarantie angelaufen ist -, mit dem die Entwicklung und Umsetzung der Operationalisierung in ausgewählten Mitgliedstaaten modelhaft erprobt wird, um beispielhafte praktikable Ansätze für alle Mitgliedstaaten zu gewinnen. Letztlich werden erst die Ergebnisse dieser Phase zeigen, ob es einen gangbaren Weg für die Definition von Anforderungen und Kriterien gibt, mit denen eine Umsetzung der Kindergarantie in den Mitgliedstaaten gesteuert, begleitet und ausgewertet werden kann. Eine entscheidende Rolle wird dabei nicht zuletzt dem begleitenden Monitoring und der Evaluation dieser Erprobungsphase zukommen.

Angesichts des zum Teil noch geringen Ausbaus von kinder- und familienbezogenen Diensten in einzelnen Regionen bzw. Ländern, insbesondere in Südosteuropa, erscheint in einer ersten Phase die Konzentration der Kindergarantie-Initiative auf armutsgefährdete Kinder und Jugendliche sinnvoll, wobei aber Stigmatisierungrisiken beachtet werden müssen. Mittel- und längerfristig sollte das Ziel allerdings sein, die Garantie auf alle Kinder auszuweiten. Wichtig wird es zudem sein, die jeweiligen nationalen Kindergarantieprogramme in ein Gesamtkonzept der Bekämpfung von Kinderarmut einzubetten, wie es die jüngste Kommissionsempfehlung vorsieht, da mit sozialen Diensten allein eine Überwindung der Kinderamut nicht möglich ist. 


\section{Mögliche Auswirkungen der Kindergarantie in Deutschland}

In Deutschland war das Interesse der öffentlichen und privaten Akteur:innen im Handlungsfeld der Kinder- und Jugendpolitik am Thema Kindergarantie bisher eher gering. Lediglich die Jugend- und Familienverbände, Kinderrechts- bzw. Kinderschutzorganisationen und einzelne Wohlfahrtsverbände, die sich auch vorher schon zum Thema Kinderarmut engagiert hatten, haben auf die europäische Initiative positiv reagiert. Dennoch ist auch hier die Überzeugung vorherrschend, dass die Initiative eher für andere Mitgliedstaaten als für Deutschland sinnvoll und hilfreich sein könnte, da hierzulande bereist ein ausgebautes und qualitativ hoch entwickeltes System von kinder- und familienbezogenen Diensten existiert.

\subsection{Die deutsche Diskussion um Grundsicherung und soziale Infrastruktur}

In der deutschen Fachdiskussion fand das Thema Kinderarmut erst relativ spät Beachtung; erst seit der Jahrtausendwende setzte eine kontinuierliche fachpolitische Diskussion dazu ein. Die Diskussion wurde maßgeblich durch die Ergebnisse der vom Bundesverband der Arbeiterwohlfahrt in Auftrag gegebene und seit 1997 durchgeführte Langzeitstudie des Instituts für Sozialarbeit und Sozialpädagogik (ISS) zu Kinderarmut beeinflusst (vgl. z. B. Holz/Laubstein/Sthamer 2012; Volf/Laubstein/Sthamer 2019).

Als Ergebnis dieser und weiterer empirischer Untersuchungen zu Kinderarmut lässt sich festhalten: Armut während der Kindheit zu erfahren hat nicht nur direkte Auswirkungen auf das gegenwärtige Leben eines Kindes, indem dieses aufgrund fehlender finanzieller Möglichkeiten von typischen altersspezifischen Aktivitäten ausgeschlossen wird, sondern wirkt darüber hinaus auch auf das weitere Leben im Jugend- und Erwachsenenalter.

Die Analysen z. B. von Tophoven et al. (2018) weisen darauf hin, dass sich die untersuchten Dimensionen sozialer Teilhabe in Abhängigkeit von den Einkommensverlaufmustern in der Kindheit unterscheiden und andauernde Armutslagen mit einer geringeren sozialen Teilhabe verbunden sind. Gleichzeitig weisen die Ergebnisse der ISS-Studien darauf hin, dass die Resilienz des Familiensystems in solchen Krisenphasen durch soziale Dienste gestärkt werden kann.

Die verfügbaren Befunde legen nahe, dass für eine Armutsprävention und Sicherung des Wohlbefindens von Kindern und Jugendlichen eine Kombination von materieller Grundsicherung und infrastrukturellen Hilfen erforderlich sein dürfte. Für die Regierungskoalitionen auf Bundesebene stand allerdings nicht erst seit den 2000er-Jahren die Verbesserung des Zugangs zum Arbeitsmarkt für Familien mit Kindern im Vordergrund, während Verbesserungen der monetä- 
ren oder der Realtransfers von nachrangiger Bedeutung waren (vgl. z. B. BMAS 2017). Bei der Gestaltung monetärer Transfers standen eher andere Ziele als die Armutsbekämpfung im Vordergrund.

Bezeichnend hierfür ist die bis heute sehr restriktive Ausgestaltung der sozialen Mindestsicherungsleistungen im Rahmen der SGB II und XII sowie des Asylbewerberleistungsgesetzes einschließlich der nach einem Urteil des Bundesverfassungsgerichts 2011 eingeführte Leistungen für Bildung und Teilhabe, die unbar als Sach- und Dienstleistungen erbracht werden. Auch bei der Weiterentwicklung der sozialen Infrastruktur standen andere Ziele als die Bekämpfung der Kinderarmut im Vordergrund (Hanesch 2013 und 2017).

Vor diesem Hintergrund entwickelte sich das Thema Kinderarmut im vergangenen Jahrzehnt zu einem Brennpunkt der sozialpolitischen Debatte. Vor allem die unzureichenden Grundsicherungsleistungen für Kinder und Jugendliche führten zur Forderung nach einer eigenständigen Kindergrundsicherung, die heute nicht nur von Expert:innen, sondern auch von Sozialverbänden, Gewerkschaften, Linken, Grünen und SPD getragen wird und auch im Bundesrat Unterstützung findet. ${ }^{6}$ Mit dem Starke-Familien-Gesetz von 2019 wie auch mit den jüngsten Reformen in der Covid-19-Pandemie haben Bundesregierung und das SPD-geführte Familienministerium allerdings unterstrichen, dass sie zu einer nachhaltigen Reform noch nicht bereit sind.

Im Kontext der Debatte um Kinderarmut sind monetäre Transfers und soziale Dienste zeitweilig als konkurrierende Ansätze gehandelt worden. In den letzten Jahren besteht jedoch weitgehend Konsens, dass es sich um ergänzende statt konkurrierende Ansätze handelt, also sowohl eine Verbesserung der materiellen Absicherung durch Anhebung bzw. Umgestaltung der Grundsicherung zu einer Kindergrundsicherung als auch ein Ausbau der sozialen Infrastruktur erforderlich ist, um die Lage armutsgefährdeter Kinder und Jugendlicher in Deutschland zu verbessern.

6 | So hat die Arbeits- und Sozialministerkonferenz in ihrer 97. Sitzung im Sinne der »Investitionen in Kinder«-Empfehlung festgestellt: Für eine kindgerechte Entwicklung sind neben einer aktiven Arbeitsmarktpolitik, um die Elternarmut zu verringern, eine qualitativ hochwertige Infrastruktur, die gleiche Bildungschancen und soziale Teilhabe ermöglicht, aber auch eine soziale Absicherung von Kindern und Jugendlichen erforderlich, die über eine reine Armutsbekämpfung hinausgeht.

Darüber hinaus hat die Konferenz mehrheitlich beschlossen: »Die Ministerinnen und Minister, Senatorinnen und Senatoren für Arbeit und Soziales appellieren an die Bundesregierung, gemeinsam mit den Ländern konkrete Umsetzungsschritte zur Einführung einer Kindergrundsicherung einzuleiten« (ASMK 2020; vgl. auch Niedersächsisches Ministerium für Soziales, Gesundheit und Gleichstellung 2019a und b; Ministerium für Soziales und Integration des Landes Baden-Württemberg 2020). 


\subsection{Defizite der sozialen Dienste für armutsgefährdete Kinder und Jugendliche}

Das System sozialer Dienste ist in Deutschland zwar breit differenziert und gut ausgebaut, vor allem wenn man es mit den süd- bzw. südosteuropäischen Ländern vergleicht. Allerdings weist es auch charakteristische Schwächen auf, was die Versorgung von armutsgefährdeten Kindern betrifft (vgl. Hanesch 2017 und 2019):

- Zwar hat sich das Angebot an frühkindlicher Bildung und Betreuung im letzten Jahrzehnt dank gemeinsamer Anstrengungen von Bund, Ländern und Gemeinden stark erhöht, dennoch reicht das Angebot bis heute nicht aus, um die Nachfrage der Eltern zu decken. Die Dienste werden nach wie vor vorrangig von Eltern mit mittlerem und höherem Einkommen in Anspruch genommen, während Kinder aus Niedrigeinkommenshaushalten und Migrantenhaushalten deutlich unterrepräsentiert sind. Nach wie vor gibt es große Unterschiede in der regionalen Versorgungsstruktur.

Zudem gibt es bis heute keine einheitlichen Qualitätsstandards, die erfüllt sein müssen (AGF 2017; Bock-Famulla et al. 2019). Die Tatsache, dass bis heute drei von vier Kindern eine Kita mit zu wenig Personal besuchen, verweist auf die Tatsache, dass als Zweck der Kinderbetreuung im Vordergrund steht, eine verbesserte Vereinbarkeit von Familie und Erwerbstätigkeit zu erreichen. Die gezielte frühe Bildungsförderung insbesondere von Kindern aus benachteiligten Milieus hat dagegen - wenn überhaupt - nur einen nachrangigen Stellenwert.

- Auch die schulische Bildung im Primar-und Sekundarbereich weist charakteristische Mängel auf, ist doch das deutsche Schulsystem vorrangig auf eine frühe Selektion und Separation statt auf eine frühe Förderung und Integration der Kinder ausgerichtet. Bezeichnenderweise kommt die Einführung von Ganztagsschulen kaum voran, zudem klaffen die Lernbedingungen der Schüler:innen je nach Bundesland und Schulform eklatant auseinander. Bildungsforscher:innen sprechen von einem »Dschungel « verschiedener Bestimmungen zur Verteilung von Ressourcen im Ganztag, wobei die riesigen Unterschiede offenlegen, wie sehr es hier an gemeinsamen Standards mangelt.

Der nach wie vor hohe Anteil an nicht gebundenen »Pseudo-Ganztagsschulen« zeigt, dass auch hier die Vereinbarkeitszielsetzung gegenüber der individuellen Förderung von Bildung und Teilhabe von Kindern aus benachteiligten Milieus eindeutig dominiert (vgl. z.B. Berkemeyer 2015; Bertelsmann Stiftung 2019). Die geplante Einführung eines Rechtsanspruchs auf Ganztagsbetreuung für Grundschulkinder wird daran nichts ändern, solange nicht die Bildung gegenüber der Betreuung in den Vordergrund rückt und 
dieser "Bildungs-Betreuung« nicht verbindliche und einheitliche Standards zugrunde gelegt werden.

- Seit Jahren warnen Ernährungswissenschaftler:innen in Deutschland vor einem Teufelskreis der Armut im Hinblick auf Kinderernährung. In wiederholten Stellungnahmen haben sie darauf hingewiesen, dass mit den geltenden Regelleistungen der sozialen Mindestsicherung nach SGB II und XII eine gesunde und vollwertige Ernährung für Kinder im Grundsicherungsbezug nicht möglich ist. Fehlernährung im Kindesalter kann zu körperlichen und geistigen Entwicklungsstörungen führen, und diese Kinder haben dann ein höheres Risiko, auch als Erwachsene in Armut zu leben.

Notwendig ist daher unter anderem eine angemessene Anhebung der Kinderregelleistungen (vgl. z. B. Society of Nutrition and Food Science/Universität Hohenheim 2018), aber auch ein erleichterter Zugang zu einer kostenfreien vollwertigen Mittagsverpflegung in Kitas und Schulen ist für diese Kinder dringend erforderlich. Auch hier erweist sich das Bildungs- und Teilhabepaket nach wie vor eher als Zugangsbarriere denn als Zugangshilfe (Paritätischer Gesamtverband 2019).

- Die Kommunen sind ordnungs- und sozialrechtlich verpflichtet, Maßnahmen zur Vermeidung bzw. Überwindung von Wohnungslosigkeit zu ergreifen. In einer aktuellen Studie für das BMAS wurde dazu festgestellt, dass diese Aufgabe der Wohnungssicherung in sehr heterogener Weise umgesetzt wird. Häufig fehlt es an einem abgestimmten Verhalten der beteiligten Akteur:innen und Stellen. Zudem sind die Maßnahmen bei vielen Hilfebedürftigen nicht bekannt und erreichen sie daher nicht. Maßnahmen zur Prävention werden nicht konsequent genug umgesetzt, auch wenn bei Familien mit Kindern besondere Anstrengungen unternommen werden. Angesichts der wachsenden Diskrepanz zwischen Bedarf und Angebot an bezahlbarem Wohnraum in städtischen Ballungszentren sind die Wohnungssicherungsdienste zunehmend überfordert (Busch-Geertsema/Henke/Steffen 2019).

- Zugangsprobleme zu Gesundheitsleistungen haben in Deutschland lediglich Kinder, deren Eltern nicht krankenversichert sind (hierzulande weniger als 1 Prozent) oder als Wohnungslose auf der Straße leben. Für Flüchtlingskinder ist der Zugang in einer ersten Phase auf notwendige Basisleistungen beschränkt. Demgegenüber haben Kinder, deren Eltern gesetzlich versichert sind oder die durch ihre Eltern kostenpflichtig privat versichert sind, freien Zugang zu Früherkennungsuntersuchungen und Gesundheitsleistungen.

Die europäische Initiative zur Kindergarantie könnte dazu beitragen, die Diskussion zu dieser Thematik auch in Deutschland zu beleben und Anstöße für 
eine bedarfsgerechte Weiterentwicklung der sozialen Infrastruktur für Kinder und Jugendliche zu geben. Einen ersten Beitrag hierzu könnte und sollte die von UNICEF durchgeführte Bestandsaufnahme der Politik zu Armut und Ausgrenzung von Kindern und Jugendlichen im Rahmen der testweisen Umsetzung der Kindergarantie in Deutschland leisten (siehe Abschnitt 3.2).

\section{Literatur}

AGF $=$ Arbeitsgemeinschaft der deutschen Familienorganisationen e.V. (2017): Gemeinsame Erklärung zu Kitaqualitätsstandards, Berlin.

Alber, J. (1995): A framework for the comparative study of social services, in: Journal of European Social Policy 5(2), S. 131-149.

ASMK = Arbeits- und Sozialministerkonferenz (2020): Externes Ergebnisprotokoll der 97. Konferenz der Ministerinnen und Minister, Senatorinnen und Senatoren für Arbeit und Soziales der Länder, Ministerium für Soziales und Integration des Landes Baden-Württemberg, Stuttgart.

Bahle, T./Pfenning, A. (2001): Angebotsformen und Trägerstrukturen sozialer Dienste im europäischen Vergleich, Mannheimer Zentrum für Europäische Sozialforschung, Arbeitspapier Nr. 34/2001, Mannheim.

Berkemeyer, N. (2015): Ausbau von Ganztagsschulen. Regelungen und Umsetzungsstrategien in den Bundesländern, Bertelsmann Stiftung, Gütersloh.

Bertelsmann Stiftung (Hrsg.) (2019): Gute Ganztagsschulen entwickeln. Zwischenbilanz und Perspektiven, Gütersloh.

BMAS = Bundesministerium für Arbeit und Soziales (2017): Lebenslagen in Deutschland. Der Fünfte Armuts- und Reichtumsbericht der Bundesregierung, Berlin.

Bock-Famulla, K./Münchow, A./Frings, J./Kempf, F./Schütz, J. (2019): Länderreport Frühkindliche Bildungssysteme 2019. Transparenz schaffen - Governance stärken, Bertelsmann Stiftung, Gütersloh.

Bruckmayer, M./Picken, N./Janta, B. (2020): Developments in Child and Family Policy in the EU in 2019, European Platform for Investing in Children: annual thematic report, Brüssel.

Busch-Geertsema, V./Henke, J./Steffen, A. (2019): Entstehung, Verlauf und Struktur von Wohnungslosigkeit und Strategien zu ihrer Vermeidung und Behebung, Gesellschaft für innovative Sozialforschung und Sozialplanung e. V, Bremen.

Eichhorst, W./Rinne, U. (2017): The European Youth Guarantee: A Preliminary Assessment and Broader Conceptual Implications, IZA Policy Paper No. 128, Bonn. 
Europäische Kommission (2013): Investitionen in Kinder: Den Kreislauf der Benachteiligung durchbrechen, Empfehlung der Kommission vom 20. Februar 2013, 2013/112/EU, Brüssel.

Europäisches Parlament (2015): Entschließung vom 24. November 2015 zur Verringerung von Ungleichheit mit besonderem Schwerpunkt auf Kinderarmut, 2014/2237(INI), Brüssel.

Europäisches Parlament/Rat der Europäischen Union/Europäische Kommission (2017): Europäische Säule sozialer Rechte, Brüssel.

European Commission (2011): Study on social services of general interest. Final report, Brüssel.

European Commission (2017): Taking stock of the 2013 Recommendation on »Investing in children: breaking the cycle of disadvantage«, Commission Staff Working Document, Brüssel.

European Commission (2020): Evaluation of the ESF and YEI Support to Youth Employment, Commission Staff Working Document, Brüssel.

European Commission (2021a): EU strategy on the rights of the child, Communication from the European Parliament, the Council, the European Economic and Social Committee and the Committee of the Regions, Brüssel.

European Commission (2021b): Proposal for a Council Recommendation establishing a European Child Guarantee, Brüssel.

European Commission (2021c): The European Pillar of Social Rights Action Plan, Brüssel.

Eurostat (2021): Von Armut oder sozialer Ausgrenzung bedrohte Bevölkerung nach Alter und Geschlecht, Tabellen-Code ILC_PEPS01, https://ec.europa. eu/eurostat/ (Abruf am 10.6.2021).

Felgueroso, F./Jansen, M. (2015): The Youth Guarantee: Theory or reality?, in: Dolado, J.J. (Hrsg.): No Country for Young People? Youth Labour Market Problems in Europe, Centre for Economic Policy Research, London.

Frazer, H./Guio, A.-C./Marlier, E. (Hrsg.) (2020): Feasibility Study for a Child Guarantee: Final Report, Feasibility Study for a Child Guarantee (FSCG), European Commission, Brüssel.

Frazer, H./Marlier, E. (2017): Progress across Europe in the implementation of the 2013 EU Recommendation on »Investing in children: Breaking the cycle of disadvantage«. A study of national policies, European Social Policy Network (ESPN), Brüssel.

Guio, A.-C./Frazer, H./Marlier, E. (2021): Study on the Economic Implementing Framework of a Possible EU Child Guarantee Scheme Including its Financial Foundation, Second Phase of the Feasibility Study for a Child Guarantee (FSCG2), European Commission, Brüssel (im Erscheinen). 
Hacker, B. (2018): Die Europäische Säule sozialer Rechte: Nutzung und Nutzen, in: Integration 4/2018, S. 259-272.

Hanesch, W. (2013): Investing in Children: Breaking the cycle of disadvantage. A Study of National Policies. Country report Germany, EU Network of Independent Experts on Social Inclusion, Brüssel

Hanesch, W. (2017): ESPN Thematic Report on Progress in the Implementation of the 2013 EU Recommendation on »Investing in Children: Breaking the Cycle of Disadvantage«. Country Report Germany, European Social Policy Network (ESPN), Brüssel.

Hanesch, W. (2019): Feasibility Study for a Child Guarantee: Country Report Germany, Internal document, Feasibility Study for a Child Guarantee (FSCG).

Holz, G./Laubstein, C./Sthamer, E. (2012): Lebenslagen und Zukunftschancen von (armen) Kinder und Jugendlichen in Deutschland. 15 Jahre AWO-ISSStudie, Frankfurt am Main.

Ministerium für Soziales und Integration des Landes Baden-Württemberg (2020): Einführung einer Kindergrundsicherung, Bericht der länderoffenen Arbeitsgruppe zur 97. Arbeits- und Sozialministerkonferenz 2020 am 26./27. November 2020, Stuttgart.

Munday, B. (2003): European social services: A map of characteristics and trends, Council of Europe, Straßburg.

Niedersächsisches Ministerium für Soziales, Gesundheit und Gleichstellung (2019a): Einführung einer Kindergrundsicherung, Bericht der länderoffenen Arbeitsgruppe zur 96. Arbeits- und Sozialministerkonferenz 2019 am 27./28. November 2019, Hannover.

Niedersächsisches Ministerium für Soziales, Gesundheit und Gleichstellung (2019b): Grobkonzept Kindergrundsicherung, Hannover.

Paritätischer Gesamtverband = Deutscher Paritätischer Wohlfahrtsverband Gesamtverband e.V. (2019): Empirische Befunde zum Bildungs- und Teilhabepaket: Teilhabequoten im Fokus, Berlin.

Society of Nutrition and Food Science e. V./Universität Hohenheim (2018): Kinder in Ernährungsarmut. Wissenschaftliche Stellungnahme zu den Auswirkungen von Armut auf den Ernährungsstatus von Kindern in Deutschland, Hohenheim.

The Social Protection Committee (2010): A voluntary quality framework for social services, Brüssel.

Tophoven, S./Lietzmann, T./Reiter, S./Wenzig, C. (2018): Aufwachsen in Armutslagen. Zentrale Einflussfaktoren und Folgen für die soziale Teilhabe, Bertelsmann Stiftung, Gütersloh. 
UNICEF, Europe and Central Asia Regional Office (2020a): European Commission and UNICEF launch pilot programme in 7 Member States to break the cycle of child poverty and social exclusion. »Child Guarantee« to introduce innovative approaches to national budgeting and planning, Press release 08-October-2020, Genf.

UNICEF, Europe and Central Asia Regional Office (2020b): The Child Guarantee: Breaking the Cycle of Disadvantage. Phase III of the Preparatory Action for a Child Guarantee, Genf.

Volf, I./Laubstein, C./Sthamer, E. (2019): Wenn Kinderarmut erwachsen wird ... AWO-ISS-Langzeitstudie zu (Langzeit-)Folgen von Armut im Lebensverlauf, Institut für Sozialarbeit und Sozialpädagogik e. V., Frankfurt am Main. 

Fazit 



\section{Den Sozialstaat komplett machen}

Die Grundsicherung weiterdenken und "weiter denken"

Florian Blank, Claus Schäfer, Dorothee Spannagel

Die Beiträge in diesem Band bearbeiten ein gemeinsames Thema aus unterschiedlichen Blickwinkeln: eine Grundsicherung, die sich nicht auf ein Transfersystem für die Ärmsten in der Gesellschaft beschränkt. Die Autor:innen gehen der Frage nach, wie eine Grundsicherung aussehen müsste, die sowohl die bisherigen Grundsicherungsleistungen in verbesserter Form umfasst als auch andere Sozialleistungen und Infrastrukturen in den Blick nimmt, die zusammen materielle Absicherung und soziale Teilhabe ermöglichen. Ein Fazit aus diesem Band kann und soll nicht die einzelnen Ergebnisse wiederholen, aber auch mit Blick auf politische Handlungsimpulse können einige übergreifende Punkte herausgegriffen werden.

Im häufig sperrigen Sozialrecht finden sich wenig Themen, die so viel öffentliche und politische Aufmerksamkeit auf sich ziehen wie die Grundsicherung im herkömmlichen bzw. engeren Sinn: Die Einführung der Grundsicherung für Arbeitsuchende (Hartz IV) führte 2005 zu heftigen Auseinandersetzungen, die zur Gründung der »Wahlalternative Arbeit \& soziale Gerechtigkeit» (WASG) und später zur gesamtdeutschen Partei Die Linke beigetragen haben. Die Kritik an den Regelsätzen, der Praxis des "Förderns und Forderns", den Sanktionen und dem »Absturz« von Arbeitslosen nach dem Auslaufen der Sozialversicherungsleistung Arbeitslosengeld I dauert auch angesichts der anhaltenden Kluft zwischen Anspruch und Wirklichkeit der Hartz-IV-Praxis immer noch an - und wurde durch die Coronakrise noch einmal zusätzlich befeuert.

Auch die anderen Bereiche der herkömmlichen Grundsicherung sind im gesellschaftlichen Fokus geblieben: Die Entwicklung der Bezieher:innen von Grundsicherung im Alter und bei Erwerbsminderung sorgt immer wieder für Schlagzeilen und schürt die Angst vor Altersarmut.

Vor dem Hintergrund dieser offenkundigen oder vermeintlichen Miseren wurden Forderungen immer lauter, die mit unterschiedlichen politischen Moti- 
ven für ein grundlegend anderes Verständnis von Grundsicherung werben: für ein bedingungsloses Grundeinkommen, das entweder als emanzipatorisches Projekt für einen besseren Sozialstaat und mehr individuelle Unabhängigkeit von Erwerbsarbeit aufgefasst wird - oder aber als Ausprägung eines »schlanken Staates«, der sich auf diese Weise aller Verantwortung für die Verbesserung spezifischer sozialer Probleme entledigen kann. Diese Utopie ist zwar viel älter als Hartz IV und die entsprechende Debatte, wird aber von dieser nicht nur wiederbelebt, sondern aktuell auch bis zu einem wissenschaftlich begleiteten Modellversuch eines privaten Vereins getrieben (Mein Grundeinkommen o.J.).

All diese Diskussionen und Reaktionen haben das politische Feld in Bewegung gesetzt; insbesondere in den Parteien des linken Spektrums wird eine grundlegende Reform des Systems gefordert. Und die Coronakrise hat die Debatte erneut aufgemischt, als die problematischen Antragsbedingungen für die Grundsicherung einer breiten Öffentlichkeit bewusst und weitere Lücken des Netzes der sozialen Sicherung für viele sichtbar wurden.

In dieser Situation zielen die in diesem Sammelband enthaltenen wissenschaftlichen Analysen darauf ab, Argumente für ebenso weitreichende wie zielführende Veränderungen der Grundsicherung zu liefern. Dafür genügt es unserer Überzeugung nach nicht, allein über Verbesserungen in der Grundsicherung für Arbeitsuchende und die weiteren Transferleistungen nachzudenken. Die Grundsicherung muss nicht nur weitergedacht werden, sie muss auch »weiter gedacht« werden.

Der in diesem Band verfolgte Ansatz bringt Elemente zusammen, die in der politischen Debatte bisher nicht immer miteinander verbunden werden. Diese Elemente wollen wir im Folgenden noch einmal kurz zusammenfassen, um damit Bausteine für ein politisches Reformprogramm bereitzustellen. Die gemeinsame Diskussion solcher Bausteine, die sich nicht nur auf eine einfache Verbesserung der materiellen Absicherung der verwundbarsten Mitglieder unserer Gesellschaft konzentrieren, hat aus unserer Sicht drei Vorteile:

- Erstens soll damit die Gefahr vermieden werden, den Trend in der Sozialpolitik zu einer immer stärkeren Bedeutung der eigentlich nachgelagerten Grundsicherungssysteme noch zu verstärken.

- Zweitens soll das Reformprogramm diverse Grundsicherungen für weite Teile der Bevölkerung bereitstellen und nicht nur für Personen, die gegenwärtig Leistungen nach SGB II und SGB XII beziehen, um insgesamt ein menschenwürdiges Existenzminimum und eine effektive soziale Teilhabe sicherzustellen. Damit ist das Programm an die Lebenslagen vieler anschlussfähig, sodass 
problematische Gegenüberstellungen von Bevölkerungsgruppen vermieden werden.

- Drittens soll ein solches Reformprogramm neben monetären Sozialtransfers auch viele öffentliche Infrastrukturen bzw. Angebote von realen öffentlichen Gütern aufeinander abstimmen und in ausreichendem Ausmaß integrieren.

Mit diesen Maßgaben kann das Programm letztlich vielleicht sogar sowohl von vielen Verfechter:innen einer »einfachen« Reform der Grundsicherung als auch von Vertreter:innen eines bedingungslosen Grundeinkommens mitgetragen werden. In diesem Zusammenhang soll noch einmal betont werden, dass die Herausgeber:innen und die meisten Autor:innen dieses Bandes ein bedingungsloses Grundeinkommen aus verschiedenen Gründen nicht für die Lösung drängender sozialer Probleme halten; die inhaltliche Auseinandersetzung mit diesem Konzept ist jedoch nicht die Zielsetzung dieses Bandes.

Was sind nun die Elemente einer "weiter gedachten« Grundsicherung? Aus unserer Sicht lassen sie sich mit folgenden Punkten benennen:

- Zunächst geht es um die Reformen der Grundsicherung im engeren Sinn. Wie in anderen Ländern auch zerfällt das deutsche System in mehrere Teile mit unterschiedlichen Regeln, Leistungshöhen und Zielgruppen: Grundsicherung für Arbeitsuchende, Grundsicherung im Alter und bei Erwerbsminderung, Hilfe zum Lebensunterhalt und das Asylbewerberleistungsgesetz. Zu nennen sind außerdem BAföG und Wohngeld, die in diesem Sammelband aber nicht weiter behandelt werden. Hier geht es darum, die Leistungen und Bezugsbedingungen zu verbessern, was vor allem bedeutet, die schon lange erhobene Forderung nach einer rationalen Bestimmung von mindestsichernden Regelbedarfen endlich umzusetzen.

- Der zweite Ansatz betriff die weitere Sozialpolitik, speziell die Sozialversicherung. Während beim vorhergehenden Punkt das bisher »letzte Netz der sozialen Sicherung im Mittelpunkt steht, geht es hier um die Systeme, die für die weit überwiegende Bevölkerungsmehrheit den Regelfall der sozialen Sicherung darstellt. In einem nicht an formalen Bezeichnungen orientierten Sinn leisten sie die Grundsicherung bei Einkommensausfall, Krankheit und Pflegebedürttigkeit.

Der Blick auf die Sozialversicherung ist auch deshalb notwendig, weil Kürzungen von Geldleistungen und Bezugsdauer des Arbeitslosengeldes I oder eine Absenkung des Rentenniveaus Menschen verstärkt auf die Grundsicherung im engeren Sinn verweisen. Diese beiden Systeme existieren nicht unabhängig voneinander, und diese Abhängigkeit muss aus politischer Sicht auch in um- 
gekehrter Richtung berücksichtigt werden: Eine Anhebung der Leistungen in der Grundsicherung und eine Erleichterung ihrer Bezugsbedingungen führt automatisch dazu, dass sich die Grundsicherung in die Sozialversicherung "hineinschiebt« - sofern letztere nicht ebenfalls verbessert wird.

- Der dritte Punkt betrifft die Rolle von Arbeit. In Deutschland ist gesellschaftliche Integration nach wie vor eng mit Erwerbsbeteiligung verbunden. Daher sollte die Diskussion einer weit verstandenen Grundsicherung auch die Arbeitsmarktpolitik nicht aus der Verantwortung entlassen, damit die Menschen durch ihre Arbeit materielle Sicherheit und Unabhängigkeit, soziale Teilhabe und auch Erfüllung durch sinnvolle Tätigkeit erfahren.

Allerdings kann es dabei nicht darum gehen, Erwerbsbeteiligung um jeden Preis zu erzwingen. Die Regulierung des Arbeitsmarktes - insbesondere eine weitgehende Verdrängung des Niedriglohnsektors -, sozialpolitische Absicherung der Arbeit auch im Sinne zeitweiser Unabhängigkeit vom Arbeitsmarkt (»Dekommodifizierung« im sozialwissenschaftlichen Jargon) und neue Rechte wie das auf eine »bedingungslose Grundzeit« neben der Erwerbszeit (Schäfer 2011) müssen dieses Vorhaben rahmen. Zugleich müssen Regelungen gefunden werden, wie Menschen sich für gesellschaftlich notwendige, aber nicht über den Arbeitsmarkt vermittelte und dort entlohnte Arbeit zum Beispiel Sorgearbeit - entscheiden können, ohne deswegen in materielle Bedrängnis zu geraten.

- Der vierte Punkt betrifft öffentliche Strukturen für Bildung, Wohnen, Verkehr, soziale Dienstleistungen und vieles mehr. Aus unserer Sicht stellt sich der $\mathrm{Zu}-$ sammenhang zwischen Grundsicherung und genereller öffentlicher Tätigkeit in mehrfacher Hinsicht dar. Zunächst sind öffentliche Infrastrukturen schlicht Geld wert - etwas vereinfacht gesagt kann die Lebenslage von Menschen sowohl durch höhere individuelle Geldtransfers verbessert werden als auch durch eine allgemeine Subventionierung von realen Gütern, zum Beispiel vergünstigte oder kostenlose Verkehrstickets. Außerdem sind bestimmte Güter und Dienstleistungen nicht in der notwendigen Menge oder Qualität vorhanden, insbesondere und schon seit Langem Wohnraum.

Und schließlich ist eine Teilhabe am öffentlichen Leben erst voll erfüllt, wenn eine begründete individuelle Chance besteht, verschiedene Angebote für Bildung, Kultur, Unterhaltung etc. zu nutzen, die ohne staatliche Intervention nicht individuell mit eigenen Mitteln auf dem Markt erworben werden könnten.

- Fünftens hat die Diskussion um Grundsicherung auch eine europäische Dimension, die sich etwa darin manifestiert, dass viele (unzureichende) nationale Regulierungen mittlerweile auf ihre Übereinstimmung mit dem (wei- 
tergehenden) europäischen Recht geprüft werden müssen. Dies schlägt sich inzwischen auch in etlichen Initiativen für eine bessere Grundsicherung nieder, die von der EU-Ebene selbst ausgehen.

Der unterschiedliche Zuschnitt und Zugang der Beiträge in diesem Sammelband zeigt deutlich, dass eine ganze Reihe von politischen Themenfeldern mit dem Ziel einer besseren Grundsicherung - verstanden als umfassende Grundversorgung der Bevölkerung - verknüptt werden sollten. Dabei sind weitere Themen hier noch nicht berücksichtigt; sowohl auf einzelne Sozialleistungen (wie Leistungen im Pflegefall) als auch auf Infrastrukturen (etwa der Mobilität) konnte hier nicht eingegangen werden. In diesem Sinne ist dieser Band kein Kompendium, sondern ein wissenschaftlicher Impuls für eine Debatte, der hoffentlich bald politisches Handeln folgt.

\section{Literatur}

Mein Grundeinkommen e. V. (o.J.): Pilotprojekt Grundeinkommen, www.pilot projekt-grundeinkommen.de/ (Abruf am 26.7.2021).

Schäfer, C. (2011): Wir brauchen die »Bedingungslose Grundzeit«, in: WSI-Mitteilungen 2/2011, S. 91-94. 



\section{Autorinnen und Autoren}

Prof. Dr. Gerhard Bäcker arbeitet als Seniorprofessor am Institut Arbeit und Qualifikation der Universität Duisburg-Essen. Er ist zugleich Senior Fellow der Hans-Böckler-Stiftung. Seine Forschung umfasst die Probleme und Perspektiven der einzelnen Bereiche des Systems der sozialen Sicherung.

E-Mail: gerhard.baecker@uni-due.de

PD Dr. Thomas Bahle arbeitet am Mannheimer Zentrum für Europäische Sozialforschung der Universität Mannheim. Seine Forschungsinteressen liegen in den Bereichen Sozialstaat, Familienpolitik, Soziale Dienste, Mindestsicherung und Armut.

Dr. Ulf Banscherus ist Sozial- und Bildungswissenschaftler und leitet die Kooperationsstelle Wissenschaft und Arbeitswelt in der Zentraleinrichtung Wissenschaftliche Weiterbildung und Kooperation (ZEWK) der Technischen Universität Berlin. Seine Arbeitsschwerpunkte liegen in den Bereichen Hochschulforschung und Hochschulentwicklung sowie Wandel der Arbeitswelt.

E-Mail: ulf.banscherus@tu-berlin.de

Dr. Irene Becker ist freiberufliche Wissenschaftlerin. Ihre Arbeitsschwerpunkte liegen auf dem Gebiet der Einkommens- und Vermögensverteilung und der sozialen Sicherung.

E-Mail: i-h.becker@t-online.de

Prof. Dr. Benjamin Benz arbeitet zu Fragen der Politikwissenschaft/Sozialpolitik (Schwerpunkte: Armut/soziale Ausgrenzung, Mindestsicherung, politische Interessenvertretung im Sozialwesen, Europa) am Fachbereich Soziale Arbeit, Bildung und Diakonie der Evangelischen Hochschule Rheinland-Westfalen-Lippe in Bochum.

E-Mail: benz@evh-bochum.de 
Dr. Florian Blank ist Leiter des Referats Sozialpolitik am Wirtschafts- und Sozialwissenschaftlichen Institut (WSI) der Hans-Böckler-Stiftung. Er beschäftigt sich u.a. mit aktuellen Problemen der Sozialversicherung und der Alterssicherung und geht Fragen der vergleichenden Wohlfahrtsstaatsforschung nach. E-Mail: florian-blank@boeckler.de

Prof. Dr. Sebastian Dullien ist wissenschaftlicher Direktor des Instituts für Makroökonomie und Konjunkturforschung der Hans-Böckler-Stiftung und Professor für Volkswirtschaftslehre an der Hochschule für Technik und Wirtschaft Berlin. Sein Arbeitsschwerpunkt liegt in der Makroökonomie.

E-Mail: Sebastian-Dullien@boeckler.de

Prof. Dr. Dr. Thomas Gerlinger ist Professor für Gesundheitssysteme, Gesundheitspolitik und Gesundheitssoziologie an der Fakultät für Gesundheitswissenschaften der Universität Bielefeld. Seine Arbeitsschwerpunkte liegen auf dem Gebiet der Gesundheitspolitik- und Gesundheitssystemforschung im Hinblick auf Deutschland, die Europäische Union und den internationalen Vergleich. E-Mail: thomas.gerlinger@uni-bielefeld.de

Prof. Dr. Walter Hanesch ist Professor (seit 2014 im Ruhestand) für Sozialpolitik und Sozialverwaltung an der Hochschule Darmstadt. Er ist seit 2011 beratend tätig für die Europäische Kommission, zuletzt als nationaler Koordinator des Europäischen Netzwerks für Sozialpolitik (ESPN).

E-Mail: walter.hanesch@h-da.de

Prof. Dr. Tanja Klenk ist Professorin für Verwaltungswissenschaft an der Helmut-Schmidt-Universität Hamburg. Ihre Arbeitsschwerpunkte liegen im Bereich der Organisation und Steuerung der (sozial)staatlichen Leistungserbringung vor dem Hintergrund von politischem, sozialem und technischem Wandel.

E-Mail: tanja.klenk@hsu-hh.de

Prof. Dr. Matthias Knuth war Leiter der Forschungsabteilung Arbeitsmarkt Integration - Mobilität im Institut Arbeit und Qualifikation der Universität Duisburg-Essen und ist dem Institut mit Eintritt in den Ruhestand als Research Fellow verbunden geblieben. Seine Arbeitsschwerpunkte sind Arbeitsmarktmobilität, Arbeitsmarktpolitik, international vergleichende Sozialpolitik, Erwerbstätigkeit von Älteren und von Personen mit Migrationshintergrund. Von 2011 bis 2017 war er Vorsitzender der Deutschen Vereinigung für Sozialwissenschaftliche Arbeitsmarktforschung (SAMF). 
Prof. Tom Krebs, Ph. D., ist Professor für Makroökonomik und Wirtschaftspolitik an der Universität Mannheim. Er arbeitet zu den Auswirkungen von Wirtschaftskrisen und Reformen auf Wachstum, Ungleichheit und die Lebensqualität.

E-Mail: tkrebs@uni-mannheim.de

Prof. Dr. Matthias Meißner ist Professor im Fachgebiet Rechtswissenschaften des Fachbereichs Sozial- und Kulturwissenschaften der Hochschule Düsseldorf. Er leitet die Forschungsstelle DIFA (Düsseldorfer Integrationsförderung in [Aus] Bildung und Arbeit) und ist Mitglied des Instituts für lebenswerte und umweltgerechte Stadtentwicklung (IN-LUST).

Dr. Claus Schäfer hat von 1972 bis 2013 am Wirtschafts- und Sozialwissenschaftlichen Institut (WSI) der Hans-Böckler-Stiftung geforscht mit den Schwerpunkten Öffentliche Finanzen - und vor allem Verteilung; dazu hat er insbesondere den jährlichen WSI-Verteilungsbericht begründet. Von 2009 bis 2013 war er Leiter der Abteilung WSI in der Hans-Böckler-Stiftung.

Dr. Dorothee Spannagel ist Referatsleiterin für Verteilungsanalyse und Verteilungspolitik am Wirtschafts- und Sozialwissenschaftlichen Institut (WSI) der Hans-Böckler-Stiftung. Ihre Arbeitsschwerpunkte liegen auf dem Gebiet der sozialen Ungleichheit und der Verteilung von Armut und Reichtum.

E-Mail: dorothee-spannagel@boeckler.de 


\section{Soziologie}
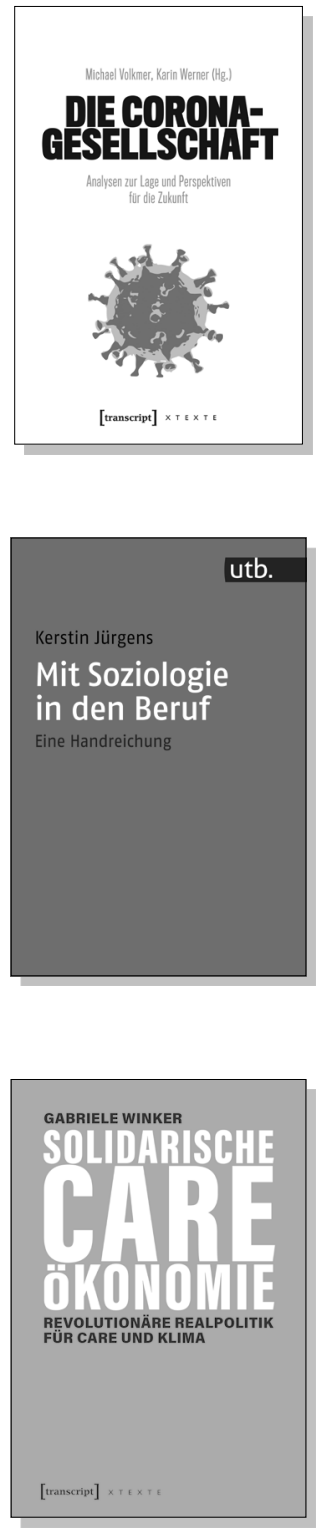

Michael Volkmer, Karin Werner (Hg.)

Die Corona-Gesellschaft

Analysen zur Lage und Perspektiven für die Zukunft

2020, 432 S., kart., Dispersionsbindung, 2 SW-Abbildungen

$24,50 €(D E), 978-3-8376-5432-5$

E-Book:

PDF: 21,99 € (DE), ISBN 978-3-8394-5432-9

EPUB: $21,99 €$ (DE), ISBN 978-3-7328-5432-5

Kerstin Jürgens

Mit Soziologie in den Beruf

Eine Handreichung

September 2021, 160 S., kart., Dispersionsbindung $18,00 €(D E), 978-3-8376-5934-4$

E-Book:

PDF: $15,99 €$ (DE), ISBN 978-3-8394-5934-8

Gabriele Winker

\section{Solidarische Care-Ökonomie}

Revolutionäre Realpolitik für Care und Klima

März 2021, 216 S., kart.

$15,00 €(D E), 978-3-8376-5463-9$

E-Book:

PDF: 12,99€ (DE), ISBN 978-3-8394-5463-3 


\section{Soziologie}

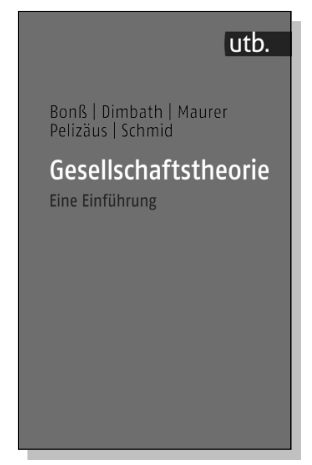

Wolfgang Bonß, Oliver Dimbath,

Andrea Maurer, Helga Pelizäus, Michael Schmid

\section{Gesellschaftstheorie}

Eine Einführung

Januar 2021, 344 S., kart.

25,00€ (DE), 978-3-8376-4028-1

E-Book:

PDF: $21,99 €$ (DE), ISBN 978-3-8394-4028-5

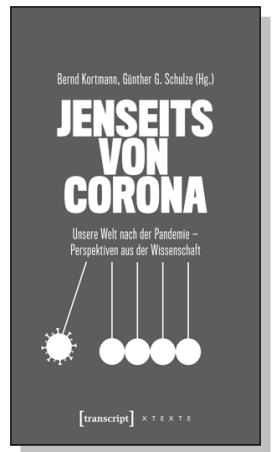

Bernd Kortmann, Günther G. Schulze (Hg.)

\section{Jenseits von Corona}

Unsere Welt nach der Pandemie -

Perspektiven aus der Wissenschaft

2020, 320 S.,

Klappbroschur, Dispersionsbindung, 1 SW-Abbildung

22,50€ (DE), 978-3-8376-5517-9

E-Book:

PDF: $19,99 €$ (DE), ISBN 978-3-8394-5517-3

EPUB: $19,99 €(D E)$, ISBN 978-3-7328-5517-9

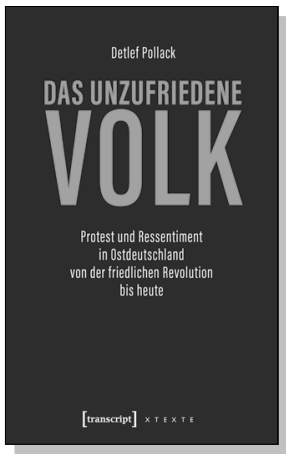

Detlef Pollack

\section{Das unzufriedene Volk}

Protest und Ressentiment in 0stdeutschland von der friedlichen Revolution bis heute

2020, 232 S.,

Klappbroschur, Dispersionsbindung, 6 SW-Abbildungen 20,00€ (DE), 978-3-8376-5238-3

E-Book:

PDF: $17,99 €$ (DE), ISBN 978-3-8394-5238-7

EPUB: $17,99 €(D E)$, ISBN 978-3-7328-5238-3 
